Rico Defila

20
n
11
20
2

Antonietta Di Giulio Hrsg.

Transdisziplinär und

transformativ forschen,

Band 2

Eine Methodensammlung 
Transdisziplinär und transformativ forschen, Band 2 
Rico Defila · Antonietta Di Giulio (Hrsg.)

\section{Transdisziplinär und transformativ forschen, Band 2}

Eine Methodensammlung

Mit einem Geleitwort von Prof. Dr. Ortwin Renn 
Herausgeber(in)

Rico Defila

Universität Basel

Basel, Schweiz
Antonietta Di Giulio

Universität Basel

Basel, Schweiz

Gefördert vom Ministerium für Wissenschaft, Forschung und Kunst Baden-Württemberg

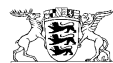

Baden-Württemberg

MINISTERIUM FÜR WISSENSCHAFT, FORSCHUNG UND KUNST
WISSENSCHAFT FÜR
NACHHALTIGKEIT

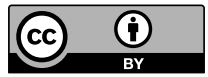

ISBN 978-3-658-27134-3

https://doi.org/10.1007/978-3-658-27135-0

Die Deutsche Nationalbibliothek verzeichnet diese Publikation in der Deutschen Nationalbibliografie; detaillierte bibliografische Daten sind im Internet über http://dnb.d-nb.de abrufbar.

\section{Springer VS}

(C) Der/die Herausgeber bzw. der/die Autor(en) 2019. Dieses Buch ist eine Open-Access-Publikation. Open Access Dieses Buch wird unter der Creative Commons Namensnennung 4.0 International Lizenz (http://creativecommons.org/licenses/by/4.0/deed.de) veröffentlicht, welche die Nutzung, Vervielfältigung, Bearbeitung, Verbreitung und Wiedergabe in jeglichem Medium und Format erlaubt, sofern Sie den/die ursprünglichen Autor(en) und die Quelle ordnungsgemäß nennen, einen Link zur Creative Commons Lizenz beifügen und angeben, ob Änderungen vorgenommen wurden.

Die in diesem Buch enthaltenen Bilder und sonstiges Drittmaterial unterliegen ebenfalls der genannten Creative Commons Lizenz, sofern sich aus der Abbildungslegende nichts anderes ergibt. Sofern das betreffende Material nicht unter der genannten Creative Commons Lizenz steht und die betreffende Handlung nicht nach gesetzlichen Vorschriften erlaubt ist, ist für die oben aufgeführten Weiterverwendungen des Materials die Einwilligung des jeweiligen Rechteinhabers einzuholen.

Die Wiedergabe von allgemein beschreibenden Bezeichnungen, Marken, Unternehmensnamen etc. in diesem Werk bedeutet nicht, dass diese frei durch jedermann benutzt werden dürfen. Die Berechtigung zur Benutzung unterliegt, auch ohne gesonderten Hinweis hierzu, den Regeln des Markenrechts. Die Rechte des jeweiligen Zeicheninhabers sind zu beachten.

Der Verlag, die Autoren und die Herausgeber gehen davon aus, dass die Angaben und Informationen in diesem Werk zum Zeitpunkt der Veröffentlichung vollständig und korrekt sind. Weder der Verlag, noch die Autoren oder die Herausgeber übernehmen, ausdrücklich oder implizit, Gewähr für den Inhalt des Werkes, etwaige Fehler oder Äußerungen. Der Verlag bleibt im Hinblick auf geografische Zuordnungen und Gebietsbezeichnungen in veröffentlichten Karten und Institutionsadressen neutral.

Springer VS ist ein Imprint der eingetragenen Gesellschaft Springer Fachmedien Wiesbaden GmbH und ist ein Teil von Springer Nature

Die Anschrift der Gesellschaft ist: Abraham-Lincoln-Str. 46, 65189 Wiesbaden, Germany 


\section{Geleitwort}

Transdisziplinäre Forschung ist zu einem Schlüsselwort in der wissenschaftlichen Diskussion um Nachhaltigkeit und gesellschaftliche Transformation geworden. Strategische Optionen für Politik zu entwickeln und Empfehlungen für die jeweils relevanten Akteure aus wissenschaftlicher Sicht zu artikulieren oder vorzubereiten, reicht für eine auf gesellschaftliche Wirksamkeit abzielende Forschung nicht aus. Natürlich gibt es bei vielen Sachfragen keinen wissenschaftlichen Konsens darüber, was gesellschaftlich wünschenswert wäre, und selbstverständlich ist Wissenschaft nicht per se legitimiert, der gesellschaftlichen Entwicklung die Richtung vorzugeben. ${ }^{1}$ Es existieren aber auch Sachfragen, zu denen - wie in der Klimafrage - seit Jahrzehnten ein gewisser wissenschaftlicher Konsens besteht, dem auch starke Kräfte in der Gesellschaft entsprechen, bei denen jedoch die Brücke zum Handeln nicht geschlagen wird, weil die Prozesse zur Transformation nicht effektiv sind oder durch gesellschaftspolitische Konflikte gelähmt werden. ${ }^{2}$ Angesichts dieser Situation ist gesellschaftlich wirksame Wissenschaft auf einen Prozess der Co-Kreation von Wissen mit Akteuren aus anderen gesellschaftlichen Bereichen angewiesen. Transdisziplinäre Forschung ist auf eine gemeinsam getragene, auf Integration ausgerichtete Zusammenführung unterschiedlicher Wissensformen, die für die Analyse eines Sachverhaltes und/oder zur Problemlösung benötigt werden, ausgerichtet. ${ }^{3,4}$

1 Defila, R., \& Di Giulio, A. (2019). Eine Reflexion über Legitimation, Partizipation und Intervention im Kontext transdisziplinärer Forschung. In M. Ukowitz \& R. Hübner (Hrsg.), Interventionsforschung. Band 3: Wege der Vermittlung. Intervention - Partizipation (S. 85-108). Wiesbaden: Springer VS. doi: 10.1007/978-3-658-22048-8_4.

2 Nanz, P., Renn, O., \& Lawrence, M. (2017). Der transdisziplinäre Ansatz des Institute for Advanced Sustainability Studies (IASS): Konzept und Umsetzung. GAIA, 26 (3), (S. 293-296). doi: 10.14512/gaia.26.3.19.

3 Lang, D. J., Wiek, A., Bergmann, M., Stauffacher, M., Martens, P., Moll, P., Swilling, M., \& Thomas, C. J. (2012). Transdisciplinary research in sustainability science: practice, principles, and challenges. Sustainability Science, 7 (Supplement 1), (S. 25-43). doi: 10.1007/s11625-011-0149-x.

4 Defila, R., \& Di Giulio, A. (1996). Interdisziplinäre Forschungsprozesse In R. Kaufmann-Hayoz \& A. Di Giulio (Hrsg.), Umweltproblem Mensch. Humanwissenschaftliche Zugänge zu umweltverantwortlichem Handeln (S. 79-129). Bern, Stuttgart, Wien: Haupt. https://bit.ly/2Zde3VZ. Zugegriffen am 20.05.2019. 
Im Begriff der Transdisziplinarität sind drei wesentliche Aspekte vereint ${ }^{5}$ : Zum Ersten passt solche Forschung über den Geltungsbereich der beteiligten Disziplinen hinaus Forschungsgegenstände, Methoden und Vorgehensweisen an außerwissenschaftliche Problemlagen und Fragestellungen an und entwickelt interdisziplinär Lösungen für gesellschaftlich komplexe Probleme. ${ }^{6,7}$ Zum Zweiten beruht solche Forschung auf einem intensiven Austausch zwischen denen, die Wissen produzieren, und denen, die dieses Wissen für politische Entscheidungen nutzen und konkret anwenden wollen, und zwar über alle Phasen des Forschungsprozesses hinweg. ${ }^{8}$ Letztere lernen so nicht nur die Ergebnisse der Forschung kennen, sondern auch die Kontextbedingungen und die Geltungsbereiche, ohne deren Kenntnis eine sachgerechte Interpretation der Ergebnisse nicht möglich ist. Zum Dritten bindet solche Forschung bewusst Wissensträger außerhalb der Wissenschaft ein. ${ }^{9}$ So findet auch Erfahrungswissen und Kontextwissen der mit der entsprechenden Frage beschäftigten Gruppierungen in der Gesellschaft Eingang in die Forschung, was gerade bei komplexen Fragen relevant ist, um nicht nur theoretisch schlüssige, sondern auch praktisch umsetzbare Lösungsvorschläge zu entwickeln. Transformative Forschung geht über konventionelle Forschung hinaus, indem sie direkt transformativ tätig wird. Dies erhöht das Legitimationsproblem, es erhöht aber auch das Wirkungspotenzial - in diesem Spannungsfeld muss sich transformative Wissenschaft bewegen.

Wie man solche transdisziplinären oder sogar transformativen Verständigungsprozesse zwischen Forschenden und Praxisakteuren konkret organisieren kann und soll, ist noch nicht geklärt. ${ }^{8,}{ }^{10}$ Wesentliche Kennzeichen solcher Prozesse sind die frühzeitige Einbindung aller relevanter Wissensträger, der forschungsbegleitende Diskurs mit denen, die das Wissen nutzen, sowie der Einsatz von kommunikativen Verfahren, die einen intensiven Austausch von Argumenten, Beobachtungen und Erfahrungen ermöglichen.

5 In Anlehnung an: Renn, O. (2019). Die Rolle(n) transdisziplinärer Wissenschaft bei konfliktgeladenen Transformationsprozessen. GAIA, 28 (1), (S. 44-51). doi: 10.145 12/gaia.28.1.11.

6 Mittelstraß, J. (1992). Auf dem Wege zur Transdisziplinarität. GAIA, 1 (5), (S. 250).

7 Mittelstraß, J. (2018). Forschung und Gesellschaft. Von theoretischer und praktischer Transdisziplinarität. GAIA, 27 (2), (S. 201-204). doi: 10.14512/gaia.27.2.4.

8 Krohn, W., Grunwald, A., \& Ukowitz, M. (2017). Transdisziplinäre Forschung revisited. Erkenntnisinteresse, Forschungsgegenstände, Wissensform und Methodologie. GAIA, 26 (4), (S. 341-347). doi: 10.14512/gaia.26.4.11.

9 Jahn, T. (2008). Transdisziplinarität in der Forschungspraxis. In M. Bergmann \& E. Schramm (Hrsg.), Transdisziplinäre Forschung. Integrative Forschungsprozesse verstehen und bewerten (S. 21-37). Frankfurt a. M., New York: Campus.

10 von Wissel, C. (2015). Die Eigenlogik der Wissenschaft neu verhandeln. Implikationen einer transformativen Wissenschaft. GAIA, 24 (3), (S. 152-155). doi: 10.14512/ gaia.24.3.4. 
In der Literatur zu transdisziplinärer Forschung findet sich (bislang) kaum eine Systematik zu den unterschiedlichen Ansätzen, Methoden und Vorgehensweisen, wie transdisziplinäre Forschung theoretisch gehaltvoll und methodisch valide praktisch umgesetzt werden kann. Mit den beiden Bänden zur Methodik der Transdisziplinarität wird ein Beitrag zur Schließung dieser Lücke geleistet. Schon mit dem ersten Band der Methodensammlung haben die beiden Herausgebenden Rico Defila und Antonietta Di Giulio neben eher konzeptionellen und Erfahrungen integrierenden Artikeln auch eine Fülle von methodischen Vorgehensweisen gesammelt, die eine Vielfalt transdisziplinären Arbeitens und transformativer Experimentierfelder aus Reallaboren verkörpern. In dem jetzt vorliegenden zweiten Band geht es bei den konzeptionellen Texten vor allem um die Zusammenarbeit mit kommunalen Akteuren auf lokaler Ebene.

Wie beim ersten Band werden im Teil 1 gemeinsame Fragen und Praktiken sowie geteilte Erfahrungen reflektiert, während die Artikel im Teil 2 Vorgehensweisen in Reallaboren widerspiegeln. Dabei zeigt sich, dass die praktische Arbeit von Reallaboren unter kreativer Mitwirkung der verschiedenen Akteure inzwischen einen hohen Stand der Ausdifferenzierung und des methodischen Kenntnisstandes erreicht hat. Gleichzeitig zeigen die Beiträge in Teil 1 auch, wo Grenzen und Schwächen des Forschungsformats Reallabor zu verorten sind. Auch ein dezidiert transdisziplinär und transformativ angelegtes Forschungsdesign wird bei vielen der berühmten „wicked problems“ nicht viel mehr als eine gemeinsam getragene Problemdefinition erreichen können. Aber auch das ist schon ein wichtiger Schritt in der gemeinsamen Aufarbeitung der Gegenwart im Hinblick auf eine nachhaltige Entwicklung der Gesellschaft.

Festzuhalten gilt, dass es nicht ausreicht, Stakeholder um einen runden Tisch zu versammeln und darauf zu hoffen, dass sich allein aus der Tatsache des gemeinsamen Gespräches ein Mehrwert ergeben würde. Es bedarf eines strukturierten und vor allem reflektierten Prozessvollzugs, der auf eigenem Prozesswissen über die Gelingensbedingungen von transdisziplinären Ansätzen beruht. Dies muss theoretisch fundiert, empirisch geprüft und methodisch reproduzierbar sein. Wie das gelingen kann, zeigen die Beiträge dieser Methodensammlung exemplarisch auf.

Prof. Dr. Dr. sc. tech. h. c. Ortwin Renn Institut für Transformative Nachhaltigkeitsforschung Potsdam (IASS) und Universität Stuttgart 


\section{Inhaltsverzeichnis}

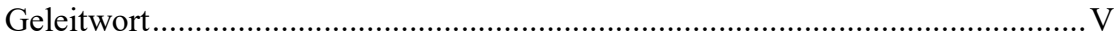

Wie Reallabore für Herausforderungen und Expertise in der Gestaltung transdisziplinären und transformativen Forschens sensibilisieren - eine Einführung...... 1

Rico Defila \& Antonietta Di Giulio

\section{Teil 1 Worauf achten: Heuristiken zum Verstehen der Akteure und ihrer Handlungsbedingungen}

Kommunale Akteure verstehen: Vorgeschichte der Arbeit in Kommunen beachten.

Jochen Eckart, Elke Häußler, Thorsten Erl, Monika Gonser \& Jan Riel

Unterschiedliche Handlungslogiken in transdisziplinären und transformativen Forschungsprojekten - Welche Risikokulturen entwickeln sich daraus und wie lassen sie sich konstruktiv einbinden?

Monika Gonser, Jochen Eckart, Charlotte Eller, Katharina Köglberger, Elke Häußler \& Felix M. Piontek

Kommunale Akteure verstehen: Komplexe Entscheidungswege in Kommunen beachten.

Monika Gonser, Jan Riel, Jochen Eckart, Thorsten Erl \& Elke Häußler

Schutz in der Exposition, Schutz für die Exposition - Wie man in transdisziplinären und transformativen Forschungsformaten mit Ungewohntem und erhöhter Aufmerksamkeit umgeht.

Katharina Köglberger, Raphael Dietz, Charlotte Eller, Felix M. Piontek, Marius Albiez \& Thomas Potthast 
Kommunale Akteure verstehen: Verteilte Wissensbestände in

Kommunen beachten.

Thorsten Erl, Monika Gonser, Jochen Eckart, Elke Häußler \& Jan Riel

\section{Teil 2 Wie vorgehen: Methoden für Co-Design und Co-Produktion}

Die „Exchange-Veranstaltungsreihe“ - ein Planungs- und

Steuerungsinstrument für transdisziplinäre Forschungsprojekte.

Christina Benighaus, Belen Zevallos, Charlotte Eller, Martina Hilligardt, Mandana Alimardani \& Ludger Benighaus

Die „TraSy-Methode“ - ein Vorgehen für die transdisziplinäre Entwicklung soziotechnischer Systeme.

Laura Gebhardt \& Alexandra König

„Barcamp adapted“ - gemeinsam zu neuem Wissen

Editha Marquardt \& Ulrike Gerhard

„UrbanUtopiaLAB“ - einen Möglichkeitsraum zur Produktion von

Transformationswissen schaffen

Christina West \& Svenja Kück

Das „Transformative Projektseminar“ - didaktische Ansätze und methodische Umsetzung 293

Richard Beecroft

\section{Anhang}

Steckbriefe der BaWü-Labs Asylsuchende, R131, Schorndorf, SRB, Urban Office.

Autorinnen und Autoren

Externe Reviewerinnen und Reviewer 


\title{
Wie Reallabore für Herausforderungen und Expertise in der Gestaltung transdisziplinären und transformativen Forschens sensibilisieren - eine Einführung
}

\author{
Rico Defila \& Antonietta Di Giulio
}

\section{Worum es geht - eine Fortsetzungsgeschichte}

Das vorliegende Buch ist eine Fortsetzung des Buches Transdisziplinär und transformativ forschen - Eine Methodensammlung (Defila und Di Giulio 2018c), die teils dieselben, teils andere Akzente setzt, die damals geführte Diskussionen teils weiterschreibt, teils ergänzt. Entsprechend gilt vieles von dem, was in der Einführung zum ersten Band steht (Defila und Di Giulio 2018b), auch für den nun vorliegenden zweiten Band. So gesehen enthält die Einführung in das zweite Buch auch eine Bilanz dessen, was sich verändert hat, was gleichgeblieben ist, und welche Gesichtspunkte neu hinzukommen.

Gleich geblieben ist der Hintergrund des Buches, die Forschung in Reallaboren, in einem Forschungsformat also, in dem transdisziplinär geforscht wird und gleichzeitig ein expliziter transformativer Anspruch verfolgt wird. Auch ein gutes Jahr nach dem ersten Buch gilt, dass eine solche Forschung innerhalb der Wissenschaft nicht vorbehaltlose Anerkennung findet, sondern auf eine gewisse $\mathrm{Zu}$ rückhaltung, wenn nicht sogar Skepsis stößt - ungeachtet dessen, dass sich dieses Forschungsformat mittlerweile einer gewissen Beliebtheit auch bei Fördergebern wie dem Deutschen Bundesministerium für Bildung und Forschung (BMBF) erfreut, wie sich beispielsweise an neueren Ausschreibungen zeigt, wie etwa der Ausschreibung von Projekten zum Thema „MobilitätsWerkStadt 2025“, die im Februar 2019 veröffentlicht wurde (s. dazu auch die Dokumentation „Förderung von Reallaboren und Citizen Science in Deutschland" der Wissenschaftlichen Dienste des Deutschen Bundestags vom März 2018, WD 2018). Nach wie vor gilt es deshalb, die Standards zur Beurteilung guter Forschung so weiter zu entwickeln, dass sie sowohl dazu dienlich sind, die Glaubwürdigkeit wissenschaftlicher Ergebnisse solcher Forschung zu beurteilen, als auch, den Besonderheiten 
dieser Forschung Rechnung zu tragen (s. Defila und Di Giulio 2018b), ein Bedarf, auf den auch Ortwin Renn in seinem Geleitwort zu diesem Buch hinweist.

Entsprechend möchte das vorliegende Buch, wie bereits das vorausgehende, einen Beitrag leisten zur Methodik transdisziplinärer, d. h. interdisziplinärer und partizipativer, Forschung, und zwar auch für solche, die nicht transformativ vorgeht - diese doppelte Ansprache ist im Folgenden durch Klammersetzung ausgedrückt: „transdisziplinär (und transformativ)“. Dieser Beitrag ist unverändert ein zweifacher:

- Erneut werden in Teil 2 des Buches ausgewählte Methoden der Wissenserzeugung, Wissensintegration und Transformation, die in Reallaboren entwickelt und umgesetzt wurden und die sich besonders bewährt haben, für die transdisziplinäre (und transformative) Forschung innerhalb und außerhalb von Reallaboren zur Verfügung gestellt. Beide Bände zusammengenommen sind es damit nun insgesamt elf Methoden, die in analoger Art und Weise aufbereitet wurden, wovon zwei auf Lehre fokussieren (s. Liste in Anlage a im Anhang dieses Beitrags).

- Einen anderen Akzent setzen hingegen die Beiträge in Teil 1 des Buches. Während 2018 Kriterien, Fragen und Prinzipien mit Blick auf die Gestaltung transdisziplinärer (und transformativer) Forschung vorgestellt wurden, richtet sich der Blick im vorliegenden Buch mehr auf die Akteure selbst und auf die Bedingungen ihres Handelns (s. Liste in Anlage a im Anhang dieses Beitrags). Das Ziel besteht dabei nicht darin, Ansatzpunkte zur Veränderung des Handelns zu benennen, sondern darin, das Handeln der Akteure zu verstehen sowie die Irritationen zu erkennen, die ihnen durch die Arbeit und Zusammenarbeit in solchen Projekten entstehen (können), positiven wie negativen. Entsprechend ist diesen Beiträgen ein reflexiver Zugang gemeinsam und dass sie Instrumente an die Hand geben, die eine auf einer vertieften Reflexion basierende Gestaltung der Zusammenarbeit in transdisziplinären (und transformativen) Projekten erleichtern.

Das Buch richtet sich in erster Linie an Personen, die transdisziplinär forschen (wollen) und dabei auch einen transformativen Anspruch verfolgen (wollen), und zwar innerhalb wie außerhalb des Formats Reallabor. Es richtet sich aber auch an Personen, die ohne einen solchen expliziten transformativen Anspruch transdisziplinär forschen (wollen). 


\section{Transdisziplinarität, Transformation und Reallabore - die begrifflichen Grundlagen}

Die begrifflichen Grundlagen haben sich gegenüber dem ersten Band nicht geändert, d. h., auch im vorliegenden Buch sind die Begriffe transdisziplinäre Forschung, transformative Forschung und Reallabore zentral. Und die Frage, ob und inwieweit das Forschungsformat Reallabor an das Ziel einer Nachhaltigen Entwicklung gekoppelt ist, hat nicht an Aktualität verloren. Deshalb werden zentrale Aussagen aus dem ersten Band an dieser Stelle wiederholt (die nachstehenden wörtlichen Zitate sind allesamt aus Defila und Di Giulio 2018b, S. 10-12):

„Transdisziplinäre Forschung wird hier akteurorientiert verstanden als Variante einer auf eine Synthese ausgerichteten interdisziplinären Forschung (s. auch Defila und Di Giulio 2018a). Bei dieser Variante beteiligen sich nicht nur Forscher(innen) aus verschiedenen wissenschaftlichen Disziplinen am Forschungsprozess, sondern zusätzlich auch Praxisakteure. Praxisakteure sind dabei substantiell am Projekt beteiligt, sei es als gleichberechtigte Mitglieder eines Projektteams oder als externe Beteiligte, die punktuell am Projekt mitwirken. Wenn Praxisakteure lediglich Untersuchungsgegenstand, Zielpublikum oder ,Echoraum' der Forschung sind, handelt es sich gemäß diesem Verständnis nicht um eine transdisziplinäre Zusammenarbeit (zum Diskurs s. z. B. auch Burton et al. 2008; Defila und Di Giulio 1998; Klein 2014; Mobjörk 2010).“ Mit dem Begriff der transformativen Forschung wiederum wird eine Forschung bezeichnet, ,die gesellschaftliche Veränderungen nicht nur untersucht und entsprechendes System-, Ziel- und Transformationswissen zur Verfügung stellt (CASS und ProClim- 1997), sondern auch gezielt auf eine gesellschaftliche Transformation“ hinwirkt und ,in diesem Sinne transformativ tätig wird (WBGU 2011 und in der Folge z. B. Schneidewind und Singer-Brodowski 2014; s. aber z. B. auch Bäckstrand 2003; Mielke et al. 2016; Otero et al. 2017; Scholz 2017).“ Das Forschungsformat Reallabor schließlich „stellt einen Versuch dar, transformative Forschung in ein spezifisches Forschungsformat zu kleiden (für einen Überblick u. a. auch über die internationale Landschaft s. z. B. TATuP-Schwerpunkt 2016; Schäpke et al. 2017; BGL-Themenheft 2017; GAIA Special Issue 2018).“ Reallabore verfolgen im idealen Fall eine dreifache Zielsetzung (s. Beecroft et al. 2018): Sie produzieren Erkenntnisse und neues Wissen (Forschungsziele), sie stoßen Transformationsprozesse an (Praxisziele), und sie unterstützen individuelle und kollektive Lernprozesse, die sie durch ihre Aktivitäten anregen (Bildungsziele). Um Transformationsprozesse anzustoßen und zu begleiten, bedienen sich Reallabore oft eines experimentellen Ansatzes (s. dazu z. B. Arnold und Piontek 2018). 
Obwohl transformative Forschung und das Forschungsformat Reallabor oft gleichgesetzt werden mit einer Forschung, die zu einer Nachhaltigen Entwicklung beiträgt (so z. B. auch in Rose et al. 2018), gibt es dafür keinen zwingenden im Forschungsformat angelegten Grund. Transformative Forschung und das Format Reallabor können vielmehr auch andere Ziele als Nachhaltige Entwicklung anvisieren, das können jedoch keine beliebigen Ziele sein: „Erstens ist eine Forschung, die aktiv und gezielt eine gesellschaftliche Veränderung anstoßen will, in besonderer Weise begründungs- und rechenschaftspflichtig, was die Ziele einer solchen Transformation betrifft. Zweitens ist Forschung, soweit sie aus öffentlichen Mitteln finanziert wird, dieser Öffentlichkeit gegenüber verpflichtet.“ An ein Ziel, auf das sich transformative Forschung ausrichtet, sollten deshalb drei Kriterien angelegt werden: (1) Das Ziel muss in ,gesellschaftspolitischer Hinsicht legitimiert" sein. (2) Für das Ziel müssen sich ,überzeugende ethische Argumente vorbringen lassen“. (3) Das Ziel muss gemeinwohlorientiert sein, d. h., es darf nicht ,auf einzelne gesellschaftliche Gruppen ausgerichtet“ sein. Transformative Forschung im Allgemeinen und das Format Reallabor im Speziellen werden hier also verstanden als eine Forschung, die gesellschaftlich legitimierte, ethisch gut begründete und gemeinwohlorientierte Ziele verfolgt. ${ }^{1}$

Damit richtet sich das vorliegende Buch, wie bereits das Buch von 2018, auch an Personen, die zwar transformativ forschen (möchten), dies aber nicht mit Blick auf eine Nachhaltige Entwicklung tun, sondern mit Blick auf ein anderes gesellschaftliches Ziel.

\section{Wie dieses Buch entstand - Förderkontext und Entstehungsprozess}

Das Ministerium für Wissenschaft, Forschung und Kunst Baden-Württemberg (MWK) förderte in den Jahren 2015 bis 2019 in zwei zeitlich leicht versetzten Förderlinien, den Förderlinien „Reallabore“ und „Reallabore Stadt", insgesamt 14 Reallabore (diese werden im Folgenden als „BaWü-Labs“ bezeichnet; s. auch die Liste in Anlage b im Anhang dieses Beitrags). ${ }^{2}$ Zusätzlich zu den 14 BaWü-Labs wurden zwei Begleitforschungs-Projekte gefördert (s. Schäpke et al. 2016; Defila

1 Was jedoch nicht davon entbindet, die Frage nach der Legitimität von Forschenden, transformativ tätig zu werden, kritisch zu reflektieren; s. dazu Defila und Di Giulio 2019 sowie Eckart et al. 2018, aber auch die seit 2014 in der Zeitschrift GAIA geführte Diskussion zu den Themen ,Transformative Wissenschaft“ und ,Theorie transdisziplinärer Forschung“.

2 Für Informationen zu den Förderlinien „Reallabore“ und „Reallabore Stadt“ sowie eine Kurzbeschreibung aller geförderten BaWü-Labs siehe http://www.reallabore-bw.de (zugegriffen am 20.05.2019). 
und Di Giulio 2018d). Für eines der beiden Begleitforschungs-Projekte zeichnen die Autorin und der Autor dieses Beitrags verantwortlich (im Folgenden als „BFBasel" bezeichnet). Das vorliegende Buch ist, wie bereits das vorausgehende Buch, ein Ergebnis, das im Rahmen der Aktivitäten der BF-Basel entstand (s. zu diesen Aktivitäten auch Defila und Di Giulio 2018d).

Der im Jahr 2018 erschienene erste Band der Methodensammlung war maßgeblich durch die BaWü-Labs der ersten Förderlinie geprägt, während das vorliegende Buch maßgeblich durch die BaWü-Labs der zweiten Förderlinie geprägt ist. Weder der Kreis der Beteiligten noch die Erfahrungen, die in die beiden Bände flossen, sind aber disjunkt. Vielmehr beteiligten sich am ersten Band auch Personen der zweiten Förderlinie und am vorliegenden zweiten Band auch solche der ersten Förderlinie, sei es als Autor(inn)en, sei es, indem sie Illustrations-Beispiele aus ihren BaWü-Labs für die Beiträge in Teil 1 des Buches beisteuerten. ${ }^{3}$ Auch in dieser Hinsicht handelt es sich bei diesen beiden Büchern also um eine Fortsetzungsgeschichte mit teils gleichen, teils anderen Akzenten.

Die BF-Basel richtete in regelmäßigen Abständen (ca. alle 9 Monate) zweitägige Workshops aus, die darauf abzielten, den BaWü-Labs einen Raum und eine methodische Struktur für die gemeinsame Reflexion, die wechselseitige Beratung, das gegenseitige Lernen und die syntheseorientierte Zusammenarbeit zur Verfügung zu stellen (s. Abb. 1). Die BF-Basel konzipierte den methodischen Aufbau dieser Workshops, moderierte sie und sorgte für die Ergebnissicherung, während die Wahl der Themen für die einzelnen Workshops in Abstimmung von BaWüLabs und BF-Basel erfolgte. Das übergeordnete Format für diese Workshops war das „Diskussionsforum“, d. h., die Workshops waren „Treffen des Diskussionsforums“, wobei es ein Diskussionsforum für die BaWü-Labs der ersten Förderlinie („Reallabore“) und eines für die BaWü-Labs der zweiten Förderlinie („,Reallabore Stadt") gab. Im Rahmen des Diskussionsforums wurden Themen und Fragen erörtert, die alle BaWü-Labs der jeweiligen Förderlinie gleichermaßen betrafen, es wurden Erfahrungen ausgetauscht und verglichen, und auf der Grundlage von Gemeinsamkeiten und Unterschieden wurden Schlüsse für die Gestaltung der Arbeit und Zusammenarbeit in einem Reallabor gezogen, die über das individuelle BaWü-Lab hinausgingen. Im Zuge dieser Diskussionen schälten sich auch die Synthese-Themen heraus, die in Beiträge im ersten Teil des vorliegenden Buches mündeten, d. h. auch beim vorliegenden Buch war es so, dass an dessen Entstehung auch Personen mitwirkten, die keinen Beitrag im Buch

3 Die Autor(inn)en sind im Verzeichnis der Autorinnen und Autoren aufgelistet, die Liste der Personen, die Beispiele aus ihrem BaWü-Lab beigesteuert haben, sind jeweils im Dank am Ende der Beiträge aufgeführt (sofern sie nicht zum Autor(inn)enTeam gehören). 


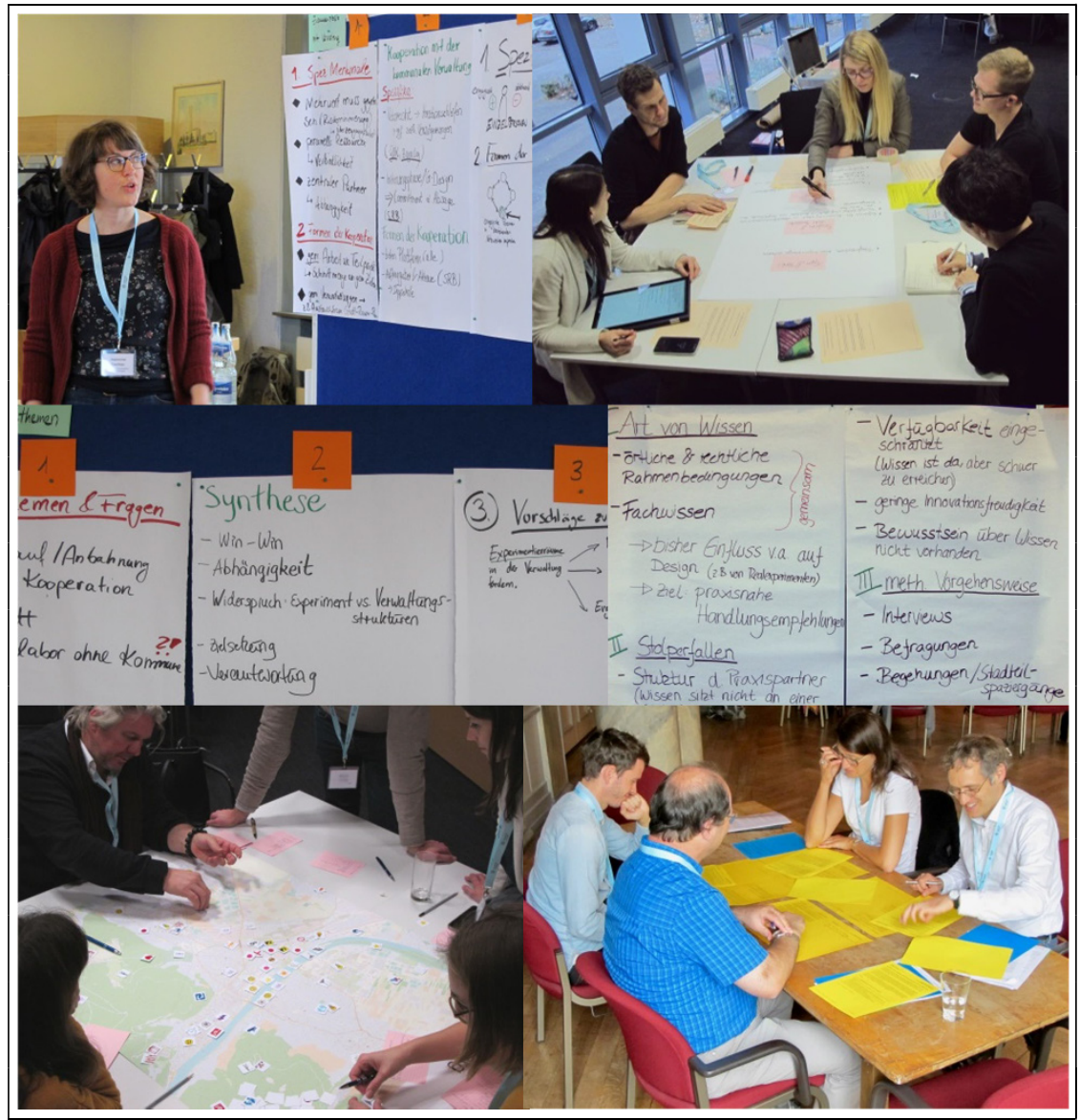

Abbildung 1: Die Treffen des Diskussionsforums waren Orte der moderierten Reflexion und Diskussion. Sie erfüllten vielfältige Funktionen, insbesondere Erfahrungsaustausch, individuelles und gemeinsames Lernen, wechselseitige Beratung und Produktion integrierten Wissens. (C) BF-Team Basel.

(mit-)verfassten. ${ }^{4}$ Das im Rahmen des Diskussionsforums aufgebaute gegenseitige Vertrauen ermöglichte es, wie bereits beim ersten Band, sämtliche Beiträge in Teil 1 und in Teil 2 des Buches nicht nur einem externen, sondern auch einem internen Review zu unterziehen, was sowohl die Qualität der Beiträge erhöhte als auch das gegenseitige und gemeinsame Lernen zusätzlich unterstützte.

4 Siehe die Liste der Personen im Dank am Ende dieses Beitrags. 


\section{Nachdenken über Bedingungen des Forschens und der Akteure - zwei sich ergänzende Perspektiven auf die Methodik transdisziplinärer (und transformativer) Forschung}

Aus den Diskussionen, die rund um den 2018 erschienenen Band geführt worden waren, schälten wir, d.h. die Autorin und der Autor des vorliegenden Beitrags, Fragen heraus, die unserer Wahrnehmung nach über den Kreis der Personen, die sich daran abarbeiteten, hinaus von Interesse sein dürften - und zwar unabhängig davon, ob diese im Rahmen eines Reallabors forschen (wollen) oder im Rahmen eines anderen transdisziplinären (und transformativen) Forschungsformats. Diese Fragen waren:

1) Wer sind die an transdisziplinärer (und transformativer) Forschung partizipierenden Akteure?

2) Wie innovativ sind die in transdisziplinärer (und transformativer) Forschung eingesetzten Methoden?

3) Können die Forschungsziele und die Praxisziele bei transdisziplinärer (und transformativer) Forschung ausbalanciert werden?

Diese Fragen prägten auch viele der Diskussionen, die rund um das vorliegende Buch geführt wurden, d. h., sie waren weiterhin mindestens implizit präsent: Durch die teilweise Überschneidung der Autor(inn)en-Teams (und der ,BaWüLab-Beispiel-Lieferant(inn)en') in beiden Bänden, durch die geographische Nähe und teilweise personelle Verflochtenheit zwischen den BaWü-Labs beider Förderlinien sowie durch die zeitliche Überschneidung der beiden Diskussionsforen, die auch erlaubte, dass die BF-Basel als ,Botin' für Erfahrungen und Einsichten agieren konnte, diffundierten die Themen, die rund um den ersten Band verhandelt wurden, auch in die Diskussionen rund um das nun vorliegende Buch. Diese drei Fragen können deshalb hier erneut verwendet werden, um auch diese jüngeren Diskussionen zu verorten. Gleichzeitig wurden in diesen klar andere Akzente gesetzt, erstens dadurch bedingt, dass die Debatten durch andere Personen, BaWü-Labs und Erfahrungen geprägt waren, zweitens dadurch bedingt, dass mit dem Förderformat Reallabor nicht mehr gleichermaßen Neuland betreten wurde, wie dies bei der ersten Förderlinie der Fall gewesen war, und drittens dadurch bedingt, dass die BaWü-Labs der zweiten Förderlinie, im Unterschied zu denen der ersten Förderlinie, über das Forschungsformat und den thematischen Kontext der Nachhaltigkeit hinaus eine weitere Gemeinsamkeit aufwiesen: alle fokussierten, der Ausschreibung entsprechend, auf Kommunen und arbeiteten eng mit kommunalen Akteuren zusammen. Insgesamt verschob sich das Gewicht von allgemeinen grundlegenden Fragen rund um das Forschungs- und Förderformat 
zu spezifischeren, wenn auch nicht minder grundlegenden Fragen. Mit Blick auf die Methodik transdisziplinärer (und transformativer) Forschung fokussierten die Reflexionen in der jüngeren Diskussion auf die beteiligten Akteure und dabei insbesondere auf die Zusammenarbeit mit kommunalen Akteuren.

\section{Frage 1: Wer sind die an transdisziplinärer (und transformativer) Forschung partizipierenden Akteure?}

Bezogen auf diese Frage wurde im Diskussionsforum der ersten Förderlinie vor allem diskutiert, wie eigentlich die Akteure zu bezeichnen sind, die an transdisziplinärer (und transformativer) Forschung partizipieren, und welche Unterscheidungen hier sinnvoll sind. Dies beinhaltet immer ein Ringen um das angemessene Verhältnis zwischen Wissenschaft und Gesellschaft, zwischen Akteuren aus der Wissenschaft und Akteuren aus der Praxis. Die entsprechenden Debatten mündeten im Buch von 2018 u. a. in einen Beitrag zur Terminologie rund um das Reallaborformat (Arnold und Piontek 2018), in einen Vorschlag zur Erfassung der Akteurskonstellation in Reallaboren (Seebacher et al. 2018) und in eine Erörterung der Implikationen von Begriffen, anhand derer die an transdisziplinärer (und transformativer) Forschung beteiligten Akteure bezeichnet werden können (s. Defila und Di Giulio 2018b, S. 16ff.).

Die Diskussionen im Diskussionsforum der zweiten Förderlinie führten zu ergänzenden Antworten auf diese Frage, indem gefragt wurde, welche Perspektiven und Handlungsweisen prägend sind für verschiedene Akteure und daher in Reallaboren oder in anderen transdisziplinären (und transformativen) Projekten systematisch aufeinandertreffen. Ein Ergebnis, das aus diesen Diskussionen hervorgegangen ist, ist der Beitrag in diesem Buch, in dem die Frage der Perspektiven und Handlungsweisen anhand eines systematischen Zugangs reflektiert wird, der den Blick nicht auf die Individuen richtet, sondern diese als Angehörige gesellschaftlicher Teilsysteme behandelt (Gonser et al. 2019a). Ein besonderes Augenmerk im gemeinsamen Ringen galt kommunalen Akteuren. Kommunen sind oft wichtige Akteure in solchen Projekten, manchmal stärker eingebunden als Praxispartner im ,inneren Kreis“, manchmal in einer lockereren Form als Praxisakteure im ,äußeren Kreis“ beteiligt (Seebacher et al. 2018). Dieser Wichtigkeit steht ein oft unterkomplexes Verständnis dessen gegenüber, was diese Akteursgruppe in ihrem Handeln kennzeichnet. Dieser Kontrast führte zu vier Beiträgen in diesem Buch, in denen das Handeln kommunaler Akteure analysiert wird (Gonser et al. 2019a) bzw. in denen Hinweise gegeben werden dazu, wie sich dieses Handeln mit Blick auf die konkrete Zusammenarbeit in einem Projekt entschlüsseln lässt (Eckart et al. 2019; Erl et al. 2019; Gonser et al. 2019b). 


\section{Frage 2: Wie innovativ sind die in transdisziplinärer (und transformativer) Forschung eingesetzten Methoden?}

Bezogen auf diese Frage führten die Debatten in den Diskussionsforen beider Förderlinien zum Schluss, dass es nicht sinnvoll ist, den in transdisziplinärer (und transformativer) Forschung verwendeten Methoden pauschal zuzuschreiben, sie seien innovativ (oder dies pauschal einzufordern). Auch die Gleichsetzung von ,innovativ ${ }^{6}$ mit ,besser ${ }^{6}$ wurde in beiden Diskussionsforen als gefährlicher Trugschluss bezeichnet, dem man aber oft begegne, und zwar innerhalb wie außerhalb der Wissenschaft.

Im Diskussionsforum der ersten Förderlinie wurde auf die Methodenwahl fokussiert und darauf, inwiefern die Zuschreibung, innovativ' irreführend wäre und unerwünschte Effekte zur Folge hätte (s. Defila und Di Giulio 2018b, S. 22ff.). Die Schlussfolgerung bestand, kurz gesagt, darin, dass es angesichts der Bedeutung, die der Methodenfrage mit Blick auf die innerwissenschaftliche Akzeptanz von Forschung zukommt, dienlicher ist, sich mit Fragen zu befassen wie der, „wofür sich welche Methode unter welchen Bedingungen eignet, wie sichergestellt werden kann, dass bei der Umsetzung von Methoden die erforderliche Qualität der Prozesse und Ergebnisse erreicht wird, und wie Vorbehalten gegenüber den erzielten Ergebnissen in der Wissenschaft begegnet werden könnte“, und zwar vor dem Hintergrund, dass die ,sorgfältige Reflexion des methodischen Vorgehens [...], die auf Nachvollziehbarkeit ausgerichtete Darstellung des methodischen Vorgehens und die kritische Methodendiskussion“ im Forschungsalltag oft vernachlässigt werden (Defila und Di Giulio 2018b, S. 23f.).

Die Diskussion im Diskussionsforum der zweiten Förderlinie schlug hier eine andere Richtung ein insofern, als nicht die wissenschaftliche Qualität und Anerkennung den Ausgangspunkt der Erörterungen bildete, sondern dass es in einem Reallabor wesentlich darum geht, zu experimentieren. Dies wiederum führte zur Frage nach den Voraussetzungen, die gegeben sein müssen, damit dies möglich wird. Die an einem Reallabor beteiligten Akteure müssen, darüber bestand Einigkeit, bereit und in der Lage sein, ihre eigene Komfortzone zu verlassen, sich von ihren Denk- und Entscheidungsmechanismen zu lösen und Dinge zu denken und auszuprobieren, die sie so noch nicht gedacht und ausprobiert haben (unabhängig davon, ob diese neu und einzigartig sind oder nicht). Das Forschungssetting wiederum, also das Reallabor oder ein anderes transdisziplinäres und transformatives Projekt, muss den Akteuren einen Rahmen bieten, der es ihnen ermöglicht, Dinge auszuprobieren, die sie sich sonst nicht auszuprobieren wagen würden. Um dafür einen geeigneten Rahmen zu bieten, muss erstens berücksichtigt werden, dass es von den konkreten Akteuren abhängt, was für diese ungewohnt ist, dass es also nicht möglich ist, eine abschließende und für alle Akteure und Projekte geltende Liste des Ungewohnten zu erstellen. Zweitens muss bedacht werden, dass das Beschreiten ungewohnter Wege für die Akteure zwingend mit sich bringt, dass 
sie sich exponieren und dass dies negative Folgen für sie haben kann, und zwar über das Projekt hinaus. Dies führt für Reallabore oder analoge Projekte zur Notwendigkeit, Experimentierräume zu schaffen, die es sowohl allen beteiligten Akteuren erlauben, für sie Ungewohntes auszuprobieren, als auch allen Akteuren einen Schutz bieten, wenn sie sich mit dem Ungewohnten exponieren. Diese gemeinsamen Überlegungen mündeten in einen Beitrag in diesem Buch, in dem anhand paradigmatischer Erfahrungen aus den BaWü-Labs der Begriff der Exposition als Folie für die Reflexion der Arbeit und Zusammenarbeit verwendet wird (Köglberger et al. 2019).

\section{Frage 3: Können die Forschungsziele und die Praxisziele bei transdisziplinärer (und transformativer) Forschung ausbalanciert werden?}

Im Diskussionsforum der ersten Förderlinie wurde bezogen auf diese Frage vor allem die Herausforderung beleuchtet, die sich daraus ergibt, dass Realexperimente (oder analoge Interventionen), die zumeist ,nicht allein aus der Forschungslogik und aus Forschungsbedarfen heraus konzipiert" werden, sondern stark auch ,aus der Logik und den Bedarfen der ,realen Welt' heraus, in die sie eingebettet sind“, nicht in jedem Fall ,in Ausrichtung und Konzeption erlauben, Fragen zu erforschen, die zum wissenschaftlichen Fortschritt beitragen" (Defila und Di Giulio 2018b, S. 24f.).

Auch bezogen auf diese Frage richtete sich der Blick im Diskussionsforum der zweiten Förderlinie primär auf das Handeln der beteiligten Akteure. Damit kommt, ergänzend zur Ebene der Projektziele, die Ebene der Chancen und Risiken, die ein Projekt aus der Sicht der beteiligten Akteure bietet, ins Spiel. Es wurde erörtert, inwiefern in einem transdisziplinären (und transformativen) Projekt wie einem Reallabor unterschiedliche Risikowahrnehmungen aufeinandertreffen und inwiefern dies den Handlungs- und Experimentierraum in einem Projekt bedeutend vergrößern kann, bedeutend verkleinern kann bzw. zu Konflikten führen kann. Als Ursache für die negativen Effekte wurde nicht die Unterschiedlichkeit der Risikowahrnehmungen gesehen, sondern dass damit nicht reflektiert umgegangen bzw. dass diese oft sogar negiert wird. Diese Unterschiedlichkeit nicht zu reflektieren bzw. sogar zu negieren wiederum kann, so die Vermutung, zur Folge haben, dass alle Beteiligten je für sich darauf hinwirken, das, was sie je aus ihrer Logik heraus als Risiko wahrnehmen, zu minimieren, bzw. dass die Chancen und Risiken im Projekt unfair verteilt sind. Die Unterschiedlichkeit als Fakt zu akzeptieren und in einem Projekt bewusst damit umzugehen, wurde als Bedingung gesehen dafür, die verschiedenen Ziele in einem transdisziplinären (und transformativen) Projekt ausbalancieren zu können und damit auch einen differenzierteren Blick zu gewinnen auf das Gelingen und Misslingen solcher Projekte. Dieses Thema wurde vertieft und mündete in einen durch viele Erfahrungen aus den BaWü-Labs unterfütterten Beitrag in diesem Buch (Gonser et al. 2019a). 
In der Einführung in den ersten Band steht zu Beginn von Kapitel 4, dass in „,diesem Kapitel [...] drei der Fragen skizziert [werden], die uns im Zuge der über zweijährigen Diskussionen rund um dieses Buch in besonderer Weise umtrieben. Dabei sind mit ,uns' alle gemeint, die an der Entstehung des Buches mitwirkten, sei es als Autor(innen)en, sei es als Mitdiskutierende an den Treffen des Diskussionsforums. In den Diskussionen wurden erste vorläufige Antworten auf diese Fragen gefunden - manche davon sind bereits ,fertiger' als andere, bei denen sich eine Antwort erst herauszuschälen beginnt" (Defila und Di Giulio 2018b, S. 15). Ein gutes Jahr und viele Diskussionen später darf gesagt werden, dass die Akzente, die zusätzlich gesetzt wurden, und die Aspekte, die nun durch die Beiträge in diesem Buch hinzukommen, die Fragen zwar weiterhin nicht abschließend beantworten, die vorläufigen Antworten dadurch jedoch noch ein Stück ,fertiger ${ }^{6}$ und differenzierter sind.

\section{Zum Aufbau des Buches}

Ziel des vorliegenden Buches ist es, wie eingangs erwähnt, einen Beitrag zur Methodik transdisziplinärer (und transformativer) Forschung zu leisten. Dies gliedert sich in zwei Teile, auf die nachstehend kurz eingegangen wird (für die bibliographischen Angaben s. Anlage a im Anhang dieses Beitrags).

\section{Teil 1}

Im ersten Teil des Buches werden Ansätze und Einsichten vorgestellt, die aus dem Bestreben entstanden, das Handeln der Akteure, die an transdisziplinärer (und transformativer) Forschung mitwirken, zu verstehen und zu verstehen helfen. Diese gründen in den Erfahrungen der BaWü-Labs und sind das Ergebnis des Austauschs zwischen BaWü-Labs, bei dem die Erfahrungen verglichen und Gemeinsamkeiten jenseits der Unterschiedlichkeit der Erfahrungen identifiziert wurden. Diese Beiträge wurden durch gemischte Teams aus mindestens drei BaWü-Labs verfasst. Die Reihenfolge, in der im Folgenden die Beiträge vorgestellt werden, entspricht nicht der Reihenfolge, wie sie im Buch abgedruckt sind.

Zwei Beiträge spiegeln einen systematischen Zugang:

- Der Beitrag Unterschiedliche Handlungslogiken in transdisziplinären und transformativen Forschungsprojekten - Welche Risikokulturen entwickeln sich daraus und wie lassen sie sich konstruktiv einbinden? von Monika Gonser, Jochen Eckart, Charlotte Eller, Katharina Köglberger, Elke Häußler und Felix M. Piontek verwendet einen systemtheoretischen Ansatz, um das Handeln der Akteure zu analysieren. Ausgehend von der Annahme, dass in Reallaboren Akteure aufeinandertreffen, die unterschiedlichen gesellschaftlichen 
Teilsystemen zugehören, wird reflektiert, was dies für die Arbeit in einem Reallabor bedeutet mit Blick auf potenzielle Reibungsflächen, auf die Risiken, die eingegangen werden (können), und auf die Verteilung von Risiken und Chancen. Zudem werden Folgerungen für das Risikomanagement in solchen Projekten gezogen.

- Der Beitrag Schutz in der Exposition, Schutz für die Exposition - Wie man in transdisziplinären und transformativen Forschungsformaten mit Ungewohntem und erhöhter Aufmerksamkeit umgeht von Katharina Köglberger, Raphael Dietz, Charlotte Eller, Felix M. Piontek, Marius Albiez und Thomas Potthast geht von der Notwendigkeit aus, in Reallaboren Neues auszuprobieren. Diese Notwendigkeit führt dazu, dass die Akteure je für sich mit Dingen konfrontiert werden, die für sie ungewohnt sind, und dass sie sich dadurch exponieren (müssen). Er beleuchtet, was dies für die einzelnen Akteure und für das Design eines Projekts bedeutet, und diskutiert das Potenzial eines Denkens in Expositionen.

Drei Beiträge vertiefen Aspekte der Akteursanalyse bezogen auf kommunale Akteure. Ausgangspunkt ist, dass eine solche Analyse nur dann eine gute Grundlage liefert für eine fruchtbare Zusammenarbeit in einem Projekt, wenn den Besonderheiten der analysierten Akteure Rechnung getragen und nicht mit verkürztem Blick an die Analyse herangegangen wird.

- Der Kurz-Beitrag Kommunale Akteure verstehen: Vorgeschichte der Arbeit in Kommunen beachten von Jochen Eckart, Elke Häußler, Thorsten Erl, Monika Gonser und Jan Riel ist dem ,blinden Flecken' gewidmet, der entsteht, wenn ausgeblendet wird, dass es in der Kommune höchstwahrscheinlich Erfahrungen aus anderen Projekten und Aktivitäten gibt, die für die (Zusammen-) Arbeit im Projekt von Bedeutung sind. Der Beitrag erörtert, welche Aspekte der Vorgeschichte kommunaler Akteure zu erkunden sind und weshalb es wichtig ist, sich mit dieser Vorgeschichte auseinanderzusetzen.

- Der Kurz-Beitrag Kommunale Akteure verstehen: Komplexe Entscheidungswege in Kommunen beachten von Monika Gonser, Jan Riel, Jochen Eckart, Thorsten Erl und Elke Häußler ist dem ,blinden Flecken' gewidmet, der entsteht, wenn ausgeblendet wird, dass die Entscheidungswege in Kommunen komplex sind und Entscheidungen von verschiedenen Akteuren getroffen werden, je nachdem, um welche Art Entscheidung es geht. Der Beitrag erörtert, welche Aspekte der Entscheidungswege innerhalb kommunaler Strukturen zu beleuchten sind und weshalb es wichtig ist, diese zu kennen.

- Der Kurz-Beitrag Kommunale Akteure verstehen: Verteilte Wissensbestände in Kommunen beachten von Thorsten Erl, Monika Gonser, Jochen Eckart, Elke Häußler und Jan Riel ist dem ,blinden Flecken' gewidmet, der entsteht, wenn ausgeblendet wird, dass sich das projektrelevante Wissen in der 
Kommune nicht bei einer Stelle oder Person bündeln, sondern auf verschiedene Stellen oder Personen verteilt sein dürfte. Der Beitrag erörtert, auf welche Aspekte der Wissensverteilung in einer Kommune zu achten ist, und zeigt, weshalb es wichtig ist, darüber Bescheid zu wissen.

\section{Teil 2}

Im zweiten Teil des Buches werden ausgewählte Methoden, die in BaWü-Labs entwickelt und umgesetzt wurden und die sich besonders bewährt haben, für die transdisziplinäre (und transformative) Forschung innerhalb und außerhalb von Reallaboren zur Verfügung gestellt. Dabei wurde besonders Wert gelegt auf Methoden, die nicht allein Forschungszielen oder Praxiszielen oder Bildungszielen dienen, sondern Zielen in mehr als einer dieser Dimensionen. Diese Methoden werden ausführlich beschrieben, und zwar so, dass Dritte sie umsetzen können. Dabei wurden die Gütekriterien zugrunde gelegt, die auch bereits im ersten Band angewendet wurden (Defila und Di Giulio 2018a). Zudem wurde darauf geachtet, die Vorgehensweisen so aufzubereiten, dass sie in vielfältigen Kontexten umsetzbar sind. Die Beiträge sind keine Fallstudien, d. h., die Erfahrungen aus dem jeweiligen BaWü-Lab dienen, sofern solche dargestellt werden, allein dem Zweck der Illustration. Die Reihenfolge, in der im Folgenden die Beiträge vorgestellt werden, entspricht der Reihenfolge, wie sie im Buch abgedruckt sind.

Die „Exchange-Veranstaltungsreihe“ - ein Planungs- und Steuerungsinstrument für transdisziplinäre Forschungsprojekte von Christina Benighaus, Belen Zevallos, Charlotte Eller, Martina Hilligardt, Mandana Alimardani und Ludger Benighaus

Mittels einer „Exchange-Veranstaltungsreihe“ kann die Arbeit in einem Forschungsprojekt gegliedert werden, in dem die transdisziplinäre Arbeit maßgeblich mit externen Beteiligten erfolgt, die punktuell in die Forschungsarbeit involviert werden. Die „Exchange-Veranstaltungsreihe“ besteht aus mehreren aufeinander aufbauenden transdisziplinären Veranstaltungen. Jede davon umfasst ein öffentliches Symposium und einen sich daran anschließenden projektinternen Reflexionsworkshop. Jede der Veranstaltungen schafft einen Ort der transdisziplinären Wissensproduktion.

Die „TraSy-Methode“ - ein Vorgehen für die transdisziplinäre Entwicklung soziotechnischer Systeme von Laura Gebhardt und Alexandra König

Bei soziotechnischen Systemen sind technische und nicht-technische Elemente eng verknüpft. Die „TraSy-Methode“ dient dazu, die Bedürfnisse heterogener Gruppen von künftigen Nutzer(inne)n und anderen lokalen Praxisakteuren für ein 
soziotechnisches System transdisziplinär zu erarbeiten. Das Vorgehen sieht mehrere Etappen vor, in denen interdisziplinäre und transdisziplinäre Arbeiten unterschiedlich gewichtet werden. Die transdisziplinäre Arbeit konzentriert sich auf Workshops, in denen gemeinsames Wissen produziert wird.

„Barcamp adapted“ - gemeinsam zu neuem Wissen von Editha Marquardt und Ulrike Gerhard

„Barcamp adapted“ ist ein Vorgehen, das insbesondere in der Entstehungs- und Anfangsphase eines transdisziplinären Forschungsprojekts eingesetzt werden kann, um Kooperationen zu initiieren, zukünftige Partner für das Projekt sowie deren Interessen und Erfahrungen kennenzulernen, und Impulse zu generieren für die Ziele und Fragen des Projekts. Das Vorgehen besteht aus einem semioffenen Workshop und einer öffentlichen Veranstaltung. In diesen zwei Teilen werden zuerst transdisziplinär Wissen und Ideen für das (künftige) Projekt erzeugt, die dann in einem größeren Kreis validiert und weiterentwickelt werden.

„UrbanUtopiaLAB“ - einen Möglichkeitsraum zur Produktion von Transformationswissen schaffen von Christina West und Svenja Kück

Ein „UrbanUtopiaLAB“ lässt sich anwenden, wenn es um die Entwicklung von urbanen Räumen wie Quartier, Stadt, Region geht, in denen häufig auf engem Raum multiple Bedeutungszuschreibungen und Raumerfahrungen sowie unterschiedliche Vorstellungen über die Nutzung des öffentlichen Raums bestehen. Das Vorgehen setzt bei individuellen Wahrnehmungen, Nutzungen und Visionen an. Auf dieser Grundlage werden in mehreren Phasen praktisch umsetzbare Vorhaben oder politische Forderungen und Handlungsempfehlungen für Politik, Verwaltung, Planung und Wissenschaft entwickelt.

Das „Transformative Projektseminar" - didaktische Ansätze und methodische Umsetzung von Richard Beecroft

Ein „Transformatives Projektseminar“ dient dazu, in der Lehre parallel Bildungs-, Praxis- und Forschungsziele zu verfolgen. Es verbindet mehrere didaktische Ansätze zu einem transdisziplinären Prozess, der in sechs Phasen gegliedert ist. Die Praxispartner eines Forschungsprojekts sind als Mit-Lehrende beteiligt, und die Studierenden arbeiten an studentischen Projekten, die auf Bedarfe der Praxispartner antworten und für diese nützlich sein sollen. Die Praxispartner geben diese Projekte jedoch nicht vor, sondern formulieren lediglich Bedarfe und reagieren auf die Ideen der Studierenden. 


\section{Expertise aufbauen - Bedingungen des Lernens aus Reallaboren für transdisziplinäre (und transformative) Forschung}

Reallabore haben es, das wurde bereits im ersten Band konstatiert, mit kumulierten Herausforderungen zu tun, weil sie gleichzeitig interdisziplinär sind, transdisziplinär sind und sowohl transformativ wie forschend tätig sind (Defila und Di Giulio 2018b, S. 24) - und die Erfahrungen auch in anderen Reallaboren als den BaWü-Labs bestätigen es (z. B. Rose et al. 2018 oder auch die Beiträge in der GAIA Special Issue 2018, in denen aus anderen Reallaboren als den BaWü-Labs berichtet wird). In solchen Projekten gilt es, Ziele in verschiedenen Dimensionen (Forschungsziele, Praxisziele, Bildungsziele, s. Beecroft et al. 2018) auszubalancieren, sich dynamisch an Entwicklungen und Erfordernisse in der ,realen Welt ${ }^{*}$ anzupassen, ohne dabei aber das Projekt und dessen Fokus aus den Augen zu verlieren, mit einer Vielzahl von Akteuren in komplexen Konstellationen professionell zu interagieren (s. Seebacher et al. 2018) sowie die Forschungs-, Transformations- und Bildungsprozesse methodisch angemessen und nach allen Regeln der Kunst partizipativen, interdisziplinären und transdisziplinären Forschens zu gestalten (s. dazu auch Defila und Di Giulio 2018a).

Mit diesen kumulierten Herausforderungen umzugehen, stellt hohe Anforderungen an das Projektdesign, an die Ressourcenallokation und an die Methodenwahl. ${ }^{5}$ Das vorliegende Buch will, wie bereits der vorausgehende Band, einen Beitrag dazu leisten, mit diesen Herausforderungen umzugehen, indem Hinweise zum Projektdesign gegeben werden und indem bewährte Methoden ,pfannenfertig' aufbereitet werden.

Die Beiträge in beiden Bänden sowie die Diskussionen, die rund um deren Entstehung stattfanden, zeigen aber auch die Grenzen eines solchen eher ,technischen'Zugangs. Die Reflexionen am Ende vieler Methoden-Beiträge in Teil 2 sowohl des ersten Bandes wie auch des vorliegenden Buches machen deutlich, wie hoch die Anforderungen an diejenigen sind, die für die Gestaltung der Prozesse in Reallaboren oder in anderen transdisziplinären (und transformativen) Projekten (mit-)verantwortlich zeichnen (diese werden im Folgenden als „Managementverantwortliche" bezeichnet, unabhängig von ihrem akademischen Status und von der Beschreibung ihrer Funktion in Projektorganigrammen, s. Defila et al. 2006, S. 16f.). Das hohe Anforderungsniveau tritt aber insbesondere auch zutage in den Diskussionen erstens zur Frage, welche Handlungslogiken und

5 Nicht zu unterschätzen, aber nicht Thema dieses Beitrags ist, dass das Format Reallabor (und andere transdisziplinäre und transformative Formate) auch für die Fördergeber, die die Rahmenbedingungen für diese Forschung schaffen müssen, eine Herausforderung darstellt (s. dazu z. B. Parodi et al. 2018). 
Risikokulturen in solchen Projekten aufeinandertreffen können (Gonser et al. 2019a), die es zu erfassen und denen es gerecht zu werden gilt, und zweitens zur Frage, welche Expositionen solche Projekte für die beteiligten Akteure mit sich bringen (Köglberger et al. 2019), die es zu verstehen und zu ermöglichen gilt. Vieles davon ist nicht neu, aber die Schärfe, mit der es in Reallaboren zutage tritt, erlaubt einen noch klareren Blick auf die Expertise, die dafür erforderlich ist.

Für die professionelle Gestaltung inter- und transdisziplinärer Prozesse ist eine Expertise erforderlich, die sich zusammenfassend als „inhaltsreiche Moderation“ (Defila et al. 2006, S. 126) bezeichnen lässt. Diese Expertise, die Managementverantwortliche von Projekten (unabhängig davon, ob es sich um kleinere Projekte oder größere Projektverbünde handelt) aufweisen müssen, lässt sich folgendermaßen beschreiben (s. dazu insbesondere Defila und Di Giulio 2015; 2017; aber auch Defila et al. 2015; für eine weitere Differenzierung s. auch die weiteren Beiträge im Forschung-Schwerpunktheft 2015 zu „Managementverantwortliche inter- und transdisziplinärer Verbünde"): Managementverantwortliche tragen die Verantwortung dafür, dass der für inter- und transdisziplinäre Forschung zentrale Qualitätsanspruch der Integration, d. h. der Synthesebildung, eingelöst wird. Diese Verantwortung hat eine thematische Dimension (bezogen auf das erforschte Thema), eine methodische Dimension (bezogen auf die Gestaltung der kognitiven Syntheseprozesse) sowie eine sozial-kommunikative Dimension (bezogen auf die beteiligten Akteure). Entsprechend vielfältig sind die (Detail-)Expertisen, die für eine erfolgreiche inhaltsreiche Moderation erforderlich sind.

In thematischer Hinsicht müssen Managementverantwortliche inter- und transdisziplinärer Projekte die Synthesebildung moderieren können, sie müssen gleichzeitig aber auch selbst einen wesentlichen inhaltlichen Beitrag zur Synthese leisten können. Sie müssen deshalb zum einen die Beiträge anderer (Personen und/oder Teilprojekte) zur Synthese beurteilen und einordnen, d. h. etwa Widersprüche oder sogar Unvereinbarkeiten herausarbeiten, Vorschläge für die Überwindung inhaltlicher Dissense vorlegen, Inkonsistenzen und Lücken aufdecken, Ergebnisse gegenseitig verständlich machen helfen. Dazu erforderlich sind insbesondere die Fähigkeit, sinnvoll mit Personen aus verschiedenen Fachgebieten innerhalb wie außerhalb der Wissenschaft zu interagieren (,interactional expertise“; englische Terminologie aus Collins und Evans 2002), die Fähigkeit, zwischen den beteiligten Fachgebieten zu übersetzen (,ability to translate“), und die Fähigkeit, Gemeinsamkeiten, Differenzen, Anknüpfungspunkte und Muster zu erkennen, und zwar auch solche, die die Beteiligten selbst vielleicht nicht erkennen, um das Potenzial aller Beteiligten für das Thema zu heben (,ability to discriminate“). Zum anderen müssen sie selbst Wissen für die Synthese aufbereiten und inhaltliche Schritte der Integration übernehmen. Dazu erforderlich ist eine Expertise, die daraus gewonnen wird, dass Beiträge zum Forschungsdiskurs geleistet werden, und zwar eine, die direkt aus Beiträgen zum erforschten Thema erwächst (,,contributory 
expertise“), mindestens aber eine, die daraus erwächst, zu wissen, was es bedeutet, solche Beiträge zu leisten, die also erlaubt, auf eine „contributory expertise“ $\mathrm{zu}$ anderen Themen zu referieren (,,referred contributory expertise"). Um Syntheseprozesse $\mathrm{zu}$ moderieren, müssen Managementverantwortliche ergebnisoffen sein - dafür ist eine gewisse Distanz zum Thema sinnvoll. Um inhaltliche Beiträge zu leisten, die Hand und Fuß haben, ist demgegenüber eine gewisse Nähe zum Thema sinnvoll (und auf längere Sicht wohl auch unvermeidlich). Managementverantwortliche müssen deshalb, bezogen auf den Inhalt des Projekts, einen guten Umgang finden im Spiel zwischen Nähe und Distanz. Sind Projekte nicht ,nur interdisziplinär und nicht, nur' transdisziplinär, sondern zusätzlich auch transformativ, vervielfacht sich die notwendige Expertise insofern, als sich diese themenbezogene Expertise auch auf die gesellschaftliche Einbettung der Ziele, auf die das Projekt ausgerichtet ist, erstreckt, also v. a. darauf, wie es um die gesellschaftspolitische Legitimation dieser Ziele steht, um deren ethische Begründung und Gemeinwohlorientierung.

In methodischer Hinsicht müssen Managementverantwortliche inter- und transdisziplinärer Projekte zum Ersten den Prozess des Ringens um das Gemeinsame und des Herausarbeitens der Differenzen gestalten können, d. h., sie müssen Methoden anwenden, die eine echte Auseinandersetzung zwischen den Beteiligten erzwingen, und zwar eine, die gleichzeitig sowohl Unterschiede in der Denk- und Arbeitsweise zutage fördert, als auch Anknüpfungspunkte und geteilte Sichtweisen sichtbar werden lässt. Zum Zweiten müssen sie den methodischen Prozess der Synthesebildung gestalten können, d. h., sie müssen Vorschläge für Verfahren der Wissensintegration generieren, den anderen im Projekt unterbreiten und deren Umsetzung begleiten, wobei sie sicherstellen müssen, dass die Umsetzung nach allen Regeln der Kunst erfolgt und zu nachvollziehbaren Erkenntnissen führt. Sowohl mit Blick auf das Fördern der Auseinandersetzung der Beteiligten untereinander als auch mit Blick auf die Gestaltung der Syntheseprozesse müssen Managementverantwortliche oftmals selbst neue Methoden entwickeln oder Methoden für das eigene Projekt anpassen, d. h., sie müssen fähig sein, Methoden zu erfinden, die wissenschaftlich Hand und Fuß haben und zu qualitativ robusten Ergebnissen führen. Diese Methoden wiederum, selbst erfundene wie übernommene, müssen sie auch dann einsetzen, wenn sie nicht zu $100 \%$ sicher sind, dass sie funktionieren - und dabei stehen sie gewissermaßen auf der Bühne. Managementverantwortliche müssen also bereit sein, ihre eigene Komfortzone zu verlassen. Um im Projekt einen echten Austausch und eine echte Zusammenarbeit zu fördern, müssen die Managementverantwortlichen aber gleichzeitig die anderen Beteiligten dazu anregen, ihre Komfortzone $\mathrm{zu}$ verlassen. Sind Projekte nicht ,nur' interdisziplinär und nicht ,nur ${ }^{6}$ transdisziplinär, sondern zusätzlich auch transformativ, vervielfacht sich die notwendige Expertise insofern, als es gilt, zusätzlich zu den Prozessen der Synthesebildung auch die Prozesse der Transformation zu gestalten. Und bezogen auf die Auseinandersetzung der Beteiligten 
untereinander gilt es, Methoden anzuwenden, die nicht nur eine Reflexion der im Projekt vertretenen fachlichen Denk- und Arbeitsweisen erlauben, sondern auch eine Reflexion der im Projekt vertretenen Risikowahrnehmungen, und die zu einem umfassenden Risikomanagement führen.

In sozial-kommunikativer Hinsicht müssen Managementverantwortliche interund transdisziplinärer Projekte tatsächliche oder potenzielle Kommunikationsbarrieren zwischen den Beteiligten erkennen und abbauen können (s. dazu auch Lefroy 2013), und sie müssen Konflikte, die sich aus deren unterschiedlichen Denk- und Arbeitsweisen ergeben (könnten), erkennen (bzw. antizipieren) können und überwinden helfen (bzw. vermeiden helfen). Mit Blick auf das Erste müssen sie in der Lage sein, einen Dialog zwischen den Beteiligten nicht nur zu initiieren, sondern diesen auch zu pflegen und aufrechtzuerhalten. Dabei ist es wichtig, dass sie darauf achten, Kommunikationen und Interaktionen in die Wege $\mathrm{zu}$ leiten, die auch unabhängig von ihnen funktionieren - je komplexer die Akteurskonstellation in einem Projekt ist, desto wichtiger ist dieser Punkt. Mit Blick auf das Zweite müssen sie in der Lage sein, zu unterscheiden zwischen Differenzen, die in der Sache begründet sind, solchen, die durch die fachlichen Denk- und Arbeitsweisen bedingt sind, und solchen, die in der Person liegen. Dabei ist es wichtig, Arbeitsbeziehungen einzurichten, die auch dann funktionieren, wenn sich die Beteiligten auf der persönlichen Ebene nicht gut verstehen. Managementverantwortliche müssen das gegenseitige Verständnis fördern und zu diesem Zweck Prozesse der Reflexion und des gegenseitigen Lernens in die Wege leiten können - und zwar auch wenn sich die Beteiligten unwillig zeigen. Managementverantwortliche müssen sowohl das soziale Gefüge des Projekts als auch die daran beteiligten Einzelpersonen und deren Befindlichkeiten im Auge behalten und auf eine für alle angemessene Balance zwischen Nähe und Distanz achten. Dazu wiederum müssen sie unterscheiden können zwischen einer persönlichen Ebene der Interaktion und einer privaten Ebene und sensibilisiert sein dafür, dass erstere im Projekt erreicht und die Grenze zu letzterer nicht überschritten wird. Sind Projekte nicht ,nur' interdisziplinär und nicht ,nur' transdisziplinär, sondern zusätzlich auch transformativ, vervielfacht sich die notwendige Expertise insofern, als es gilt, zusätzlich die jeweils spezifischen Expositionen, die die Beteiligten im Projekt (bzw. durch die Aktivitäten im Projekt) eingehen (könnten), zu erkennen (bzw. zu antizipieren) und entsprechende Schutzvorkehrungen zu bieten. Zudem ist bei der Gestaltung der sozialen und kommunikativen Prozesse im Projekt zu bedenken, welche Beziehungen die Akteure außerhalb des Projekts untereinander haben und wie die Zusammenarbeit im Projekt deren Rolle in der gesellschaftlichen Transformation beeinflussen kann.

Bezogen auf alle drei Dimensionen kommt bei Projekten, die nicht ,nur ${ }^{6}$ interdisziplinär und nicht ,nur' transdisziplinär sind, sondern, wie Reallabore, zusätzlich auch transformativ, ein weiterer Aspekt ins Spiel, mit dem Management- 
verantwortliche professionell umgehen müssen, die Nähe und Distanz bezogen auf die gesellschaftlichen Ziele, auf die das Projekt ausgerichtet ist. Fragen, mit denen sich Managementverantwortliche hier konfrontiert sehen, sind z. B.: Wie stark muss bzw. darf die Identifikation mit diesen Zielen sein (ihre und die des Projekts), und wie beeinflusst dies die Arbeit im Projekt? Wie stark muss bzw. darf sich das Projekt (bzw. müssen oder dürfen sie selbst sich) auf das Wertsystem und die Zielvorstellung der Praxisakteure einlassen und es sich zu eigen machen, und wie beeinflusst dies die Arbeit im Projekt? Bei diesem Aspekt der Nähe und Distanz bezogen auf die gesellschaftlichen Ziele gibt es eine klare Grenze der Verantwortung insofern, als Managementverantwortlichen keine Verantwortung dafür zukommt, dass die transformativen Ziele des Projekts erreicht werden. Auf die Frage, wo und wie diese Grenze im konkreten Fall zu ziehen ist, kann keine generell geltende Antwort gegeben werden, d. h., diese ist projektspezifisch zu beantworten. Die Expertise, die Managementverantwortliche dazu haben müssen, besteht darin, für diese Frage sensibilisiert zu sein.

Um angemessen mit den kumulierten Herausforderungen umzugehen, die sich in Reallaboren (oder anderen transdisziplinären und transformativen Projekten) stellen, müssen diejenigen, die für solche Projekte (mit-)verantwortlich sind, in ihrem Handeln unterstützt werden. Dafür wiederum müssen Managementverantwortliche durch Handeln und Reflexion gewonnene Einsichten zur Gestaltung der Prozesse in solchen Projekten aufbereiten und weitergeben - und so nicht nur selbst als Individuen Expertise in der Gestaltung solcher Prozesse entwickeln und pflegen, sondern durch das Tradieren von Wissen auch zum Aufbau der Expertise Dritter beitragen.

Das vorliegende Buch reiht sich, wie bereits der erste Band der Methodensammlung, hier ein, d. h., es will dazu einen Beitrag leisten, indem direkt anwendbare Methoden sowie Erfahrungen aus den BaWü-Labs in reflektierter und aggregierter Form zur Verfügung gestellt werden. Dem Bedarf nach Wissensaufbereitung und -tradierung kann jedoch nur entsprochen werden, wenn es für Managementverantwortliche einen Ort gibt, in dem sie Erfahrungen austauschen und vergleichen können und so Einsichten produzieren können, die über ihre jeweiligen projektspezifischen Erfahrungen hinausgehen. Es braucht, mit anderen Worten, eine Synthese der Erfahrungen von Managementverantwortlichen. Und dies wiederum erfordert einen darauf ausgerichteten Prozess der Synthesebildung. ${ }^{6}$ Die Diskussionsforen für die BaWü-Labs waren solche Orte, und deren Ergebnis sind das vorausgehende und das vorliegende Buch. Nur wenn solche Orte der moderierten Reflexion zur Verfügung gestellt werden, und nur, wenn darin strukturierte

6 Davon, dass ein solcher Bedarf besteht, zeugt auch das 2019 gegründete „Netzwerk Reallabore der Nachhaltigkeit“" (https://www.reallabor-netzwerk.de, zugegriffen am 20.05.2019). 
Prozesse der Wissensintegration stattfinden, werden Reallabore das ganze Potenzial entfalten können, das sie als Quelle für die Methodik transdisziplinären und transformativen Forschens haben.

\section{Dank}

Dieses Buch indirekt mitgeprägt haben die Personen, die an den Diskussionen rund um den Zweck, den Charakter und den Aufbau des ersten Bands von 2018 beteiligt waren. Diesen Personen, die dort in der Einführung namentlich erwähnt sind, sei an dieser Stelle nochmals gedankt. Direkt geprägt sind Inhalt und Charakter dieses Buches von den Personen, die unabhängig davon, ob sie am Ende selbst als Autor(inn)en tätig wurden, mitdiskutiert haben bei der Frage, welches sinnvolle und interessante Themen sein könnten für die Synthesen im vorliegenden Buch. Ohne deren Beiträge und Kreativität und ohne deren Bereitschaft, ihre eigenen Erfahrungen mit Dritten zu teilen und mit denen Dritter zu vergleichen, wäre das Buch ärmer geworden. Diese Personen sind: Marius Albiez, Mareike Bahn, Robert Blaszczyk, Fabian Dembski, Jochen Eckart, Charlotte Eller, Havva Engin, Thorsten Erl, Monika Gonser, Sarah Hausmann, Elke Häußler, Martina Hilligardt, Christoph Hupfer, Sebastian Kapser, Monika Pröbster, Tim Sippel, Jeannine Tischler, Belen Zevallos, Philip Zwernemann.

Ein großer Dank geht an alle Personen, die sich im Rahmen des externen Reviews intensiv mit dem Ziel des Buches und den einzelnen Beiträgen befassten. Ihre Einlassung auf die Idee des Buches und ihre wohlwollend-kritische Prüfung trugen wesentlich zur Qualität des Buches bei. Um die Anonymität des Reviews zu wahren, kann den Gutachter(inne)n bei den einzelnen Beiträgen nicht namentlich, sondern lediglich abstrakt gedankt werden. Die Namen aller Gutachter(innen) sind aber im Verzeichnis der Review-Personen im Anhang zu diesem Buch aufgelistet.

Einen speziellen Dank möchten wir erstens Angelika Schulz aussprechen für den Satz und für ihre Bereitschaft, in beiden Bänden immer auch dem individuellen Charakter der Beiträge Rechnung zu tragen, und zweitens Britta Fietzke für das gründliche Korrektorat dieses Buches. Wir danken beiden auch für die Flexibilität, mit der sie sich auf den dynamischen Zeitplan der Buchentstehung eingelassen haben, die aber nie zulasten der Sorgfalt ging.

Ein besonderer Dank geht schließlich an das Ministerium für Wissenschaft, Forschung und Kunst Baden-Württemberg (MWK), ohne dessen Förderung weder die Arbeit in den BaWü-Labs noch die Arbeit der BF-Basel möglich gewesen wären und ohne dessen Unterstützung auch dieses Buch nicht entstanden wäre. 


\section{Literatur}

Arnold, A., \& Piontek, F. M. (2018). Zentrale Begriffe im Kontext der Reallaborforschung. In R. Defila \& A. Di Giulio (Hrsg.), Transdisziplinär und transformativ forschen. Eine Methodensammlung (S. 143-154). Wiesbaden: Springer VS. doi: 10.1007/ 978-3-658-21530-9_8.

Bäckstrand, K. (2003). Civic Science for Sustainability: Reframing the Role of Experts, Policy-Makers and Citizens in Environmental Governance. Global Environmental Politics, 3 (4), (S. 24-41).

Beecroft, R., Trenks, H., Rhodius, R., Benighaus, C., \& Parodi, O. (2018). Reallabore als Rahmen transformativer und transdisziplinärer Forschung: Ziele und Designprinzipien. In R. Defila \& A. Di Giulio (Hrsg.), Transdisziplinär und transformativ forschen. Eine Methodensammlung (S. 75-100). Wiesbaden: Springer VS. doi: 10.1007/978-3-65821530-9_4.

BGL-Themenheft (2017). Reallabore als Forschungsformat nachhaltiger Stadtentwicklung. Berichte. Geographie und Landeskunde, 91 (1).

Burton, R., Ronningen, K., \& Wedderburn, L. (2008). Conducting integrated research. A critical literature review of interdisciplinary and transdisciplinary research. Report 12/08. Trondheim: Centre for Rural Research, Norwegian University of Science and Technology.

CASS \& ProClim- (1997). Forschung zu Nachhaltigkeit und Globalem Wandel - Wissenschaftspolitische Visionen der Schweizer Forschenden. Bern: ProClim-/SANW. https:// naturwissenschaften.ch/service/publications/75640-visionen-der-forschenden. Zugegriffen am 20.05.2019.

Collins, H. M., \& Evans, R. (2002). The Third Wave of Science Studies: Studies of Expertise and Experience. Social Studies of Science, 32 (2), (S. 235-296). doi: 10.1177/ 0306312702032002003.

Defila, R., \& Di Giulio, A. (2019). Eine Reflexion über Legitimation, Partizipation und Intervention im Kontext transdisziplinärer Forschung. In M. Ukowitz \& R. Hübner (Hrsg.), Interventionsforschung. Band 3: Wege der Vermittlung. Intervention - Partizipation (S. 85-108). Wiesbaden: Springer VS. doi: 10.1007/978-3-658-22048-8_4.

Defila, R., \& Di Giulio, A. (2018a). Partizipative Wissenserzeugung und Wissenschaftlichkeit - ein methodologischer Beitrag. In R. Defila \& A. Di Giulio (Hrsg.), Transdisziplinär und transformativ forschen. Eine Methodensammlung (S. 39-67). Wiesbaden: Springer VS. doi: 10.1007/978-3-658-21530-9_2.

Defila, R., \& Di Giulio, A. (2018b). Reallabore als Quelle für die Methodik transdisziplinären und transformativen Forschens - eine Einführung. In R. Defila \& A. Di Giulio (Hrsg.), Transdisziplinär und transformativ forschen. Eine Methodensammlung (S. 9-35). Wiesbaden: Springer VS. doi: 10.1007/978-3-658-21530-9_1.

Defila, R., \& Di Giulio, A. (Hrsg.). (2018c). Transdisziplinär und transformativ forschen. Eine Methodensammlung. Wiesbaden: Springer VS. doi: 10.1007/978-3-658-21530-9. 
Defila, R., \& Di Giulio, A. (2018d). What is it good for? Reflecting and systematizing accompanying research to research programs. GAIA, 27 (S1), (S. 97-104). doi: 10. 14512/gaia.27.S1.17.

Defila, R., \& Di Giulio, A. (2017). Managing Consensus in Inter- and Transdisciplinary Teams: Tasks and Expertise. In R. Frodeman, J. Th. Klein \& R. Pacheco (Hrsg.), The Oxford Handbook of Interdisciplinarity (2. ed) (S. 332-337). Oxford, New York: Oxford University Press. doi: 10.1093/oxfordhb/9780198733522.013.27.

Defila, R., \& Di Giulio, A. (2015). Integrating knowledge: challenges raised by the „Inventory of Synthesis“. Futures, 65, Special Issue Transdisciplinarity revisited, (S. 123135). doi: 10.1016/j.futures.2014.10.013.

Defila, R., \& Di Giulio, A. (1998). Interdisziplinarität und Disziplinarität. In J.-H. Olbertz (Hrsg.), Zwischen den Fächern - über den Dingen? Universalisierung versus Spezialisierung akademischer Bildung (S. 111-137). Opladen: Leske \& Budrich. doi: 10.1007/ 978-3-322-90935-0_6.

Defila, R., Di Giulio, A., \& Scheuermann, M. (2015). Managementverantwortliche interund transdisziplinärer Verbünde - Rollen, Kompetenzen, Karrieren. Einführung in das Schwerpunktheft „Managementverantwortliche inter- und transdisziplinärer Verbünde. Managers of inter- and transdisciplinary research groups". Forschung, 8 (3/4), S. 64 69. https://www.universitaetsverlagwebler.de/forschung. Zugegriffen am 20.05.2019.

Defila, R., Di Giulio, A., \& Scheuermann, M. (2006). Forschungsverbundmanagement. Handbuch für die Gestaltung inter- und transdisziplinärer Projekte. Zürich: vdf Hochschulverlag an der ETH Zürich.

Eckart, J., Häußler, E., Erl, Th., Gonser, M., \& Riel, J. (2019). Kommunale Akteure verstehen: Vorgeschichte der Arbeit in Kommunen beachten. In R. Defila \& A. Di Giulio (Hrsg.), Transdisziplinär und transformativ forschen, Band 2. Eine Methodensammlung (S. 33-38). Wiesbaden: Springer VS.

Eckart, J., Ley, A., Häußler, E., \& Erl, Th. (2018). Leitfragen für die Gestaltung von Partizipationsprozessen in Reallaboren. In R. Defila \& A. Di Giulio (Hrsg.), Transdisziplinär und transformativ forschen. Eine Methodensammlung (S. 105-135). Wiesbaden: Springer VS. doi: 10.1007/978-3-658-21530-9_6.

Erl, Th., Gonser, M., Eckart, J., Häußler, E., \& Riel, J. (2019). Kommunale Akteure verstehen: Verteilte Wissensbestände in Kommunen. In R. Defila \& A. Di Giulio (Hrsg.), Transdisziplinär und transformativ forschen, Band 2. Eine Methodensammlung (S. 139145). Wiesbaden: Springer VS.

Forschung-Schwerpunktheft (2015). Managementverantwortliche inter- und transdisziplinärer Verbünde. Managers of inter- and transdisciplinary research groups. Forschung, 8 (3/4). https://www.universitaetsverlagwebler.de/forschung. Zugegriffen am 20.05.2019.

GAIA Special Issue (2018). Labs in the real world. Advancing transdisciplinary research and sustainability transformations. GAIA, 27 (S1). https://www.ingentaconnect.com/ content/oekom/gaia/2018/00000027/a00101s1. Zugegriffen am 20.05.2019. 
Gonser, M., Eckart, J., Eller, C., Köglberger, K., Häußler, E., \& Piontek F. M. (2019a). Unterschiedliche Handlungslogiken in transdisziplinären und transformativen Forschungsprojekten - Welche Risikokulturen entwickeln sich daraus und wie lassen sie sich konstruktiv einbinden? In R. Defila \& A. Di Giulio (Hrsg.), Transdisziplinär und transformativ forschen, Band 2. Eine Methodensammlung (S. 39-83). Wiesbaden: Springer VS.

Gonser, M., Riel, J., Eckart, J., Erl, Th., \& Häußler, E. (2019b). Kommunale Akteure verstehen: Komplexe Entscheidungswege in Kommunen. In R. Defila \& A. Di Giulio (Hrsg.), Transdisziplinär und transformativ forschen, Band 2. Eine Methodensammlung (S. 85-91). Wiesbaden: Springer VS.

Klein, J. T. (2014). Interdisciplinarity and Transdisciplinarity: Keyword Meanings for Collaboration Science and Translational Medicine. Journal of Translational Medicine \& Epidemiology, 2 (2), (Nr. 1024).

Köglberger, K., Dietz, R., Eller, C., Piontek, F. M., Albiez, M., \& Potthast, T. (2019). Schutz in der Exposition, Schutz für die Exposition - Wie man in transdisziplinären und transformativen Forschungsformaten mit Ungewohntem und erhöhter Aufmerksamkeit umgeht. In R. Defila \& A. Di Giulio (Hrsg.), Transdisziplinär und transformativ forschen, Band 2. Eine Methodensammlung (S. 93-138). Wiesbaden: Springer VS.

Lefroy, T. (2013). Interdisciplinary Research is about People as well as Concepts and Methods. In G. Bammer (Hrsg.), Disciplining Interdisciplinarity (S. 365-373). Canberra: ANU E Press. https://www.jstor.org/stable/j.ctt2jbkj5.52. Zugegriffen am 20.05.2019.

Mielke, J., Vermeßen, H., Ellenbeck, S., \& Fernandez Milan, B. (2016). Stakeholder Involvement in sustainability science - a critical view. Energy Research \& Social Science (ERSS), 17, (S. 71-81). doi: 10.1016/j.erss.2016.04.001.

Mobjörk, M. (2010). Consulting versus participatory transdisciplinarity: A refined classification of transdisciplinary research. Futures, 42 (8), (S. 866-873). doi: 10.1016/ j.futures.2010.03.003.

Otero, I., Niewöhner, J., Krueger, T., Doğmus, Ö. C., Himmelreich, J., Sichau, C., \& Hostert, P. (2017). The position of scientists in transformations of human-environment systems. An inquiry into IRI THESys research practices. THESys Discussion paper No. 2017-1. Berlin: Humboldt-Universität zu Berlin. doi: 10.18452/3136.

Parodi, O., Ley, A., Fokdal, J., \& Seebacher, A. (2018). Empfehlungen für die Förderung und Weiterentwicklung von Reallaboren. Erkenntnisse aus der Arbeit der BaWü-Labs. GAIA, 27 (1), (S. 178-179). doi: 10.14512/gaia.27.1.15.

Rose, M., Wanner, M., \& Hilger, A. (2018). Das Reallabor als Forschungsprozess und -infrastruktur für nachhaltige Entwicklung. Konzepte, Herausforderungen und Empfehlungen. NaWiKO Synthese Working Paper No. 1. https://nachhaltigeswirtschaftensoef.de/synthese-reallabore. Zugegriffen am 20.05.2019.

Schäpke, N., Stelzer, F., Bergmann, M., \& Lang, D. J. (2016). Tentative theses on transformative research in real-world laboratories: First insights from the accompanying research ForReal. Technikfolgenabschätzung - Theorie und Praxis, 25 (3), (S. 10-15). https://www.tatup-journal.de/weiterleitung8676.php. Zugegriffen am 20.05.2019. 
Schäpke, N., Stelzer, F., Bergmann, M., Singer-Brodowski, M., Wanner, M., Caniglia G., \& Lang, D. (2017). Reallabore im Kontext transformativer Forschung. Ansatzpunkte zur Konzeption und Einbettung in den internationalen Forschungsstand. (No. 1/2017). Leuphana Universität Lüneburg, Institut für Ethik und Transdisziplinäre Nachhaltigkeitsforschung. http://hdl.handle.net/10419/168596. Zugegriffen am 20.05.2019.

Schneidewind, U., \& Singer-Brodowski, M. (2014). Transformative Wissenschaft. Klimawandel im deutschen Wissenschafts- und Hochschulsystem. 2. Aufl. Marburg: Metropolis.

Scholz, R. (2017). The Normative Dimension in Transdisciplinarity, Transition Management, and Transformation Sciences: New Roles of Science and Universities in Sustainable Transitioning. Sustainability, 9. (S. 991). doi: 10.3390/su9060991.

Seebacher, A., Alcántara, S., \& Quint, A. (2018). Akteure in Reallaboren - Reallabore als Akteure. In R. Defila \& A. Di Giulio (Hrsg.), Transdisziplinär und transformativ forschen. Eine Methodensammlung (S. 155-159). Wiesbaden: Springer VS. doi: 10. 1007/978-3-658-21530-9_9.

TATuP-Schwerpunkt (2016). Reallabore als Orte der Nachhaltigkeitsforschung und Transformation. Technikfolgenabschätzung - Theorie und Praxis, 25 (3), (S. 4-51). https:// www.tatup-journal.de/tatup163.php. Zugegriffen am 20.05.2019.

WBGU (Wissenschaftlicher Beirat für Globale Umweltveränderungen) (2011). Welt im Wandel. Gesellschaftsvertrag für eine Große Transformation. Hauptgutachten 2011. Berlin: WBGU. Verfügbar unter: http://www.wbgu.de. Zugegriffen am 20.05.2019.

WD (Wissenschaftliche Dienste des Deutschen Bundestags) (2018). Förderung von Reallaboren und Citizen Science in Deutschland. Dokumentation. Aktenzeichen WD 8 3000 - 017/18, Abschluss der Arbeit 9. März 2018. Fachbereich WD 8: Umwelt, Naturschutz, Reaktorsicherheit, Forschung und Bildung. https://www.bundestag.de/ resource/blob/550742/8f269b6399b3098eabf9d5b09f31f88f/wd-8-017-18-pdf-data.pdf. Zugegriffen am 20.05.2019. 


\section{Anhang}

Anlage a: Beiträge in der Methodensammlung

(Band 1 2018, Band 2 2019)

\section{Einführung in die beiden Bände}

Defila, R., \& Di Giulio, A. (2018). Reallabore als Quelle für die Methodik transdisziplinären und transformativen Forschens - eine Einführung. In R. Defila \& A. Di Giulio (Hrsg.), Transdisziplinär und transformativ forschen. Eine Methodensammlung (S. 9-35). Wiesbaden: Springer VS. doi: 10.1007/978-3-658-21530-9_1.

Defila, R., \& Di Giulio, A. (2019). Wie Reallabore für Herausforderungen und Expertise in der Gestaltung transdisziplinären und transformativen Forschens sensibilisieren eine Einführung. In R. Defila \& A. Di Giulio (Hrsg.), Transdisziplinär und transformativ forschen, Band 2. Eine Methodensammlung (S. 1-30). Wiesbaden: Springer VS.

\section{Worauf achten: Kriterien, Prinzipien, Fragen für Planung und Reflexion (Teil 1 2018)}

Alcántara, S., Quint, A., \& Seebacher, A. (2018). Der Partizipationsmythos „Partizipation in Reallaboren muss repräsentativ sein“. In R. Defila \& A. Di Giulio (Hrsg.), Transdisziplinär und transformativ forschen. Eine Methodensammlung (S. 137-141). Wiesbaden: Springer VS. doi: 10.1007/978-3-658-21530-9_7.

Arnold, A., \& Piontek, F. M. (2018). Zentrale Begriffe im Kontext der Reallaborforschung. In R. Defila \& A. Di Giulio (Hrsg.), Transdisziplinär und transformativ forschen. Eine Methodensammlung (S. 143-154). Wiesbaden: Springer VS. doi: 10.1007/ 978-3-658-21530-9_8.

Beecroft, R., Trenks, H., Rhodius, R., Benighaus, C., \& Parodi, O. (2018). Reallabore als Rahmen transformativer und transdisziplinärer Forschung: Ziele und Designprinzipien. In R. Defila \& A. Di Giulio (Hrsg.), Transdisziplinär und transformativ forschen. Eine Methodensammlung (S. 75-100). Wiesbaden: Springer VS. doi: 10.1007/978-3-65821530-9_4.

Defila, R., \& Di Giulio, A. (2018). Partizipative Wissenserzeugung und Wissenschaftlichkeit - ein methodologischer Beitrag. In R. Defila \& A. Di Giulio (Hrsg.), Transdisziplinär und transformativ forschen. Eine Methodensammlung (S. 39-67). Wiesbaden: Springer VS. doi: 10.1007/978-3-658-21530-9_2.

Eckart, J., Ley, A., Häußler, E., \& Erl, Th. (2018). Leitfragen für die Gestaltung von Partizipationsprozessen in Reallaboren. In R. Defila \& A. Di Giulio (Hrsg.), Transdisziplinär und transformativ forschen. Eine Methodensammlung (S. 105-135). Wiesbaden: Springer VS. doi: 10.1007/978-3-658-21530-9_6.

Quint, A., Alcántara, S., \& Seebacher, A. (2018). Der Partizipationsmythos „Partizipation in Reallaboren ist per se transparent und muss es auch sein“. In R. Defila \& A. Di Giulio (Hrsg.), Transdisziplinär und transformativ forschen. Eine Methodensammlung (S. 69-73). Wiesbaden: Springer VS. doi: 10.1007/978-3-658-21530-9_3. 
Seebacher, A., Alcántara, S., \& Quint, A. (2018). Akteure in Reallaboren - Reallabore als Akteure. In R. Defila \& A. Di Giulio (Hrsg.), Transdisziplinär und transformativ forschen. Eine Methodensammlung (S. 155-159). Wiesbaden: Springer VS. doi: 10.1007/ 978-3-658-21530-9_9.

Seebacher, A., Alcántara, S., \& Quint, A. (2018). Der Partizipationsmythos „Partizipation bedeutet, alle immer an allem zu beteiligen“. In R. Defila \& A. Di Giulio (Hrsg.), Transdisziplinär und transformativ forschen. Eine Methodensammlung (S. 101-104). Wiesbaden: Springer VS. doi: 10.1007/978-3-658-21530-9_5.

\section{Worauf achten: Heuristiken zum Verstehen der Akteure und ihrer Handlungsbedingungen (Teil 1 2019)}

Eckart, J., Häußler, E., Erl, Th., Gonser, M., \& Riel, J. (2019). Kommunale Akteure verstehen: Vorgeschichte der Arbeit in Kommunen beachten. In R. Defila \& A. Di Giulio (Hrsg.), Transdisziplinär und transformativ forschen, Band 2. Eine Methodensammlung (S. 33-38). Wiesbaden: Springer VS.

Erl, Th., Gonser, M., Eckart, J., Häußler, E., \& Riel, J. (2019). Kommunale Akteure verstehen: Verteilte Wissensbestände in Kommunen. In R. Defila \& A. Di Giulio (Hrsg.), Transdisziplinär und transformativ forschen, Band 2. Eine Methodensammlung (S. 139145). Wiesbaden: Springer VS.

Gonser, M., Eckart, J., Eller, C., Köglberger, K., Häußler, E., \& Piontek F. M. (2019). Unterschiedliche Handlungslogiken in transdisziplinären und transformativen Forschungsprojekten - Welche Risikokulturen entwickeln sich daraus und wie lassen sie sich konstruktiv einbinden? In R. Defila \& A. Di Giulio (Hrsg.), Transdisziplinär und transformativ forschen, Band 2. Eine Methodensammlung (S. 39-83). Wiesbaden: Springer VS.

Gonser, M., Riel, J., Eckart, J., Erl, Th., \& Häußler, E. (2019). Kommunale Akteure verstehen: Komplexe Entscheidungswege in Kommunen. In R. Defila \& A. Di Giulio (Hrsg.), Transdisziplinär und transformativ forschen, Band 2. Eine Methodensammlung (S. 85-91). Wiesbaden: Springer VS.

Köglberger, K., Dietz, R., Eller, C., Piontek, F. M., Albiez, M., \& Potthast, T. (2019). Schutz in der Exposition, Schutz für die Exposition - Wie man in transdisziplinären und transformativen Forschungsformaten mit Ungewohntem und erhöhter Aufmerksamkeit umgeht. In R. Defila \& A. Di Giulio (Hrsg.), Transdisziplinär und transformativ forschen, Band 2. Eine Methodensammlung (S. 93-138). Wiesbaden: Springer VS.

\section{Wie vorgehen: Methoden für Co-Design und Co-Produktion (Teil 22018 und 2019)}

Alcántara, S., Arnold, A., Lindner, D., Busch, S., Dietz, R., Friedrich, M., Ritz, C., \& Sonnberger, M. (2018). Zwischen Wunsch und Wirkung - Ein transdisziplinärer Visionsworkshop mit Bürgerinnen und Bürgern. In R. Defila \& A. Di Giulio (Hrsg.), Transdisziplinär und transformativ forschen. Eine Methodensammlung (S. 269-299). Wiesbaden: Springer VS. 
Bachinger, M., Bleher, D., Rau, H., \& Prieß, R. (2018). Die „WiNo-Methode“ zur Identifikation von Wissen in transdisziplinären Netzwerken mithilfe der Wissensbilanzierung. In R. Defila \& A. Di Giulio (Hrsg.), Transdisziplinär und transformativ forschen. Eine Methodensammlung (S. 301-327). Wiesbaden: Springer VS.

Beecroft, R. (2019). Das „Transformative Projektseminar“ - didaktische Ansätze und methodische Umsetzung. In R. Defila \& A. Di Giulio (Hrsg.), Transdisziplinär und transformativ forschen, Band 2. Eine Methodensammlung (S. 293-337). Wiesbaden: Springer VS.

Benighaus, C., Zevallos, B., Eller, C., Hilligardt, M., Alimardani, M., \& Benighaus, L. (2019). Die „Exchange-Veranstaltungsreihe“ - ein Planungs- und Steuerungsinstrument für transdisziplinäre Forschungsprojekte. In R. Defila \& A. Di Giulio (Hrsg.), Transdisziplinär und transformativ forschen, Band 2. Eine Methodensammlung (S. 149-189). Wiesbaden: Springer VS.

Gebhardt, L., \& König, A. (2019). Die „TraSy-Methode“ - ein Vorgehen für die transdisziplinäre Entwicklung soziotechnischer Systeme. In R. Defila \& A. Di Giulio (Hrsg.), Transdisziplinär und transformativ forschen, Band 2. Eine Methodensammlung (S. 191235). Wiesbaden: Springer VS.

Marquardt, E., \& Gerhard, U. „Barcamp adapted“ - gemeinsam zu neuem Wissen. In R. Defila \& A. Di Giulio (Hrsg.), Transdisziplinär und transformativ forschen, Band 2. Eine Methodensammlung (S. 237-257). Wiesbaden: Springer VS.

Puttrowait, E., Dietz, R., Gantert, M., \& Heynold, J. (2018). Der Weg zum Realexperiment - Schlüsselakteure identifizieren, Kooperationsstrukturen aufbauen, Projektideen auswählen. In R. Defila \& A. Di Giulio (Hrsg.), Transdisziplinär und transformativ forschen. Eine Methodensammlung (S. 195-232). Wiesbaden: Springer VS.

Rhodius, R., \& Pregernig, M. (2018). Per „Wissensmesse“ zum Forschungsprogramm Arbeitsphasen und Abstimmungsprozesse zwischen Wissenschaft und Praxis. In R. Defila \& A. Di Giulio (Hrsg.), Transdisziplinär und transformativ forschen. Eine Methodensammlung (S. 163-194). Wiesbaden: Springer VS.

Trenks, H., Waitz, C., Meyer-Soylu, S., \& Parodi, O. (2018). Mit einer Realexperimentreihe Impulse für soziale Innovationen setzen - Realexperimente initiieren, begleiten und beforschen. In R. Defila \& A. Di Giulio (Hrsg.), Transdisziplinär und transformativ forschen. Eine Methodensammlung (S. 233-268). Wiesbaden: Springer VS.

West, C. (2018): „Wissen to Go“ - Transdisziplinär-transformative Lehre als „Reallabor im Kleinen”. In R. Defila \& A. Di Giulio (Hrsg.), Transdisziplinär und transformativ forschen. Eine Methodensammlung (S. 329-373). Wiesbaden: Springer VS.

West, C., \& Svenja Kück, S. (2019). „UrbanUtopiaLAB“ - einen Möglichkeitsraum zur Produktion von Transformationswissen schaffen. In R. Defila \& A. Di Giulio (Hrsg.), Transdisziplinär und transformativ forschen, Band 2. Eine Methodensammlung (S. 259291). Wiesbaden: Springer VS. 
Anlage b: Im Rahmen der Förderlinien „, Reallabore“ und „, Reallabore Stadt" des Ministeriums für Wissenschaft, Forschung und Kunst Baden-Württemberg (MWK) geförderte Reallabore (,,BaWü-Labs“)

\section{Förderlinie „Reallabore“}

EnSign RealLabor - Klimaneutrale Hochschule als Partner der Region Projektleitung: Prof. Dr. Ursula Eicker (Hochschule für Technik Stuttgart)

www.hft-stuttgart.de/Forschung/Reallabor

Future City Lab-Stuttgart - Reallabor für nachhaltige Mobilitätskultur * Projektleitung: Prof. Antje Stokman (Universität Stuttgart)

www.r-n-m.net

Nachhaltige Transformation der Textilwirtschaft am Standort Dietenheim

Projektleitung: Prof. Dr. Martin Müller (Universität Ulm), Prof. Dr. Matthias Freise (Hochschule Reutlingen)

dietenheim-zieht-an.de

Reallabor 131: KIT findet Stadt*/**

Projektleitung: Dr. Oliver Parodi, Dr. Andreas Seebacher (Karlsruher Institut für Technologie)

www.quartierzukunft.de

Space Sharing - Nutzungsintensivierung des Gebäudebestands durch Mehrfachnutzung und dynamische Programmierung

Projektleitung: Prof. Matthias Rudolph (bis 2018), Prof. Mark Blaschitz (ab 2018), Prof. Marianne Mueller (ab 2018) (Staatliche Akademie der Bildenden Künste Stuttgart)

www.spacesharing.org

Urban Office - Nachhaltige Stadtentwicklung in der Wissensgesellschaft */** Projektleitung: Prof. Dr. Ulrike Gerhard, Dr. Editha Marquardt, Universität Heidelberg

www.uni-heidelberg.de/urbanoffice

Wissensdialog Nordschwarzwald - ein Reallaborprojekt * Projektleitung: Prof. Dr. Barbara Koch, Universität Freiburg www.wissensdialog-nordschwarzwald.de0 


\section{Förderlinie „Reallabore Stadt“}

Asylsuchende in der Rhein-Neckar-Region **

Projektleitung: Prof. Dr. Birgit Werner (Pädagogische Hochschule Heidelberg), PD Dr. Friedhelm Pfeiffer (Leibniz-Zentrum für Europäische Wirtschaftsforschung GmbH Mannheim), Dr. Christina West (Universität Heidelberg, Hochschule Darmstadt), Dr. Georg Mildenberger (Universität Heidelberg)

www.reallabor-asyl.de

BUGA:log - Logistische Nahversorgung im urbanen Raum mit automatisierten Transporteinheiten im Rahmen der Bundesgartenschau 2019 mit Quartiers- und Stadtteilentwicklung „Neckarbogen“"

Projektleitung: Prof. Dr. Tobias Bernecker, Prof. Dr. Nicola Marsden, Prof. Dr.Ing. Raoul Zöllner (Hochschule Heilbronn)

www.bugalog.de

Energielabor Tübingen - Potenziale, Partizipation, Perspektiven

Projektleitung: Prof. Dr. Volker Hochschild (Universität Tübingen)

www.gemeinsam-zur-energiewende.de

GO Karlsruhe - Partizipative Forschung für den Fußgängerverkehr Projektleitung: Prof. Dr.-Ing. Christoph Hupfer, Prof. Dr. Jochen Eckart, Prof. Dr.-Ing. Jan Riel, Prof. Dr.-Ing. Thomas Schlegel (Hochschule Karlsruhe - Technik und Wirtschaft)

www.imm.hs-karlsruhe.de/gokarlsruhe

Reallabor Schorndorf: Zukunftsweisender Öffentlicher Verkehr - Bürgerorientierte Optimierung der Leistungsfähigkeit, Effizienz und Attraktivität im Nahverkehr **

Projektleitung: Mascha Brost, Laura Gebhardt (Deutsches Zentrum für Luft- und Raumfahrt) 
Reallabor Stadt:Quartiere 4.0 - Frühzeitige gestaltende Bürgerbeteiligung für eine nachhaltige Entwicklung in Baden-Württemberg Projektleitung: Mike Letzgus (Universität Stuttgart)

digitale-mitwirkung.de

STADT-RAUM-BILDUNG - Reallabor für die nachhaltige Planung von Bildungslandschaften und die Integration von Aus- und Umbauten von Schulgebäuden $* *$

Projektleitung: Prof. Dr. Marc Kirschbaum, Prof. Andreas Bartels (SRH Hochschule Heidelberg)

www.stadt-raum-bildung.de

* s. auch Steckbrief im Anhang zu Defila und Di Giulio (2018), doi: 10.1007/978-3-65821530-9.

** s. auch Steckbrief im Anhang zu diesem Buch.

Open Access Dieses Kapitel wird unter der Creative Commons Namensnennung 4.0 International Lizenz (http://creativecommons.org/licenses/by/4.0/deed.de) veröffentlicht, welche die Nutzung, Vervielfältigung, Bearbeitung, Verbreitung und Wiedergabe in jeglichem Medium und Format erlaubt, sofern Sie den/die ursprünglichen Autor(en) und die Quelle ordnungsgemäß nennen, einen Link zur Creative Commons Lizenz beifügen und angeben, ob Änderungen vorgenommen wurden.

Die in diesem Kapitel enthaltenen Bilder und sonstiges Drittmaterial unterliegen ebenfalls der genannten Creative Commons Lizenz, sofern sich aus der Abbildungslegende nichts anderes ergibt. Sofern das betreffende Material nicht unter der genannten Creative Commons Lizenz steht und die betreffende Handlung nicht nach gesetzlichen Vorschriften erlaubt ist, ist für die oben aufgeführten Weiterverwendungen des Materials die Einwilligung des jeweiligen Rechteinhabers einzuholen.

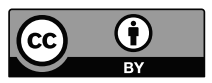


Teil 1

Worauf achten:

Heuristiken zum Verstehen

der Akteure und ihrer

Handlungsbedingungen 


\title{
Kommunale Akteure verstehen: Vorgeschichte der Arbeit in Kommunen beachten
}

\author{
Jochen Eckart, Elke Häußler, Thorsten Erl, Monika Gonser \& Jan Riel
}

\section{Herausforderungen bei der Analyse kommunaler Akteure in Reallaboren}

Kommunen spielen in Reallaboren und in der transdisziplinären und transformativen Forschung allgemein oft eine zentrale Rolle. Aufbau und Organisation einer Kommune sind durchaus komplex und können von jeweils ortsspezifischen Besonderheiten geprägt sein. Insofern ist ein besseres Verständnis von Kommunen und insbesondere den einzelnen Akteuren innerhalb einer Kommune (Fachverwaltung, Verwaltungsspitze, politische Entscheidungsträger) von maßgeblicher Bedeutung für das Gelingen der Zusammenarbeit in solchen Projekten.

Für das bessere Verständnis der kommunalen Akteure in Reallaboren und in anderen transdisziplinären und transformativen Forschungsvorhaben bietet sich das Instrument der Akteursanalyse an (Eckart et al. 2018). Die Akteursanalyse hat sich aus Ansätzen der Politikfeldanalyse (Blum und Schubert 2011), des Strategic Management (Freeman 1984) sowie der Partizipationsforschung (Reed et al. 2009; Zimmermann 2006) entwickelt. Auch im Forschungskontext wird die Akteursanalyse bereits angewandt (Butterworth et al. 2011). Sie dient als Instrument, um die Einbindung der Praxisakteure in transdisziplinären und transformativen Forschungsvorhaben im Detail zu planen. Im Rahmen der Akteursanalyse sind die für die Forschungs-, Praxis- und Bildungsziele eines Vorhabens erforderlichen Akteure (Beecroft et al. 2018) zu identifizieren. Die Akteursanalyse befasst sich zudem mit den Erwartungen der Akteure darüber, wie und in welchem Umfang sie sich an einem Vorhaben beteiligen können oder sollen sowie mit den Möglichkeiten und Grenzen der Akteure. Damit eine Akteursanalyse gelingen kann und in der Folge die Zusammenarbeit in einem Projekt fruchtbar verläuft, ist es wichtig, den Besonderheiten der analysierten Akteure Rechnung zu tragen und nicht mit verkürztem Blick an die Analyse heranzugehen. Es ist also, mit anderen Worten, ein Bewusstsein nötig für mögliche ,blinde Flecken', die die Qualität einer Akteursanalyse beeinträchtigen könnten. 
Basierend auf Erfahrungen aus den vom Ministerium für Wissenschaft, Forschung und Kunst Baden-Württemberg (MWK) seit 2015 geförderten 14 Reallaboren der Förderlinien „Reallabore“ und „Reallabore Stadt" (im Folgenden BaWü-Labs) ${ }^{1}$ und bezogen auf den Akteur Kommune wird dargestellt, inwiefern ,blinde Flecken' existieren können, also Wissenslücken auf Seiten der Wissenschaftler(innen) über die Funktionsweise von und die Arbeit mit verschiedenen kommunalen Akteuren. Diese ,blinden Flecken' können in der Akteursanalyse sowie in der eigentlichen Forschungsarbeit zu empfindlichen Komplikationen führen - sei es, weil nicht die richtigen Ansprechpartner gefunden, weil vorgegebene Entscheidungswege nicht beachtet oder weil ohnehin vorbelastete Beziehungen zwischen Akteuren aus Unkenntnis überstrapaziert werden.

Diesen ,blinden Flecken' sind in diesem Buch drei Kurzbeiträge gewidmet: Ein Beitrag beschreibt die Verteilung von Wissensbeständen innerhalb von Kommunen und inwiefern Kommunen nicht nur als ein (homogener) Ansprechpartner (miss)zu verstehen sind (Erl et al. 2019 in diesem Buch). Ein Beitrag stellt die komplexen Entscheidungswege innerhalb kommunaler Strukturen dar, deren Kenntnis für das Gelingen der Zusammenarbeit von besonderer Bedeutung ist (Gonser et al. 2019 in diesem Buch). Der vorliegende Beitrag widmet sich der Vorgeschichte kommunaler Akteure und zeigt, warum es wichtig ist, sich mit dieser auseinanderzusetzen.

\section{Kontinuierliches Arbeiten in der Kommune}

Die Arbeit in Reallaboren oder in anderen transdisziplinären oder transformativen Forschungsvorhaben ist durch deren Projektcharakter mit einem klaren Anfangsund Endpunkt versehen. Die Arbeit der kommunalen Akteure zeichnet sich hingegen häufig durch ein kontinuierliches Arbeiten vor Ort an einem Thema aus. Thematische Arbeit ist daher aus Sicht der kommunalen Akteure meist kein einmaliges Projekt ohne Bezüge zur Vergangenheit oder zur Zukunft, sondern durch ein zeitliches und organisatorisches Vor-, Neben-, Mit- und Nacheinander geprägt (Streich 2005). Daher ist auch die Mitarbeit in Reallaboren und in verwandten Projekten aus kommunaler Sicht in vorherige und zukünftige Aktivitäten und Projekte eingebunden.

Zwei Teilaspekte sind hierbei zu unterscheiden: Die Kontinuität der Arbeit in einem Thema sowie die Kontinuität der Beziehung zwischen den beteiligten Akteuren. In den Kommunen gibt es bei den meisten Themen bereits einen historischen Vorlauf, an den sich die Akteure aus der Kommune bzw. in der Öffentlichkeit erinnern und worauf sie Bezug nehmen. Viele Themen sind daher in der

1 Für Informationen zu den beiden Förderlinien siehe http://www.reallabore-bw.de (zugegriffen am 20.05.2019). 
Einschätzung und dem Handeln vorgeprägt. Zudem ist das Verhältnis der handelnden Akteure auch durch Vorgeschichten geformt. So kann das für eine konstruktive Zusammenarbeit erforderliche Vertrauen zwischen den Wissenschaftler(inne)n und kommunalen Akteuren nicht innerhalb von Wochen oder wenigen Monaten entstehen, sondern benötigt eine längere Zeit und kann durch vergangene Interaktionen beeinflusst sein. Wenn solche Vorgeschichten negiert werden, entstehen ,blinde Flecken` in der Akteursanalyse.

\section{Die Vorgeschichte als Herausforderung für die Arbeit in und mit Kommunen}

Für die Arbeit in transdisziplinären und transformativen Projekten können sich Hürden ergeben, wenn diese thematisch sensible Punkte oder historisch vorbelastete Orte tangieren. In Themen, in denen hingegen positive Erfahrungen gemacht wurden, sehen sie sich eher mit hohen Erwartungen konfrontiert.

In der Kommune vorbelastete Themen (, heiße Eisen', ,Tabus'), werden von kommunalen Akteuren mit Vorsicht behandelt oder gar gänzlich gemieden. Dadurch kann es zu einem erhöhten Arbeitsaufwand durch zusätzlich erforderliche Kommunikationsprozesse oder zu Verzögerungen beim Projektfortschritt kommen. Dabei können Konflikte, die ihren Ursprung nicht in dem aktuellen Projekt selbst haben, die Zusammenarbeit mit den kommunalen Akteuren in diesem Projekt aber dennoch beeinflussen.

Im Reallabor Nachhaltige Transformation der Textilwirtschaft am Standort Dietenheim (,BaWü-Lab Dietenheim zieht an“) war die Kommunalverwaltung zeitweisen Vorwürfen von Fehlentscheidungen im Hinblick auf ein anderes, vom Reallabor unabhängiges, Projekt vor Ort ausgesetzt. Es entstand für die wissenschaftliche Seite der nicht zu belegende Eindruck, dass man daher seitens der Kommune zu dieser Zeit ,auf Nummer sicher' gehen wollte. So geriet die Planung einer Reallabor-Veranstaltung ins Stocken und konnte erst nach einer Aussprache erfolgreich fortgesetzt werden.

Im Reallabor Asylsuchende in der Rhein-Neckar-Region (,,BaWü-Lab Asylsuchende“) (s. auch Steckbrief im Anhang zu diesem Buch) sollten Fragen der dezentralen Unterbringung von Geflüchteten in einzelnen Stadt- und Ortsteilen wissenschaftlich untersucht werden. Zeitgleich waren die Kommunen auf der sehr konkreten Suche nach Gebäuden und Flächen, die sich zur Unterbringung oder zum Bau von Unterbringungen von Geflüchteten eignen würden. Der kommunale Suchprozess wurde von teils überaus kritischen Diskussionen unter den Bürger(inne)n begleitet. Dieser externe Konflikt wirkte sich auf den Ablauf des Reallabors aus, da die Kommunen teilweise eher zögerlich dem Forschungsanliegen zustimmten. So bestand wohl die Sorge, dass durch das Agieren mehrerer Akteure oder eine möglicherweise unklare Kommunikation der Ziele des Reallabors noch mehr Aufruhr oder möglicherweise Missverständnisse in die 
öffentliche Debatte eingebracht werden könnten. Um diesen Befürchtungen zu begegnen, wurden die einzelnen Forschungsschritte in enger Kommunikation mit den Kommunen vorgenommen und Kommunikationsprozesse detailliert abgesprochen.

Zudem können sich historische Konflikte auf die Zusammenarbeit mit weiteren Praxisakteuren in der Kommune auswirken und zu Verzögerungen oder Hindernissen im Projektverlauf führen.

Im Reallabor GO Karlsruhe - Partizipative Forschung für den Fußgängerverkehr (,,BaWü-Lab GO Karlsruhe“) bestand in einem Stadtteil ein Konflikt durch vorherige fehlgeschlagene Partizipationsaktivitäten. Die Ergebnisse einer weiteren externen Forscher(innen)gruppe trafen in einem Beteiligungsprozess auf starke Vorbehalte seitens der bürgerschaftlichen Akteure vor Ort. Die ablehnende Haltung war jedoch nicht durch die Wissenschaftler(innen) selbst verursacht, sondern begründete sich durch weitere externe Konflikte in einem Neubaugebiet des Stadtteils und durch die Unzufriedenheit mit der kommunalen Verwaltung. Aufgrund dieser historischen Konflikte zwischen externer Forscher(innen)gruppe, kommunaler Verwaltung und bürgerschaftlichen Akteuren verlief die auf das Reallabor bezogene Partizipation in diesem Stadtteil deutlich langsamer und weniger erfolgreich als in anderen Stadtteilen ohne negative Vorgeschichte.

Weiterhin können die Beziehungen zwischen den verschiedenen Akteuren in einem Projekt durch die Vorgeschichte geprägt sein (Eckart et al. 2018; Butterworth et al. 2011). Eine wichtige Grundlage für eine erfolgreiche Zusammenarbeit zwischen den Wissenschaftler(inne)n und den kommunalen Akteuren ist das gegenseitige Vertrauen. Es kann verschieden stark belastbare Vertrauensverhältnisse zwischen den Akteuren geben, die sich auf den Gestaltungsspielraum der Zusammenarbeit in einem Projekt auswirken können.

In manchen BaWü-Labs gab es bestehende und gut funktionierende Arbeitsbeziehungen zwischen den Wissenschaftler(inne)n und den kommunalen Akteuren, die auf eine Zeit vor dem Reallabor zurückgingen. Dadurch bestand bereits ein gegenseitiges Vertrauen und damit verbunden die Bereitschaft der kommunalen Akteure, auch über das gewohnte Verwaltungshandeln hinausgehende Aktivitäten anzugehen. Ist das Vertrauensverhältnis hingegen durch frühere Erfahrungen belastet, kann dies ein deutliches Hindernis in der Zusammenarbeit bilden.

Im Fall des BaWü-Labs GO Karlsruhe war die Konstellation insofern günstig, als einer der Wissenschaftler zuvor viele Jahre in einem der Fachämter in der Kommune beschäftigt war. Die Entscheidungsstrukturen sowie die inhaltlichen Positionen von Verwaltungsakteuren waren daher zum einen den Wissenschaftler(inne)n bekannt, zum anderen konnten aber auch die Akteure der Verwaltung die Anfragen der Wissenschaftler(innen) von vorneherein besser einschätzen. Die fachliche Position und die Arbeitsweise der Wissenschaftler(innen) waren den Mitarbeitenden der Verwaltung ebenfalls bekannt, so dass in verschiedenen Fällen keine besondere Überzeugungsarbeit zu leisten war. Eine solche Ausgangslage ist sicherlich selten. Unabhängig davon erscheinen der Weg über bestehende Verbindungen und das Aufbauen auf bereits vorhandene Vertrauensverhältnisse sinnvoll. 
In Projekten entstehen neue Arbeitsbeziehungen zwischen Wissenschaftler(inne)n und kommunalen Akteuren. Hier ist besondere Aufmerksamkeit auf den Vertrauensaufbau zu legen, um eine produktive Arbeitsbeziehung zu schaffen. Dafür erforderlich sind insbesondere die gegenseitige Akzeptanz der Fachkenntnis sowie ein offener, fairer Umgang miteinander.

Im BaWü-Lab Asylsuchende waren mit Blick auf die Zusammenarbeit mit einigen Akteuren zunächst der gemeinsame Besuch einer Fachveranstaltung sinnvoll, gefolgt von der Konzeption und Durchführung einer Einzelveranstaltung, hin zur Konzeption einer Veranstaltungsreihe und eines ersten experimentellen Projektsettings etc., um schrittweise die Möglichkeit zu geben, das Vertrauen auszuweiten. Auch die ,Lieferung' zunächst einseitiger Beiträge wie z. B. die Aufbereitung von Fachinformationen, die nur bedingt mit dem konkreten Reallabor zu tun hatten, für den kommunalen Akteur aber gerade relevant waren, konnte Vertrauen schaffen.

\section{Berücksichtigung der Vorgeschichte in der Akteursanalyse}

Die Vorgeschichte der Arbeit in einem Thema sowie des Verhältnisses zwischen den Akteuren ist zu analysieren, da sich durch deren Unkenntnis Schwierigkeiten im Ablauf eines Projekts ergeben können. Bewährt hat sich im Rahmen der Akteursanalyse, diese nicht allein auf die gegenwärtigen Rahmenbedingungen der Akteure zu konzentrieren, sondern auch die Vorgeschichte des Themas sowie der Beziehung der Akteure in den Blick zu nehmen. Mögliche Fragen für eine Akteursanalyse, die diesen , blinden Fleck' adressieren, sind:

- Welche Ergebnisse oder Erfahrungen aus vorangegangenen Prozessen oder Projekten im sozialräumlichen und thematischen Gebiet des Projekts gibt es?

- Wie etabliert sind die Arbeitsbeziehungen und das Vertrauen zwischen den Akteuren aus vorherigen Prozessen oder Projekten?

Diese Fragen können im Rahmen von Interviews mit kommunalen Insidern geklärt werden. Dies kann weiter durch Interviews mit ausgewählten externen Praxisakteuren ergänzt werden, die mit dem entsprechenden kommunalen ,Feld“ vertraut sind und eine Einschätzung, von außen‘ bieten können.

\section{Literatur}

Beecroft, R., Trenks, H., Rhodius, R., Benighaus, C., \& Parodi, O. (2018). Reallabore als Rahmen transformativer und transdisziplinärer Forschung: Ziele und Designprinzipien. In R. Defila \& A. Di Giulio (Hrsg.), Transdisziplinär und transformativ forschen. Eine Methodensammlung. (S. 75-100). Wiesbaden: Springer VS. doi: 10.1007/978-3-65821530-9 4.

Blum, S., \& Schubert, K. (2011). Politikfeldanalyse. Wiesbaden: Springer VS. 
Butterworth, J., McIntyre, P., \& da Silva Wells, C. (Hrsg.). (2011). Switch in the City: Putting Urban Water Management to the Test. Den Haag: IRC International Water and Sanitation Centre.

Eckart, J., Ley, A., Häußler, E., \& Erl, Th. (2018). Leitfragen für die Gestaltung von Partizipationsprozessen in Reallaboren. In R. Defila \& A. Di Giulio (Hrsg.), Transdisziplinär und transformativ forschen. Eine Methodensammlung (S. 105-135). Wiesbaden: Springer VS. doi: 10.1007/978-3-658-21530-9_6.

Freeman, R. E. (1984). Strategic Management: A Stakeholder Approach. Boston: Pitman.

Reed, M. S., Graves, A., Dandy, N., Posthumus, H., Hubacek, K., \& Morris, J. (2009). Who's in and why? A typology of stakeholder analysis methods for natural resource management. Journal of Environmental Management, 90, (S. 1933-1949).

Streich, B. (2005). Stadtplanung in der Wissensgesellschaft. Ein Handbuch. Wiesbaden: VS Verlag für Sozialwissenschaften.

Zimmermann, A. (2006). Instrumente zur Akteursanalyse - 10 Bausteine für die partizipative Gestaltung von Kooperationssystemen. Eschborn: Deutsche Gesellschaft für Technische Zusammenarbeit (GTZ).

\section{Die zwei weiteren Beiträge in diesem Buch zu ,blinden Flecken ‘}

Erl, Th., Gonser, M., Eckart, J., Häußler, E., \& Riel, J. (2019). Kommunale Akteure verstehen: Verteilte Wissensbestände in Kommunen. In R. Defila \& A. Di Giulio (Hrsg.), Transdisziplinär und transformativ forschen, Band 2. Eine Methodensammlung (S. 139145). Wiesbaden: Springer VS.

Gonser, M., Riel, J., Eckart, J., Erl, Th., \& Häußler, E. (2019). Kommunale Akteure verstehen: Komplexe Entscheidungswege in Kommunen. In R. Defila \& A. Di Giulio (Hrsg.), Transdisziplinär und transformativ forschen, Band 2. Eine Methodensammlung (S. 85-91). Wiesbaden: Springer VS.

Open Access Dieses Kapitel wird unter der Creative Commons Namensnennung 4.0 International Lizenz (http://creativecommons.org/licenses/by/4.0/deed.de) veröffentlicht, welche die Nutzung, Vervielfältigung, Bearbeitung, Verbreitung und Wiedergabe in jeglichem Medium und Format erlaubt, sofern Sie den/die ursprünglichen Autor(en) und die Quelle ordnungsgemäß nennen, einen Link zur Creative Commons Lizenz beifügen und angeben, ob Änderungen vorgenommen wurden.

Die in diesem Kapitel enthaltenen Bilder und sonstiges Drittmaterial unterliegen ebenfalls der genannten Creative Commons Lizenz, sofern sich aus der Abbildungslegende nichts anderes ergibt. Sofern das betreffende Material nicht unter der genannten Creative Commons Lizenz steht und die betreffende Handlung nicht nach gesetzlichen Vorschriften erlaubt ist, ist für die oben aufgeführten Weiterverwendungen des Materials die Einwilligung des jeweiligen Rechteinhabers einzuholen.

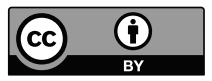




\title{
Unterschiedliche Handlungslogiken in transdisziplinären und transformativen Forschungsprojekten - Welche Risikokulturen entwickeln sich daraus und wie lassen sie sich konstruktiv einbinden?
}

\author{
Monika Gonser, Jochen Eckart, Charlotte Eller, Katharina Köglberger, \\ Elke Häußler \& Felix M. Piontek
}

\section{$1 \quad$ Einleitung}

Transdisziplinäre (und transformative) ${ }^{1}$ Forschungsprojekte finden an institutionellen Schnittstellen gesellschaftlicher Teilsysteme statt, wie beispielsweise Wissenschaft, Recht, Politik, Wirtschaft sowie Zivilgesellschaft. Vertreter(innen) aus den verschiedenen Teilsystemen arbeiten und forschen gemeinsam. Ein transdisziplinäres Vorgehen setzt per se eine Zusammenarbeit von Vertreter(inne)n mindestens zweier verschiedener Teilsysteme voraus, wovon das eine immer das Teilsystem der Wissenschaft sein muss. Systemtheoretisch betrachtet (bspw. Luhmann 1984; Schimank 2007) folgen Vertreter(innen) der einzelnen Teilsysteme jeweils spezifischen Handlungslogiken. ${ }^{2}$ Durch die Zusammenarbeit in einem Projekt werden die unterschiedlichen Handlungslogiken der Teilsysteme spürbar. Eine solche Zusammenarbeit kann und soll Synergien erzeugen, sie kann jedoch auch zu Konflikten zwischen den Institutionen oder Akteuren der Teilsysteme führen, die es zu erkennen und zu beheben gilt.

Sowohl Synergien als auch Konflikte im Zuge der Zusammenarbeit unterscheiden sich in ihrer Intensität je nach Zusammensetzung des Projektteams. Zudem bringt ein transformativer Forschungsansatz mit seinem Anspruch, Veränderungs-

1 Die Autor(innen) möchten einen Beitrag zur Methodik transdisziplinärer Forschung leisten, die auch transformativ vorgehen kann, aber nicht muss. Diese doppelte Ansprache wird im Beitrag durch die Klammersetzung ,transdisziplinär (und transformativ)“" ausgedrückt.

2 Das Verhältnis von Teilsystem und Akteur wird in Kapitel 2 erklärt. 
prozesse anzustoßen, sowie der damit einhergehenden Notwendigkeit, meist experimentelle Anwendungen (z. B. Realexperimente ${ }^{3}$ ) in der Praxis durchzuführen, ein intensiviertes Aufeinandertreffen unterschiedlicher Teilsysteme mit sich. Denn die transformativen Aktivitäten beschränken sich oft nicht allein auf ein gesellschaftliches Teilsystem. Sie tangieren meist, und häufig auch gewollt, mehrere Teilsysteme. Um in einem Projekt eine hohe Synergie zu erzielen und Konflikte zu vermeiden bzw. zu lösen, empfiehlt sich eine vertiefte Analyse der Handlungslogiken der am Projekt beteiligten Teilsysteme. Durch den transdisziplinären Charakter eines Forschungsprojekts werden zudem auch unterschiedliche Definitionen, Wahrnehmungen und Einschätzungen von Risiken sowie unterschiedliche Bereitschaften, diese zu übernehmen, deutlich und bedeutsam. Diese sogenannten Risikokulturen, die sich je nach Handlungslogik des Teilsystems unterscheiden, werden in diesem Beitrag hergeleitet und erklärt.

Der Beitrag bietet einen Analyserahmen, der bei der Konzipierung von transdisziplinären (und transformativen) Forschungsprojekten helfen soll, die Synergien zwischen den Teilsystemen zu stärken und mögliche Konflikte frühzeitig zu erkennen. Vor Beginn eines Projekts können damit bereits potenzielle Konflikte je nach beteiligten Vertreter(inne)n der verschiedenen Teilsysteme abgeschätzt, mögliche Vorkehrungen getroffen und die nötige Transparenz innerhalb des Projektteams hergestellt werden. Ebenso kann die analytische Betrachtung auch bei bereits laufenden Forschungsprojekten helfen, Konflikte zu verstehen und $\mathrm{zu}$ lösen. Sollten während der Durchführung eines Projekts (unerwartete) Konflikte auftreten, kann dieser Analyserahmen eine Hilfestellung zur Selbstreflexion sein, indem er dazu beiträgt, dass die Handlungslogiken der beteiligten Vertreter(innen) der verschiedenen Teilsysteme nachvollziehbar werden. Für die Leser(innen) soll dieser Beitrag folglich eine Hilfestellung sowohl bei der Planung als auch während der Durchführung eines transdisziplinären (und transformativen) Forschungsprojekts sein.

Die analytische Aufarbeitung der Erfahrungen mehrerer Reallabore (BaWüLabs $^{4}$ ) und ein Quervergleich dieser Erfahrungen bilden die Basis für diesen Beitrag. Diese BaWü-Labs wurden in unterschiedlichen Kontexten realisiert und widmeten sich unterschiedlichen Forschungsthemen. Konflikte zwischen den Institutionen und Akteuren verschiedener Teilsysteme sowie Risiken und Chancen

3 Realexperimente sind Interventionen, die gemeinsam mit Akteuren aus der Praxis konzipiert und durchgeführt werden, und die im Rahmen eines Reallabors oder verwandten Forschungsformats durchgeführt und wissenschaftlich begleitet werden (s. Arnold und Piontek 2018, S. 146ff.).

4 Das Ministerium für Wissenschaft, Forschung und Kunst Baden-Württemberg fördert(e) seit 2015 in zwei Förderlinien 14 sogenannte Reallabore, die im Folgenden als ,BaWü-Labs' bezeichnet werden (s. auch http://www.reallabore-bw.de, zugegriffen am 20.05.2019). 
werden mit Beispielen aus diesen BaWü-Labs illustriert, und Lösungsansätze, die in den BaWü-Labs gewählt wurden, werden reflektiert. Ein Reallabor ist eines von vielen möglichen transdisziplinären (und transformativen) Forschungsformaten, und zwar eines, das gesellschaftswissenschaftliche Forschung anknüpfungsfähig für die Praxis macht, indem es Lösungswissen zu gesellschaftlichen Fragen durch häufig experimentelle Anwendungen erprobt und Faktoren einer potenziellen Übertragbarkeit reflektiert (z. B. Schneidewind und Singer-Brodowski 2014; Arnold und Piontek 2018). Die Beispiele im vorliegenden Text stammen aus den BaWü-Labs, da die Autor(inn)en in diesem Kontext gearbeitet haben. Der präsentierte Analyserahmen sowie die dargelegte Aufarbeitung der BaWü-Labs dürften jedoch für transdisziplinäre (und transformative) Forschungsprojekte im Allgemeinen gelten, eine Verengung auf die BaWü-Labs oder das Format Reallabor wurde deshalb bewusst nicht vorgenommen.

Im Folgenden werden zunächst der Analyserahmen, der zur Entfaltung der Handlungslogiken verwendet wird, dargestellt und der Begriff Risiko eingeführt. Für die Teilsysteme Wissenschaft, Politik, Recht, Wirtschaft und Zivilgesellschaft werden dann unter anderem deren Funktionen, Leitwerte, Leitdifferenzen, Entscheidungsstrukturen, Finanzierungen sowie Risikoverständnis und Risikomanagement erklärt. Die aus den Handlungslogiken resultierenden Risikokulturen und damit einhergehende Differenzen bezüglich der Wahrnehmung von Risiken und der Bereitschaft, diese einzugehen, werden dargestellt. Ebenso werden das Verständnis von und der Umgang mit Misserfolgen erläutert. In der transdisziplinären Realität ergeben sich häufig spezifische Chancen und Konflikte aus dem Zusammentreffen der partizipierenden Vertreter(innen) der Teilsysteme. Diese werden mithilfe von Beispielen aus den BaWü-Labs kurz diskutiert. Die in den BaWü-Labs gewählten Lösungsansätze dienen den Leser(inne)n als Anregung bei der Durchführung des eigenen transdisziplinären (und transformativen) Forschungsprojekts.

\section{Analyserahmen}

Um die unterschiedlichen Kontexte von Akteuren in solchen Forschungsprojekten $\mathrm{zu}$ verdeutlichen und damit diskutierbar zu machen, wird an dieser Stelle das systemtheoretische Konzept der Handlungslogiken von funktional definierten gesellschaftlichen Teilsystemen eingeführt. Anschließend wird der Analyserahmen der Risikokultur dargestellt. Obwohl in der Alltagskommunikation und in der Wissenschaft oft verwendet, gibt es kein allgemein gültiges Verständnis, wie der Begriff Risiko definiert und worauf er angewendet wird, dies variiert je nach Disziplin, gesellschaftlichem Teilsystem und sozialem Kontext (Weißensteiner 2014). Nach Fischer (2016) ist Risiko ein „,contested concept“", welches zwischen den Disziplinen sowie teilweise auch innerhalb der Disziplinen unterschiedlich definiert wird. Die darüber hinaus auch in der Anwendung vielfältige Begriffs- 
auffassung erklären Bechmann und Stehr (2000) damit, dass Risiko ein soziales Konstrukt ist, das abhängig vom sozialen Kontext unterschiedliche Anwendungen findet. Auch Fischer (2016) sowie Rayner (1992) gehen davon aus, dass Risiken sozial vermittelt sind und nicht unabhängig vom gesellschaftlichen Kontext verstanden werden. Ausgehend vom Verständnis, dass Risiko sozial konstruiert ist, werden von individuellen und kollektiven Akteuren in transdisziplinären (und transformativen) Forschungsprojekten jeweils andere Definitionen von Risiko genutzt und der Begriff unterschiedlich angewendet.

\subsection{Was wird unter Teilsystemen und Handlungslogiken verstanden?}

Systemtheoretische Ansätze gehen in Anlehnung an Luhmann (1984) von einer funktionalen Differenzierung von Gesellschaften aus (beispielsweise Baecker 2016; Reichel 2012; Schimank 2012). In diesem Ausdifferenzierungsprozess bilden sich in Abhängigkeit von der je spezifisch zu erfüllenden Funktion arbeitsteilig vorgehende Teilsysteme heraus. $\mathrm{Zu}$ diesen Teilsystemen gehören etwa das politische System, das Rechtssystem, das Wissenschaftssystem oder das Wirtschaftssystem. Diese funktionieren selbstreferenziell, das heißt, sie beziehen sich in ihrer Handlungslogik einzig auf sich selbst. Luhmann analysiert dabei ausschließlich Kommunikation, also die Frage, wo ein Austausch von Informationen stattfindet und anschlussfähig ist und wo nicht. Individuen - und somit auch Akteure - kommen in seiner Analyseperspektive nicht vor. Der vorliegende Beitrag nimmt jedoch keine Analyseperspektive im strengen Luhmann'schen Sinne ein. Vielmehr folgt er Schimank, der aufbauend auf Luhmann bezüglich der Teilsysteme auch die in ihnen handelnden Akteure thematisiert. Schimank erklärt diese Ausweitung damit, dass die funktionale Ausdifferenzierung einer Gesellschaft auf lange Sicht nur dann erhalten bleibt, wenn es in der Gesellschaft im ausreichenden Maß Akteure gibt, die sich die jeweils verschiedenen Handlungslogiken zu eigen machen und ihre jeweilige Aufrechterhaltung unterstützen (Schimank 2007). ${ }^{5}$

5 Schimank erläutert das Eingebundensein in Teilsysteme und die darin vorhandenen unterschiedlichen Akteursebenen folgendermaßen: „Aus der Sicht eines individuellen Gesellschaftsmitglieds heißt funktionale Differenzierung: All sein Streben, Denken und Handeln wird je nach Ausprägung seines Inklusionsprofils, also seines Einbezugs in die verschiedenen Teilsysteme, von etwa einem Dutzend Leitsternen gelenkt - faktisch sind es in den allermeisten Fällen nicht mehr als ein halbes Dutzend, die das Gros der Lebensführung nachhaltig prägen [...]. Für Organisationen als zweiten Typ von Akteur in der modernen Gesellschaft gilt das noch viel zugespitzter. Während Individuen durch multiple Partialinklusion in einer Mehrzahl von Teilsystemen charakterisiert sind, gehören die meisten Organisationen einem und nur einem Teilsystem an [...]. Unternehmen beispielsweise sind Wirtschaftsorganisationen und folgen als solche 
Die jeweilige Handlungslogik eines Teilsystems leitet sich aus einem nur für dieses Teilsystem gültigen Leitwert ab. Dieser Leitwert bringt „die fraglose oberste Orientierung allen Handelns" (Schimank 2007, S. 116) im betreffenden Teilsystem zum Ausdruck. Aus dem jeweiligen Leitwert wiederum ergibt sich die zugehörige Leitdifferenz, die als „Weichensteller“ (Schimank 2007, S. 116) dient. Die Leitdifferenz hat zwei Ausprägungen, beispielsweise für das Wissenschaftssystem ,wahr' und ,unwahr'. Nur die Kommunikationsinhalte, die im Wissenschaftssystem als (vorläufig), wahr ${ }^{`}$ angenommen werden, sind zur weiteren Erkenntnisproduktion anschlussfähig (Egner 2008). Mithilfe des Leitwerts wird die Arbeitsteilung in der Gesellschaft ausdifferenziert und ausbalanciert. Allerdings führt dies auch dazu, dass ,die Belange der anderen Teilsysteme nur höchst widerwillig berücksichtigt" werden (Schimank 2012, S. 114).

In Kapitel 3 werden die zentralen Eigenschaften der in den BaWü-Labs vertretenen Teilsysteme Wissenschaft, Politik, Recht, Wirtschaft und Zivilgesellschaft dargestellt. Abgeleitet aus dem systemtheoretischen Analyseansatz werden Funktion, Leitwert und Leitdifferenz des jeweiligen Teilsystems dargestellt. Zudem werden Qualitätssicherung, Entscheidungsstrukturen und Finanzierungen für die Teilsysteme kurz beschrieben. Diese zentralen Aspekte werden anhand folgender Leitfragen für jedes Teilsystem dargestellt:

- Was ist die Funktion des Teilsystems, an welchem Leitwert und an welcher Leitdifferenz orientiert es sich?

- Wie sieht Qualitätssicherung im jeweiligen Teilsystem aus?

- Wie werden Entscheidungen im jeweiligen Teilsystem getroffen?

- Wie gestalten sich die Finanzierungszusammenhänge des jeweiligen Teilsystems?

Abschließend wird die Relevanz der jeweiligen Handlungslogik für die Zusammenarbeit in transdisziplinären (und transformativen) Forschungsprojekten in Abgrenzung zu Handlungslogiken anderer Teilsysteme dargestellt.

dem binären Code dieses Teilsystems gleichsam monomanisch - Gesichtspunkte anderer Teilsysteme wie etwa rechtliche Regelungen oder wissenschaftliche Erkenntnisse tauchen, wie bereits erwähnt, immer nur als untergeordnete Randbedingungen der Orientierung an Zahlungsfähigkeit auf. [...] Nicht einzelne Akteure jeweils für sich, sondern erst deren handelndes Zusammenwirken erzeugt, im Wechselspiel mit handlungsprägenden Strukturen, soziale Dynamiken. Dieses handelnde Zusammenwirken kann zwar prinzipiell dauerhaft mäandern, ohne dass sich feste Konstellationsstrukturen spieltheoretisch als Gleichgewicht konzipierbar - herausbilden; der Regelfall, gerade im Rahmen engmaschiger teilsystemischer Programmstrukturen, sind jedoch dauerhafte, manchmal sehr langlebige Strukturmuster" (Schimank 2007, S. 208). 
Obwohl nach Schimank (2007) deutlich wird, dass Organisationen in ihrer Handlungslogik zumeist nur einem Teilsystem zuzuordnen sind, gibt es immer wieder auch solche, die mehrere Handlungslogiken in sich vereinen und damit einen hybriden Akteur darstellen. Im Kontext der BaWü-Labs traf dies vor allem auf Kommunen zu. Diese vereinen Aspekte sowohl der Handlungslogik des Teilsystems Recht als auch solche des Teilsystems Politik in sich, da sie sowohl die Rechtspflege zur Aufgabe haben als auch, über die kommunale Hierarchie, Gestaltungsaufgaben aus der Politik - beispielsweise durch den oder die (Ober-)Bürgermeister(in) oder den Gemeinderat - übertragen bekommen.

\subsection{Was wird unter Risiko und Risikokulturen verstanden?}

Wie eingangs des Kapitels dargelegt, ist die Auffassung von Risiko sozial konstruiert. Da die Akteure in transdisziplinären (und transformativen) Forschungsprojekten aus verschiedenen gesellschaftlichen Teilsystemen stammen, haben sie auch jeweils eine spezifische Auffassung von Risiko, die in solchen Projekten aufeinandertreffen. Für die Analyse der verschiedenen Risikoauffassungen wird auf das begriffliche Instrumentarium der Risikoforschung zurückgegriffen.

Vereinfacht können eine sozialwissenschaftliche und eine mathematische Definition von Risiko unterschieden werden. In den Ingenieurwissenschaften und der Versicherungswirtschaft wird ein mathematischer (technologischer und ökonomischer) Begriff von Risikoverwendet. So beschreibt die VDI-Richtlinie 4006 Risiko als die Kombination aus der Häufigkeit und der Auswirkungen eines zu einem Schaden führenden Ereignisses (VDI 4006 Blatt 1). Risiko ist so verstanden ein Produkt aus der Wahrscheinlichkeit und der Schadenshöhe eines Ereignisses. Damit verbunden ist eine mathematische Auffassung von Risiko, die versucht, das Risiko zu kalkulieren und damit zu managen sowie zu kontrollieren (Bonß 2011). Motel und Richter (2016) bieten eine sozialwissenschaftliche Definition von Risiko: alle potenziellen Bedrohungen für die Zielerreichung eines individuellen oder kollektiven Akteurs. In einer solchen Auffassung von Risiko werden auch die aus Sicht des mathematischen Verständnisses häufig paradoxen Entscheidungen von Akteuren, ein Risiko einzugehen, berücksichtigt (Bonß 2011). Das für beide Auffassungen von Risiko zentrale Verständnis von Schäden wird im Nachfolgenden dargestellt.

Aus der sozialwissenschaftlichen Auffassung von Risiko ergibt sich das Konzept der Risikokultur, das unterschiedlich definiert wird (z. B. Hoitsch et al. 2005; Fischer 2016; Jahner und Kremar 2005). So bezeichnet der Begriff Risikokultur ein Gerüst aus Praktiken und Deutungen, durch die Risiken analysiert und gemanagt werden. Bei der Analyse von Risikokulturen wird betrachtet, wie die Akteure das Risiko wahrnehmen, welche Haltung die Akteure zum Risiko haben und wie die Akteure mit dem Risiko umgehen (Jahner und Kremar 2005; Fischer 2016). 
Die für die spezifische Analyse der Risikokultur von Akteuren in transdisziplinären (und transformativen) Forschungsvorhaben wie Reallaboren relevanten Fragestellungen werden im Folgenden hergeleitet.

Die Akteure berücksichtigen unterschiedliche Phänomene als Schäden. Nach Auffassung der Versicherungswirtschaft sind Schäden unfreiwillige Einbußen, die ein Akteur an einem geschützten Gut oder Interesse erleidet (Möller und Bruck 1980). Aus Sicht des Risikomanagements versteht Link (2001) unter Schäden die von einem Akteur negativ bewerteten Folgen eines Ereignisses. Schäden sind somit alle Beeinträchtigungen von Zielen oder Gütern von Akteuren durch externe Ereignisse; diese Beeinträchtigungen können ökonomischer, rechtlicher oder politischer Natur sein. Ein Schaden kann also auch ein Misserfolg von transdisziplinären (und transformativen) Forschungsprojekten oder Teilen davon sein, wenn diese aus Sicht mindestens der Akteure eines Teilsystems, im Sinne der jeweiligen Handlungslogik, schlechte, enttäuschende oder negative Ergebnisse oder Konsequenzen haben.

Zudem ist zu unterscheiden, ob sich die Auffassung von Risiko allein auf Schäden konzentriert oder ob auch Chancen betrachtet werden. So fokussiert die Definition von Motel und Richter (2016) auf Schäden. Kromschröder (2003) unterscheidet hingegen beim Risiko eine negative Seite der Schäden, die durch ein Ereignis entstehen können, und eine positive Seite der Chance, ein Ziel zu erreichen. Im Kontext des Risikomanagements wird unter Chance ein ungewisses Ereignis beschrieben, das sich, sollte es eintreffen, positiv auf das Erreichen eines Ziels auswirkt (Link 2001). Eine Chance stellt, so gesehen, der Erfolg von transdisziplinären (und transformativen) Forschungsprojekten oder Teilen davon dar, wenn diese gemäß der Handlungslogik mindestens eines Teilsystems gut gelaufen sind. Im Kontext des Risikomanagements besteht häufig die Annahme, dass Chancen nicht ohne die Gefahr von Schäden zu haben sind. Folglich führt die Meidung von Schäden zu einem Verzicht auf Chancen (Kromschröder 2003). Für das Verständnis der Risikokultur verschiedener gesellschaftlicher Teilsysteme ist zu analysieren, welche Schäden nach deren Handlungslogik berücksichtigt werden, und ob die Akteure allein die Gefahr von Schäden oder auch die Abwägung von Schäden und Chancen berücksichtigen.

Bei der Analyse der Risikokultur ist zudem zu betrachten, welche Eintrittswahrscheinlichkeit bei Schäden und welche Schadenshöhe ein Akteur gewillt ist, einzugehen, um eine Chance der Zielerreichung wahrzunehmen (Jahner und Kremar 2005). Um die Risikokultur verschiedener gesellschaftlicher Teilsysteme zu analysieren, ist also die sich aus der Handlungslogik ergebende Risikoaffinität oder Risikoaversion zu beschreiben. Risikoaffinität besteht, wenn bei einer Auswahl die Alternative mit der höchsten erwarteten Zielerreichung bevorzugt wird, auch wenn damit höhere Risiken verbunden sind. Risikoaversion ist die Wahl der 
Alternativen mit dem geringeren Risiko hinsichtlich des Eintretens des Ereignisses bzw. des geringstmöglichen Schadens.

Die Risikokultur umfasst auch das Risikomanagement als die Steuerungsaufgabe der Identifikation, des Bewertens, der Steuerung sowie der Kommunikation von Risiken z. B. einer Organisation (DIN ISO 31000). Die Risikoidentifikation stellt die erste Phase des Risikomanagementprozesses dar. Mittels einer systematischen Identifikation werden mögliche Risiken festgehalten. Darauf aufbauend erfolgt die Risikobewertung, um anschließend Maßnahmen zur Risikosteuerung zu ergreifen (Weißensteiner 2014, S. 18ff.). Risikomanagement hat zum Ziel, die Handlungsfähigkeit von Akteuren gegenüber eventuellen Hindernissen und Krisen zu stärken (Schmitz und Wehrheim 2006). Dies kann auch Maßnahmen des Projektmanagements umfassen, soweit sich diese auf den Umgang mit Risiken beziehen. Das Risikomanagement von Akteuren hängt von der jeweiligen Risikokultur von Organisationen (im weiteren Sinne auch gesellschaftlichen Teilsystemen) ab (Douglas 1992).

Die identifizierten Fragen für die Analyse der Risikokultur der verschiedenen gesellschaftlichen Teilsysteme sind:

- Welche Schäden und Chancen werden im Teilsystem berücksichtigt?

- Wie risikoaffin oder risikoavers ist die Handlungslogik des Teilsystems?

- Welches Risikomanagement wird im Teilsystem üblicherweise betrieben?

Diese Fragen werden im folgenden Kapitel für die verschiedenen Teilsysteme ebenfalls beantwortet, wobei jeweils Bezug darauf genommen wird, wie sich die Risikokultur eines Teilsystems bezogen auf transdisziplinäre (und transformative) Forschungsprojekte manifestiert.

\section{Teilsystemspezifische Risikokulturen in transdisziplinären (und transformativen) Forschungsprojekten}

Im Folgenden wird analysiert, wie die durch die Handlungslogiken der gesellschaftlichen Teilsysteme geprägten Risikokulturen der Akteure deren Wahrnehmung und Handeln in Bezug auf Risiken in Reallaboren und anderen transdisziplinären (und transformativen) Forschungsprojekten leiten. Dabei werden die Teilsysteme Wissenschaft, Politik, Recht, Wirtschaft und Zivilgesellschaft berücksichtigt. Die Analyse orientiert sich an den aus dem Analyserahmen hergeleiteten Fragen (s. Kapitel 2). Nicht eingegangen wird auf Bewertungen von Risiken aufgrund von Persönlichkeitsstrukturen und anderen Handlungsvoraussetzungen, die sich aus der Person und nicht aus der Handlungslogik eines Teilsystems ergeben. 


\subsection{Wissenschaft}

Wissenschaft - Der Begriff der Wissenschaft bezieht sich hier zum einen auf Einrichtungen, die aufgrund ihrer gesellschaftlichen Aufgabe, ihrer Struktur und ihrer diskursiven Einbindung der Forschung zuzurechnen sind. Lehre hingegen wird im vorliegenden Zusammenhang ebenso wenig reflektiert wie Forschung, die überwiegend auf ein kommerzielles Ziel hin betrieben wird. Zum anderen bezieht sich der Begriff auf individuelle Gesellschaftsmitglieder, die aufgrund individueller formaler Qualifikation und institutioneller Anbindung an Wissenschaftseinrichtungen der Wissenschaft zugerechnet werden.

Funktion, Leitwert, Leitdifferenz: Nach Schimank (2012) übernimmt das Teilsystem Wissenschaft die Funktion des Wissenserwerbs und der Wissensreflexion einer Gesellschaft. Entsprechend richtet sich die wissenschaftliche Handlungslogik am Leitwert der Wissenschaft aus, dem Streben nach Wahrheit und Erkenntnis. Im wissenschaftlich forschenden Sinn wird anhand der im Teilsystem Wissenschaft zugrunde gelegten Leitdifferenz , wahr/unwahr' entschieden, welche Informationen für das Teilsystem anschlussfähig sind. ,Unwahr“ ist dabei als ,falsifizierbar' zu verstehen. Sowohl die Veröffentlichung, wahrer' als auch ,unwahrer' Informationen oder Interpretationen kann wissenschaftlich produktiv sein - beispielsweise, wenn sie anschließend widerlegt werden. Das heißt, ein in diesem Sinne unwahres, weil eine Hypothese falsifizierendes, aber auch ein die Hypothese nicht bestätigendes oder unerwartetes Ergebnis ist aus Sicht der Wissenschaft erstmal unproblematisch, weil es für den Erkenntnisgewinn trotzdem relevant ist, indem es wissenschaftliches Denken auf neue Wege leitet.

Qualitätssicherung: Im Teilsystem Wissenschaft hat sich ein System der Qualitätssicherung entwickelt, das im Wesentlichen in zwei Ansätzen zum Ausdruck kommt. Erstens wird versucht, über ein Ausbildungssystem mit klarer Hierarchie in Form unterschiedlicher akademischer Statusgruppen, Umfang und Qualität der erworbenen inhaltlichen und methodischen Kompetenzen abzubilden, um daraus ein weiteres Mitwirken am Teilsystem Wissenschaft abzuleiten. Zweitens werden über ein umfassendes System der Peer-Begutachtung und des Peer-Diskurses Forschungsprojekte und -ergebnisse vor- und nachbereitend reflektiert, eingeordnet, verworfen oder weiterentwickelt. Dadurch wird Vorgehensweisen und Ergebnissen die notwendige Qualität zugesprochen. Durch die Anzahl der Veröffentlichungen und Zitate in peer-reviewten Formaten kann die Anschlussfähigkeit und Verwertbarkeit des forschenden Handelns aus der Perspektive des Teilsystems Wissenschaft nachgewiesen werden.

Entscheidungsstrukturen: Was die hierarchische Strukturierung des Teilsystems Wissenschaft betrifft, so sind Führungspositionen und Entscheidungsmacht an akademische Erfolge und Qualitätssiegel geknüpft, mit dem Professorentitel als dem weitestreichenden Status- und Hierarchiemerkmal. Zuständigkeiten und 
Verantwortungsumfang sind üblicherweise von der akademischen Qualifikationsstufe abhängig. Daneben sind aber auch die Anzahl der Zitierungen und Publikationen, die Höhe eingeworbener Förderungen etc. Indikatoren akademischen Erfolgs. Führungs- und Arbeitsstrukturen in der Wissenschaft unterscheiden sich zum Beispiel in Abhängigkeit von der Disziplin. Verallgemeinernd lässt sich sagen, dass strategische und grundsätzliche inhaltliche Entscheidungen zumeist der professoralen Ebene zugeordnet werden, operativ-inhaltliche eher der akademischen, nicht-professoralen Ebene. Nicht-inhaltliche, also administrative $\mathrm{Zu}-$ ständigkeiten fallen häufig, aber nicht ausschließlich, der nicht-akademischen Ebene zu.

Finanzierung: Forschung wird üblicherweise aus zwei Quellen finanziert, die aus der gleichen Institution kommen können, aber nicht müssen. Die meisten Forschungseinrichtungen erhalten eine Grundförderung, aus der feste Stellen, Räume, Anlagen etc. finanziert werden und in deren Rahmen (nebst Lehre) auch Forschung betrieben wird. Der größere Anteil der Forschungsfinanzierung geht über Drittmittelförderungen ein, die die Akteure forschungsprojektbezogen einwerben müssen. Darüber hinaus gibt es auch „Inter-Systembeziehungen“ (Schimank 2007, S. 119) beispielsweise zum Teilsystem Wirtschaft oder dem Teilsystem Politik. Diese tragen - oft über Forschungsfinanzierungen - Nutzungserwartungen und Ergebniserwartungen an die Wissenschaft heran.

Schäden und Chancen: Ein Schaden entsteht für die Wissenschaft nur, wenn im Rahmen eines transdisziplinären (und transformativen) Forschungsprojekts kein neues Wissen gewonnen werden kann und daher keine Aussagen im Sinne der Leitdifferenz von ,wahr/unwahr' getroffen werden können. Wie bereits dargelegt, bildet es für die Wissenschaft keinen Schaden, wenn durch die Forschung in einem solchen Projekt nicht das erwartete Ergebnis erzeugt wird. Dies wird als Erkenntnisschritt akzeptiert. Ein Schaden entsteht damit vor allem, wenn beispielsweise ein geplantes Realexperiment nicht umgesetzt wird und in der Folge keinerlei Daten zum Untersuchungsgegenstand vorliegen. Das Risiko des Schadens, dass kein Wissen gewonnen werden kann, ist bei transdisziplinären (und transformativen) Forschungsprojekten aufgrund der zahlreichen externen Einflussfaktoren sowie Unwägbarkeiten von Forschung in der , realen Welt' höher als bei Forschung unter kontrollierten Bedingungen. Akteure aus der Wissenschaft nehmen jedoch das mit transdisziplinären (und transformativen) Forschungsprojekten verbundene höhere Risiko von Schäden in Kauf, da sie höhere Chancen sehen, bestimmte Wissensbestände zu gewinnen sowie die Ergebnisse der Forschung schneller und direkter zur Umsetzung zu bringen.

Im Reallabor für nachhaltige Mobilitätskultur (,,BaWü-Lab RNM“) wurden ursprünglich sechs verschiedene Realexperimente zur Durchführung ausgewählt (vgl. Puttrowait et al. 2018). Aus verschiedenen Gründen kam es bei zwei Realexperimenten schließlich doch nicht zur Umsetzung. Während bei einem Fall relativ frühzeitig ein 
Schlussstrich gezogen werden konnte, flossen im anderen Fall über einen längeren Zeitraum Ressourcen in die Bearbeitung des Experiments. Die durch das Nichtzustandekommen der Realexperimente freiwerdenden Ressourcen konnten zum einen für die Durchführung der übrigen Realexperimente eingesetzt werden, zum anderen konnten Forschungsdaten auf anderem Wege erhoben werden, wodurch eine teilweise Kompensation gelang.

Im Reallabor GO Karlsruhe - Partizipative Forschung für den Fußgängerverkehr (, BaWü-Lab GO Karlsruhe“) wurden zusammen mit Akteuren aus der Praxis zahlreiche mögliche Realexperimente zur Förderung des Fußverkehrs identifiziert. Von den zunächst angedachten Realexperimenten konnte im Verlauf des Reallabors jedoch nur ca. die Hälfte auch realisiert werden. Die anderen Realexperimente wurden in verschiedenen Planungsstadien abgebrochen. Verschiedene im BaWü-Lab beteiligte Wissenschaftler(innen) hatten daher den Schaden, dass sie Arbeit in die Planung von Realexperimenten investiert hatten, ohne dass dadurch Ergebnisse gewonnen wurden. Jedoch konnten andere geplante Realexperimente umgesetzt werden, so dass insgesamt das Projektziel des Erkenntnisgewinns zum Fußverkehr erreicht werden konnte. In der Gesamtbilanz wurde daher der Schaden durch einzelne abgebrochene Realexperimente durch den realisierten Erkenntnisgewinn des Gesamtvorhabens aufgewogen.

Risikoaversion und Risikoaffinität: Forschung als ein langfristiger und mit Unsicherheiten verbundener Prozess ist per se risikobehaftet (Hanebuth et al. 2015). Auch Huber (2013) stellt Risikobereitschaft als einen Kern von Wissenschaft heraus, die Fragen formuliert und Projekte verfolgt, deren Ausgang ungewiss ist. Die Handlungslogik des Teilsystems Wissenschaft setzt demnach generell eine gewisse Risikoaffinität der Akteure voraus. Allgemein bietet das Teilsystem Wissenschaft Rahmenbedingungen (wie z. B. einen weitgehenden Haftungsausschluss oder eine erfolgsunabhängige Vergütung), die es Wissenschaftlern erlauben, risikoaffin zu handeln (Hanebuth et al. 2015).

Im Reallabor Space Sharing - Nutzungsintensivierung des Gebäudebestands durch Mehrfachnutzung und dynamische Programmierung (,BaWü-Lab Space Sharing“) wurde die Innovation Space Sharing (für ausführliche Informationen s. RLSS 2018) an einem Standort im Betrieb getestet. Dabei gab es zu Beginn viele Unbekannte in unterschiedlichen Bereichen, mit denen umgegangen werden musste, die zum Teil aber auch Forschungsfragen waren, die im Laufe des Projekts geklärt werden sollten. Diese zwei Arten von Unbekannten konnten jedoch nicht isoliert betrachtet werden, sondern standen in Wechselwirkung zueinander. So hätten eine Fehlentscheidung oder ein negativer Verlauf in einem Bereich bewirken können, dass der Betrieb gar nicht hätte durchgeführt werden können. Folglich hätten auch die Forschungsfragen in den anderen Bereichen nicht bearbeitet werden können. Folgende Unbekannte stellten unter anderem zu Beginn ein Risiko dar: Standortwahl (,Welcher Standort ist für Space Sharing geeignet?“), Bedarf (,Wie groß ist die Nachfrage aus der Stadtbevölkerung nach Space Sharing?“), Art der Nutzungen („Für welche Nutzungen wird Space Sharing angefragt? Wie lassen sich diese kombinieren?“), bauliche Adaptionen (,Welche baulichen Adaptionen sind für Space Sharing notwendig? Lassen sich diese am gewählten Standort umsetzen?“). Diese Risiken in Kauf zu nehmen, setzte eine gewisse Risikobereitschaft voraus. 
Das Reallabor BUGA:log - Logistische Nahversorgung im urbanen Raum mit automatisierten Transporteinheiten im Rahmen der Bundesgartenschau 2019 mit Quartiersund Stadtteilentwicklung ,Neckarbogen“ (,,BaWü-Lab BUGA:log “) hätte ohne eine hohe Risikobereitschaft aller Akteure gar nicht umgesetzt werden können. Bereits der Bezugsrahmen des Reallabors, das autonome Fahren, ist per se mit einer hohen Unsicherheit behaftet. Dann gab es zum Zeitpunkt des Aufsetzens des Reallabors noch zahlreiche unbekannte Umgebungsparameter (,,die Stadt im Werden“ als Teil der Ausstellungskonzeption der Bundesgartenschau 2019). Verschiedene Risiken, die mit den technischen und rechtlichen Voraussetzungen des autonomen Fahrbetriebs in Zusammenhang stehen, konnten erst im Laufe des Projekts adressiert und gelöst werden. Dass dies so sein würde, war den Partnern des Reallabors vorab bewusst und wurde explizit als ein Teil des gemeinsamen Projekts definiert und gelebt. Um dieses Reallabor umzusetzen, mussten nicht nur die eher risikoaffinen Wissenschaftler(innen) hohe Risiken in Kauf nehmen, sondern auch die eher risikoaversen Akteure aus der Kommune.

Risikomanagement: Das Risikomanagement in Bezug auf den wissenschaftlichen Erkenntnisgewinn ist bei transdisziplinären (und transformativen) Forschungsprojekten vergleichbar mit Forschung im Allgemeinen und ist insbesondere auf die Wahrung der Leitdifferenz von ,wahr/unwahr' für die Bewertung der Ergebnisse ausgelegt. Das Risikomanagement erfolgt zum einen durch vorab im Forschungsdesign berücksichtigte alternative Forschungspfade, zum anderen durch die Orientierung an den Standards guter wissenschaftlicher Praxis. Überprüft wird Letzteres durch die oben beschriebenen Mechanismen zur Veröffentlichung der Ergebnisse und der Peer-Begutachtung. In transdisziplinären (und transformativen) Forschungsprojekten besteht jedoch das höhere Risiko, dass gewonnene Erkenntnisse aufgrund des neuen Forschungsformats durch einige Peers aus der Wissenschaft nicht anerkannt werden. Darüber hinaus müssen die Akteure der Wissenschaft in solchen Projekten das Risiko des Ausfallens von Forschungsschritten insbesondere in Realexperimenten und den damit verbundenen Ausfall oder die Beeinträchtigung des Erkenntnisgewinns managen. Um mit diesem Risiko umzugehen, nutzen die Akteure der Wissenschaft Risikomanagementstrategien aus anderen Teilsystemen wie zum Beispiel Strategien des Projektmanagements aus der Wirtschaft.

Im BaWü-Lab GO Karlsruhe wurden, um den Erfolg des Reallabors zu gewährleisten und das Risiko von Schäden zu reduzieren, zahlreiche Instrumente des Projektmanagements wie bspw. interne Projektgruppen, Beirat von externen Partnern oder Monitoring von Projektschritten eingesetzt. Diese Maßnahmen wurden zwar nicht als Risikomanagement bezeichnet, erfüllten aber auch diese Funktion. So bestand bei den geplanten Realexperimenten im öffentlichen Straßenraum ein hohes Risiko, dass diese bereits frühzeitig in der Planungsphase aufgrund externer Einflussfaktoren nicht umgesetzt würden, wodurch keinerlei Forschungsergebnisse hätten gewonnen werden können. Durch die enge Abstimmung mit den externen Partnern im Beirat wurde versucht, frühzeitig zu identifizieren und zu bewerten, für welche geplanten Realexperimente ein hohes Ausfallrisiko besteht. Als Risikosteuerungsmaßnahmen wurde 
entweder darauf hingewirkt, die externen Hindernisse für diese Realexperimente zu beseitigen, oder es wurde der Arbeitsaufwand für deren Planung reduziert, um den Verlust an Arbeitsaufwand zu verringern.

Das Energielabor Tübingen - Potenziale, Partizipation, Perspektiven (,,BaWü-Lab Energielabor Tübingen“) agierte unter dem Motto „Gemeinsam zur Energiewende“ und wollte die Stadtgesellschaft, v. a. die Bewohner(innen) bestimmter Quartiere, für die Partizipation an Maßnahmen des Reallabors gewinnen. Entsprechend anberaumte Veranstaltungen für alle Bürger(innen) fanden aber zu wenig Anklang, so dass weder von echter breiter Partizipation hätte gesprochen werden können noch wissenschaftlich brauchbare Erkenntnisse hätten erzeugt werden können. Die getroffene Maßnahme des Risikomanagements bestand darin, dass Abstand genommen wurde vom Ziel, die gesamte Bevölkerung anzusprechen. Darauf wurden dann „Wendepunkt-Projekte“ als Realexperimente entworfen, die sich spezifischer auf die bereits im Bereich Energiewende Aktiven richteten. Diese Art von Partizipation konnte dann erfolgreich vor Ort durchgeführt werden.

\subsection{Politik}

Politik - Der Begriff der Politik bezieht sich auf Einrichtungen, deren Aufgaben die politische Gestaltung und die Vertretung individueller Gesellschaftsmitglieder sind, also beispielsweise Parlamente und Regierungen, aber auch demokratisch legitimierte Interessenverbände auf den verschiedenen föderalen Ebenen. Entsprechend sind auch einzelne Politiker(innen) als Angehörige derartiger Einrichtungen der Politik zuzurechnen.

Funktion, Leitwert und Leitdifferenz: Nach Schimank (2007) ist die Funktion des Teilsystems Politik das Treffen belastbarer kollektiver Entscheidungen über die Repräsentation von Interessen und Werten durch gewählte Mandatsträger im demokratischen System. Unter Interessen sind hierbei handlungsstiftende, individuelle und kollektive Ziele und Bedürfnisse zu verstehen (Rudzio 2015). Werte wiederum sind hier dem Individuum übergeordnete, normenstiftende Ideen eines guten gesellschaftlichen Zusammenlebens (Joas 2005). Der Leitwert des Teilsystems Politik sind möglichst umfassende Gestaltungsmöglichkeiten gesellschaftlicher Lebensumstände. Die Leitdifferenz ist ,Macht/keine Macht.

Qualitätssicherung: In Demokratien muss sich der Gestaltungswille des Teilsystems Politik über Wahlen legitimieren und unterliegt somit auch einer Qualitätssicherung im Sinne der Wähler(innen). Politische Entscheidungsstrukturen sind von der Legitimation durch Wahlen abhängig. In einem weiter gefassten Sinne lassen sich auch Aspekte indirekter politischer Partizipation von Bürger(inne)n als Qualitätssicherung verstehen, beispielsweise wenn in politischen Gestaltungsprozessen über Jurys oder Beteiligungsräte qualitative Einschätzungen zu verschiedenen Gestaltungsoptionen eingeholt werden. 
Entscheidungsstrukturen: Im Teilsystem Politik gilt typischerweise ein repräsentativer Ansatz. Der Gestaltungswunsch wird also durch die Wähler(innen) an die Politik delegiert. Im Unterschied zur Zivilgesellschaft (s. Abschnitt 3.5) kann bei ausreichender Unterstützung durch die Mehrheit eine Gestaltung auch für die nicht zustimmende Minderheit vorgenommen werden. Die Handlungslogik der Politik unterscheidet sich von der Handlungslogik der Zivilgesellschaft insofern, als die Leitdifferenz ,Macht/keine Macht' über Repräsentation und Mehrheitsentscheid gewonnen werden muss und den Akteuren diese Legitimation ihres Handelns auch immer wieder entzogen werden kann. Die Frage des Machterhalts oder der Machtausweitung zwischen den Wahlen hängt jedoch nur bedingt vom Wahlergebnis selbst ab und kann sich zwischen den Wahlen auch schwankend aus Netzwerken, politischer Kommunikation, Informationszugriff, Koalitionen etc. ergeben. Als gesellschaftliche Erwartung wird jedoch der Wunsch an politische Repräsentant(inn)en und Einheiten herangetragen, dass sich Macht möglichst ausschließlich und transparent aus Wahlergebnissen ableitet und dieses auch nach der Wahl von Politiker(inne)n als Grundlage für inhaltliche Gewichtungen während der Legislaturperiode herangezogen wird. Mit welcher Reichweite dieser Legitimationsmechanismus in der Realität tatsächlich greift (Crouch 2008), geht über den vorliegenden Beitrag hinaus.

Finanzierung: Politische Strukturen sind üblicherweise so aufgebaut, dass der Wählerwille über das Haushaltsrecht auch die finanziellen Mittel verleiht, um politische Schwerpunktsetzungen vorzunehmen. Die Finanzierung erfolgt in Deutschland über Steuergelder von Land, Bund und der Europäischen Union. Das Teilsystem Politik wird aber auch durch Mittel finanziert, die sich nicht aus der staatlichen Steuerungsstruktur vor allem über Steuern ergeben. Deshalb spielen auch Spenden, Sponsoring von Unternehmen oder beispielsweise Stiftungsgelder insbesondere bei Modellprojekten eine Rolle und führen zu der Entstehung von bereits genannten „Inter-Systembeziehungen“ namentlich zum Teilsystem Wirtschaft. Entstehen daraus Überschneidungen zwischen den Leitzielen der so verbundenen Teilsysteme, können daraus Konflikte aufgrund von Widersprüchen zwischen den Leitwerten resultieren.

Schäden und Chancen: Das zentrale Risiko, das sich aus der Handlungslogik des Teilsystems Politik ergibt, besteht darin, dass eine vorgenommene Handlung die Macht verkleinern könnte. Die betrachteten Schäden beziehen sich weniger auf ein transdisziplinäres (und transformatives) Forschungsprojekt als Ganzes, sondern konzentrieren sich auf die in der ,realen Welt' umgesetzten und wahrnehmbaren Maßnahmen, insbesondere Realexperimente. Für das Teilsystem Politik ist damit insbesondere die Außenwirkung beispielsweise eines Reallabors von Relevanz. Für die Politik entstehen Schäden, wenn die darin umgesetzten Realexperimente zwar wie erwartet funktionieren, die Wirkungen jedoch politisch (z. B. von einem Teil der Wählerschaft) nicht gewollt sind. Wenn die Maßnahme nicht dem 
Wählerwillen als Machtlegitimation der Politik entspricht, ergibt sich ein Konflikt in der Leitdifferenz ,Macht/keine Macht ${ }^{\star}$. Ein Schaden aus Sicht der Politik kann zudem dann eintreten, wenn beispielsweise die in einem Reallabor umgesetzten Realexperimente nicht die gewünschten Ergebnisse erzielen. Wie ausgeführt kann in der Wissenschaft auch eine fehlende Bestätigung einer Hypothese zu einem akzeptierten Wissensgewinn führen. Im Teilsystem Politik dagegen werden nicht erfüllte Erwartungen und das Lernen aus Fehlern nur sehr eingeschränkt akzeptiert. Dabei ist aus der Handlungslogik der Politik nicht so sehr problematisch, dass die Gestaltung der gesellschaftlichen Lebensumstände nicht wie gewünscht gelingt, sondern vielmehr, dass dies den Schaden eines Machtverlusts bewirken kann. So werden Fehler entsprechend der Leitdifferenz ,Macht/keine Macht' von der politischen Gegenseite häufig zur Kritik genutzt. Dies kann Vorwürfe umfassen wie z. B., mit öffentlichen Geldern nicht verantwortlich umgegangen zu sein oder Externe dem Risiko von Schäden ausgesetzt zu haben. Jedoch werden von der Politik auch Chancen von transdisziplinären (und transformativen) Forschungsprojekten wahrgenommen. Diese liegen meist darin, dass sie helfen, die verfolgten Gestaltungsziele umzusetzen. Wie bereits erwähnt, spielte die Handlungslogik des Teilsystems Politik im Rahmen der BaWü-Labs insbesondere für die Akteure aus kommunalen Verwaltungen eine relevante Rolle.

In Heilbronn wurde das BaWü-Lab BUGA:log von der Verwaltung auch gezielt genutzt, um - auch über die Fragestellungen des Reallabors hinausgehend - den Dialog zwischen der Stadt und den Anbietern innovativer Lösungen im Bereich Mobilität und Logistik zu intensivieren. Dies wurde als Chance gesehen, auch in anderen Bereichen die Transformation der Stadt im Mobilitätsbereich voranzubringen.

Der 2012 beschlossene Verkehrsentwicklungsplan Karlsruhe formuliert als politisches Ziel die verstärkte Förderung des Fußverkehrs. Das BaWü-Lab GO Karlsruhe, welches ab 2015 ebenfalls dieses Ziel verfolgte, wurde daher von der Kommunalpolitik der Stadt Karlsruhe positiv aufgenommen. Es wurde als eine Chance gesehen, die bereits beschlossene politische Zielsetzung umzusetzen. Dafür wurde das Risiko von politisch nicht gewollten Teilergebnissen und damit verbundenen Kontroversen in der Kommunalpolitik eingegangen.

Risikoaversion und Risikoaffinität: Die Risikobereitschaft des Teilsystems Politik wird durch zwei gegensätzliche Kräfte beeinflusst. Einerseits verfolgt es langfristige Gestaltungsabsichten, die es erforderlich machen, zumindest partiell Neues zu wagen und dafür auch Risiken einzugehen. Andererseits kann sich Politik Misserfolge bei der Umsetzung ihrer Gestaltungsabsichten ohne erhebliche Nachteile durch Kritik seitens politischer Gegner nicht leisten. Die Politik wägt daher die Chancen und Schäden von Maßnahmen, wie zum Beispiel die Beteiligung an einem Reallabor, sorgfältig ab. Nach Auffassung von Kepplinger (2009) gehen Akteure der Politik, wenn diese Erfolgsaussichten sehen, relativ große Risiken ein (auch im Vergleich zu anderen Akteuren). So zeigt eine Befragung von Kepplinger (2009), dass die meisten befragten Politiker(innen) der Ansicht sind, dass 
Politik irrational handeln würde, wenn Politiker(innen) das Risiko von Fehlschlägen scheuten. Dieser Widerspruch zwischen vergleichsweise hohen Kosten im Fall von Kritik und der Selbsteinschätzung als risikoaffine Menschen erklärt sich möglicherweise aus der Frage, wie gut entstandene Schäden auf einzelne Handelnde zurückgeführt und Konsequenzen einzelnen Personen zugeordnet werden können. Alles in allem ist das Teilsystem Politik risikoaffin. Dies zeigte sich beispielsweise auch in den BaWü-Labs, die vielfach von der Kommunalpolitik bereits in der Anfangsphase unterstützt wurden, obwohl aufgrund der zahlreichen Unsicherheiten ein nicht geringes Risiko bestand.

Gegenstand des Reallabors Wissensdialog Nordschwarzwald (,BaWü-Lab WiNo“) war der Nationalpark Schwarzwald, der erste Nationalpark in Baden-Württemberg. Obwohl dessen Einrichtung politisch umstritten war, unterstützten einzelne Bürgermeister(innen) der Region das Reallabor ausgesprochen engagiert, z. B. durch die Mitwirkung bei öffentlichen Veranstaltungen. Dies kann zum einen damit begründet werden, dass die kommunalen Vertreter(innen) davon ausgingen, dass ein Scheitern dieser Veranstaltungen bzw. des gesamten Projekts nicht ihnen persönlich angelastet werden würde. Zum anderen betraf ihr Engagement nicht originäre Verwaltungs- bzw. hoheitliche Aufgaben ihrer Kommunen, sondern lag im Bereich freiwilliger Leistungen. So stehen Gemeinden beispielsweise im Tourismus auch in Konkurrenz zueinander und streben die Herausarbeitung von Alleinstellungsmerkmalen an. Ein Teilprojekt des Reallabors wiederum befasste sich damit, nachhaltige Tourismusprodukte auf Basis des Waldes zu entwickeln. Bürgermeister(innen), die sich engagierten, nahmen daher auch eine unternehmerische Haltung ein, die zu höherer Risikoaffinität führte.

Risikomanagement: Das Teilsystem Politik kann aufgrund seiner Risikoaffinität auf ein breites Spektrum von Risikomanagementstrategien zurückgreifen. Dies umfasst vorsorgende Strategien wie den Verantwortungstransfer auf andere Akteure durch „Schwarze Peter-Spiele“ bis hin zu Strategien zum Umgang mit Schäden wie z. B. das „Framing von negativen Meldungen“. In den BaWü-Labs konnte als eine Risikomanagementstrategie insbesondere beobachtet werden, dass die Politik die Kontrolle darüber bewahrte, in welchem Umfang sie öffentlich mit dem Projekt assoziiert wurde.

Im Rahmen des Reallabors Stadt:Quartiere 4.0 - Frühzeitige gestaltende Bürgerbeteiligung für eine nachhaltige Entwicklung in Baden-Württemberg (,,BaWü-Lab Stadt: Quartiere 4.0") entstand im Zuge der Arbeit eine Differenz zwischen den Forschungsergebnissen und den politischen Zielen der beteiligten politischen Akteure, so dass sich deren anfängliche Risikoaffinität über den Projektzeitraum hin zu größerer Zurückhaltung bis hin zur Risikoaversion veränderte. Die Risikomanagementstrategie der politischen Akteure bestand darin, darauf hinzuwirken, dass Resultate aus der wissenschaftlichen Arbeit nur insoweit kommuniziert werden sollten, als sie politischen Zielen zuträglich schienen, und die Veröffentlichung von Ergebnissen, die diesen abträglich schienen, zumindest nicht zu unterstützen. Dies wiederum schränkte den Handlungsspielraum des Reallabors insofern ein, als einige der Arbeiten im Reallabor eine umfängliche Kommunikation der Ergebnisse an die breite Öffentlichkeit vorgesehen hatten. 
Im BaWü-Lab BUGA:log beteiligte sich die Stadt Heilbronn, vertreten durch die Person des Oberbürgermeisters und den Gemeinderat, sowohl über eine erhebliche finanzielle Unterstützung am Reallabor als auch durch die aktive Mitarbeit an der Konzeption und Umsetzung des Reallabors. Durch die intensive Mitarbeit in der Konzeptionsund Vorbereitungsphase betrieb die Stadt Heilbronn für sich selbst auch aktives Risikomanagement, indem sie ihre Positionen und Bedürfnisse (z. B. im Bereich des Verkehrsrechts, aber auch im Bereich der Lade- und Versorgungsinfrastruktur) früh einbrachte, so dass von Beginn an Risiken mit den Partnern aus der Wissenschaft diskutiert und Lösungen dafür entwickelt werden konnten.

\subsection{Recht}

Recht - Der Begriff des Rechts bezieht sich vor allem auf Einrichtungen, die der Rechtsprechung und Rechtspflege zuzuordnen sind, also beispielsweise Gerichte oder auch Genehmigungsbehörden, die vor allem die Einhaltung geltenden Rechts gewährleisten sollen. Entsprechend institutionell angebundene, individuelle Gesellschaftsmitglieder, deren Aufgabe in der Rechtsprechung und Rechtspflege liegt, werden ebenfalls dem Teilsystem Recht zugeordnet.

Funktion, Leitwert und Leitdifferenz: Die Funktion des Teilsystems Recht ist es, normative Erwartungen zu stabilisieren, indem es für die Gesellschaft verlässliche Verhaltensregeln entwickelt, festhält und Sanktionen für deren Nichteinhaltung festlegt (Callies 2006). Leitwert ist die Feststellung von Recht mit der Leitdifferenz ,Recht/Unrecht'. Rechtmäßig ist eine Handlung, wenn sie geltenden Rechtsvorgaben des in einer Gesellschaft dem Teilsystem Recht zugrundeliegenden Gesetzeskörpers entspricht. Aufgrund dieses über alle gesellschaftlichen Teilsysteme hinweg geltenden Gesetzeskörpers spielt das Teilsystem Recht für alle anderen Teilsysteme eine begrenzende, aber auch eine ermöglichende Rolle. Kein Teilsystem kann außerhalb des vom Teilsystem Recht festgelegten Rahmens also außerhalb des Gesetzes - agieren, selbst wenn sein jeweils eigener Leitwert und seine eigenen Leitdifferenzen dies durchaus zulassen würden. Umgekehrt ermöglicht das Teilsystem Recht jedoch auch über die Teilsysteme hinweg verlässliche Handlungsabläufe durch eine klare, regulatorische und normenbasierte Rahmung. Das würde sich bei einer nicht rechtsgeleiteten Auseinandersetzung allein auf der Grundlage der verschiedenen Handlungslogiken der anderen Teilsysteme nicht ergeben.

Qualitätssicherung: Qualitätssicherung erfolgt im Teilsystem Recht über den Abgleich eines Sachverhalts mit geltenden Gesetzen, die Rechtsaufsicht durch Gerichte sowie die Sanktionierung im Fall einer Nichteinhaltung von Recht.

Entscheidungsstrukturen: Die Prüfung, ob ein Sachverhalt geltendem Recht entspricht, erfolgt oft mehrstufig, von einer ersten laienhaften Einschätzung bis hin zu einer richterlichen Entscheidung. Die Entscheidung, welche Informationen im Sinne des Leitwerts, Recht/Unrecht' anschlussfähig an das Teilsystem sind, un- 
terliegt im Teilsystem Recht einer starken Professionalisierung. Die Professionalisierung besteht in einer notwendigen juristischen (Teil-)Ausbildung und dem stark formalisierten Nachweis juristischen Könnens. Es gibt jedoch auch Positionen, wie beispielsweise das deutsche Verfassungsgericht, in das Richter(innen) durch die Politik berufen werden, womit es hier eine „Inter-Systembeziehung“ zum Teilsystem Politik gibt.

Finanzierung: Die Finanzierung von Rechtsstrukturen wie Ämtern und Gerichten erfolgt in erster Linie aus Steuermitteln, während der individuelle Zugang zum Rechtssystem auch aus privaten Mitteln gewährleistet wird. Dabei wird im Teilsystem Recht stark darauf geachtet, dass der Zugang grundsätzlich allen Bürger(inne)n offensteht: Um diesen für jedermann zu gewährleisten, werden, in Abhängigkeit von Vorleistungen und individuellen Vermögenslagen, kollektiv gestützte Prozesskostenhilfen über Versicherungen sowie über Transferzahlungen angeboten.

Schäden und Chancen: Das Teilsystem Recht ist in transdisziplinären (und transformativen) Forschungsprojekten insbesondere durch die jeweiligen (z. B. kommunalen) Verwaltungen repräsentiert. Der Extremfall eines Schadens aus Sicht des Teilsystems Recht ist, wenn im Rahmen von beispielsweise Realexperimenten rechtlich nicht zulässige Maßnahmen umgesetzt werden. Dies kollidiert klar mit der Leitdifferenz ,Recht/Unrecht' und widerspricht dem Selbstverständnis der kommunalen Verwaltung, Regeln einzuhalten und geltendem Recht zu entsprechen (Hill 2016). Schäden aus Sicht der Verwaltung ergeben sich zudem aus Haftungsrisiken in derartigen Projekten. Risiken entstehen, wenn beispielsweise kommunale Verwaltungen innovative Maßnahmen für die Umsetzung von Realexperimenten genehmigen müssen, eventuell für diese Entscheidung in die Haftung genommen werden und somit die Leitdifferenz von ,Recht/Unrecht` zum Tragen kommt. Dies kann in einem Reallabor beispielsweise zur Folge haben, dass selbst wenn die fachlich Zuständigen in einer kommunalen Verwaltung willens wären, eine innovative Maßnahme zu unterstützen, diese durch die hausinternen Jurist(inn)en mit Verweis auf die Amtshaftung gebremst werden. Transdisziplinäre (und transformative) Forschungsprojekte bieten kommunalen Verwaltungen aber auch die Chance, Maßnahmen auszuprobieren, die im üblichen Verwaltungshandeln nicht möglich wären. Dies, indem Reallabore einen Transfer von Risiken von der kommunalen Verwaltung auf die Wissenschaft, die die rechtliche Verantwortung übernimmt und ggf. haftet, ermöglichen.

In Karlsruhe ergab sich im Laufe des BaWü-Labs GO Karlsruhe die rechtliche Möglichkeit, neuartige Fahrbahnmarkierungen zur Fußgängerförderung auszuprobieren. Die Kommune bat daher das BaWü-Lab, diese neuen Möglichkeiten in Form eines Realexperiments umzusetzen und deren Wirkung wissenschaftlich zu analysieren, bevor diese generell in der Stadt umgesetzt würden. Neben der Möglichkeit der schnellen Umsetzung der Maßnahmen spielte hierfür auch der teilweise Risikotransfer auf die Akteure der Wissenschaft eine Rolle. 
Beim Realexperiment „Parklets für Stuttgart“ (s. auch Köglberger et al. 2019 in diesem Buch, Kapitel 4) des BaWü-Labs RNM wurden straßenbegleitende Parkplätze zu Aufenthaltsflächen für Fußgänger(innen) umgestaltet. Hierdurch entstand zum einen das Unfallrisiko eines Zusammenstoßes zwischen Fahrzeugen und einem Parklet. Zum anderen hätten beim Aufbau oder bei der Nutzung der Parklets Fußgänger(innen) auf die Straße treten können, so dass es auf diese Weise zu Unfällen mit Personenschäden hätte kommen können. Für diese möglichen Schäden hätte die Universität als Selbstversicherer gehaftet. Um diese Risiken zu minimieren, wurden im Rahmen der Genehmigung durch das Amt für öffentliche Ordnung Auflagen erlassen, für deren Einhaltung die Wissenschaftler(innen) Sorge tragen mussten. So wurde die Verkehrssicherheit für vorbeifahrende PKW durch das Anbringen von reflektierenden Folien erhöht, beim Aufbau der Parklets wurde die Baustelle sichtbar markiert und abgesperrt, und die Aufbauenden trugen Warnwesten. Die Parklets durften nur vom Bürger(innen)steig aus zugänglich sein, um ein Hinaustreten auf die Straße zu verhindern. Da keine Partei über Erfahrungen mit einer vergleichbaren Intervention hatte, wurde die Risikobewertung teilweise auch nachträglich noch angepasst, woraus kurzfristige Nachbesserungsforderungen entstanden.

Risikoaversion und Risikoaffinität: Im Vergleich zu den anderen Akteuren in transdisziplinären (und transformativen) Forschungsprojekten verhalten sich Verwaltungen eher risikoavers. Hill (2016) und Fischer (2016) zeigen auf, dass ein vorsichtiger Umgang mit Risiken das Verhalten von Verwaltungen generell kennzeichnet. Die Folge ist ein auf Abstimmung und Absicherung bedachter Umgang mit Risiken. Risiken werden nur akzeptiert, wenn diese erforderlich sind, um das Allgemeinwohl zu gewährleisten. Nach dieser Handlungslogik ist ein Risiko nur dann akzeptabel, wenn institutionelle Regeln bestehen, um diese zu kontrollieren (Renn 2008).

Im BaWü-Lab GO Karlsruhe sollten im Rahmen eines Realexperiments temporäre Bauteile zur Geschwindigkeitsreduktion verbaut werden. Für diese Bauteile wurde von der kommunalen Verwaltung entsprechend geltendem Recht gefordert, dass sie einschlägigen Prüfsiegeln entsprechen. Jedoch ergab eine umfassende Recherche, dass für Bauteile dieser Art in der Planungspraxis keine Prüfsiegel oder Qualitätsnormen bestehen. Der Anforderung des Rückgriffs auf etablierte Regelwerke konnte daher nicht entsprochen werden. Daraufhin erfolgte durch die Verwaltung eine ausführliche Abfrage bei anderen Kommunen, welche Erfahrungen bisher mit solchen Bauteilen gemacht worden waren und welche institutionellen Regeln zur Anwendung gekommen waren. Erst gestützt darauf erfolgte die Entscheidung der Verwaltung, das Realexperiment zu ermöglichen.

Risikomanagement: Als generelle Risikomanagementstrategien von Verwaltungen nennt Fischer (2016) das ausgeprägte Festhalten an Regeln und Hierarchien. Daher wird in einer Verwaltung jedes von den Regeln abweichende Verhalten tendenziell als bedrohlich aufgefasst. Die Arbeit in transdisziplinären (und transformativen) Forschungsprojekten, insbesondere die Umsetzung innovativer Maßnahmen im Rahmen z. B. von Realexperimenten, hat jedoch häufig zur Folge, dass das Festhalten an etablierten institutionellen Regeln als Risikomanagement- 
strategie nicht möglich ist. Bei der Mitarbeit in derartigen Projekten muss die kommunale Verwaltung daher gewillt sein, sich über ihre etablierten Risikomanagementstrategien hinwegzusetzen. Umgekehrt müssen die Akteure aus der Wissenschaft gewillt sein, sich den Risikomanagementstrategien der Verwaltung durch institutionelle Regeln anzupassen, soweit es solche gibt.

Im BaWü-Lab BUGA:log in Heilbronn zeigte sich, dass komplexen rechtlichen Anforderungen, die sich aus dem autonomen Fahren im öffentlichen Straßenraum ergaben, am besten mit einem in zeitlicher Hinsicht parallel zum Projekt stattfindenden Dialogprozess begegnet werden konnte. In diesen Dialog wurden früh alle in rechtlicher Hinsicht relevanten Entscheidungsebenen (,,von der Kommune bis zum Bund“) einbezogen. Früh wurde auch das Ziel formuliert, eine gemeinsame Lösung erarbeiten zu wollen, also nicht rechtliche Verantwortlichkeiten zwischen Ebenen hin und her zu schieben. Als Leitprinzip diente dabei der Wunsch, den Interessen des Reallabors so weit wie möglich entsprechen zu können, aber gleichzeitig auch ohne Einschränkungen die Möglichkeiten und Grenzen aller beteiligten Verwaltungsebenen bei der Einhaltung und Durchsetzung des geltenden Rechtsrahmens zu respektieren.

Im Rahmen des BaWü-Labs Stadt:Quartiere 4.0 waren die Wissenschaftler(innen) des Projekts zwar in die Konzeption von Veranstaltungen im Rahmen des Integrierten Mobilitätsentwicklungsplans (IMEP) der Stadt Herrenberg eingebunden, jedoch gab es klare Grenzen in Bezug auf die Visionen nachhaltiger Mobilität, die dabei entwickelt werden konnten. So konnten gewisse Visionen, die durch die Simulationen und Visualisierungen des Reallabors hätten generiert werden können, nicht weiterverfolgt werden, weil sie nicht im Interesse der Kommune lagen. Das Risiko für die Kommune wurde durch die kommunalen Akteure dadurch reduziert, dass eine Konzentration auf die konkrete Umsetzung von Maßnahmen stattfand und die Kontrolle des Prozesses sowie der Öffentlichkeitsbeteiligung soweit als möglich bei der Verwaltung und dem Gemeinderat gelassen wurden.

\subsection{Wirtschaft}

Wirtschaft - Unter dem Begriff der Wirtschaft werden Einrichtungen und individuelle Gesellschaftsmitglieder verstanden, deren Ziel es ist, über Marktmechanismen ihren individuellen oder gruppenbezogenen Nutzen in Form von Profit zu steigern. Dazu gehören primär Unternehmen.

Funktion, Leitwert und Leitdifferenz: Als Funktion der Wirtschaft gelten die Allokation $^{6}$ von Gütern beziehungsweise, die „Kommunikation von Knappheit [...] im Medium des Markts“ beziehungsweise die Verknüpfung zukunftsfähiger Vorsorge mit je gegenwärtigen Bedürfnissen (Luhmann 1988, S. 63). Dabei befreit sie die Produzent(inn)en vom unwahrscheinlichen Finden der Nachfrage nach ihren Produkten, indem sie Angebot und Nachfrage zusammenkommen lässt.

6 Allokation ist die Zuweisung von finanziellen Mitteln, Materialien und Produktivkräften. 
Gleichzeitig ermöglicht sie eine vergleichsweise konfliktfreie Vornahme von Tauschhandlungen, da der Erwerb von Gütern über die Zahlung von Geld erfolgt, das nur in Form einer Weitergabe nutzbar ist (Luhmann 1984). Unterschiedliche Gesellschaften fokussieren auf unterschiedliche Knappheiten und wählen unterschiedliche Ansätze, um „diese Knappheit durchzusetzen“, beispielsweise über einen Plan oder über den Markt (Baecker 2016, S. 169). Die handlungslogische Rahmung des Teilsystems mit dem Leitwert der Erhaltung und des Zuwachses der Zahlungsfähigkeit eines Unternehmens oder eines individuellen Gesellschaftsmitglieds sowie der Leitdifferenz ,Zahlung/keine Zahlung' ist breit diskutiert. Preise erlauben dabei eine Kommunikation über zu erwartende Zahlungen und eine Reduktion des Tauschvorgangs auf Zahlungen, ohne anderweitige Verpflichtungen entstehen zu lassen. Preise sind also als „Informationsraffung und -verkürzung“ (Luhmann 1988, S. 19) zu verstehen. Innerhalb der Handlungslogik des Teilsystems Wirtschaft ist eine Handlung nur anschlussfähig, wenn ein Austausch weitere Zahlungen ermöglicht, also Gewinn verspricht. Jedoch agieren nicht alle Akteure aus der Wirtschaft, die einem Unternehmen zuzuordnen sind, ausschließlich nach einer wirtschaftlichen Handlungslogik. Vielmehr wird diese oft ergänzt durch die Logik der Corporate Social Responsibility, die eine am Gemeinwohl orientierte Verantwortung als ergänzenden Leitwert postuliert und damit das Kriterium ,gesellschaftlich wertvoll'. So haben Unternehmen die Wahl, ob sie sich aufgrund von Gewinnerwartungen oder, ähnlich wie zivilgesellschaftliche Akteure (s. Abschnitt 3.5), aufgrund der gesellschaftlichen Verantwortung an einer Unternehmung beteiligen.

Qualitätssicherung: Die Entstehung von Profit ist als Qualitäts- und Erfolgsindikator für das Teilsystem Wirtschaft zu betrachten, da sie den Handlungsspielraum des Akteurs, der den Profit empfängt, im Hinblick auf zukünftige Zahlungen erweitert.

Entscheidungsstrukturen: Hierarchien und Entscheidungsstrukturen erklären sich aus der Zahlungsfähigkeit, der Liquidität, die einem Akteur im Teilsystem Wirtschaft gegenwärtig oder ggf. auch zukünftig zugeschrieben wird. Dies kann direkt der Fall sein, indem auf das eigene Vermögen des Akteurs abgestellt wird, die Zuschreibung kann auch indirekt erfolgen, indem auf die Zuschreibung der Befähigung zur Zahlungsfähigkeit durch Banken abgestellt wird.

Finanzierung: Wirtschaft ist das einzige Teilsystem, in dem Entscheidungen über Finanzierungen nicht als Produkt der Umwelt zu verstehen sind, sondern im Teilsystem selbst entstehen. Das Medium Geld stellt den Mittelpunkt von Kommunikationsabläufen dar. Das erklärt, warum der vielbemühte ,Business Case ${ }^{7}$ von

7 „Ein Business Case ist ein Szenario zur betriebswirtschaftlichen Beurteilung einer Investition. Auch ein Projekt stellt eine Investition dar und muss deshalb seine Vorteilhaftigkeit gegenüber der Geschäftsleitung unter Beweis stellen“ (Brugger 2009, S. 11). 
solcher Relevanz ist: Aus Teilsystemperspektive muss eine Zahlung eine weitere Zahlung ermöglichen, um das selbstreferenzielle System aufrechtzuerhalten.

Schäden und Chancen: Die Akteure aus dem Teilsystem Wirtschaft beurteilen die mit Schäden und Chancen verbundenen Risiken von transdisziplinären (und transformativen) Forschungsprojekten vorrangig nach der Leitdifferenz ,Zahlung/keine Zahlung' bzw. dem daraus abgeleiteten Profit. Dabei sind die Akteure der Wirtschaft bereit, das Risiko von Schäden in Form von monetären Verlusten in Kauf zu nehmen, wenn die Chance besteht, (ggf. später) Gewinne zu erzielen.

Im Reallabor Schorndorf: Zukunftsweisender Öffentlicher Verkehr - Bürgerorientierte Optimierung der Leistungsfähigkeit, Effizienz und Attraktivität im Nahverkehr (,,BaWü-Lab Schorndorf") (s. auch Steckbrief im Anhang zu diesem Buch) waren der Verkehrs- und Tarifverbund Stuttgart (VVS), der lokale Busbetreiber sowie die Kommune als Projektpartner in das Projekt eingebunden. Alle Beteiligten sahen in der Erprobung eines neuen, bedarfsgerechten Bussystems (ohne festen Fahrplan und fixe Haltestellen) die Chance herauszufinden, ob ein solches Bussystem die Zukunft des ÖPNV sein könnte. Um dieses zukünftige Potenzial ausloten zu können, brachten die Praxispartner eigene Ressourcen dafür ein, die zunächst einmal eine ,Mehrbelastung ‘ darstellten. Diese wurden beispielsweise dazu eingesetzt, im Rahmen des Testbetriebs im Reallabor den Kund(inn)en ein vergünstigtes Busticket anzubieten.

Im BaWü-Lab Energielabor Tübingen mit der Zielrichtung „Nachhaltige Energiewende" wurde u. a. mit einem lokalen Energieversorger kooperiert. Dieser musste, wie alle anderen Energieversorger auch, mit der laufenden Transformation des Energiesystems umgehen. Diese soll, nach unverändert geltender Auffassung, im Sinne der Nachhaltigkeit vor allem dezentral ablaufen, was aktuelle Geschäftsmodelle in Frage stellt, neue rechtliche Fragestellungen aufwirft und die technische Komplexität steigen lässt. Demgegenüber verfügte der Energieversorger nur über ein begrenztes Budget für Investitionen in Forschung und Entwicklung. Solange aber noch niemand weiß, wo genau die Reise hingeht, sind alle Beteiligten zurückhaltend, wenn es darum geht, in eine bestimmte Richtung - hier in Richtung neuer dezentraler Energieversorgungssysteme - zu gehen, so auch der Praxispartner im Reallabor. Das wirkte sich auch auf die Arbeit im Reallabor aus. Zum einen schränkte es den Handlungsspielraum ein, zum anderen waren die betriebswirtschaftlichen Erwägungen seitens des Energieversorgers zu berücksichtigen.

Im Reallabor Urban Office - Nachhaltige Stadtentwicklung in der Wissensgesellschaft (,,BaWü-Lab Urban Office“) (s. auch Steckbrief im Anhang zu diesem Buch) wurde mit einem Unternehmen der Stadt Heidelberg zusammengearbeitet. Es war zunächst schwierig, das Unternehmen für die Kooperation zu gewinnen, da der Geschäftsführer keinen direkten Profit damit verbinden konnte. Für ihn stellte sich das Projekt zunächst ausschließlich als Risiko dar, da Ressourcen investiert werden mussten, ohne einen möglichen Gewinn abschätzen zu können. Erst im Projektverlauf gelang es, nach einigen Gesprächen und ersten Arbeitsschritten, dem Geschäftsführer die Chancen des gemeinsamen Projekts aufzuzeigen und ihn so von den potenziellen Chancen einer $\mathrm{Zu}$ sammenarbeit zu überzeugen. 
Risikoaversion und Risikoaffinität: Akteure aus der Wirtschaft weisen generell eine gewisse Risikoaffinität auf. Sie folgen dabei einer entrepreneurhaften Logik und sind bereit, Risiken im Austausch für entstehende Chancen zu akzeptieren (Renn 2008; Hill 2016). Diese generelle Risikoaffinität von Akteuren aus der Wirtschaft konnte auch in den BaWü-Labs beobachtet werden. Für transdisziplinäre (und transformative) Forschungsprojekte bedeutet dies, dass wirtschaftliche Akteure bereit sind, Ressourcen wie Zeit und Geld in die Arbeit an einem derartigen Projekt zu investieren. Dabei nehmen sie das Risiko in Kauf, dass die Investitionen verloren gehen können, solange die Chance besteht, von den Ergebnissen des Projekts zu profitieren.

Im BaWü-Lab Space Sharing wurden, als ein Teilaspekt, rechtliche Fragestellungen bearbeitet. Um das tun zu können, wurde ein Praxispartner aus der Wirtschaft mit Fachexpertise im Immobilienrecht ins Projektteam integriert. Dieser war insofern bereit, ein Risiko einzugehen, als er Zeit und auch Geld in Form von Mitarbeiter(innen)stunden investierte, ohne damit direkten Gewinn zu erzielen. Er sah aber die Chance, aus den gewonnenen Erkenntnissen zukünftig wirtschaftlichen Nutzen ziehen zu können, indem er diese im ziemlich jungen Markt von Immobilien für Mehrfachund Parallelnutzung - mit entsprechend geringen Erfahrungswerten - würde anwenden können.

Im BaWü-Lab BUGA:log engagierten sich mehrere Industriepartner größtenteils unentgeltlich mit der Bereitstellung von Komponenten für das Fahrzeug und die Reallabor-Umgebung. Sogar erheblicher Umbau- und Anpassungsaufwand an eigenen Komponenten wurde teilweise in Kauf genommen, weil die möglichen Chancen, die sich aus einem Erfolg des Reallabors ergeben könnten, als sehr hoch bewertet wurden.

Risikomanagement: Akteure aus der Wirtschaft nutzen für ihr Risikomanagement häufig Ansätze aus dem mathematischen Risikoverständnis. Das monetäre Risiko wird dabei als Produkt aus Schadenshöhe und Eintrittswahrscheinlichkeit betrachtet. Im Ergebnis können die Akteure für ihr Risikomanagement eine klare Grenze ableiten, welches finanzielle Risiko sie für einen erwarteten Gewinn durch ein transdisziplinäres (und transformatives) Forschungsprojekt akzeptieren. Ein Potenzial derartiger Projekte ist, dass die Akteure der Wirtschaft die Möglichkeit haben, einen Teil des Risikos auf andere Akteure (z. B. aus der Wissenschaft) zu verlagern. Die Risiken von Schäden werden so aus der jeweils eigenen Gewinnund Verlustrechnung herausgehalten und anderen Akteuren überlassen (Kromschröder 2003). In vielen transdisziplinären (und transformativen) Forschungsprojekten übernehmen die Akteure der Wissenschaft das höhere finanzielle Risiko, da sich diese am Ziel Wissensgewinnung und nicht am Ziel Profit orientieren. 


\subsection{Zivilgesellschaft}

Zivilgesellschaft - Unter dem Begriff Zivilgesellschaft werden Einrichtungen und individuelle Gesellschaftsmitglieder verstanden, deren Handeln sich nicht primär aus einem rechtlichen oder politischen Auftrag oder aus den Zielen Wissensgewinnung oder Profit erklärt, sondern vor allem aus dem Ziel, sich individuell oder als Gruppe an gemeinwohlorientierten, also nicht an Partikularinteressen orientierten, gesellschaftlichen Gestaltungsprozessen zu beteiligen.

Funktion, Leitwert und Leitdifferenz: Ob die Zivilgesellschaft ein Teilsystem im Sinne Luhmanns und Schimanks ist, ob sich also eine durchgängige Handlungslogik herausarbeiten lässt, kann nicht eindeutig beantwortet werden. Dies hat sicher zum einen mit der teilweise überaus breiten Definition von Zivilgesellschaft zu tun (Reichel 2012, S. 56), die mitunter den gesamten gesellschaftlichen Raum zwischen Staat und Wirtschaft erfasst, und zum anderen mit der Betonung der ,Verteidigungsrolle' und damit Abwehrhaltung der Zivilgesellschaft gegenüber Staat und Wirtschaft. So umfasst der Begriff häufig sowohl den Sportverein als auch die Bürgerrechtsbewegung. Dies verdeutlicht intuitiv, weshalb die Frage, ob tatsächlich ein kohärentes gesellschaftliches Teilsystem vorliegt, noch nicht abschließend diskutiert ist. Wer davon ausgeht, dass die Zivilgesellschaft ein gesellschaftliches Teilsystem ist, setzt keinen gemeinsamen, dem gesamten Teilsystem zugrunde liegende Wertekonsens nach Reichel voraus. Das heißt, es ist nicht relevant, ob sich die Werte der einzelnen Akteure unterscheiden oder sogar widersprechen, wichtig ist nur, dass sie Werte als die wesentliche Grundlage ihres Handelns mit dem Ziel gesellschaftlicher Gestaltung erachten. Als Leitwert wird also die gemeinnützige, das heißt über den persönlichen Nutzen hinausgehende und auf der Grundlage von Werten erfolgende Gestaltung gesellschaftlicher Bedingungen gesehen.

Qualitätssicherung: Eine Handlung ist demnach im Sinne des Teilsystems Zivilgesellschaft qualitativ gut und erfolgreich, wenn sie Werte umsetzt oder gesellschaftliche Anschlüsse an diese verstärkt oder erschließt. Dies wird erreicht, indem Menschen wertebezogen davon überzeugt werden, sich einer Gruppe oder Organisation von wertemäßig Gleichgesinnten anzuschließen oder indem sich Gleichgesinnte zu neuen wertebasierten Gruppen oder Organisationen zusammenfinden. In jedem Fall besteht der unbedingte Anspruch der Akzeptanz der entsprechenden grundliegenden Werte durch die Mitglieder der Gruppe bzw. der Organisation.

Entscheidungsstrukturen: Hierarchien in zivilgesellschaftlichen Zusammenhängen leiten sich häufig daraus ab, wie authentisch wertebezogen jemand lebt bzw. wie authentisch jemand für die Werte der Gruppe oder Organisation wirbt. Durch Gleichgesinnte bekommen Akteure, innerhalb geltenden Rechts, einen größeren Handlungs- und Entscheidungsspielraum zugesprochen. 
Finanzierung: Die Zivilgesellschaft übernimmt in geringerem Maße festgelegte und definierte staatliche Aufgaben - außer in Trägerschaft - und verfügt dadurch meist über eine nur geringfügige langfristige, institutionelle Förderung. Ein häufiges Finanzierungsmodell sind Spenden durch Gleichgesinnte als Ausdruck einer wertemäßigen Übereinstimmung der spendenden Akteure mit dem jeweiligen Organisationszweck oder eher kürzere Förderungen in Form von Fördermitteln, die beispielsweise von staatlichen Einrichtungen kommen können oder von anderen gemeinnützigen Organisationen. Dies gilt insbesondere für kleine Initiativen und Bewegungen ohne langfristige Aufgabe und gesellschaftlich zugeschriebene Rolle und kann dazu führen, dass langfristige Planungen in den Hintergrund treten.

Schäden und Chancen: Schäden können sich ergeben, wenn durch Maßnahmen im Rahmen eines transdisziplinären (und transformativen) Forschungsprojekts die Werte des Akteurs aus der Zivilgesellschaft angestrebt, diese dann aber in der Umsetzung verfehlt werden. Der Schaden für die Akteure der Zivilgesellschaft besteht darin, dass die von der Teilnahme an einem solchen Projekt erhoffte Unterstützung der eigenen Werte nicht erfolgt. Wenn die angestrebten Ergebnisse verfehlt werden, verlieren die betroffenen Akteure die Legitimation für die eingesetzten Ressourcen. Ein weiterer Schaden kann entstehen, wenn in einem transdisziplinären (und transformativen) Forschungsprojekt den Werten der Akteure widersprechende Ergebnisse erzielt werden. Zivilgesellschaftliche Akteure müssen sich dann insbesondere innerhalb ihrer jeweiligen Organisationen rechtfertigen, warum sie an einem solchen Projekt mitgewirkt und das Ergebnis nicht verhindert haben. Die Ergebnisoffenheit zivilgesellschaftlicher Akteure ist also vergleichsweise geringer als beispielsweise die der Akteure aus der Wissenschaft. Die Chancen für zivilgesellschaftliche Akteure sind, dass durch das transdisziplinäre (und transformative) Forschungsprojekt die Akzeptanz und Verbreitung der eigenen Werte gefördert werden. Zudem besteht in solchen Projekten die Chance, Gleichgesinnte für die zu vertretenden Werte zu finden. Insbesondere transdisziplinäre (und transformative) Forschungsprojekte, deren Themenstellungen aus einer Werteperspektive anschlussfähig sind, können solche Chancen für die Zivilgesellschaft eröffnen.

In Heidelberg eröffnete die Zusammenarbeit mit Wissenschaftler(inne)n den zivilgesellschaftlichen Akteuren im BaWü-Lab Urban Office neue Chancen für die Thematisierung von alternativen Perspektiven auf aktuelle Stadtentwicklungsprozesse. Unterschiedliche Akteure wie Architekt(inn)en, Städteplaner(innen), Student(inn)en und Stadtforscher(innen) fanden sich zusammen und gründeten den Verein „Urban Innovation - Stadt neu denken! e. V.“ mit Sitz in Heidelberg. Der Verein konnte ein Urban Innovation Center in einem zur Zwischennutzung freigegebenen Gebäude eröffnen und stellte dort, über die Laufzeit des Reallabors hinaus, Co-Working-Arbeitsplätze zur Verfügung, mit dem Ziel, Bürgerschaft, Wirtschaft, Wissenschaft und Kommunalpolitik zusammenzubringen, um gemeinsam innovative Lösungen für Herausforderungen in Heidelberg zu entwickeln. 
Im Reallabor 131: KIT findet Stadt (,BaWü-Lab R131“) (s. auch Steckbrief im Anhang zu diesem Buch) zeigten sich die Chancen und Risiken für zivilgesellschaftliche Akteure auf unterschiedlichen Projektebenen. Zivilgesellschaftliche Akteure ergriffen in Form von Realexperimenten (,Dein NachhaltigkeitsExperiment“, vgl. Trenks et al. 2018) die Chance, die Umsetzung eigener Werte und Zielvorstellungen in der Praxis zu testen. Im Zentrum standen dabei Themen wie Gemeinschaftsbildung, Schutz der Biodiversität oder Entschleunigung. Des Weiteren brachten sie sich als Praxisakteure in transdisziplinäre und transformative Bildungsprozesse ein und nutzten die Möglichkeit, gemeinsam mit Studierenden die eigene Arbeit voranzutreiben und ihre Sichtbarkeit zu stärken (s. Beecroft 2019 in diesem Buch). Für zivilgesellschaftliche Akteure stellten die eingebrachten personellen Ressourcen den Engpass dar, der mit Blick auf die Chancen und Risiken einer gemeinsamen Aktivität entscheidend war, da die Wirksamkeit der einzelnen Aktivitäten und deren positive öffentliche Resonanz hoch sein konnten, aber nicht garantiert waren. Ein weiteres Risiko konnte sich aus einer zu hohen Intensität der im Reallabor umgesetzten Interventionen ergeben, da diese durchaus zu Ergebnissen führen konnten, die dann im normalen Arbeitsmodus der zivilgesellschaftlichen Akteure kaum aufrechtzuhalten waren.

Risikoaversion, Risikoaffinität: Aus der Handlungslogik des Teilsystems Zivilgesellschaft ergibt sich keine klare Präferenz für die Bereitschaft zum Risiko. Beispielsweise waren die in BaWü-Labs eingebundenen zivilgesellschaftlichen Akteure überaus divers, was deren Risikobereitschaft anbelangt. Das deutet darauf hin, dass die Risikobereitschaft zivilgesellschaftlicher Akteure stärker als in den anderen Teilsystemen von den involvierten Personen abhängt und weniger von der Handlungslogik des Teilsystems. Die zivilgesellschaftlichen Gruppen und Personen tendieren dazu, jeweils individuell abzuwägen, ob die sich durch ein Projekt ergebenden Chancen das mit einer Beteiligung verbundene Risiko rechtfertigen.

Risikomanagement: Das Gleiche trifft auf die Frage des Risikomanagements zu. Ein über alle Akteure der Zivilgesellschaft hinweg ähnliches Risikomanagement ist nicht erkennbar.

\section{Risiken, Schäden und Chancen durch das Zusammentreffen teilsystemspezifischer Handlungslogiken}

Wie in den vorangegangenen Kapiteln dargestellt wurde, wirken unterschiedliche teilsystembezogene Handlungslogiken und Risikokulturen in transdisziplinäre (und transformative) Forschungsprojekte hinein. Dabei muss beachtet werden, dass die Handlungen, Schwerpunkte und Positionen einzelner Personen nicht ausschließlich von ihrer Teilsystemzugehörigkeit und von den Leitwerten des jeweiligen Teilsystems abgeleitet werden können, sondern auch auf persönlichen Vorlieben, eigenen Erfahrungen und anderen individuellen Eigenschaften beruhen. Gleichwohl werden die Akteure, um kommunikativ an ihr Teilsystem anschluss- 
fähig bleiben zu können, die jeweilige Handlungslogik im Blick haben und sich auch im Sinne der jeweils spezifischen Risikokultur verhalten. Im Folgenden wird zunächst beleuchtet, was geschieht, wenn unterschiedliche Handlungslogiken aufeinandertreffen. Im Anschluss wird diskutiert, welche Chancen und Risiken sich daraus ergeben können und welche Rolle Risikoaffinität und -aversion in diesem Zusammenhang spielen.

\subsection{Was geschieht, wenn die Handlungslogiken unterschiedlicher Teilsysteme aufeinandertreffen?}

Wenn die Handlungslogiken unterschiedlicher Teilsysteme aufeinandertreffen, bedeutet dies zunächst, dass die gleiche in einem Projekt geplante Aktivität unterschiedliche Bewertungen erfährt hinsichtlich der Passung zu einem Leitwert, der Anschlussfähigkeit an die bisherige Arbeit, der notwendigen Maßnahmen zur Qualitätssicherung, der Art und des Ausmaßes bestehender Risiken und/oder des möglichen Risikomanagements. Das folgende Beispiel beleuchtet das Zusammentreffen unterschiedlicher Handlungslogiken hinsichtlich der Anschlussfähigkeit einer Befragung an unterschiedliche Teilsysteme, die über die jeweilige Leitdifferenz ersichtlich wird.

Im Reallabor „Asylsuchende in der Rhein-Neckar-Region“ (,,BaWü-Lab Asylsuchen$d e^{\text {") }}$ (s. auch Steckbrief im Anhang zu diesem Buch) wurden Daten zur Einstellung von Anwohner(inne)n in Bezug auf die Einrichtung von Unterkünften für Geflüchtete durch die Wissenschaft als besonders anschlussfähig erachtet. Untersuchungen dazu hatten zum damaligen Zeitpunkt ein besonders hohes Erkenntnispotenzial, da die dezentrale Unterbringung von Geflüchteten in verschiedenen Stadtteilen einen neuen, noch wenig erforschten Steuerungsansatz darstellten. Es war davon auszugehen, dass die Peer-Diskussion und wissenschaftlichen Begutachtungen das Potenzial derartiger Ergebnisse als vielversprechend einstufen würden. Entsprechend groß war das Interesse der beteiligten Wissenschaftler(innen) an der Datengewinnung. Da zum geplanten Befragungszeitpunkt jedoch in der betreffenden Kommune die Standortfrage von Unterkünften noch nicht entschieden war, musste auf kommunaler Seite zunächst geklärt werden, welches Signal eine derartige Befragung in die Stadt- oder Ortsteildiskussion geben könnte. Hier bestand die Sorge, dass Fragen zu Einstellungen gegenüber einer potenziellen Unterbringung von Geflüchteten in den entsprechenden Stadtteilen als Ankündigung einer tatsächlichen Unterbringung gewertet werden würden und die Stadtverwaltung deswegen mit Gegenwehr aus den befragten Stadtteilen zu rechnen hätte. Die Befürchtung war, dass ein schwieriger Dialogeinstieg mit der Bürgerschaft eine möglicherweise tatsächlich irgendwann einzurichtende Unterbringung verhindern würde, weil das Thema, verbrannt` wäre. Gesellschaftliche Gestaltung basierend auf kollektiven Entscheidungen wäre dann unmöglich, weil die durch die Befragung vorab ausgelöste Diskussion mit den Bürger(inne)n den politischen Gestaltungsspielraum verringert hätte. Das Vorgehen wurde also aus Sicht des Teilsystems Politik als wenig anschlussfähig erachtet. 
An diesem Beispiel lässt sich ein durch das Zusammentreffen zweier unterschiedlicher Handlungslogiken entstehender Widerspruch sehr schön erkennen: Die wissenschaftliche Anschlussfähigkeit einer Erkenntnis wird durch ihre Zitierung in relevanten Journals belegt. Eine Erkenntnis ist dabei insbesondere dann anschlussfähig, wenn sie im Teilsystem Wissenschaft so zum ersten Mal dargestellt wird, also wenn beispielsweise Daten präsentiert und analysiert werden, die in dieser Form noch nicht vorliegen. Im Teilsystem Politik wiederum ist die Übersetzbarkeit einer Maßnahme in politische Unterstützung ein Qualitätsmerkmal. Droht durch die Durchführung einer Maßnahme vor allem der Entzug politischer Unterstützung in der Wählerschaft, so wird sie entsprechend der Leitdifferenz von ,Macht/keine Macht' nicht als qualitativ wertvoll eingestuft.

Fragen der multiperspektivischen Qualität einer Maßnahme können also sehr unmittelbar den Weg in die Diskussion leiten, ob ein Akteur in einem transdisziplinären (und transformativen) Forschungsprojekt eine Maßnahme unterstützt und wenn ja, in welchem Umfang. Dabei kann die Beurteilung aus der Perspektive von mehr als nur zwei Teilsystemen erfolgen. Hierzu ein Beispiel, bei dem Akteure aus Wissenschaft, Politik und Zivilgesellschaft an der Diskussion einer Maßnahme beteiligt waren, bei der es um die Erweiterung des Forschungsgegenstands ging.

Im Reallabor STADT-RAUM-BILDUNG - Reallabor für die nachhaltige Planung von Bildungslandschaften und die Integration von Aus- und Umbauten von Schulgebäuden (,,BaWü-Lab SRB“) (s. auch Steckbrief im Anhang zu diesem Buch) wurden gemeinsam mit Schulträgern (kommunalen Verwaltungen) und mehreren Schulen anhand von Simulationsexperimenten mögliche zukünftige, vor allem bauliche Veränderungen (Transformationen) der Schulen und des Quartiers erarbeitet. Um ein fruchtbares Resultat mit Blick auf die Nutzbarkeit der Forschungsergebnisse zu erzielen, war es wichtig, auch eine zunächst strukturelle Erweiterung der Schule für die Oberstufe und den Abschluss der allgemeinen Hochschulreife mitzudenken, die sich auch baulich auswirken würde. Eine solche Erweiterung wird jedoch auf politischer Ebene und in der Öffentlichkeit sehr kontrovers diskutiert, da solche Schulen meist als Konkurrenz zu Gymnasien empfunden werden. Einige Elternvertreter(innen) als Akteure aus der Zivilgesellschaft wie auch Akteure aus der Verwaltung und aus der Wissenschaft begrüßten die Erweiterungsoption. Die Eltern setzten sich dafür ein, weil die dann möglichen, neuen Bildungsschwerpunkte den von ihnen unterstützten Werten in der Schulbildung entsprachen, die kommunale Verwaltung, weil sich die planerische Sicherheit im Umgang mit steigenden Schülerzahlen erhöhen würde, und die Wissenschaft, weil sie Erkenntnisse aus den neuen Bildungssettings gewinnen könnte. In allen drei Teilsystemen erwies sich die Erweiterungsoption also als grundsätzlich anschlussfähig. Die tatsächliche Entscheidung über eine Erweiterung lag jedoch beim demokratisch gewählten Gremium, dem Gemeinderat, also bei einem weiteren Akteur aus dem Teilsystem Politik. Hier wurde ein Abrücken von bisherigen Bildungsschwerpunkten nicht unbedingt als eine Maßnahme erachtet, die größte Wählerunterstützung erfahren würde, womit sich die Anschlussfähigkeit ans Teilsystem Politik letztlich als schwieriger erwies. Um diesen möglichen Konflikt zwischen Vertreter(inne)n der genannten 
Teilsysteme von vornherein abzumildern, wurde eine Erweiterung für die Oberstufe als zusätzliche Option gestaltet, so dass die Transformation in zwei Stufen - mit und ohne Erweiterung - diskutiert werden konnte.

Schwierig an der Teilsystemvielfalt ist bei diesem Beispiel unter anderem die Einschätzung, welcher Akteur welchem Teilsystem zuzuordnen ist und ob diese Zuordnung durchgängig durch die Debatte eingehalten wird. So können beispielsweise die Elternvertreter(innen) sowohl als Akteure der Zivilgesellschaft als auch als Akteure im politischen System handeln, indem sie sich entweder wertebezogen für bestimmte Bildungsschwerpunkte einsetzen oder sich als potenzielle Wähler(innen) verhalten. Realitätsnah dürfte in solchen Fällen, insbesondere an der ,Grenze' zwischen Zivilgesellschaft und Politik, die Annahme sein, dass es eine Mischung der beiden Handlungslogiken ist, die die Wahrnehmung, die Bewertung und das Handeln der Beteiligten steuert.

Ein weiteres Beispiel für unterschiedliche Perspektiven auf und Bewertungen desselben Sachverhalts in Abhängigkeit des Teilsystems ist der hinlänglich diskutierte Umgang mit dem Faktor Zeit. Während beispielsweise im Teilsystem Politik verstreichende Zeit bis zur Entwicklung einer Lösung für ein gesellschaftliches Problem in einem Machtverlust resultieren kann, ist ein so direkter Zusammenhang im Teilsystem Wissenschaft nicht herzustellen, d. h. der Faktor Zeit ist der wissenschaftlichen Qualität untergeordnet.

So beteiligten sich Akteure aus der Kommune, und damit aus den Teilsystemen Recht und Politik, aufgrund eines akuten Lösungsbedarfs für gesellschaftspolitische Fragen am BaWü-Lab Asylsuchende. Hier war es die Notwendigkeit der Integration von Geflüchteten nach 2015. Dieser Lösungsbedarf war im Sinne des Teilsystems Politik akut, da unzufriedene Wähler(innen) zu Machtverlust führen können. Auch im Teilsystem Recht spielte der Faktor Zeit eine wichtige Rolle, da im Gesetz verankerte, einzuhaltende Fristen die Grenze zwischen Recht und Unrecht markieren können. Für die Wissenschaft aber, die auf theoretischen Fragestellungen aufbaute, war dieser Faktor von untergeordneter Bedeutung. Wichtiger war den Akteuren aus diesem Teilsystem, dass Aussagen zu Effekten und ,Good Practices', die als Lösungen in Frage kommen, möglicherweise erst nach Abschluss des gesamten Forschungsprozesses, also nach drei Jahren getroffen werden können. Grund für diese Vorsicht war der Zeitbedarf, der üblicherweise nötig ist für die intensive Beobachtung beispielsweise mehrerer Fälle sowie für eine wissenschaftlichen Ansprüchen genügende Generierung und Auswertung von Daten.

Eine Herausforderung kann auch der Umgang mit rechtlichen Risiken bieten. So ist für die meisten Vertreter(innen) aus der Wissenschaft die Berücksichtigung von rechtlichen Risiken eine ungewohnte Aufgabe. Dies im Gegensatz zu beispielsweise kommunalen Verwaltungen. Erschwerend kommt oft hinzu, dass sich Realexperimente in rechtlich und verfahrenstechnisch nicht durchdeklinierten Handlungsräumen abspielen, da sie aufgrund ihres experimentellen Charakters außerhalb standardisierter Genehmigungs- und Gestaltungsprozesse abgewickelt werden müssen. 
Im BaWü-Lab Space Sharing mussten sich die Akteure aus der Wissenschaft als Betreiber des Realexperiment-Standorts Space Sharing mit rechtlichen Anforderungen der Liegenschaftseigentümer sowie der Nutzer(innen) auseinandersetzen. Das bedeutete, dass es für sie auf einmal die Leitwertdifferenz ,Recht/Unrecht' zu berücksichtigen galt, die im Teilsystem Wissenschaft nur am Rande eine Rolle spielt. Gleichzeitig gab es für das Modell der parallelen und zeitversetzten Nutzung derselben Räumlichkeiten durch unterschiedliche Personen und für verschiedene Nutzungen noch kein vorgefertigtes rechtliches Vertragsrahmenwerk oder entsprechende Erfahrungswerte. Deswegen wurde dieser Aspekte gezielt als eigene Fragestellung herausgearbeitet und als Teil des Forschungsprojekts durch einen Praxispartner mit entsprechender Fachexpertise bearbeitet.

Nicht immer muss das Aufeinandertreffen von Handlungslogiken zu einem Konflikt führen, auch ein bloßes Feststellen eines Spannungsfelds bzw. einer Überlagerung kann mit Blick auf einen konstruktiven Umgang damit schon hilfreich sein.

\subsection{Welche Chancen und Grenzen ergeben sich aus dem Zusammentreffen unterschiedlicher Handlungslogiken?}

In allererster Annäherung ergibt sich aus dem Zusammentreffen unterschiedlicher Handlungslogiken einerseits die Chance, dass sich der Handlungsspielraum eines Projekts und somit sein Erfolgspotenzial vergrößern, und andererseits das Risiko, dass sich der Handlungsspielraum und somit sein Erfolgspotenzial verringern. Beides soll im Folgenden illustriert werden.

Das Zusammentreffen zweier oder mehrerer Teilsysteme und ihrer Handlungslogiken kann zu einer Chance werden, wenn beispielsweise eine Handlung in den Bereich des Möglichen rückt, die in der Handlungslogik nur eines der beteiligten Teilsysteme zwar wünschenswert, aber nicht möglich wäre. Dies kann der Fall sein, wenn z. B. für ein Vorhaben ein bestimmtes, neues Wissen notwendig ist, dessen Produktion sich jedoch nicht mit dem Leitwert des entsprechenden Teilsystems vereinbaren lässt und deswegen keine Mittel dafür bereitgestellt werden können. Vergleichsweise häufig tritt dieser Fall bei Realexperimenten auf, die beispielsweise für eine kommunale Verwaltung oder einen zivilgesellschaftlichen Akteur durchgeführt und wissenschaftlich begleitet werden.

Im BaWü-Lab Asylsuchende wurden in einer Kooperation zwischen einer Hochschule und einem großen Bildungsträger sogenannte Lerntheken entwickelt. Dabei handelt es sich um teildigitalisierte Lernstationen, an denen - unterstützt von einer Fachlehrkraft und/oder ehrenamtlichen Sprachhelfer(inne)n - erste Deutschlernschritte unternommen werden können. Diese Lerntheken wurden insbesondere für die Lebenssituation in Erstaufnahmezentren entwickelt, in denen sich Deutschunterricht im Klassenverband aufgrund der Terminstreuung der Geflüchteten, des kurzen und versetzten Verbleibs in der Aufnahmestelle und gegebenenfalls fehlender Mittel nicht umsetzen lässt. Der 
Bildungsträger verfügte weder über die personellen Möglichkeiten noch über die wissenschaftliche Expertise, die Lerntheken ohne Unterstützung aus dem Teilsystem Wissenschaft zu entwickeln. Eine auf Forschung ausgerichtete wissenschaftliche Arbeit mit dem Ziel des Erkenntnisgewinns, wie solche Lerntheken aufzusetzen sind, entsprach nicht seinen bildungsbezogenen Kernanliegen. Umgekehrt fehlten der wissenschaftlichen Seite für die Reflexion der Lernsituation Erstaufnahmestelle und für ihre pädagogische Begleitung die Perspektive auf die praktischen Notwendigkeiten, das Wissen um die Rahmenbedingungen und generell die Einbindung in das Aufnahmesystem von Geflüchteten. Die tatsächliche Schaffung außeruniversitärer Bildungssettings entspricht nicht dem Leitwert des Teilsystems Wissenschaft. Erst durch die Kooperation rückte für beide Akteure die Entwicklung der Lerntheken in den Bereich des Möglichen.

Im BaWü-Lab Energielabor Tübingen trafen zwei Handlungslogiken aufeinander. Die eine folgte der Devise ,Alle Ressourcen in die praktische Umsetzung konkreter Energiewende-Maßnahmen stecken" und wurde insbesondere von einem zivilgesellschaftlichen Praxispartner eingebracht, der seit Langem daran arbeitete. Die andere folgte der Devise „Es gilt, die Ursachen der aktuellen Nichtumsetzung besser zu verstehen“ und wurde von den Forscher(inne)n stark gemacht. Zur Vermittlung zwischen diesen Logiken erwies es sich als sehr hilfreich, die politischen Gegebenheiten (Reform des Erneuerbare-Energien-Gesetzes, keine Internalisierung externer Kosten bei Treibhausgasen etc.), an denen das Reallabor nicht ansetzen konnte, als problematische Rahmenbedingung der Arbeit ausdrücklich auszuweisen und zu akzeptieren. Dies wirkte handlungsentlastend, weil sich damit das Reallabor diesen Fragen nicht spezifisch widmen musste, sie also aus der Arbeit ausklammern konnte. Zugleich wurden aber im Bereich der Energiesuffizienz, der dem Praxispartner am Herzen lag, konkrete Experimente zum Thema Klimaschutz und Gutes Leben (Wendepunktprojekte) durchgeführt, die die Dimension des direkten und indirekten Energiebedarfs und -konsums adressierten. Die wissenschaftliche Begleitung und Auswertung dieser Wendepunktprojekte ermöglichten eine Adressierung beider Handlungslogiken.

Der Handlungsspielraum kann sich insbesondere dann verringern, wenn die potenziellen Schäden aus der Perspektive mehrerer gesellschaftlicher Teilsysteme ins Blickfeld geraten. So können insbesondere Risikowahrnehmungen aus dem Teilsystem Recht relevant werden.

Das Realexperiment „Stäffele Gallery“ des BaWü-Labs RNM beschäftigte sich mit der Frage, wie die zahlreichen Treppen, die bei der bewegten Stuttgarter Topografie wichtige Wegverbindungen für Fußgänger(innen) darstellen, verbessert werden können. Dafür wurden verschiedene Interventionen geplant und durchgeführt, die sich nicht nur auf die Treppen selbst, sondern auch auf die ihnen angegliederten oder von ihnen gekreuzten Straßenräume bezogen. Um das Risiko von Unfällen mit Personenschäden zu vermeiden, untersagte die genehmigende Behörde jedoch verschiedene der angedachten Maßnahmen, wie beispielsweise Markierungen, die Zebrastreifen ähnelten, da diese bei Fußgänger(inne)n ein trügerisches Sicherheitsgefühl hervorrufen könnten. Hier war es klar die Risikowahrnehmung aus dem Teilsystem Recht, die zu einer Einschränkung des Handlungsspielraums führte. 
Aufgrund der großen Ausdifferenzierung und Regulierungsdichte im Teilsystem Recht besteht häufig nur sehr wenig Spielraum für Kosten-Nutzen-Abwägungen bei innovativen oder von der Norm abweichenden Prozessen. Für Akteure, die dem Teilsystem nicht angehören, ist oft nicht zu erkennen, wie sie in einer innovativen, also nicht oder wenig regulierten Situation rechtlich abgesichert handeln können. Die Zuschaltung eines Akteurs aus dem Teilsystem Recht kann jedoch aufgrund des Professionalisierungsgrads in diesem Teilsystem die Kosten für den Prozess deutlich erhöhen. Das wiederum kann dazu führen, dass eine Intervention, deren Durchführung in einem rein wissenschaftlichen, experimentellen Setting unproblematisch wäre, in der ,realen Welt' nicht durchführbar ist, und zwar auch dann nicht, wenn die Durchführung des Realexperiments geltendem Recht - beispielsweise der Straßenverkehrsordnung - nicht explizit widerspricht. Aufgrund des engen Regelsystems des Teilsystems Recht ist es bereits ausreichend für die Unzulässigkeit des Realexperiments, wenn eine experimentelle Situation rechtlich nicht geregelt ist.

Weitere Begrenzungen des Handlungsspielraums eines Projekts können durch unterschiedliche rechtliche Sanktionsformen beispielsweise im Zusammenhang mit Haftungsregelungen entstehen. Mit unterschiedlichen Risikobewertungen verbunden ist hier die Frage der Haftung. Im Teilsystem Wissenschaft wird die Haftung im Normalfall nicht von einzelnen Wissenschaftler(inne)n, sondern von deren Institution getragen. ${ }^{8}$ Für Schäden, die durch die Verwendung oder Bekanntgabe von Forschungsergebnissen entstehen, besteht eine weitreichende Haftungsfreiheit für lege artis gewonnene Erkenntnisse mit einer Verpflichtung zur sofortigen Berichtigung anerkannter Irrtümer und unter Ausschluss des Tatbestands leichter Fahrlässigkeit (Heldrich 2015). Aufgrund der bei Forschung in der ,realen Welt' potenziell höheren Eintrittswahrscheinlichkeit sowie höheren Schadenshöhe von Ereignissen hingegen kann es erforderlich sein, dass Akteure der Wissenschaft vorsichtiger agieren, dass der Handlungsspielraum also verringert wird, um Risiken auszuschließen, die zwar nicht aus der Sicht des Teilsystems Wissenschaft ein Risiko darstellen, sehr wohl aber aus der Sicht des Teilsystems Recht.

Im BaWü-Lab GO Karlsruhe wurden verschiedene Realexperimente im öffentlichen Verkehrsraum durchgeführt. Damit bestand das Risiko, dass durch die Realexperimente Verkehrsunfälle mit Sach- oder Personenschäden entstehen. Daher wurde von den Akteuren aus der Wissenschaft besonders sorgfältig darauf geachtet, sicherzustellen, dass durch die Realexperimente keine über die übliche Unfallgefahr hinausgehenden

8 Jedoch kann eine Schadensersatzpflicht für individuelle Versuchsleiter(innen) entstehen, wenn vorab nicht ausreichend über die Risiken eines (Real-)Experiments informiert wurde oder wenn es zu Verletzungen der Sorgfaltspflicht kam. 
Gefährdungen entstehen. Als an einem Standort während der Versuchsphase ein Verkehrsunfall passierte, war es wichtig, dass aus der Unfallbeschreibung nachvollzogen werden konnte, dass dieser nicht ursächlich auf das Realexperiment zurückzuführen war. Andernfalls wäre die Fortsetzung der Realexperimente bedroht gewesen.

\subsection{Was bedeuten Risikoaversion und Risikoaffinität beim Zusammentreffen unterschiedlicher Handlungslogiken?}

Wenn sich Akteure in der Handlungslogik unterschiedlicher gesellschaftlicher Teilsysteme bewegen, richten sie ihr Augenmerk auf jeweils unterschiedliche Risiken. Durch die abweichende Risikoidentifikation können Verständnisschwierigkeiten entstehen, wenn beispielsweise zwar die aus einem anderen Teilsystem stammende Risikoeinschätzung zur Kenntnis genommen, aber die damit einhergehende Risikoaversion zum Vorwurf gemacht wird.

Im BaWü-Lab GO Karlsruhe fasste ein Teil der zivilgesellschaftlichen Akteure das von rechtlichen Risiken geleitete Verhalten der Kommune als Verweigerungshaltung auf, frei nach dem Motto, dass die Stadt sich nicht traue, den geäußerten Bürgerwillen umzusetzen, sich also nicht - wie die Vertreter(innen) der Zivilgesellschaft - wertorientiert verhalte. Nicht bei allen Akteuren aus der Zivilgesellschaft konnte ein Verständnis dafür geweckt werden, dass das regelkonforme Risikoverhalten der Kommune der auf sie einwirkenden Handlungslogik des Teilsystems Recht entsprach und daher zu respektieren war.

Aus dem Zusammenwirken unterschiedlicher, den Handlungslogiken der jeweiligen Teilsysteme folgenden Risikokulturen können sich Chancen ergeben. So kann beispielsweise ein in der risikoaversen Handlungslogik seines Teilsystems handelnder Akteur seinen Handlungsspielraum indirekt, durch die Kooperation mit einem in der risikoaffinen Handlungslogik eines anderen Teilsystems handelnden Akteur erweitern. Dies ist häufig für kommunale Verwaltungen als risikoaversem Akteur der Fall, wenn sie mit Vertreter(inne)n der Wissenschaft, die in einer risikoaffinen Handlungslogik agieren, kooperieren. So kann es innovative Infrastrukturmaßnahmen geben, die als ,Regelmaßnahmen“ für eine Kommune nicht möglich sind, weil es für sie (noch) keine allgemeine Regelung gibt. Werden sie jedoch wissenschaftlich begleitet und damit als ,Experiment" gekennzeichnet, so können sie über das experimentelle Setting und die dem Teilsystem Wissenschaft zugeordnete Verantwortung im Testlauf durchgeführt werden. In einem nächsten Schritt können dann positive Ergebnisse aus solchen experimentellen Settings als Argument für eine rechtliche Anpassung und allgemeine Regelung solcher innovativer Infrastrukturmaßnahmen dienen. Dadurch wird es den Kommunen möglich, nach einem Testlauf derartige Maßnahmen als Normalfall umzusetzen. Hier stellen sich also eine Risikobetrachtung, die das Gesamtprojekt in den Blick nimmt, und die dann daraus resultierende Zuordnung des Risikos auf den jeweils risikoaffineren Akteur als Chance der Kooperation dar. 
On-Demand Mobilitätsangeboten, wie dem Bedarfsbus im BaWü-Lab Schorndorf, wird ein hohes Potenzial mit Blick auf die Gestaltung der Mobilität von morgen zugesprochen. Die rechtlichen und regulatorischen Randbedingungen erlauben allerdings die flexible Bedienform nicht ohne Weiteres. Für Experimente jedoch erlaubt das Personenbeförderungsgesetz (PBefG) eine praktische Erprobung für einen beschränkten Zeitraum von maximal vier Jahren (gestützt auf die sogenannte „Experimentierklausel“, § 2 Abs. 7 PBefG). Durch die Zusammenarbeit im Reallabor wurde es möglich, das Potenzial einer innovativen Betriebsweise zu erproben. Ob eine Umsetzung als Normalfall möglich wäre, müsste geprüft werden (gestützt auf die „Auffangklausel“", $\S 2$ Abs. 6 PBefG).

Wird umgekehrt die Risikobewertung durch die Akteure jeweils einzeln vorgenommen, ist für die Entscheidung meist das geringste akzeptierte Risiko der jeweils vertretenen Teilsysteme ausschlaggebend. Ein Realexperiment kann beispielsweise nicht realisiert werden, wenn das Risiko schon nur eines Akteurs überschritten wird. Der ,kleinste gemeinsame Risikonenner ${ }^{6}$ wird dann selbst zum Risiko für das Projekt. Aufgrund des unterschiedlichen Verständnisses von Risiko kann diese Entscheidung zudem für die anderen Akteure nur schwer nachvollziehbar sein, was zu Spannungen im Projekt führen kann.

Im BaWü-Lab GO Karlsruhe hatten die wissenschaftlichen und die kommunalen Akteure teilweise unterschiedliche Einschätzungen zum akzeptablen Risiko von Realexperimenten zu Maßnahmen zur Fußgängerförderung im öffentlichen Straßenraum. Beide Akteure waren sich einig, dass der Verkehrssicherheit ein hohes Gewicht einzuräumen ist. Uneinigkeit bestand hingegen darüber, welche Folgewirkungen der Maßnahmen für andere Verkehrsteilnehmer(innen) (Umwege, Parkraum etc.) akzeptabel seien. Das Risiko von Folgewirkungen wurde entsprechend der den beteiligten Teilsystemen innewohnenden Risikoaffinität und -aversion unterschiedlich eingeschätzt. Da die Kommune die verkehrsrechtlichen Anordnungen erlässt, war deren Risikobewertung letztendlich ausschlaggebend.

\section{Welches Risikomanagement ist in Anbetracht unterschiedlicher Handlungslogiken und Risikokulturen zu empfehlen?}

In Kapitel 3 dieses Beitrags wurden die Handlungslogiken ausgewählter in transdisziplinären (und transformativen) Forschungsprojekten wie Reallaboren vertretenen gesellschaftlichen Teilsysteme anhand zentraler Charakteristika herausgearbeitet. Zudem wurden Risikobewertungen, Risiko und übliche Risikomanagementansätze in diesen Teilsystemen dargestellt. Auf dieser Grundlage wurde in Kapitel 4 anhand von Beispielen gezeigt, wie sich das Zusammentreffen der unterschiedlichen Handlungslogiken und Risikokulturen in Projekten auswirken kann. Im Folgenden werden nun drei Empfehlungen gegeben, wie sich aufbauend auf den Erfahrungen der BaWü-Labs das Zusammentreffen der unterschiedlichen 
Handlungslogiken und Risikokulturen in transdisziplinären (und transformativen) Forschungsprojekten konstruktiv begleiten lässt. Diese sind ein aktives Risikomanagement (Abschnitt 5.1), die Analyse und Anpassung der Risikoverteilung (Abschnitt 5.2) sowie das bewusste Antizipieren von Risiken (Abschnitt 5.3).

\subsection{Aktives Risikomanagement betreiben}

Die Literatur (vgl. u. a. Motel und Richter 2016; Link 2001; Hill 2016) empfiehlt für das Zusammenspiel von Akteuren mit unterschiedlichen Handlungslogiken die Anwendung eines aktiven Risikomanagements. Diese Empfehlung kann auch auf transdisziplinäre (und transformative) Forschungsprojekte übertragen werden, und sie lässt sich auch aus den Erfahrungen der BaWü-Labs ableiten. Ein Risikomanagement erweitert den Handlungsspielraum für die Kooperation der verschiedenen Akteure, da Risiken früher identifiziert werden können und daher mehr Zeit zur Verfügung steht, mit diesen Risiken proaktiv umzugehen (Motel und Richter 2016). Ein umfassendes Risikomanagement mit den Schritten Risikoidentifikation, Risikobewertung und Risikosteuerung wurde in keinem der BaWü-Labs aktiv realisiert, in einzelnen BaWü-Labs wurden jedoch einzelne Schritte eines Risikomanagements durchgeführt, die zeigen, dass ein solches bewusstes Risikomanagement möglich ist.

In einer sehr frühen Projektphase des BaWü-Labs BUGA:log fand ein erster Workshop mit allen beteiligten Akteuren statt, in dem es darum ging, eine gemeinsame Projektzeitplanung aufzusetzen sowie kritische Pfade und Abhängigkeiten zwischen einzelnen Arbeitspaketen zu benennen. Dieser Plan enthielt also auch Elemente der Risikoidentifikation. Er diente im weiteren Projektverlauf allen Akteuren des Reallabors als Orientierung und gab gleichzeitig eine Struktur für die regelmäßigen Projektsitzungen vor. Diese Form der Strukturierung wurde von allen Projektpartnern akzeptiert und für erforderlich gehalten, insbesondere auch, weil der Zeitraum, in dem das Realexperiment durchgeführt werden sollte - die Bundesgartenschau 2019 -, von Beginn an klar und fest vorgegeben war. Da damit ein Verschieben des Realexperiments unmöglich war und da gleichzeitig die Nichtumsetzung des Realexperiments Konsequenzen für die Bundesgartenschau gehabt hätte, war es von zentraler Bedeutung, die Unwägbarkeiten sehr bewusst in die Projektplanung aufzunehmen.

Zum Ersten zeigt die Erfahrung der BaWü-Labs bezüglich der Fragen des Risikomanagements, dass es wichtig ist, nicht nur auf Risiken im Sinne von Schäden $\mathrm{zu}$ fokussieren, sondern auch Chancen sprachlich und analytisch durchgehend im Blick zu haben. Eine solche Chance ist beispielsweise eine mögliche Erweiterung des Handlungsspielraums der Akteure im Zusammenspiel der Handlungslogiken und Risikokulturen (s. die Beispiele in den Abschnitten 4.2 und 4.3). Die systematische Analyse von Chancen und Risiken aus der Perspektive der jeweils wirkenden Handlungslogiken und Risikokulturen erlaubt es, diese Chancen und Risiken nicht aus dem Blick zu verlieren und damit die Handlungssicherheit aller 
Beteiligten und des Projekts insgesamt zu erhöhen. Die verschiedenen Akteure haben aufgrund der für sie relevanten Handlungslogiken oft auch unterschiedliche Kompetenzen entwickelt. Werden diese Kompetenzen im gesamten Projektverlauf im Blick behalten, ist es möglich, jeweils den Akteur mit einer Aufgabe zu betrauen, für den diese nicht neu ist, weil sie in besonderer Weise seiner Handlungslogik entspricht. Wenn beispielsweise ein Reallabor dazu beitragen will, bestimmte Veränderungen im Verhalten einer Stadtbevölkerung im Sinne einer nachhaltigeren Lebensführung zu bewirken, gilt es, die Kompetenzen der Akteure aus den Teilsystemen Politik und Zivilgesellschaft bezüglich der Gestaltung der Gesellschaft zu nutzen. Das Teilsystem Politik kann hierbei z. B. sowohl auf Mechanismen der Macht als auch der Vorbildwirkung zurückgreifen, während das Teilsystem Zivilgesellschaft z. B. über Beispiele, Vernetzung und die Ansprache von Werten Einfluss nehmen kann.

Zum Zweiten sind in den unterschiedlichen Handlungslogiken Erfolg und Misserfolg nicht gleichartig definiert. Erfolg und Misserfolg sind eine nachträgliche Bewertung, die sich an der ursprünglichen Zielsetzung des jeweiligen Akteurs bemisst (siehe Kapitel 2). Es empfiehlt sich, ein besonderes Augenmerk auf die unterschiedlichen, aus den verschiedenen Handlungslogiken resultierenden Auffassungen von Schaden zu legen - insbesondere mit Blick auf die Langzeitfolgen, die diese mit sich bringen können. Hier sind beispielsweise Schäden aus Sicht des Teilsystems Recht zu nennen, da diese oft Haftungsfragen beinhalten und somit im Eintrittsfall des Schadens schwerwiegende Auswirkungen auf die Träger des Risikos, auch über den Projektzeitraum hinaus, haben können. Klare Absprachen sowie Bewusstmachung dieser rechtlichen Risiken bei allen Beteiligten sind notwendig. Gegebenenfalls kann es sinnvoll sein, parallel zu oder vor Realexperimenten das entsprechende rechtliche Setting auszuarbeiten. Ein weiteres relevantes Beispiel sind politische Schäden, die gebührend beachtet werden sollten. Ein Realexperiment, das zum Beispiel die Bevölkerung zu stark polarisiert, kann die Umsetzung eines politischen Projekts auf Jahre hinaus unmöglich machen, weswegen ein detailliertes Risikomanagement hier von Vorteil wäre.

Zum Dritten erlaubt es eine nach Handlungslogiken differenzierte Analyse von Erfolg und Misserfolg, die verengte Erfolgsbewertung im Sinne von ,erfolgreich/ gescheitert" für das Gesamtprojekt zu überwinden. So kann beispielsweise ein vorab entwickeltes, nach Handlungslogiken ausdifferenziertes Chancen- und Risikenraster dazu beitragen, über das gesamte Projekt hinweg einen detaillierten Überblick über Erfolgs- und Misserfolgsaspekte des Projekts zu haben. Ein solches Raster ist für die Steuerung wie für die interne und externe Kommunikation eines Projekts dienlich.

Konkrete Maßnahmen des Risikomanagements lassen sich leicht verständlich für alle Beteiligten umsetzen (ohne die Notwendigkeit, vorher ein komplexes Risikomanagement-Regelwerk verstehen zu müssen). Dies ist bei Bedarf auch kurzfristig 
und mit beschränktem Zeitbudget möglich. Aufgrund der für transdisziplinäre (und transformative) Forschungsprojekte wichtigen Vorteile empfiehlt es sich daher, solche unabhängig von Größe oder zeitlichem Umfang des Forschungsprojekts durchzuführen. Je größer und komplexer das Projekt wird, umso sinnvoller kann es sein, ein bewusstes Risikomanagement über das Gesamtprojekt aufzubauen. Teil eines umfassenden Risikomanagements eines transdisziplinären (und transformativen) Forschungsprojekts sollte sein, dass zu Beginn eine Reflexion über die im Projekt vorhandenen Handlungslogiken und Risikokulturen stattfindet und dass Maßnahmen ergriffen werden, die das Verständnis zwischen den Vertreter(inne)n der verschiedenen Teilsysteme für die jeweils anderen Handlungslogiken und Risikokulturen erhöhen.

Bei der Planung und Umsetzung des Risikomanagements sowie einzelner Maßnahmen sollten auf jeden Fall der zeitliche sowie der personelle Aufwand berücksichtigt werden sowie Überlegungen zum Verhältnis von Aufwand und Nutzen angestellt werden. Maßnahmen zum Aufbau von Verständnis für die unterschiedlichen Handlungslogiken und die damit einhergehenden Risikokulturen sind kurzfristig aufwendig, können aber zu einem späteren Zeitpunkt im Projekt Konflikte und zeitliche oder finanzielle Verluste ersparen. Auch längerfristige Zerwürfnisse zwischen Projektbeteiligten können auf diese Weise verhindert werden. Ein komplexes Regelwerk zum Risikomanagement oder zeitaufwendige Verpflichtungen, wie die Teilnahme an umfangreichen Workshops zur gemeinsamen Risikoidentifikation und zur Definition von Maßnahmen zur Risikostreuung, können aber potenzielle Akteure auch von einer Beteiligung abschrecken.

\subsection{Risikoverteilung analysieren und bei Bedarf anpassen}

In Reallaboren sollten, wie generell in Innovationskooperationen, die Chancen und Risiken fair auf die Akteure aufgeteilt werden. Während beim traditionellen Risikomanagement die getrennt vorgenommenen Einzelrisikobewertungen der verschiedenen Akteure zum Risiko des Projekts insgesamt zusammengefügt werden, bietet sich nach Link (2001) bei Innovationskooperationen ein umgekehrtes Vorgehen an. Dabei wird beispielsweise in einem Reallabor zuerst das Risiko eines Realexperiments aus übergeordneter Sicht betrachtet. In einem nächsten Schritt wird dann analysiert, wie dieses Risiko auf die einzelnen Akteure im Reallabor aufgeteilt wird. Als Ausgangslage sind in transdisziplinären (und transformativen) Forschungsprojekten Risiken oft insofern ungleich verteilt, als das, was aus Sicht eines Teilsystems als Risiko zu werten ist, von Akteuren aus einem anderen Teilsystem verursacht wird.

In Reallaboren besteht zum Beispiel, abhängig vom Forschungsgegenstand, häufig eine ungleiche Risikoverteilung zwischen Forscher(inn)en und kommunalen Akteuren, da die Forscher(innen) Risiken verursachen, die von der Verwaltung 
mitgetragen werden müssen. Dabei kommt noch hinzu, dass der Nutzen, der aus einer Inkaufnahme des Risikos entsteht, in der Handlungslogik des einen Teilsystems relevant ist, der Schaden jedoch in der eines anderen. Insbesondere bei Projekten, die technische Strukturen aufbauen, ist der Frage besondere Aufmerksamkeit zu widmen, ob Nutzen und Schaden jeweils einseitig in einer bestimmten Handlungslogik zu verorten sind. Bei Realexperimenten, die im Stadtraum erfolgen, können auch Externe und eventuell dem Forschungsgegenstand gegenüber Ablehnende von den Auswirkungen betroffen sein. Dies kann besonders dann zu Problemen führen, wenn Wissenschaftler(innen) ein Realexperiment mit dem Ziel wissenschaftlichen Erkenntnisgewinns durchführen, in der Öffentlichkeit aber die Kommune als Eignerin der technischen Struktur und somit als Verantwortliche wahrgenommen wird. Hier entsteht eine Diskrepanz zwischen jenen, die einen Gewinn aus dem Realexperiment ziehen (bspw. in Form von Daten für Publikationen), und jenen, die Risiken (bspw. Bürgerbeschwerden oder sogar Klagen) tragen.

Im BaWü-Lab GO Karlsruhe wurden Maßnahmen zur Verbesserung der Situation des Fußverkehrs gemeinsam mit zu Fuß Gehenden entwickelt und provisorisch umgesetzt und im Sinne einer wissenschaftlichen Handlungslogik getestet. Um die Chance der Wissensgenerierung und der Förderung des Fußverkehrs zu haben, waren Eingriffe in den öffentlichen Verkehrsraum erforderlich. Als Maßnahme zur Temporeduzierung in Wohnstraßen kam der Wunsch auf, im Rahmen eines Realexperiments die Wirksamkeit von Fahrbahnschwellen zu testen. Diese werden deutschlandweit eingesetzt, verfügen aber nicht über eine explizite Zulassung, da es für Fahrbahnschwellen und Kissen keine eindeutigen Anforderungen in Form von technischen Lieferbedingungen oder Ausführungsbestimmungen gibt. Der Einbau muss daher von der jeweiligen Straßenverkehrsbehörde genehmigt werden, die damit aber auch potenziell Verantwortung übernimmt, falls z. B. der Sturz eines Radfahrers durch einen solchen Einbau verursacht werden sollte. Dieses Risiko lag also bei einem kommunalen Akteur, der einer rechtlichen Handlungslogik folgt. Das Reallabor steckte dadurch in einer Zwickmühle: Um die Wirkung der Einbauten zu testen, musste schon vorher deren Wirkung bekannt sein und durch das Reallabor nachgewiesen werden. Die Abklärung, wie die Haftungsfrage geregelt werden kann, stellte einen langwierigen Prozess dar.

Im BaWü-Lab SRB stellte sich vor Projektbeginn die Frage, inwiefern Realexperimente in Schulen oder in deren direkter Umgebung realisiert werden können. Schulen stellen einen sensiblen Kontext dar, da die Sicherheit der Kinder und Jugendlichen höchste Priorität genießt. Temporäre physische Interventionen (ähnlich wie Parklets) sind an Schulen nicht denkbar, da sich die Kinder verletzen könnten. Im Falle eines Unfalls wären die Schulleitung und der Schulträger (die Kommune) haftbar, nicht jedoch die Wissenschaftler(innen). Das heißt, die Schule würde im Sinne einer rechtlichen Handlungslogik den Schaden tragen müssen, während die Wissenschaft durch den potenziellen Erkenntnisgewinn den Nutzen aus dem Realexperiment ziehen würde. Das Risiko läge damit ausschließlich bei den Praxispartnern (Schulleitung und Schulträger). Um diesen Konflikt zu vermeiden, wurde im Reallabor ein spezielles methodisches Vorgehen als Alternative zum Realexperiment entwickelt. 
Deswegen sollte bei einer Anpassung der Risikoaufteilung das Verursacherprinzip beachtet werden, indem möglichst diejenigen Akteure, die ein Risiko verursachen, dieses auch tragen. Wenn eine Anwendung des Verursacherprinzips nicht möglich ist, sollte der Akteur, der das Risiko tragen müsste, diesem zustimmen können, so dass eine bewusste Übernahme stattfinden könnte. Dabei sind die Grenzen der Möglichkeit zur Übernahme von Risiken, die sich bei den verschiedenen Akteuren aufgrund ihrer jeweiligen Handlungslogiken ergeben, zu berücksichtigen. Weiterhin bietet sich der Risikotransfer zu denjenigen Akteuren zum Beispiel in einem Reallabor an, die eine höhere Risikoaffinität besitzen. So kann in einem Reallabor für bestimmte risikobehaftete Realexperimente ein risikoaffiner Akteur die Verantwortung übernehmen und dies in klaren Regelungen zur Verantwortungsübernahme festhalten.

Im BaWü-Lab GO Karlsruhe wurde bei der Öffentlichkeitsarbeit zu den Realexperimenten in den Vordergrund gestellt, dass diese durch Akteure aus der Wissenschaft vorbereitet, umgesetzt und begleitet werden. Damit wurde versucht zu gewährleisten, dass das Risiko der Kritik von Bürger(inne)n an den Realexperimenten sich nicht auf die Kommune, sondern auf die Wissenschaftler(innen) konzentriert. Diese haben die Kritik gesammelt und mussten auf diese auch auf Bürgerveranstaltungen reagieren.

Teile eines Forschungsprojekts, wie zum Beispiel ein bestimmtes Realexperiment, können schließlich von einem Teil der Projektpartner in Abhängigkeit der jeweils wirkenden Handlungslogik als Misserfolg angesehen werden. Dies kann von den Akteuren aus der Wissenschaft berücksichtigt werden, indem entsprechende Vorsorgestrategien entwickelt werden, wie dem von einem Schaden betroffenen Teilsystem vermittelt werden kann, dass für ein anderes Teilsystem derselbe Projektteil positiv verlaufen ist. Auch hier gilt, dass der Blick auf das Projekt als Ganzes eine differenziertere Sicht erlaubt, als wenn die einzelnen Akteure nur auf ihre eigenen Risiken und Chancen fokussieren.

\subsection{Risiken bewusst antizipieren - Prävention und Nachsorge vereinbaren}

Eine bewusste Auseinandersetzung mit verschiedenen Risikokulturen kann den Eintritt von Schäden reduzieren, indem sie ein Risikomanagement ermöglicht. Ein solcher Analyseprozess kann zudem eine positive Teamerfahrung für ein Projekt sein. Aus diesen Gründen ist es wichtig, Risiken bewusst zu antizipieren sowie Prävention und Nachsorge zu vereinbaren.

$\mathrm{Zu}$ relevanten Zeitpunkten im Ablauf eines transdisziplinären (und transformativen) Forschungsprojekts, zum Beispiel bei der konkreten Planung eines Realexperiments, ist es wichtig, das jeweils unterschiedliche Verständnis der Akteure für die Identifikation, Bewertung und Steuerung von Risiken offenzulegen und zu diskutieren. Dies kann beispielsweise im Rahmen eines Workshops geschehen. 
Zudem sind Entscheidungsmechanismen zur Risikosteuerung vorab mit allen Projektpartnern einvernehmlich zu vereinbaren. Zwischen den Akteuren kann es unterschiedliche Präferenzen geben, welche Risikosteuerungsstrategien bevorzugt werden. Risikoaverse Akteure wie Kommunen bevorzugen tendenziell ursachenbezogene Maßnahmen, mit der die Eintrittswahrscheinlichkeit von Schäden reduziert wird, da angestrebt wird, diese wenn immer möglich ganz zu vermeiden. Risikoaffinere Akteure wie zum Beispiel Akteure aus der Wissenschaft berücksichtigen hingegen eher wirkungsbezogene Maßnahmen, die darauf zielen, die Konsequenzen möglicher Schäden zu reduzieren.

Solche unterschiedlichen Präferenzen können zum Beispiel zu Differenzen zwischen Akteuren bezüglich Realexperimenten führen. So können Verwaltungseinheiten eine Nichtgenehmigung von Realexperimenten bevorzugen, wenn sie diese als zu risikoreich einstufen, während die Wissenschaftler(innen) eine Setzung von Maßnahmen zur Risikoreduktion anstreben würden. In solchen Aushandlungsprozessen zwischen den Akteuren ist die unterschiedliche Beurteilung von Schäden zu berücksichtigen. Wissenschaftler(innen) stufen die Nichtumsetzung eines Realexperiments als Schaden ein, da somit die Möglichkeit für Erkenntnisgewinn ausbleibt. Die Verwaltung sieht dagegen den Schaden in der Umsetzung rechtlich unzulässiger Maßnahmen.

Trotz sorgfältiger Planung, Konfliktprävention und Risikomanagement kann es geschehen, dass Realexperimente oder andere Projektteile für die Akteure eines oder mehrerer Teilsysteme zum Misserfolg werden. Was Misserfolg ist beziehungsweise was als Misserfolg empfunden wird, kann dabei je nach Teilsystem sehr unterschiedlich ausfallen. Relevant bei der Bewertung von Erfolg und Misserfolg ist vor allem die Handlungslogik der unterschiedlichen gesellschaftlichen Teilsysteme. So kann beispielsweise eine Intervention im öffentlichen Raum in einem Projekt zwar nicht den von der Stadtverwaltung gewünschten Effekt haben, aber dabei helfen, umfangreiche Daten zu erheben. In einem solchen Fall steht die nach außen als verantwortlich wahrgenommene Verwaltung möglicherweise in der Kritik durch Anwohner(innen) oder die Presse, obwohl durch die Intervention wertvolle Erkenntnisse für Veröffentlichungen oder weitere Interventionen gewonnen wurden. Nur durch einen offenen internen Dialog oder den vorher einkalkulierten Eintritt eines Schadenfalls ist es möglich, anschließend noch ähnliche Aktionen durchzuführen und auch weiterhin vertrauensvoll kooperieren zu können. Umgekehrt kann es auch geschehen, dass eine öffentliche Intervention den von den Teilsystemen Politik und/oder Zivilgesellschaft erwünschten Effekt erzielt, aber keine wissenschaftlichen Erkenntnisse erbringt. In einem solchen Fall führt die Forschung z. B. nicht zu Publikationen, wodurch die beteiligten Wissenschaftler(innen) in die Kritik beispielsweise seitens des Fördergebers (und der anderen Akteure im Teilsystem Wissenschaft) geraten können. Hier könnte die Prävention in einem offenen Dialog über Qualitätskriterien mit dem Fördergeber bestehen. 


\section{Was sonst noch zu sagen wäre ...}

Der vorliegende Beitrag wurde auf Grundlage der Erfahrungen aus verschiedenen BaWü-Labs entwickelt. Während der gemeinsamen Arbeit und der Reflexion über diese Erfahrungen wurde den Autor(inn)en erneut deutlich, dass es in jedem BaWü-Lab wichtig war, Konflikte zwischen unterschiedlichen Akteuren und Akteursgruppen zu vermeiden. Dabei waren weder die Konflikte noch der Umgang damit identisch. Ein Grund für diese Diversität war, dass jedes BaWü-Lab verschiedene Zusammensetzungen von Vertreter(inne)n der beschriebenen Teilsysteme mit ihren jeweiligen Handlungslogiken aufwies. Außerdem hatte jedes BaWü-Lab, bezogen auf die Verteilung von inhaltlichen, finanziellen, organisatorischen, rechtlichen und kommunikativen Verantwortlichkeiten auf die Beteiligten der verschiedenen Teilsysteme, eine eigene Struktur gewählt. Aufgrund der Bandbreite der Erfahrungen aus den BaWü-Labs liefert dieser Beitrag eine recht umfassende Darstellung möglicher Risiken und möglicherweise auftretender Konflikte. Jedoch und trotzdem ist der Beitrag nicht abschließend und erhebt auch keinen Anspruch auf Vollständigkeit, denn jedes transdisziplinäre (und transformative) Forschungsprojekt hat seine eigene Konstellation von Teilsystemen und wird entsprechend seinen eigenen Lösungsweg hinsichtlich potenzieller Risiken und Konflikte finden müssen.

Gemeinsam war den BaWü-Labs jedoch ein ständig anhaltender dynamischer Prozess, der kaum vorhersehbar oder planbar war. Dieser ging meist einher mit Änderungen von Bedingungen und Verschiebungen in der Ausgangslage. So zogen beispielsweise politische Wahlen Veränderungen der Rahmenbedingungen nach sich. Dies konnte zu personellen Änderungen oder zu inhaltlichen Neuausrichtungen einzelner Vertreter(innen) der Teilsysteme führen. Mit Blick darauf wird von den Autor(inn)en ein wiederholtes Überprüfen von Risiken und Konflikten auch während der Projektlaufzeit empfohlen.

Bei der Betrachtung der Teilsysteme unserer funktionalen Gesellschaft wurde deutlich, dass es sich um ein sehr effizientes - über die Historie hinweg entwickeltes - Gesellschaftssystem handelt. Dieses scheint jedoch eher unflexibel zu sein und im Widerspruch zu Innovation und vor allem zur Transformation zu stehen. Insofern wird ein großer Mehrwert in der transdisziplinären (und transformativen) Forschung gesehen. Denn erst durch das Ausloten dieser funktionalen ,Grenzen' zwischen den gesellschaftlichen Teilsystemen und das Infragestellen von Setzungen innerhalb dieser ,Grenzen' entstehen Konflikte, aber auch Synergien zwischen den Teilsystemen. Dieser Prozess kann - losgelöst vom eigentlichen Inhalt eines transdisziplinären Projekts - transformativ wirken für ein Teilsystem, indem andere Leitwerte, Leitdifferenzen und Risikokulturen Berücksichtigung finden. Die Herausforderung besteht also darin, diese Chance der 
transdisziplinären (und transformativen) Forschung zu nutzen, gleichzeitig mit den Risiken umzugehen und mögliche Konflikte nicht zu scheuen, sondern diese offen anzusprechen und produktiv anzugehen. Dies soll durch diesen Beitrag unterstützt werden.

\section{Dank}

Ein besonderer Dank der Autor(inn)en geht an Marius Albiez, Monika Bachinger, Richard Beecroft, Tobias Bernecker, Fabian Dembski, Raphael Dietz, Laura Gebhardt, Mike Letzgus, Editha Marquardt, Thomas Potthast, Regina Rhodius und Michael Ruddat für die Bereitstellung von Beispielen aus ihren jeweiligen Reallaboren. Sie danken Marius Albiez, Fabian Dembski, Raphael Dietz, Thorsten Erl und Jan Riel für die Diskussion über eine frühere Textfassung im Rahmen des internen Reviews. Die Autor(inn)en möchten zudem den beiden anonymen externen Gutachter(inne)n für die konkreten Hinweise zur Verbesserung des Textes danken. Schließlich danken sie den beiden Herausgebenden, Rico Defila und Antonietta Di Giulio, für ihre Rückmeldungen zum Text.

\section{Literatur}

Arnold, A., \& Piontek, F. (2018). Zentrale Begriffe im Kontext der Reallaborforschung. In R. Defila \& A. Di Giulio (Hrsg.), Transdisziplinär und transformativ forschen. Eine Methodensammlung (S. 143-154). Wiesbaden: Springer VS. doi: 10.1007/978-3-65821530-9_8.

Baecker, D. (2016). Wirtschaft als funktionales Teilsystem. In A. Maurer (Hrsg.), Handbuch der Wirtschaftssoziologie (S. 163-180). Wiesbaden: Springer VS.

Bechmann, G., \& Stehr, N. (2000). Risikokommunikation und die Risiken der Kommunikation wissenschaftlichen Wissens: Zum gesellschaftlichen Umgang mit Nichtwissen. GAIA, 9 (2), (S. 113-121).

Beecroft, R. (2019). Das „Transformative Projektseminar“ - didaktische Ansätze und methodische Umsetzung. In R. Defila \& A. Di Giulio (Hrsg.), Transdisziplinär und transformativ forschen, Band 2. Eine Methodensammlung (S. 293-337). Wiesbaden: Springer VS.

Bonß, W. (2011). (Un-)Sicherheit in der Moderne. In P. Zoche, S. Kaufman \& R. Haverkamp (Hrsg.), Zivile Sicherheit. Gesellschaftliche Dimensionen gegenwärtiger Sicherheitspolitiken (S. 43-109). Bielefeld: transcript.

Brugger, P. (2009). Der IT Business Case. Wiesbaden: Springer. 
Callies, G.-P. (2006). Systemtheorie: Luhmann/Teubner. In S. Buckel, R. Christensen \& A. Fischer-Lescano (Hrsg.), Neue Theorien des Rechts (S. 57-74). Stuttgart: Lucius \& Lucius.

Crouch, C. (2008). Postdemokratie. Frankfurt a. M.: Suhrkamp.

DIN ISO 31000 (2018). Risikomanagement - Leitlinien (ISO 31000:2018). doi: 10.31030/ 2880923.

Douglas, M. (1992). Risk and blame essays in cultural theory. London: Routledge.

Egner, H. (2008). Warum konnte das nicht verhindert werden? Über den (Nicht-)Zusammenhang von wissenschaftlicher Erkenntnis und politischen Entscheidungen. In C. Felgentreff \& T. Glade (Hrsg.), Naturrisiken und Sozialkatastrophen (S. 421-433). Berlin, Heidelberg: Springer.

Fischer, S. (2016). Risiko und Risikokultur - Konzepte für die Beschreibung und Analyse des gesellschaftlichen Umgangs mit Unsicherheit. Zeitschrift für Außen- und Sicherheitspolitik, 9 (2), (S. 191-200).

Hanebuth, A., Lee, R., Meschke, S., \& Nicklas, M. (2015). Schlussbetrachtung. In A. Hanebuth, R. Lee, S. Meschke, \& M. Nicklas (Hrsg.), Forschungskooperationen zwischen Wissenschaft und Praxis. Erkenntnisse und Tipps für das Management (S. 225233). Wiesbaden: Springer Gabler.

Heldrich, A. (2015). Die zivilrechtliche Haftung für Schäden durch wissenschaftliche Betätigung. Ordnung der Wissenschaft, (3), (S. 155-164).

Hill, H. (2016). Innovationsmanagement in der Verwaltung. In Innovativer Staat 2017. Das Jahrbuch für die Verwaltung der Zukunft (S. 66-67). Berlin: Wegweiser Media \& Conferences $\mathrm{GmbH}$.

Hoitsch, H. J., Winter, P., \& Bächle, R. (2005). Risikokultur und risikopolitische Grundsätze: Strukturierungsvorschläge und empirische Ergebnisse. Controlling und Management, 49 (2), (S. 125-133).

Huber, I. (2013). Wissenschaft als Beruf? Das Paradox des heutigen Wissenschaftsverständnisses. Bulletin der Vereinigung der Schweizerischen Hochschuldozierenden, 3 (4), (S. 34-37).

Jahner, S., \& Kremar, H. (2005). Risikokultur als zentraler Erfolgsfaktor für ein ganzheitliches IT Risk Management. IM Information Management \& Consulting, (2), (S. 4754).

Joas, H. (2005). Die kulturellen Werte Europas. Eine Einleitung. In: H. Joas \& K. Wiegandt (Hrsg.), Die kulturellen Werte Europas (S. 11-41). Frankfurt: Fischer Taschenbuch.

Kepplinger, H.-M. (2009). Politikvermittlung. Wiesbaden: Verlag für Sozialwissenschaften. 
Köglberger, K., Dietz, R., Eller, C., Piontek, F. M., Albiez, M., \& Potthast T. (2019). Schutz in der Exposition, Schutz für die Exposition - Wie man in transdisziplinären und transformativen Forschungsformaten mit Ungewohntem und erhöhter Aufmerksamkeit umgeht. In R. Defila \& A. Di Giulio (Hrsg.), Transdisziplinär und transformativ forschen, Band 2. Eine Methodensammlung (S. 93-138). Wiesbaden: Springer VS.

Kromschröder, B. (2003). Risiko - Risikoforschung - Risikokommunikation. In J. Geiss, D. Wortmann \& F. Zuber (Hrsg.), Nachhaltige Entwicklung - Strategie für das 21. Jahrhundert? (S. 123-142). Wiesbaden: VS Verlag für Sozialwissenschaften.

Link, P. (2001). Risikomanagement in Innovationskooperationen. Ein Ansatz zur fairen Aufteilung von Chancen und Risiken. Dissertation, ETH Zürich.

Luhmann, N. (1984). Die Wirtschaft der Gesellschaft als autopoietisches System. Zeitschrift für Soziologie, 13 (4), (S. 308-327).

Luhmann, N. (1988). Die Wirtschaft der Gesellschaft. Frankfurt a. M.: Suhrkamp.

Motel, J., \& Richter, M. (2016). Risikomanagement in einer Bundesbehörde. VM Verwaltung \& Management, 22 (2), (S. 73-82).

Möller, H., \& Bruck, E. (1980). Kommentar zum Versicherungsvertragsgesetz und zu den Allgemeinen Versicherungsbedingungen unter Einschluss des Versicherungsvermittlungsrechtes. Berlin: De Gruyter.

Puttrowait, E., Dietz, R., Gantert, M., \& Heynold, J. (2018). Der Weg zum Realexperiment - Schlüsselakteure identifizieren, Kooperationsstrukturen aufbauen, Projektideen auswählen. In R. Defila \& A. Di Giulio (Hrsg.), Transdisziplinär und transformativ forschen. Eine Methodensammlung (S. 195-232). Wiesbaden: Springer VS.

Rayner, S. (1992). Cultural Theory and risk analysis. In S. Krimsky \& D. Golding (Hrsg.), Social theories of risk (S. 83-115). Santa Barbara: Praeger Publishers.

Reichel, A. (2012). Die Zivilgesellschaft der Gesellschaft? Systemtheoretische Beobachtungen eines unruhigen Funktionssystems. In S. Jansen, E. Schröter \& N. Stehr (Hrsg.), Bürger. Macht. Staat? - Neue Formen der gesellschaftlichen Teilhabe, Teilnahme und Arbeitsteilung (S. 53-73). Wiesbaden: Springer Fachmedien.

Renn, O. (2008). Concepts of risk: an interdisciplinary review part 1 - disciplinary risk concepts. GAIA, 17 (1), (S. 50-66).

RLSS (Reallabor Space Sharing, ABK Stuttgart) (Hrsg.). (2018). One Space Fits All Space Sharing Report. Erfahrungsbericht und Empfehlungen aus zwei Jahren Betriebserfahrung des Reallabor Space Sharing-Pilotprojekts. Staatliche Akademie der Bildenden Künste Stuttgart.

Rudzio, W. (2015). Das politische System der Bundesrepublik Deutschland. Wiesbaden: Springer Verlag.

Schimank, U. (2007). Theorien gesellschaftlicher Differenzierung. Wiesbaden: VS Verlag für Sozialwissenschaften. 
Schimank, U. (2012). Wissenschaft als gesellschaftliches Teilsystem. In S. Maasen, M. Kaiser, M. Reinhart \& B. Sutter (Hrsg.), Handbuch Wissenschaftssoziologie (S. 113-123). Wiesbaden: Springer Fachmedien.

Schmitz, T., \& Wehrheim, M. (2006). Risikomanagement. Grundlagen-Theorie-Praxis. Stuttgart: Kohlhammer.

Schneidewind, U., \& Singer-Brodowski, M. (2014). Transformative Wissenschaft: Klimawandel im deutschen Wissenschafts- und Hochschulsystem. Marburg: Metropolis.

Trenks, H., Waitz, C., Meyer-Soylu, S., \& Parodi, O. (2018). Mit einer Realexperimentreihe Impulse für soziale Innovationen setzen - Realexperimente initiieren, begleiten und beforschen. In R. Defila \& A. Di Giulio (Hrsg.), Transdisziplinär und transformativ forschen. Eine Methodensammlung (S. 233-268). Wiesbaden: Springer VS.

VDI 4006 Blatt 1 (2015). Menschliche Zuverlässigkeit - Ergonomische Forderungen und Methoden der Bewertung. VDI Richtlinie, hrsg. vom Verein Deutscher Ingenieure e. V. Düsseldorf.

Weißensteiner, C. (2014). Reputation als Risikofaktor in technologieorientierten Unternehmen. Status Quo - Reputationstreiber - Bewertungsmodell. Wiesbaden: Springer Fachmedien.

Open Access Dieses Kapitel wird unter der Creative Commons Namensnennung 4.0 International Lizenz (http://creativecommons.org/licenses/by/4.0/deed.de) veröffentlicht, welche die Nutzung, Vervielfältigung, Bearbeitung, Verbreitung und Wiedergabe in jeglichem Medium und Format erlaubt, sofern Sie den/die ursprünglichen Autor(en) und die Quelle ordnungsgemäß nennen, einen Link zur Creative Commons Lizenz beifügen und angeben, ob Änderungen vorgenommen wurden.

Die in diesem Kapitel enthaltenen Bilder und sonstiges Drittmaterial unterliegen ebenfalls der genannten Creative Commons Lizenz, sofern sich aus der Abbildungslegende nichts anderes ergibt. Sofern das betreffende Material nicht unter der genannten Creative Commons Lizenz steht und die betreffende Handlung nicht nach gesetzlichen Vorschriften erlaubt ist, ist für die oben aufgeführten Weiterverwendungen des Materials die Einwilligung des jeweiligen Rechteinhabers einzuholen.

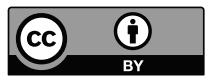




\title{
Kommunale Akteure verstehen: Komplexe Entscheidungswege in Kommunen beachten
}

\author{
Monika Gonser, Jan Riel, Jochen Eckart, Thorsten Erl \& Elke Häußler
}

\section{Herausforderungen bei der Analyse kommunaler Akteure in Reallaboren}

Kommunen spielen in Reallaboren und in der transdisziplinären und transformativen Forschung allgemein oft eine zentrale Rolle. Aufbau und Organisation einer Kommune sind durchaus komplex und können von jeweils ortsspezifischen Besonderheiten geprägt sein. Insofern ist ein besseres Verständnis von Kommunen und insbesondere den einzelnen Akteuren innerhalb einer Kommune (Fachverwaltung, Verwaltungsspitze, politische Entscheidungsträger) von maßgeblicher Bedeutung für das Gelingen der Zusammenarbeit in solchen Projekten.

Für das bessere Verständnis der kommunalen Akteure in Reallaboren und in anderen transdisziplinären und transformativen Forschungsvorhaben bietet sich das Instrument der Akteursanalyse an (Eckart et al. 2018). Die Akteursanalyse hat sich aus Ansätzen der Politikfeldanalyse (Blum und Schubert 2011), des Strategic Management (Freeman 1984) sowie der Partizipationsforschung (Reed et al. 2009; Zimmermann 2006) entwickelt. Auch im Forschungskontext wird die Akteursanalyse bereits angewandt (Butterworth et al. 2011). Sie dient als Instrument, um die Einbindung der Praxisakteure in transdisziplinären und transformativen Forschungsvorhaben im Detail zu planen. Im Rahmen der Akteursanalyse sind die für die Forschungs-, Praxis- und Bildungsziele eines Vorhabens erforderlichen Akteure (Beecroft et al. 2018) zu identifizieren. Die Akteursanalyse befasst sich zudem mit den Erwartungen der Akteure darüber, wie und in welchem Umfang sie sich an einem Vorhaben beteiligen können oder sollen sowie mit den Möglichkeiten und Grenzen der Akteure. Damit eine Akteursanalyse gelingen kann und in der Folge die Zusammenarbeit in einem Projekt fruchtbar verläuft, ist es wichtig, den Besonderheiten der analysierten Akteure Rechnung zu tragen und nicht mit verkürztem Blick an die Analyse heranzugehen. Es ist also, mit anderen Worten, ein Bewusstsein nötig für mögliche ,blinde Flecken', die die Qualität einer Akteursanalyse beeinträchtigen könnten. 
Basierend auf Erfahrungen aus den vom Ministerium für Wissenschaft, Forschung und Kunst Baden-Württemberg (MWK) seit 2015 geförderten 14 Reallaboren der Förderlinien „Reallabore“ und „Reallabore Stadt" (im Folgenden BaWü-Labs) ${ }^{1}$ und bezogen auf den Akteur Kommune wird dargestellt, inwiefern ,blinde Flecken' existieren können, also Wissenslücken auf Seiten der Wissenschaftler(innen) über die Funktionsweise von und die Arbeit mit verschiedenen kommunalen Akteuren. Diese ,blinden Flecken' können in der Akteursanalyse sowie in der eigentlichen Forschungsarbeit zu empfindlichen Komplikationen führen - sei es, weil nicht die richtigen Ansprechpartner gefunden, weil vorgegebene Entscheidungswege nicht beachtet oder weil ohnehin vorbelastete Beziehungen zwischen Akteuren aus Unkenntnis überstrapaziert werden.

Diesen ,blinden Flecken' sind in diesem Buch drei Kurzbeiträge gewidmet: Ein Beitrag widmet sich der Vorgeschichte kommunaler Akteure und zeigt, warum es wichtig ist, sich mit dieser auseinanderzusetzen (Eckart et al. 2019 in diesem Buch). Ein Beitrag beschreibt die Verteilung von Wissensbeständen innerhalb von Kommunen und inwiefern Kommunen nicht nur als ein (homogener) Ansprechpartner (miss)zu verstehen sind (Erl et al. 2019 in diesem Buch). Im vorliegenden Beitrag werden die komplexen Entscheidungswege innerhalb kommunaler Strukturen dargestellt, deren Kenntnis für das Gelingen der Zusammenarbeit von besonderer Bedeutung ist.

\section{Entscheidungsinstanzen in der Kommune}

Zum Verständnis der inneren Strukturen einer Kommune ist zunächst deren grundsätzlicher Aufbau, wie er durch die rechtlichen Grundlagen gegeben ist, zu verstehen. Dieser Aufbau besteht in aller Regel aus mehreren Ebenen, die getrennt, aber auch in Zusammenarbeit handeln. Dies wird vorliegend gezeigt am Beispiel von Kommunen in Baden-Württemberg, da die Erfahrungen der BaWüLabs in diesem Bundesland gewonnen wurden. Typische Kommunen in BadenWürttemberg sind in drei Ebenen gegliedert:

Gemeinderat/Stadtrat: Der Gemeinde- bzw. Stadtrat setzt sich aus gewählten Bürger(inne)n zusammen. Diese gewählten Gemeinde- bzw. Stadträt(inn)e(n) sind meistens parteigebunden und in Fraktionen oder Fraktionsgemeinschaften organisiert. Auf Basis von Informationen vor allem durch die Fachämter entscheidet der Gemeinde- bzw. Stadtrat in letzter (kommunaler) Instanz über wesentliche kommunale Angelegenheiten.

1 Für Informationen zu den beiden Förderlinien siehe http://www.reallabore-bw.de (zugegriffen am 20.05.2019). 
(Ober-)Bürgermeister(in): Die (Ober-)Bürgermeister(innen) sind je nach Gebietskörperschaft und Größe verschieden benannt, sie agieren jedoch stets als Verwaltungsspitze und als Vorsitzende des Gemeinde- bzw. Stadtrats. In Stadtkreisen und großen Kreisstädten werden Oberbürgermeister(innen) und mehrere nachgeordnete Bürgermeister(innen) mit spezifischen Zuständigkeitsgebieten (Dezernaten) gewählt. Während Oberbürgermeister(innen) direkt von Bürger(inne)n gewählt werden, werden nachgeordnete Bürgermeister(innen) vom Gemeinde- bzw. Stadtrat gewählt. (Ober-)Bürgermeister(innen) genießen große Entscheidungskompetenzen für ihre jeweiligen Dezernate, auch wenn sie an die Beschlüsse des Gemeinde- bzw. Stadtrats gebunden sind.

Verwaltung und Fachämter: Die Fachämter betreiben die eigentliche inhaltliche Arbeit in den Kommunen. Je größer die Kommune, desto größer und spezialisierter sind in der Regel die Fachämter. Sie sind auf der Fachebene nach Fachthemen untergliedert und werden von einer Amtsleitung geführt. Die Fachämter haben innerhalb ihres thematischen Zuständigkeitsbereichs eine beachtliche Entscheidungskompetenz und können Prozesse maßgeblich unterstützen oder blockieren.

\section{Entscheidungsprinzipien in der Kommune}

Die Vergegenwärtigung des Aufbaus einer Kommune hilft bei der Beantwortung der Frage, welche kommunalen Akteure für die betrachtete Themenstellung eines Projekts die relevanten Entscheidungen treffen. Die Antwort auf diese Frage richtet sich dabei weniger nach inhaltlichen, als vielmehr nach Kriterien der Reichweite von Entscheidungen und danach, ob es sich um legislative, exekutive oder operative Entscheidungen handelt. Die Zuordnung kann fließend sein und wird aufgrund lokaler Gegebenheiten nicht in Gänze der hier vorgestellten Schematisierung entsprechen.

Grundsatzentscheidungen durch das oberste politische Gremium: Grundsatzentscheidungen werden vom Gemeinde- bzw. Stadtrat unter Vorsitz der jeweiligen (Ober-)Bürgermeister(innen) als oberstem Entscheidungsgremium der Kommune getroffen. Langfristige politische Schwerpunkte sowie der städtische Haushalt werden vom Gemeinde- bzw. Stadtrat entschieden. Dieser beschäftigt sich meist nicht im Detail mit Themenstellungen (auch nicht von Reallaboren), sondern überlässt dies den (Ober-)Bürgermeister(inne)n und insbesondere den Fachämtern der Verwaltung. In der Antragsphase eines Projekts ist eine Interessenbekundung der Kommune zur Zusammenarbeit wichtig. Im besten Fall wird diese als Grundsatzentscheidung durch den Gemeinde- bzw. Stadtrat oder als strategische Entscheidung durch den bzw. die (Ober-)Bürgermeister(in) getroffen. 
Strategische Entscheidungen durch Führungspositionen: Die Quervernetzung zwischen verschiedenen (Zuständigkeits-)Bereichen wird im Alltag von Personen in Führungspositionen wahrgenommen. Je nach Größe der Kommunen können dies Amtsleiter(innen), Bürgermeister(innen) oder auch Oberbürgermeister(innen) sein. Dieser Personenkreis ist es, der strategische Entscheidungen trifft und auf deren Konsistenz achtet. Unter strategische Entscheidungen können sowohl inhaltliche Setzungen fallen als auch die Zuteilung von Ressourcen im Rahmen des vorgegebenen Haushalts.

Entscheidungen im Arbeitsprozess in den Fachämtern: Entscheidungen über alltägliche, rein operative und routinemäßige Fragen werden in den Fachämtern durch die zuständigen Sachbearbeiter(innen) getroffen, weitreichendere inhaltliche Entscheidungen durch die Abteilungs- oder Amtsleiter(innen). Dabei können die Fachämter - neben der fachlichen Einbindung - auch eine moderierende Rolle einnehmen. Eine moderierende Rolle kommt der Kommune vor allem dann zu, wenn diese nicht der direkte Ansprechpartner ist, sondern nur bei strategischen Fragen eingeschaltet wird (z. B. unterstehen in Baden-Württemberg die Schulgebäude der Kommune als Schulbauträger, während die Lehrer(innen) beim Land angestellt sind).

\section{Herausforderungen im Hinblick auf Entscheidungswege in Kommunen}

Ist ein Projekt wie z. B. ein Reallabor bzw. die Zusammenarbeit mit einem Projekt entschieden, wird die operative Zusammenarbeit in der Regel mit der ,tiefsten' zuständigen Ebene erfolgen, also z. B. mit den Fachämtern. Die Entscheidungswege spielen aber auch in der operativen Umsetzung eine Rolle, es kann also ein Fehler sein, diese nur bezogen auf die Grundsatzentscheidung im Blick zu haben.

Relevanz von Hierarchien: Allgemein sind kommunale Verwaltungen stark hierarchieorientiert. Deshalb ist es besonders wichtig, keinen relevanten Entscheidungsträger zu übergehen. Generell ist eine Entscheidung weniger angreifbar, wenn sie sich aus Entscheidungen einer höheren Entscheidungsinstanz ableiten lässt. Aus diesem Grund ist es sinnvoll, anstehende (Teil-)Projekte jeweils mit der nächsthöheren Entscheidungsinstanz abzusprechen, die dann den Gestaltungsoder Arbeitsauftrag an die Ebene gibt, mit der externe Akteure wie Wissenschaftler(innen) zusammenarbeiten. Gleichzeitig sollte die Entscheidung zur Unterstützung eines (Teil-)Projekts nicht nur ,top down' und - im ungünstigsten Fall gegen den Willen der operativen Ebene - getroffen werden, da das Engagement in der Zusammenarbeit dann möglicherweise auf ein Minimum reduziert wird. Es kann also durchaus sinnvoll sein, mit möglichst vielen kommunalen Akteuren auf 
allen Ebenen im Gespräch zu sein, um Verständnis und Unterstützung für das (Teil-)Projekt zu schaffen. Dabei sollte der korrekte Entscheidungsprozess jedoch durchgängig eingehalten werden.

Entwicklung einer einheitlichen Verwaltungsposition durch die übergeordnete Ebene: Grundsätzlich können in allen Phasen eines Projekts unterschiedliche Positionen von unterschiedlichen Akteuren innerhalb der Kommune zutage treten. Lassen sich Differenzen innerhalb einer Ebene nicht ausräumen, muss eine Entscheidung durch eine ,höhere Instanz' getroffen werden. Dieser Zustand sollte jedoch möglichst vermieden werden, da der , unterlegene' Akteur dann möglicherweise weniger engagiert oder sogar gegen das Projekt arbeiten könnte.

Kompetenz zur Zuordnung von Ressourcen: Insbesondere für Tätigkeiten, die sich auf der operativen Ebene nicht im Rahmen des Tagesgeschäfts erledigen lassen, ist eine Klärung der Ressourcenzuteilung (für Arbeitszeit, Materialien, Veranstaltungen etc.) wichtig. Dies kann häufig nicht auf der Arbeitsebene, sondern nur von den übergeordneten Führungspositionen entschieden werden. Dabei sind vor allem in kleinen Gemeinden mit kleinen Ämtern die Kommunikations- und Entscheidungswege kurz. Die Bürgermeister(innen) sind in der Regel über aktuelle Tätigkeiten der Fachämter inhaltlich informiert und können beurteilen, welche zusätzlichen Aktivitäten möglich sowie mit der Verwaltungshaltung vereinbar sind. In größeren Gemeinden dagegen sind Bürgermeister(innen) - oder gar Oberbürgermeister(innen) - weniger in das Tagesgeschäft der Fachämter eingebunden.

Existenz von Vetoplayern: Schließlich sollte bei der Analyse der Akteure in einer Kommune beachtet werden, dass vor allem aus den Ebenen Gemeinde- bzw. Stadtrat sowie (Ober-)Bürgermeister(in) Vetoplayer kommen können: Diese zeichnen sich dadurch aus, dass sie ein Vorhaben komplett blockieren können, weil ohne deren Unterstützung und Beteiligung das Vorhaben nicht umgesetzt werden kann (Zimmermann 2006). So kann z. B. der Gemeinde- bzw. Stadtrat durch seine Entscheidungen von der Verwaltung vorgeschlagene Aktivitäten bestätigen, modifizieren oder auch gegen deren Wunsch verhindern bzw. durchsetzen, weswegen es sinnvoll sein kann, auch mit einzelnen Gemeinde- bzw. Stadträt(inn)en oder mit den Fraktionen im Gespräch zu sein.

\section{Berücksichtigung der Entscheidungswege in der Akteursanalyse}

Anhand der Akteursanalyse ist zu verstehen, wie Entscheidungen in der Kommune zustande kommen und welche Akteure dabei welche Rolle spielen. Hierbei ist beispielsweise die Identifikation von Vetoplayern ein wesentlicher Baustein, da diese in der Lage sind, ein Vorhaben erheblich zu verzögern bzw. gänzlich zu 
blockieren. Im Idealfall hingegen wirken diese unterstützend auf das Projekt ein. Weiter ist der Frage, an welcher Stelle zeitliche, finanzielle und personelle Ressourcen für das Projekt genutzt werden können, Aufmerksamkeit zu schenken, um gegebenenfalls gezielt an dieser Stelle Entscheidungswege zu beeinflussen. Um Entscheidungswege nachvollziehen zu können und gegebenenfalls auf diese einzuwirken, ist es sinnvoll, sich mit den Entscheidungsprinzipien und Entscheidungsinstanzen in der Kommune vertraut zu machen. Hierbei kann natürlich das Organigramm der Kommune eine Hilfe sein, das beispielsweise die kommunalen Verwaltungshierarchien abbildet. Sich lediglich auf das Organigramm zu stützen, wäre aber nicht sinnvoll, sondern würde zu ,blinden Flecken' führen. Es gilt, auch dem ungeschriebenen ,Protokoll‘ einer Kommune nachzuspüren. Dieses wiederum kann nur im direkten Gespräch erfragt werden, indem man sich nach den in der Kommune üblichen Vorgehens- und Entscheidungswegen erkundigt. Besteht bereits Kontakt zu einzelnen Mitarbeiter(inne)n in der Kommune, fällt dies leichter, als wenn die Kontakte zuerst noch aufgebaut werden müssen.

\section{Literatur}

Beecroft, R., Trenks, H., Rhodius, R., Benighaus, C., \& Parodi, O. (2018). Reallabore als Rahmen transformativer und transdisziplinärer Forschung: Ziele und Designprinzipien. In R. Defila \& A. Di Giulio (Hrsg.), Transdisziplinär und transformativ forschen. Eine Methodensammlung. (S. 75-100). Wiesbaden: Springer VS. doi: 10.1007/978-3-65821530-9 4 .

Blum, S., \& Schubert, K. (2011). Politikfeldanalyse. Wiesbaden: Springer VS.

Butterworth, J., McIntyre, P., \& da Silva Wells, C. (Hrsg.). (2011). Switch in the City: Putting Urban Water Management to the Test. Den Haag: IRC International Water and Sanitation Centre.

Eckart, J., Ley, A., Häußler, E., \& Erl, Th. (2018). Leitfragen für die Gestaltung von Partizipationsprozessen in Reallaboren. In R. Defila \& A. Di Giulio (Hrsg.), Transdisziplinär und transformativ forschen. Eine Methodensammlung (S. 105-135). Wiesbaden: Springer VS. doi: 10.1007/978-3-658-21530-9_6.

Freeman, R. E. (1984). Strategic Management: A Stakeholder Approach. Boston: Pitman.

Reed, M. S., Graves, A., Dandy, N., Posthumus, H., Hubacek, K., \& Morris, J. (2009). Who's in and why? A typology of stakeholder analysis methods for natural resource management. Journal of Environmental Management, 90, (S. 1933-1949).

Zimmermann, A. (2006). Instrumente zur Akteursanalyse - 10 Bausteine für die partizipative Gestaltung von Kooperationssystemen. Eschborn: Deutsche Gesellschaft für Technische Zusammenarbeit (GTZ). 


\section{Die zwei weiteren Beiträge in diesem Buch zu ,blinden Flecken ‘}

Eckart, J., Häußler, E., Erl, Th., Gonser, M., \& Riel, J. (2019). Kommunale Akteure verstehen: Vorgeschichte der Arbeit in Kommunen beachten. In R. Defila \& A. Di Giulio (Hrsg.), Transdisziplinär und transformativ forschen, Band 2. Eine Methodensammlung (S. 33-38). Wiesbaden: Springer VS.

Erl, Th., Gonser, M., Eckart, J., Häußler, E., \& Riel, J. (2019). Kommunale Akteure verstehen: Verteilte Wissensbestände in Kommunen. In R. Defila \& A. Di Giulio (Hrsg.), Transdisziplinär und transformativ forschen, Band 2. Eine Methodensammlung (S. 139145). Wiesbaden: Springer VS.

Open Access Dieses Kapitel wird unter der Creative Commons Namensnennung 4.0 International Lizenz (http://creativecommons.org/licenses/by/4.0/deed.de) veröffentlicht, welche die Nutzung, Vervielfältigung, Bearbeitung, Verbreitung und Wiedergabe in jeglichem Medium und Format erlaubt, sofern Sie den/die ursprünglichen Autor(en) und die Quelle ordnungsgemäß nennen, einen Link zur Creative Commons Lizenz beifügen und angeben, ob Änderungen vorgenommen wurden.

Die in diesem Kapitel enthaltenen Bilder und sonstiges Drittmaterial unterliegen ebenfalls der genannten Creative Commons Lizenz, sofern sich aus der Abbildungslegende nichts anderes ergibt. Sofern das betreffende Material nicht unter der genannten Creative Commons Lizenz steht und die betreffende Handlung nicht nach gesetzlichen Vorschriften erlaubt ist, ist für die oben aufgeführten Weiterverwendungen des Materials die Einwilligung des jeweiligen Rechteinhabers einzuholen.

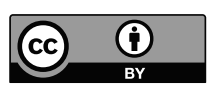




\section{Schutz in der Exposition, Schutz für die Exposition - Wie man in transdisziplinären und transformativen Forschungsformaten mit Ungewohntem und erhöhter Aufmerksamkeit umgeht}

Katharina Köglberger, Raphael Dietz, Charlotte Eller, Felix M. Piontek, Marius Albiez \& Thomas Potthast ${ }^{1}$

\section{$1 \quad$ Einleitung}

Transdisziplinäres und transformatives ${ }^{2}$ Forschen bietet viele Chancen und Vorteile. So zeichnet sich transdisziplinäres Forschen dadurch aus, dass neben Wissenschaftler(inne)n verschiedener Disziplinen auch Praxisakteure ${ }^{3}$ unterschiedlicher Hintergründe substantiell am Forschungsprozess mitwirken (Defila und Di Giulio 2018a, S. 33). Durch den Einbezug von Praxisakteuren kann eine höhere Anschlussfähigkeit der Forschung an das praktische Handeln in der Gesellschaft erzielt werden. Die unterschiedlichen Akteure erweitern anhand des gegenseitigen Austauschs ihren methodischen Werkzeugkasten sowie ihren Erkenntnishorizont.

1 Lead Autor(inn)en: Katharina Köglberger und Raphael Dietz, die anderen in Reihenfolge der Beispiele im Text.

2 Die Autor(inn)en möchten einen Beitrag zur Methodik transdisziplinärer Forschung leisten, die auch transformativ vorgehen kann, aber nicht muss. Diese doppelte Ansprache wird im Beitrag an den Stellen, an denen dies zutrifft, durch Klammersetzung ausgedrückt: ,transdisziplinär (und transformativ)'. An Stellen, an denen der transformative Aspekt allerdings im Vordergrund steht (z. B. Kapitel 4), wird auf die Klammersetzung verzichtet.

3 ,Akteur' bezeichnet ein handelndes Individuum. ,Akteursgruppe' bezeichnet mehrere Individuen, die aus dem gleichen Kontext (also z. B. Wirtschaft) stammen. Die Akteure werden nach der Funktion bezeichnet, in der sie am Forschungsprojekt mitwirken. Die Definition erfolgt nach den Sektoren des 3-Kreise-Modells von Seebacher et al. (2018): Als ,Wissenschaftler(innen)` werden dem Wissenschaftssystem Angehörige 
Um diese Vorteile und Chancen ausschöpfen zu können, ist es notwendig, sich zugleich den damit verbundenen Herausforderungen zu stellen und sich auch auf „Neues einzulassen“ (Jahn 2008, S. 33). Das Arbeiten über das angestammte Arbeitsumfeld hinaus kann beinhalten, dass die Beteiligten sich unter (kritischer) Beobachtung durch andere Akteure Neuem stellen, indem sie für die eigene Tätigkeit unübliche Aufgaben oder ungewohnte Rollen übernehmen. Vor allem bei Forschungsprojekten, die neben einem transdisziplinären auch einen transformativen Ansatz verfolgen, kommt hinzu, dass dies oft nicht nur unter Beobachtung durch andere Akteure im Projekt oder dessen Umfeld, sondern auch unter erhöhter Aufmerksamkeit der Öffentlichkeit erfolgt. So kann es beispielsweise vorkommen, dass Wissenschaftler(innen) im Rahmen eines transdisziplinären (und transformativen) Forschungsprojekts zum ersten Mal in ihrer beruflichen Laufbahn auf einer Bürger(innen)versammlung ihr Projekt vorstellen und gegen ein ablehnendes oder möglicherweise sogar aufgebrachtes Publikum verteidigen müssen. Während dies für erfahrene Moderator(inn)en eine Routinesituation darstellt, kann es für die Wissenschaftler(innen) eine ungewohnte Situation sein, in der sie sich einer Exposition im Sinne des vorliegenden Beitrags ausgesetzt sehen.

Die Autor(inn)en verstehen Exposition hier wie folgt: Die Akteure sind bei der Durchführung einer Tätigkeit im Rahmen eines transdisziplinären (und transformativen) Forschungsprojekts einer erhöhten Aufmerksamkeit durch Dritte ausgesetzt und zwar in einem Bereich, in dem sie das so nicht kennen. Dies stellt für sie eine ungewohnte Situation dar, zu deren Bewältigung sie nicht einfach auf ihre professionellen Routinen zurückgreifen können. Ungewohnt können für den betroffenen Akteur sowohl die durchzuführende Tätigkeit als auch die dabei erfahrene Aufmerksamkeit sein. Von Exposition wird hier gesprochen, wenn mindestens einer der beiden Aspekte zutrifft. Die Exposition ist häufig eine Notwendigkeit, der sich die Akteure zur Durchführung des geplanten Forschungsprojekts stellen müssen. Sie kann aber zusätzlich einen Mehrwert für das Projekt

bezeichnet, die in dieser Funktion am Forschungsprojekt teilnehmen. Alle weiteren am Forschungsprojekt Beteiligte, die nicht als Angehörige des Wissenschaftssystems im Projekt mitwirken, werden als ,Praxisakteure' bezeichnet. ,Wirtschaftliche Praxisakteure' bezeichnet Akteure, die als Vertreter(innen) eines Wirtschaftsunternehmens am Forschungsprojekt teilnehmen. ,Praxisakteure der öffentlichen Verwaltung ${ }^{`}$ bezeichnet Akteure, die als Vertreter(innen) der öffentlichen Verwaltung am Forschungsprojekt teilnehmen. ,Zivilgesellschaftliche Praxisakteure` bezeichnet Akteure, die als Vertreter(innen) einer zivilgesellschaftlichen Organisation oder als Individuen am Forschungsprojekt teilnehmen. Diese Bezeichnung fasst aus Gründen der Übersichtlichkeit Akteure zusammen, die verschiedenen Sektoren wie „lokale Gemeinschaft“, „organisierte Zivilgesellschaft“ oder „Kultur“ zugeordnet werden können (so in Abb. 1 in Seebacher et al. 2018, S. 156). 
oder die Akteure mit sich bringen. Um mögliche nachteilige Auswirkungen einer Exposition zu vermeiden oder möglichst gering zu halten, bedarf es Vorkehrungen, die ausreichend Schutz vor diesen Auswirkungen bieten. Dies gilt sowohl für den Schutz der betroffenen Akteure gegenüber der Öffentlichkeit als auch innerhalb des Projekts. Solche Schutzvorkehrungen können beispielsweise Fortbildungsmaßnahmen sein, die über die üblichen Aufgaben der Akteure hinausgehen und entsprechend bei der Projektplanung und Finanzierung zusätzlich zu berücksichtigen sind.

Bisherige Ansätze, die eine Reflexion von Erfahrungen in transdisziplinären (und transformativen) Forschungsprojekten erlauben, thematisieren beispielsweise Methoden der Prozessgestaltung (z. B. Bergmann et al. 2010; Defila und Di Giulio 2018b; McDonald et al. 2009) oder Managementaufgaben, die bei solchen Projekten wahrgenommen werden müssen (z. B. Defila et al. 2006; Pohl und Hirsch Hardorn 2006; Schophaus et al. 2004). Andere fokussieren auf unterschiedliche Phasen, die in Projekten unterschieden werden können (z. B. Jahn 2008; Jahn et al. 2012), oder auf Qualitätskriterien und Evaluation (z. B. Bergmann et al. 2005; Boix Mansilla et al. 2006; Defila und Di Giulio 1999; Klein 2006). Auch Lang et al. (2012) beschäftigen sich mit idealtypischen Gestaltungsprinzipien in transdisziplinären Forschungsprojekten sowie den damit möglicherweise einhergehenden Herausforderungen. Aus der Exposition resultierende Herausforderungen bleiben allerdings in allen diesen Ansätzen unberücksichtigt. Der vorliegende Beitrag möchte mit dem Thema der Exposition einen alternativen Zugang bieten, über Erfahrungen in transdisziplinären (und transformativen) Forschungsprojekten zu reflektieren. Die Reflexion über Expositionen kann in der Vorbereitung auf ein Projekt oder in der Vorbereitung auf einzelne Aufgabenbereiche eines Projekts wie etwa der Öffentlichkeitsarbeit oder öffentlichen Interventionen von Nutzen sein. Eine solche Reflexion erlaubt es, frühzeitig ein Bewusstsein dafür zu erzeugen, welche ungewohnten Situationen für wen auftreten könnten. Dieses Wissen hilft zunächst bei der Abwägung, ob jemand bereit ist, sich auf die entsprechenden Situationen einzulassen. Sollte dies der Fall sein, ermöglicht die Reflexion, sich schon im Vorfeld effektiver auf den Umgang mit Expositionen vorzubereiten sowie die nötigen Ressourcen einzuplanen. Die Reflexion hilft, mögliche negative Folgen ${ }^{4}$, wie etwa die Überforderung von Akteuren, frühzeitig zu erkennen und somit vorausschauend Schutzvorkehrungen ergreifen zu können. Diese Schutzvorkehrungen können entweder auf die Vermeidung bzw. Verringerung der negativen Folgen oder aber auf den Umgang mit ihnen abzielen.

4 Negative Folgen spielen in das Thema Erfolg und Misserfolg hinein. Für eine fundierte Auseinandersetzung mit Risiko, Erfolg und Misserfolg in transdisziplinären (und transformativen) Forschungsprojekten siehe Gonser et al. 2019 (in diesem Band). 
Im Beitrag werden anhand der in den beteiligten BaWü-Labs ${ }^{5}$ gesammelten Erfahrungen verschiedene Expositionen aufzeigt, von denen die Autor(inn)en vermuten, dass sie bei vergleichbaren Settings auch in anderen transdisziplinären (und transformativen) Forschungsprojekten auftreten können. Wie die Ausgestaltung von Schutzvorkehrungen aussehen kann, wird im Beitrag exemplarisch aufgezeigt. Wichtig für die Entscheidung, ob es sich lohnt, sich zu exponieren, ist auch das Wissen über mögliche positive Auswirkungen. Diese sammelt und beleuchtet der Beitrag deshalb ebenfalls, wobei von ,Mehrwert' gesprochen wird, wenn positive Auswirkungen direkt mit den beschriebenen Expositionen einhergehen, und von ,positiven Effekten', wenn sie den jeweiligen Schutzvorkehrungen entspringen. Dabei gilt, dass sich die beiden zusammenhängenden Aspekte nicht immer eindeutig trennen lassen. Zudem besteht nicht der Anspruch, dass der Mehrwert oder die positiven Effekte hier völlig neue Aspekte beschreiben. Der Zweck liegt lediglich in der Zusammenführung und Zuordnung zu den jeweiligen Expositionen.

Grundlage für die Entfaltung der Expositionen sind die in den BaWü-Labs gesammelten Erfahrungen der Autor(inn)en. Deshalb werden die Expositionen schwerpunktmäßig, aber nicht ausschließlich, aus der Perspektive der Wissenschaftler(innen) betrachtet. Zur Erweiterung der Perspektive wurde an zwei Zeitpunkten im Schreibprozess von verschiedenen Praxisakteuren eine Rückmeldung eingeholt - zur ersten Fassung des Beitrags sowie zu einer späteren Fassung. Die Rückmeldungen dienten erstens der Auswahl der dargelegten Expositionen. Zweitens konnten auf diese Weise Aussagen über Expositionen, die Praxisakteure betreffen, validiert werden.

Der Beitrag ist in sechs Kapitel gegliedert. Nach der Einleitung (Kapitel 1) werden in den Kapiteln 2 bis 5 verschiedene Expositionen, die in transdisziplinären (und transformativen) Forschungsprojekten vorkommen können, dargelegt. $\mathrm{Zu}-$ nächst werden Expositionen behandelt, die sich durch das Forschungsdesign und den Forschungsgegenstand ergeben können (Kapitel 2), gefolgt von solchen, die aus einer verstärkten Öffentlichkeitsarbeit entstehen können (Kapitel 3). Anschließend wird auf Expositionen eingegangen, die sich durch Öffentliche Interventionen (Kapitel 4), sowie solche, die sich durch die Integration von Lehre in das Forschungsprojekt (Kapitel 5) ergeben können. Kapitel 6 gibt einen zusammenfassenden Überblick und reflektiert, wie eine Betrachtung mithilfe der Expo-

5 Das Ministerium für Wissenschaft, Forschung und Kunst Baden-Württemberg fördert(e) seit 2015 in zwei Förderlinien 14 sogenannte Reallabore, die im Folgenden als ,BaWü-Labs' bezeichnet werden (s. auch http://www.reallabore-bw.de, zugegriffen am 20.05.2019). Reallabore sind ,ein Forschungsformat, in dem transdisziplinär geforscht wird und gleichzeitig ein expliziter transformativer Anspruch verfolgt wird" (Defila und Di Giulio 2018c, S. 9). 
sitionsthematik sowohl für die einzelnen Akteure als auch für das Projektmanagement von Nutzen sein kann. Zudem werden mögliche Weiterentwicklungen des Expositionsthemas mit punktuellen Anknüpfungen an die zu Beginn des Beitrags dargelegten bisher gängigen Reflexionszugänge aufgezeigt.

Die Kapitel 2-5 gliedern sich in Abschnitte (2.1, 2.2 etc.), welche sich mit einzelnen Expositionen befassen. Diese Abschnitte wiederum sind anhand einer gleichbleibenden Struktur aufgebaut: Zunächst gibt ein kurzer Text einen Überblick, welche Expositionen behandelt werden. Dann werden die Expositionen durch ein Beispiel aus einem BaWü-Lab illustriert. Im Anschluss an das Beispiel folgt die allgemeine Beschreibung der daran sichtbar werdenden Expositionen. Hierbei werden Antworten auf die folgenden Fragen gegeben:

- Was ist die Ursache für die Expositionen?

- In welchen ungewohnten Tätigkeiten und/oder in welcher Aufmerksamkeit drücken sich die Expositionen aus und wer exponiert sich gegenüber wem?

- Zu welchem Zeitpunkt im Projekt kommen die Expositionen zum Tragen?

- Welche möglichen negativen Folgen können sich aus den Expositionen ergeben?

Nach der Beantwortung dieser Fragen werden Schutzvorkehrungen dargelegt, wie mit den zuvor beschriebenen Expositionen umgegangen werden kann. Hierbei wird insbesondere darauf eingegangen, wann und was durch wen getan werden muss, um diese Schutzvorkehrungen wirkungsvoll zu gestalten. Abschließend beleuchtet jeder Abschnitt auch den Mehrwert, der durch die beschriebenen Expositionen entsteht, sowie die positiven Effekte, die mit den dazu passenden Schutzvorkehrungen einhergehen.

\section{Expositionen durch das Forschungsdesign und den Forschungsgegenstand}

Um sowohl Wissenschaftler(innen) aus verschiedenen Disziplinen als auch Praxisakteure aus verschiedenen Praxisfeldern in der transdisziplinären (und transformativen) Forschung zu integrieren, ist oft ein besonderes Forschungsdesign notwendig, das beispielsweise spezielle Formen der Kommunikation innerhalb des Projektteams ${ }^{6}$, zwischen den beteiligten Akteuren untereinander oder gegen-

6 ,Projektteam‘ beschreibt jene Personen, die für Konzeption und Steuerung des Forschungsprojekts verantwortlich sind, sowie jene, die sich besonders intensiv an den Aktivitäten des Projekts beteiligen. Entsprechend dem 3-Kreise-Modell von Seebacher et al. (2018) sind dies Personen des „Kernbereichs“ und des ,inneren Akteurskreises“. 
über Externen mit sich bringt. Damit geht oft ein neues, meist ungewohntes Rollenverständnis für die Wissenschaftler(innen) einher, da sie die Kommunikation begleiten und steuern und dabei ungewohnte Aufgaben zur Gestaltung des Kommunikationsprozesses wahrnehmen müssen.

Transdisziplinäre (und transformative) Forschungsprojekte können es beispielsweise mit sich bringen, dass nicht nur das Vorgehen früher als sonst üblich transparent dargestellt werden muss, sondern sogar die Anonymität von Personen oder Institutionen, die Gegenstand der Forschung sind, aufgehoben werden muss. Weiterhin widmet sich transdisziplinäre und insbesondere transformative Forschung häufig auch politisch und gesellschaftlich sensiblen Themen, die zumeist schon von vorneherein mit einer hohen Aufmerksamkeit belegt sind. Daraus ergeben sich Expositionen für die Wissenschaftler(innen) wie auch für die Praxisakteure oder Institutionen. Im Folgenden werden die Expositionen, die durch das Gestalten von nicht anonymisierter Forschung sowie durch das Forschen in gesellschaftlich und politisch sensiblen Kontexten auftreten können, erläutert.

\subsection{Gestalten von nicht anonymisierter Forschung}

Bereits zu Beginn der Durchführung eines transdisziplinären (und transformativen) Forschungsprojekts kann es sinnvoll sein, die Anonymität von Fallbeispielen aufzuheben, um eine Transparenz des Forschungsprozesses nach außen ${ }^{7}$ herzustellen. Daraus resultieren Expositionen für Wissenschaftler(innen) und Praxisakteure.

\section{Beispiel BaWü-Lab SRB: Forschen an und mit Schulen bei Aufhebung der Anonymität}

Für die Forschung im Reallabor STADT-RAUM-BILDUNG - Reallabor für die nachhaltige Planung von Bildungslandschaften und die Integration von Aus- und Umbauten von Schulgebäuden („,BaWü-Lab SRB“) (s. auch Steckbrief im Anhang zu diesem Buch) wurden einzelne Sekundarschulen als beispielhafte Schulen (,Next Practice') ausgewählt; diese Schulen waren Praxispartner im Projekt. Ziel war es, aus den Erfahrungen der bereits stattgefundenen oder anstehenden strukturellen und physisch-räumlichen Transformation an diesen Schulen und deren Umgebung zu lernen, um diese Erkenntnisse wiederum auf andere Schulen und deren Umgebung anwenden zu können. Diese ,Next Practice'-Beispiele wurden nicht anonymisiert, wie es aus forschungsethischen Gründen üblich wäre, sondern wurden namentlich, inklusive Standortnennung, dargestellt. Dadurch war es auch für Personen, die nicht am BaWü-Lab beteiligt waren, transparent, welche Schulen als Beispielschulen dienten. Aufgrund der Vielzahl an Schulen, die als

7 ,Außen' beschreibt jene Personen, die nicht oder nur punktuell an der Forschung beteiligt sind. Entsprechend dem 3-Kreise-Modell von Seebacher et al. (2018) sind dies Personen des ,äußeren Akteurskreises“" oder der ,umgebenden Welt“. 
Beispielschulen infrage kamen, war es wichtig, die Auswahl dieser Beispielschulen zu begründen. Ausgewählt wurden ausschließlich Schulen einer bestimmten Schulart (sog. Gemeinschaftsschulen), die schon seit einigen Jahren ein besonderes pädagogisches Konzept verfolgten. Gleichzeitig spielten deren Schulgebäude und Standorte eine wesentliche Rolle bei der Auswahl. Gesucht wurden anhand dieser drei Aspekte wenige, aber möglichst verschiedene Beispielschulen, um ein breites Spektrum abzudecken. Durch die Transparenz der Auswahlkriterien und die Aufhebung der Anonymität der Beispielschulen konnten Personen, die nicht oder nur punktuell am Forschungsprojekt beteiligt waren, den Forschungsprozess und getroffene Entscheidungen nachvollziehen sowie den Stand der Forschung und erste Erkenntnisse bezogen auf die konkreten Fälle mitverfolgen.

Aufgrund der fehlenden Anonymität wurde also eine Art Bühne erzeugt. Auf dieser Bühne wurden nun nicht nur die Forschenden, sondern auch die Vertreter(innen) der gewählten ,Next Practice'-Schulen exponiert. Die Exposition der Schulen führte dazu, dass diese eine gewisse Prominenz erhielten. Es handelte sich um Schulen, die meist ungewöhnliche pädagogische Konzepte verfolgten. Da sie gleichzeitig als ,Next Practice definiert wurden, führte das beispielsweise bei Skeptiker(inne)n gegenüber diesen pädagogischen Konzepten dazu, dass sie ihre Kritik hierzu direkt kundtaten. Dies hatte rege Diskussionen bei öffentlichen Symposien des BaWü-Labs zur Folge. Die Aufhebung der Anonymität der Schulbeispiele ermöglichte aber auch, dass die Schulen tatsächlich als Vorbilder wirkten, da sich andere Schulen dort direkt Rat für den eigenen Transformationsprozess holen konnten. Einem Rechtfertigungszwang bereits zu Beginn und während der laufenden Forschung ausgesetzt zu sein, war sowohl für die Praxispartner als auch für die Wissenschaftler(innen) eine ungewohnte Situation. Für letztere bezog sich dies vorwiegend auf die Auswahlkriterien und Datenerhebungsmethoden.

Das Mitwirken der Vertreter(innen) der Beispielschulen sowohl bei der Generierung als auch bei der Auswertung der erhobenen Daten zur eigenen Schule führte zu punktgenauen Analysen und möglichen Lösungsansätzen. Die erzeugten Ergebnisse stellten, weil sie so spezifisch zugeschnitten waren, eine Art Kompensation für die Exposition der Vertreter(innen) der Beispielschulen dar. Sie wurden von den Beteiligten als großer Mehrwert für die weitere Praxisarbeit wahrgenommen.

Für transdisziplinäre (und transformative) Forschung kann es sinnvoll (oder sogar unvermeidlich) sein - entgegen forschungsethischer Gepflogenheiten - Fallbeispiele, Interviewpartner oder Ähnliches offenzulegen und somit deren Anonymität aufzuheben. Dadurch wird nicht nur Transparenz innerhalb des Projektteams hergestellt, sondern auch nach außen gegenüber Praxisakteuren und sogar Externen $^{8}$, die nur punktuell oder eben auch gar nicht an der Forschung beteiligt sind.

8 ,(Projekt)extern' bezeichnet in Abgrenzung zum Begriff ,außen“ nur jene Personen, die laut 3-Kreise-Modell von Seebacher et al. (2018) außerhalb der Kreise, also in der umgebenden Welt, stehen und sich somit nicht bewusst an Aktivitäten des Forschungsprojekts beteiligen. 
Daraus kann ein forschungsethischer Konflikt entstehen, da nun die beforschten Institutionen und/oder Personen für alle transparent sind. Eine solche Transparenz führt zu einer Exposition der Wissenschaftler(innen) und der beteiligten Praxisakteure.

Wissenschaftler(innen) exponieren sich gegenüber Kolleg(inn)en der Wissenschaft mehr als bei den meisten Forschungsprojekten üblich. Durch die Aufhebung der Anonymität und die stärkere Transparenz des Forschungsprozesses müssen die Wissenschaftler(innen) bereits zu Beginn des Forschungsprojekts oder jedenfalls früh im Prozess - also nicht erst nach Abschluss der Forschung beispielsweise ihre wissenschaftlichen Kriterien oder gewählten Methoden rechtfertigen.

Die Praxisakteure exponieren sich gegenüber Praxis und Wissenschaft. Sie müssen ihren Sonderstatus gegenüber anderen Akteuren aus der Praxis rechtfertigen und sich Kritiker(inne)n stellen. Gleichzeitig exponieren sie sich gegenüber der Wissenschaft, da sie sich deren Analyse und Bewertung aussetzen. Dies geschieht natürlich bei jeder Forschung. Bei einer Aufhebung der Anonymität geschieht dies jedoch in besonderer Weise, da dadurch zum Beispiel auch eventuell kritische Befunde direkt mit dem öffentlich bekannten Fallbeispiel in Verbindung gebracht werden können.

Durch die laufende inhaltliche und konzeptionelle Transparenz entsteht folglich eine öffentliche Bewertungs- und Angriffsfläche. Wissenschaftler(innen) und Praxisakteure finden sich dadurch in einer ungewohnten Situation wieder.

Eine Schutzvorkehrung für die Praxisakteure bietet das Format der transdisziplinären Forschung an sich, da die Praxisakteure, wenn sie zugleich Praxispartner sind, gleichberechtigt mitforschen und dadurch aktiv und direkt bei der Prozessgestaltung und der Produktion der Ergebnisse mitwirken. Sie können so nicht nur den Status quo gemeinsam mit den Wissenschaftler(inne)n reflektieren und bewerten, sondern auch bei der Darstellung der Inhalte nach außen aktiv mitwirken, so dass sie sich auf eine mögliche Konfrontation durch Kritiker(innen) vorbereiten können. Dies ermöglicht das Erzielen von passgenauen Forschungsergebnissen, die direkt von den Praxispartnern verwertet oder angewandt werden können. Dies wiederum kann jedoch zu einer weiteren Exposition der Wissenschaftler(innen) führen, wenn aufgrund der Mitwirkung der Praxispartner bei den Veröffentlichungen der Ergebnisse die Wissenschaftlichkeit der Befunde infrage gestellt wird.

Einen Mehrwert der beschriebenen Transparenz und Aufhebung der Anonymität stellt die Möglichkeit der direkten Kontaktaufnahme oder ,Besichtigung ' der Fallbeispiele dar. Diese können sogar als Vorbilder wahrgenommen werden und das Initial für Transformationen anderer Orte, Objekte oder Sachverhalte darstellen, so dass sie durch die Exposition transformative Wirkung erzeugen können. 
Darüber hinaus wird die Forschung durch die Aufhebung der Anonymität der Fallbeispiele ,greifbar', so dass vor allem abstrakte Inhalte leichter auch an Externe vermittelt werden können.

\subsection{Forschen in gesellschaftlich und politisch sensiblen Kontexten}

Herausforderungen eines transdisziplinären (und transformativen) Forschungsprojekts können durch das Agieren in gesellschaftlich und politisch sensiblen Kontexten aufgrund starker Emotionen der betroffenen Akteursgruppen und von Vorbehalten untereinander verstärkt werden. Sie münden dadurch punktuell in Expositionen einzelner Personen oder Gruppen.

\section{Beispiel BaWü-Lab SRB: Transformative Forschung im Kontext von Kindern und Ju- gendlichen}

Das BaWü-Lab SRB war an verschiedenen Sekundarschulen unterwegs. Die Schulgemeinschaft, bestehend aus Kollegium, Schülerschaft, Eltern und Bildungspartnern ${ }^{9}$, der Schulträger (die Kommune) sowie das Gebäude selbst und das umgebende Quartier bildeten den Forschungskontext. Die Schule sowie deren konzeptionelle und räumliche Transformation stellten den Forschungsgegenstand dar.

Gerade der Kontext von Kindern und Jugendlichen ist besonders sensibel, denn diese genießen ein hohes Maß an Aufmerksamkeit und Schutz in unserer Gesellschaft. Bei der Zusammenarbeit mit den Akteuren vor Ort (z. B. bei der Durchführung von Workshops) wurden die verschiedenen Interessengruppen mit ihren zum Teil konträren Haltungen deutlich. Diese Haltungen waren häufig mit starken Emotionen verbunden, was eine sachliche Diskussion erschwerte. So lagen die Interessen der Eltern vorwiegend auf der Behütung sowie auf einer erfolgreichen Schulkarriere ihres Kindes, was zur Formulierung entsprechender Forderungen gegenüber dem Lehrpersonal oder auch gegenüber dem Schulträger führte. Der Fokus der Lehrkräfte lag auf guten Lern- und Arbeitskonditionen, damit sowohl sie selbst als auch die Kinder und Jugendlichen optimale Bedingungen zum Lehren und Lernen vorfänden. Der Schulträger verfolgte meist übergreifende Interessen, wie die Quartiersentwicklung oder die Ermittlung von perspektivischen Schüler(innen)zahlen.

Damit die gemeinsame Arbeit in den Workshops möglich war und zur Entwicklung von gemeinsam getragenen Lösungsansätzen führen konnte, mussten beispielsweise die Lehrkräfte Abstand nehmen von ihrer eigenen Perspektive der Pädagogik und Didaktik und sich in das fremde Themenfeld der Architektur und Stadtplanung hineindenken, um ihre eigenen Interessen für alle verständlich formulieren zu können. Dazu mussten

9 ,Bildungspartner' sind Personen außerhalb des Lehrer(innen)kollegiums, die das pädagogische Angebot der Schule ergänzen, wie beispielsweise Sozialpädagog(inn)en oder Vertreter(innen) von Unternehmen. 
Vorbehalte gegenüber den anderen Beteiligten, beispielsweise gegenüber dem Schulträger, abgelegt werden, um sachlich argumentieren zu können. Gleichzeitig mussten Wissenslücken offen angesprochen und gemeinsam diskutiert und geklärt werden. Dies führte zu einer Exposition der Lehrkräfte gegenüber den anderen Beteiligten. Dasselbe galt für alle Beteiligten in unterschiedlichem Maße. Da der Schulträger vor allem bei der baulichen Transformation einer Schule die wichtigste Rolle innehat, war es für die zuständigen Vertreter(innen) dieses Akteurs besonders schwierig, wenn sie sich durch Wissenslücken gegenüber den anderen Beteiligten exponierten.

Um eine produktive Kooperation zwischen den Vertreter(inne)n der verschiedenen Akteursgruppen sicherzustellen, mussten die Wissenschaftler(innen) zwischen den jeweils verschiedenen Interessen vermitteln. Sie übernahmen die ungewohnte Rolle der Moderation oder gar die der Mediation, um mögliche Missverständnisse frühzeitig zu klären und eventuell aufkeimende Konflikte zu lösen. Sie agierten unparteiisch und unterstützten die Akteursgruppen dabei, jenseits von potenziellen Konflikten oder gegensätzlichen Interessen gemeinsamen Boden zu suchen und zu finden. Das Einnehmen dieser ungewohnten Rolle führte meist zu einer Exposition gegenüber den lokalen Praxisakteuren.

Zusätzlich exponierten sich die Wissenschaftler(innen) durch das Forschungsthema und den Forschungsgegenstand gegenüber der Politik, beispielsweise gegenüber dem Gemeinderat. Denn durch die Betrachtung von Gemeinschaftsschulen wurden diese als zukunftsfähige und ,gute' Schulart definiert. Dies wurde jedoch von den politischen Lagern, abhängig von den lokalen Gemengelagen, kontrovers diskutiert. Das Projektteam agierte also nicht nur im sensiblen Kontext von Kindern und Jugendlichen, sondern zudem im Spannungsfeld politischer Lager. Je nach lokalen politischen Mehrheiten in einer Kommune oder nach Prägungen aufgrund möglicher vorangegangener Zerwürfnisse bezüglich der Einführung von Gemeinschaftsschulen, exponierten sich die Wissenschaftler(innen) in diesen Auseinandersetzungen, so dass sie einem gewissermaßen ,politischen' Rechtfertigungszwang ausgesetzt waren. Um diesem vorzubeugen, wurden mehrere Termine im Gemeinderat vorgesehen. Diese fanden vor Beginn der Kooperation statt, um das Vorhaben zu erläutern und gemeinsam zu diskutieren. Eventuelle Einwände wurden aufgegriffen und die Planung gegebenenfalls angepasst. Direkt nach Abschluss wurden die Ergebnisse dem Gemeinderat vorgestellt und gemeinsam diskutiert. Die Anzahl der Termine variierte je nach Kooperationspartner.

Das Forschen in gesellschaftlich und politisch sensiblen Kontexten kann zweierlei Expositionen erzeugen: erstens Expositionen aufgrund gesellschaftlicher Spannungsfelder und zweitens Expositionen aufgrund politischer Polaritäten. Aus dem gesellschaftlichen Kontext können sich starke Interessenlagen ergeben, die konträr sein können und aufgrund der Sensibilität mit einer überaus starken Haltung und Emotion vertreten werden können. Das wiederum kann eine sachliche Kommunikation erschweren und Nährboden für Missverständnisse erzeugen. Begeben sich Wissenschaftler(innen) in dieses Spannungsfeld und erarbeiten mit Praxisakteuren Lösungsansätze, wie im vorangegangenen Beispiel beschrieben, führt dies zu Expositionen aller Beteiligten. 
Praxisakteure müssen sich nicht nur in für sie fremde Themengebiete einarbeiten, sondern exponieren sich gegenüber den jeweils anderen Interessengruppen aufgrund von fehlendem Wissen. Denn um ein gesamtheitliches Bild auf ein System zu erhalten, muss das Wissen aller Beteiligten zusammengeführt werden. Dafür wiederum ist es erforderlich, zuerst das spezialisierte Wissen von allen zu identifizieren. Dadurch werden aber auch Wissenslücken Einzelner hinsichtlich dieses Systems für alle offensichtlich. Zusätzlich kann es individuell auch an Expertise und Erfahrung mangeln, was im Prozess ebenfalls zu einer Exposition führen kann. Diese für transdisziplinäre Forschung übliche Herausforderung kann in einem gesellschaftlich sensiblen Kontext aufgrund der mitschwingenden Emotionen und möglicher gegenseitiger Voreingenommenheit zur Exposition werden. Je nach Gemengelage kann es einzelnen Praxisakteuren besonders schwerfallen, Wissenslücken oder fehlende Expertise einzugestehen, zu kommunizieren und sich damit den anderen Praxisakteuren gegenüber zu exponieren. Besonders betroffen sind davon meist Akteure mit einer besonderen Rolle im Transformationsprozess. Im vorangegangenen Beispiel wäre das der Schulträger, da er bei der Finanzierung eines Schulumbaus die entscheidende Schlüsselrolle innehat und deshalb die Situation für seine Rolle als unangemessen wahrnimmt.

Die Wissenschaftler(innen) exponieren sich erstens dadurch, dass sie häufig in dem beschriebenen gesellschaftlichen Spannungsfeld die für sie meist ungewohnte Rolle der Moderation oder gar Mediation (wie im Beispiel zuvor beschrieben) einnehmen. ${ }^{10}$ Zweitens führt auch der politische Kontext zu einer Exposition der Wissenschaftler(innen). Sie müssen sich aufgrund der Normativität, die durch das Setzen des Forschungsthemas erzeugt werden kann, je nach lokalen politischen Mehrheiten möglicherweise gegenüber Politiker(inne)n einzelner Fraktionen rechtfertigen. So entstehen „Episoden, in denen neben der internen Berücksichtigung von erfolgreichen Lernschritten, die zu angestrebten Lösungen beitragen, immer auch die politische Relevanz bestehender Ziele einer externen Bewertung ausgesetzt ist" (Groß et al. 2005, S. 211).

Starke Auseinandersetzungen zwischen den Praxisakteuren können mögliche negative Auswirkungen dieser Expositionen sein, sofern diese durch die Wissenschaftler(innen) nicht verhindert werden können. Eine ungünstige politische

10 Idealerweise sollte die Moderation oder Mediation eigentlich von Dritten übernommen werden, so dass kein Rollenkonflikt der Wissenschaftler(innen) entsteht, wenn diese als Moderator(inn)en oder Mediator(inn)en aktiv in den Prozess eingreifen, den sie untersuchen und experimentell transformieren. In mehreren BaWü-Labs zeigte sich jedoch, dass derartige Moderationen oder Mediationen zum einen oft spontan vonnöten waren, so dass die Wissenschaftler(innen) kurzfristig in diese Rolle schlüpfen mussten. Zum anderen konnten aufgrund fehlender Ressourcen meist keine externen Moderator(inn)en oder Mediator(inn)en eingesetzt werden. 
Gemengelage könnte zudem die Durchführung eines Teils des Projekts beziehungsweise der Datenerhebung erschweren oder gar verhindern. ${ }^{11}$

Die Schutzvorkehrung für die Exposition der Praxisakteure wird durch das Agieren der Wissenschaftler(innen) als Moderator(inn)en und Mediator(inn)en hergestellt. Somit stellt die Schutzvorkehrung für die Praxisakteure gleichzeitig die zuvor erläuterte Exposition der Wissenschaftler(innen) dar. Die Notwendigkeit von Moderations- und Mediationstätigkeiten kann sich während des gesamten Zusammenarbeitszeitraums mit den Praxisakteuren ergeben. Um diese Schutzvorkehrung gewährleisten zu können, bedarf es wiederum einer Schutzvorkehrung für die damit einhergehende Exposition der Wissenschaftler(innen) als Moderator(inn)en und Mediator(inn)en. Entsprechend können Aus- und Fortbildungsangebote für die Wissenschaftler(innen) eine mögliche Schutzvorkehrung darstellen, weil diese ermöglichen, die ungewohnte Rolle bereits im Vorfeld zu erlernen und zu trainieren. Einen Schutz der Wissenschaftler(innen) vor der Exposition gegenüber der Politik bieten Analysen der politischen Polaritäten bezogen auf den jeweiligen Kontext, in den diese sich begeben, so dass eine Vorbereitung auf kritische Diskussionen möglich ist. Eine Vorstellung und Erläuterung des Vorhabens beispielsweise im Gemeinderat wiederum kann eine mögliche Beoder Verhinderung des Vorhabens durch einzelne Politiker(innen) minimieren. Weitere Termine zur Vorstellung von Zwischenergebnissen und zur Präsentation des Ergebnisses können dies unterstützen.

Ein Mehrwert in der beschriebenen Exposition der Praxisakteure liegt - wie bei den meisten transdisziplinären Forschungsprojekten - darin, dass diese gegenseitig die jeweils anderen Sichtweisen, Interessen, Zielvorstellungen und Expertisen kennen sowie hoffentlich schätzen lernen. Im Zuge der Kooperation lernen sie außerdem den Fachjargon der anderen Akteure zu verstehen und anzuwenden. Gerade in einem sensiblen Kontext kann das Moderieren und Schlichten durch die Wissenschaftler(innen) verhärtete Konflikte lösen, so dass die dann eingeübte Zusammenarbeit die bestehenden Vorbehalte vorerst überbrücken kann. Dies bietet die Möglichkeit, dass die Zusammenarbeit der Praxisakteure gegebenenfalls über das Forschungsprojekt hinaus weitergeführt werden kann und mögliche Auseinandersetzungen aufgrund der geschaffenen gemeinsamen Kommunikationsbasis selbstständig ausgefochten werden können.

11 Weitere Ausführungen hierzu siehe Kapitel 4. 


\section{Expositionen durch Öffentlichkeitsarbeit}

An die BaWü-Labs wurde seitens des Fördergebers die Vorgabe gestellt, vom „Wissen zum Handeln zu kommen“ (MWK 2013, S. 31). Schäpke et al. (2017) sehen in solchen Anforderungen, die darin bestehen, neben der Wissensgenerierung Erkenntnisse in die ,gesellschaftliche Breite“ zu tragen, ein grundsätzliches Charakteristikum von Reallaboren. Um diese Anforderung zu erfüllen, fand in vielen BaWü-Labs ein Austausch mit der Öffentlichkeit und/oder eine Zusammenarbeit mit (Teilen) der Bevölkerung statt. Durch die breite öffentliche Sichtbarkeit entstanden für die beteiligten Akteure Expositionen. Im Folgenden werden die Expositionen erläutert, die durch das Organisieren von und Repräsentieren auf Veranstaltungen sowie durch das mediale Aufrufen für das Austesten von Innovationen entstehen.

\subsection{Organisieren von und Repräsentieren auf Veranstaltungen}

Im Rahmen von transdisziplinären (und transformativen) Forschungsprojekten kann es vorkommen, dass (Groß-)Veranstaltungen mit Teilnehmer(inne)n aus verschiedenen Akteursgruppen durchgeführt werden, in denen das Projektteam die tragende Rolle einnimmt. Organisieren, Veranstalten und Repräsentieren exponiert die am Projekt Beteiligten auf mehrfache Weise gegenüber den anderen Beteiligten und gegenüber externen Besucher(inne)n.

Beispiel BaWü-Lab Dietenheim zieht an: Organisation und Durchführung von Großveranstaltungen in Form der Messen „Dietenheim zieht an“

Das Reallabor Nachhaltige Transformation der Textilwirtschaft am Standort Dietenheim (,BaWü-Lab Dietenheim zieht an“) führte im Verlauf der Förderphase mehrere Messeveranstaltungen durch. In die Planung und Ausgestaltung waren alle Projektpartner und somit alle beteiligten Akteursgruppen (Wissenschaftler(innen), lokale Unternehmen und Verbände sowie die Stadtverwaltung und der Bürgermeister) involviert. Für alle Akteure war bereits im Vorfeld klar, dass vom Erfolg dieser Veranstaltungen viel abhing, da diese die größten nach außen hin sichtbaren Aktivitäten des BaWü-Labs waren. Besonders die kommunalen Akteure erhofften sich davon auch eine positive Außendarstellung. Durch die große Anzahl an erwarteten Besucher(inne)n und die Berichterstattung durch regionale Medien waren alle Beteiligten gegenüber einer breiten Öffentlichkeit exponiert. Eine weitere Exposition ergab sich dadurch, dass Unternehmen akquiriert wurden, bei „Dietenheim zieht an“ auszustellen. So waren alle im Projektteam vertretenen Akteursgruppen sowohl gegenüber den Bewohner(inne)n der Stadt und weiteren externen Besucher(inne)n als auch gegenüber den ausstellenden, teilweise neu gewonnenen Akteuren exponiert. Letzteres galt im Besonderen für einen Unternehmer, der einige seiner Geschäftspartner als Aussteller gewinnen konnte. 
Die Rolle als öffentlich auftretende Verantwortliche einer großen öffentlichen Veranstaltung war für die Wissenschaftler(innen) im Projektteam, anders als für einige Praxispartner, ungewohnt. Ziel der Veranstaltungen war eine positive Berichterstattung und somit ein erhöhter Bekanntheitsgrad sowohl des BaWü-Labs als auch der beforschten Konzepte nachhaltigen Textilkonsums.

Um die Wahrscheinlichkeit eines Misserfolgs zu mindern und von Beginn an viele Besucher(innen) anzuziehen, fand die erste Messe gleichzeitig mit dem etablierten Stadtfest „Dietenheim leuchtet“ statt. Bei allen Veranstaltungen wurden örtliche Vereine und Verbände integriert, um eine breite Öffentlichkeit anzusprechen und anzuziehen. In enger Absprache und Zusammenarbeit mit der kommunalen Verwaltung konnte eine Vielzahl regionaler Akteure gewonnen werden. Hinzu kamen umfangreiche Werbemaßnahmen in Form von Plakatwerbung in umliegenden Orten, Anzeigen in Zeitungen und sozialen Medien sowie Flyer. Die größtenteils positive, in Einzelfällen aber auch kritische Berichterstattung sowie die Wirksamkeit unterschiedlicher Werbekanäle wurden sowohl im Team der Wissenschaftler(innen) reflektiert als auch mit den Praxisakteuren diskutiert.

Trotz der umfangreichen Herausforderungen boten die Veranstaltungen eine hervorragende Gelegenheit, den Forschungsgegenstand des BaWü-Labs bekannter zu machen, und ermöglichten gleichzeitig, sowohl transformative Prozesse anzustoßen als auch Daten zu erheben.

Transdisziplinäre (und transformative) Forschungsprojekte können auf vielfache Weise in der Öffentlichkeit stehen. Projektinhalte und Teilprojekte werden häufig durch verschiedene Akteure nach außen repräsentiert. Dies kann in unterschiedlichen Formaten geschehen - von Informationsveranstaltungen in kleinem Rahmen für interessierte Kleingruppen bis hin zu Großveranstaltungen. Das führt häufig zu Expositionen gegenüber der Öffentlichkeit, unabhängig davon, ob nur vor einer kleinen Gruppe betroffener Anwohner(innen) präsentiert wird oder ob (über-)regionale Medien über eine Veranstaltung des Forschungsprojekts berichten. Kritische Fragen und negative Rückmeldungen können besonders für unvorbereitete und unerfahrene Akteure problematisch werden. Mit zunehmender Breite der angesprochenen Öffentlichkeit steigt zudem die Wahrscheinlichkeit, dass auch ein Publikum erreicht wird, das dem Projekt oder Teilen davon ablehnend gegenübersteht. Ebenfalls steigt die Wahrscheinlichkeit, dass Vertreter(innen) aller politischen Parteien und/oder Journalist(inn)en teilnehmen, und zwar unabhängig davon, welche Nähe oder Haltung zu den Projektthemen sie haben. Die daraus resultierende Exposition kann alle Akteure eines Forschungsprojekts betreffen und kann, abhängig von der Erfahrung und vom Hintergrund der Person, unterschiedlich stark ausfallen, andere Expositionen hingegen betreffen nur einzelne Akteursgruppen. 
Im Rahmen der BaWü-Labs übernahmen vorwiegend die Wissenschaftler(innen) die Organisation solcher Veranstaltungen und traten so nach außen hin als Verantwortliche und Ansprechpartner auf, sie exponierten sich also entsprechend. Präsentieren Wissenschaftler(innen) Ergebnisse ihrer Forschungsarbeit, bewegen sie sich in der Regel auf ihnen vertrautem Terrain. Dies ändert sich, wenn sie Veranstaltungen organisieren, die einer (mehr oder weniger breiten) Bekanntmachung des Forschungsgegenstands dienen - so zum Beispiel im Fall von „Dietenheim zieht an". Dann ist für die Wissenschaftler(innen) eine Exposition möglich, weil sie ungewohnte Tätigkeiten übernehmen und sich Kritik und Fragen aussetzen, die nicht nur das Projekt oder dessen Teile betreffen, sondern auch das Thema an sich.

Bei den Praxisakteuren exponieren sich jene Akteure, die nur in einem Teil des Forschungsprojekts involviert sind, aber beispielsweise im Rahmen von Veranstaltungen plötzlich auf Kritik zu anderen Teilen des Projekts reagieren sollen. Die Exposition ist umso unangenehmer, je mehr ihnen das nötige Hintergrundwissen beziehungsweise der Gesamtüberblick über das Projekt fehlen.

Die Exposition wirtschaftlicher Akteure kann davon abhängen, inwieweit sie mit den normativen Grundsätzen des Forschungsprojekts (z. B. Nachhaltige Entwicklung oder Gemeinwohl) im Einklang stehen, da sie durch ihre Teilnahme am Projekt an den entsprechenden Grundsätzen gemessen werden. Unternehmen können sich Kritik von außen aussetzen, wenn ihr unternehmerisches Handeln als nicht mit den Grundsätzen des Forschungsprojekts übereinstimmend wahrgenommen und ihre Teilnahme daher als unglaubwürdig eingestuft wird. Des Weiteren können Größe und Bekanntheit eines Unternehmens eine Rolle spielen, weil gerade größere Unternehmen mögliche nachteilige Folgen durch eine Exposition sowie die finanziellen Folgen (also inwiefern sich der Aufwand einer Teilnahme für sie beispielsweise finanziell lohnt) genau abwägen.

Ähnlich exponiert sich die öffentliche Verwaltung, und zwar insbesondere, wenn sie sich an der Einführung neuer Veranstaltungsformate beteiligt und sich somit hinsichtlich des Themas und der damit verbundenen normativen Grundsätze positioniert. Die durch das Projekt betroffene Bevölkerung nimmt häufig die Verwaltung als verantwortlich wahr und adressiert Kritik entsprechend (zum Thema Umgang mit Beschwerden an die Verwaltung s. Abschnitt 4.1).

Zivilgesellschaftliche Akteure schließlich können sich durch die Teilnahme an Großveranstaltungen transdisziplinärer (und transformativer) Forschungsprojekte gegenüber einer breiten Öffentlichkeit und kommunalen Akteuren exponieren. So kann beispielsweise eine Nachbarschaftsinitiative durch die Teilnahme an einer solchen Veranstaltung gleichzeitig größere Aufmerksamkeit erfahren als bisher und mehr Kritik ausgesetzt sein, als sie es gewohnt ist. 
Eine sehr grundlegende Schutzvorkehrung besteht darin, von Beginn eines transdisziplinären (und transformativen) Projekts an allen Akteuren die wissenschaftliche, praktische und gesellschaftliche Relevanz des Forschungsgegenstands aus Sicht jeder beteiligten Akteursgruppe zu erläutern und sicherzustellen, dass alle Beteiligten einen Überblick über das gesamte Projekt haben. Dadurch können alle Beteiligten jederzeit die Hintergründe erläutern. Durch ein transparentes und einheitliches Auftreten der beteiligten Akteure gegenüber Interessierten wie auch gegenüber Kritiker(inne)n kann zudem eine positive Außenwahrnehmung gefördert werden. Eine Absprache der wichtigen Positionen und eine Vergegenwärtigung möglicher Kritikpunkte sowie der Antworten darauf kann vor einer Großveranstaltung für die exponierten Akteure hilfreich sein. Kritik (aber auch Lob) aus den Medien und von Dritten wiederum sollte mit allen Beteiligten reflektiert werden, um zu verhindern, dass sich die Beteiligten abwertende Bemerkungen oder persönliche Angriffe zu sehr zu Herzen nehmen. Es sollte erörtert werden, weshalb etwas kritisiert wurde, welche Verbesserungen vorgenommen werden sollen oder ob die Kritik überhaupt eine Relevanz für das Projekt hat. Ein einheitliches Erscheinungsbild aller Teilprojekte mit entsprechendem Wiedererkennungswert kann eine weitere Schutzvorkehrung sein, indem es den Blick von den einzelnen Akteuren weg und zum Projekt hin lenkt und gleichzeitig die Zugehörigkeit zum Projekt zeigt. Eine umfangreiche Bewerbung von Großveranstaltungen wie etwa „Dietenheim zieht an“, um möglichst viele Besucher anzulocken, ist ebenfalls eine Schutzvorkehrung. Dadurch wird die Chance erhöht, dass beteiligte Akteure die Veranstaltung als Erfolg wahrnehmen. Zusätzlich können Presserundgänge und -mitteilungen die mediale Berichterstattung positiv beeinflussen.

Abhängig vom Forschungsgegenstand, Ziel und Setting eines Projekts können große Veranstaltungen einen hohen Mehrwert bieten. Wenn Forschungsprojekte das Ziel haben, die Bevölkerung zu integrieren und zu aktivieren, kann die Durchführung öffentlichkeitswirksamer Maßnahmen und Veranstaltungen schnell zu einem wesentlichen Teil der Projektarbeit werden. Durch Großveranstaltungen können auf einen Schlag viele Menschen erreicht und informiert oder zur Teilnahme an weiteren Veranstaltungen und/oder öffentlichen Interventionen (s. Kapitel 4) gewonnen werden. Durch erfolgreiche Veranstaltungen im Rahmen der BaWü-Labs wurde oft eine positive Berichterstattung in den Medien erreicht, die dazu führte, dass weitere Teilprojekte einfacher durchgeführt werden konnten. Erfahrungsgemäß waren auch viele beteiligte (z. B. kommunale) Akteure eher bereit, an weiteren Veranstaltungen mitzuwirken und sich weiter zu exponieren, wenn die Resonanz der Öffentlichkeit positiv war. Auch im Hinblick auf die Verstetigung von Teilprojekten (z. B. durch Übernahme seitens kommunaler oder zivilgesellschaftlicher Akteure) kann ein hoher Bekanntheitsgrad hilfreich sein. Schließlich wird ein positives Medienecho auch durch den Fördergeber wahrgenommen und kann möglicherweise sogar eine Weiterförderung erleichtern. 


\subsection{Mediales Aufrufen für das Austesten von Innovationen}

In transformativen (und transdisziplinären) Forschungsprojekten kann es zum Austesten von Innovationen notwendig sein, mediale Aufrufe durchzuführen, um den Bedarf zu erheben sowie die Umsetzung und Auswirkungen erproben zu können. Den Wissenschaftler(inne)n und beteiligten Praxisakteuren können dabei durch den öffentlichen Charakter des medialen Aufrufs und durch die Spielregeln der medialen Kommunikation Expositionen entstehen.

\section{Beispiel BaWü-Lab Space Sharing: Nutzer(innen)aufruf für den Pilotstandort}

Im Reallabor Space Sharing - Nutzungsintensivierung des Gebäudebestands durch Mehrfachnutzung und dynamische Programmierung („BaWü-Lab Space Sharing“) sollte getestet werden, wie Räume im Laufe eines Tages mehrfach genutzt werden können. Um den Pilotstandort im Calwerkopfbau am Rotebühlplatz in Stuttgart zu betreiben, musste zu Projektbeginn ein Nutzer(innen)aufruf an die Stuttgarter Stadtbevölkerung gestartet werden. Um ein Bild zu erhalten, für welche Nutzungen in der Stadt von wem Räume gesucht werden, musste dieser Aufruf so angelegt sein, dass potenzielle Interessierte aus einem möglichst großen Teil der Bevölkerung angesprochen wurden. Außerdem konnte nur durch vielfältige Rückmeldungen ausgetestet werden, welche Nutzungen unter welchen Bedingungen nacheinander und/oder parallel stattfinden könnten. Um Nutzer(innen) für den Standort zu finden, musste das Thema Space Sharing ansprechend und leicht verständlich sowie überzeugend von Projektbeginn an über die Medien vermittelt werden. Der Standort, der anfangs nicht im öffentlichen Bewusstsein verankert war, musste sichtbar gemacht werden.

Die am medialen Aufruf beteiligten Wissenschaftler(innen) der Fachgruppe Architektur der Staatlichen Akademie der Bildenden Künste Stuttgart (ABK Stuttgart) exponierten sich in diesem Prozess des Aufrufs vor allem gegenüber am Forschungsprojekt Interessierten und potenziellen Nutzer(inne)n sowie gegenüber den Kolleg(inn)en und der Öffentlichkeit. Der öffentliche Charakter des medialen Aufrufs ermöglichte es allen, die Arbeit der Wissenschaftler(innen) zu beobachten und von Projektbeginn an kritisch zu hinterfragen. Bei ausbleibender Resonanz hätten Vorwürfe wie unprofessionelles Vorgehen aufkommen können. Des Weiteren hätte der Pilotstandort im Calwerkopfbau nicht betrieben werden können.

Der mediale Aufruf stellte sich als komplexer, umfangreicher und langwieriger als ursprünglich geplant heraus, was zu Verzögerungen führte. Ein breit gestreuter Rückgriff auf unterschiedliche Medien war notwendig, wobei das Anliegen in der jeweils adäquaten Form bekannt gemacht werden musste. Der mediale Aufruf reichte schließlich von digitalen Formaten wie Homepage, Facebook und Ansprechpartner per E-Mail über Printmedien (Plakate, Infoflyer, Artikel in lokaler Presse) bis zur persönlichen Präsenz bei Veranstaltungen Dritter. Entscheidend für die Resonanz aus der Bevölkerung war die Entwicklung eines identitätsstiftenden Kommunikationskonzepts - ähnlich wie eine Corporate Identity $(\mathrm{Cl})$ bei Firmen - in Kooperation mit der Fachgruppe Kommunikationsdesign der ABK Stuttgart. Es bestand aus einem leicht wiedererkennbaren Logo 
(s. dazu Abb. 1), das von digitalen Medien über Printmedien bis zu Visualisierungen und Umsetzungen im Raum zur Anwendung kam, der Wortmarke "Calwerkopfbau" und einem einheitlichen Leitsystem (s. dazu Abb. 2), das bereits vor dem Gebäude, im Treppenhaus und in den Space-Sharing-Räumlichkeiten als ,physisches Handbuch' diente. Um Interessierte, die sich vom Nutzer(innen)aufruf angesprochen fühlten, die Entscheidung zur Teilnahme zu erleichtern, wurden zu Beginn der Standortbetreibung

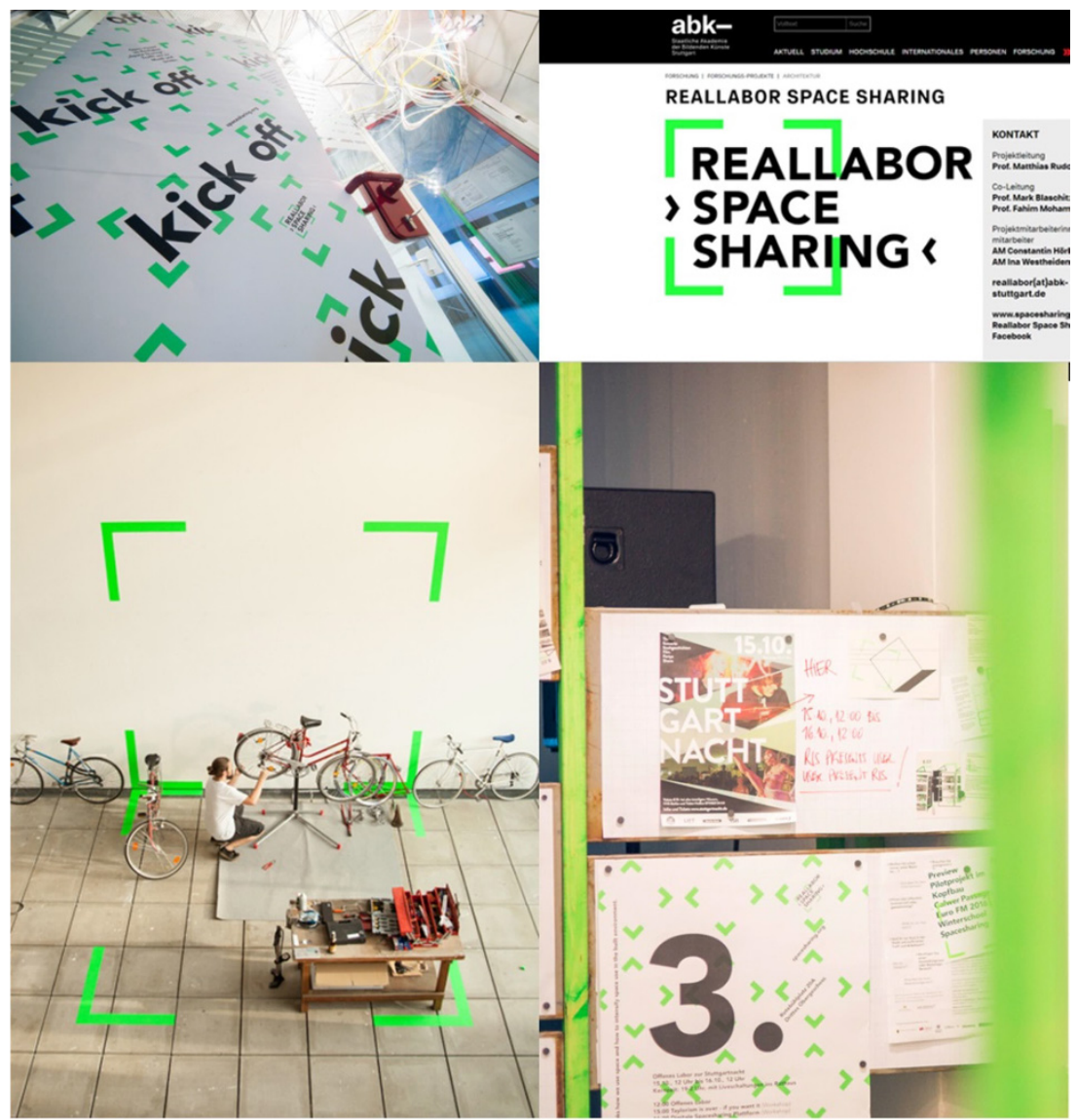

Abbildung 1: Das Logo wurde bei Plakatgestaltungen, auf der Webseite und als Umsetzung im Raum beim 24/7-Realexperiment angewendet, und die Farbe des Logos fand in der Gestaltung der Räumlichkeiten Anwendung. (C) Oben und rechts unten Reallabor Space Sharing, ABK Stuttgart; links unten Master Stegreif Spacesharing 24/7, ABK Stuttgart; Plakatgestaltung: Marlene Kehle, Erika Mai, Juliane Windbiel, Tina Zeltwanger. 
Informationsabende veranstaltet. An diesen wurde erstens ausführlich über das Projekt informiert und auf den experimentellen Charakter des Standorts hingewiesen. Zweitens gab es auch die Möglichkeit zur Diskussion sowie zum persönlichen Kennenlernen der Wissenschaftler(innen) sowie der anderen Interessierten. Diese Diskussionsveranstaltungen wurden später zum Austausch mit den Nutzer(inne)n in regelmäßigen Abständen fortgeführt. Des Weiteren erwiesen sich Multiplikatoren - z. B. Studierende oder bereits beteiligte Nutzer(innen) - als hilfreich bei der Kommunikation mit Interessierten (zu Kommunikationsstrategien im BaWü-Lab Space Sharing s. RLSS 2018, S. 39ff.).

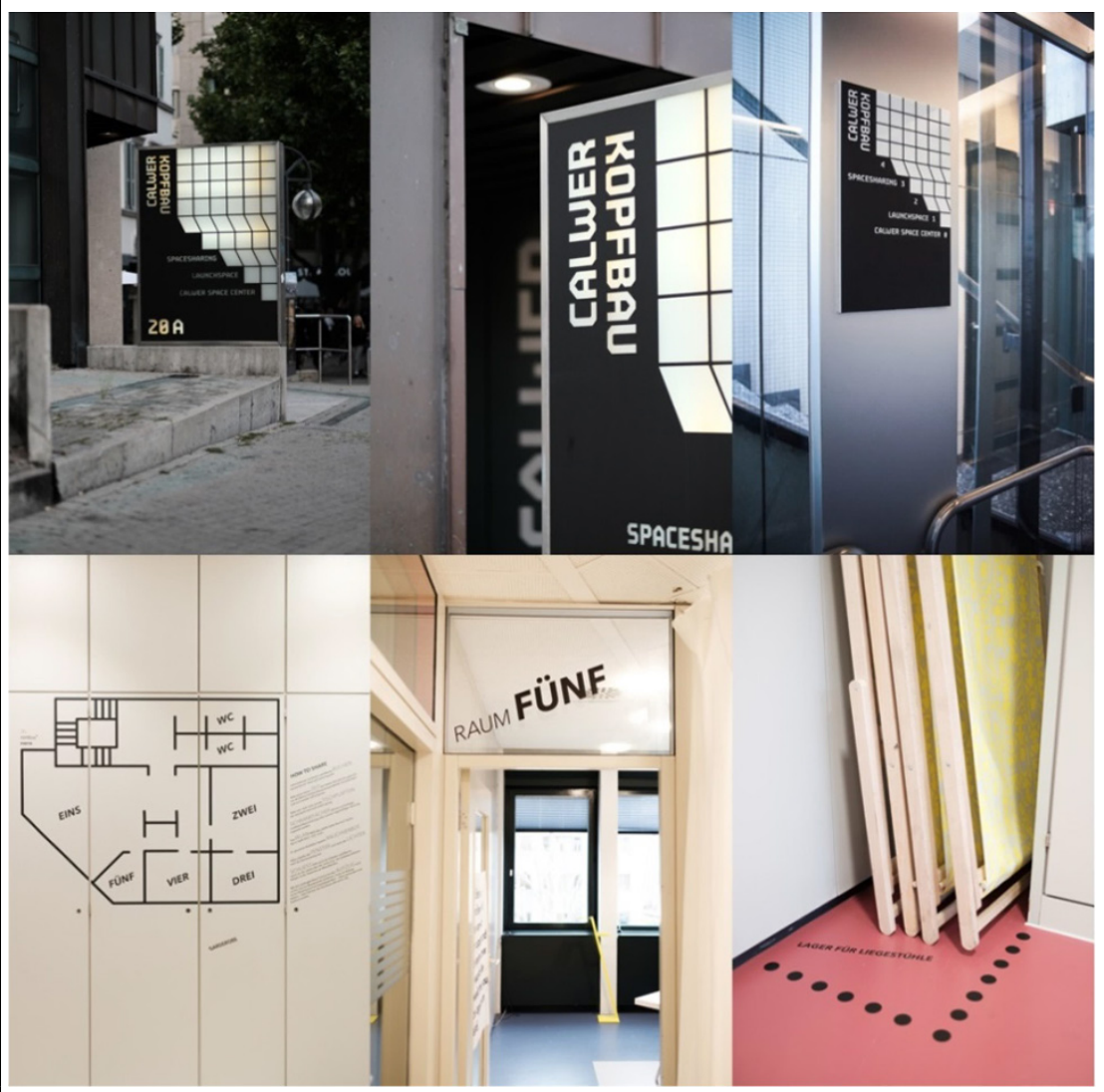

Abbildung 2: Das Leitsystem zog sich von vor dem Gebäude über Aufzug und Treppenhaus bis in die Space-Sharing-Räumlichkeiten im 3. Obergeschoss. Es bewirkte die Präsenz des Projekts im Stadtraum und diente den Nutzer(inne)n vom Eingang weg bis zum Möbel als , physisches Handbuch'. (C) Marlene Kehle, Erika Mai, Juliane Windbiel, Tina Zeltwanger. 
Für das umfassende Austesten einer Innovation (Bedarfserhebung, Erprobung der Umsetzung, Überprüfung der Auswirkungen etc.) im gesellschaftlichen Kontext kann es notwendig sein, einen medialen Aufruf durchzuführen, um den Bedarf zu erheben und Teilnehmer(innen) zu finden. Dabei können für die Wissenschaftler(innen) - sowie gegebenenfalls auch für involvierte Praxisakteure - durch den öffentlichen Charakter des medialen Aufrufs und die Spielregeln der medialen Kommunikation Expositionen entstehen.

Da die Erhebung von Bedarf und Zielpublikum der Innovation Teil der Forschungsfrage ist, kann der mediale Aufruf nicht fokussiert werden. Vielmehr muss er möglichst breit gestreut - also öffentlich über eine große Bandbreite an Medien - erfolgen. Zum Beispiel kann nicht gezielt nach 100 sportlichen Personen zwischen 50 und 60 Jahren gesucht werden, sondern es muss, wie im Beispiel des BaWü-Labs Space Sharing, möglichst die komplette Stadtbevölkerung angesprochen werden, um zu erforschen, wer wie Bedarf für die Innovation (in diesem Fall Space Sharing) hat. Zudem kann es wegen des transformativen Anspruchs notwendig sein, eine möglichst große Vielfalt gesellschaftlicher Gruppen anzusprechen. Um wissenschaftlich brauchbare Rückmeldungen aus der Bevölkerung zu erhalten, kann jedoch in der Kommunikation über die Medien die Bedarfserhebung für die Innovation nicht als Untersuchungsfrage formuliert werden. Stattdessen ist es in solchen Fällen notwendig, durch eine überzeugende Darstellung der Innovation Interesse und Bewusstsein für den Bedarf zu generieren, um mögliche und möglichst viele Interessierte zur Teilnahme zu motivieren. Es müssen also Aspekte, die erst im Laufe des Projekts nach erfolgreichem medialem Aufruf untersucht werden können, bereits zu Projektbeginn als affirmative Aussagen in der Öffentlichkeit präsentiert werden.

Die Notwendigkeit der ungerichteten und breiten Ansprache sowie die Mechanismen der medialen Kommunikation (Stichwort Vermarktung) widersprechen in einem solchen Prozess des medialen Aufrufs also den üblichen Vorgehensweisen in der Forschung. Die verantwortlichen Wissenschaftler(innen) exponieren sich erstens, weil sie mit und über die Medien arbeiten müssen. Dies entspricht nicht ihrem üblichen Aufgabenfeld und bringt eine große öffentliche Aufmerksamkeit mit sich. Die Kommunikation über die Medien ermöglicht es de facto jedem Interessierten von Beginn des medialen Aufrufs an die Arbeit im Forschungsprojekt zu verfolgen und zu kritisieren. Je nach Tätigkeitsfeld und Erfahrung betrifft dies auch am medialen Aufruf beteiligte Praxisakteure. Zweitens machen sich Wissenschaftler(innen) durch die Anpassung an die Spielregeln der medialen Kommunikation gemessen am Konsens zu wissenschaftlichen Vorgehensweisen im Wissenschaftssystem angreifbar. Mögliche negative Folgen können daher sein, dass ihnen unprofessionelles Handeln vorgeworfen wird - sowohl bezogen auf die Wissenschaftlichkeit als auch bezogen auf die Medienkommunikation. 
Außerdem ist das Austesten der Innovation vom Rücklauf auf den medialen Aufruf abhängig. Bleibt der Rücklauf aus, können folglich der Testlauf nicht wie geplant stattfinden und die damit einhergehenden Forschungsfragen nicht beantwortet werden. Erschwerend kommt hinzu, dass der Verlauf medialer Kommunikationsprozesse aufgrund komplexer Zusammenhänge und Eigendynamiken erstens schwer plan- und steuerbar sowie zweitens unzureichend analysierbar ist. Eine korrekte Analyse bei fehlendem Rücklauf, ob kein Bedarf vorhanden ist oder ob der mediale Aufruf nicht adäquat durchgeführt wurde, ist daher schwierig. Bei zu geringem Rücklauf muss mit der schon grundsätzlich schwierigen Herausforderung, das (Teil-)Projekt zur Innovation nicht wie geplant durchführen zu können, auch noch unter einer breiten Aufmerksamkeit agiert werden. Handlungsmöglichkeiten können dadurch eingeschränkt sein. Zum Beispiel kann gegebenenfalls nicht innerhalb des Projektteams nach Lösungsstrategien wie Neuausrichtung oder Ersatzmaßnahmen gesucht werden, ohne diese nach außen rechtfertigen zu müssen.

Um Wissenschaftler(innen) - sowie gegebenenfalls am Medienaufruf beteiligte Praxisakteure - nicht ungeschützt dem für sie normalerweise fremden Bereich der Medienkommunikation auszusetzen, ist es zumindest notwendig, sie für diese Aufgabe zu schulen. Als Schutzvorkehrung empfehlenswerter ist es jedoch, Akteure mit entsprechender Medienexpertise von Anfang an ins Projektteam zu integrieren. Sie können so bereits in der Projektkonzeption das notwendige Wissen einbringen, wie über welche Medienkanäle wer erreicht werden kann und dazu passende Kommunikationskonzepte entwickeln. Um erstens ein Ausbleiben des Rücklaufs auf Grund von Fehlern beim medialen Aufruf zu vermeiden und zweitens korrekte Schlüsse aus dem Rücklauf in die Bedarfsanalyse einfließen lassen zu können, empfiehlt es sich zudem, mit einem medialen Aufruf von Anfang an gleich bewusst wie mit der Wahl sämtlicher anderer Methoden umzugehen. Auch die zeitliche Komponente sollte in der Projektplanung berücksichtigt werden - so bedarf der mediale Aufruf eines ausreichend langen zeitlichen Vorlaufs für die Vorbereitung und dann für die Verbreitung über Medien und Mundpropaganda. Mediale Eigendynamiken können hierbei sowohl beschleunigend als auch verzögernd wirken. Um kritischen Stimmen sowie zweifelnden Interessierten die Möglichkeit zu geben, Zweifel und Kritik zielgerichtet zu äußern (statt ungerichtet in sozialen Medien), sollte ihnen die Möglichkeit gegeben werden, mit den betroffenen Akteuren direkt zu diskutieren. Hierzu empfehlen sich Kommunikations- und Veranstaltungsformate, auf denen ausdrücklich ein persönlicher Kontakt hergestellt werden kann - also zum Beispiel die Einrichtung einer E-Mail-Adresse, über die in kurzen Zeitfenstern Anfragen beantwortet werden, oder das Gesprächsangebot bei Veranstaltungen. Es gilt die Spanne zwischen breiter Öffentlichkeitsansprache einerseits und dem Bedürfnis nach dem persönlichen Gesprächsangebot andererseits abzudecken. 
Ein breiter medialer Auftritt zur Mitwirkung kann auch einen Mehrwert für ein Projekt darstellen. Durch die beschriebene Kommunikation des Forschungsvorhabens über verschiedene Medien entsteht die Notwendigkeit, bereits frühzeitig über die Forschungsfragen, -ziele und -methoden zu reflektieren und diese auf den Punkt gebracht für unterschiedliche Kanäle medientauglich zu formulieren. Dies kann zu einer inhaltlichen Schärfung und Fokussierung des Vorhabens beitragen, da sich alle Projektverantwortlichen über die essenziellen Aspekte einig werden müssen. Darüber hinaus kann Mehrwert durch den medialen Aufruf entstehen, wenn es gelingt, die Innovation so ansprechend zu kommunizieren, dass ein Beitrag zur Ingangsetzung des transformativen Prozesses geleistet wird. Des Weiteren können sich Interessierte, die von Seiten der Projektverantwortlichen nicht in Betracht gezogen worden waren, auf den medialen Aufruf hin melden und neue Inhalte einbringen. Schließlich kann ein positiver Effekt durch die ergriffenen Schutzvorkehrungen für die am Kommunikationsprozess beteiligten Wissenschaftler(innen) sowie Praxisakteure entstehen, indem sie sich Medienkompetenzen aneignen.

\section{Expositionen durch Öffentliche Interventionen}

Die Anforderung an transformative Forschung, gesellschaftliche Veränderungsprozesse anzustoßen, kann es mit sich bringen, dass Interventionen, beispielsweise in Form von Realexperimenten ${ }^{12}$, durchgeführt werden. Im Folgenden werden Expositionen erläutert, die aus der Durchführung von Interventionen im öffentlichen Raum entstehen. Unter solchen Interventionen werden gezielte Veränderungen des Status quo verstanden, die öffentlich sichtbar und wirksam sind und in der Regel die Genehmigung durch eine zuständige Behörde benötigen. Expositionen können durch öffentlich formulierte Kritik wie auch durch Beschwerden entstehen, die an das Projekt- bzw. Interventionsteam ${ }^{13}$ gerichtet wer-

12 Bei Realexperimenten handelt es sich um „Experimentierprozesse, die nicht in der Sonderwelt der wissenschaftlichen Laboratorien, sondern in der Gesellschaft stattfinden. [...] In Realexperimenten geht es zwar ebenso um gezielte Eingriffe und um Erkenntnisgewinn, jedoch nicht in erster Linie um Forschung und neues Wissen. [...] Da jedoch selten über die genaue Abfolge und die Erfolgsaussichtungen [sic] ein unzweifelhafter Konsens besteht, kommt der Frage entscheidende Bedeutung zu, wie man bei gegebenen Wissenslücken und Unsicherheiten dennoch zum Handeln kommen kann“ (Groß et al. 2005, S. 11).

13 Das Realexperiment, das hier als Beispiel dient, wurde in enger Kooperation zwischen Wissenschaftler(inne)n und den initiierenden Praxisakteuren durchgeführt. Da sich in diesem Prozess die Rollenverteilung nicht immer klar abgrenzen ließ und viele Aktivitäten gemeinsam geplant, durchgeführt und evaluiert wurden und da angenommen werden darf, dass dies auch in anderen Fällen so sein könnte, wird von den an der 
den. Diese Kritik kann sich auf unterschiedliche Aspekte der Intervention beziehen. Anhand des Realexperiments „Parklets für Stuttgart“ werden Expositionen beleuchtet, die durch die Kritik an Genehmigung und Durchführung von Interventionen sowie durch die Rechtfertigung für die Involvierung von projektexternen Personen entstehen.

\section{Beispiel BaWü-Lab RNM: „Parklets für Stuttgart“ - Hintergrund des Realexperiments}

Im Rahmen des Realexperiments „Parklets für Stuttgart“ (Lazarova et al. 2018) wurden über einen Zeitraum von drei Monaten an verschiedenen Standorten in der Stuttgarter Innenstadt öffentliche Parkplätze zu sogenannten Parklets ${ }^{14}$ umgestaltet. Ziel des Vorhabens war es, eine öffentliche Diskussion über Flächengerechtigkeit und die Gestaltung des öffentlichen Raums anzuregen sowie gleichzeitig Alternativen erlebbar zu machen. Initiiert wurde das Realexperiment durch zivilgesellschaftliche Praxisakteure ${ }^{15}$, deren Vorhaben in einem Ideenwettbewerb von einer transdisziplinär besetzten Jury zur Umsetzung ausgewählt wurde (zum Auswahlprozess s. Puttrowait et al. 2018). Das Reallabor für nachhaltige Mobilitätskultur („BaWü-Lab RNM“) unterstützte das Vorhaben finanziell und durch wissenschaftliche Begleitung. Daraus ergab sich ein intensiver Kooperationsprozess zwischen den zivilgesellschaftlichen und weiteren Praxisakteuren und den für das Realexperiment zuständigen Wissenschaftler(inne)n. Die Stadt Stuttgart, vertreten durch das Amt für öffentliche Ordnung, erarbeitete eine Genehmigungsgrundlage. Geplant und gebaut wurden die Parklets von Studierenden der Architektur und Stadtplanung der Universität Stuttgart im Rahmen einer eigens eingerichteten Lehrveranstaltung, die durch das Interventionsteam betreut wurde. Sie entwarfen die Parklets in Abstimmung mit lokalen Pat(inn)en, also an den Standorten anliegenden Privatpersonen oder Gewerbetreibenden, die während der Standzeit der Parklets auch für die Pflege und als Ansprechpartner, ihres' jeweiligen Parklets verantwortlich waren.

Durchführung einer solchen Intervention Beteiligten im Folgenden als ,Interventionsteam' gesprochen.

14 Als ,Parklet' bezeichnet man die räumliche Erweiterung des Gehwegs mittels einer temporären oder dauerhaften Installation, die anstelle einer Parkfläche für PKW Raum für Menschen schafft. Parklets können vielfältig gestaltet sein und unterschiedliche Funktionen wie Sitzgelegenheiten, Spielflächen oder Urban Gardening anbieten (vgl. San Francisco Planning Department 2018).

15 Dies waren drei Studierende der Architektur und Stadtplanung der Universität Stuttgart, zusammen mit weiteren zivilgesellschaftlichen Akteuren. Da die Studierenden aus privaten Motiven und außerhalb universitärer Verpflichtungen handelten, werden die Initiator(inn)en im Weiteren als zivilgesellschaftliche Praxisakteure bezeichnet. 


\subsection{Kritik an Genehmigung und Durchführung von Interventionen}

Öffentliche Interventionen können Beschwerden und öffentlich formulierte Kritik aufgrund einer inhaltlichen Ablehnung der mit der Intervention verbundenen Ziele oder der Vorgehensweise in der Umsetzung hervorrufen, wodurch sich sowohl die durchführenden Akteure als auch die genehmigenden Behörden exponiert sehen.

\section{Beispiel BaWü-Lab RNM: „Parklets für Stuttgart“ - Genehmigung und Beschwerde- management}

Das Realexperiment zog sowohl ein breites mediales Echo als auch eine öffentliche Diskussion in den sozialen Medien nach sich, wobei neben Zustimmung auch Kritik laut wurde. Zudem wurden an das Interventionsteam und die Verwaltung auch konkrete Beschwerden gerichtet. Die Beschwerden und Kommentare bewegten sich dabei auf unterschiedlichen inhaltlichen Ebenen. So wurde die Sinnhaftigkeit der Intervention generell infrage gestellt, da darin eine Verschlimmerung der Parkplatzsituation gesehen wurde. Andere kritisierten dagegen die Ästhetik oder Funktionalität einzelner Parklets. Teilweise wurde auch die Rechtmäßigkeit der Genehmigung der Intervention angezweifelt, weswegen es essenziell gewesen war, eine rechtlich nicht anfechtbare Begründung für die Genehmigung zu finden. Die meisten Beschwerden wurden direkt an die Stadtverwaltung und das Interventionsteam herangetragen. In einigen der betroffenen Stadtbezirke forderten auch die Bezirksbeiräte eine persönliche Vorstellung in einer öffentlichen Sitzung, da sie die Befürchtung äußerten, dass sie von den Bürger(inne)n auf die Intervention angesprochen und Beschwerden an sie adressiert werden würden. Für das Interventionsteam stellten diese Art und Menge der Kritik eine neue Erfahrung dar, in der sich die Mitglieder exponiert sahen. Die involvierten Vertreter(innen) der Stadtverwaltung hingegen verfügten zwar schon über eine gewisse Erfahrung mit Beschwerden, doch auch bei ihnen wurde deutlich, dass die quantitative Zunahme einen zusätzlichen Aufwand darstellte.

Dem Interventionsteam war daran gelegen, zu verhindern, dass sich die öffentliche Meinung lediglich aufgrund mangelnder Kommunikation und nicht aufgrund sachlicher Argumente gegen das Vorhaben wendete und somit eine gegebenenfalls sinnvolle Verstetigung aus sachfremden Gründen behindert würde. Zudem stand die Möglichkeit im Raum, dass die Genehmigung bei zu großem Widerstand widerrufen worden wäre, was den Abbruch des Realexperiments zur Folge gehabt hätte. Daher wurde als Schutzvorkehrung ein zwischen dem Interventionsteam und der Verwaltung gemeinsam abgestimmtes Beschwerdemanagement eingerichtet. Das Interventionsteam veröffentlichte eine eigene E-Mail-Adresse sowie die telefonischen und postalischen Kontaktdaten eines Ansprechpartners. An den Parklets selbst wurden erklärende Tafeln angebracht, die das Realexperiment erläuterten und auf Möglichkeiten zur Rückmeldung hinwiesen.

Persönlich beim Amt für öffentliche Ordnung vorgetragene Beschwerden wurden dort direkt bearbeitet. Schriftliche Beschwerden wurden beim Interventionsteam gesammelt und durch dieses beantwortet, wofür ein Verhaltenskodex vereinbart wurde. Jeder 
Person, die sich beschwerte, wurde zudem ein persönliches Gespräch angeboten. Bei konkreten Mängeln bezüglich der Gestaltung der Parklets wurde schnellstmöglich Abhilfe geschaffen (z. B. durch notwendige Reparaturen). Um die Politiker(innen) zu informieren, wurde das Vorhaben wie gewünscht durch das Interventionsteam präsentiert. Im Laufe des Realexperiments wurde zudem ein Rundgang mit interessierten Fraktionen durchgeführt, der es den Teilnehmenden ermöglichte, sich über Ergebnisse und Erfahrungen mit den Parklets auszutauschen.

Das Beschwerdemanagement war mit einem erheblichen Aufwand verbunden, doch durch das Ergreifen der beschriebenen Maßnahmen konnten ein möglicher Abbruch vermieden und das Ziel einer Diskussion über die Sache erreicht werden. Die Beschwerden wurden schließlich sogar Teil des Datenmaterials, anhand dessen diese Diskussion systematisch ausgewertet wurde.

Obwohl bei der Vorstellung des Projekts in den Bezirksbeiräten einige Fraktionen das Vorhaben inhaltlich ablehnten, zeigten sich die Politiker(innen) insgesamt dankbar über die Information, da diese sie in die Lage versetzte, Auskunft geben und Beschwerden weiterleiten zu können. Für das Interventionsteam wurde dagegen sichtbar, welche politischen Gruppierungen das Projekt unterstützten und welche es aus welchen Gründen ablehnten. Zudem lieferten die Politiker(innen) wertvolle Hinweise dazu, wie die Parklets besser in den lokalen Gemeinschaften verankert werden konnten.

Wie das Beispiel „Parklets für Stuttgart“ zeigt, kann bei einer öffentlichen Intervention mit Kritik oder konkreten Beschwerden aus der Öffentlichkeit oder der Politik gerechnet werden, die schon während der Planungsphase, vor allem aber ab Sichtbarwerdung der Intervention eintreten kann.

Durch die Durchführung der Intervention im öffentlichen Raum entsteht eine Exposition für die verantwortlichen Wissenschaftler(innen) und Praxisakteure gegenüber den Teilen der Öffentlichkeit und der Politik, die die Ziele und Inhalte sowie das Vorgehen der Intervention kritisieren. Der öffentliche Charakter kann dazu führen, dass eine große Menge an einzelnen Beschwerden das Interventionsteam erreicht. Die Menge der Beschwerden, aber auch die Art der Kritik kann für das Interventionsteam eine ungewohnte Situation darstellen, weil die Kritik nicht nur innerhalb eines fachlichen (und zumeist strukturierten) Austauschs, sondern über öffentliche Kanäle formuliert wird. Zudem konzentrieren sich die Argumente der Kritiker(innen) nicht immer auf sachliche Argumente, sondern können sogar persönliche Angriffe beinhalten. Gegenüber der Verwaltung exponiert sich das Interventionsteam durch die Verpflichtungen, wie zum Beispiel Genehmigungsauflagen, die mit der Intervention einhergehen und deren Einhaltung von der Verwaltung kritisch überwacht werden kann.

Die Erfahrung mit den Parklets zeigte, dass die Verwaltung zudem sehr auf die öffentliche Meinung achtet und in Folge dieser Meinung zusätzlichen Druck auf das Interventionsteam ausüben beziehungsweise selbst erfahrenen Druck an das 
Interventionsteam weitergeben kann. Als mögliche negative Folge der Art und Menge von Kritik und Beschwerden kann sich erstens eine inhaltliche Ablehnung der Intervention in Öffentlichkeit und Politik ergeben. Zweitens kann der Eindruck unprofessionellen Vorgehens entstehen und sich ein negatives öffentliches Meinungsbild lediglich aufgrund suboptimaler Kommunikation und Handhabe von Beschwerden entwickeln. Dieses negative Meinungsbild kann den Zielvorstellungen der Praxisakteure zuwiderlaufen und die wissenschaftlichen Akteure in ihrem Bestreben nach Wissenserwerb behindern, da kostbare Ressourcen auf das Beantworten von Beschwerden verwendet werden müssen.

Die Akteure der öffentlichen Verwaltung wiederum sehen sich gegenüber der Öffentlichkeit exponiert, da sie für die Genehmigungserteilung kritisiert und im schlimmsten Fall juristisch belangt werden können. Zusätzlich sehen sie sich dem Druck von Politiker(inne)n ausgesetzt, die eigene oder durch die Bürgerschaft an sie herangetragene Kritik am Genehmigungsprozess, wegen Fehlern in der Durchführung und Kommunikation oder an den Zielen der Intervention an die Verwaltung herantragen. Ungewohnt an dieser Situation sind für die Verwaltung vor allem der wissenschaftliche und experimentelle Kontext sowie mögliche spezifische Eigenschaften der Intervention, die das Erarbeiten und spätere Verteidigen einer Genehmigung im Vergleich zu Themen, bei denen Erfahrungen und Routinen vorhanden sind, erschweren können. Zudem kommt es zu einer quantitativen Zunahme der Beschwerden, wodurch es zu zusätzlichen Belastungen kommen kann, wenn dafür keine personellen Ressourcen zur Verfügung gestellt werden. Eine mögliche negative Konsequenz von alldem kann sein, dass die Verwaltung zukünftigen Kooperationen ablehnend gegenübersteht. Im schlimmsten Fall droht auch der vorzeitige Abbruch der Intervention durch Entzug der Genehmigung oder aufgrund einer erfolgreichen Klage.

Lokale Politiker(innen) werden von Beginn der Intervention an durch das Interventionsteam gegenüber den Bürger(inne)n exponiert, da sie von diesen auf die Intervention angesprochen werden können. Dies kann dazu führen, dass die Politiker(innen) daraufhin Druck auf die öffentliche Verwaltung ausüben, der dann an das Interventionsteam weitergegeben wird. Das Interventionsteam und die öffentliche Verwaltung sehen sich also wiederum der Reaktion durch die Politiker(innen) ausgesetzt.

Die Erfahrung des BaWü-Labs RNM zeigt schließlich, dass es kaum gelingt, eine Differenzierung zwischen einer einzelnen Intervention und dem Forschungsprojekt als Ganzes in der öffentlichen Wahrnehmung zu erreichen, weswegen die Kritik an der Intervention auch immer auf das Gesamtprojekt zurückfallen kann, wodurch sich dieses ebenfalls exponiert sieht. Eine erste Schutzvorkehrung für alle Akteure besteht darin, die Öffentlichkeit über das Projekt adäquat zu informieren, wodurch der Kritik entgegengewirkt werden kann, welche lediglich aus Unkenntnis und der ,Überraschung' über die Intervention entsteht. Eine Schutz- 
vorkehrung vor ablehnender inhaltlicher Kritik kann während der Intervention in einer aktiven, mit guten Argumenten untermauerten Beteiligung an der öffentlichen Diskussion bestehen. Hierfür können die verschiedenen Medien regelmäßig auf entsprechende Inhalte gescannt und Beiträge verfasst werden. Auch können persönliche Gespräche oder Informationsveranstaltungen vor Ort angeboten werden.

Eine weitere wichtige Schutzvorkehrung stellt ein zwischen den Akteuren der öffentlichen Verwaltung und dem Interventionsteam abgestimmtes Verfahren für den Umgang mit den Beschwerden dar, welches darlegt, welche Kommunikationskanäle zum Einreichen von Beschwerden angeboten werden, wer für die Beantwortung verantwortlich ist, welcher Verhaltenskodex für die Beantwortung gilt und wie mit Beschwerden verfahren wird, die sich nicht durch eine Antwort oder Auskunft erledigen lassen. Als Schutzvorkehrung gegen juristische Konsequenzen sollte die Verwaltung im Zuge der Planung der Intervention, wie für jede andere Maßnahme im öffentlichen Raum auch, eine juristisch sichere Grundlage für die Genehmigung finden. Als Schutzvorkehrung für lokale Politiker(innen) sind eine umfassende Information oder ein persönlicher Austausch über die Intervention mit dem Interventionsteam dienlich.

Als Mehrwert ergibt sich, dass mit der Durchführung von öffentlichen Interventionen viele projektexterne Personen in die Thematik einbezogen oder darauf aufmerksam gemacht werden sowie wissenschaftliche Fragestellungen in einem gesellschaftlichen Kontext untersucht werden können, wie es dem Wesenskern von Realexperimenten entspricht. Eine erfolgreiche Genehmigung kann einen Präzedenzfall schaffen, der es ermöglicht, vergleichbare Interventionen auch an anderen Orten durchzuführen, woraus sich ein transformativer Verstetigungseffekt ergeben kann. Positive Effekte der Schutzvorkehrungen liegen darin, dass die in den Beschwerden und der öffentlichen Diskussion enthaltenen Informationen als Daten genutzt werden können, die dazu beitragen, die mit der Intervention verbundenen Forschungsfragen zu beantworten. Werden diese analytisch ausgewertet, können sich darüber hinaus Hinweise ergeben, welche Probleme auftreten und welche Aspekte gut funktionieren. Dies kann im Sinne des „rekursiven Lernens“ (vgl. Groß et al. 2005) dazu genutzt werden, die mit der Intervention untersuchte Maßnahme zu verbessern und die Akzeptanz zu steigern. Ein weiterer positiver Effekt ergibt sich aus dem Austausch mit den Politiker(inne)n, da lokales Wissen erschlossen werden kann, das erlaubt, die Intervention noch besser auf lokale Gegebenheiten anzupassen. Bei inhaltlicher Zustimmung können die Politiker(innen) zudem als Multiplikator(inn)en für eine positive Haltung gegenüber der Intervention dienen. 


\subsection{Rechtfertigung für die Involvierung von projektexternen Personen}

Durchführende Akteure und Verantwortliche von öffentlichen Interventionen können in Kritik geraten, weil projektexterne Personen betroffen sind, ohne ihre Zustimmung zur Involvierung in ein Forschungsprojekt gegeben zu haben. Sie exponieren sich sowohl gegenüber den Betroffenen als auch im forschungsethischen Diskurs.

\section{Beispiel BaWü-Lab RNM: „Parklets für Stuttgart“ - Umgang mit Anliegenden}

Die Parklets wurden auf öffentlichen Parkplätzen errichtet, die vor allem durch Anwohner(innen) und Kund(inn)en von Gewerbetreibenden genutzt wurden. Dies führte zu einer kontroversen Diskussion, bei der die Argumente öffentlich ausgetauscht oder als direkte Rückmeldung an das Interventionsteam gerichtet wurden. Neben der zuvor beschriebenen inhaltlichen Auseinandersetzung über Sinn und Ziele der Intervention meldeten sich auch unmittelbar Betroffene zu Wort. Vor allem Anwohner(innen) beschwerten sich, die sich von den Parklets zum Beispiel durch Lärm belästigt sahen oder denen ,ihr Parkplatz' (den sie gewohnheitsmäßig für sich beanspruchten, obwohl es sich um öffentliche Flächen handelt) abhandengekommen war. Anliegende Gewerbetreibende hatten Sorge, dass aufgrund des Parkplatzmangels Kund(inn)en ausbleiben könnten. Ein anliegender Gewerbetreibender beauftragte aus diesem Grund sogar einen Anwalt, um die Rechtmäßigkeit der Genehmigung zu prüfen, da er negative Auswirkungen auf sein Geschäft fürchtete. Das Interventionsteam und die genehmigende Verwaltung sahen sich also auch hier durch Kritik und Beschwerden exponiert, allerdings bewegte sich diese Kritik nicht mehr auf der Ebene der Forschungsfrage, sondern auf der Ebene der persönlichen Betroffenheit.

Um etwaige Gefährdungen durch die Parklets auszuschließen und mögliche Störungen von Anliegenden auf ein vertretbares Maß zu beschränken, wurden als Schutzvorkehrung mit der Genehmigung entsprechende Auflagen verbunden und bei der Gestaltung der Parklets berücksichtigt. Projektexterne waren nur in einem solchen Maß Veränderungen ausgesetzt, wie es mit herkömmlichen Gestaltungsmaßnahmen vergleichbar war. Die Intervention wurde somit durch das Interventionsteam und die genehmigende Verwaltung als vertretbar eingestuft.

Der größte Teil der öffentlichen Diskussion lief über soziale Medien und die lokale Presse, worauf das Interventionsteam nur sehr begrenzt Einfluss nehmen konnte. Um die Anliegenden nicht mit der Veränderung zu überraschen, wurde vorbeugend mittels eines Einwurfschreibens und Beschilderungen an den Parkplätzen über die bevorstehende Intervention informiert. Um auch einen persönlichen Austausch zu ermöglichen, wurden verschiedene Diskussions- und Informationsformate, wie eine Podiumsdiskussion oder ein Eröffnungsrundgang, angeboten. Es zeigte sich jedoch, dass diese Angebote von den Betroffenen kaum wahrgenommen wurden. Stattdessen richteten sie ihre Beschwerden vor allem direkt an die Verwaltung, die daraufhin vom Interventionsteam und den Pat(inn)en der Parklets konkrete Nachbesserungen wie das Einhalten der Sperrstunde auf den Parklets verlangte. 
Aufgrund der Durchführung von Interventionen im öffentlichen Raum können projektexterne Personen betroffen sein, die nicht vorher um ihre Zustimmung zum Einbezug in das Forschungsprojekt gebeten werden können. So kamen im Beispiel „Parklets für Stuttgart“ Anwohner(innen) und Kund(inn)en mit den Parklets in Berührung. Dies bedeutet nicht, dass sich aus öffentlichen Interventionen eine nachteilige Auswirkung auf die Betroffenen ergeben muss, aber sie verursachen zumindest eine Veränderung der üblichen Umstände. Die ungewohnte Situation sowie gegebenenfalls nachteilige Auswirkungen können bei den Betroffenen negative Gefühle wie Irritation, Unmut oder Überforderung hervorrufen. Hierbei kann auch die persönliche Wahrnehmung einer Veränderung als individueller Nachteil eine wichtige Rolle spielen.

Infolgedessen kann es in erster Linie zu einer Exposition der die Intervention durchführenden und genehmigenden Akteure (aus Wissenschaft und Praxis) gegenüber den Betroffenen kommen, wenn diese ihre negativen Gefühle sowie persönlichen Wahrnehmungen und Standpunkte an sie adressieren. Hierbei liegt nicht die sachliche Kritik oder Beschwerde im Vordergrund (dazu s. Abschnitt 4.1), sondern die (als nachteilig empfundenen) Veränderungen der persönlichen Lebensumstände sowie die Infragestellung, warum man in ein Forschungsprojekt involviert werde.

In zweiter Linie exponieren sich auch die verantwortlichen Akteure wie Interventions- und Projektleitende, da sie das grundsätzliche Vorgehen im Forschungsprojekt, die konkrete Intervention sowie die damit einhergehenden Auswirkungen im Diskurs mit den Betroffenen rechtfertigen müssen.

Verantwortliche Wissenschaftler(innen) exponieren sich darüber hinaus gegenüber Wissenschaft und Öffentlichkeit. Laut forschungsethischem Konsens werden Personen üblicherweise über Inhalte, Methoden und Ziele eines Forschungsprojekts informiert, dann wird ihre Zustimmung zur Beteiligung eingeholt, und erst danach werden sie in das Projekt involviert. Wenn dies, wie bei Interventionen im öffentlichen Raum, nicht möglich ist, sehen sich die verantwortlichen Wissenschaftler(innen) mit einem forschungsethischen Konflikt konfrontiert. Vor allem dann, wenn Beteiligte Auswirkungen als negativ einstufen, können nicht nur das konkrete Forschungsprojekt, sondern transformative Forschungsformate per se in Kritik geraten. Negative Folgen für das einzelne Forschungsprojekt bestehen darin, dass heftige Kritik von Betroffenen oder Fachkolleg(inn)en schwerwiegende Auswirkungen auf die Durchführung des Projekts haben kann (z. B. Abbruch der Intervention, Verhinderung von darauf aufbauenden Schritten, Teilprojekten sowie Verstetigung) oder auf dessen Rezeption (z. B. Diskreditierung der Ergebnisse). 
Als vorbeugende Schutzvorkehrung sollten daher Reflexionsrunden eingebaut werden, bei denen geplante Interventionen kritisch geprüft und gegebenenfalls angepasst werden. Die Zumutbarkeit von Auswirkungen von Interventionen auf projektexterne Personen sollte besonders sorgfältig überprüft werden. Die Genehmigung durch die öffentliche Verwaltung stellt hierbei eine gute Prüfinstanz dar. Des Weiteren sollten die beteiligten Akteure vor dem Schritt in die Öffentlichkeit im Rahmen einer Reflexionsrunde projektintern über das Vorhaben und allfällige Kritikpunkte reflektieren und sich gegenseitig austauschen, um bereits vor der Umsetzung der Intervention einen Prozess des bewussten Umgangs mit ablehnenden und emotionalen Haltungen $\mathrm{zu}$ starten. Solche InformationsReflexionsrunden mögen aufgrund des Umfangs der Vorbereitungen für die Durchführung sowie dem Enthusiasmus, die Intervention nach dem oft langwierigen und umständlichen Vorbereitungsprozess (Planung, Genehmigungen, Finanzierung etc.) endlich umsetzen zu können, eine zeitliche und organisatorische Herausforderung sein. Sie ermöglichen aber, gut vorbereitet zu sein für klärende, sachliche Gespräche und Schriftverkehr, wenn während der Durchführung der Intervention oder im Nachgang ablehnende und/oder emotionale Reaktionen sowie Unverständnis von Betroffenen an die Adresse der ausführenden Akteure gerichtet werden. Es empfiehlt sich, sich bewusst darauf vorzubereiten, dass voraussichtlich nicht alle projektexternen Personen mit ihrer Involvierung in das Projekt einverstanden sein werden (bezüglich Informationsangebot vor Ort und Beschwerdemanagement s. Abschnitt 4.1). Des Weiteren sollte als Schutzvorkehrung für die Wissenschaftler(innen) daran gearbeitet werden, in der Bevölkerung wie in der Wissenschaft ein Bewusstsein dafür zu schaffen, dass der transformative Anspruch von Forschung je nach Thema und Ziel ein Wirken über die Projektgrenzen hinaus erfordern und damit zum Beispiel die Involvierung von projektexternen Personen nötig machen kann.

Aus der beschriebenen Exposition geht kein direkter Mehrwert hervor, der über den zuvor beschriebenen (s. Abschnitt 4.1), mit der Durchführung der Intervention zusammenhängenden, hinausgeht. Ein positiver Effekt aus den Schutzvorkehrungen kann für das Forschungsprojekt durch die vorausschauende Reflexion sowie den kritischen Diskurs mit projektexternen Personen entstehen, wenn diese konstruktiv zur Verbesserung der Intervention und somit zur Qualitätssteigerung im Forschungsprojekt genutzt werden. Des Weiteren kann ein gut vorbereiteter Dialog mit projektexternen Betroffenen zum transformativen Prozess beitragen, wenn es in dessen Rahmen gelingt, einen Reflexionsprozess bei den Gesprächspartner(inne)n anzustoßen und somit die Anliegen des Forschungsprojekts in die Bevölkerung hineinzutragen. 


\section{Expositionen durch die Integration von Lehre in das Forschungsprojekt}

Lehre ist neben der Forschung - idealtypisch verstanden als Einheit - die zentrale Aufgabe von Universitäten und Hochschulen; entsprechend ist sie auch für transdisziplinäre und transformative Forschungsprojekte zumindest programmatisch, oft aber auch praktisch bedeutsam. Dies kann eine große Bandbreite an Lehr- und Lernformaten betreffen, von eher fachspezifischen bis hin zu fächerübergreifenden Veranstaltungen mit Praxisakteuren. In diesen findet Exposition von Studierenden, Praxisakteuren und Lehrenden statt, sowohl gegenüber der Öffentlichkeit als auch untereinander. Die nachfolgenden Ausführungen beziehen sich auf Lehrerfahrungen aus dem Energielabor Tübingen - Potenziale, Partizipation, Perspektiven (,BaWü-Lab Energielabor Tübingen“) einschließlich der mit diesem BaWü-Lab assoziierten Lehr- und Lernaktivitäten (vgl. Albiez et al. 2018). ${ }^{16}$ Die Lehrenden waren gleichzeitig als Wissenschaftler(innen) im BaWü-Lab Energielabor Tübingen tätig. Anhand dieser Erfahrungen werden die Expositionen beleuchtet, die sich ergeben durch Erwartungen im Hinblick auf Strukturen der Wissensvermittlung und das Verhältnis von Empirie und normativer Orientierung sowie durch die Mitwirkung an transdisziplinären und transformativen Lehrveranstaltungen.

\subsection{Erwartungen im Hinblick auf Strukturen der Wissensvermittlung und das Verhältnis von Empirie und normativer Orientierung}

Lehrende exponieren sich durch das transdisziplinäre und transformative Forschungsformat Reallabor. Dies gilt sowohl für den Umgang mit Erwartungen der Studierenden zum vorhandenen Fachwissen der Lehrenden als auch für die Bestimmung des Verhältnisses zwischen ,Fakten', Werten und Normen.

Beispiel BaWü-Lab Energielabor Tübingen: Umgang mit Erwartungen zu wertorientierter Forschung in Lehrveranstaltungen

Neben fachspezifischen Seminaren, die sich beispielsweise an angehende Lehrkräfte im naturwissenschaftlichen Bereich an der Universität Tübingen richteten, wurden einzelne Lehreinheiten für angehende Ingenieur(inn)e(n) an der Universität Stuttgart angeboten. In allen Lehrveranstaltungen wurde bereits zu Beginn auf das Forschungsformat Reallabor und die damit einhergehenden transdisziplinären und transformativen For-

16 Gemeint sind hier Lehrveranstaltungen, die im Zusammenhang mit dem Forschungsprojekt „Wissenschaftliche Bildung und gesellschaftliche Verantwortung“ standen. Hierbei flossen Know-how zu transdisziplinärer Lehre sowie personelle Ressourcen aus dem BaWü-Lab Energielabor Tübingen in die Lehraktivitäten ein. 
schungsansätze eingegangen. Zudem wurde das Verhältnis zu anderen Forschungsansätzen aufgezeigt. Die Lehrenden exponierten sich zunächst durch die Breite des Gegenstands Energiewende. So herrschte bei den Studierenden teilweise die Vorstellung vor, transdisziplinäre Nachhaltigkeitswissenschaftler(innen) verfügten über disziplinäre Fachkenntnis in allen für das Thema Energiewende relevanten Feldern. Sie müssten beispielsweise zugleich Expert(inn)en für die Funktion technischer Anlagen sein, für die sozio-technische Transformation des Mobilitätssektors und für Lebenszyklusanalysen in der Umweltbilanzierung. Des Weiteren exponierten sich die Lehrenden dadurch, dass sie ausdrücklich auf die Verknüpfung von Empirie und Normen hinwiesen. Dies stand im Gegensatz zur Erwartung mancher Studierender, dass Wissenschaft rein evidenz- und faktenbasiert betrieben werden müsse. Um dieses Spannungsfeld zu thematisieren, wurden beispielsweise aktionsorientierte Lehrmethoden wie "Six Thinking Hats" von de Bono (1999) ${ }^{17}$ angepasst und genutzt. Die Studierenden versetzten sich in die Rolle von Projektmanager(inne)n und beschäftigten sich in Übernahme unterschiedlicher Sichtweisen und Diskussionstypen (quantitativ faktenbasiert, emotional, risikobewusst, synergie-/konsensorientiert) mit dem Thema Energiewende. Ziel war es, bei den Studierenden ein Bewusstsein dafür zu fördern, dass im Zuge der praktischen Umsetzung technische und gesellschaftliche Fragestellungen sowie Werte und Normen nicht getrennt voneinander betrachtet werden können. Das Verhältnis von Empirie und normativer Orientierung wurde zudem im Rahmen der Lehrer(innen)bildung aufgegriffen und vor dem Hintergrund des im sogenannten „Beutelsbacher Konsens" beschriebenen ideologischen Überwältigungsverbots ${ }^{18}$ diskutiert. Die Frage war, inwiefern die Vermittlung der Zustimmung zu Zielen einer Nachhaltigen Entwicklung zu einer Überforderung von Schüler(inne)n führen könnte und welche Rolle dabei die Lehrpersonen spielen.

17 „Six Thinking Hats“ ist eine systematische Anleitung zur Perspektivenübernahme: Probleme werden von sechs verschiedenen Blickwinkeln aus betrachtet und beschrieben, was insgesamt dazu beiträgt, sie besser zu analysieren und in der Folge möglichst gut zu lösen.

18 Der Beutelsbacher Konsens wurde 1976 auf einer Tagung der „Landeszentrale für politische Bildung Baden-Württemberg“ erarbeitet und stellt heute einen anerkannten Standard Politischer Bildung an allen Schulen dar, der auch außerhalb Deutschlands in vielen Ländern beachtet wird (s. a. https://www.lpb-bw.de/beutelsbacher-konsens. html, zugegriffen am 19.05.2019). Er setzt sich zusammen aus den drei Prinzipien „1. Überwältigungsverbot ... 2. Was in Wissenschaft und Politik kontrovers ist, muss auch im Unterricht kontrovers erscheinen ... 3. Der Schüler muss in die Lage versetzt werden, eine politische Situation und seine eigene Interessenlage zu analysieren“ (Wehling 1977, S. 179f.). Das Überwältigungsverbot lautet wie folgt: „Es ist nicht erlaubt, den Schüler - mit welchen Mitteln auch immer - im Sinne erwünschter Meinungen zu überrumpeln und damit an der, Gewinnung eines selbständigen Urteils` zu hindern. Hier genau verläuft nämlich die Grenze zwischen Politischer Bildung und Indoktrination. Indoktrination aber ist unvereinbar mit der Rolle des Lehrers in einer demokratischen Gesellschaft und der - rundum akzeptierten - Zielvorstellung von der Mündigkeit des Schülers“ (Wehling 1977, S. 179f.). 
Vorweg sei angemerkt, dass die im Kontext der Lehre handelnden Personen sich zwar spezifische Rollen zuschreiben können, diese jedoch von der Rollenerwartung der eingebundenen Akteure abweichen können. Zudem können sich die Rollenzuschreibungen während des Prozesses verändern (s. Beecroft 2019 in diesem Band).

Die sich im Kontext der Lehre ergebenden Expositionen von Lehrenden sind daher insbesondere von Rollenerwartungen v. a. seitens der Lernenden geprägt, weniger von der selbstständigen Zuschreibung durch die Lehrenden selbst. Dadurch können Stereotype an Bedeutung gewinnen, etwa bei der Exposition der Lehrenden durch das Forschungsformat. Die Offenheit für Themen und Ergebnisse sowie eine gesamtheitliche Herangehensweise können den Eindruck verstärken, die Lehrenden müssten Fachexpert(inn)en in (möglichst) vielen Disziplinen sein. Als weitere Exposition für die Lehrenden ist die bereits angesprochene Zusammenführung von empirischen und normativen Aspekten zu sehen (vgl. Potthast 2015). Diese entsteht, weil bei Lernenden oft die Vorstellung vorherrscht, wissenschaftliche Arbeit müsse per se unabhängig oder ,neutral' sein, was in Spannung geraten kann zu einer explizit normen- und wertorientierten transdisziplinären und transformativen Arbeit z. B. im Kontext von Nachhaltiger Entwicklung. Diese Erwartungen können zur Überforderung der Lehrenden führen.

Eine Schutzvorkehrung für die Lehrenden ist die Transparenz des Lehr- ebenso wie des Forschungsformats gegenüber den Studierenden. Bereits zu Beginn einer entsprechenden Lehraktivität kann den Studierenden dargelegt werden, welche Fragestellungen in einem transdisziplinären und transformativen Projekt wie beforscht werden. In diesem Prozess kann auch auf die Besonderheit von transdisziplinärer Forschung als Integrationsforschung eingegangen werden, und dies kann mit den fachlichen Hintergründen der Studierenden verglichen werden. Zudem kann ausdrücklich auf den normativen Rahmen aufmerksam gemacht werden. Etwaigen ,Neutralitätserwartungen' an Wissenschaft seitens der Studierenden kann mit Übungssituationen zum Umgang mit explizit normativen Settings begegnet werden (vgl. Beispiel „Methode der Six Thinking Hats“).

Der Mehrwert der vorliegenden Exposition liegt in der Vorbereitung der Studierenden für die anschließende transdisziplinäre Praxis. Normative Perspektiven spielen hier durchwegs eine wichtige Rolle, dies gilt für die gemeinsame Initiierung der Forschungsfrage ebenso wie für die Erarbeitung neuer Wissensbestände wie auch für die Integration der Ergebnisse in Wissenschaft und Gesellschaft. Darüber hinaus können Studierende für den Umgang mit Werten und Normen, die sich durch die Verknüpfung von Forschung mit gesellschaftlichen Anliegen ergeben, in einem geschützten Umfeld geschult werden. Die Lehrenden wiederum können die Erfahrungen aus solchen Veranstaltungen zur Weiterentwicklung von Lehrkonzepten nutzen, sei es thematisch, wie zum Beispiel die Ausgestaltung des Konzepts der Bildung für Nachhaltige Entwicklung, sei es von solchen, die 
sich generell auf transdisziplinäre sowie transformative Lehre beziehen. Als positiven Nebeneffekt können die Studierenden Einblicke in potenzielle Berufsfelder erhalten. Zudem können die Studierenden lernen, ihre eigene Fachdisziplin und ihre Kompetenzen zu reflektieren.

\subsection{Mitwirkung an transdisziplinären und transformativen Lehrveranstaltungen}

Expositionen können entstehen, wenn Studierende, Praxisakteure und Lehrende gemeinsam transdisziplinäre und transformative Lehraktivitäten mit einem starken Praxisbezug gestalten, beispielsweise im Rahmen von Projektseminaren. Expositionen ergeben sich hier vor allem durch den starken Interventionscharakter, das Betätigungsfeld des Praxisakteurs sowie durch unterschiedliche Rollenerwartungen der Akteure.

\section{Beispiel BaWü-Lab Energielabor Tübingen: Durchführung transdisziplinärer und trans- formativer Lehraktivitäten im Kontext des BaWü-Labs}

Im Kontext des BaWü-Labs Energielabor Tübingen wurden in Verbindung mit dem Forschungsprojekt „Wissenschaftliche Bildung und gesellschaftliche Verantwortung" überfachliche Veranstaltungen mit unterschiedlicher thematischer Ausrichtung im Rahmen des überfachlichen Nachhaltigkeitszertifikats „Studium Oecologicum“ durchgeführt.

Im Zuge von Lehrveranstaltungen mit starkem Projektcharakter exponierten sich die Studierenden beispielsweise durch Interventionen auf dem Campus. Hiervon waren auch rechtliche Fragestellungen betroffen. So musste geklärt werden, ob und unter welchen Bedingungen von den Studierenden eine ,Blumenwiese' aus gebrauchten Pappbechern vor der Bibliothek aufgestellt werden durfte. Dabei waren im Vorfeld Abstimmungen mit dem Eigentümer sowie dem Betreiber notwendig. Beispielsweise musste gewährleistet werden, dass zur Schonung der Bäume naturschutzrechtliche Vorgaben zum Wurzelwerk eingehalten und Fragen der späteren Entsorgung geklärt wurden. In einem anderen Fall musste außerdem sichergestellt werden, dass eine öffentliche Kunstperformance vor Gebäuden nicht gegen den Brandschutz oder ähnliche Bestimmungen verstieß. Neben der beabsichtigten Intervention gegenüber der Öffentlichkeit rief die Aktion auch einzelne für diese Gebäude zuständige Mitarbeitende auf den Plan. Aufgrund der Vorgespräche mit den Studierenden zur Rechtslage sowie zur Einhaltung der notwendigen Vorschriften konnten die Studierenden die Situation vor Ort letztendlich selbstständig bewältigen.

Des Weiteren exponierten sich Praxisakteure als Ansprechpersonen für die Studierenden. Auf der operativen Ebene musste beispielsweise geklärt werden, in welchem Umfang Einzelpersonen aus der Praxis den Studierenden während den Arbeitsphasen zur Verfügung standen und welche Erwartungen erfüllt werden konnten. Nicht zuletzt exponierten sich die Praxisakteure in den Veranstaltungen durch ihre Betätigungsfelder 
außerhalb der Lehrveranstaltung. Beispielsweise nutzten manche Studierende die Gelegenheit, um mit dem Verantwortlichen der Cafeterien intensiv über deren Geschäftspraxis zu diskutieren. Dies bezog sich beispielsweise auf das Angebot an veganen Speisen oder den Umgang mit Lebensmittelabfällen.

Studierende können sich in Form von Projektseminaren mit starkem Praxisbezug auf vielfältige Weise exponieren, beispielsweise gegenüber den Praxisakteuren und gegebenenfalls der Öffentlichkeit, wenn sie Aktivitäten wie Befragungen oder Interventionen durchführen oder wenn sie Forschungsergebnisse präsentieren (und je nachdem auch das übergeordnete Forschungsprojekt repräsentieren). Die Rolle von öffentlichen Interventionen wurde im vorangegangenen Kapitel bereits erläutert, soll vor dem Hintergrund der Lehre jedoch noch einmal aufgegriffen werden, da ordnungsrechtliche oder Haftungsfragen nicht zum Tagesgeschäft von Lehrenden zählen, Studierende aber, die im öffentlichen Raum agieren und sich exponieren, dabei einen rechtssicheren Rahmen benötigen.

Des Weiteren exponieren sich Lehrende in ihrer Funktion als Hauptverantwortliche für die Lehrveranstaltung als auch als transdisziplinäre und transformative Wissenschaftler(innen) gegenüber Studierenden und Praxisakteuren.

Außerdem kann Exposition im Fall von Rollenerwartungen, die an die Praxisakteure gerichtet sind, auftreten. Sie können eher operative Dinge betreffen, wie nicht erfüllbare Erwartungen seitens der Studierenden, was zeitliche Ressourcen der Praxisakteure angeht, es können aber auch sehr grundsätzlich Dinge betroffen sein, wenn es etwa um Eingriffe in und Erwartungen an die Arbeit von Praxisakteuren geht. Praxisakteure exponieren sich also möglicherweise während der Veranstaltungen durch ihr Betätigungsfeld.

Eine Schutzvorkehrung für die Studierenden können Lehrende dadurch schaffen, dass sowohl im Vorfeld als auch im Prozess rechtliche Fragestellungen mit den Verantwortlichen geklärt werden. Dies sind hier beispielsweise Gespräche mit Eigentümer(inne)n, Hausmeister(inne)n von Gebäuden oder dem Ordnungsamt. Zudem können in diesem Rahmen die Studierenden für rechtliche Belange sensibilisiert werden. Der zeitliche Aufwand, der für die Klärung rechtlicher Sachverhalte gegebenenfalls benötigt wird, ist dann bereits während der Konzeptionsphase der Lehrveranstaltung zu berücksichtigen. Eine Schutzvorkehrung für Lehrende und Studierende stellt die bewusste Auswahl der Praxisakteure vor der eigentlichen Veranstaltung dar. Die Lehrenden sollten sich im Vorfeld Fragen stellen wie: Welche Erfahrungen mit Expositionsbezug haben wir bereits mit den Praxisakteuren in der Vergangenheit gemacht? Wurden gemeinsame öffentliche Veranstaltungen durchgeführt? Welche positiven oder negativen Positionen haben die infrage kommenden Akteure gegenüber dem Forschungsprojekt? Eine Schutzvorkehrung für die Praxisakteure stellt deren Einbindung während der Konzeption der Lehrveranstaltung dar. Die Vorgespräche können im Vorfeld und 
im kleinen Rahmen ohne Studierende stattfinden. Hier ist zu klären, zu welchen Zeiten die Praxisakteure an der Lehrveranstaltung teilnehmen können, und in welchen Phasen bei Bedarf während der Veranstaltung mit ihnen über den laufenden Prozess diskutiert wird, etwa um unerwünschte Überraschungen gegen Ende zu vermeiden. Die Einbindung von Praxisakteuren in die Konzeption kann auch dazu beitragen, Zielsetzung, Interessen und Erwartungen dieser Akteure bereits im Vorfeld zu diskutieren. Dies ist vor allem dann relevant als Schutzvorkehrung, wenn die Studierenden öffentliche Interventionen realisieren, die die Praxisakteure direkt betreffen. Was die Exposition der Praxisakteure durch deren Betätigungsfeld angeht, ist es Aufgabe der Lehrenden, während der Veranstaltung für Schutz zu sorgen, kritische Konstellationen zu erkennen, zu moderieren und wenn nötig in Diskussionen einzugreifen. Um sich hierauf vorzubereiten, können die Lehrenden bereits bei den Vorgesprächen mit den Praxisakteuren auf mögliche Rollenbilder und Kritikpunkte eingehen.

Ein Mehrwert, den transdisziplinäre und transformative Lehr- und Lernformate haben, besteht in der Durchführung von Interventionen, da etwa die Einbindung weiterer Akteure möglich wird. Ein weiterer Mehrwert liegt in der Erschließung neuer gesellschaftlich relevanter Themen für die Forschung. Zum Ersten können Studierende zusätzliche Kompetenzen und Arbeitskraft einbringen, zum Zweiten haben diese aufgrund ihrer persönlichen Rolle möglicherweise Zugang zu weiteren Akteuren, was den Handlungsrahmen erweitert. Lehrveranstaltungen können so auch Testfelder für die weitere transdisziplinäre und transformative Arbeit sein: Formen der Intervention können ausprobiert oder Hinweise zu neuen Handlungsfeldern gewonnen werden, die im späteren Verlauf eines Forschungsprojekts weiterverfolgt werden können. Ein möglicher positiver Effekt kann zudem sein, dass Studierende für die Komplexität sozio-technischer Themen sensibilisiert werden und institutionelle sowie rechtliche Belange mitdenken.

Durch die entsprechenden Schutzvorkehrungen kann das Vertrauensverhältnis zwischen den unterschiedlichen Akteuren verbessert werden. Dies ermöglicht es beispielsweise den Studierenden, tiefere Einblicke in das Betätigungsfeld der Praxisakteure zu erhalten. Als weiterer positiver Effekt für die Praxisakteure kann die Unterstützung für deren Vorhaben und Interessen gesehen werden. Hier ergeben sich auch Querbezüge zum Lehrformat Service Learning ${ }^{19}$, bei welchem Studierende gemeinwohlorientierte Akteure bei deren Arbeit unterstützen und sich durch die Akteurskonstellation ähnliche Fragen zu Exposition und Schutzvorkehrung ergeben können (vgl. Albiez und Potthast 2018).

19 ,Service Learning - Lernen durch Engagement ist eine Unterrichtsmethode, die gesellschaftliches Engagement von Schülerinnen und Schülern mit fachlichem Lernen im Unterricht verbindet" (Seifert und Zentner 2010, S. 3). In Deutschland wurde die Methode zunächst von Schulen adaptiert und wird inzwischen auch an verschiedenen Universitäten vermehrt angeboten. 


\section{Schlussreflexionen}

In den vorausgegangenen Kapiteln wurde anhand von Beispielen aus verschiedenen BaWü-Labs aufgezeigt, dass sich Akteure im Rahmen von transdisziplinären (und transformativen) Forschungsprojekten in für sie ungewohnten Situationen, ausgelöst durch ungewohnte Aufmerksamkeit und/oder Tätigkeiten, wiederfinden können. Für diese Situationen wurde der Begriff der Exposition eingeführt. Verschiedene Formen der Exposition wurden dargestellt. So wurde zunächst auf Expositionen eingegangen, die aus dem Forschungsdesign und dem Forschungsgegenstand in BaWü-Labs entstanden waren (Kapitel 2), gefolgt von Expositionen, die aus einer verstärkten Öffentlichkeitsarbeit resultierten (Kapitel 3). Daran schlossen sich Expositionen an, deren Ursachen in der Durchführung von Öffentlichen Interventionen lagen (Kapitel 4), um abschließend jene Exposition zu beleuchten, die sich durch die Integration von Lehre in das Forschungsprojekt ergaben (Kapitel 5).

Wie in Kapitel 1 eingeführt, stellt die Reflexion über Expositionen einen anwendungsorientierten, ergänzenden Zugang zu etablierten Ansätzen der Forschung über transdisziplinäre und transformative Forschung dar. Der Schwerpunkt des Beitrags liegt in der Darstellung von verschiedenen Expositionen und möglichen Strategien, mit diesen umzugehen. Im Folgenden wird dargelegt, wie der Zugang über Expositionen in transdisziplinären (und transformativen) Forschungsprojekten zum Tragen kommen kann. Des Weiteren werden Möglichkeiten zur Weiterentwicklung dieses Zugangs aufgezeigt und punktuelle Verknüpfungen zu etablierten Forschungsansätze herausgearbeitet.

\subsection{Expositionen bei Planung und Durchführung eines transdisziplinären und transformativen Forschungsprojekts: die Anwendung}

Den einzelnen Akteuren bietet die Reflexion über Expositionen eine Hilfestellung, um sich auf ihr Mitwirken in einem Forschungsprojekt vorzubereiten. Dazu empfiehlt es sich, die in Kapitel 1 genannten Fragen von einem persönlichen Standpunkt und der jeweiligen spezifischen Situation aus betrachtet anzuwenden. Insbesondere eine bewusste Auseinandersetzung mit bevorstehenden, ungewohnten Tätigkeiten (im Gegensatz zur üblichen Arbeitsroutine) und mit ungewohnter Aufmerksamkeit kann helfen, Expositionen im Vorhinein zu erkennen und sich entsprechend darauf vorzubereiten. Das frühzeitige Erkennen des Mehrwerts von Expositionen und positiven Effekten von Schutzvorkehrungen kann ein Motivationsfaktor für die Arbeit im Forschungsprojekt sein. Beispielsweise kann die Erkenntnis, dass eine zusätzlich notwendige Schulung längerfristig eine (vielleicht schon seit langem angestrebte) Zusatzqualifikation bringt, die Einsatzbereitschaft 
für das Forschungsprojekt fördern. Aber auch während der Projektlaufzeit oder im Nachgang kann eine solche Reflexion helfen, den individuellen Lernprozess zu unterstützen.

Auf der Ebene von Projektleitung und -management hilft eine vorausschauende Reflexion anhand der in Kapitel 1 dargelegten Fragen zu Expositionen, um kritische Situationen, die durch Expositionen ausgelöst werden könnten, zu antizipieren, die Ursachen zu analysieren und sowohl in der Konzeptionsphase als auch bei Zwischenevaluationen zielgerichtete Strategien zu entwickeln. Dabei sollte der Aspekt der Exposition von Seiten aller Akteursgruppen beleuchtet werden, damit alle notwendigen Kompetenzen im Projektteam vertreten sind - spätestens nach Durchführung der Schutzvorkehrungen, die der Aneignung von Kompetenzen dienen. So können die Aufgaben entsprechend den Kompetenzen verteilt, Schutzvorkehrungen mit zeitlichem Vorlauf rechtzeitig in die Wege geleitet, ähnliche Schutzvorkehrungen gebündelt und ausreichend finanzielle Ressourcen für Schutzvorkehrungen wie Schulungen oder das ,Einkaufen' spezieller Expertise oder die Integration von weiteren Akteuren ins Team vorgesehen werden. Ebenso können Mehrwert und positive Effekte in der Projektplanung bewusst berücksichtigt werden. Wichtig ist es bei der Reflexion über Expositionen aus Sicht von Projektleitung und -management, die beteiligten Akteure auch als Individuen zu berücksichtigen, da manche Schutzvorkehrungen vom individuellen Wissens- und Erfahrungsschatz abhängen. So kann beispielsweise eine Weiterbildung im Bereich Medien zur Durchführung eines medialen Aufrufs für eine(n) spezielle(n) Wissenschaftler(in) überflüssig sein, wenn diese(r) bereits im Rahmen von vorhergehenden Projekten Medienkompetenzen erworben hat.

Bezüglich der zeitlichen Komponente empfehlen die Autor(inn)en, in transdisziplinären (und transformativen) Forschungsprojekten eine vorausschauende Reflexion über Expositionen zu Projektbeginn im Anschluss an eine Akteursanalyse durchzuführen. Diese hat zum Ziel, Akteure sowie deren Interessen und Konflikte zu identifizieren und entsprechende Strategien zu entwickeln (zu Akteursanalyse bei Reallaboren vgl. Eckart et al. 2018). Die gewonnenen Erkenntnisse zu Fragen wie etwa, welche Akteure im Projekt beteiligt sind, welche Relevanz und Gewichtung im Forschungsprojekt sie jeweils einnehmen oder wo mögliche Konfliktlinien verlaufen, können beim Erkennen von Expositionen und beim Abwägen von Schutzvorkehrungen hilfreich sein. Eine Reflexion über mögliche auftretende Expositionen kann auch parallel zur Rollenklärung und -festlegung stattfinden. Eine solche wird von Jahn (2008) als notwendiger Teil interdisziplinärer Integration angesehen. Weiter empfiehlt es sich, die Reflexion im Laufe eines Forschungsprojekts zu wiederholen, um Schutzvorkehrungen anzupassen. Durch die Analyse von antizipierten und eingetretenen Expositionen, deren Mehrwert und den positiven Effekten der Schutzvorkehrungen können zudem Lerneffekte im Projekt erhöht werden. 


\subsection{Expositionen im Kontext transdisziplinärer und transformativer Forschung: ein Ausblick}

Wie im vorhergehenden Abschnitt dargelegt, gibt die Reflexion über Expositionen dem Projektmanagement ein Mittel an die Hand, um kritische Situationen, verursacht durch ungewohnte Tätigkeiten und/oder ungewohnte Aufmerksamkeit, antizipieren und entsprechende Schutzvorkehrungen entwickeln zu können. Eine mögliche Weiterentwicklung des im vorliegenden Beitrag dargelegten Zugangs liegt daher in einer Untersuchung, bezogen auf welche Managementansätze für transdisziplinäre (und transformative) Forschungsprojekte die Reflexion über Expositionen in welcher Art und Weise einen Beitrag leisten kann zur Weiterentwicklung beziehungweise zu welchen Managementansätzen diese Reflexion eine sinnvolle Ergänzung darstellt. Als ein mögliches Beispiel seien hier Schophaus et al. (2004) mit ihrem Ansatz des transdisziplinären Kooperationsmanagements genannt. Sie legen einen Schwerpunkt auf den Kooperationscharakter also die Schnittstellen zwischen Akteuren - und geben Handreichungen, wie mit diesen umgegangen werden kann. Hierbei könnte die Betrachtung von Expositionen neue Erkenntnisse bringen, da sie beleuchtet, wo und wie an den Schnittstellen durch ungewohnte Tätigkeiten und/oder Aufmerksamkeit besondere Herausforderungen ausgelöst werden.

Dass eine vertiefte Auseinandersetzung mit dem Thema der Exposition auf der Ebene des Forschungsprojektmanagements zu einem produktiven Forschungsverlauf beitragen kann, lassen auch Erkenntnisse aus der Analyse von Prozessabläufen schließen. So erläutert Jahn (2008), dass das Ausbalancieren von Spannungen entscheidend den Ertrag eines Forschungsprojekts beeinflusst. Ausgehend davon, dass Expositionen Teil der angesprochenen Spannungen sind, bedeutet der Umkehrschluss, dass der bewusste Umgang mit Expositionen für die Ausbalancierung von Spannungen und somit den Projektertrag wichtig sein kann.

Es zeigen sich zudem Anknüpfungspunkte an Konstellationsfragen, wie sie Defila et al. formulieren: „Praxispartner und Forschende bilden eine mehr oder weniger geschlossene Gesellschaft" (2016, S. 58). Hier wäre die Frage nach Expositionen einerseits so zu verstehen, dass ausgehend davon, dass ein transdisziplinäres Team von außen als hermetisch geschlossener Expert(inn)enzirkel wahrgenommen werden könnte, geprüft wird, welche Exposition dies für wen verursachen kann. Andererseits wäre zu fragen, ob nicht eine solche Geschlossenheit auch Schutz vor Exposition Einzelner bietet.

In diesem Beitrag steht der Unterschied zwischen transdisziplinärer und transformativer Forschung nicht im Vordergrund der Expositions-Analysen. Es wäre genauer zu untersuchen, ob sich Forschende insgesamt weniger exponieren, die vermeintlich ,nur' ein Praxisproblem in transdisziplinären Teams bearbeiten, als diejenigen, die explizit und programmatisch damit auch Wissenschaft und Gesell- 
schaft maßgeblich verändern, also transformieren wollen. Für die Frage des Auftretens in der Öffentlichkeit ist diese Unterscheidung möglicherweise nicht besonders relevant, aber innerhalb der Wissenschaftsgemeinschaft hat die Diskussion um transformative Forschung erhebliche Wellen geschlagen (initiiert durch Strohschneider 2014), so dass hier Exposition zu erwarten ist. Dies zeigt sich ansatzweise in der Exposition, die durch transdisziplinäre (und transformative) Lehre entsteht (s. Kapitel 5).

Die Reflexion über Expositionen kann auch als Teilaspekt in die Evaluierung von transdisziplinären (und transformativen) Forschungsprojekten und die Qualitätssicherung für solche Projekte integriert werden. Im Diskurs über Qualitätskriterien und Evaluierung interdisziplinärer, transdisziplinärer und transformativer Forschungsprojekte gibt es Vertreter(innen), die die Relevanz von Lernen und Coachen im Gegensatz zu einem reinen beurteilenden Zugang betonen. So streicht Klein (2006) den Wert eines ,coaching model“" hervor (S. 78), und Bergmann et al. (2005) schlagen eine diskursive, formative Evaluation vor. In solchen diskursiven und coachenden Evaluationsprozessen kann die Analyse von Expositionen und des Umgangs damit zum Lernprozess beitragen, indem besondere Herausforderungen in Form von Ungewohntem erkannt und geplante, durchgeführte und mögliche Strategien des Umgangs reflektiert werden. Des Weiteren kann die Analyse von Expositionen dazu beitragen, die notwendigen Grundlagen für Lernprozesse im Team zu schaffen, da sie machtbedingte Abhängigkeiten („Wer exponiert sich gegenüber wem?“) aufzeigen kann. Machtgefüge haben laut Jahn et al. (2012) einen wichtigen Einfluss auf den wechselseitigen Lernprozess. Schutzvorkehrungen könnten darüber hinaus zur Abdeckung von Lücken in der Qualitätssicherung dienen, wenn zum Beispiel zusätzliche Fortbildungen gewährleisten, dass die notwendige Expertise im Team gegeben ist. Wichtig scheint eine solche Untersuchung auch in Anbetracht von kritischen Stimmen, die in der Forderung nach umfassender, angleichender Kompetenzerweiterung aller Akteure keine Lösung sehen: „Der Umgang mit vorhandenen Ausgangs-, Qualifikationsund Funktionsunterschieden im Kooperationsprozess erwies sich allerdings [...] als kompliziert. Der Anspruch der gleichberechtigten Kooperation scheint dazu zu verleiten, bei Forschern und Praktikern die gleichen Kompetenzen vorauszusetzen oder den Erwerb derselben zum Ziel zu erheben“" (Prengel et al. 2008, S. 186).

Im vorliegenden Beitrag wurden Expositionen ausschließlich anhand von Beispielen aus BaWü-Labs analysiert und dargelegt. Bei einer Analyse von Expositionen im Rahmen von anderen transdisziplinären (und transformativen) Forschungsformaten müssen deren gegebenenfalls anderen Rahmenbedingungen und Grundvoraussetzungen berücksichtigt werden. Diese können Einfluss auf Ursachen, Auswirkungen und Häufigkeit von Expositionen haben. So spielt zum Beispiel der Fördergeber eine wichtige Rolle in der im vorhergehenden Absatz 
bereits angesprochenen Machtstruktur. Die vorgegebene normative Zielrichtung hat ebenfalls Auswirkungen auf die Ursachen, negativen Folgen und den Mehrwert von Expositionen. Eine mögliche Weiterentwicklung wäre daher eine vergleichende Analyse von Expositionen in verschiedenen transdisziplinären (und transformativen) Forschungsformaten unter Berücksichtigung von Rahmenbedingungen der Förderung. Ziel wäre, systematisches Wissen zu Interdependenzen zwischen Rahmenbedingungen, Ursachen, Auswirkungen und Häufigkeit von Expositionen in transformativen (und transdisziplinären) Forschungsprojekten aufzubauen. Expositionen könnten so systematisch erfasst, analysiert und zu einer Datenbank ausgebaut werden. Eine solche Datenbank könnte dazu beitragen, den Aufbau sogenannter „transformative Literacy“ (Schneidewind 2013) voranzutreiben.

Eine weitere Richtung wäre eine Analyse, ob gewisse Methoden in der transdisziplinären (und transformativen) Forschung mehr Expositionen als andere mit sich bringen und wie verhindert werden kann, dass sich dies negativ auf die Prozessqualität auswirkt: „Dienlicher als eine Debatte über die Neuartigkeit der Methoden, die bei transdisziplinärer (und transformativer) Forschung eingesetzt werden, scheint daher eine Auseinandersetzung mit Fragen wie der, wofür sich welche Methode unter welchen Bedingungen eignet, wie sichergestellt werden kann, dass bei der Umsetzung von Methoden die erforderliche Qualität der Prozesse und Ergebnisse erreicht wird, [...]“" (Defila und Di Giulio 2018c, S. 23). Es wäre zu untersuchen, ob eine Reflexion über die zur Wahl stehenden Methoden anhand von Expositionen hierzu beitragen kann. Dies könnte beispielsweise helfen, eine Methode zu wählen, die im Vergleich zu anderen bei gleichem Ertrag mit weniger Expositionen und somit weniger Aufwand für Schutzvorkehrungen einhergeht.

Schließlich möchten die Autor(inn)en eine Untersuchung der Überlagerung und Akkumulation von Expositionen sowie möglicher Strategien der Entzerrung und Resistenz gegen diese anregen. Der vorliegende Beitrag konzentriert sich darauf, einzelne Expositionen möglichst isoliert darzustellen, um die gesammelten Erfahrungen entsprechend konsequent analysieren zu können und das Thema greifbar zu vermitteln. Wie so oft ist die Realität aber komplexer und es kann zu Überlagerungen oder Wechselwirkungen zwischen verschiedenen Expositionen kommen, welche noch einer zusätzlichen Betrachtung bedürfen. Als Beispiel sei an dieser Stelle an die Darlegung in Abschnitt 2.2 erinnert, in der die Schutzvorkehrung für die Praxisakteure die Exposition für die Wissenschaftler(innen) darstellt. 


\subsection{Schutz in der Exposition, Schutz für die Exposition}

Im vorliegenden Beitrag wurde deutlich, dass sich die Beschäftigung mit Exposition lohnt: Die Erfahrungen der BaWü-Labs haben gezeigt, dass Exposition ein konstitutives Merkmal von transdisziplinärer und transformativer Forschung ist. Gleichzeitig können sich aus den Expositionen direkter Mehrwert und/oder aus den Schutzvorkehrungen positive Effekte ergeben, die sich für das Projekt ausschöpfen lassen. So bestehen, zusammengefasst, der Mehrwert der Expositionen, die sich durch das Forschungsdesign und den Forschungsgegenstand ergeben, sowie die positiven Effekte der dazu passenden Schutzvorkehrungen vor allem in der Entwicklung eines gegenseitigen Verständnisses der Akteure, in der Möglichkeit, verhärtete Konflikte aufzulösen, und in der leichteren Verständlichkeit von Forschung (s. Kapitel 2). Durch Öffentlichkeitsarbeit verursachte Expositionen und die dazu passenden Schutzvorkehrungen wiederum können durch eine breite öffentliche Präsenz und damit einhergehende größere Bekanntheit zur Verstetigung beitragen sowie bei der Gewinnung neuer (und unerwarteter) Akteure hilfreich sein (s. Kapitel 3). Expositionen im Rahmen von öffentlichen Interventionen und die passenden Schutzvorkehrungen können Mehrwert und positive Effekte mit sich bringen, indem sie das Forschungsthema in die Bevölkerung tragen und somit breite transformative Prozesse unterstützen (s. Kapitel 4), jene, die durch die Einbindung von Lehre in das Forschungsprojekt verursacht werden, können Praxiserfahrungen für Studierende, Unterstützung von Praxisakteuren durch Studierende sowie weitere Möglichkeiten zum Experimentieren für das Projekt mit sich bringen (s. Kapitel 5).

Ziel einer vorausschauenden Reflexion über Expositionen ist es, ausreichend Schutz in der Exposition zu bieten, um negativen Auswirkungen von Expositionen zu begegnen und den damit verbundenen Mehrwert nutzbar zu machen. Eine mögliche, grundsätzliche Form von Schutzvorkehrung können der bewusste, offene Umgang mit Fehlern und eine entsprechende projektexterne Kommunikation sein, bei der auch über Ergebnisse berichtet wird, die aus Sicht der Beteiligten nicht wie gewünscht verliefen. Schließlich und endlich ist anzumerken, dass das Forschungsformat Reallabor beziehungsweise die Förderung von transdisziplinären (und transformativen) Projekten per se Schutz für die Forschung und für die beteiligten Personen bietet, weil diese Formate damit von den Mittelgebern legitimiert werden. Eine solche Förderung gemeinsam mit entsprechenden Schutzvorkehrungen bieten also im Allgemeinen und im Speziellen Schutz für die Exposition. 


\section{Dank}

Die Autor(inn)en danken Ludger Eltrop für seinen inhaltlichen Input bei der Erstellung des Beitrags und Fabian Dembski, Jochen Eckart, Thorsten Erl, Monika Gonser, Elke Häußler und Jan Riel für die Diskussion über eine frühere Textfassung im Rahmen des internen Reviews. Besonderer Dank gebührt Thomas Becker (Freies Lastenrad Stuttgart), Sarah Behrens (ABK Stuttgart), Timo Dreher (BUND Neckar-Alb), Frank Gwildis (Sachgebiet Stadtentwicklungsplanung der Stadt Stuttgart), Johannes Heynold (JoHey - Studio für Dialog im Raum), Barbara Lupp (BUND Neckar-Alb), Ina Westheiden (ABK Stuttgart) und einem anonymen Praxispartner von STADT-RAUM-BILDUNG für die Feedbacks aus ihrer Sicht als Praxisakteure. Herzlichen Dank an Matthias Rudolph und Juliane Windbiel für die Recherche und Bereitstellung des Bildmaterials sowie an Constantin Hörburger für die Recherche von Unterlagen (alle drei ABK Stuttgart). Insbesondere möchten die Autor(inn)en auch den beiden anonymen externen Gutachter(inne)n für die konkreten Hinweise zur Verbesserung des Textes danken. Schließlich danken sie den beiden Herausgebenden, Rico Defila und Antonietta Di Giulio, für ihre Rückmeldungen zum Text.

\section{Literatur}

Albiez, M., König, A., \& Potthast, T. (2018). Transdisziplinarität und Bildung für Nachhaltige Entwicklung in der Lehre an der Universität Tübingen: Konzeptionelle Fragen mit Bezug auf Lehraktivitäten des „Energielabors Tübingen“. In W. Leal Filho (Hrsg.), Nachhaltigkeit in der Lehre. Eine Herausforderung für Hochschulen (S. 189-206). Berlin, Heidelberg: Springer Spektrum.

Albiez, M., \& Potthast, T. (2018). Gemeinsam lernen, forschen, engagieren. Alles eins? In: N. Neuber, W. Paravicini \& M. Stein (Hrsg.), Forschendes Lernen. The Wider View. Eine Tagung des Zentrums für Lehrerbildung der Westfälischen Wilhelms-Universität Münster vom 25. bis 27.09.2017 (Schriften zur Allgemeinen Hochschuldidaktik) (S. 139-142). Münster: WTM-Verlag.

Beecroft, R. (2019). Das „Transformative Projektseminar“ - didaktische Ansätze und methodische Umsetzung. In R. Defila \& A. Di Giulio (Hrsg.), Transdisziplinär und transformativ forschen, Band 2. Eine Methodensammlung (S. 293-337). Wiesbaden: Springer VS.

Bergmann, M., Brohmann, B., Hoffmann, E., Loibl, M. C., Rehaag, R., Schramm, E., \& Voss, J.-P. (2005). Qualitätskriterien transdisziplinärer Forschung. Ein Leitfaden für die formative Evaluation von Forschungsprojekten. Frankfurt a. M.: Institut für sozialökologische Forschung. https://www.isoe.de/ftp/evalunet_leitfaden.pdf. Zugegriffen am 15.04.2019.

Bergmann, M., Jahn, T., Knobloch, T., Krohn, W., Pohl, C., \& Schramm, E. (2010). Methoden transdisziplinärer Forschung. Ein Überblick mit Anwendungsbeispielen. Frankfurt a. M., New York: Campus. 
Boix Mansilla, V., Feller, I., \& Gardner, H. (2006). Quality assessment in interdisciplinary research and education. Research evaluation, 15 (1), (S. 69-74). doi: 10.3152/1471544 06781776057.

De Bono, E. (1999). Six thinking hats. 1. Back Bay Paperback Aufl., überarbeitet und aktualisiert. Boston: Back Bay Books.

Defila, R., \& Di Giulio, A. (1999). Transdisziplinarität evaluieren - aber wie? Evaluationskriterien für inter- und transdisziplinäre Forschung. Panorama. Sondernummer 1999. https://forschungsverbundmanagement.net/publikationen/Sondernummer.Pano.1. 99.pdf. Zugegriffen am 15.04.2019.

Defila, R., Di Giulio, A., \& Scheuermann, M. (2006). Forschungsverbundmanagement. Handbuch für die Gestaltung inter- und transdisziplinärer Projekte. Zürich: vdf Hochschulverlag an der ETH Zürich.

Defila, R., Di Giulio, A., \& Schäfer, M. (2016). Hotspots der transdisziplinären Kooperation - Ausgangslagen von besonderer Bedeutung. In R. Defila \& A. Di Giulio (Hrsg.), Transdisziplinär forschen - zwischen Ideal und gelebter Praxis. Hotspots, Geschichten, Wirkungen (S. 27-89). Frankfurt a. M., New York: Campus.

Defila, R., \& Di Giulio, A. (2018a). Transdisziplinarität und Reallabore. In Reallabor für nachhaltige Mobilitätskultur Universität Stuttgart (Hrsg.), Stuttgart in Bewegung. Berichte von unterwegs (S. 31-37). Berlin: Jovis. doi: 10.18419/opus-10234.

Defila, R., \& Di Giulio, A. (Hrsg.). (2018b). Transdisziplinär und transformativ forschen. Eine Methodensammlung. Wiesbaden: Springer VS. doi: 10.1007/978-3-658-21530-9.

Defila, R., \& Di Giulio, A. (2018c). Reallabore als Quelle für die Methodik transdisziplinären und transformativen Forschens - eine Einführung. In R. Defila \& A. Di Giulio (Hrsg.), Transdisziplinär und transformativ forschen. Eine Methodensammlung (S. 9-35). Wiesbaden: Springer VS. doi: 10.1007/978-3-658-21530-9_1.

Eckart, J., Ley, A., Häußler, E., \& Erl, Th. (2018). Leitfragen für die Gestaltung von Partizipationsprozessen in Reallaboren. In R. Defila \& A. Di Giulio (Hrsg.), Transdisziplinär und transformativ forschen. Eine Methodensammlung (S. 105-135). Wiesbaden: Springer VS. doi: 10.1007/978-3-658-21530-9_6.

Gonser, M., Eckart, J., Eller, C., Köglberger, K., Häußler, E., \& Piontek F. M. (2019). Unterschiedliche Handlungslogiken in transdisziplinären und transformativen Forschungsprojekten - Welche Risikokulturen entwickeln sich daraus und wie lassen sie sich konstruktiv einbinden? In R. Defila \& A. Di Giulio (Hrsg.), Transdisziplinär und transformativ forschen, Band 2. Eine Methodensammlung (S. 39-83). Wiesbaden: Springer VS.

Groß, M., Hoffmann-Riem, H., \& Krohn, W. (2005). Realexperimente. Ökologische Gestaltungsprozesse in der Wissensgesellschaft. Bielefeld: transcript.

Jahn, T. (2008). Transdisziplinarität in der Forschungspraxis. In M. Bergmann \& E. Schramm (Hrsg.), Transdisziplinäre Forschung. Integrative Forschungsprozesse verstehen und bewerten (S. 21-37). Frankfurt a. M., New York: Campus.

Jahn, T., Bergmann, M., \& Keil, F. (2012). Transdisciplinarity: Between mainstreaming and marginalization. Ecological Economics, 79, (S. 1-10). doi: 10.1016/j.ecolecon. 2012.04.017. 
Klein, J. T. (2006). Afterword: the emergent literature on interdisciplinary and transdisciplinary research evaluation. Ecological Economics, 15 (1), (S. 75-80). doi: 10.3152/ 147154406781776011.

Lang, D. J., Wiek, A., Bergmann, M., Stauffacher, M., Martens, P., Moll, P., Swilling, M., \& Thomas, C. J. (2012). Transdisciplinary research in sustainability science: practice, principles, and challenges. Sustainability Science, 7 (Supplement 1), (S. 25-43). doi: 10.1007/s11625-011-0149-x.

Lazarova, K., Helfenstein, B. S., Dietz, R., \& Alcántara, S. (2018). Parklets für Stuttgart. Ein Realexperiment. Stuttgart: Reallabor für nachhaltige Mobilitätskultur Universität Stuttgart. http://www.r-n-m.net/wp-content/uploads/2018/03/Parklets-fuer-Stuttgart_ web.pdf. Zugegriffen am 27.02.2019.

McDonald, D., Bammer, G., \& Dean, P. (2009). Research integration using dialogue methods. Canberra, Australia: ANU E Press, The Australian National University. https://press-files.anu.edu.au/downloads/press/p60381/pdf/book.pdf. Zugegriffen am 15.04.2019.

MWK (Ministerium für Wissenschaft, Forschung und Kunst Baden-Württemberg) (Hrsg.). (2013). Wissenschaft für Nachhaltigkeit. Herausforderung und Chance für das badenwürttembergische Wissenschaftssystem. Stuttgart. https:/www.baden-wuerttemberg.de/ fileadmin/redaktion/dateien/PDF/Broschüre_Wissenschaft_für_Nachhaltigkeit.pdf. Zugegriffen am 19.05.2019.

Pohl, C., \& Hirsch Hadorn, G. (2006). Gestaltungsprinzipien für die transdisziplinäre Forschung. Ein Beitrag des td-net. München: oekom.

Potthast, T. (2015). Ethics and Sustainability Science beyond Hume, Moore and Weber. Taking Epistemic-Moral Hybrids Seriously. In S. Meisch, J. Lundershausen, L. Bossert \& M. Rockoff (Hrsg.), Ethics of Science in the Research for Sustainable Development (S. 129-152). Baden-Baden: Nomos.

Prengel, A., Heinzel, F., \& Carle, U. (2008). Methoden der Handlungs-, Praxis- und Evaluationsforschung. In W. Helsper \& J. Böhme (Hrsg.), Handbuch der Schulforschung (S. 181-197). Wiesbaden: Springer VS.

Puttrowait, E., Dietz, R., Gantert, M., \& Heynold, J. (2018). Der Weg zum Realexperiment - Schlüsselakteure identifizieren, Kooperationsstrukturen aufbauen, Projektideen auswählen. In R. Defila \& A. Di Giulio (Hrsg.), Transdisziplinär und transformativ forschen. Eine Methodensammlung (S. 195-232). Wiesbaden: Springer VS. doi: 10. 1007/978-3-658-21530-9_11.

RLSS (Reallabor Space Sharing, ABK Stuttgart) (Hrsg.). (2018). One Space Fits All Space Sharing Report. Erfahrungsbericht und Empfehlungen aus zwei Jahren Betriebserfahrung des Reallabor Space Sharing-Pilotprojekts. Stuttgart: Staatliche Akademie der Bildenden Künste.

San Francisco Planning Department (2018). San Francisco - Parklet Manual. San Francisco: City of San Francisco. http://groundplaysf.org/wp-content/uploads/Parklet_Manual_ 2018-FINAL_upload.pdf. Zugegriffen am 21.05.2019.

Schäpke, N., Stelzer, F., Bergmann, M., Singer-Brodowski, M., Wanner, M., Caniglia G., \& Lang, D. (2017). Reallabore im Kontext transformativer Forschung. Ansatzpunkte zur Konzeption und Einbettung in den internationalen Forschungsstand. (No. 1/2017). 
Leuphana Universität Lüneburg, Institut für Ethik und Transdisziplinäre Nachhaltigkeitsforschung. http://hdl.handle.net/10419/168596. Zugegriffen am 20.05.2019.

Schneidewind, U. (2013). Transformative Literacy. Gesellschaftliche Veränderungsprozesse verstehen und gestalten. GAIA, 22 (2), (S. 82-86).

Schophaus, M., Schön, S., \& Dienel, H.-L. (Hrsg.). (2004). Transdisziplinäres Kooperationsmanagement. Neue Wege in der Zusammenarbeit zwischen Wissenschaft und Gesellschaft. München: oekom.

Seebacher, A., Alcántara, S., \& Quint, A. (2018). Akteure in Reallaboren - Reallabore als Akteure. In R. Defila \& A. Di Giulio (Hrsg.), Transdisziplinär und transformativ forschen. Eine Methodensammlung (S. 155-159). Wiesbaden: Springer VS. doi: 10. 1007/978-3-658-21530-9_9.

Seifert, A., \& Zentner, S. (2010). Service-Learning - Lernen durch Engagement: Methode, Qualität, Beispiele und ausgewählte Schwerpunkte. Eine Publikation des Netzwerks Lernen durch Engagement. Weinheim: Freudenberg Stiftung. http://buendnis. augsburg.de/fileadmin/buendnis-aug/dat/2_projekte/tu_was/3Pub_Wissen_LdE.pdf. Zugegriffen am 27.02.2019.

Strohschneider, P. (2014). Zur Politik der Transformativen Wissenschaft. In: A. Brodocz, D. Herrmann, R. Schmidt, D. Schulz \& J. Schulze Wessel (Hrsg.), Die Verfassung des Politischen (S. 175-192). Wiesbaden: Springer VS. doi: 10.1007/978-3-658-04784-9_10.

Wehling, H.-G. (1977). Konsens à la Beutelsbach? Nachlese zu einem Expertengespräch. In S. Schiele \& H. Schneider (Hrsg.), Das Konsensproblem in der politischen Bildung (S. 173-184). Stuttgart: Ernst Klett.

Open Access Dieses Kapitel wird unter der Creative Commons Namensnennung 4.0 International Lizenz (http://creativecommons.org/licenses/by/4.0/deed.de) veröffentlicht, welche die Nutzung, Vervielfältigung, Bearbeitung, Verbreitung und Wiedergabe in jeglichem Medium und Format erlaubt, sofern Sie den/die ursprünglichen Autor(en) und die Quelle ordnungsgemäß nennen, einen Link zur Creative Commons Lizenz beifügen und angeben, ob Änderungen vorgenommen wurden.

Die in diesem Kapitel enthaltenen Bilder und sonstiges Drittmaterial unterliegen ebenfalls der genannten Creative Commons Lizenz, sofern sich aus der Abbildungslegende nichts anderes ergibt. Sofern das betreffende Material nicht unter der genannten Creative Commons Lizenz steht und die betreffende Handlung nicht nach gesetzlichen Vorschriften erlaubt ist, ist für die oben aufgeführten Weiterverwendungen des Materials die Einwilligung des jeweiligen Rechteinhabers einzuholen.

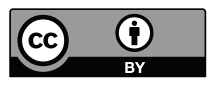




\title{
Kommunale Akteure verstehen: Verteilte Wissensbestände in Kommunen beachten
}

\author{
Thorsten Erl, Monika Gonser, Jochen Eckart, Elke Häußler \& Jan Riel
}

\section{Herausforderungen bei der Analyse kommunaler Akteure in Reallaboren}

Kommunen spielen in Reallaboren und in der transdisziplinären und transformativen Forschung allgemein oft eine zentrale Rolle. Aufbau und Organisation einer Kommune sind durchaus komplex und können von jeweils ortsspezifischen Besonderheiten geprägt sein. Insofern ist ein besseres Verständnis von Kommunen und insbesondere den einzelnen Akteuren innerhalb einer Kommune (Fachverwaltung, Verwaltungsspitze, politische Entscheidungsträger) von maßgeblicher Bedeutung für das Gelingen der Zusammenarbeit in solchen Projekten.

Für das bessere Verständnis der kommunalen Akteure in Reallaboren und in anderen transdisziplinären und transformativen Forschungsvorhaben bietet sich das Instrument der Akteursanalyse an (Eckart et al. 2018). Die Akteursanalyse hat sich aus Ansätzen der Politikfeldanalyse (Blum und Schubert 2011), des Strategic Management (Freeman 1984) sowie der Partizipationsforschung (Reed et al. 2009; Zimmermann 2006) entwickelt. Auch im Forschungskontext wird die Akteursanalyse bereits angewandt (Butterworth et al. 2011). Sie dient als Instrument, um die Einbindung der Praxisakteure in transdisziplinären und transformativen Forschungsvorhaben im Detail zu planen. Im Rahmen der Akteursanalyse sind die für die Forschungs-, Praxis- und Bildungsziele eines Vorhabens erforderlichen Akteure (Beecroft et al. 2018) zu identifizieren. Die Akteursanalyse befasst sich zudem mit den Erwartungen der Akteure darüber, wie und in welchem Umfang sie sich an einem Vorhaben beteiligen können oder sollen sowie mit den Möglichkeiten und Grenzen der Akteure. Damit eine Akteursanalyse gelingen kann und in der Folge die Zusammenarbeit in einem Projekt fruchtbar verläuft, ist es wichtig, den Besonderheiten der analysierten Akteure Rechnung zu tragen und nicht mit verkürztem Blick an die Analyse heranzugehen. Es ist also, mit anderen Worten, ein Bewusstsein nötig für mögliche ,blinde Flecken', die die Qualität einer Akteursanalyse beeinträchtigen könnten. 
Basierend auf Erfahrungen aus den vom Ministerium für Wissenschaft, Forschung und Kunst Baden-Württemberg (MWK) seit 2015 geförderten 14 Reallaboren der Förderlinien „Reallabore“ und „Reallabore Stadt" (im Folgenden BaWü-Labs) ${ }^{1}$ und bezogen auf den Akteur Kommune wird dargestellt, inwiefern ,blinde Flecken' existieren können, also Wissenslücken auf Seiten der Wissenschaftler(innen) über die Funktionsweise von und die Arbeit mit verschiedenen kommunalen Akteuren. Diese ,blinden Flecken' können in der Akteursanalyse sowie in der eigentlichen Forschungsarbeit zu empfindlichen Komplikationen führen - sei es, weil nicht die richtigen Ansprechpartner gefunden, weil vorgegebene Entscheidungswege nicht beachtet oder weil ohnehin vorbelastete Beziehungen zwischen Akteuren aus Unkenntnis überstrapaziert werden.

Diesen ,blinden Flecken' sind in diesem Buch drei Kurzbeiträge gewidmet: Ein Beitrag stellt die komplexen Entscheidungswege innerhalb kommunaler Strukturen dar, deren Kenntnis für das Gelingen der Zusammenarbeit von besonderer Bedeutung ist (Gonser et al. 2019 in diesem Buch). Ein Beitrag widmet sich der Vorgeschichte kommunaler Akteure und zeigt, warum es wichtig ist, sich mit dieser auseinanderzusetzen (Eckart et al. 2019 in diesem Buch). Der vorliegende Beitrag beschreibt die Verteilung von Wissensbeständen innerhalb von Koтmunen und inwiefern Kommunen nicht nur als ein (homogener) Ansprechpartner (miss)zu verstehen sind.

\section{Strukturierung von Wissen in Fachämtern}

Wissensbezogene, inhaltliche Arbeit in Kommunen wird in Deutschland auf der operativen Ebene von den sogenannten „Fachämtern“ geleistet (analoge Einrichtungen gibt es auch in anderen Ländern, hier wird der in Deutschland gebräuchliche Begriff verwendet). Je größer die Kommune, desto größer und spezialisierter sind in der Regel die Fachämter. Die Fachämter haben innerhalb ihres thematischen Zuständigkeitsbereichs eine beachtliche Entscheidungskompetenz und können Prozesse maßgeblich unterstützen oder blockieren. Folgende Strukturprinzipien werden dabei deutlich, die analog auch für andere Länder als Deutschland gelten dürften:

Kommunale Entscheidungen werden auf der Grundlage von durch die Fachämter aufbereitetem Wissen getroffen: Der Gemeinde- oder Stadtrat, aber auch (Ober-) Bürgermeister(innen) treffen weitreichende Entscheidungen vor allem auf Basis von durch die Fachämter bereitgestelltem Wissen. Informations- bzw. Beschlussvorlagen des Gemeinde- oder Stadtrats werden zum Beispiel durch Fachämter vorbereitet, wobei meist einem Fachamt die Federführung bei der Bearbeitung

1 Für Informationen $\mathrm{zu}$ den beiden Förderlinien siehe http://www.reallabore-bw.de (zugegriffen am 20.05.2019). 
zugewiesen wird. Handelt es sich um eine Maßnahme bzw. um ein Thema beispielsweise eines städtischen Freiraums, wird das Landschaftsamt federführend sein, handelt es sich um eine Mobilitätsfrage, wird die Verkehrsplanung maßgeblich sein etc. Die Wissensauswahl und -bewertung durch die Fachämter ist deswegen von großer politischer Bedeutung. Welches Wissen Fachämtern zur Verfügung steht, auf das sie dabei greifen (können), ist daher auch bedeutsam, wenn es darum geht, Informations- oder Beschlussvorlagen mit Blick auf die Zusammenarbeit mit einem transdisziplinären und transformativen Forschungsprojekt wie einem Reallabor vorzubereiten.

Vernetzungen zwischen den in verschiedenen Fachämtern vorliegenden Wissensbeständen werden durch Methoden des Verwaltungsmanagements hergestellt: Kommunale Verwaltungen sind hierarchisch nach Zuständigkeiten organisiert. Einen Einblick in die Zuständigkeitsverteilung z. B. für Deutschland bietet die allgemeine, bundesweit geltende Struktur, die durch die Kommunale Gemeinschaftsstelle für Verwaltungsmanagement (KGSt) nach dem 2. Weltkrieg aufgestellt wurde. Ein System von Ordnungskennziffern unterscheidet hier auf erster Ebene die übergeordneten Ressorts: Hauptverwaltung (1), Finanzverwaltung (2), Rechts-, Sicherheits- und Ordnungsverwaltung (3), Schul- und Kulturverwaltung (4), Sozial-, Jugend- und Gesundheitsverwaltung (5), Bauverwaltung (6), Verwaltung für öffentliche Einrichtungen (7) und Verwaltung für Wirtschaft und Verkehr (8). Auf einer zweiten Ebene werden diese Ressorts weiter differenziert, wie z. B. in Schulverwaltung (40) und Kulturamt (41) oder in Stadtplanung (61), Bauordnungsamt (63) und Grünflächenamt (67). Dieses System ist für die Kommunen in Deutschland nicht zwingend anzuwenden, jedoch häufig vorzufinden. Im Ergebnis verfügen die Mitarbeitenden in der kommunalen Verwaltung nur in ihrem jeweils thematisch eingeschränkten (Zuständigkeits-)Bereich über vertieftes Wissen. Den Akteuren der kommunalen Verwaltung ist diese ,Wissensordnung' wohlbekannt, für Wissenschaftler(innen) hingegen ist sie zwar wichtig, wenn sie mit Kommunen zusammenarbeiten, aber zumeist nicht oder nur marginal bekannt.

Im Reallabor GO Karlsruhe - Partizipative Forschung für den Fußgängerverkehr (,,BaWü-Lab GO Karlsruhe“) war das für die Arbeit im Projekt erforderliche kommunale Fachwissen über Stadtplanungsamt (Verkehrsplanung, inhaltliche Arbeit an der Bürgerbeteiligung), Tiefbauamt, Ordnungsamt (in der Rolle als Straßenverkehrsbehörde), eine ämterübergreifende Baustellenkoordination, Stadtmarketing sowie das Amt für Stadtentwicklung (als organisierende Stelle für Bürgerbeteiligung) breit verteilt. Aufgrund der früheren Mitarbeit eines Mitglieds des Forscher(innen)teams in der Stadtverwaltung waren jedoch Kenntnisse über die Organisation der Ämter wie auch über persönliche Ansprechpartner vorhanden, so dass der Zugriff auf das relevante Fachwissen auch ohne vorhergehende umfangreiche Akteursanalyse einfach möglich war. Wäre das nicht der Fall gewesen, wäre eine umfassende Analyse nötig gewesen, um herauszufinden, welche Fachämter über das jeweils relevante Wissen verfügen. 
Bei kleineren Kommunen besteht dagegen möglicherweise aufgrund der Bündelung verschiedener Ressorts in einer Person (Bürgermeister(in), Amtsleitung) bereits bei dieser Person umfangreiches Querschnittswissen, was es entsprechend einfacher macht, an dieses Wissen heranzukommen.

Das Reallabor STADT-RAUM-BILDUNG - Reallabor für die nachhaltige Planung von Bildungslandschaften und die Integration von Aus- und Umbauten von Schulgebäuden (,,BaWü-Lab SRB“) (s. auch Steckbrief im Anhang zu diesem Buch) arbeitete mit unterschiedlich großen Kommunen. Während in Heidelberg eine kontinuierliche Kommunikation mit drei Amtsleitungen (Stadtplanungsamt, Gebäudemanagement, Amt für Schule und Bildung) aufgeteilt auf zwei Dezernate (Dezernat für Bauen und Verkehr, Dezernat für Familie, Soziales und Kultur) wichtig war, kommunizierten die Wissenschaftler(innen) in der Landeshauptstadt Stuttgart mit dem Schulverwaltungsamt, das eine eigene Planungsabteilung für den Schulbau unterhält. In Müllheim wiederum wurde hauptsächlich mit der Leitung des Baudezernats zusammengearbeitet.

Die Vernetzung zwischen verschiedenen (Zuständigkeits-)Bereichen und Wissensbeständen wird im Verwaltungsalltag meist nur von Personen in Führungspositionen wahrgenommen. Über die Vernetzung durch Führungspositionen hinaus gibt es in kommunalen Verwaltungen noch andere Werkzeuge zur Wissensverknüpfung, von denen einige hier beispielhaft genannt seien: Der Austausch zwischen den einzelnen Ressorts bzw. Fachämtern funktioniert gemeinhin über den sogenannten „Ämterumlauf“. Mittels einer Akte zum Sachverhalt werden die entsprechenden Ämter in Kenntnis gesetzt und um Stellungnahme gebeten. Diese Stellungnahmen werden nach einer gewissen Bearbeitungszeit wieder an das federführende Amt zurückgeschickt. Daraufhin muss dieses Amt alle Hinweise, das heißt, das Wissen der anderen Ämter, aufarbeiten und in die Vorlage einpflegen. Zudem versuchen einige Verwaltungen, die hierarchischen Strukturen über sogenannte ,agile“ Arbeitsgruppen fachämterübergreifend themen- bzw. projektbezogen aufzubrechen und neu zu strukturieren. Damit werden in der Verwaltung erste Schritte unternommen, um gerade auch für z. B. Reallabore relevantes Querschnittswissen zu erzeugen. Für ein transdisziplinäres und transformatives Projekt bedeutet das, dass nur an wenigen Stellen in einer kommunalen Verwaltung integriertes Querschnittswissen vorliegen dürfte und dass es nicht nur herauszufinden gilt, wo sich welches Wissen bündelt, sondern auch, wie innerhalb der Kommune Wissen ausgetauscht und gebündelt wird.

\section{Herausforderungen im Umgang mit kommunalem Wissen}

Das Arbeiten mit kommunalen Wissensbeständen gestaltet sich aufgrund der beschriebenen komplexen und hierarchischen kommunalen Verwaltungsstrukturen in Teilen als langwierig bzw. ,schwerfällig'. Für ,Externe', wie etwa Wissenschaftler(innen) in einem Reallabor, kann es sich überaus schwierig gestalten, zu 
erkennen, welche Wissensbestände in den Fachämtern vorhanden sind, wie sich diese gliedern und wo und wie Wissen gebündelt wird. Weiter wird dies durch die fast wie ,Geheimcodes' erscheinende Verwaltungsgliederung erschwert, die für außenstehende Wissenschaftler(innen) auf den ersten Blick nur schwer erfassbar ist.

Probleme entstehen insbesondere dann, wenn in einem transdisziplinären und transformativen Projekt wie einem Reallabor davon ausgegangen wird, dass alle Akteure in der kommunalen Verwaltung über das gleiche Wissen verfügen bzw. dass alles für das Reallabor relevante Wissen bei einem Akteur innerhalb der kommunalen Verwaltung zu finden ist. Das ,unterkomplexe' Verständnis der Verteilung von Wissensbeständen innerhalb einer Kommune wird so zu einem ,blinden Fleck'.

\section{Berücksichtigung der verteilten Wissensbestände in der Akteursanalyse}

Im Rahmen der Akteursanalyse ist deshalb zu identifizieren, welche Akteure in der kommunalen Verwaltung über welche Teilaspekte des für das Projekt relevanten Wissens verfügen. Zudem ist zu klären, ob es Stellen in der Verwaltung gibt, die das für das Projekt relevante Querschnittswissen zusammenfassen. Dabei ist davon auszugehen, dass die Wissensbestände auf eine Vielzahl von Akteuren verteilt sind und nicht mit einem Ansprechpartner allein erschlossen werden können. Für Deutschland kann die oben erwähnte Zuständigkeitsverteilung gemäß KGSt erste Ansatzpunkte liefern, die relevanten Ansprechpartner innerhalb der kommunalen Verwaltung zu identifizieren. Teilweise werden allerdings in den Kommunen die Zuschnitte der einzelnen Ressorts den örtlichen Besonderheiten angepasst (und auch anders benannt). Diese lokalen Anpassungen wiederum können im Organigramm der Kommune sowie durch die genauere Beschreibung der Zuständigkeitsverteilung innerhalb der Verwaltung in Erfahrung gebracht werden.

Um als transdisziplinäres und transformatives Projekt auf kommunale (Querschnitts-)Wissensbestände zugreifen zu können, ist es notwendig, auch innerhalb der Kommune Ansprechpartner oder Teams zu identifizieren bzw. zu bilden, die den seitens der Wissenschaft gewählten gesamtheitlichen Zugang angemessen spiegeln. Insbesondere bestehende fachämterübergreifende Teams können helfen, innerhalb der Verwaltung Querschnittswissen zu erschließen und in das gemeinsame Forschungsprojekt einzubringen. Falls es keine solchen gibt, lohnt es sich, in Erfahrung zu bringen, ob eine solche Arbeitsgruppe eingerichtet werden könnte. Im besten Fall ist das transdisziplinäre Team ,symmetrisch' organisiert, d. h., dass sich jeweils ein(e) Vertreter(in) aus einem kommunalen Fachamt und ein(e) 
Vertreter(in) aus der Wissenschaft entsprechen, was Themenschwerpunkte der Arbeit und Wissenschaftskultur betrifft. In diesem Fall ist zumindest gewährleistet, dass die gemeinsame Arbeit auf der Grundlage ähnlicher Sprachen und ähnlicher Arbeitskulturen aufbauen kann. Das erhöht theoretisch die Möglichkeit, im Verlauf des Projekts zwischen kommunalen Akteuren und Wissenschaftler(inne)n ,auf Augenhöhe' diskutieren und forschen zu können. Kritisch ist vor diesem Hintergrund ein ,asymmetrisches" Verhältnis, d. h., wenn Einzelpersonen aus sehr unterschiedlichen Wissenschaftskulturen (z. B. Geisteswissenschaften und Ingenieurwissenschaften) aufeinandertreffen. In solchen Fällen sollte versucht werden, breiter aufgestellte Teams einzusetzen, so dass das ausgetauschte Fachwissen auch von allen Beteiligten richtig verstanden und interpretiert wird. In einer umfassenden Akteursanalyse sollte deshalb auch beleuchtet werden, welchen fachlichen Hintergrund die zuständigen Personen in der kommunalen Verwaltung haben, d. h., auch diesem möglichen ,blinden Fleck' sollte Aufmerksamkeit geschenkt werden.

\section{Literatur}

Beecroft, R., Trenks, H., Rhodius, R., Benighaus, C., \& Parodi, O. (2018). Reallabore als Rahmen transformativer und transdisziplinärer Forschung: Ziele und Designprinzipien. In R. Defila \& A. Di Giulio (Hrsg.), Transdisziplinär und transformativ forschen. Eine Methodensammlung. (S. 75-100). Wiesbaden: Springer VS. doi: 10.1007/978-3-65821530-9_4.

Blum, S., \& Schubert, K. (2011). Politikfeldanalyse. Wiesbaden: Springer VS.

Butterworth, J., McIntyre, P. \& da Silva Wells, C. (Hrsg.). (2011). Switch in the City: Putting Urban Water Management to the Test. Den Haag: IRC International Water and Sanitation Centre.

Eckart, J., Ley, A., Häußler, E., \& Erl, Th. (2018). Leitfragen für die Gestaltung von Partizipationsprozessen in Reallaboren. In R. Defila \& A. Di Giulio (Hrsg.), Transdisziplinär und transformativ forschen. Eine Methodensammlung (S. 105-135). Wiesbaden: Springer VS.

Freeman, R. E. (1984). Strategic Management: A Stakeholder Approach. Boston: Pitman.

Reed, M. S., Graves, A., Dandy, N., Posthumus, H., Hubacek, K., \& Morris, J. (2009). Who's in and why? A typology of stakeholder analysis methods for natural resource management. Journal of Environmental Management, 90, (S. 1933-1949).

Zimmermann, A. (2006). Instrumente zur Akteursanalyse - 10 Bausteine für die partizipative Gestaltung von Kooperationssystemen. Eschborn: Deutsche Gesellschaft für Technische Zusammenarbeit (GTZ). 


\section{Die zwei weiteren Beiträge in diesem Buch zu, blinden Flecken ‘}

Eckart, J., Häußler, E., Erl, Th., Gonser, M., \& Riel, J. (2019). Kommunale Akteure verstehen: Vorgeschichte der Arbeit in Kommunen beachten. In R. Defila \& A. Di Giulio (Hrsg.), Transdisziplinär und transformativ forschen, Band 2. Eine Methodensammlung (S. 33-38). Wiesbaden: Springer VS.

Gonser, M., Riel, J., Eckart, J., Erl, Th., \& Häußler, E. (2019). Kommunale Akteure verstehen: Komplexe Entscheidungswege in Kommunen. In R. Defila \& A. Di Giulio (Hrsg.), Transdisziplinär und transformativ forschen, Band 2. Eine Methodensammlung (S. 85-91). Wiesbaden: Springer VS.

Open Access Dieses Kapitel wird unter der Creative Commons Namensnennung 4.0 International Lizenz (http://creativecommons.org/licenses/by/4.0/deed.de) veröffentlicht, welche die Nutzung, Vervielfältigung, Bearbeitung, Verbreitung und Wiedergabe in jeglichem Medium und Format erlaubt, sofern Sie den/die ursprünglichen Autor(en) und die Quelle ordnungsgemäß nennen, einen Link zur Creative Commons Lizenz beifügen und angeben, ob Änderungen vorgenommen wurden.

Die in diesem Kapitel enthaltenen Bilder und sonstiges Drittmaterial unterliegen ebenfalls der genannten Creative Commons Lizenz, sofern sich aus der Abbildungslegende nichts anderes ergibt. Sofern das betreffende Material nicht unter der genannten Creative Commons Lizenz steht und die betreffende Handlung nicht nach gesetzlichen Vorschriften erlaubt ist, ist für die oben aufgeführten Weiterverwendungen des Materials die Einwilligung des jeweiligen Rechteinhabers einzuholen.

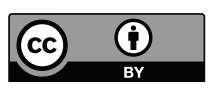




\section{Teil 2}

\section{Wie vorgehen: \\ Methoden für Co-Design \\ und Co-Produktion}




\section{Die „Exchange-Veranstaltungsreihe“ - ein Planungs- und Steuerungsinstrument für transdisziplinäre Forschungsprojekte}

Christina Benighaus, Belen Zevallos, Charlotte Eller, Martina Hilligardt, Mandana Alimardani \& Ludger Benighaus

\section{Einleitung und Kontext}

Kern eines jeden Reallabors ist die Zusammenarbeit von Akteuren aus der Wissenschaft (Forscher(innen)) mit Akteuren aus der für das Projekt relevanten Praxis, um gemeinsam Wissen zu erzeugen (Forschungsziel), Transformationsprozesse $\mathrm{zu}$ fördern und $\mathrm{zu}$ gestalten (Praxisziel) und individuelle und gesellschaftliche Lernprozesse anzustoßen (Bildungsziel) (Beecroft et al. 2018, S. 79ff.). Reallabore bedürfen daher geeigneter Formate für die Zusammenarbeit verschiedenster Akteure (Schäpke et al. 2017; Arnold und Piontek 2018). Dies gilt analog ebenso für andere transdisziplinäre (und transformative) Forschungsformate und -projekte. Die in diesem Beitrag erläuterte Planung einer Veranstaltungsreihe ist ein Werkzeug zur detaillierten Gliederung und Steuerung eines transdisziplinären Forschungsprojekts, bei dem die transdisziplinäre Arbeit maßgeblich mit externen Beteiligten erfolgt, die punktuell in die Forschungsarbeit involviert werden (s. dazu Defila et al. 2006, S. 214ff.). Es führt zu mehreren transdisziplinären Veranstaltungen, die in Form von öffentlichen Symposien mit jeweils anschließendem projektinternen Reflexionsworkshop durchgeführt werden. Mithilfe jeder dieser Veranstaltungen wird ein abgegrenzter Ort der transdisziplinären Wissensproduktion zur Verfügung gestellt. Die Verknüpfung mehrerer solcher Veranstaltungen zu einer Veranstaltungsreihe - der „Exchange-Veranstaltungsreihe" - gibt die Struktur für die Zusammenarbeit zwischen Akteuren aus der Wissenschaft und Akteuren aus der Praxis im Forschungsprojekt vor. Sie ermöglicht es, die gesamte Projektplanung entlang der Veranstaltungsreihe zu organisieren, zu steuern sowie eine aktive Schnittstelle zwischen Wissenschaft und Praxis zu schaffen.

Die Planung der Exchange-Veranstaltungsreihe geht in Anlehnung an Eckart et al. (2018, S. 127) von vier Phasen eines Forschungsprozesses aus: Einstiegsphase, Orientierungsphase, Arbeitsphase und Abschlussphase. Diese spiegeln sich 
in den einzelnen Veranstaltungen der Reihe wider. Die Veranstaltungen dienen für die jeweilige Phase als Meilensteine und wirken dadurch Struktur gebend sowohl für die einzig an den Veranstaltungen beteiligten Akteure als auch für das Projektteam selbst. Gleichzeitig dienen sie auf inhaltlicher Ebene der Verknüpfung der verschiedenen Erkenntnisse - beispielsweise aus mehreren Teilprojekten - im Forschungsprojekt, indem diese Erkenntnisse aus den einzelnen Teilen des Projekts im Zuge der Veranstaltungen gemeinsam reflektiert und angereichert werden. ${ }^{1}$ Die Exchange-Veranstaltungsreihe stellt ein Instrument dar, durch welches externe Akteure insbesondere aus der Praxis (Anwender(innen), Nutzer(innen) im Themenbereich des Projekts), aber auch aus der Wissenschaft (für das Projekt relevante Forscher(innen)) punktuell im Zuge der Projektlaufzeit beteiligt werden. Als ,externe Akteure' werden hier jene Akteure aus dem ,äußeren Akteurskreis“ im Sinne von Seebacher et al. (2018, S. 157) bezeichnet, die nicht aktiv im Forschungsprojekt eingebunden sind (also nicht Teil des Projektteams sind, weder als Praxispartner noch als Forscher(innen)), sondern sich in der jeweiligen Veranstaltung entsprechend einbringen. Das im Projekt generierte Wissen wird im Rahmen der Veranstaltungen einem größeren Kreis an Akteuren präsentiert, gemeinsam mit diesen externen Akteuren reflektiert und durch die gemeinsame Wissensproduktion von Projektteam, externen Forscher(inne)n und Praxisakteuren erweitert. Durch die Verknüpfung der Veranstaltungen wird gleichzeitig eine Lernumgebung geschaffen, die den Beteiligten einen kontinuierlichen, geschützten Rahmen für Information, Austausch, Kooperation sowie Evaluation und Reflexion bietet (Beecroft et al. 2018, S. 83).

Das in diesem Beitrag beschriebene Werkzeug wurde im Rahmen des Reallabors „STADT-RAUM-BILDUNG - Reallabor für die nachhaltige Planung von Bildungslandschaften und die Integration von Aus- und Umbauten von Schulgebäuden“ (Reallabor SRB, s. auch Steckbrief im Anhang zu diesem Buch) entwickelt. Im Reallabor SRB standen Sekundarschulen in Baden-Württemberg im Fokus, die aktuellen Umwälzungen auf politischer Ebene sowie notwendigen strukturellen und baulichen Veränderungen ausgesetzt waren. Die hohe gesellschaftliche und politische Aktualität veranlasste das Projektteam, bereits im Antrag mehrere öffentliche Symposien in regelmäßigem zeitlichem Abstand vorzusehen. Da Bildungsthemen in Baden-Württemberg auf (politischer) Landesebene entschieden werden, sollte ein möglichst breiter Kreis an Akteuren aus dem ganzen Bundes-

1 Im Beitrag wird die gängige Projektstruktur zugrunde gelegt, dass inter- und transdisziplinäre Forschungsprojekte aus mehreren Teilen bestehen, die auf gemeinsame Ziele und Ergebnisse ausgerichtet sind. In diesen Teilen, die hier als ,Teilprojekte' bezeichnet werden (und die selbst disziplinär, interdisziplinär oder transdisziplinär sein können), finden „Forschungsarbeiten statt, die zu planen und aufeinander abzustimmen sind, die aber gleichzeitig einen eigenen Stellenwert haben und auch eigenständige Ergebnisse hervorbringen" (Defila et al. 2006, S. 38). 
land und darüber hinaus beteiligt werden. Hierzu wurden gezielt Personen eingeladen, die sich kurz vor oder mitten in einem Veränderungsprozess einer Schule befanden. Im Fokus standen zunächst Schlüsselpersonen, die bei diesen Veränderungen an Schulen eine wesentliche Rolle einnahmen. Zu diesen Personen gehörten Vertreter(innen) der kommunalen Verwaltungen, Schulleitungen und Politiker(innen) vorwiegend aus den Stadt- und Gemeinderäten. Des Weiteren wurden Personen aus den Kollegien einzelner Schulen, Architekt(inn)en verschiedener Büros sowie Wissenschaftler(innen) aus thematisch verwandten Forschungsprojekten adressiert, so dass eine breite Mischung verschiedener Akteure mit unterschiedlichen Wissensbeständen und Interessen entstand (s. hierzu Benighaus und Renn 2016, S. 70ff.). Durch landesweite Einladungen wurden nicht nur verschiedene Akteursgruppen, sondern auch Personen der gleichen Akteursgruppe, jedoch aus unterschiedlichen Kontexten, zusammengebracht. Somit konnten sich beispielsweise Vertreter(innen) verschiedener Kommunen über ihre jeweiligen individuellen Herausforderungen austauschen. Das wiederholte Zusammentreffen der teilnehmenden Personen führte zu einem zunehmenden Vertrauensverhältnis und einer Zusammenarbeit zwischen den Teilnehmer(inne)n, die zum Teil auch jenseits der vom Reallabor SRB organisierten Veranstaltungen weitergeführt wurde.

Das im Folgenden beschriebene Werkzeug der Planung einer Veranstaltungsreihe eignet sich - abgeleitet aus den Erfahrungen des Reallabors SRB - bei gesellschaftlich aktuellen und politisch kontrovers diskutierten sowie in den aktuellen Medien vielfach besprochenen Themen. Die aus diesen Rahmenbedingungen entstehende hohe Dynamik im Feld - sowohl hinsichtlich neuer Erfahrungen und Praktiken als auch hinsichtlich der sich verändernden Akteurslandschaft - kann $\mathrm{zu}$ einer chancenreichen, aber auch zu einer konfliktbehafteten Zusammenarbeit zwischen den Akteuren sowie zwischen dem Forschungsprojekt und den Akteuren im Feld, die nicht am Projekt beteiligt sind, führen. Dieser Herausforderung kann durch eine Veranstaltungsreihe Rechnung getragen werden, da die jeweils aktuellen Akteure mit ihrem jeweils aktuellen Wissen wiederholt über die gesamte Projektlaufzeit hinweg einbezogen werden. Dies führt bezogen auf die Forschung im Projekt zu Iterationsschleifen im Prozess, so dass das jeweils neue Praxiswissen immer wieder reflektiert und in die Forschung integriert werden kann und so die Forschungsergebnisse mit der Dynamik im Feld Schritt halten können.

Bei einem Forschungsthema, das die vorher beschriebenen Merkmale erfüllt, kann es zudem wichtig sein, dass sich das Forschungsprojekt als Akteur im Feld präsentiert und legitimiert, um das Interesse der Akteure am Projekt und an der gemeinsamen Arbeit zu wecken und zu halten (Quint et al. 2018, S. 71). Zudem eignet sich das Instrument einer Veranstaltungsreihe speziell für Forschungsprojekte, die so angelegt sind, dass sie sich nicht nur auf einen Ort beziehen, 
sondern ein Thema bearbeiten, das mehrere Orte oder eine ganze Region betrachtet. Gerade wenn der Fokus der Forschung eher auf einer allgemeinen Problemstellung und weniger auf der lokalen Verortung des Problems liegt, ist das Instrument besonders geeignet. Denn um eine Vielfalt an Erfahrung und Wissen aus Wissenschaft und Praxis zu integrieren, können durch eine Exchange-Veranstaltungsreihe externe Forscher(innen) und Praxisakteure aus verschiedenen Kontexten punktuell integriert werden (s. zur Einbindung von Akteuren in Reallaboren Alcántara et al. 2018, S. 138ff.). Dadurch kann die notwendige Bandbreite des Wissens für das Projekt fruchtbar gemacht werden.

Einen besonderen Nutzen bietet der vorliegende Beitrag für alle, die sich in der Planung eines transdisziplinären Forschungsprojekts befinden. Diese Planung wird meist von denjenigen Wissenschaftler(inne)n übernommen, die auch nach Genehmigung des Projekts und Start der Forschung zum „Kernbereich“ gehören werden, also Teil des für die „Konzeption, inhaltliche Kohärenz, Prozessgestaltung und organisatorische Steuerung verantwortlichen Teams" sein werden (Seebacher et al. 2018, S. 157). Der Beitrag stellt eine Anleitung zur Planung einer transdisziplinären Veranstaltungsreihe als ,Gerüst ${ }^{`}$ des transdisziplinären Projektdesigns dar. Die Exchange-Veranstaltungsreihe wird im Folgenden an einem allgemeinen Projektablauf vorgestellt, der natürlich in jedem transdisziplinären Forschungsprojekt individuell variieren kann, so dass das Instrument entsprechend angepasst werden muss.

Im Folgenden wird die Exchange-Veranstaltungsreihe erläutert, wobei zuerst auf die Planung der Veranstaltungsreihe eingegangen wird (Kapitel 2), bevor die einzelnen Veranstaltungen vertieft dargestellt werden (Kapitel 3). Abschließend werden das Instrument und seine Anwendung im Reallabor SRB noch einmal reflektiert (Kapitel 4). Einzelne Beispiele aus dem Reallabor SRB in Kapitel 3 zeigen die Anwendung in diesem Forschungsprojekt und deren Mehrwert (s. die SRBBoxen).

\section{Die Exchange-Veranstaltungsreihe im Überblick und ihre Planung}

Durch die Exchange-Veranstaltungsreihe wird die Projektlaufzeit in mehrere Veranstaltungen, bestehend aus öffentlichen Symposien mit jeweils angeschlossenem internem Reflexionsworkshop, gegliedert (s. Tab. 1). Ein wichtiges Ziel der Symposien ist es, die Transparenz des Forschungsprojekts zur gewährleisten und einer breiteren Öffentlichkeit die Möglichkeit zu geben, die Inhalte des Forschungsprojekts zu erfassen und zu kommentieren. Die am nächsten Tag stattfindenden, internen Reflexionsworkshops wiederum bieten einen gesteuerten Austausch innerhalb des Projektteams. Dieses Vorgehen führt zu einer klaren Konstituierung 
von ,Innen“ und ,Außen“ (s. dazu Quint et al. 2018, S. 71ff.). Zum ,inneren“ Kreis zählen die aktiv am Forschungsprojekt Beteiligten. Der regelmäßige Austausch in den Reflexionsworkshops und das dort stattfindende Reflektieren des Symposiums vom Vortag fördern das Vertrauensverhältnis im Projektteam und eine konstruktive Zusammenarbeit im weiteren Forschungsverlauf. Zusätzlich führt das gemeinsam Erlebte und Reflektierte der Symposien zu gemeinsamen Erfahrungen des Projektteams und unterstützt dadurch die Konsens- und Synthesebildung (Defila et al. 2006, S. 35-38). Damit dies gelingen kann, wird die Veranstaltungsreihe bereits im Forschungsantrag verankert und bildet die Grundlage des Forschungsdesigns.

Die folgenden Ausführungen erläutern, wie die Veranstaltungsreihe noch vor Beginn der eigentlichen Forschung geplant wird. Dabei sind einzelne Veranstaltungen zwingend, während andere optional sind, d. h. abhängig von der Dauer des Forschungsprojekts und von den gewünschten Schwerpunkten vorgesehen oder weggelassen werden können. Wie die einzelnen Veranstaltungen dieser Reihe also die Symposien mit jeweils anschließendem Reflexionsworkshop - im Speziellen geplant und durchgeführt werden können, wird in Kapitel 3 ausgeführt.

\subsection{Die Exchange-Veranstaltungsreihe im Überblick}

Die Exchange-Veranstaltungsreihe gliedert die gesamte Projektlaufzeit in zeitliche Abschnitte, definiert Meilensteine im Projekt und erlaubt es, die Reihe als ein Steuerungsinstrument zu nutzen. Beispielsweise können so Teilprojekte, aus denen das Forschungsprojekt bestehen kann, sinnvoll miteinander vernetzt werden. Dadurch wird bereits zu Beginn des Forschungsprozesses sichergestellt, dass im Verlauf des Projekts Wissen aus den Teilprojekten zusammengeführt wird, um die Synthesebildung zu unterstützen. Zentral ist dabei, dass sich die beteiligten Akteure ihrer gleichzeitigen Rollen als Expert(inn)en und Laien im Kommunikationsprozess bewusst sind und die Sichtweise des ,fachfremden' Gegenübers annehmen (s. dazu z. B. Godemann 2005, S. 85f.).

Sowohl die einzelnen Symposien als auch die jeweils anschließenden Reflexionsworkshops dienen der interdisziplinären Verknüpfung der Wissensbestände der beteiligten Forscher(innen) und der transdisziplinären Integration von Praxiswissen. Über die Symposien wird das Praxiswissen, aktiviert' und ,abgerufen'. In den Reflexionsworkshops diskutieren die Forscher(innen) die Erkenntnisse aus dem jeweils vorausgegangenen Symposium quer zu den beteiligten Disziplinen, werten sie anschließend wissenschaftlich aus und integrieren sie in die laufende Forschung. Zudem wird der Stand der Arbeit in den einzelnen Teilprojekten innerhalb der Projektteams besprochen, und unter Berücksichtigung der durch die externen Akteure neu gewonnenen Erkenntnisse werden die Fragestellungen und Ziele des Gesamtprojekts und der Teilprojekte überarbeitet. 
Tabelle 1: Die Veranstaltungen, aus denen die Exchange-Veranstaltungsreihe besteht.

\begin{tabular}{|c|c|}
\hline $\begin{array}{l}\text { Veranstaltung 1: } \\
\text { Tür-Öffnerin }\end{array}$ & $\begin{array}{l}\text { Steht zu Beginn des Projektverlaufs in der Einstiegsphase: } \\
\text { Zur Etablierung des Forschungsprojekts und Gewinnung } \\
\text { von (weiteren) Praxisakteuren für die transdisziplinäre } \\
\text { Zusammenarbeit. Das Forschungsprojekt und das Projekt- } \\
\text { team werden vorgestellt, das im Forschungsantrag dar- } \\
\text { gelegte Zielwissen wird durch externe Akteure reflektiert } \\
\text { und ergänzt. }\end{array}$ \\
\hline $\begin{array}{l}\text { Veranstaltung } 2 \\
\text { (optional): } \\
\text { Weg-Bereiterin }\end{array}$ & $\begin{array}{l}\text { Optionale Veranstaltung in der Orientierungsphase zwi- } \\
\text { schen Anfang und Mitte des Projektverlaufs: Den Fokus } \\
\text { bilden die transparente Darstellung des Forschungsgegen- } \\
\text { stands, Berichte der Praxispartner (z. B. aus Fallbeispielen } \\
\text { des Projekts) sowie die Integration von Praxiswissen in } \\
\text { den Forschungsprozess. }\end{array}$ \\
\hline $\begin{array}{l}\text { Veranstaltung 3: } \\
\text { Weg-Begleiterin }\end{array}$ & $\begin{array}{l}\text { Findet circa zur Halbzeit der Projektlaufzeit statt, also } \\
\text { während der Arbeitsphase: Sie dient einer ersten Diskus- } \\
\text { sion der praktischen Anwendbarkeit erster Eindrücke aus } \\
\text { der Forschung und der Rückkopplung der Erfahrungen mit } \\
\text { dem wissenschaftlichen Diskurs. }\end{array}$ \\
\hline $\begin{array}{l}\text { Veranstaltung } 4 \\
\text { (optional): } \\
\text { Ziel-Erspäherin }\end{array}$ & $\begin{array}{l}\text { Steht zwischen Mitte und Ende des Projektverlaufs, also } \\
\text { ebenfalls in der Arbeitsphase: Sie dreht sich um die Vor- } \\
\text { stellung erster Teilergebnisse und die Rückspiegelung der } \\
\text { Erkenntnisse aus der Forschung an die externen Praxis- } \\
\text { akteure sowie um die gemeinsame Erarbeitung von Trans- } \\
\text { formationswissen. }\end{array}$ \\
\hline $\begin{array}{l}\text { Veranstaltung 5: } \\
\text { Ziel-Beschreiterin }\end{array}$ & $\begin{array}{l}\text { Findet am Ende des Projektverlaufs statt, also in der Ab- } \\
\text { schlussphase: Hier geht es um die Darstellung der Ge- } \\
\text { samtergebnisse und um deren Umsetzung. Generiertes } \\
\text { Transformationswissen, das im Projekt entstanden ist, } \\
\text { kann so in die Praxis und in folgende Forschungen inte- } \\
\text { griert werden. }\end{array}$ \\
\hline \multicolumn{2}{|c|}{$\begin{array}{l}\text { Reflexionsworkshop: An jeder der Veranstaltungen folgt nach dem öffentlichen Sym- } \\
\text { posium ein interner Reflexionsworkshop, in dem das Projektteam die Beiträge und } \\
\text { Impulse der externen Praxisakteure und ggf. der externen Forscher(innen) aufbereitet } \\
\text { und auswertet. }\end{array}$} \\
\hline
\end{tabular}

Die Exchange-Veranstaltungsreihe dient somit der regelmäßigen Vernetzung und Integration der Ergebnisse aus den Teilprojekten. Die Veranstaltungen fungieren als ,Verknüpfungspunkte' eines Netzes an verschiedenen Wissensbeständen. Während sich in der Einstiegs- und Orientierungsphase erste gemeinsame Themen für die Synthese konkretisieren, werden die Schnittstellen in der Arbeitsphase zunehmend interessanter. Das konsequente Herstellen von Transparenz be- 
zogen auf Forschungsprozesse der Teilprojekte erhöht diese Dynamik und unterstützt dadurch die Bildung von Querschnittswissen und das Entstehen einer Synthese. Welche Arbeitsschritte zur Umsetzung der, Verknüpfungsfunktion' bei den einzelnen Veranstaltungen notwendig sind, wird in Kapitel 3 unter Aufgabenpaket 6 „Dokumentation und Auswertung“ erläutert.

Der folgende Abschnitt beschreibt, wie die Planung der Exchange-Veranstaltungsreihe und die darauf bezogene Steuerung der Vernetzung von Teilprojekten innerhalb des Forschungsprojekts realisiert werden.

\subsection{Die Planung der Exchange-Veranstaltungsreihe}

Für die Planung der Exchange-Veranstaltungsreihe wird, wie bereits erwähnt, von einer vierstufigen Gliederung eines transdisziplinären Forschungsprojekts ausgegangen: Einstiegsphase, Orientierungsphase, Arbeitsphase und Abschlussphase (Eckart et al. 2018, S. 127), die mithilfe der Veranstaltungsreihe strukturiert werden.

\section{Schritt 1: Erstellung des Zeitplans für die Exchange-Veranstaltungsreihe}

Um den Zeit- und Arbeitsplan festzulegen, muss entschieden werden, aus wie vielen Veranstaltungen die Reihe besteht und welche Projektschritte dadurch definiert werden. Dies kann in jedem Forschungsprojekt variieren. Zur Vorbereitung der jeweils nächsten Veranstaltung hat sich im Reallabor SRB ein Rhythmus von neun Monaten bewährt. Ein kürzerer Rhythmus, beispielsweise von einem halben Jahr, kann zur Überforderung der externen Akteure (vor allem der Praxisakteure) aufgrund deren begrenzter Zeitbudgets führen und erfordert zusätzliche Ressourcen im Projektteam, da in diesem Fall die Aufgaben des Veranstaltungsmanagements und der inhaltlichen Forschungsarbeit personell getrennt werden müssen. Ein längerer Rhythmus als neun Monate kann dagegen eine Reduktion des Interesses der externen Praxisakteure mit sich bringen und bietet gleichzeitig eine zu schmale Kommunikationsbasis für den internen Kreis, was wiederum den Aufbau des Vertrauensverhältnisses für eine gemeinsame konstruktive Arbeit gefährdet. Ausgehend von einem neunmonatigen Rhythmus ergibt sich ein Projektverlauf, der im Folgenden exemplarisch für ein dreijähriges und für ein fünfjähriges Forschungsprojekt dargestellt wird.

Für ein dreijähriges Forschungsprojekt sind drei bis vier Veranstaltungen sinnvoll, je nachdem, wie viel Bedarf für die projektinterne Vor- und Nachbereitung zu Beginn und zum Ende des Forschungsprojekts besteht. Bei drei Veranstaltungen plant man die erste Veranstaltung am Ende der Einstiegsphase (Veranstaltung 1: Tür-Öffnerin, s. Abschnitt 3.1), die zweite in der Arbeitsphase (Veranstaltung 3: Weg-Begleiterin, s. Abschnitt 3.3) und die dritte in der Abschlussphase 


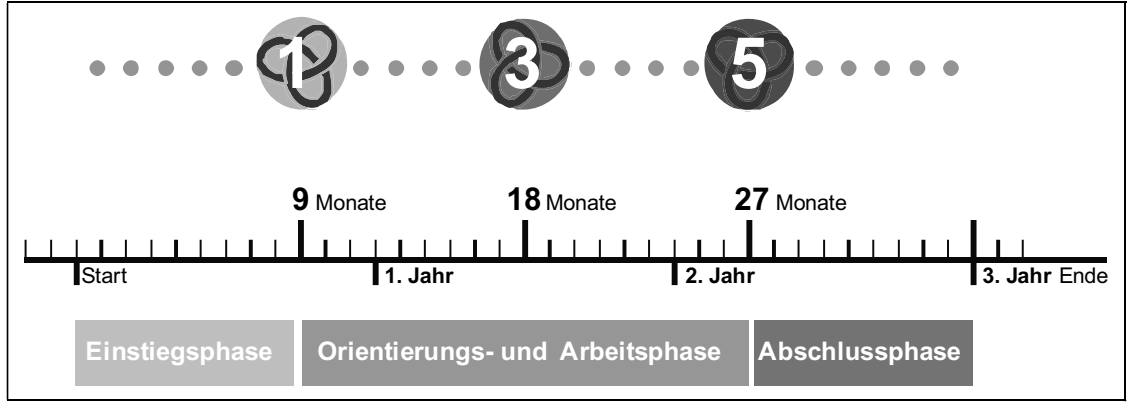

Abbildung 1: Zeitplan eines dreijährigen Forschungsprojekts mit drei Veranstaltungen. (C) Reallabor SRB.

(Veranstaltung 5: Ziel-Beschreiterin, s. Abschnitt 3.5). Dadurch stehen sowohl zu Beginn als auch zum Ende des Forschungsprojekts jeweils neun Monate für die Vor- und Nachbereitung zur Verfügung (s. Abb. 1).

Für ein dreijähriges Forschungsprojekt mit zweistufigem Antragsverfahren sind vier Veranstaltungen zu empfehlen. Je nachdem kann zu den bereits genannten Veranstaltungen optional Veranstaltung 2: Weg-Bereiterin (s. Abschnitt 3.2) oder Veranstaltung 4 Ziel-Erspäherin (s. Abschnitt 3.4) eingesetzt werden (s. Abb. 2). Die Weg-Bereiterin ermöglicht eine Rückkopplung über die Ausrichtung des Projekts mit externen Akteuren und die Integration von deren Wissen noch während der Orientierungsphase. Die Ziel-Erspäherin dagegen unterstützt die gemeinsame Wissensproduktion und betont somit die Arbeitsphase. Aufgrund des zweistufigen Antragsverfahrens muss die Einstiegsphase kürzer ausfallen, so dass sie bereits nach vier Monaten durch die erste Veranstaltung abzuschließen ist. Entsprechend verbleiben nach der letzten Veranstaltung noch fünf Monate für die Finalisierung des Forschungsprojekts.

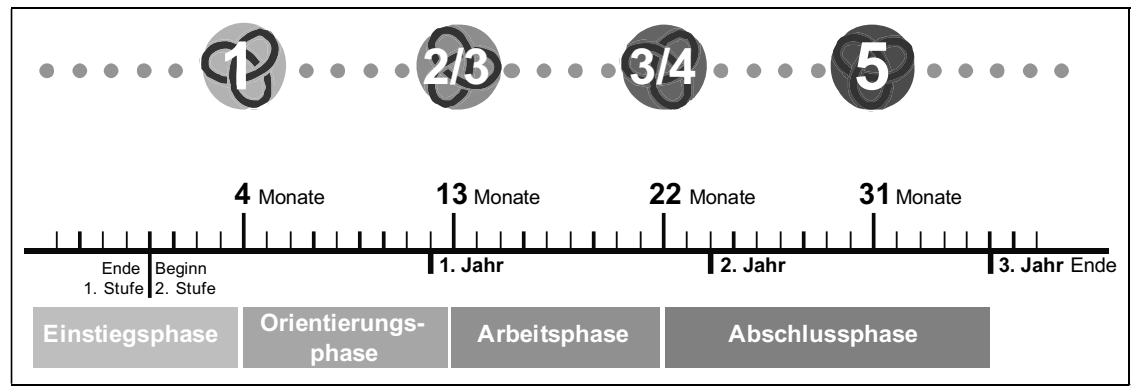

Abbildung 2: Zeitplan eines dreijährigen Forschungsprojekts mit vier Veranstaltungen. (C) Reallabor SRB. 


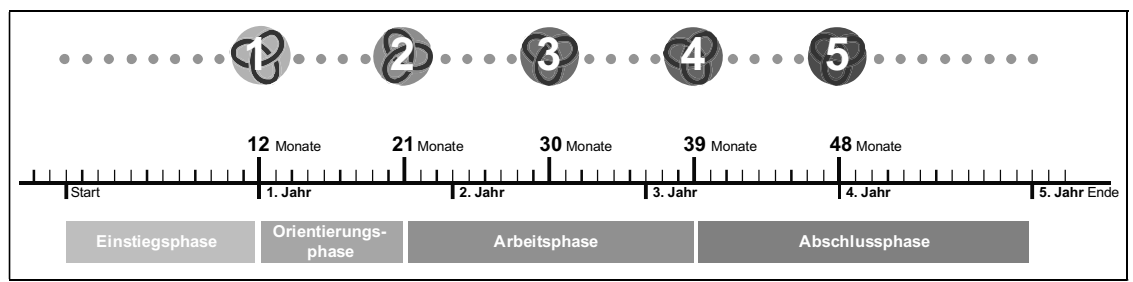

Abbildung 3: Zeitplan eines fünfjährigen Forschungsprojekts mit fünf Veranstaltung. (C) Reallabor SRB.

Für ein fünfjähriges Forschungsprojekt sind fünf bis sechs Veranstaltungen möglich (s. Abb. 3 und 4). Somit wird jede Projektphase durch mindestens eine Veranstaltung definiert, wobei die Arbeitsphase durch zwei oder drei Veranstaltungen gegliedert werden kann, das heißt, Veranstaltung 4: Ziel-Erspäherin (s. Abschnitt 3.4) wird bei insgesamt sechs Veranstaltungen doppelt eingesetzt (s. Abb. 4). Durch die Dopplung ist es möglich, jeweils verschiedene Themenschwerpunkte für die gemeinsame Wissensproduktion mit den externen Akteuren zu setzen.

Eine zusätzliche, sechste Veranstaltung wird insbesondere für Forschungsprojekte mit einem zweistufigen Antragsverfahren empfohlen. Bei fünf Veranstaltungen ergibt sich eine Einstiegsphase (und damit Vorbereitungszeit) von einem Jahr bis zur ersten Veranstaltung. Gleiches gilt für die Abschlussphase am Ende. Bei sechs Veranstaltungen verkürzt sich die Zeit bis zur ersten Veranstaltung auf sieben Monate, und am Ende verbleiben noch acht Monate für die Finalisierung des Forschungsprojekts.

Durch den bewussten Einsatz der Veranstaltungen als Meilensteine zur Definition der Projektphasen sowie durch die entsprechende Kommunikation nach innen und außen wird die Strukturierung des Forschungsprozesses sowohl für das interne Projektteam als auch für die externen Akteure transparent und nachvollziehbar.

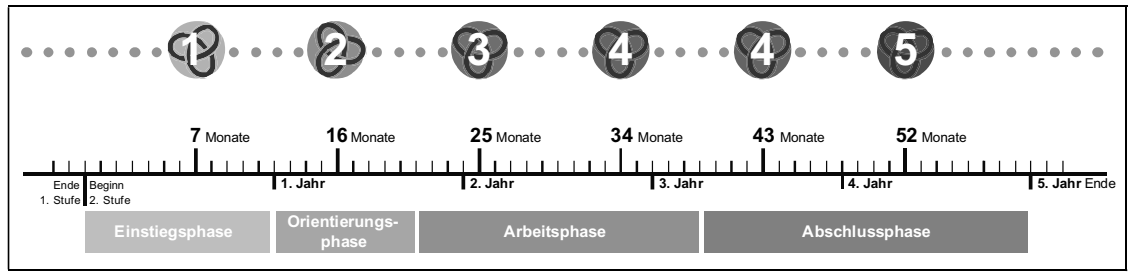

Abbildung 4: Zeitplan eines fünfjährigen Forschungsprojekts mit sechs Veranstaltungen. (C) Reallabor SRB. 


\section{Schritt 2: Definition von Ort, Raum und Zeit der Veranstaltungen}

Jede Veranstaltung ist zweitägig, wobei das Symposium am ersten Tag und der Reflexionsworkshop am zweiten Tag stattfinden. Es empfiehlt sich, bereits zu Beginn des Projekts festzulegen, wo und wann genau die Symposien und internen Reflexionsworkshops stattfinden sollen. Neben der frühzeitigen Suche und Reservierung geeigneter Räumlichkeiten ist dabei vor allem relevant, dass die Veranstaltungsorte für alle Beteiligten (interne wie externe) gut erreichbar sein müssen. Bei der Festlegung des Zeitpunkts sind Wochentag und Uhrzeiten von Beginn und Ende mit besonderer Sorgfalt unter Berücksichtigung der relevanten internen und externen Akteure aus Wissenschaft und Praxis zu wählen. Die Parameter bezüglich Ort, Raum und Zeit sollten möglichst zu Beginn des Forschungsprojekts oder bereits während der Antragstellung definiert werden, weil sie ein wesentlicher Bestandteil der Forschungsplanung und der Budgetierung sind.

\section{Schritt 3: Inhaltliche Vorbereitung jeder Veranstaltung als Meilenstein}

Die Veranstaltungen sind Meilensteine und dienen der regelmäßigen Standortbestimmung innerhalb des Forschungsverlaufs. Das heißt, die Forscher(innen) müssen für jeden Meilenstein ihren aktuellen Stand des jeweiligen Teilprojekts und des Gesamtprojekts dokumentieren und präsentieren können. Dieser Stand findet Eingang in die Vorträge der Symposien. Dafür müssen die Vorträge in einer Sprache formuliert werden, die für alle externen Akteure verständlich ist. Zudem werden der Stand der Arbeit, aktuelle Schwierigkeiten sowie die geplanten weiteren Schritte im internen Reflexionsworkshop dargelegt und gemeinsam besprochen. Die dadurch entstehende Pflicht einer regelmäßigen Reflexion, Aufbereitung und Berichterstattung unterstützt die Steuerung des Forschungsprozesses und die inhaltliche Vernetzung der Teilprojekte. Damit die Veranstaltungsreihe diese Funktion erfüllen kann, gilt es, die Erwartungen an die Vorbereitung der einzelnen Veranstaltungen im Antrag oder spätestens zu Beginn der Einstiegsphase auszuformulieren.

\section{Schritt 4: Dokumentation der Veranstaltungen}

Im Rahmen der einzelnen Veranstaltungen gilt es, ,Produkte ‘ und Diskussionen des Symposiums sowohl fotografisch als auch mittels Ton- und Videoaufnahmen $\mathrm{zu}$ dokumentieren, um sie als (gemeinsam) generiertes Wissen in die Forschung einfließen zu lassen und um sie für Pressearbeit und Dokumentationszwecke zu nutzen, z. B. für Präsentationen des Projekts auf der Webseite. Im Zuge der Projektplanung sind ggf. entsprechende forschungsethische Genehmigungen einzuholen, und es ist festzulegen, wie das diesbezügliche Einverständnis der Teilneh- 
mer(innen) eingeholt und dokumentiert wird. Die genaue Art der Dokumentation wiederum ist abhängig von den Methoden, die während der Veranstaltungen eingesetzt werden. Näher erläutert wird die Dokumentationsart im Folgenden jeweils in den Beschreibungen der einzelnen Veranstaltungen unter Aufgabenpaket 6 „Dokumentieren und Auswerten“ in den Abschnitten 3.1-3.5. Die Dokumentation der internen Reflexionsworkshops wiederum erfolgt nur zur internen Verwendung. Sie erfolgt, indem protokolliert wird, was im Team erarbeitet und besprochen wird. Auch darauf wird, soweit Bedarf besteht, jeweils unter Aufgabenpaket 6 ,Dokumentieren und Auswerten“ näher eingegangen.

\section{Schritt 5: Erstellung des Projektplans (Zusammenfassung)}

Aus den zuvor erläuterten Meilenstein- und Verknüpfungsfunktionen der nun ins Auge gefassten Exchange-Veranstaltungsreihe ergibt sich der Projektplan zur Durchführung der transdisziplinären Arbeit und der Synthesebildung im Forschungsprojekt. Abb. 5 zeigt einen idealisierten Projektplan für ein Forschungsprojekt, das aus zwei Teilprojekten besteht. Senkrecht in der Mitte ist die Veranstaltungsreihe, die dem Ganzen Halt und Struktur gibt. Die fünf Veranstaltungen bilden die Verknüpfungspunkte der Wissensintegration. Die Pfeile stellen die Wissensbestände und Impulse dar, die aus den Teilprojekten in die Veranstaltungen einfließen. Zusätzlich zu den Inhalten der Teilprojekte wirken auch die Erfahrungen der externen Akteure aus Wissenschaft und Praxis sowie die Dynamik im Praxisfeld auf die Veranstaltungen ein (gepunktete Pfeile). Die Erkenntnisse aus den Veranstaltungen wiederum fließen zurück in die Teilprojekte. Die Grafik veranschaulicht, wie das Design eines transdisziplinären Forschungsprojekts zu denken ist, wenn es mit einer Exchange-Veranstaltungsreihe strukturiert wird. Bei der Erstellung des Projektplans für ein konkretes Projekt sind die Wissensbestände und Impulse, die in der Grafik durch die Pfeile nur symbolisiert werden, inhaltlich zu beschreiben. Diese Inhalte sind im Projektverlauf regelmäßig fortzuschreiben. 


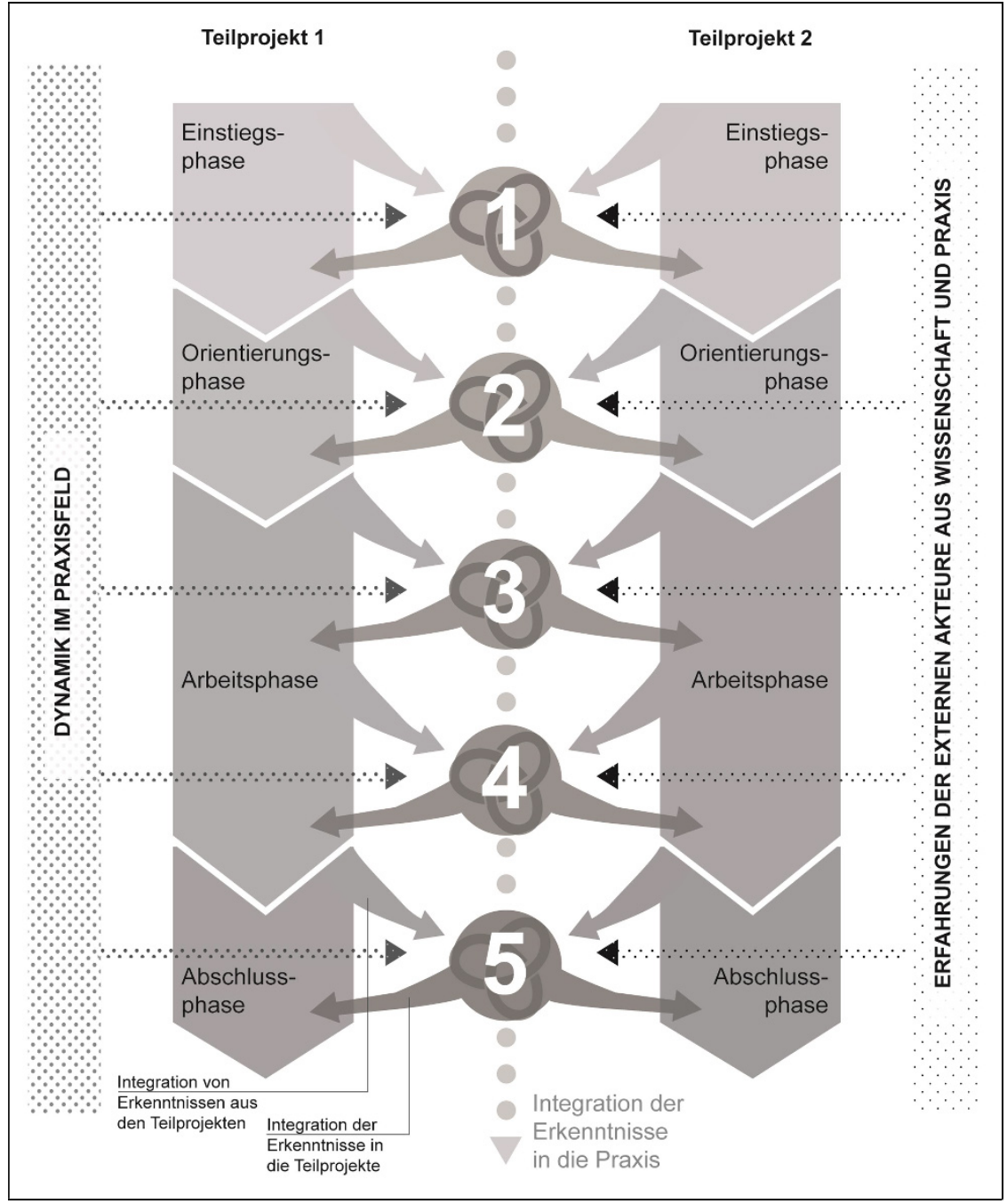

Abbildung 5: Idealisierter Projektplan, bei dem eine Exchange-Veranstaltungsreihe das ,Gerüst‘ für die Forschungsplanung bildet. (C) Reallabor SRB. 


\section{Die einzelnen Veranstaltungen der Exchange-Veranstaltungsreihe}

In diesem Kapitel wird die Planung der einzelnen Veranstaltungen der ExchangeVeranstaltungsreihe dargestellt. Jede Veranstaltung setzt einen eigenen Schwerpunkt und dient spezifischen Zielen (s. Abschnitte 3.1-3.5). Sie besteht immer aus zwei Teilen: einem öffentlichen Symposium am ersten Tag und einem internen Reflexionsworkshop am folgenden Tag (zu letzterem s. Abschnitt 3.6). In der Exchange-Veranstaltungsreihe werden fünf Veranstaltungen unterschieden, deren Kombination abhängig von der Dauer und den Schwerpunkten eines Projekts festgelegt wird (s. Abschnitt 2.2), wobei Veranstaltung 2 und Veranstaltung 4 bei einem dreijährigen Forschungsprojekt optional sind:

- Veranstaltung 1: Tür-Öffnerin (Abschnitt 3.1)

- Veranstaltung 2: Weg-Bereiterin (optional) (Abschnitt 3.2)

- Veranstaltung 3: Weg-Begleiterin (Abschnitt 3.3)

- Veranstaltung 4: Ziel-Erspäherin (optional) (Abschnitt 3.4)

- Veranstaltung 5: Ziel-Beschreiterin (Abschnitt 3.5)

Gemeinsam sind allen Veranstaltungen der grundlegende zeitliche Aufbau, die Intention des Reflexionsworkshops sowie, dass die Symposien und die Reflexionsworkshops dokumentiert werden (s. Kapitel 2).

Während alle Symposien einen ähnlichen Start haben, unterscheiden sie sich nicht nur hinsichtlich ihrer Ziele und Themen, sondern auch hinsichtlich der eingesetzten Beteiligungsmethoden (s. zu den Unterschieden auch Tab. 2 in Abschnitt 3.7). Aus diesen Unterschieden ergeben sich innerhalb von sechs immer gleichen Aufgabenpaketen spezifische Planungsaufgaben für jede Veranstaltung. Diese sechs Aufgabenpakete (AP) sind:

- AP 1: Identifizieren und Erreichen der Akteure

- AP 2: Inhaltliche Impulse aus dem Projekt bestimmen

- AP 3: Vorbereitungen speziell für externe Akteure treffen

- AP 4: Beteiligungsmethodik definieren

- AP 5: Wertschätzung durch Atmosphäre und Stimmung vermitteln

- AP 6: Dokumentieren und Auswerten 
Im Folgenden werden Ausrichtung und Planung der fünf Veranstaltungen beschrieben. Wobei letzteres anhand dieser sechs Aufgabenpakete erfolgt (bei AP 4 jeweils ergänzt um ein Beispiel aus dem Reallabor SRB, s. SRB-Boxen).

\subsection{Veranstaltung 1: Tür-Öffnerin}

Zur Gewinnung von Praxispartnern bedarf es einer Vorstellung der Inhalte und Ziele des Forschungsprojekts, um dieses zu bewerben und Praxisakteure über die aktuellen wissenschaftlichen Fragestellungen rund um das Projektthema zu informieren. Hier setzt die Veranstaltung $1 \mathrm{zu}$ Beginn des Projektverlaufs (Einstiegsphase) an. Ziel dieser Veranstaltung ist zum einen die Gewinnung und Einbindung von (weiteren) Praxisakteuren für die und in die transdisziplinäre Zusammenarbeit und Generierung von Wissen (s. zur Generierung von Wissen Eckart et al. 2018, S. 116). Zum anderen ist es die Vorstellung des Projektteams und dessen Expertise. Aus der Projektperspektive besteht der Hauptnutzen dieser Veranstaltung in der Legitimierung des Projekts als Akteur in der Forschungs- und Praxislandschaft sowie im Aufbau eines Netzwerks für einen späteren Austausch. Zudem wird der Grundstein für eine vertrauensvolle und kooperative Zusammenarbeit im Projekt gelegt.

\subsubsection{Steckbrief Tür-Öffnerin}

Wissensintegration und Bildungsziele: Durch den Einsatz dieser Veranstaltung wird insbesondere das Zielwissen externer Akteure in das Projekt und dessen Zielstellungen integriert. Externe Akteure (Praxisakteure, externe Forscher(innen), Student(inn)en, ggf. weitere Personen) werden sowohl informiert als auch konsultiert. Gleichzeitig profitieren diese von einem persönlichen Wissenserwerb sowie vom Aufbau neuer Kontakte und vom Austausch nicht nur mit dem Projektteam, sondern auch untereinander.

Ablauf und Rollen: Zu Beginn geben Kurzvorträge aus dem Projektteam Einblicke in das Forschungsprojekt. Anschließend erhalten die Teilnehmer(innen) die Möglichkeit, sich selbst ein Bild des Projekts zu machen und mit dem Projektteam zu diskutieren. Rolle der verschiedenen Akteure:

- Moderation: Projektteam

- Impulsreferate: Forscher(innen) aus dem Projektteam

- Teilnehmer(innen): Nehmen als interessiertes Publikum teil, haben darüber hinaus keine spezifische Rolle

Nutzen für externe Praxisakteure:

- Erweiterung und Pflege ihres Netzwerks, Austausch mit Peers

- Horizonterweiterung, Erwerb neuen Wissens 
Fragen, die die Inhalte der Tür-Öffnerin definieren: Was ist das Forschungsthema? Was ist das Forschungsvorhaben? Wer ist das Projektteam? Welche Expertise bringt das Projektteam mit? Welche Rolle wird das Projektteam im Feld einnehmen? Wie reagieren die externen Akteure auf das Projekt?

Voraussetzungen, die für den Einsatz der Tür-Öffnerin erfüllt sein müssen:

- Projektteam steht fest.

- Forschungsthemen und Fragestellungen sind definiert.

- Relevante Akteure im Feld (Praxis und Forschung) sind identifiziert.

\subsubsection{Planungsaufgaben Tür-Öffnerin}

AP 1 (Identifizieren und Erreichen der Akteure): Das Projektteam führt eine Akteursanalyse durch (s. Eckart et al. 2018, S. 120ff.; von Interesse sind sowohl Praxisakteure als auch Forscher(innen), die nicht im Projektteam involviert sind). Um die Akteure bestmöglich zu erreichen, werden verschiedene Formen der breiten Bewerbung des Symposiums eingesetzt: persönliche Einladungen per Post; Einladungen per E-Mail; Ankündigen über Online-Portale sowie auf den Webseiten der beteiligten Institutionen; Aushänge und Flyer in Hochschulen, Schulen, Läden, Gemeindeverwaltungen etc.

AP 2 (Inhaltliche Impulse aus dem Projekt bestimmen): Vertreter(innen) des Projektteams geben Einblicke in die Forschung zum Thema und stellen das Projekt, die Relevanz der Forschung und die Forschungsfragen dar. Sie erläutern die Projektstruktur und die Arbeitsweise im Projektteam. Die Nutzung von Zeitungsartikeln und prägnanten Zitaten aus der Presse dienen dazu, die Relevanz und die Aktualität der Thematik herauszustellen. In der Planung gilt es, die Elemente und Botschaften zu identifizieren, die präsentiert werden sollen, und dazu Konsens im Projektteam herzustellen.

AP 3 (Vorbereitungen speziell für externe Akteure treffen): Vorträge von Expert(inn)en aus dem Projektteam können als Publikumsmagnet fungieren, sofern bekannte Forscher(innen) oder Praxispartner zum Team gehören. Dies dient sowohl dazu, die Attraktivität der Veranstaltung zu steigern, als auch dazu, die Legitimierung und Anerkennung des Projekts als Ganzes beziehungsweise der Beteiligten als Expert(inn)en im Themenbereich zu erhöhen. Fehlt im Team ein solcher ,Magnet', gilt es zu überlegen, mit welchen anderen Mitteln diese erste Veranstaltung für externe Akteure besonders attraktiv gemacht werden könnte.

AP 4 (Beteiligungsmethodik definieren): Wichtig ist es bei dieser Veranstaltung, einen Ausgleich zu schaffen zu den passiv-aufnehmenden Formen der Kommunikation, d. h., Raum zu schaffen und Zeit zu bieten für informelle Gespräche und spontane Begegnungen. Eine gute Möglichkeit, sich selbst ein Bild von dem Pro- 
jekt zu verschaffen, bietet eine Ausstellung (siehe SRB-Box 1). Während Vorträge und Diskussionsrunden Inhalte und den Rhythmus vorgeben, eignet sich zur ,Aktivierung' der Teilnehmer(innen) eine Beteiligungsmethode, die speziell den Austausch der Teilnehmer(innen) untereinander und mit dem Projektteam anregt sowie den Rhythmus des Geschehens den Teilnehmer(inne)n anpasst. Für die Tür-Öffnerin eignet sich im Besonderen ein Markt der Möglichkeiten. Dies ist ein offenes Format, das allen Beteiligten einen Raum für selbstgesteuerte Kommunikation bietet. Der informelle Charakter der ,Marktstände', die verschiedene Aspekte des Forschungsthemas und Projekts zeigen, befördert eine entspannte Atmosphäre, die zum Austausch zwischen allen Beteiligten einlädt. Auf diese Weise können zum einen Forschungsinhalte individuell kennengelernt werden, zum anderen erhalten die einzelnen Akteure die Möglichkeit, sich persönlich untereinander zu vernetzen.

SRB-Box 1: Sich gegenseitig kennen und schätzen lernen in entspannter Atmosphäre: Akteure unterhalten sich über denkbare Ansätze für die Weiterentwicklung von Schulen

Im Reallabor SRB wurde eine Ausstellung als Markt der Möglichkeiten organisiert, um das Projekt im Rahmen des Symposiums zu präsentieren (s. Abb. 6). Der Markt der Möglichkeiten konnte vor, während und nach dem Symposium von den teilnehmenden externen Akteuren besucht werden. An Posterwänden, die thematisch den einzelnen Elementen des Projekts zugeordnet waren, standen Mitglieder des Projektteams für Diskussionen bereit. Sie kamen mit den ,Marktbesucher(inne)n' ins Gespräch und konnten sich so mit diesen über das Forschungsvorhaben, die Relevanz der Fragestellungen in der Praxis sowie mögliche weitere relevante, aber im Projekt noch nicht enthaltene Aspekte austauschen. Es wurden Arbeiten eines studentischen Wettbewerbs ausgestellt, die mögliche Ansätze für ein Transformationsvorhaben einer Schule zeigten, in Form von Plänen und sinnlich erfahrbaren dreidimensionalen Modellen. Die Student(inn)en präsentierten dazu selbst an ihrem ,Marktstand'ihre Ideen und standen für Fragen Interessierter bereit. Die interessengesteuerten Diskussionen zwischen Teammitgliedern und ,Marktbesucher(inne)n' stellten einen niederschwelligen Zugang zu dem Thema dar und unterstützten eine lockere Atmosphäre. Der Markt der Möglichkeiten wurde im Programm mit einer längeren Pause verbunden. Durch die Nähe der ,Marktstände' zu Stehtischen, an denen gegessen werden konnte, entstand ein ,reges Treiben', und zusätzlich unterstützte die Verbindung von Verpflegung und Diskussion eine entspannte Atmosphäre. Um der Pause eine Struktur zu verleihen, wurde ein Gong (analog zur Schulglocke) eingesetzt. Dieser ertönte drei Mal, nach jeweils 15 Minuten. Wer wollte, konnte dann an einen anderen ,Marktstand' oder Stehtisch zum informellen Gespräch wechseln. Dieser Gong ermöglichte eine zwanglose Strukturierung der Pause, so dass sich die Teilnehmer(innen) zurückziehen und später wieder zum ,Marktgeschehen“ dazustoßen konnten. An einzelnen ,Marktständen' gab es auch kurze Vorträge, die jeweils kurz nach dem Gongschlag begannen. Die Veranstaltung stellte so einen gelungenen Einstieg als ,Tür-öffner' in das Forschungsthema und das Forschungsprojekt für die externen Akteure dar. 

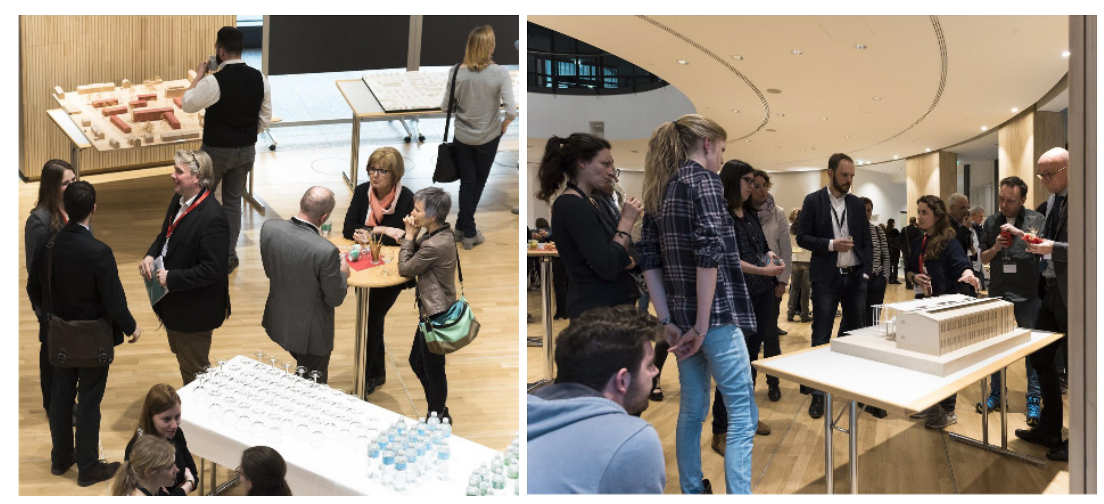

Abbildung 6: Austausch zwischen den Teilnehmer(inne)n und Teammitgliedern auf dem Markt der Möglichkeiten der Veranstaltung „Old School - Neues Lernen Vol. 2" des Reallabors SRB. Die auch räumliche Verbindung von Verpflegung und Diskussion erlaubte nicht nur eine Vielzahl von Gesprächen, sondern auch eine individuell gesteuerte Auseinandersetzung mit dem Thema. (C) Reallabor SRB 2017.

AP 5 (Wertschätzung durch Atmosphäre und Stimmung vermitteln): Die erste Veranstaltung dient dem Aufbau eines abgegrenzten Ortes, der Vertrauen und Offenheit unter allen Akteuren schafft. Da die Kommunikationsform den Charakter der Situation prägt, eignet sich ein Markt der Möglichkeiten in besonderer Weise, weil er sich dazu anbietet, eine offene Beteiligung sicherzustellen und soziale Ausschlussformen zu vermeiden (s. zur Wahl der Methoden Bergold und Thomas 2010, S. 338; zu offener Beteiligung/Transparenz Benighaus und Renn 2016, S. 80ff.). In der Durchführung ist ein besonderes Augenmerk auf Teilnehmer(innen) zu richten, die ,verloren' herumstehen. Für solche Fälle gilt es, bei der Planung eine Vorgehensweise und Zuständigkeiten zu vereinbaren.

AP 6 (Dokumentieren und Auswerten): Für die Ergebnisdokumentation des Markts der Möglichkeiten eignen sich Videoaufnahmen und Fotos (s. zum Einsatz solcher Dokumentationsmethoden Hofmann 2014, S. 45ff.). Fotos dienen im Falle der Tür-Öffnerin vorwiegend der Dokumentation der Veranstaltung mit Blick auf die Kommunikation nach außen. Um die inhaltlichen Ergebnisse zu erfassen, bieten sich vor allem Gedächtnisprotokolle an, die im Nachgang von jenen verfasst werden, die an den ,Marktständen“ als Ansprechpersonen bereitstanden. In der Planung gilt es, Kriterien und die inhaltliche Stoßrichtung dieser Protokolle festzulegen. 


\subsection{Veranstaltung 2: Weg-Bereiterin (optional)}

Gezielt die Erfahrungen, Erkenntnissen und Herausforderungen der externen Praxisakteure einzuholen und zu diskutieren, erlaubt es, die Praxisrelevanz der Forschungsthemen $\mathrm{zu}$ erhöhen, indem diese mit den Bedürfnissen der Praxis abgestimmt werden. Dem dient in der Orientierungsphase (zwischen Anfang und Mitte des Projektverlaufs) die Veranstaltung 2. Ziel dieser Veranstaltung ist es, Praxisakteure im Forschungsprozess mitzunehmen und die Forschungsfragen auf die aktuelle Praxissituation anzupassen. Der Nutzen dieser Veranstaltung für das Projekt liegt also vor allem darin, die Bedürfnisse der Praxis wahrzunehmen. Da$\mathrm{zu}$ ist eine transparente Darstellung des Forschungsstands notwendig, die ergänzt wird um Impulsvorträge von Praxispartnern, die die Teilnehmer(innen) aus ihrer Perspektive über die Auswahl und Begründung der einzelnen Schwerpunkte im Projekt informieren, über die Forschungsmethoden sowie ggf. die Auswahl und Bedeutung der Fallbeispiele.

\subsubsection{Steckbrief Weg-Bereiterin}

Wissensintegration und Bildungsziele: Durch den Einsatz dieser Veranstaltung werden Wissen und Erfahrungen der Praxispartner (z. B. aus den im Projekt behandelten Fallbeispielen), die im besten Fall durch eigene vorausgegangene Transformationsprozesse bereits Erfahrungen sammeln konnten, zur Verfügung gestellt. Dieses Wissen kann inner- wie außerhalb des Projekts sowohl in der Forschung als auch in der Praxis integriert werden. Die anwesenden Forscher(innen) können ihre Kompetenzen mithilfe der Lösungsansätze aus der Praxis entwickeln, und umgekehrt können die anwesenden Praxisakteure ihre Kompetenzen bezogen auf das Wissen der im Projekt vertretenen (inter-)disziplinären Perspektiven verfolgen.

Ablauf und Rollen: Im ersten Symposiumsteil geben Vorträge den Teilnehmer(inne)n Einblicke in Forschung und Praxis. Im zweiten Teil werden die Teilnehmer(innen) mit in die Diskussion einbezogen. Raum für Gespräche in kleineren Gruppen bietet diesmal ein anschließendes Catering. Rolle der verschiedenen Akteure:

- Moderation: Projektteam

- Impulsreferate: Praxispartner (wenn möglich solche, die Fallbeispiele vertreten)

- Teilnehmer(innen): Nehmen an einer gesteuerten Diskussionsrunde teil

Nutzen für externe Praxisakteure:

- Pflege ihres Netzwerks, Austausch mit Peers

- Einblicke in die Perspektive und Erfahrungen Dritter, daraus Impulse für die eigene Arbeit 
Fragen, die die Inhalte der Weg-Bereiterin definieren: Wie wird geforscht? Wie soll der Forschungsprozess aussehen? Welche Methoden sollen eingesetzt werden (ggf.: Welche Fallbeispiele bilden den Untersuchungsgegenstand?) und sind diese geeignet?

Voraussetzungen, die für den Einsatz der Weg-Bereiterin erfüllt sein müssen:

- Falls mit Fallbeispielen gearbeitet wird: Fallbeispiele für die Forschung sind ausgewählt.

- Erste Forschungserfahrungen, erste Transformationserfahrungen liegen vor.

\subsubsection{Planungsaufgaben Weg-Bereiterin}

AP 1 (Identifizieren und Erreichen der Akteure): Der Kreis der Teilnehmer(innen) entspricht dem Kreis, der für Veranstaltung 1 eingeladen wurde. Falls Veranstaltung 2 realisiert wird, kann der Kreis gezielt um Akteure erweitert werden, die in Veranstaltung 1 , gefehlt' haben.

AP 2 (Inhaltliche Impulse aus dem Projekt bestimmen): In der Veranstaltung stellen Praxispartner ihre für das Projekt relevanten Fallbeispiele/Themen/Erfahrungen vor. Diese sollten so aufbereitet und vorgetragen werden, dass sie leicht verständlich und spannend erzählt werden und das deutlich wird, weshalb diese für das Projekt ausgewählt wurden. Letztendlich ist das Ziel der Vorstellung nicht nur, dass die Teilnehmer(innen) Einblicke in das Projekt gewinnen, sondern auch, dass sie von Erfahrungen, die aus existierenden Beispielen stammen, lernen und sie in ihre Kontexte übertragen können. In der Vorbereitung muss geklärt werden, welcher Fokus sich eignet, um das Wissen und die Erfahrungen der Teilnehmer(innen) zu aktivieren, die eine Anpassung der Forschung an die Praxis erlauben. Ein wichtiger Schritt ist die Wahl der Themenschwerpunkte bzw. Fallbeispiele für die Veranstaltung. Welche Fallbeispiele oder Themen sind eine gute Grundlage für die Darstellung der Forschungsfragen? Es ist nützlich, dafür, wie bereits bei Veranstaltung 1, die Praxispartner einzubinden, die besonders bekannt oder im Feld etabliert sind. Einen wichtigen Stellenwert hat das Briefing, damit die Impulse in das Symposium den gewünschten Fokus haben.

AP 3 (Vorbereitungen speziell für externe Akteure treffen): Fallbeispiele von Praxispartnern oder aus dem Projektteam können als Publikumsmagnet fungieren, sofern sie spannend aufbereitet und vorgetragen werden. Sie dienen sowohl dazu, die externen Praxispartner als Kooperationspartner stärker einzubinden, als auch, die Praxisnähe, Anwendbarkeit und Verständlichkeit des Projekts zu erhöhen. Gerade zu Projektbeginn, wenn erst wenige oder keine Ergebnisse vorliegen, steigern Fallbeispiele aus existierenden Projekten die Veranstaltungsattraktivität für externe Akteure und zeigen die Vernetzung zu unterschiedlichen Kontexten auf. 
AP 4 (Beteiligungsmethodik definieren): Die Praxispartner können ihre Fallbeispiele in Vorträgen präsentieren. Aber auch eine Ausstellung ist eine ansprechende Methode, um die Fallbeispiele/Themen/Erfahrungen den Teilnehmer(inne)n in visueller Form näherzubringen. Als Methode ebenfalls geeignet ist das sogenannte "Storytelling“, in dem das Fallbeispiel/Thema in eine ,Geschichte“ verpackt wird. Da die Praxisakteure auch in einen strukturierten Austausch treten sollen, werden sie nach den Präsentationen in die Diskussion mit einbezogen. Hierzu sind Beteiligungsformate sinnvoll, die es ermöglichen, Plenumspräsentationen damit zu verbinden, dass Teilnehmer(innen) ihre Erfahrungen einbringen und Rückmeldungen zu den Präsentationen geben (s. zum Diskurs sowie zum Vorgehen bei Beteiligungsformaten Renn und Benighaus 2003, S. 108ff.). Für die Weg-Bereiterin bietet sich daher zum Beispiel das Fishbowl-Format an (auch Aquarium- oder Innen-/Außenkreis-Methode genannt, für eine Beschreibung der Methode s. z. B. United Nations Human Rights 2012; s. SRB-Box 2). Dies ist ein offenes Format, das eine Diskussion vor und mit einem Plenum ermöglicht, dabei aber im Vergleich z. B. zur Podiumsdiskussion die Hierarchie zwischen Podiumsgästen und Publikum aufhebt, indem alle Anwesenden Teil eines Sitzkreises werden. Diese Methode kann eine unbefangene Kommunikation im ,Aquarium“ unterstützen. In der Diskussion können nicht nur die Beispiele der Referent(inn)en diskutiert werden, es ist auch möglich, den Fokus der Diskussion auf Fragen zu richten, wie die Erfahrungen aus der Praxis in der Forschung angewendet werden könnten, so dass die Diskussionsergebnisse in den laufenden Forschungsprozess integriert werden können. Für eine noch stärker auf Wissensintegration zielende Methode ist zum Beispiel das Expertenpuzzle sinnvoll (auch Gruppenpuzzle oder Jigsaw-Methode genannt, für eine Beschreibung der Methode s. z. B. https://www.jigsaw.org, zugegriffen am 30.04.2019), das sich für die zunächst getrennte und dann integrierende Erarbeitung von Wissen zu einem Thema eignet. In einem ersten Schritt arbeiten dabei Praxisakteure in Gruppen zu jeweils einem Teilthema zusammen. Danach werden gemischte Gruppen gebildet, in die jeweils mindestens ein Praxisakteur eines jeden Teilthemas eingebunden ist, und diese Gruppen fügen die Erkenntnisse zu allen Teilthemen zusammen.

\section{SRB-Box 2: Auseinandersetzung auf gleicher Augenhöhe in großer Runde: Durch den Einbezug verschiedener Expertisen entstehen kontroverse Diskussionen zu aktuellen Schulentwicklungen}

In einem der Symposien des Reallabors SRB wurde für die Diskussionsrunde mit Praxisakteuren die Fishbowl-Methode angewandt (s. Abb. 7). Übergreifendes Thema der Diskussion war die Frage „Architektur und Schulkonzepte - eine Symbiose?“ Für den Einstieg in das Thema hielten eine Schulleiterin, ein Schulleiter und ein Architekt Impulsvorträge. Es handelte sich hierbei um Praxispartner aus dem Projekt, die gleichzeitig Vertreter(innen) der im Projekt bearbeiteten Fallbeispiele waren. Sie präsentierten ihre Erfahrungen in Bezug auf die von ihnen angewandten innovativen pädagogischen Kon- 
zepte bzw. auf die Tätigkeit als Schulbauberater. Diese praxisnahen Vorträge boten für die Teilnehmer(innen) einen leichten Einstieg, um die Themen schnell zu erfassen. Nach den Impulsvorträgen ging die Diskussion in großer Runde unter Einbezug des Plenums weiter. Das Format Fishbowl, das als innerer Sitzkreis von Hockern und als mehrere äußere Sitzkreise mit Stühlen eingerichtet war, bot für alle Beteiligten die Möglichkeit, sich unkompliziert einzubringen, Nachfragen zu stellen oder Ergebnisse zu kommentieren und auf die aktuelle Situation im Schulbau zu übertragen.

Zu Beginn der Diskussionsrunde nahmen die drei Referent(inn)en im inneren Kreis Platz und zwei Personen aus dem Projektteam setzten sich zusätzlich dazu. Sie erläuterten zuerst die ,Spielregeln' der Fishbowl und moderierten diese im weiteren Verlauf. Drei Hocker im inneren Kreis blieben für Praxisakteure aus dem Plenum frei. Diese freien Plätze füllten sich schnell, und viele der Teilnehmer(innen) wechselten während des Verlaufs zeitweise in den inneren Kreis, um sich an der Diskussion zu beteiligen. Sie stellten den Referent(inn)en und/oder dem Projektteam Fragen. Die Dynamik der Diskussion veränderte sich positiv, d. h. die Anzahl Fragen nahm im Verlauf der Diskussion deutlich zu, und die Zahl der Fragenden erweiterte sich kontinuierlich. Wertvoll an dieser Vorgehensweise war, dass die verschiedenen Perspektiven und Meinungsbilder der Akteure deutlich wurden und die unterschiedlichen Disziplinen sich untereinander ,erkannten', schätzen lernten und die Meinung der anderen antizipiert wurde. Durch die Methode wurde zudem deutlich, welche Impulse im Anschluss fokussiert und verdichtet werden sollten.

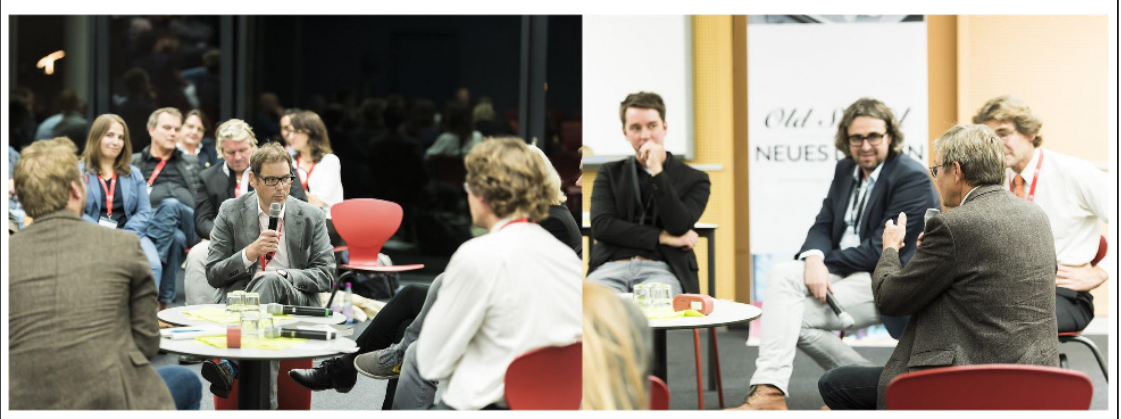

Abbildung 7: Sitzkreise mit Stühlen und Hockern bei der Fishbowl der Veranstaltung „Old School - Neues Lernen Vol. 3“. (C) Reallabor SRB 2017.

AP 5 (Wertschätzung durch Atmosphäre und Stimmung vermitteln): Durch die Impulse von Praxispartnern, die in einer alltagsnahen Sprache vorgetragen werden, kann die Brücke zu den Erfahrungen der Praxisakteure geschlagen werden. Kombiniert mit offenen, hierarchiefernen Diskussionsformaten (s. SRB-Box 2) erhöht dies die aktive Beteiligung der Teilnehmer(innen). Dies wiederum verstärkt die Wahrnehmung der Veranstaltung als Netzwerk- und Bildungsplattform. 
AP 6 (Dokumentieren und Auswerten): Um die Kommentare und Geschichten der Teilnehmer(innen) zu dokumentieren, eignen sich Ton- und Videoaufnahmen. Die Tonaufnahmen bzw. die Tonspur der Videoaufzeichnung sollten transkribiert und im Nachgang ausgewertet werden. Zusätzlich hilfreich sind Notizen zur Diskussion, die am nächsten Tag im Reflexionsworkshop direkt einfließen können. Fotos dienen im Fall der Weg-Bereiterin vorwiegend der Dokumentation der Veranstaltung für die Kommunikation nach außen. Im Reflexionsworkshop werden vor allem die Beiträge aus der Diskussion im Plenum ausgewertet. Die am Projekt Beteiligten nutzen die Feedbackschleife, um in dieser Projektphase nochmals die Themen zu priorisieren und den Projektfokus einzuengen. Damit dies gut gelingt, gilt es, sich im Vorfeld über die Art und Weise sowie den Fokus der Notizen während des Symposiums zu verständigen.

\subsection{Veranstaltung 3: Weg-Begleiterin}

Die Veranstaltung 3 liegt in der Mitte des Projektverlaufs, also in der Arbeitsphase. Ziel dieser Veranstaltung ist es, anhand der Darstellung des Forschungsstandes, d. h. der Erläuterung erster Forschungseindrücke und Erfahrungsberichte aus dem bisherigen Projektverlauf, die externen Akteure zum Nachdenken über die Projektergebnisse anzuregen, diese auf die eigene Arbeit zu übertragen und damit deren Anwendbarkeit zu bewerten. Ein besonderer Fokus liegt hier auf der Rückkoppelung mit dem wissenschaftlichen Diskurs. Diese Rückkopplung der Ergebnisse soll einen Beitrag leisten für eine innovative und dynamische Forschung, die auf die aktuelle Situation der Praxisakteure eingeht. Ziel und Nutzen ist insbesondere die Legitimierung des Projekts als Akteur in der Wissenschaft und ein Testen der Anwendungsorientierung der ersten Projektergebnisse.

\subsubsection{Steckbrief Weg-Begleiterin}

Wissensintegration und Bildungsziele: Veranstaltung 3 ,aktiviert ${ }^{\star}$ vor allem das Wissen der externen Forscher(innen), die durch ihre eigene Forschung bereits über Erfahrungen verfügen, die für das Projekt relevant sind. Dieses Wissen kann sowohl im Projekt als auch in der Praxis integriert werden. Gleichzeitig können damit alle Beteiligten ihr Wissen erweitern.

Ablauf und Rollen: In Inputs geben externe Forscher(innen) einen Überblick über das Forschungsfeld, während die internen Forscher(innen) anhand von Praxisbeispielen die Anwendung und Übertragbarkeit der ersten Forschungsergebnisse aus dem Projekt aufzeigen. Nach der, Vortragsrunde' bietet sich ein Beteiligungsformat an, das eine Übertragung der präsentierten Inhalte auf die Arbeit der Praxisakteure in gemischten Kleingruppen erlaubt. Rolle der verschiedenen Akteure: 
- Moderation: Projektteam, externe Forscher(innen)

- Impulsreferate: Vertreter(innen) des Projektteams, externe Forscher(innen)

- Teilnehmer(innen): Übertragung der Impulse aus der Forschung auf die eigene Arbeit im Praxisfeld

Nutzen für externe Praxisakteure:

- Pflege ihres Netzwerks, Austausch mit Peers

- Impulse aus der Forschung und Einblicke in die Forschung inner- und außerhalb des Projekts

- Impulse für das praktische Handeln in den eigenen Problemstellungen

Fragen, die die Inhalte der Weg-Begleiterin definieren: Wie ist der aktuelle Forschungsstand? Was sind die ersten Erkenntnisse und Erfahrungen, die das Projektteam gesammelt hat? Gibt es weitere Forschungsprojekte oder Initiativen im selben Themenbereich?

Voraussetzungen, die für den Einsatz der Weg-Begleiterin erfüllt sein müssen:

- Erste Erkenntnisse und Erfahrungen aus dem Projekt liegen vor.

- Es besteht Klarheit darüber, welches die für das Projekt besonders spannenden Forschungsfelder und -projekte sind, von denen gelernt werden kann.

\subsubsection{Planungsaufgaben Weg-Begleiterin}

AP 1 (Identifizieren und Erreichen der Akteure): Grundsätzlich wird derselbe Kreis an Akteuren eingeladen wie bei der/den vorangegangenen Veranstaltung(en). Zusätzlich gilt es, gezielt externe Forscher(innen) einzuladen, die dem Projekt spannende neue Impulse geben können. Im Hinblick darauf ist es von Nutzen, kontinuierlich und mit genügend Vorlauf eine Datenbank möglicher Referent(inn)en aufzubauen. Dafür bietet sich eine Literaturrecherche an, die einen Überblick über den aktuellen Stand der Forschung, aber vor allem auch über die aktiven und relevanten Akteure in der Forschungslandschaft gibt. Kriterien zur Auswahl von externen Forscher(inne)n müssen äußerst früh im Projektverlauf gemeinsam festgelegt werden.

AP 2 (Inhaltliche Impulse aus dem Projekt bestimmen): Vertreter(innen) aus dem Projektteam präsentieren den Zwischenstand und erste praxisrelevante Forschungsergebnisse des Projekts. Zur Vorbereitung gehört es, herauszuarbeiten, wie die mittlerweile vorliegenden Teilergebnisse aus dem Projekt so in Bezug zueinander gesetzt werden, dass den Teilnehmer(innen) ein Gesamtbild vermittelt werden kann. 
AP 3 (Vorbereitungen speziell für externe Akteure treffen): Um eine gute Mischung der Teilnehmer(innen) in den Kleingruppen zu unterstützen und ihre spezifische Expertise in Wert zu setzen, hilft es, die Teilnehmer(innen) nach Akteursgruppen ,aufzuteilen' sowie die Zuordnung der Individuen auf den Namensschildern durch kleine Aufkleber mit unterschiedlichen Farben auszuweisen. Die Aufteilung in unterschiedliche Akteursgruppen ist für das spätere Beteiligungsformat wichtig, um eine Mischung der Perspektiven, Erfahrungen und Expertisen zu gewährleisten. Die Zuordnung zu Akteursgruppen fällt leichter, wenn die Teilnehmer(innen) von Beginn an (also ab Veranstaltung 1) entsprechend kategorisiert erfasst werden, wenn also Daten zu den Teilnehmer(inne)n erhoben werden, die Auskunft über Parameter geben, die aus Projektsicht relevant sind.

AP 4 (Beteiligungsmethodik definieren): Um möglichst viele Praxisperspektiven fruchtbar zu machen, bietet sich an, in der Veranstaltung Weg-Begleiterin ein World Café umzusetzen (für eine Beschreibung der Methode s. Brown und Isaacs 2007; Benighaus und Benighaus 2016, S. $216 \mathrm{ff}$; http://www.theworldcafe.com, zugegriffen am 22.04.2019). Dieses Format nutzt die ungezwungene Kaffeehausatmosphäre, um die verschiedenen Teilnehmer(innen) einer Veranstaltung ins Gespräch zu bringen. Das Format kann gut eingesetzt werden, um die Anwendbarkeit der Forschungsergebnisse in der Praxis zu überprüfen. Tische mit jeweils unterschiedlichem Fokus geben die Themen, nach denen sich das Projekt inhaltlich strukturieren lässt, wieder. In der ersten Runde des World Cafés wird auf eine Mischung der Teilnehmer(innen) aus den unterschiedlichen Akteursgruppen geachtet (anhand der Farbcodierung der Namensschilder, s. dazu AP 3). In den folgenden Runden ist jedoch auch eine Zuordnung ausschließlich nach individuellen Neigungen möglich. Die Moderator(inn)en der Café-Tische (,Gastgeber(innen) ${ }^{6}$ ) übernehmen die Aufgabe, innerhalb der Tischgruppe die Diskussion in Fluss zu bringen und die Ergebnisse festzuhalten (s. SRB-Box 3). Mit dem Einsatz von Papiertischdecken, die als Schreibunterlage dienen, werden die Diskussionsbeiträge festgehalten. Die so dokumentierten Ergebnisse in Form von Skizzen, Zeichnungen und Mitschriften können in das Projekt einfließen, zitiert oder auch als solche übernommen werden.

\section{SRB-Box 3: Erste Projektergebnisse mit Praxisakteuren in Kleingruppen reflektieren und auf Anwendbarkeit überprüfen - Diskussion von Handlungsempfehlungen in der Schulbauplanung}

Für das Symposium zum Thema „Planungsprozesse von Schulen - neue Wege für neues Lernen" sollten Handlungsempfehlungen als erste praktische Ergebnisse diskutiert und kommentiert werden. Dazu wurde ein World Café eingesetzt (s. Abb. 8), mit dem eine Kaffeehausatmosphäre erzeugt wurde. Runde Tischinseln, die mit Papiertischdecken gedeckt wurden, dienten sowohl der Dekoration als auch als Schreibunterlage, um die wesentlichen Gedanken direkt mit Buntstift oder Marker festzuhalten. Blumenvasen auf 
den Tischen erzeugten einen informellen Charakter, der zum Verweilen einlud. Eine Café-Etikette, die die Kommunikationsregeln erläuterte, ermunterte die Teilnehmer(innen), ihre eigenen Ansichten und Ideen einzubringen und einander zuzuhören. Von den Teilnehmer(inne)n wurde diese entspannte Atmosphäre positiv wahrgenommen und der Raum zum offenen, ungezwungenen Gespräch sehr geschätzt.

Um die Tischgruppen möglichst heterogen zusammenzusetzen, wurden die Namensschilder anhand der Anmeldung kategorisiert. So erhielten beispielsweise alle Architekt(inn)en einen schwarzen Punkt auf dem Namensschild, alle Mitarbeiter(innen) kommunaler Verwaltungen einen grünen, alle Lehrer(innen) einen blauen Punkt und so weiter. Auf diese Weise konnten sich die Teilnehmer(innen) selbstständig in gemischte Gruppen zusammenfinden. Durch die Begrüßung der jeweiligen Tischgruppe durch die 'Gastgeber(innen)' und die kleine Gruppengröße von sechs bis acht Personen wurde ein intimer Raum geschaffen, um gemeinsam über das jeweilige Thema zu diskutieren. Es war zu beobachten, dass sich die Gruppen schnell in konstruktive Gespräche vertieften. Nach 30 Minuten gab es einen Wechsel der Gruppen. Die ,Gastgeber(innen)' fassten die Diskussion für die neue Gruppe, die sich am betreffenden Tisch einfand, zusammen. Auf diese Weise wurden die Themen und Fragen in den aufeinanderfolgenden Gesprächsrunden immer weiter vertieft. Aus Sicht der Diskussionsteilnehmer(innen) wichtige Überlegungen und Ideen wurden unverzüglich auf den Papiertischdecken festgehalten, um die Inhalte der Diskussion zu dokumentieren. Anschließend wurden im Plenum die Diskussionsergebnisse aller Thementische vorgestellt. Die beschriebenen Tischdecken wurden als Grundlage für den Reflexionsworkshop am folgenden Tag genutzt.

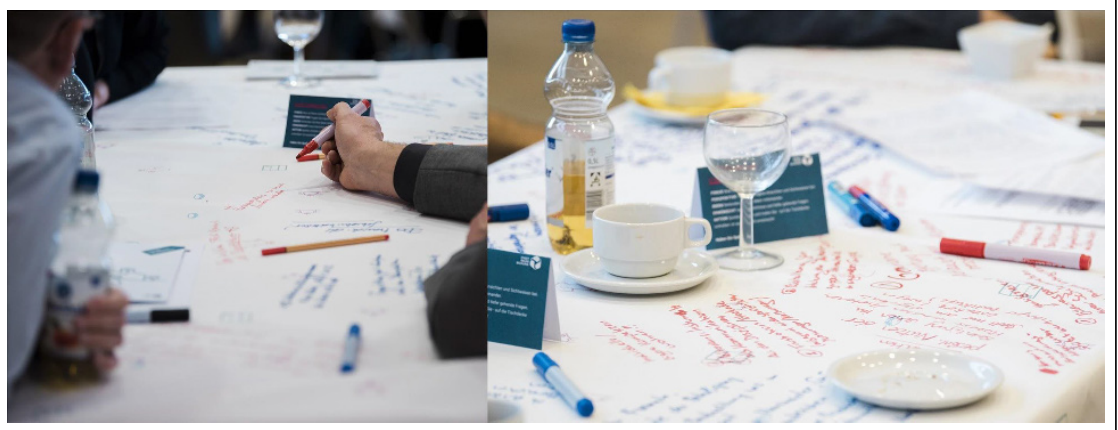

Abbildung 8: Papiertischdecken des World Cafés der Veranstaltung „Planungsprozesse von Schulen - neue Wege für neues Lernen“. Dadurch, dass die Ergebnisse spontan und von allen Diskussionsteilnehmer(inne)n aufgeschrieben werden, ist die Moderation besonders gefordert, weil sie den Gesprächsverlauf konzentriert verfolgen muss, um die Ergebnisse in nächsten World Café-Runden und dann im Plenum wiedergeben zu können.

(c) Reallabor SRB 2017. 
AP 5 (Wertschätzung durch Atmosphäre und Stimmung vermitteln): Ein World Café bietet einen guten Rahmen für einen offenen und ungezwungenen Austausch über die Projektergebnisse. Eine liebevolle Gestaltung des Cafés zeigt eine hohe Wertschätzung der Teilnehmer(innen) seitens der Verantwortlichen der Veranstaltung.

AP 6 (Dokumentieren und Auswerten): Im World Café dienen die Tischdecken der Dokumentation. Diese haben den Charakter von Protokoll-Notizen, die durch das Projektteam ausgewertet werden können. Im Reflexionsworkshop können die auf den Tischdecken festgehaltenen Ergebnisse direkt gesichtet und genutzt werden. Für eine spätere Auswertung hingegen ist es zwingend, ausführliche Protokolle zu erstellen, damit die Tischdecken-Notizen verständlich und die Ergebnisse der Diskussionen nutzbar werden. Dafür wiederum ist es sinnvoll, auch über Tonaufnahmen der an den Tischen geführten Diskussionen zu verfügen. Ebenfalls dienlich sind zeitnahe erstellte Zusammenfassungen des Diskussionsverlaufs an den Tischen durch die jeweiligen, Gastgeber(innen)' . Werden die Ergebnisse der Tische im Plenum vorgestellt und diskutiert, empfiehlt es sich zudem, dies durch Ton- und Videoaufnahmen zu dokumentieren.

\subsection{Veranstaltung 4: Ziel-Erspäherin (optional)}

Zwischen der Mitte und dem Ende des Projektverlaufs setzt Veranstaltung 4 während der Arbeitsphase ein. Diese Veranstaltung fördert vor allem die gemeinsame Wissensproduktion der beteiligten Akteure mit Blick auf die Nutzung der Ergebnisse in der Praxis. In der Weg-Begleiterin fand dieser Schritt schon im Ansatz statt, dieser wird aber in der optionalen Veranstaltung der Ziel-Erspäherin noch verstärkt, indem weitere Teilergebnisse aus dem Projekt vorgestellt und überprüft werden. Ziel der Veranstaltung ist eine systematische Rückkopplung der Erkenntnisse aus der Forschung an die Expertise der Praxisakteure. Zudem werden Praxisakteure, die bisher noch nicht im Netzwerk aufgetreten sind, gezielt eingeladen, um den Akteurskreis gegenüber den bisherigen Veranstaltungen zu erweitern.

\subsubsection{Steckbrief Ziel-Erspäherin}

Wissensintegration und Bildungsziele: In dieser Veranstaltung wird vor allem Transformationswissen der Praxisakteure für das Projekt fruchtbar gemacht, das in das Projekt integriert werden kann. Das Projektteam erwirbt so neues Wissen aus der Praxis. Der Wissensaustausch zwischen dem Projektteam und den externen Akteuren zu diesem Zeitpunkt, zu dem viele Ergebnisse vorliegen, stößt den gemeinsamen Lernprozess an. Diese Co-Produktion wiederum erleichtert es den 
Praxisakteuren, selbst einen persönlichen Wissenserwerb und eine Selbstentwicklung zu verfolgen, was auch ein Empowerment darstellt (s. dazu z. B. Eckart et al. 2018, S. 118).

Ablauf und Rollen: Der erste Teil der Ziel-Erspäherin wird durch Impulsvorträge zum Stand der Arbeit im Projekt eingeleitet. Im zweiten Teil der Veranstaltung werden in kleinen heterogenen Gruppen konkrete praxisbezogene Ansätze erarbeitet. Rolle der verschiedenen Akteure:

- Moderation: Projektteam oder extern beauftragte Moderation

- Impulsreferate: Vertreter(innen) des Projektteams

- Teilnehmer(innen): Entwickeln auf der Grundlage ihrer Expertise und für ihre Problemstellungen Ideen für mögliche Lösungen, indem sie Projektergebnisse auf ihre Situation anwenden

Nutzen für externe Praxisakteure:

- Pflege ihres Netzwerks, Austausch mit Peers

- Einblicke in und Impulse aus der Forschung

- Impulse für das praktische Handeln mit Bezug auf ihre Problemstellungen

- Empowerment und Hinweise, wie sie auch nach Ende des Projekts Transformationsprozesse gestalten können

Fragen, die die Inhalte der Ziel-Erspäherin definieren: Was sind die ersten Zwischenergebnisse der Forschung? Welche Erkenntnisse wurden bereits gewonnen? Wie werden bereits erhobene Daten bis zum Ende ausgewertet?

Voraussetzungen, die für den Einsatz der Ziel-Erspäherin erfüllt sein müssen:

- Wesentliche Teilergebnisse aus dem Projekt liegen vor

- Projektteam hat Überblick über Akteure im Feld, die bisher außen vor blieben, aber mit Blick auf die Praxisrelevanz und Legitimität der Ergebnisse beteiligt werden sollten.

\subsubsection{Planungsaufgaben Ziel-Erspäherin}

AP 1 (Identifizieren und Erreichen der Akteure): Grundsätzlich bleibt der Kreis der Teilnehmer(innen) derselbe wie bei den vorhergegangenen Veranstaltungen. In dieser Veranstaltung wird aber darüber hinaus ein Schwerpunkt in der Identifikation und ,Aktivierung' bisher zu wenig berücksichtigter Akteursgruppen gesetzt, die zusätzlich zum bisherigen Kreis gezielt angesprochen und eingeladen werden sollen. Besonderes Augenmerk liegt dabei auf marginalisierten Akteuren. 
Als ,marginalisierte Akteure" werden Akteure verstanden, die nicht über die Möglichkeiten oder Rahmenbedingungen verfügen, sich aktiv in das Forschungsprojekt einzubringen, beispielsweise Migrant(inn)en, Kinder, Senior(inn)en und andere. Die Frage in der Vorbereitung lautet also: Welche Akteursgruppen fehlen in der Betrachtung bis dato, und wie können diese erreicht werden? Durch die Auswertung der Teilnehmer(innen) der bisherigen Veranstaltungen entsteht ein Überblick über die schon beteiligten Akteure und das wiederum ist eine Grundlage zur Identifizierung der ,Lücken', die mit Blick auf eine umfassendere Beteiligung zu schließen sind.

AP 2 (Inhaltliche Impulse aus dem Projekt bestimmen): Vertreter(innen) aus dem Projektteam präsentieren Erkenntnisse aus dem Projekt, wobei vor allem solche ausgewählt werden, die aus Sicht des Teams ein besonderes Umsetzungspotenzial haben.

AP 3 (Vorbereitungen speziell für externe Akteure treffen): In dieser Veranstaltung gilt es, sorgfältig darauf zu achten, dass für marginalisierte Akteure die Materialien zielgruppenspezifisch vorbereitet werden.

AP 4 (Beteiligungsmethodik definieren): Um Kommunikationshindernisse zwischen dem Projektteam, bisherigen externen Akteuren und neu hinzukommenden marginalisierten Akteuren $\mathrm{zu}$ überwinden, werden niedrigschwellige Interaktionsebenen eingeführt und kreativ fördernde Methoden eingesetzt (s. zu solchen Methoden Hofmann 2014, S. 45ff.). Die gemeinsame Wissensproduktion kann beispielsweise durch die Anwendung der Methode Ideenwerkstatt angeregt werden (s. dazu Rogge 2000, S. 77ff.). Dieses Format motiviert die Teilnehmer(innen), nicht nur ins Gespräch zu kommen und intensiv zu diskutieren, sondern darüber hinaus gemeinsam Wissen und Ideen für die Praxis zu erzeugen (s. auch SRB-Box 4). Um kreativ arbeiten zu können, ist die Wahl des Raums für diese Veranstaltung besonders kritisch. Von Vorteil ist ein größerer Plenumssaal, in dem die Begrüßung, die Inputvorträge und die anschließende Präsentation der Gruppenergebnisse stattfinden kann. Zudem braucht es ausreichend Platz für die Gruppenarbeiten. Um zu verhindern, dass sich die Gruppen bei der Ideenwerkstatt gegenseitig stören, sollten die Werkstätten auf einer großen Fläche mit runden Stehtischen oder Sitzgruppen verteilt sein. Schließlich sollte der Raum ausreichend Platz für das Catering aufweisen. 


\section{SRB-Box 4: Ideenwerkstatt mit Kindern, Jugendlichen und Erwachsenen im Reallabor SRB: beliebte und unbeliebte Orte im Schulgebäude, auf dem Schulhof, auf dem Schulweg}

In einer Ideenwerkstatt mit Schüler(inne)n und Erwachsenen zum Thema „Beliebte und unbeliebte Orte in der Schule“ wurden im Rahmen des Symposiums „Lernen aus der Vergangenheit - Planen für die Zukunft" zwei getrennte Werkstätten durchgeführt (s. Abb. 9). Die Schüler(innen) arbeiteten in kleinen Sitzgruppen zusammen und konnten anhand von Fotografien ihre Lieblingsorte und ihre unbeliebten Orte im Schulgebäude, auf dem Schulhof und auf dem Schulweg beschreiben. Sie entwickelten anschließend Handlungsempfehlungen sowie mögliche Maßnahmen für den weiteren Umgang mit den unbeliebten Orten und zu deren Verbesserung. Sie schrieben die fünf wichtigsten Empfehlungen auf die fünf Seiten von Papphockern. Die Erwachsenen gingen ähnlich vor, hatten aber keine Fotos, die sie nutzen konnten. Sie beschrieben ebenfalls ihre beliebten und unbeliebten Orte im Schulgebäude, auf dem Schulhof und auf dem Schulweg, entwickelten Ideen zur Verbesserung und fassten ihre Ergebnisse in fünf Handlungsempfehlungen zusammen. Die Ergebnisse präsentierten die Schüler(innen) und Erwachsenen vor dem Plenum anhand der Papphocker, die sie in den Gruppen beschrieben hatten. Die entstandene Papphocker-Wand der Schüler(innen) und Erwachsenen zeigte zum Abschluss visuell die Ergebnisse und ließ die Vielfalt der Antworten deutlich werden.

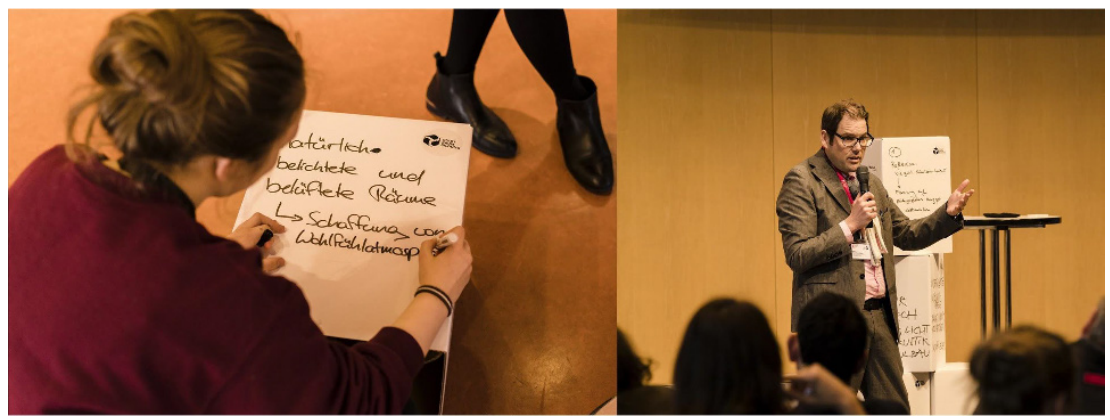

Abbildung 9: Gestaltung der Papphocker und Präsentation der Ergebnisse der Werkstätten auf der Veranstaltung „Old School - Neues Lernen Vol. 4“. Die ,dreidimensionale' Darstellung der Ergebnisse macht diese ,greifbarer'. (c) Reallabor SRB 2018.

AP 5 (Wertschätzung durch Atmosphäre und Stimmung vermitteln): Insbesondere in der Arbeit mit marginalisierten Gruppen ist die Wortwahl wichtig, damit diese nicht eingeschüchtert werden, die Aufgabenstellung verstehen und das Gefühl haben, dass ihre Meinung wertgeschätzt wird. Im Projektteam sollte diskutiert werden, mit welcher Haltung diesen Gruppen begegnet werden soll, und in welchen 
Handlungen sich dies ausdrückt. Zudem ist es wichtig, dass die Sitzordnung im Sitzkreis, um einen kleinen Tisch herum oder an Stehtischen, dazu beiträgt, Hierarchien zwischen den Akteuren abzubauen.

AP 6 (Dokumentieren und Auswerten): In der Ideenwerkstatt (oder einem vergleichbaren Format) können Videoaufnahmen und Fotos die Arbeit dokumentieren. Produkte wie die Papphocker-Wand mit Handlungsempfehlungen der Akteure fassen die Ergebnisse der Diskussion zusammen und ermöglichen im Reflexionsworkshop eine unmittelbare Nutzung der Empfehlungen. Für die weitere Forschungsarbeit sind jedoch ergänzend umfangreiche Protokolle erforderlich, die z. B. gestützt auf Tonaufnahmen der Diskussionen und Präsentationen im Plenum erstellt werden können.

\subsection{Veranstaltung 5: Ziel-Beschreiterin}

Am Ende des Projektverlaufs, in der Abschlussphase, setzt Veranstaltung 5 ein. Zentral ist die Vorstellung der Ergebnisse aus dem Projekt mit Blick auf die Diffusion sowie die Implementierung und Etablierung (Verstetigung) der Ergebnisse. Ziel der letzten Veranstaltung ist zudem ein Rückblick auf das Projekt. Im Mittelpunkt steht die Integration der Ergebnisse in die Praxis und gleichzeitig die Verabschiedung des Projekts als Akteur aus dem Feld. Wesentlich ist auch die Danksagung an die externen Akteure sowie ein gemeinsamer feierlicher Abschluss.

\subsubsection{Steckbrief Ziel-Beschreiterin}

Wissensintegration und Bildungsziele: In dieser Veranstaltung wird vor allem Transformationswissen , aktiviert' und erzeugt. Dieses wiederum kann in die Praxis, in folgende und/oder in andere Projekte integriert werden.

Ablauf und Rollen: Vertreter(innen) des Projektteams stellen die Ergebnisse des Projekts als Ganzes vor. Die gezeigten Einblicke und Erkenntnisse werden in zwei Diskussionsrunden mit unterschiedlichen Schwerpunkten diskutiert. Alle ziehen zum Abschluss Resümee. Rolle der verschiedenen Akteure:

- Moderation: Projektteam, wobei die Praxispartner eine besondere Rolle spielen sollten

- Impulsreferate: Vertreter(innen) des Projektteams

- Teilnehmer(innen): Sind hier in erster Linie als Multiplikator(inn)en angesprochen 
Nutzen für externe Praxisakteure:

- Pflege ihres Netzwerks, Austausch mit Peers

- Einblicke in und Impulse aus der Forschung

- Impulse für das eigene Handeln in der Praxis

- Anregungen, wie sie auch außerhalb des Projekts Transformationen anregen und Transformationsprozesse gestalten können

Fragen, die die Inhalte der Ziel-Beschreiterin definieren: Was sind die Ergebnisse der Forschung? Was waren die Chancen und Herausforderungen und was wurde daraus gelernt? Wie geht es weiter?

Voraussetzungen, die für den Einsatz der Ziel-Beschreiterin erfüllt sein müssen:

- Gesamtergebnisse aus dem Projekt liegen vor.

- Erste Produkte (bspw. Publikationen), die sich an die Praxis richten, liegen möglichst vor oder können jedenfalls in Aussicht gestellt werden.

\subsubsection{Planungsaufgaben Ziel-Beschreiterin}

AP 1 (Identifizieren und Erreichen der Akteure): Zu dieser Veranstaltung werden alle eingeladen, die an einer der vorangegangenen Veranstaltungen teilgenommen haben. Zusätzlich sind gezielt weitere Akteure einzuladen, die mit Blick auf die Umsetzung eine besondere Rolle spielen könnten. Bei dieser Veranstaltung ist es überaus wichtig, dass die Praxispartner des Projekts prominent auftreten (z. B. für Präsentation, Einführung in eine Ausstellung, Moderation). Deren Teilnahme sollte bereits beim Anbahnen der Kooperation sichergestellt werden.

AP 2 (Inhaltliche Impulse aus dem Projekt bestimmen): Mit Blick auf die Darstellung der Projektergebnisse gilt es in der Projektplanung, die Synthesearbeiten so zu planen, dass deren Ergebnisse vorliegen. Im Mittelpunkt der Veranstaltung steht, nebst einer umfassenden Präsentation und Diskussion der Gesamtergebnisse des Projekts, die Reflexion der Zusammenarbeit zwischen Forscher(inne)n, Praxispartnern und externen Praxisakteuren: Was hat sich als besonders schwierig herausgestellt? Was hat sich bewährt, was sind unsere Lessons Learnt? Es empfiehlt sich, dafür eine Präsentation der Erfahrungen der Praxispartner in Form eines Vortrages mit anschließender Diskussionsrunde vorzusehen, in die externe Praxisakteure eingebunden werden.

AP 3 (Vorbereitungen speziell für externe Akteure treffen): Es bewährt sich, Broschüren mit einer Zusammenfassung der Ergebnisse und des Prozesses zu erstellen, welche die Teilnehmer(innen) mitnehmen können. 
AP 4 (Beteiligungsmethodik definieren): Die Projektergebnisse können auf vielfältige Art präsentiert werden (Poster, Vorträge, Ausstellung etc.), angesichts des in aller Regel beträchtlichen Umfangs empfiehlt sich die Wahl einer ,komprimierenden' Methode. Pecha Kucha (eine Vortragstechnik, in der 20 Folien jeweils 20 Sekunden lang eingeblendet werden) zum Beispiel ermöglicht es, in einer prägnanten Präsentation das Wesentliche der Forschungsergebnisse auf den Punkt zu bringen und auf anschauliche Weise einen Einblick in den Projektverlauf sowie die Forschungsergebnisse zu geben (zu Pecha Kucha s. z. B. https://www. pechakucha.com, zugegriffen am 22.04.2019). Eine anschließende Diskussion kann z. B. als Podiumsdiskussion gestaltet werden (s. hierzu Friesen et al. 2006), die als eine Art Interview realisiert wird. Dies bietet auch den Praxispartnern, die am Forschungsprozess beteiligt waren, die Möglichkeit, sich aktiv an der Gestaltung des Symposiums zu beteiligen (s. SRB-Box 5). Die Inhalte der so gestalteten Diskussionsrunde regen die Teilnehmer(innen) wiederum an, mit den Referent(inn)en und dem Projektteam in Dialog zu treten. Für das Forschungsprojekt wiederum bietet es die Chance, dass nicht nur kritisch nachgedacht, sondern ein Prozess zur Umsetzung und zur Formulierung weiterer Forschungsfragen angeregt wird.

\section{SRB-Box 5: Abschluss des Reallabors SRB: Resümee mit Praxispartnern in einem Inter- view}

Das Abschluss-Symposium stand am Ende der dreijährigen Förderzeit des Reallabors SRB. Das Ziel war es, Erfahrungen aus dem Projektverlauf zu teilen und Einblicke in die Ergebnisse zu geben. Zwei Schulleitungen, die als Praxispartner aktiv im Forschungsprojekt mitgearbeitet hatten, reflektierten mit Forscher(inne)n des Reallabors SRB die gemeinsame, kooperative Arbeit und ihre Lessons Learnt. Das Projektteam wollte so darstellen, wie die Kooperation im Projekt ablief und welchen direkten Nutzen die Partner im Projekt hatten. Im ersten Teil der anschließenden Diskussionsrunde wurden die Podiumsgäste dazu interviewt (s. Abb. 10) und berichteten von ihren sehr persönlichen Erfahrungen, aufgrund von Fragen wie: Was funktionierte gut? Wie aufwendig war das Ganze? Was kam dabei heraus? Was hätten wir anders machen können? Auf diese Art Feedback zu erhalten, war für die Forscher(innen) wertvoll. Auch für die externen Praxisakteure, die am Symposium teilnahmen, war es interessant, Kenntnis von der Wahrnehmung und den Erfahrungen der Praxispartner, bezogen auf die Zusammenarbeit im Reallabor, zu bekommen. Im zweiten Teil der Podiumsdiskussion wurde die Diskussionsrunde für Fragen und Anmerkungen aus dem Publikum geöffnet. Da im Publikum viele externe Akteure saßen, die bereits an vorangegangenen Symposien teilgenommen hatten, entstand dank des bereits aufgebauten Vertrauensverhältnisses eine anregende und inhaltsreiche Diskussion zwischen den externen Akteuren und den Praxispartnern und Forscher(innen) auf dem Podium. 

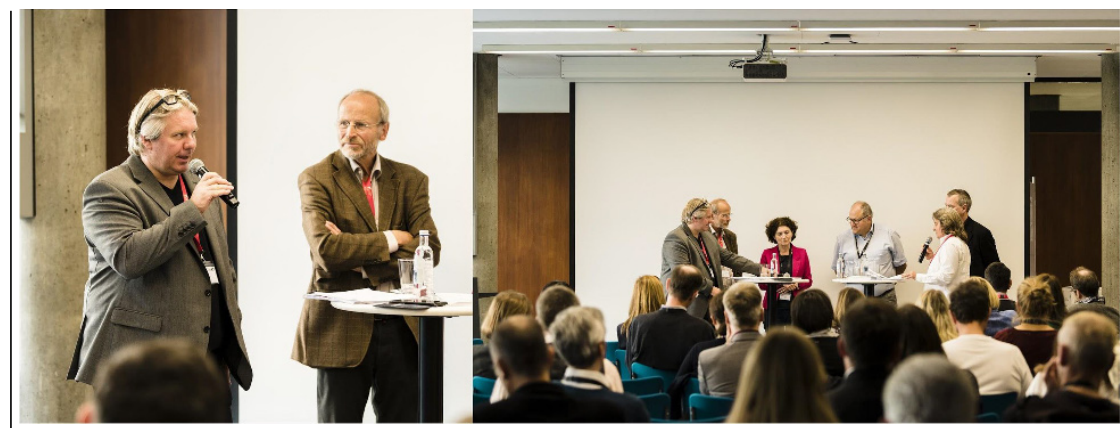

Abbildung 10: Forscher(innen) und Praxispartner bei der Podiumsdiskussion auf der Veranstaltung "Symposium STADT-RAUM-BILDUNG“". Podiumsdiskussionen können gehaltvoll sein, wenn sie sorgfältig vorbereitet und moderiert werden. (C) Reallabor SRB 2018.

AP 5 (Wertschätzung durch Atmosphäre und Stimmung vermitteln): In der Abschlussveranstaltung ist es wichtig, eine besondere Stimmung herzustellen und Wertschätzung auszudrücken. Es ist zu empfehlen, ein rückblickendes Element mit einzubauen, das auch emotionale Anknüpfungspunkte bietet und Erinnerungen wachruft an das gemeinsam Erlebte, z. B. einen Videozusammenschnitt der vorangegangenen Symposien (und weiterer Aktionen während der Forschung). Dazu können Fotos, Video- und Audioausschnitte benutzt werden, welche die Highlights des Forschungsprojekts zeigen und den Forschungsprozess zusammenfassen. Das dient nicht nur als kurzer Rückblick der vergangenen Jahre, sondern auch zur Einstimmung auf die Verabschiedung, indem die Zusammenarbeit mit den Praxispartnern und mit den externen Akteuren wertschätzend dargestellt wird. Eine Danksagung an alle Beteiligten schließt den offiziellen Teil ab. Um das Symposium ausklingen zu lassen, werden alle Anwesenden zum Umtrunk bei Musik und Häppchen eingeladen, so dass die gemeinsame Arbeit abschließend gefeiert und das Forschungsprojekt als Akteur verabschiedet werden kann.

AP 6 (Dokumentieren und Auswerten): Auch das Abschlusssymposium sollte durch Videoaufnahmen und Fotos dokumentiert werden, wobei die Tonspur der Videoaufzeichnung transkribiert und ausgewertet werden kann. Alternativ können die Interviewergebnisse protokolliert werden, womit der Transkriptionsaufwand entfällt. Im Reflexionsworkshop wird nochmals Resümee gezogen; vor allem wird an den Lessons Learnt gearbeitet. 


\subsection{Reflexionsworkshop jeweils am Tag nach dem Symposium}

Reflexion und Aufbereitung der Impulse aus den Symposien: Die Impulse der externen Akteure aus den Symposien werden im internen Reflexionsworkshop, der jeweils am nächsten Tag stattfindet, reflektiert und aufgearbeitet. Dies ersetzt natürlich nicht eine weitergehende sorgfältige Ergebnisaufbereitung, dient aber dazu, die noch frischen Eindrücke zu vergemeinschaften und die nächsten Schritte zu planen. Gestützt auf ihre Notizen und auf die Produkte aus dem jeweiligen Symposium sammeln und clustern alle Teammitglieder die aus ihrer Sicht relevanten Ergebnisse, Einschätzungen und Fragen. Anschließend diskutieren sie diese in teilprojektübergreifenden Kleingruppen von drei bis fünf Personen mit Blick auf die Konsequenzen für das Projekt als Ganzes und seine Teile, bevor sich dann die einzelnen Teams der Teilprojekte zusammensetzen und über die Integration der Ergebnisse in das jeweilige Teilprojekt beraten. Gemeinsam wird abschließend ein Arbeitsplan erstellt, in dem festgehalten wird, wie die weitere Auswertung der Symposiumsergebnisse erfolgt und wie diese im Projekt umgesetzt werden sollen. Damit dies möglich ist, gilt es, bei der Projektplanung entsprechende Flexibilität im Design vorzusehen.

Inhaltliche Vorbereitung mit Blick auf die interne Vernetzung: Damit der Reflexionsworkshop seine Funktion im Syntheseprozess erfüllen kann, ist über die Vorbereitung auf das jeweilige Symposium hinaus eine Aufbereitung des Arbeitsstands in den Teilprojekten für interne Zwecke erforderlich. Dazu legen alle Teilprojekte ihren jeweils aktuellen Zwischenstand sowie offene Fragen und Herausforderungen dar. Um die Arbeitslast insgesamt in Grenzen zu halten, empfiehlt es sich, dies anhand eines Handouts mit vorgegebener Gliederung zu tun: Ziel und Fragestellung, Arbeitsstand bis dato, erste wesentliche Erkenntnisse, Schwierigkeiten, anstehende Entscheidungen und (neue) Schnittstellen zu anderen Teilprojekten, geplante Arbeitsschritte. Ein solches Handout kann zu Beginn der Arbeit erstellt werden, dann für jeden Reflexionsworkshop von den Teilprojekten auf den aktuellen Stand gebracht und zur Vorbereitung des Workshops vorher von der Projektkoordination an alle Teammitglieder geschickt werden. 


\section{7 Übersicht über die Veranstaltungen der Exchange- Veranstaltungsreihe}

Tab. 2 gibt eine Übersicht zu allen fünf Veranstaltungen der Exchange-Veranstaltungsreihe und stellt die Schwerpunkte je Veranstaltung mithilfe von Fragen dar: Sie erläutert,

- wann die Veranstaltung im Verlauf des Projekts sinnvoll einzusetzen ist;

- wie weit das Projekt jeweils fortgeschritten sein muss, um inhaltlich wie auch strukturell Beiträge in der Veranstaltung leisten zu können;

- die möglichen Inhalte der Veranstaltung;

- das Ziel und den Nutzen der Veranstaltung für das Projekt;

- was Praxisakteure, die nicht in das Projekt eingebunden sind, von der Veranstaltung haben. 
Tabelle 2: Schwerpunkte der fünf Veranstaltungen der Exchange-Veranstaltungsreihe.

\begin{tabular}{|c|c|c|}
\hline & $\begin{array}{l}\text { Veranstaltung } 1 \\
\text { Tür-Öffnerin }\end{array}$ & $\begin{array}{l}\text { Veranstaltung } 2 \\
\text { Weg-Bereiterin (optional) }\end{array}$ \\
\hline $\begin{array}{l}\text { Wann im Projekt- } \\
\text { verlauf sollte die } \\
\text { Veranstaltung } \\
\text { eingesetzt wer- } \\
\text { den? }\end{array}$ & $\begin{array}{l}\text { Einstiegsphase } \\
\text { 3-jähriges Projekt, ohne/mit 2-stu- } \\
\text { figem Antragsverfahren: 9. Monat / } \\
\text { 4. Monat } \\
\text { 5-jähriges Projekt, ohne/mit 2-stu- } \\
\text { figem Antragsverfahren: } 12 \text {. Monat / } \\
\text { 7. Monat }\end{array}$ & $\begin{array}{l}\text { Orientierungsphase } \\
\text { 3-jähriges Projekt, ohne/mit 2-stufi- } \\
\text { gem Antragsverfahren: - / optional } \\
\text { 13. Monat } \\
\text { 5-jähriges Projekt, ohne/mit 2-stufi- } \\
\text { gem Antragsverfahren: } 21 \text {. Monat / } \\
\text { 16. Monat }\end{array}$ \\
\hline $\begin{array}{l}\text { Was setzt die } \\
\text { Veranstaltung } \\
\text { voraus, mit Blick } \\
\text { auf das, was im } \\
\text { Projekt bereits } \\
\text { erreicht wurde? }\end{array}$ & $\begin{array}{l}\text { - Projektteam steht fest. } \\
\text { - } \text { Forschungsthemen und Fragestel- } \\
\text { lungen sind definiert. } \\
\text { - } \text { Relevante Akteure im Feld (Praxis } \\
\text { und Forschung) sind identifiziert. }\end{array}$ & $\begin{array}{l}\text { - Falls mit Fallbeispielen gearbeitet } \\
\text { wird: Fallbeispiele für die For- } \\
\text { schung sind ausgewählt. } \\
\text { - Erste Forschungserfahrungen, } \\
\text { erste Transformationserfahrungen } \\
\text { liegen vor. }\end{array}$ \\
\hline $\begin{array}{l}\text { Welche Fragen } \\
\text { definieren die } \\
\text { Inhalte der Veran- } \\
\text { staltung? }\end{array}$ & $\begin{array}{l}\text { Was sind Forschungsthema und For- } \\
\text { schungsvorhaben? Wer ist das Pro- } \\
\text { jektteam? Welche Expertise bringt } \\
\text { es mit? Welche Rolle wird es im Feld } \\
\text { einnehmen? Wie reagieren die ex- } \\
\text { ternen Akteure auf das Projekt? }\end{array}$ & $\begin{array}{l}\text { Wie wird geforscht? Wie soll der For- } \\
\text { schungsprozess aussehen? Welche } \\
\text { Methoden sollen eingesetzt werden } \\
\text { (ggf.: Welche Fallbeispiele bilden den } \\
\text { Untersuchungsgegenstand?) und } \\
\text { sind diese geeignet? }\end{array}$ \\
\hline $\begin{array}{l}\text { Was sind das Ziel } \\
\text { und der Nutzen } \\
\text { der Veranstaltung } \\
\text { für das Projekt? }\end{array}$ & $\begin{array}{l}\text { - Etablierung Forschungsprojekt } \\
\text { - Gewinnung von (weiteren) Praxis- } \\
\text { akteuren für die transdisziplinäre } \\
\text { Zusammenarbeit } \\
\text { - Vorstellung des Forschungspro- } \\
\text { jekts und des Projektteams } \\
\text { - Das im Forschungsantrag darge- } \\
\text { legte Zielwissen wird durch exter- } \\
\text { ne Akteure reflektiert und ergänzt }\end{array}$ & $\begin{array}{l}\text { - Transparente Darstellung des For- } \\
\text { schungsgegenstands, Berichte der } \\
\text { Praxispartner (z. B. aus Fallbeispie- } \\
\text { len des Projekts) } \\
\text { - Die Integration von Praxiswissen in } \\
\text { den Forschungsprozess }\end{array}$ \\
\hline $\begin{array}{l}\text { Worin liegt der } \\
\text { Nutzen für Praxis- } \\
\text { akteure, die nicht } \\
\text { in das Projekt } \\
\text { eingebunden sind? }\end{array}$ & $\begin{array}{l}\text { - Erweiterung und Pflege ihres } \\
\text { Netzwerks, Austausch mit Peers } \\
\text { - Horizonterweiterung, Erwerb } \\
\text { neuen Wissens }\end{array}$ & $\begin{array}{l}\text { - Pflege ihres Netzwerks, Austausch } \\
\text { mit Peers } \\
\text { - Einblicke in die Perspektive und } \\
\text { Erfahrungen Dritter, daraus Im- } \\
\text { pulse für die eigene Arbeit }\end{array}$ \\
\hline
\end{tabular}


Forts. Tabelle 2

\begin{tabular}{|c|c|c|}
\hline $\begin{array}{l}\text { Veranstaltung } 3 \\
\text { Weg-Begleiterin }\end{array}$ & $\begin{array}{l}\text { Veranstaltung } 4 \\
\text { Ziel-Erspäherin (optional) }\end{array}$ & $\begin{array}{l}\text { Veranstaltung } 5 \\
\text { Ziel-Beschreiterin }\end{array}$ \\
\hline Arbeitsphase & Arbeitsphase & Abschlussphase \\
\hline $\begin{array}{l}\text { 3-jähriges Projekt, ohne/mit } \\
\text { 2-stufigem Antragsverfahren: } \\
\text { 18. / 13. oder 22. Monat }\end{array}$ & $\begin{array}{l}\text { 3-jähriges Projekt, ohne/mit } \\
\text { 2-stufigem Antragsverfahren: } \\
\text { - / optional 22. Monat }\end{array}$ & $\begin{array}{l}\text { 3-jähriges Projekt, ohne/mit } \\
\text { 2-stufigem Antragsverfahren: } \\
\text { 27. / 31. Monat }\end{array}$ \\
\hline $\begin{array}{l}\text { 5-jähriges Projekt, ohne/mit } \\
\text { 2-stufigem Antragsverfahren: } \\
\text { 30. / 25. Monat }\end{array}$ & $\begin{array}{l}\text { 5-jähriges Projekt, ohne/mit } \\
\text { 2-stufigem Antragsverfahren: } \\
\text { 39. / 34. und/oder 43. Monat }\end{array}$ & $\begin{array}{l}\text { 5-jähriges Projekt, ohne/mit } \\
\text { 2-stufigem Antragsverfahren: } \\
\text { 48. / 52. Monat }\end{array}$ \\
\hline $\begin{array}{l}\text { - Erste Erkenntnisse und Er- } \\
\text { fahrungen aus dem Projekt } \\
\text { liegen vor. } \\
\text { - Für das Projekt besonders } \\
\text { spannende Forschungsfel- } \\
\text { der und -projekte, von de- } \\
\text { nen gelernt werden kann, } \\
\text { sind bekannt. }\end{array}$ & $\begin{array}{l}\text { - Wesentliche Teilergebnisse } \\
\text { aus dem Projekt liegen vor. } \\
\text { - Überblick über Akteure im } \\
\text { Feld, die bisher außen vor } \\
\text { blieben, mit Blick auf die Pra- } \\
\text { xisrelevanz und Legitimität } \\
\text { der Ergebnisse aber beteiligt } \\
\text { werden sollten, besteht. }\end{array}$ & $\begin{array}{l}\text { - Gesamtergebnisse aus dem } \\
\text { Projekt liegen vor. } \\
\text { - Erste Produkte, die sich an } \\
\text { die Praxis richten, liegen } \\
\text { möglichst vor oder können } \\
\text { jedenfalls in Aussicht ge- } \\
\text { stellt werden. }\end{array}$ \\
\hline $\begin{array}{l}\text { Was sind erste Erkenntnisse } \\
\text { und Erfahrungen, die das } \\
\text { Projektteam gesammelt hat? } \\
\text { Gibt es weitere Forschungs- } \\
\text { projekte oder Initiativen im } \\
\text { selben Themenbereich? }\end{array}$ & $\begin{array}{l}\text { Was sind die ersten Zwischen- } \\
\text { ergebnisse der Forschung? } \\
\text { Welche Erkenntnisse wurden } \\
\text { bereits gewonnen? Wie werden } \\
\text { bereits erhobene Daten bis zum } \\
\text { Ende ausgewertet? }\end{array}$ & $\begin{array}{l}\text { Was sind die Ergebnisse der } \\
\text { Forschung? Was waren die } \\
\text { Chancen und Herausforderun- } \\
\text { gen und was wurde daraus } \\
\text { gelernt? Wie geht es weiter? }\end{array}$ \\
\hline $\begin{array}{l}\text { - Erste Diskussion der prak- } \\
\text { tischen Anwendbarkeit } \\
\text { erster Eindrücke aus der } \\
\text { Forschung } \\
\text { - Rückkopplung der Erfah- } \\
\text { rungen mit dem wissen- } \\
\text { schaftlichen Diskurs }\end{array}$ & $\begin{array}{l}\text { - Vorstellung erster Teilergeb- } \\
\text { nisse } \\
\text { - Rückspiegelung der Erkennt- } \\
\text { nisse aus der Forschung an } \\
\text { die externen Praxisakteure } \\
\text { - Gemeinsame Erarbeitung von } \\
\text { Transformationswissen }\end{array}$ & $\begin{array}{l}\text { - Darstellung der Gesamter- } \\
\text { gebnisse und deren Umset- } \\
\text { zung } \\
\text { - Generiertes Transforma- } \\
\text { tionswissen kann in die Pra- } \\
\text { xis und in folgende / andere } \\
\text { Projekte integriert werden }\end{array}$ \\
\hline $\begin{array}{l}\text { - Pflege ihres Netzwerks, } \\
\text { Austausch mit Peers } \\
\text { - Impulse aus der Forschung } \\
\text { und Einblicke in die For- } \\
\text { schung inner- und außer- } \\
\text { halb des Projekts } \\
\text { - Impulse für das praktische } \\
\text { Handeln in den eigenen } \\
\text { Problemstellungen }\end{array}$ & $\begin{array}{l}\text { _ Pflege ihres Netzwerks, Aus- } \\
\text { tausch mit Peers } \\
\text { _ Einblicke in und Impulse aus } \\
\text { der Forschung } \\
\text { - Impulse für das praktische } \\
\text { Handeln mit Bezug auf ihre } \\
\text { Problemstellungen } \\
\text { - Empowerment und Hinweise, } \\
\text { wie sie auch nach Ende des } \\
\text { Projekts Transformationspro- } \\
\text { zesse gestalten können }\end{array}$ & $\begin{array}{l}\text { - Pflege ihres Netzwerks, Aus- } \\
\text { tausch mit Peers } \\
\text { - Einblicke in und Impulse aus } \\
\text { der Forschung } \\
\text { - Impulse für das eigene Han- } \\
\text { deln in der Praxis } \\
\text { - Anregungen, wie sie auch } \\
\text { außerhalb des Projekts } \\
\text { Transformationen anregen } \\
\text { und Transformationsprozes- } \\
\text { se gestalten können }\end{array}$ \\
\hline
\end{tabular}




\section{Reflexion}

Die Idee, eine Veranstaltungsreihe als Planungs- und Steuerungsinstrument für transdisziplinäre Forschungsprojekte einzusetzen, entstand auf Basis der Erfahrungen aus dem Reallabor SRB. In diesem wurden schon frühzeitig, während der Auftragsphase, mehrere Symposien mit anschließenden Reflexionsworkshops für die einzelnen Projektphasen geplant und in der Laufzeit umgesetzt. Der Veranstaltungsrhythmus lag bei durchschnittlich sechs Monaten. Bei einer Gesamtdauer des Projekts von drei Jahren war dies durchaus ambitioniert, und die teilweise hohe zeitliche Belastung der Forscher(innen), die die Veranstaltungen inhaltlich ausgestalten, auswerten und deren Ergebnisse umsetzen mussten, stellte oftmals eine große Herausforderung im Reallabor SRB dar.

Im Projektverlauf des Reallabors SRB hat sich die Einbindung von externen Akteuren als wichtiger Kern für die Arbeit herausgestellt. Die Veranstaltungsreihe ermöglichte eine konsistente und kontinuierliche Kommunikation mit Praxisakteuren, so dass ,Betroffene' und Interessierte im Forschungsprozess mitgenommen und sowohl an Entscheidungen über den Forschungsprozess als auch an Ergebnissen teilhaben konnten. Über die verschiedenen öffentlichen Symposien konnten bereits zu Beginn das Forschungsvorhaben, Erfahrungsberichte, Teilergebnisse und zuletzt die Gesamtergebnisse des Projekts mit einer breiteren Öffentlichkeit reflektiert werden, was von Beginn an ermöglichte, neues Wissen und Impulse für die Forschung einzuholen. Gerade für die Schulentwicklung, in der die politische Entscheidungsfindung großen Raum einnimmt, war eine transparente, öffentliche Darstellung, Diskussion und Bewertung des Forschungsprozesses mit externen Praxisakteuren und auch mit externen Wissenschaftler(inne)n erheblich für eine hohe Qualität des Prozesses.

Die Symposien boten den Forscher(inne)n die Möglichkeit, neue Kontakte zu externen Forscher(inne)n herzustellen und diese zu verstetigen. Die Praxisakteure wiederum knüpften Kontakte untereinander, so dass sie sich gegenseitig bei ihrer beruflichen Tätigkeit unterstützen konnten und dies auch weiterhin tun können. Die Symposien förderten somit die Entstehung eines Netzwerks sowohl für die Forscher(innen) als auch für die Praxisakteure, das die Laufzeit des Reallabors SRB überdauert hat.

Die internen Reflexionsworkshops boten den Forscher(inne)n im Projektteam die Möglichkeit, die Erkenntnisse aus dem jeweils vorangegangenen Symposium quer zu den beteiligten Disziplinen und Teilprojekten zu diskutieren, zu reflektieren und zu werten. Dies ermöglichte den Transfer von hoch aktuellem Praxiswissen in die laufende Forschung. Die regelmäßige Auf- und Nachbereitung der Ergebnisse aus den Symposien nahm zeitlich viele Kapazitäten in Anspruch, führte aber dazu, dass die Ergebnisse für Akteure aus der Forschung und der Praxis des Schulbaus anschlussfähiger wurden. Dieser Erfolg ist zu großen Teilen 
auf die kombinierte Reflexion durch die Symposien und die Reflexionsworkshops zurückzuführen. Neben der Funktion der Diffusion, des Austauschs und der Vernetzung wirkte die Veranstaltungsreihe zudem strukturgebend für das Reallabor SRB, indem sie die Meilensteine setzte, an denen eine Reflexion innerhalb des Projekts stattfinden musste.

Der hohe organisatorische Aufwand für die Veranstaltungsreihe in der Planung, Durchführung und Dokumentation hat sich gelohnt, weil das Ergebnis von allen (internen und externen) Akteuren insgesamt sehr positiv aufgenommen und sehr wertgeschätzt wurde und die produktive Zusammenarbeit zwischen Wissenschaftler(inne)n und Praxisakteuren erhöhte. Eine wichtige Rolle spielten dabei auch die Vermittlung von Wertschätzung gegenüber den Praxisakteuren und das Schaffen einer vertrauensvollen Atmosphäre in den Symposien. Diese beiden Aspekte - Wertschätzung vermitteln und Vertrauen schaffen - bilden eine wesentliche Grundlage für den erfolgreichen Einsatz der Exchange-Veranstaltungsreihe als Planungs- und Steuerungsinstrument.

\section{Dank}

Die Autor(inn)en danken Richard Beecroft für die Diskussionen über eine frühere Fassung des Textes. Insbesondere möchten sie Marius Albiez, Laura Gebhardt, Andri König und Thomas Potthast sowie zwei anonymen Gutachter(inne)n für die konkreten Hinweise zur Verbesserung des Textes im Rahmen des internen und externen Reviews danken. Schließlich danken die Autor(inn)en den beiden Herausgebenden, Rico Defila und Antonietta Di Giulio, für ihre Rückmeldungen zum Text. 


\section{Literatur}

Alcántara, S., Quint, A., \& Seebacher, A. (2018). Der Partizipationsmythos „Partizipation in Reallaboren muss repräsentativ sein". In R. Defila \& A. Di Giulio (Hrsg.), Transdisziplinär und transformativ forschen. Eine Methodensammlung (S. 137-159). Wiesbaden: Springer VS. doi: 10.1007/978-3-658-21530-9_7.

Arnold, A., \& Piontek, F. M. (2018). Zentrale Begriffe im Kontext der Reallaborforschung. In R. Defila \& A. Di Giulio (Hrsg.), Transdisziplinär und transformativ forschen. Eine Methodensammlung (S. 143-154). Wiesbaden: Springer VS. doi: 10.1007/ 978-3-658-21530-9_8.

Beecroft, R., Trenks, H., Rhodius, R., Benighaus, C., \& Parodi, O. (2018). Reallabore als Rahmen transformativer und transdisziplinärer Forschung: Ziele und Designprinzipien. In R. Defila \& A. Di Giulio (Hrsg.), Transdisziplinär und transformativ forschen. Eine Methodensammlung (S. 75-100). Wiesbaden: Springer VS. doi: 10.1007/978-3-65821530-9_4.

Benighaus, C., \& Benighaus, L. (2016). Diskussion und Vorsorge von Hagelereignissen. In C. Benighaus, G. Wachinger \& O. Renn (Hrsg.), Bürgerbeteiligung - Konzepte und Lösungswege für die Praxis (S. 216-222). Berlin, Frankfurt a. M.: Wolfgang Metzner Verlag.

Benighaus, C., \& Renn, O. (2016). Teil A Grundlagen. In C. Benighaus, G. Wachinger \& O. Renn (Hrsg.), Bürgerbeteiligung - Konzepte und Lösungswege für die Praxis (S. 17102). Berlin, Frankfurt a. M.: Wolfgang Metzner Verlag.

Bergold, J., \& Thomas, S. (2010). Partizipative Forschung. In G. Mey \& K. Mruck (Hrsg.), Handbuch Qualitative Forschung in der Psychologie (S. 333-344). Wiesbaden: Springer VS.

Brown, J., \& Isaacs, D. (2007). Das World Café: Kreative Zukunftsgestaltung in Organisationen und Gesellschaft. Heidelberg: Carl-Auer-Systeme Verlag.

Defila, R., Di Giulio, A., \& Scheuermann, M. (2006). Forschungsverbundmanagement: Handbuch für die Gestaltung inter- und transdisziplinärer Projekte. Zürich: vdf Hochschulverlag an der ETH Zürich.

Eckart, J., Ley, A., Häußler, E., \& Erl, Th. (2018). Leitfragen für die Gestaltung von Partizipationsprozessen in Reallaboren. In R. Defila \& A. Di Giulio (Hrsg.), Transdisziplinär und transformativ forschen. Eine Methodensammlung (S. 105-135). Wiesbaden: Springer VS. doi: 10.1007/978-3-658-21530-9_6.

Friesen, Kaye and Associates (2006). Tips for Facilitating Panel Discussions. https://www.fka.com/files/TIPS-Facilitating-Panel-Discussions.pdf. Zugegriffen am 18.01.2019.

Godemann, J. (2005). Verständigung als Basis inter- und transdisziplinärer Zusammenarbeit. In G. Michelsen \& J. Godemann (Hrsg.), Handbuch der Nachhaltigkeitskommunikation. Grundlagen und Praxis (S. 85-95). München: oekom.

Hofmann, S. (Hrsg.) (2014). Partizipation macht Architektur. Die Baupiloten-Methode und Projekte. Berlin: Jovis. 
Quint, A., Alcántara, S., \& Seebacher, A. (2018). Der Partizipationsmythos „Partizipation in Reallaboren ist per se transparent und muss es auch sein“. In R. Defila \& A. Di Giulio (Hrsg.), Transdisziplinär und transformativ forschen. Eine Methodensammlung (S. 69-73). Wiesbaden: Springer VS. doi: 10.1007/978-3-658-21530-9_3.

Renn, O., \& Benighaus, C. (2003). Diskurs: Vorgehen, Bedingungen, Klassifikation und Chancen. In A. Ley \& L. Weitz (Hrsg.), Praxis Bürgerbeteiligung: Ein Methodenhandbuch (Arbeitshilfen für Selbsthilfe- und Bürgerinitiativen, Nr. 30) (S. 108-114). Bonn: Stiftung Mitarbeit.

Rogge, K. I. (2000). Ideenwerkstatt. In H.-W. Kuhn \& P. Massing (Hrsg.), Methoden und Arbeitstechniken (Lexikon der politischen Bildung, Bd. 3) (S. 77-78). Schwalbach am Taunus: Wochenschau Verlag.

Schäpke, N., Stelzer, F., Bergmann, M., Singer-Brodowski, M., Wanner, M., Caniglia G., \& Lang, D. (2017). Reallabore im Kontext transformativer Forschung. Ansatzpunkte zur Konzeption und Einbettung in den internationalen Forschungsstand. (No. 1/2017). Leuphana Universität Lüneburg, Institut für Ethik und Transdisziplinäre Nachhaltigkeitsforschung. http://hdl.handle.net/10419/168596. Zugegriffen am 20.05.2019.

Seebacher, A., Alcántara, S., \& Quint, A. (2018). Akteure in Reallaboren - Reallabore als Akteure. In R. Defila \& A. Di Giulio (Hrsg.), Transdisziplinär und transformativ forschen. Eine Methodensammlung (S. 155-159). Wiesbaden: Springer VS. doi: 10.1007/ 978-3-658-21530-9_9.

United Nations Human Rights, Office of the Commissioner (2012). Fishbowl: The art of active listening. http://slitoolkit.ohchr.org/data/downloads/fishbowl.pdf. Zugegriffen am 18.01.2019.

Open Access Dieses Kapitel wird unter der Creative Commons Namensnennung 4.0 International Lizenz (http://creativecommons.org/licenses/by/4.0/deed.de) veröffentlicht, welche die Nutzung, Vervielfältigung, Bearbeitung, Verbreitung und Wiedergabe in jeglichem Medium und Format erlaubt, sofern Sie den/die ursprünglichen Autor(en) und die Quelle ordnungsgemäß nennen, einen Link zur Creative Commons Lizenz beifügen und angeben, ob Änderungen vorgenommen wurden.

Die in diesem Kapitel enthaltenen Bilder und sonstiges Drittmaterial unterliegen ebenfalls der genannten Creative Commons Lizenz, sofern sich aus der Abbildungslegende nichts anderes ergibt. Sofern das betreffende Material nicht unter der genannten Creative Commons Lizenz steht und die betreffende Handlung nicht nach gesetzlichen Vorschriften erlaubt ist, ist für die oben aufgeführten Weiterverwendungen des Materials die Einwilligung des jeweiligen Rechteinhabers einzuholen.

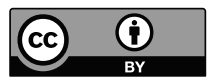




\title{
Die „TraSy-Methode“ - ein Vorgehen für die transdisziplinäre Entwicklung soziotechnischer Systeme
}

\author{
Laura Gebhardt \& Alexandra König
}

\section{$1 \quad$ Einleitung}

Der Ansatz, Nutzer(innen) ${ }^{1}$ nicht nur als passive Konsument(inn)en zu sehen, sondern sie als Mitgestalter(innen) aktiv in die Entwicklung von Produkten und Lösungsstrategien zu involvieren (Mayas et al. 2012; Pruitt und Adlin 2006), wird in der Industrie bereits seit einiger Zeit verfolgt. Auch Städte und Kommunen haben erkannt, welche Vorteile es bringt, Bürger(innen) bei der Generierung neuer Ideen sowie in Planungs- und Entwicklungsprozessen zu beteiligen (Gebhardt et al. 2014; Selle 2013). Statt Wissen über Verhaltensweisen und Präferenzen von Nutzer(inne)n zu generieren und dieses danach in die Praxis zu überführen, werden Problemlösungen gemeinsam mit der Zivilgesellschaft ${ }^{2}$ vor Ort entwickelt, wie beispielweise auf der Plattform Bremenbewegen.de ${ }^{3}$. Bei den meisten dieser Ansätze bleibt jedoch unklar, wie Wissen über die Bedürfnisse unterschiedlicher Nutzergruppen (bzw. Nutzertypen) ${ }^{4}$ generiert, in Anforderungen an zu entwickelnde Systeme übersetzt und in die Lebenswelt der Betroffenen überführt wird. Unter ,System“ wird im Folgenden ,ein Komplex von Elementen, die miteinander verbunden und voneinander abhängig sind und insofern eine strukturierte Ganzheit bilden“, verstanden (Hügli und Lübcke 1991, S. 561). ,So-

1 Als Nutzer(innen) werden Personen bezeichnet, die ein Produkt oder eine Dienstleistung in Anspruch nehmen, da sie sich davon einen Nutzen versprechen.

2 Der Begriff Zivilgesellschaft benennt den Teil der außerwissenschaftlichen Gesellschaft, der nicht durch staatliche Organe organisiert wird. Somit dient der Begriff insbesondere als Abgrenzung zum staatlichen System und zum Wissenschaftssystem (Defila und Di Giulio 2018b).

3 Siehe die Webseite http://www.bremenbewegen.de (zugegriffen am 01.03.2019).

4 Nutzergruppen umfassen jeweils mehrere Nutzer(innen), die sich bezüglich forschungsrelevanter Merkmale untereinander ähnlich sind. Gleichzeitig unterscheiden sie sich dadurch von anderen Nutzer(inne)n, die nicht dieser Gruppe angehören. Die Begriffe Nutzergruppen und Nutzertypen werden im Folgenden synonym verwendet. 
ziotechnische Systeme' sind Systeme, die sowohl technische als auch nicht-technische Elemente umfassen (Büscher und Schippl 2013). Die technischen und nicht-technischen (sozialen) Elemente sind in soziotechnischen Systemen eng verknüpft (Lösch 2012). Soziotechnische Systeme berühren gesellschaftsdynamische Prozesse und sind deshalb eng mit der Transformationsforschung verbunden (Büscher und Schippl 2013). Sie können beispielsweise ein Schul- oder ein Verkehrssystem $^{5}$ sein. So umfasst ein Verkehrssystem technische Elemente wie die Verkehrsinfrastruktur mit Verkehrsmitteln und Leitsystemen für deren Koordinierung. Aber auch nicht-technische Elemente, wie Verkehrsteilnehmer(innen), die den Verkehr verursachen, sind Teil des Verkehrssystems, ebenso wie die Einrichtungen, die den Transportprozess organisieren.

Methodische Ansätze zur Erhebung von Nutzeranforderungen an neue Systeme, die davon ausgehen, dass sich Nutzer(innen) ihrer Ziele, Bedürfnisse und Anforderungen an neue soziotechnische Systeme bewusst sind und diese explizit äußern können, greifen häufig zu kurz (Brandies et al. 2017). Stattdessen müssen methodische Herangehensweisen, die zum Ziel haben, Bedürfnisse von zukünftigen Nutzer(innen) eines Systems zu identifizieren und zu beschreiben, beachten, dass diese den Nutzer(inne)n häufig selbst nicht bewusst oder leicht zugänglich sind (Achtziger und Gollwitzer 2009) und dass diese auch häufig nicht direkt beobachtbar sind (Sanders 2003). Dementsprechend sind jene Vorgehen vielversprechend, die durch den Einsatz tiefgehender und breitgefächerter Methoden, wie beispielsweise der Methode des Lauten Denkens (van Someren et al. 1994) oder der Methode der teilnehmenden Beobachtung (Lüders 2003), die eher impliziten Denkweisen und Bedürfnisse der zukünftigen Nutzer(innen) zutage bringen. Diese Methoden erlauben es den Forschenden, in die Lebenswelt der Befragten einzudringen. Wichtige Voraussetzung für den Erfolg der Identifizierung impliziter Denkweisen und Bedürfnisse der Nutzer(innen) sind dabei die Offenheit der Teilnehmer(innen) gegenüber den genannten Methoden und ihre Bereitschaft, eigene Gedanken und Einstellungen mitzuteilen.

Im vorliegenden Beitrag wird eine Methode vorgestellt, die dazu dient, Bedürfnisse heterogener Nutzergruppen eines zu entwickelnden soziotechnischen Systems systematisch zu erheben und die erhobenen Anforderungen anschließend in die konkrete Ausgestaltung dieses Systems umzusetzen. Es wird eine Methode zur transdisziplinären Entwicklung soziotechnischer Systeme vorgestellt und reflektiert. Die ,TraSy-Methode‘ (TRAnsdisziplinäre Entwicklung soziotechnischer SYsteme) wurde im Rahmen des „Reallabors Schorndorf: Zukunftsweisender Öffentlicher Verkehr - Bürgerorientierte Optimierung der Leistungsfähigkeit,

5 In diesem Beitrag wird nicht der Anspruch erhoben, den Begriff System im Sinne der soziologischen Systemtheorie (Luhmann 1984, 1997) zu verstehen und zu operationalisieren. 
Effizienz und Attraktivität im Nahverkehr“ (Reallabor Schorndorf; s. auch Steckbrief im Anhang zu diesem Buch) entwickelt und angewendet. Die TraSy-Methode wird im Folgenden vorgestellt und am Beispiel des Reallabors Schorndorf illustriert. Ziel dieses Projekts war die Entwicklung eines bedarfsgerechten und nachhaltigen Mobilitätskonzeptes für die Stadt Schorndorf (Klötzke et al. 2018). Zentral war hierbei die Frage, welche Anforderungen die Bürger(innen) der Stadt Schorndorf an das Unterwegssein stellen und wie die zukünftigen Nutzer(innen) bei der Entwicklung und Erprobung einer Mobilitätsinnovation auf Augenhöhe involviert werden können. Das neue Verkehrssystem sollte dabei keine Standalone-Lösung darstellen, sondern in das bestehende System integriert werden (König et al. 2018).

Die TraSy-Methode ist an der Schnittstelle der drei Wissensarten (System-, Zielund Transformationswissen) nach CASS und ProClim- (1997) zu verorten. Sie hat zum Ziel, durch die Analyse der aktuellen Handlungspraktiken der Nutzer(innen) Systemwissen sowie durch die Entwicklung und Erprobung eines zukünftigen soziotechnischen Systems Zielwissen zu generieren. Dabei wird ein Transformationsprozess angestoßen, bei dem Transformationswissen erzeugt wird.

Im Folgenden wird die TraSy-Methode zuerst anhand von Fragen, die sich an der Methode Interessierte stellen könnten, generell eingeführt. Anschließend wird das Vorgehen der TraSy-Methode schrittweise erklärt, um die Anwendung der TraSyMethode zu ermöglichen.

\subsection{Welche Ergebnisse können mit der TraSy-Methode erzielt werden?}

Mithilfe der TraSy-Methode können konkrete Systeme und Wissen über deren Einbettung in einem konkreten gesellschaftlichen Kontext entwickelt werden. Im Falle des Reallabors Schorndorf war dies ein appbasiertes Rufbus-System, das im Kontext des bereits bestehenden Nahverkehrsangebots der Stadt Schorndorf entwickelt wurde. Zentrales Ergebnis nach der Durchführung der TraSy-Methode soll ein System sein, das am Markt bzw. in der Gesellschaft bestehen kann, also über den Prototypenstatus hinausgeht. Ergebnisse, die während des Prozesses der Methodendurchführung entstehen und sich im Laufe des Vorgehens weiterentwickeln, sind: 1) die Beschreibung von Nutzertypen, 2) konkrete Nutzungsszenarien, 3) eine Liste mit Anforderungen unterschiedlicher Nutzertypen an das System, 4) Konzeptentwürfe des zu entwickelnden Systems und 5) Prototypen. Das Vorgehen der TraSy-Methode wird geleitet und strukturiert von zwei zentralen Arbeitsdokumenten: 1) ein Leitplankendokument, das Fragen und Antworten in Bezug auf die konkrete Ausgestaltung des Systems umfasst, und 2) ein Spezifikationsheft, das konkrete Anforderungen an das System zusammenfasst (für eine detaillierte Beschreibung von Leitplankendokument und Spezifikationsheft, s. Kapitel 2). 


\subsection{Für welche Themenbereiche ist die TraSy-Methode geeignet?}

Zwar wurde die TraSy-Methode im Bereich der Mobilitätsforschung entwickelt, jedoch kann sie in zahlreichen weiteren Bereichen angewendet werden, in denen soziotechnische Systeme entwickelt werden sollen. Denkbar ist beispielsweise die Anwendung der TraSy-Methode in Bereichen wie Infrastruktur und Städtebau (z. B. Bau eines neuen Kindergartens), Energieerzeugung (z. B. Bau einer Windkraftanlage) oder Bildung (z. B. Entwicklung einer Online-Lernplattform).

Besonders geeignet ist die Anwendung dieser Methode bei Themenbereichen, bei denen es darum geht, Anforderungen unterschiedlicher Nutzer(innen) einer heterogenen Bevölkerung an ein neues soziotechnisches System zu explorieren. $\mathrm{Zu}-$ dem ist die Methode dann besonders geeignet, wenn das notwendige Wissen für die Beschreibung der Nutzeranforderungen über verschiedene Praxisakteure ${ }^{6}$ verteilt ist. Durch einen nutzertyporientierten Ansatz über alle Entwicklungsschritte hinweg kann der Heterogenität der Bevölkerung Rechnung getragen werden. Die TraSy-Methode kann insbesondere dann eine geeignete Methode für ein Forschungsprojekt darstellen, wenn folgende Kriterien gegeben sind:

- Nutzeranforderungen an ein noch nicht existierendes soziotechnisches System sollen identifiziert, beschrieben und validiert werden.

- Das Projektthema berührt den Alltag und die täglichen Praktiken einer heterogenen Nutzergruppe.

- Neben direkten Nutzer(inne)n werden von der Entwicklung auch indirekte Nutzer(innen), wie zum Beispiel Anwohner(innen), betroffen sein.

- Das Thema berührt die Bedürfnisse und Anforderungen diverser Interessengruppen, die teilweise im Gegensatz zueinander stehen können.

- Das Thema erfordert Entscheidungen, die langfristige Effekte auf gesellschaftlich relevante Lebensbereiche haben und gesellschaftspolitische Konflikte bewirken können.

6 Als ,Praxisakteure“ werden hier Akteure aus der Praxis bezeichnet, die punktuell am Projekt beteiligt sind, die also nicht fester Bestandteil des Projektteams sind. Die Praxisakteure können aus der Zivilgesellschaft, dem Staat, Verbänden und Verbünden oder der Wirtschaft stammen. 


\subsection{Was unterscheidet die TraSy-Methode von anderen partizipativen Methoden?}

Das Vorgehen bei der TraSy-Methode zeichnet sich v. a. durch den Einsatz qualitativ-partizipativer Dialog-Methoden aus, die einen „kommunikativen Raum“ (Bergold und Thomas 2010, S. 335) für die Wissensgenerierung schaffen sollen.

Der umfassende Partizipationsansatz unterscheidet die TraSy-Methode von Methoden der partizipativen Technologiefolgenabschätzung (pTA), die vorsieht, Betroffene und Interessierte an soziotechnischen und politischen Entscheidungsprozessen zu beteiligen, um bei politischen Entscheidungen möglichst zahlreiche Aspekte eines Problems zu berücksichtigen (Joss und Bellucci 2002). Der Fokus der pTA liegt damit auf der Bewertung von technischen Entwicklungen, um Entscheidungen zu unterstützen (Hennen 2012), während die TraSy-Methode die Entwicklung eines neuen Systems gemeinsam mit den zukünftigen Nutzer(inne)n zum Ziel hat. Anders als bei der pTA arbeiten bei der TraSy-Methode Praxisakteure (insbes. Praxispartner) ${ }^{7}$ und Wissenschaftler(innen) ${ }^{8}$ gemeinsam an Aufgaben. Somit wird insbesondere den Praxispartnern aktive Mitbestimmung eingeräumt, d. h., die Deutungshoheit liegt bei den Wissenschaftler(inne)n und den Praxispartnern.

Die TraSy-Methode weist auch eine Ähnlichkeit mit den Ansätzen der Partizipativen Technikentwicklung auf (Kucharski und Merkel 2018). Gemeinsamkeiten zwischen den Ansätzen der Partizipativen Technikentwicklung und der TraSyMethode sind im iterativen Vorgehen zu sehen sowie im symmetrischen Verhältnis zwischen den Wissenschaftler(inne)n und den zukünftigen Nutzer(inne)n, die als gleichberechtigte Forschungssubjekte und nicht als Untersuchungsobjekte angesehen werden (Bergold und Thomas 2012; Müller et al. 2014). Die TraSyMethode geht jedoch über die Beteiligung von Nutzer(inne)n in der Designphase hinaus und beteiligt sie auch in den weiteren Phasen der Systementwicklung.

Die TraSy-Methode weist schließlich Aspekte des Design Thinkings auf, da sie ebenfalls auf der Annahme basiert, dass alltägliche Probleme erfolgreicher gelöst werden können, wenn die Bedürfnisse und Motive der zukünftigen Nutzer(innen) berücksichtigt werden, indem diese in einen iterativen Entwicklungsprozess einfließen (Plattner et al. 2009). Die TraSy-Methode verwendet jedoch nur Teilaspekte des Design Thinking-Ansatzes, wie beispielsweise den iterativen Charak-

7 ,Praxispartner' sind gleichberechtigte Mitglieder eines Projektteams, die in unterschiedlicher Intensität am Projekt partizipieren, jedoch immer Entscheidungsgewalt haben und kontinuierlich im Projekt mitwirken.

8 Als ,Wissenschaftler(innen)' werden Personen bezeichnet, die dem Wissenschaftssystem angehören und forschend im Projekt involviert sind (Defila und Di Giulio 2018b). 
ter der Konzeptentwicklung. Die TraSy-Methode ist im Vergleich zu einem Design Thinking-Prozess aufwendiger, aufgrund der umfangreichen Analysen von Daten aus Primär- und Sekundärquellen.

\subsection{Wer wird in der TraSy-Methode beteiligt und welche Kompetenzen der Beteiligten sind erforderlich?}

Im Sinne der transdisziplinären Forschung entspricht die TraSy-Methode der Position, Forschung mit Menschen zu betreiben, nicht über oder für Menschen (Bergold und Thomas 2010, S. 333). Deshalb werden die Nutzer(innen) in allen Teilschritten der TraSy-Methode als aktiv Partizipierende und Expert(inn)en ihrer Lebenswelt einbezogen. Dabei ist es wichtig, zu beachten, Unterschiede zwischen Wissenschaftler(inne)n und Praxisakteuren nicht zu verwischen und Praxisakteure nicht $\mathrm{zu}$ Wissenschaftler(inne)n zu machen, sondern in ihrer Rolle als Expert(inn)en der eigenen Lebenswelt zu belassen (Defila und Di Giulio 2018a).

Das Projektteam sollte aus Wissenschaftler(inne)n und Praxispartnern bestehen. Praxispartner sind Akteure, die durch ihr Praxiswissen einen wichtigen Beitrag zur Systementwicklung beitragen, da sie den Anwendungskontext überaus gut kennen. Weiterhin sind Praxispartner Vertreter bestimmter Nutzergruppen wie beispielsweise eines Seniorenverbands. Die TraSy-Methode sieht vor, dass verschiedene wissenschaftliche Disziplinen gemeinsam an der Entwicklung des neuen Systems arbeiten. Je nach thematischem Schwerpunkt des zu entwickelnden Systems werden neben Sozial- und Verhaltenswissenschaftler(inne)n auch Informatiker(innen), Ingenieur(inn)e(n) und Designer(innen) beteiligt.

Empfehlenswert ist, wenn sowohl die wissenschaftlichen als auch die außerwissenschaftlichen Teammitglieder (Praxispartner) eine Vorstellung der jeweiligen Kompetenzen und Arbeitsbereiche der anderen Teammitglieder besitzen. Falls dies nicht der Fall sein sollte, also beispielsweise die außerwissenschaftlichen Teammitglieder noch nicht in Forschungsprojekten mit Wissenschaftler(inne)n gearbeitet haben, bietet es sich an, zu Beginn ein längeres Treffen innerhalb des Projektteams zu organisieren, mit dem Ziel, die Kompetenzen und Rollen innerhalb des Projektteams zu bestimmen und die Erwartungen an die Zusammenarbeit zu diskutieren. Eine weitere Herausforderung, die sich bei der Zusammenarbeit von Forscher(inne)n und Praxispartnern ergeben kann, ist die Verständigung auf eine gemeinsame Sprache. So ist es zum Beispiel von hoher Bedeutung, dass alle Beteiligten dasselbe unter einem Begriff wie beispielsweise „System“ verstehen. Diese Aspekte sind auch in der Interaktion mit den Praxisakteuren außerhalb des Projektteams zu beachten.

Die Definition der Rolle der Wissenschaftler(innen) stellt eine Herausforderung für das transdisziplinäre Arbeiten dar. Jaeger-Erben et al. sprechen dabei von 
einer Hybridisierung der Rollen von Wissenschaftler(inne)n, die ,[...] oft neben der Erzeugung und Systematisierung von Wissen auch noch die Rolle von Moderatoren, Mediatoren, Supervisoren, Marketing- und Kommunikationsfachleuten, Pädagogen und noch viel mehr übernehmen. Diese Vielfalt an Rollen geht oftmals weiter [sic!] über das hinaus, was Wissenschaftler(innen) qua Expertise und Profession vertreten und verantworten können“ (2018, S. 120). Es bietet sich daher an, die Rollen der beteiligten Akteure vor Projektbeginn klar zu definieren und im Projektverlauf kontinuierlich zu überprüfen, ob diese noch eingehalten werden oder ob eine Anpassung der Rollenstrukturen vorgenommen werden muss. Zudem deutet diese Herausforderung auf die Relevanz der Schulung der Wissenschaftler(innen) bezüglich der verwendeten Methoden und Soft Skills hin.

Im Falle des Reallabors Schorndorf wurde ausgehend von der Projektkonzeption ein interdisziplinäres Wissenschaftsteam aus Ingenieur(inn)en, Psycholog(inn)en, Sozial- und Kommunikationswissenschaftler(inne)n sowie Stadtplaner(inne)n zusammengestellt, das alle wissenschaftlich-empirischen Arbeitsschritte (z. B. Datenanalyse, Interviews, Workshops) der TraSy-Methode durch seine Kompetenz und sein Methodenportfolio selbst durchführen konnte.

\subsection{Wie werden Einzelmethoden zur TraSy-Methode kombiniert?}

Die TraSy-Methode besteht aus einer Kombination von bereits existierenden Einzelmethoden, die für die Entwicklung eines komplexen Systems auf Basis heterogener Nutzeranforderungen gezielt zusammengestellt wurden. Bei der Zusammenstellung der Einzelmethoden zur TraSy-Methode wurde die Stimmigkeit der Methodenintegration und -rekombination beachtet, wie von Defila und Di Giulio (2018a, S. 51) gefordert. Die TraSy-Methode wurde gezielt für ein breites Anwendungsfeld entwickelt. Damit ist die Übertragbarkeit auf andere Themenbereiche gegeben (s. Abschnitt 1.2). Für die TraSy-Methode wurden qualitative und quantitative Methoden kombiniert. So wurde im Reallabor Schorndorf beispielsweise die quantitative Methode der Datenanalyse gewählt, um den Status quo in Bezug auf die soziodemografische Zusammensetzung der Bevölkerung Schorndorfs zu analysieren (s. Abschnitt 2.1). Der Großteil der Einzelmethoden in der TraSy-Methode beruht jedoch auf qualitativen Methoden empirischer Sozialforschung, die sich besonders dann eignen, wenn es darum geht, (noch) nicht (quantitativ) messbare Sachverhalte, Themen und Entwicklungen zu erfassen und zu explorieren (Grosvenor 2000; Røe 2000). Das Aufdecken subjektiver, handlungsleitender „Relevanzstrukturen hinter dem sichtbaren Verhalten“ (Scheiner 1998, S. 62f.) und das Verstehen psychologischer und sozialer Aspekte, die das Handeln der Menschen (mit-)bestimmen, kann nur mit Hilfe qualitativer Methoden erfolgen. 


\section{Der Weg zu neuen soziotechnischen Systemen mit Hilfe der TraSy-Methode}

Die TraSy-Methode wird so beschrieben, dass auch eine Anwendung auf andere Themenbereiche als den, der im Reallabor Schorndorf bearbeitet wurde, möglich ist. Dabei ist zu beachten, dass die Beschreibung der TraSy-Methode keine starre Methodenanleitung darstellt, sondern eine, die an den jeweiligen Kontext anzupassen ist. In Abhängigkeit des ,Umfangs' der Forschungsfrage muss deshalb beispielsweise die passende Anzahl und Länge der Workshops gewählt werden.

Abb. 1 zeigt den Weg von der Analyse des Handelns der potenziellen Nutzer(innen) des zu entwickelnden Systems (1) über die Identifikation von Nutzertypen und potenziellen Nutzungsszenarien im zukünftigen System (2) hin zur Generierung von Ideen und Konzepten (3) sowie zur Erprobung und Evaluierung (4) des neuen Systems. Unter Nutzungsszenarien sind dabei potenzielle Einsatzzwecke des zu entwickelnden Systems zu verstehen. Das im Folgenden beschriebene Vorgehen bezieht sich auf die Schritte 1-3. Die Erprobung des entwickelten Systems (4) ist nicht Gegenstand dieses Beitrags. An dieser Stelle soll jedoch betont

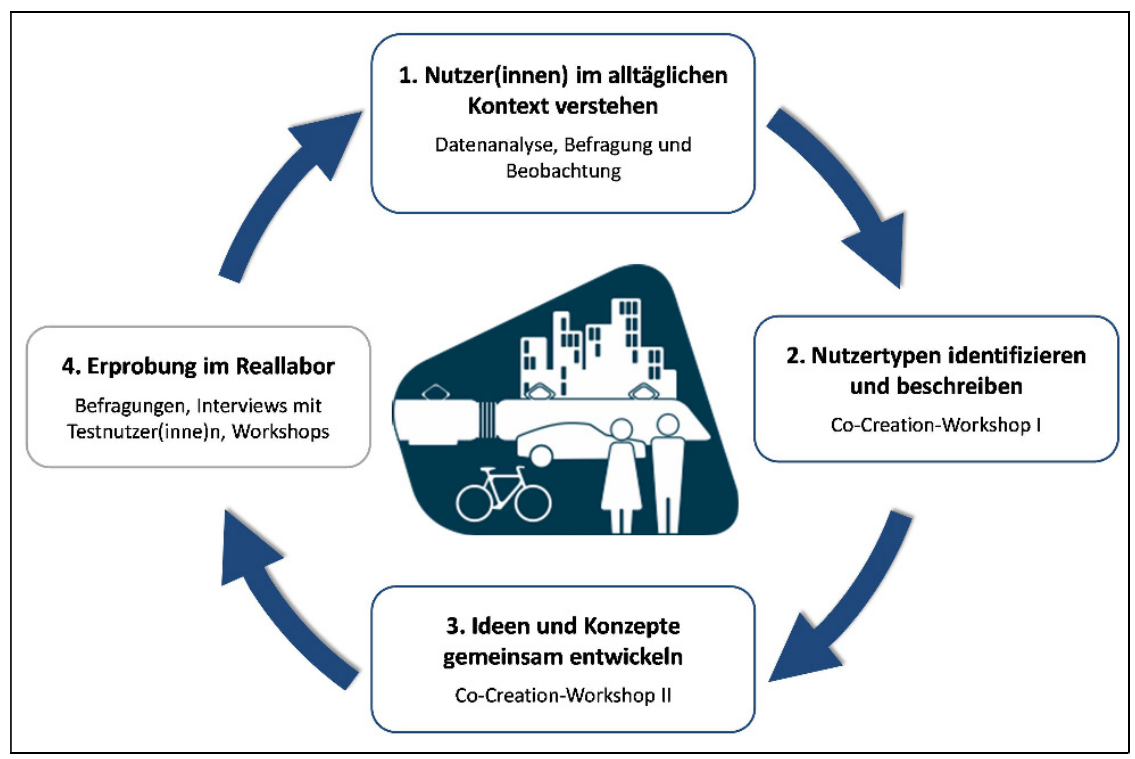

Abbildung 1: Schematische Darstellung der vier Phasen der TraSy-Methode; im Beitrag wird auf die Phasen 1-3 im Detail eingegangen. (C) Laura Gebhardt, Alexandra König. 
werden, dass ein mit der TraSy-Methode entwickeltes System unter Realbedingungen erprobt und validiert werden sollte. So testeten die Schorndorfer(innen) für neun Monate das im Reallabor Schorndorf mit der TraSy-Methode entwickelte Bussystem. In einem Pilotbetrieb vom März bis Dezember 2018 wurden die Nutzer(innen) intensiv wissenschaftlich begleitet, und das System wurde auf dieser Grundlage kontinuierlich evaluiert und angepasst (Brost et al. 2018; Gebhardt et al. 2019).

Das nachfolgend beschriebene methodische Vorgehen wird vor dem Hintergrund der gemachten Erfahrungen im Reallabor Schorndorf formuliert, es kann jedoch, wie in Abschnitt 1.2 gezeigt, auch in thematisch und räumlich andere Kontexte übertragen werden. Je nach Untersuchungsgegenstand bzw. Projektvorhaben gilt es, die Zeiträume, die für die einzelnen Phasen zur Verfügung stehen, anders zu definieren. Im Reallabor Schorndorf dauerte Phase 1 rund drei Monate, Phase 2 und Phase 3 dauerten von der Konzeption, über die Rekrutierung der Teilnehmer(innen) bis hin zur Durchführung und Auswertung jeweils rund fünf Monate, Phase 4 zehn Monate.

\subsection{Nutzer(innen) im alltäglichen Kontext verstehen}

Da die Methode stets in einem gesellschaftlichen Kontext angewendet wird, ist es wichtig, diesen Kontext annäherungsweise in seiner Komplexität zu verstehen. Ziel der ersten Phase der TraSy-Methode ist daher das Verstehen der Ausgangssituation, in die das zukünftige System eingebettet sein wird. Erfasst werden müssen zum Beispiel die raumstrukturellen Charakteristika (z. B. vorhandene Infrastruktur) und die soziodemografischen Besonderheiten des Untersuchungsgebiets. Diese Aufgabe wiederum wird von den Wissenschaftler(inne)n im Projektteam wahrgenommen. Dafür werden drei klassische Methoden empirischer Sozialforschung angewandt: 1) eine Daten- und Dokumentenanalyse, 2) eine teilnehmende Beobachtung und 3) Experteninterviews (s. Abb. 2).

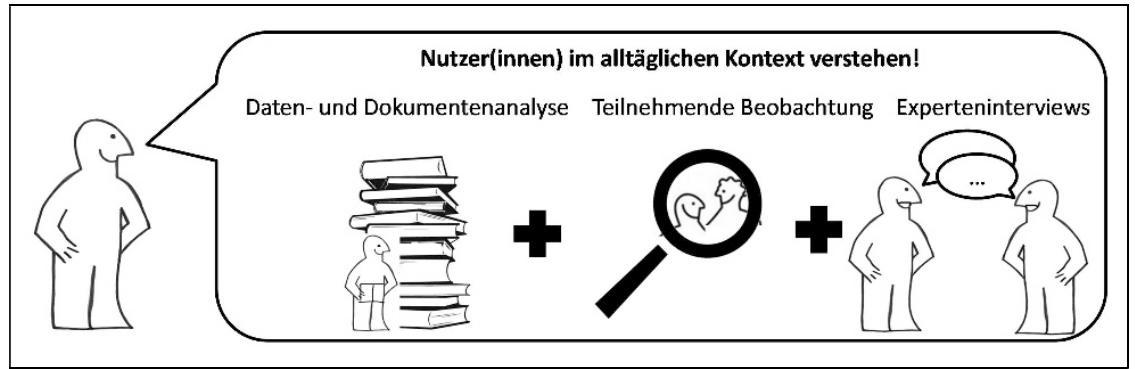

Abbildung 2: Grafische Darstellung der Arbeitsschritte in Phase 1 der TraSy-Methode. (C) Laura Gebhardt, Alexandra König. 
Alle Ergebnisse und Zwischenschritte werden systematisch dokumentiert. Dazu sieht die TraSy-Methode zwei zentrale Dokumente vor, ein Leitplankendokument sowie ein Spezifikationsheft. Im Leitplankendokument werden kontinuierlich während des gesamten Projektverlaufs Fragen gesammelt, die als relevant erachtet werden. Diese werden innerhalb des Projektteams regelmäßig diskutiert und bearbeitet. Das Leitplankendokument wird als Tabelle, mit Hilfe eines Tabellenkalkulationsprogramms o. Ä., erstellt (s. Abb. 3). Zu Beginn des Projekts ist das Leitplankendokument eine leere Tabelle. Alle zu diskutierenden Fragen werden einzeln in Tabellenzeilen notiert. In den dazugehörigen Spalten werden Ansprechpartner innerhalb oder außerhalb des Projektteams benannt. Zudem können neben der eigentlichen Beantwortung der Frage auch deren Bearbeitungsstatus sowie die Dringlichkeit der Frage festgehalten werden. Gemeinsam , abgesegnete Antworten werden hier festgehalten und dann in das Spezifikationsheft überführt.

Das Spezifikationsheft stellt eine Weiterentwicklung klassischer Lasten- und Pflichtenhefte dar, wie man sie in herkömmlichen Entwicklungs- und Produktionsprozessen kennt, d. h., es ist eine Zusammenführung aller für die Entwicklung des Systems relevanten Informationen. Es dient der Dokumentation der Ergebnisse aus allen Arbeiten, der Herleitung und Begründung der Parameterfestlegung (wie im Falle des Reallabors Schorndorf beispielsweise der Haltestellenabstände), dem Wissensaustausch zwischen den Projektbeteiligten sowie der Vereinheitlichung des Kenntnisstandes durch fortgesetztes Einarbeiten der Ergebnisse von Absprachen und Berichten.

Am Anfang des Dokuments werden die Projektziele aufgelistet und der Zeitplan eingetragen. Weiterhin werden die (z. B. von Teilprojekten) erwarteten Teilergebnisse benannt. Zu Beginn des Projekts enthält das Spezifikationsheft ausschließlich diese Inhalte. Im weiteren Verlauf wird das Herzstück des Dokuments ergänzt: die Beschreibung der Spezifikationen des zu entwickelnden Systems. Die Spezifikationen sind detaillierte Beschreibungen der Systemeigenschaften (im Reallabor Schorndorf gehörte dazu z. B. der maximale Haltestellenabstand zwischen den einzelnen Haltestellen). Die Spezifikationen werden, so detailliert wie möglich, in thematisch abgegrenzten Kapiteln festgehalten, die in ihrer Gesamtheit einen Überblick über das System als Ganzes bieten. Das Spezifikationsheft wird nach der Durchführung jeder der Einzelmethoden anhand der neuen Erkenntnisse und Ergebnisse durch das Projektteam aktualisiert. Ergebnisse werden so fortlaufend festgehalten und innerhalb des Projektteams in Treffen und regelmäßigen Telefonkonferenzen diskutiert, die bestenfalls zweiwöchentlich stattfinden sollten und deren Länge von den jeweils zu besprechenden Themen abhängt. In diesen Besprechungen wird entschieden, welche Ergebnisse, d. h. welche Spezifikationen in das Spezifikationsheft aufgenommen werden sollen. Das Spezifikationsheft dient zum Beispiel auch zur Beauftragung von Unteraufträgen (wie z. B. Programmiertätigkeiten). 


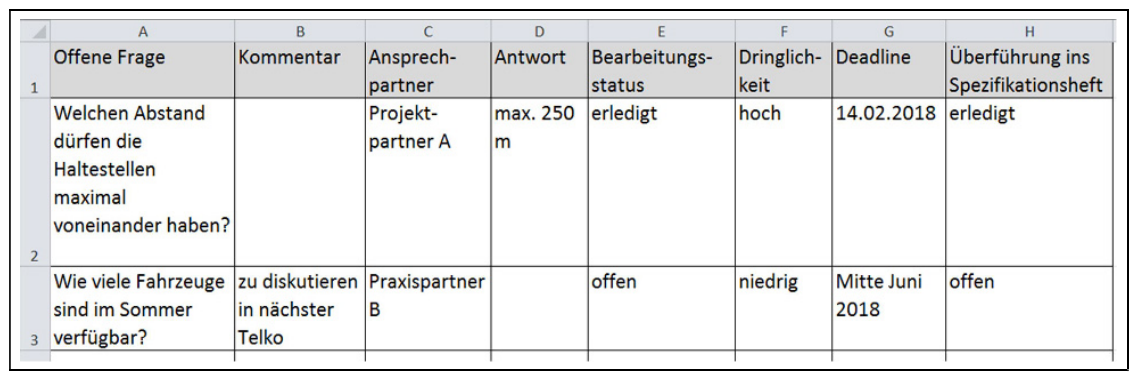

Abbildung 3: Beispiel eines Leitplankendokuments. (C) Laura Gebhardt, Alexandra König.

\subsubsection{Daten- und Dokumentenanalyse}

Ausgehend von der Annahme der handlungsorientierten Forschung, dass Menschen ihr Handeln unter den Bedingungen ihrer Lebenswelt produzieren und reproduzieren, werden das alltägliche Handeln der Nutzer(innen) und dessen Handlungsrahmen als Ausgangspunkt für die Entwicklung des neuen Systems in den Blick genommen. Konkret bedeutet dies, dass soziodemografische und räumliche Daten recherchiert, ausgewertet und interpretiert werden. Die Dokumentenanalyse sollte von der Frage geleitet sein, welche Daten wichtig sind, um den Status quo hinsichtlich des Projektziels zu verstehen. Dabei sollte aber stets dem Prinzip gefolgt werden, dass es umso besser ist, je mehr Informationen über das zu untersuchende Gebiet, seine Charakteristika und die darin handelnden Menschen gesammelt und ausgewertet werden. Für die Daten- und Dokumentenanalyse bieten die jeweiligen nationalen statistischen Ämter oder kommunalen Datenbestände, im Falle Deutschlands das Statistische Bundesamt oder das Bundesinstitut für Bau-, Stadt- und Raumforschung, eine solide Basis solcher Daten.

Zunächst gilt es, die relevanten und zu betrachtenden Parameter zu identifizieren. Da die TraSy-Methode soziotechnische Systeme entwickelt, sind soziodemografische Daten stets zu recherchieren. Alle weiteren relevanten Parameter hängen von dem zu entwickelnden System ab. Bei einem Vorhaben im Bereich Mobilität macht es beispielsweise Sinn, sich das aktuelle Mobilitätsverhalten im Untersuchungsraum anzusehen. Bei der Betrachtung ist immer auch von Bedeutung, die recherchierten Zahlen in Vergleich zu anderen Räumen (z. B. der gesamtdeutsche Durchschnitt) zu setzen, um ein besseres Verständnis für die spezifischen Charakteristika des Untersuchungsgebiets (z. B. der überdurchschnittliche Altersdurchschnitt der Bevölkerung) zu bekommen.

Zentrale Fragen im Reallabor Schorndorf waren beispielsweise: Wer wohnt hier? Welche Verkehrsmittel werden derzeit bevorzugt genutzt? Wie gut ist der öffent- 


\section{Altersstruktur:}

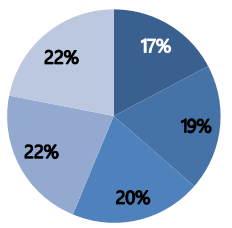

unter 18

$\square$ - 18 bis unter 35

35 bis unter 50

50 bis unter 65

65 und älter

$\mathrm{N}=39.520$

Das Durchschnittsalter in Schorndorf beträgt 51,5 Jahre.

Der Bundesdurchschnitt liegt bei 44,3 Jahren.

\section{Pkw-Besitz:}

Deutschland (gesamt)

Schorndorf

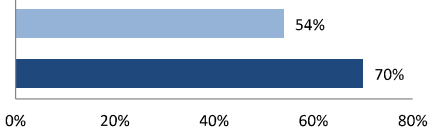

\section{Modal Split:}

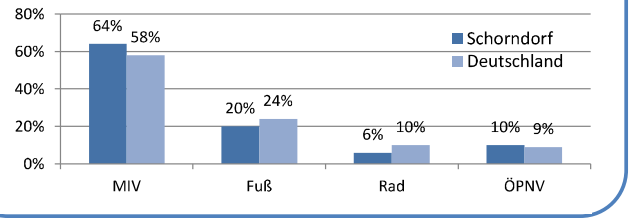

\section{Haushaltsmerkmale:}

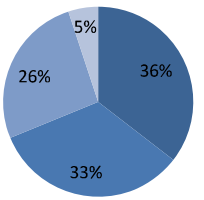

$$
\begin{aligned}
& \text { Einpersonenhaushalte } \\
& \text { Zweipersonenhaushalte } \\
& \text { Drei- und Vierpersonenhaushalte } \\
& \text { Haushalte mit } 5 \text { und mehr Personen } \\
& N=17.891
\end{aligned}
$$

Im Durchschnitt leben 2,2 Personen in einem Haushalt.

Der Bundesdurchschnitt liegt bei 2 Personen.

Abbildung 4: Relevante Kriterien für die Daten- und Dokumentenanalyse sowie beispielhafte Ergebnisse für das im Reallabor Schorndorf untersuchte Thema. Von oben: Altersverteilung der Bevölkerung in Schorndorf nach Altersgruppen; Pkw-Besitz in Schorndorf im bundesdeutschen Vergleich; Verkehrsmittelnutzung in Schorndorf im bundesdeutschen Vergleich; Haushaltsstruktur in Schorndorf. Quellen: Verband Region Stuttgart 2011, Mobilität in Deutschland 2008. C) Laura Gebhardt, Alexandra König. 
liche Verkehr? Die Abb. 4 illustriert beispielhaft für das Vorhaben in Schorndorf die relevanten Parameter sowie Ergebnisse der Dokumentenanalyse. Für die Analyse der Ausgangssituation in Schorndorf wichtige Parameter waren etwa die Altersstruktur und die aktuelle Verkehrsmittelnutzung.

\subsubsection{Teilnehmende Beobachtung}

Um ein über die Analyse von statistischen Daten hinausgehendes Verständnis für die Rahmenbedingungen vor Ort zu erlangen, finden teilnehmende Beobachtungen statt. Das Kennzeichnende dieser Methode ist die persönliche Teilnahme der Forschenden an Interaktionen im Untersuchungsraum. Dabei ist die Annahme leitend, dass durch die dabei mögliche unmittelbare Erfahrung der Situation Aspekte des Handelns und Denkens beobachtbar werden, die in Gesprächen und Dokumenten über diese Interaktionen bzw. Situationen nicht zugänglich wären (Girtler 2002; Lüders 2003).

Damit gute Ergebnisse erzeugt werden, müssen die Ziele der teilnehmenden Beobachtungen im Vorfeld klar definiert werden, so dass die Beobachter(innen) wissen, auf was sie achten müssen. Zudem muss eine Dokumentationsform bestimmt werden, mit deren Hilfe die gemachten Beobachtungen festgehalten werden. Hier bietet sich ein vorstrukturiertes Beobachtungsprotokoll an. Dieses sollte geschlossene Fragen enthalten (im Reallabor Schorndorf z. B. „Wie viele Fahrgäste waren im Bus anwesend?"), aber auch die Möglichkeit für Notizen über weiterführende Beobachtungen in Freitextfeldern lassen. Da das Vorgehen einen explorativen Charakter besitzt, muss flexibel auf Veränderungen eingegangen werden können. Das bedeutet, dass während der Beobachtung weitere Fragen in das Beobachtungsprotokoll aufgenommen werden sollten, wenn sich Hinweise auf deren Relevanz ergeben. Die teilnehmende Beobachtung sollte verteilt auf mehrere Tage stattfinden, um ein möglichst genaues Bild der Situation zu erhalten. Zudem sollten sich mindestens zwei Beobachter(innen) ein Bild von der Lage machen und ihre Ergebnisse unabhängig voneinander dokumentieren und auswerten. Die Auswertung sollte zwar von den vorher definierten Forschungsfragen geleitet werden, jedoch sollte die teilnehmende Beobachtung auch genutzt werden, um neue Hinweise zu erhalten, die in den anschließenden Experteninterviews diskutiert werden können.

Im Reallabor Schorndorf wurden Vor-Ort-Begehungen von Haltestellen und Probefahrten mit den bestehenden innerstädtischen Buslinien vorgenommen. Das Ziel der teilnehmenden Beobachtungen war die Erfassung von Informationen zu folgenden Punkten: Pünktlichkeit, Fahrgastaufkommen und -struktur, die Situation beim Ein- und Ausstieg, Möglichkeiten zum Festhalten, Gangbreite und Situation beim Durchgehen nach hinten, Platzverhältnisse (für Kinderwagen, Rollstühle etc.), Fahrkomfort, Ticketkauf sowie allgemeine Stimmung und Interaktionen zwischen den Fahrgästen. 


\subsubsection{Experteninterviews}

Um die in den vorausgehenden Schritten gesammelten Daten und Einsichten zu untermauern, werden Interviews mit Personen vor Ort geführt. Als Expert(inn)en werden Personen mit Vorwissen zum jeweiligen Themenfeld, in das das Projektvorhaben eingebettet ist, ausgewählt. Dabei gilt es, Expertengespräche mit Personen aus unterschiedlichen Bereichen zu führen, um eine möglichst große Bandbreite an Einschätzungen einzufangen. Die Gespräche haben zum Ziel, explorative Erkenntnisse über die Gegebenheiten im Untersuchungsraum zu erhalten, um den Rahmen, in dem das System angewendet werden soll, möglichst gut zu kennen. Zudem wird mit den Gesprächen die Bildung eines lokalen Netzwerkes an Expert(inn)en angestrebt, die auch für weitere Fragen konsultiert werden und die Einführung des neuen Systems unterstützen können. Das Hauptkriterium für die Auswahl der Expert(inn)en ist deren Zugang zu spezifischem Wissen, das für die Systementwicklung von Bedeutung sein könnte. Die Befragung der Expert(innen)en dauert ungefähr eine Stunde und basiert auf einem teilstrukturierten Leitfaden, der sowohl für alle identische Fragen enthält als auch solche, die zugeschnitten sind auf den Kompetenzbereich des/der jeweiligen Experten(in) (für ein Beispiel aus dem Reallabor Schorndorf, s. Anlage a im Anhang dieses Beitrags). Für diese Arbeiten ist es wichtig, Mitarbeiter(innen) mit Erfahrungen in der Anwendung von Methoden der empirischen Sozialforschung im Projekt zu haben.

Die Auswertung der Expertenbefragung erfolgt mit Blick auf die Identifizierung potenzieller Nutzertypen sowie möglicher Nutzungsszenarien. Diese fließen als Input in Phase 2 der TraSy-Methode ein. Die Erkenntnisse aus Phase 1 werden in die beiden zentralen Dokumente zur Wissensbündelung innerhalb des Gesamtprojekts - das Leitplankendokument und das Spezifikationsheft - überführt.

Im Reallabor Schorndorf wurden die Experteninterviews mit Vertreter(inne)n des Verkehrsclubs Deutschland (VCD), des Allgemeinen Deutschen Fahrrad-Clubs (ADFC), eines lokalen Gewerbevereins (Centro), von Stadtmobil, des Seniorenforums, eines lokalen Taxiunternehmens, eines lokalen Busunternehmens und des Gemeinderats durchgeführt. Mit Hilfe der Expert(inn)en wurden Erkenntnisse gesammelt über aktuelle Schwachstellen und Nutzungshürden im Zusammenhang mit dem öffentlichen Nahverkehr sowie Erkenntnisse über mögliche Zielgruppen des neuen Buskonzepts (Senior(inn)en, mobilitätseingeschränkte Personen, regelmäßige Autonutzer(innen), regelmäßige Busnutzer(innen)). 


\subsection{Nutzertypen identifizieren und beschreiben- Co-Creation-Workshop I}

In dieser Phase geht es darum, die in Phase 1 benannten möglichen Nutzertypen und Nutzungsszenarien vertiefend zu prüfen und zu charakterisieren. Dies erfolgt nun gemeinsam mit Praxisakteuren.

Von entscheidender Bedeutung für den Erfolg von Methoden, die auf die Einbeziehung von Praxisakteuren (insbes. aus der Zivilgesellschaft) ausgerichtet sind, erweisen sich Dialog-Methoden, die einen „kommunikativen Raum“ (Bergold und Thomas 2010, S. 337) für die Wissensgenerierung schaffen (Defila und Di Giulio 2018a, S. 45). Daher sieht die TraSy-Methode im Anschluss an die beschriebene Analyse der Ausgangssituation einen Co-Creation-Workshop vor. CoCreation beschreibt dabei einen kreativen und wechselseitigen Lernprozess, in den sowohl wissenschaftliche als auch außerwissenschaftliche Akteure involviert sind, mit dem Ziel, gemeinsame Ergebnisse zu generieren.

Der im Folgenden beschriebene Workshop I dient dazu, die unterschiedlichen Nutzertypen und deren Anforderungen an das zu entwickelnde System zu verstehen und potenzielle Nutzungsszenarien zu entwickeln. Zentrale Herausforderungen, deren man sich vorab bewusst sein sollte, sind dabei die Heterogenität der Akteure und die unterschiedlichen Anforderungen verschiedener Akteursgruppen, die sich zum Teil auch widersprüchlich gegenüberstehen. Dieser Herausforderung kann durch die Arbeit mit Nutzergruppen begegnet werden. Eine Nutzergruppensegmentierung stellt die Möglichkeit dar, die Komplexität heterogener Populationen durch die Identifikation von homogenen Subgruppen zu reduzieren (für eine Übersicht über Typisierungsansätze s. Hunecke 2015). Dadurch kann ein tiefgehendes Verständnis der Bedürfnisse einzelner Gruppen erzeugt und bei der Entwicklung des Systems berücksichtigt werden.

Dieser erste Workshop dient also dazu, die unterschiedlichen Nutzergruppen und deren Anforderungen an das zu entwickelnde System zu verstehen und potenzielle Nutzungsszenarien gemeinsam zu erarbeiten. Die Vorbereitung, Durchführung und Auswertung des Workshops sind im Folgenden in Bezug auf das Themenfeld Mobilität beschrieben, können jedoch analog auch in anderen Themenfeldern angewandt werden.

\subsubsection{Workshopziele}

Wie bereits dargelegt, hat der Co-Creation-Workshop I zum Ziel, die Nutzer(innen) bei der Ausgestaltung des Systems in einer frühen Entwicklungsphase zu involvieren, d. h., die nutzertypenspezifischen Anforderungen zu identifizieren. Auf Basis dieser Anforderungen können anschließend konkrete Entwürfe des Systems entstehen, die beschreiben, welche Eigenschaften es besitzen sollte, d. h., was es 
leisten muss, um den Anforderungen der unterschiedlichen Nutzertypen zu entsprechen.

Der Workshop hat folgende Ziele:

1) Spezifizierung und Validierung der Nutzertypen

2) Skizzierung einer prototypischen Nutzung des neuen Systems durch unterschiedliche Nutzertypen

3) Entwicklung und Beschreibung von Funktionsweisen des neuen Systems

Für die Entwicklung des Bussystems im Reallabor Schorndorf bedeutete dies: 1) detaillierte Beschreibung potenzieller Nutzergruppen des neuen Bussystems; 2) Skizzierung einer fiktiven Nutzung des Busses (Bedarfsermittlung, Reiseplanung und Reisedurchführung, s. Abb. 7); 3) Festlegung von Abholpunkten, Betriebszeiten und weiteren Funktionalitäten des Systems.

\subsubsection{Vorphase: Bisher identifiziertes Wissen nutzbar machen}

Die Ergebnisse der vorherigen Phase Nutzer(in) im alltäglichen Kontext verstehen (s. Abschnitt 2.1) werden in Form der im Leitplankendokument erfassten Antworten als Input für den Workshop verwendet (s. Abb. 5). Diese Antworten stellen wichtige Leitplanken dar, die der Arbeit im Workshop einen Rahmen geben sollen. Offene Fragen aus dem Leitplankendokument werden im Workshop adressiert, um Antworten zu finden. Die Leitplanken sind beispielsweise bestimmte Themen oder Fragestellungen, die durch das Projektvorhaben nicht beeinflusst werden können. Diese Restriktionen müssen zusammengestellt werden, um sie den Workshopteilnehmer(inne)n kommunizieren zu können. Im Reallabor Schorndorf war dies zum Beispiel die Frage nach dem Betriebsgebiet innerhalb der Stadt. Im Rahmen der Untersuchungen in Phase 1 hatte sich klar herausge-

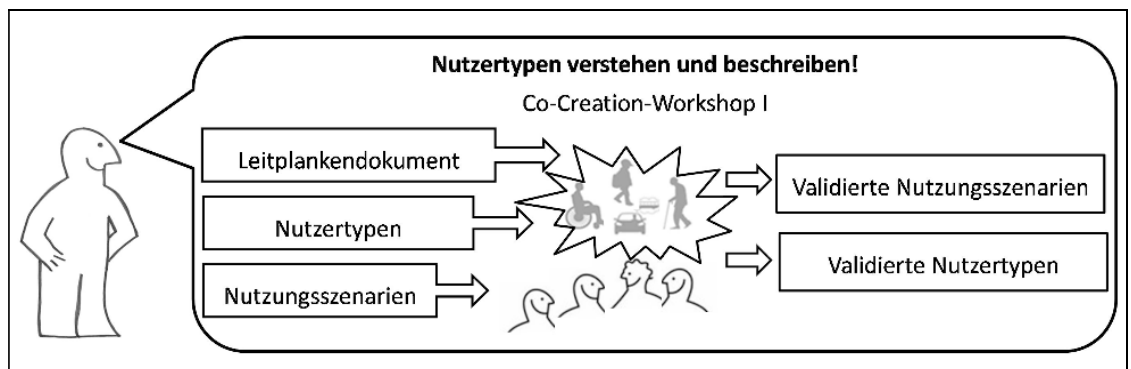

Abbildung 5: Co-Creation-Workshop I zur Validierung von Nutzertypen und Nutzungsszenarien. (C) Laura Gebhardt, Alexandra König. 
stellt, dass eine Einbindung aller Teilorte Schorndorfs aus technischen und organisatorischen Gründen nicht möglich sein würde. Diese Restriktion wurde in Form der Festlegung des in Frage kommenden Einsatzgebiets für das neue Bussystem von Anfang an kommuniziert.

Weiterhin ist das Wissen über Nutzertypen und deren Handeln, das in der vorherigen Phase (s. Abschnitt 2.1) generiert wurde, so aufzubereiten, dass es den Workshopteilnehmer(inne)n verständlich präsentiert werden kann. Dies geschieht in Form von grafisch aufbereiteten Profilen der identifizierten Nutzertypen (s. Abb. 6). Gleiches gilt für die angedachten Nutzungsszenarien (s. Abb. 7). Beide werden im Verlauf des Workshops weiter ausgestaltet.

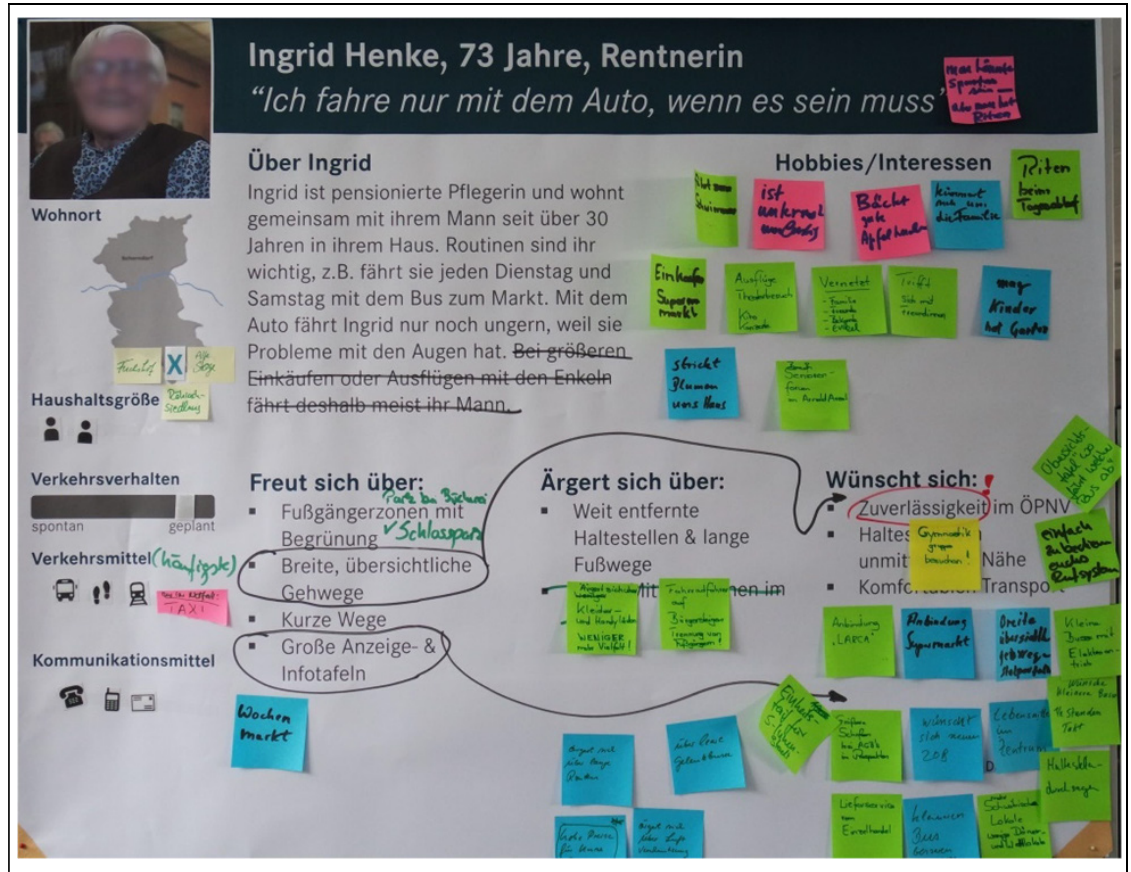

Abbildung 6: Die gedruckten Informationen basieren auf dem in Phase 1 gewonnenen Wissen über Nutzertypen. Sie dienen als Grundlage zur strukturierten Diskussion der Nutzertypen. Durch Beschriftungen, Klebezettel, Symbole (z. B. Darstellung der Verkehrsmittel) wird das Nutzerprofil gemeinsam weiterentwickelt, hier am Beispiel der Seniorin Frau Henke. (C) Salome Keller. 


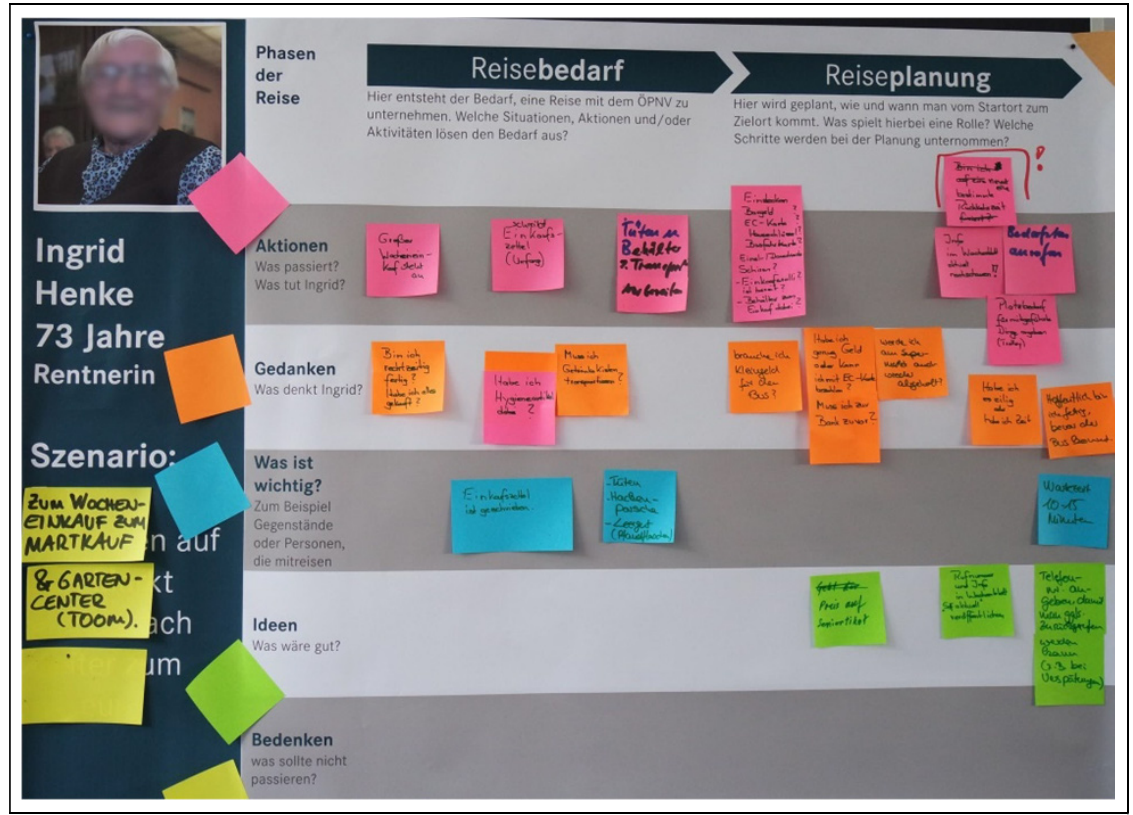

Abbildung 7: Die gedruckten Informationen basieren auf dem in Phase 1 gewonnenen Wissen über Nutzungsszenarien. Sie dienen als Vorlage zur Diskussion eines Nutzungsszenarios. Bezogen auf einen Nutzertyp - hier die Seniorin Frau Henke - wird der Reiseablauf schrittweise durchlebt und davon ausgehend prototypisch beschrieben. (C) Salome Keller.

\subsubsection{Inhaltliche und methodische Vorbereitung}

Mit Blick auf die methodische Vorbereitung sollte ein Regiebuch zur Planung des Workshopablaufs erstellt werden. Dieses Regiebuch kann eine einfache Tabelle mit Zeitangaben sowie Arbeitsaufträgen für und Leitfragen an die Teilnehmer(innen) sein, das die Moderator(inn)en unterstützt und gewährleistet, dass die Arbeit in Kleingruppen analog zueinander abläuft.

Wie bereits unter Vorphase: Bisher identifiziertes Wissen nutzbar machen (s. Abschnitt 2.2.2) genannt, gilt es vor allem, das Inputmaterial für den Workshop zielgruppenspezifisch aufzubereiten. Hier geht es in erster Linie um eine Komplexitätsreduktion. Es gilt, die relevanten Informationen und $\mathrm{zu}$ diskutierenden Themen und Fragestellungen verständlich aufzubereiten. Dies betrifft sowohl das Wissen um Nutzertypen und Nutzungsszenarien als auch die für das Projekt - und damit die Diskussion im Workshop - geltenden Restriktionen. Im Workshop des Reallabors Schorndorf erleichterte zum Beispiel ein großer Stadtplan die Diskussion um das Einsatzgebiet des Busses. 
Zur Strukturierung des Workshops sind zudem Visualisierungen zu empfehlen, die vorab erstellt werden sollten. Neben der Agenda (s. Abb. 8) können beispielsweise auch die Anleitung zur Vorstellungsrunde (Mein Name ist...; Ich wohne in...; Mein eindrucksvollstes Erlebnis im Zusammenhang mit...) oder Arbeitsaufträge entsprechend gestaltet werden.

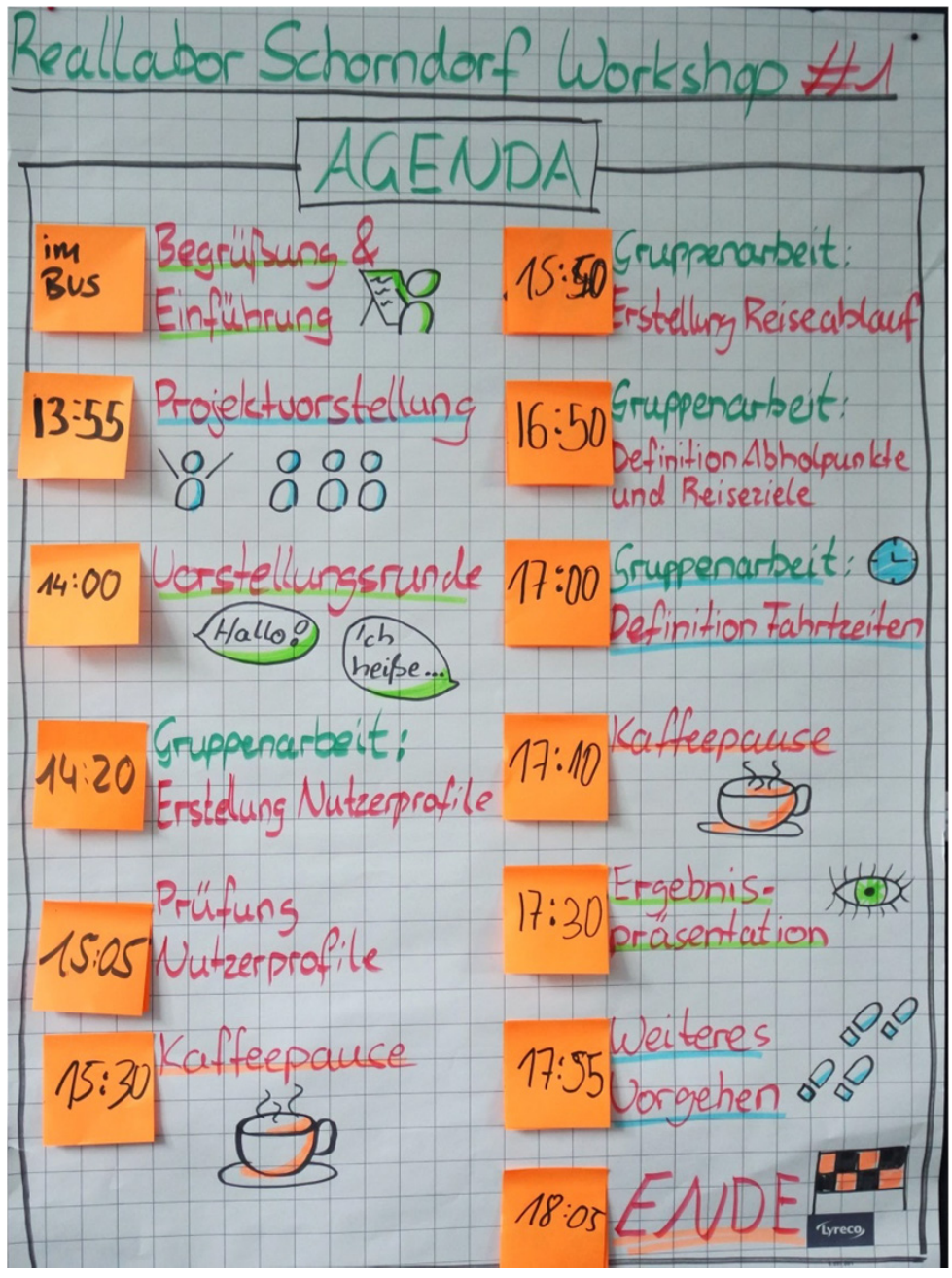

Abbildung 8: Illustrierter Ablauf des Workshops. Post-its bieten die Möglichkeit, auf Veränderungen, die nötig werden, agil zu reagieren und ggf. die Zeiten anzupassen. (C) Salome Keller. 
Für die Auswahl und Vorbereitung des Tagungsorts schließlich sind die folgenden Punkte zu bedenken: Es empfiehlt sich, zentral gelegene Räumlichkeiten zu nutzen, die für die Teilnehmer(innen) gut zu erreichen sind und genügend Platz sowohl für die Arbeit im Plenum als auch für die Arbeit in Kleingruppen bieten. Idealerweise lässt sich zusätzlich ein Eingangsbereich (Foyer) so gestalten, dass hier informelle Gespräche nach dem offiziellen Workshop stattfinden können. Das Anbringen eines beschreibbaren Posters mit der Überschrift „Themenspeicher" erweist sich als sinnvoll, um Anregungen und Fragen zu sammeln, für die im Rahmen des Workshops kein oder zu wenig Raum ist.

\subsubsection{Ansprache und Gewinnung der Teilnehmer(innen)}

Für die Motivation und Gewinnung der Teilnehmer(innen) ist es sinnvoll, Praxisakteure bei lokalen Einrichtungen anzusprechen. Zusätzlich kann die Rekrutierung durch Anzeigen in verschiedenen Medien (Wochenzeitung, Projekthomepage, Facebook, Twitter) oder durch eine Zufallsstichprobenziehung (nach Alter und Geschlecht) durch das Einwohnermeldeamt (soweit es um die Bevölkerung geht) und das Anschreiben der gezogenen Personen gelingen. Am Workshop sollten zwischen 15 und 20 Praxisakteure teilnehmen, deren Teilnahme vor dem Workshop bestätigt sein sollte. Diese Gruppengröße erlaubt es, sowohl intensiv in Kleingruppen (à 4-5 Personen) als auch im Plenum zu diskutieren.

Im Rahmen der Ansprache und Gewinnung der Teilnehmer(innen) wird kommuniziert, dass es Bild- und Tonaufnahmen geben wird, die lediglich für interne Zwecke verwendet werden. Innerhalb des Workshops wird dies den Teilnehmer(inne)n noch einmal schriftlich vorgelegt, mit der Bitte, sich durch ihre Unterschrift damit einverstanden zu erklären.

Ausreichende Informationen bezüglich des Projektvorhabens und der Projektziele sind wichtig für die Partizipationsbereitschaft der Praxisakteure. Dafür bietet es sich an, mit einer Werbeagentur eine umfangreiche Informations- und Werbekampagne zu gestalten. Hierfür sollte eine enge Zusammenarbeit zwischen Projektteam und Agentur stattfinden, um zu erörtern, welche Gruppen über welche Kanäle erreicht werden können. Ältere Menschen können beispielsweise eher über Zeitungen und Informationsveranstaltungen erreicht werden, wohingegen Kinder und Jugendliche eher über Vereine, Jugendzentren, Schulen etc. angesprochen werden können. Vereine und Verbände sind darüber hinaus wichtige Multiplikatoren. Wichtig ist, dass keine für das Thema relevante Gruppe ausgeschlossen wird und dass darauf geachtet wird, Zugangsbarrieren (z. B. sprachlicher Natur) zu vermeiden. 
Im Reallabor Schorndorf wurden alle hier genannten Kanäle zur Rekrutierung der Teilnehmer(innen) genutzt. Der Teilnehmerkreis setzte sich schließlich zur Hälfte aus Personen zusammen, die basierend auf einer Zufallsstichprobenziehung durch das Einwohnermeldeamt der Stadt Schorndorf angeschrieben wurden, zur anderen Hälfte aus Personen, die sich aufgrund der örtlichen Informationen angesprochen fühlten.

\subsubsection{Gruppeneinteilung und Moderation}

Das Workshopkonzept sieht vor, dass es innerhalb des Workshops Phasen in der Gesamtgruppe und Phasen in Kleingruppen gibt. Die Kleingruppen setzen sich zum einen aus Vertreter(inne)n der Nutzertypen zusammen (pro Nutzertyp eine Gruppe). Zusätzlich sollte es in jeder Kleingruppe eine(n) Wissenschaftler(in) sowie eine(n) Moderator(in) geben. Im Idealfall hat das Projektteam Mitglieder, die über Moderationskompetenzen verfügen. Sollte dies nicht der Fall sein, sollten externe Moderator(inn)en engagiert werden.

\subsubsection{Durchführung des Workshops}

Der Ablaufplan (s. Tab. 1) zeigt, wie sich innerhalb des rund vierstündigen Workshops Phasen in der Gesamtgruppe und Gruppenarbeitsphasen ergänzen. Tab. 1 zeigt, welche Informationen, Leitfragen bzw. Arbeitsaufträge in den unterschiedlichen Phasen relevant sind. Die Formulierung der Fragen bzw. Arbeitsaufträge hängt selbstverständlich vom jeweiligen thematischen Kontext des zu entwickelnden Systems ab.

Neben einer Feedback-Runde im Plenum am Ende des Workshops bietet sich eine (anonymisierte) Abfrage per Fragebogen im Anschluss an den Workshop an, um Hinweise zur individuell-subjektiven Bewertung der entwickelten Ideen zu erhalten.

Im Reallabor Schorndorf wurden die Teilnehmer(innen) zum Beispiel gefragt, inwieweit sie sich mit dem entworfenen Nutzertyp und dem zugehörigen Nutzungsszenario identifizieren können bzw. welche Aspekte sie gerne noch in die Diskussion und den Entwicklungsprozess eingebracht hätten (zur Veranschaulichung s. Fragebogen in Anlage b im Anhang dieses Beitrags). So ließ sich feststellen, dass der Großteil der Teilnehmer(innen) sich sowohl mit den Nutzerprofilen als auch den potenziellen Nutzungsszenarien stark identifizieren konnte. 
Tabelle 1: Ablaufplan des Co-Creation-Workshops I inklusive Leitfragen und Arbeitsanweisungen.

\begin{tabular}{|c|c|c|c|}
\hline Phase & Was ist zu tun? & $\begin{array}{l}\text { Fragen \& } \\
\text { Arbeitsanweisungen }\end{array}$ & $\begin{array}{l}\text { Zeitfenster } \\
\text { in Minuten }\end{array}$ \\
\hline $\begin{array}{l}\text { Begrüßung } \\
\text { und Projekt- } \\
\text { vorstellung }\end{array}$ & $\begin{array}{l}\text { Begrüßung, Nen- } \\
\text { nung der Veranstal- } \\
\text { tungsziele \& Agen- } \\
\text { da, Kurzpräsenta- } \\
\text { tion des Projekts }\end{array}$ & $\begin{array}{l}\text { Alltagssprache wählen, um } \\
\text { das Projektvorhaben zu } \\
\text { skizzieren (im Stil von „was } \\
\text { bisher geschah“, ohne zu } \\
\text { viele Projektdetails aufzu- } \\
\text { führen) } \\
\text { Hinweis auf Themen- } \\
\text { speicher }\end{array}$ & 10 \\
\hline $\begin{array}{l}\text { Vorstellungs- } \\
\text { runde }\end{array}$ & $\begin{array}{l}\text { Das Eis brechen: } \\
\text { Einladen zur Vor- } \\
\text { stellungsrunde; Mo- } \\
\text { derator(in) startet } \\
\text { mit einem positiven } \\
\text { Beispiel }\end{array}$ & $\begin{array}{l}\text { Mein Name ist ... / Ich woh- } \\
\text { ne in ... / Mein ein- } \\
\text { drucksvollstes Erlebnis im } \\
\text { Zusammenhang mit ... (pas- } \\
\text { send zum Themenfeld wäh- } \\
\text { len) }\end{array}$ & $\begin{array}{l}\text { 20, ca. } \\
1 \text { Min. pro } \\
\text { Teilneh- } \\
\text { mer(in) }\end{array}$ \\
\hline $\begin{array}{l}\text { Gruppenarbeit: } \\
\text { Prüfung und } \\
\text { Ausdifferen- } \\
\text { zierung Nutzer- } \\
\text { profile }\end{array}$ & $\begin{array}{l}\text { Pro Gruppe das } \\
\text { idealtypisch skiz- } \\
\text { zierte Nutzerprofil } \\
\text { diskutieren, ändern, } \\
\text { ergänzen, spezifi- } \\
\text { zieren }\end{array}$ & $\begin{array}{l}\text { Entspricht die Beschreibung } \\
\text { des Nutzertyps Ihnen? Wel- } \\
\text { che Aspekte fehlen oder } \\
\text { sind nicht stimmig? Über } \\
\text { was freut sich Frau/Herr X, } \\
\text { was ärgert sie/ihn, was } \\
\text { wünscht sie/er sich? usw. } \\
\text { (für ein Beispiel des Ergeb- } \\
\text { nisses s. Abb. 6) }\end{array}$ & 45 \\
\hline Pause & $\begin{array}{l}\text { Imbiss/Getränke } \\
\text { bereitstellen, an } \\
\text { Themenspeicher } \\
\text { erinnern }\end{array}$ & $\begin{array}{l}\text { Informelle Gespräche mit } \\
\text { den Teilnehmer(inne)n } \\
\text { suchen }\end{array}$ & 20 \\
\hline $\begin{array}{l}\text { Gruppenarbeit: } \\
\text { Prüfung und } \\
\text { Ausdifferenzie- } \\
\text { rung Nutzungs- } \\
\text { szenarien }\end{array}$ & $\begin{array}{l}\text { Pro Gruppe das für } \\
\text { den entsprechen- } \\
\text { den Nutzertyp skiz- } \\
\text { zierte Nutzungs- } \\
\text { szenario diskutie- } \\
\text { ren, ändern, ergän- } \\
\text { zen, spezifizieren }\end{array}$ & $\begin{array}{l}\text { Zu welchem Zweck könnte } \\
\text { der Nutzertyp das System } \\
\text { nutzen? Welche Gedanken } \\
\text { würden einer Person bei der } \\
\text { Nutzung durch den Kopf } \\
\text { gehen? Was ist ihr dabei } \\
\text { wichtig? Was bereitet Sor- } \\
\text { ge? usw. (für ein Beispiel } \\
\text { des Ergebnisses s. Abb. 7) }\end{array}$ & 50 \\
\hline
\end{tabular}




\begin{tabular}{|c|c|c|c|}
\hline Phase & Was ist zu tun? & $\begin{array}{l}\text { Fragen \& } \\
\text { Arbeitsanweisungen }\end{array}$ & $\begin{array}{l}\text { Zeitfenster } \\
\text { in Minuten }\end{array}$ \\
\hline $\begin{array}{l}\text { Gruppenarbeit: } \\
\text { Benennung von } \\
\text { konkreten } \\
\text { Funktionen des } \\
\text { neuen Systems }\end{array}$ & $\begin{array}{l}\text { Pro Gruppe die für } \\
\text { den Nutzertyp rele- } \\
\text { vanten Eigenschaf- } \\
\text { ten des Systems } \\
\text { definieren }\end{array}$ & $\begin{array}{l}\text { Wie müsste das System ge- } \\
\text { staltet sein, damit es den } \\
\text { Anforderungen des Nutzer- } \\
\text { typs entspricht? In welcher } \\
\text { Situation und wie würde } \\
\text { dieser Nutzertyp das System } \\
\text { nutzen? usw. }\end{array}$ & 20 \\
\hline Pause & $\begin{array}{l}\text { Imbiss/Getränke } \\
\text { bereitstellen, an } \\
\text { Themenspeicher } \\
\text { erinnern }\end{array}$ & $\begin{array}{l}\text { Informelle Gespräche mit } \\
\text { den Teilnehmer(inne)n } \\
\text { suchen }\end{array}$ & 20 \\
\hline $\begin{array}{l}\text { Ergebnisprä- } \\
\text { sentation der } \\
\text { Kleingruppen } \\
\text { im Plenum }\end{array}$ & $\begin{array}{l}\text { Präsentation der Er- } \\
\text { gebnisse der Klein- } \\
\text { gruppen }\end{array}$ & $\begin{array}{l}\text { Was zeichnet den Nutzertyp } \\
\text { aus? Für welchen Zweck und } \\
\text { wie würde der Nutzertyp } \\
\text { das System nutzen? Was } \\
\text { wäre ihm dabei wichtig? } \\
\text { usw. }\end{array}$ & 30 \\
\hline $\begin{array}{l}\text { Abschluss/ } \\
\text { Ausblick }\end{array}$ & $\begin{array}{l}\text { Zusammenfassung } \\
\text { der Erkenntnisse } \\
\text { (inkl. Themen- } \\
\text { speicher), Ausblick } \\
\text { auf das weitere } \\
\text { Vorgehen, Bitte um } \\
\text { Feedback (anonymer } \\
\text { Feedbackfrage- } \\
\text { bogen) }\end{array}$ & $\begin{array}{l}\text { Was hat Ihnen gut gefallen? } \\
\text { Was weniger gut? Welche } \\
\text { Fragen sind offengeblieben? } \\
\text { usw. }\end{array}$ & 20 \\
\hline
\end{tabular}

\subsubsection{Workshopdokumentation}

Es ist ratsam, den Workshop mit einer Videokamera oder einem Tonband aufzuzeichnen, um die Diskussion und Interaktion sowohl aus dem Plenum als auch aus den Kleingruppen bei der anschließenden Auswertung nachvollziehen zu können. Die Daten werden nach Ablauf des Projekts gelöscht, was den Teilnehmer(inne)n so auch kommuniziert wird.

Die unterschiedlichen Visualisierungen, wie zum Beispiel die Nutzerprofile (s. Abb. 6), die im Rahmen des Workshops gemeinsam erarbeitet werden, stellen zudem eine Dokumentation der Diskussion und v. a. der in der Gruppe herausgearbeiteten Ergebnisse dar. Insbesondere in interdisziplinären Teams ist es ratsam, das Material (Skizzen, bunte Klebezettel, Artikuliertes) für alle im Projektteam gut verständlich und lebensnah aufzubereiten, um dann ausgehend davon die weitere Konzeption vorzunehmen. 


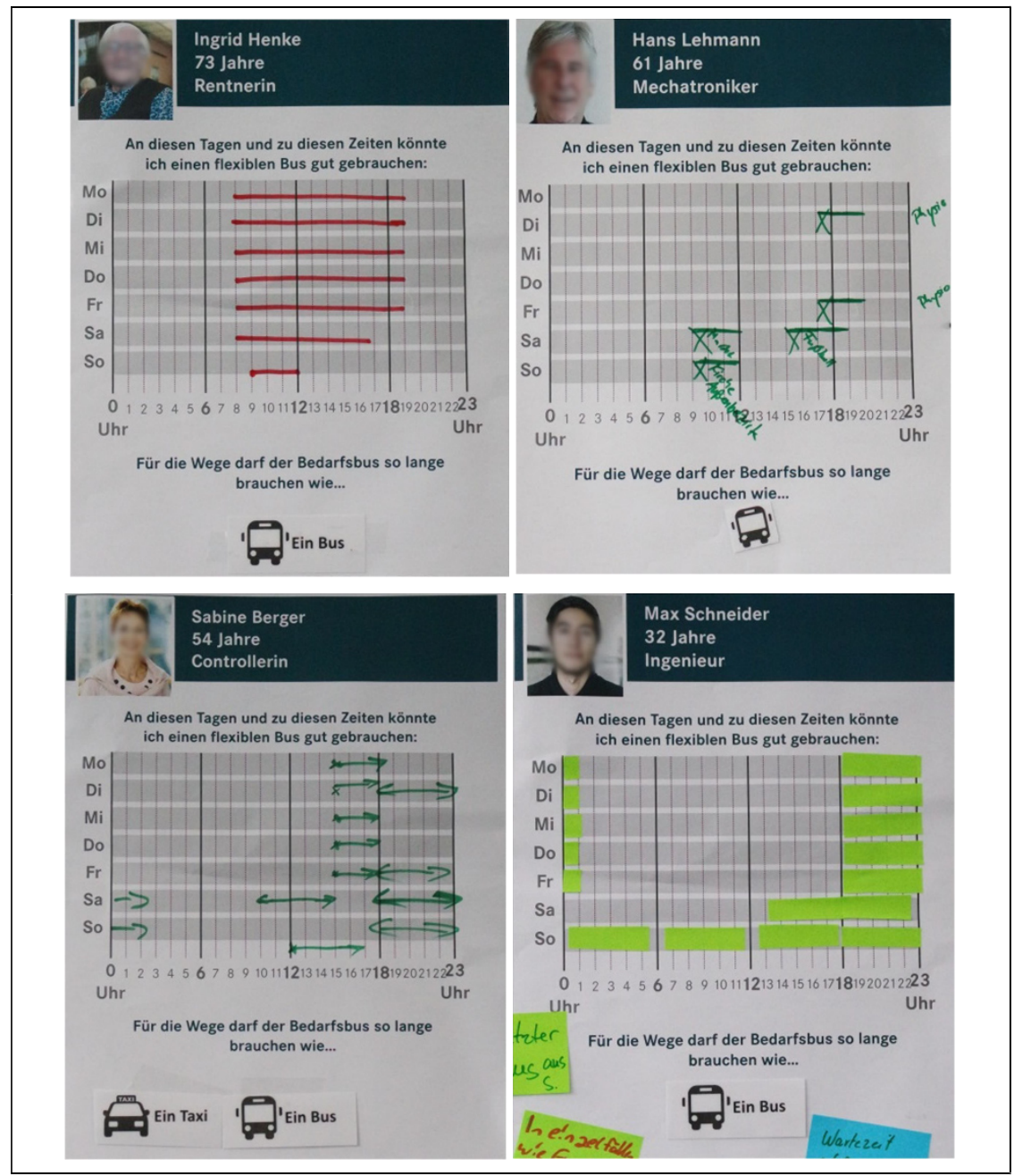

Abbildung 9: Die unterschiedlichen Präferenzen der Nutzertypen bezüglich der Betriebszeiten des Busses, kenntlich gemacht durch das Einzeichnen oder Bekleben der gewünschten Zeitfenster. (C) Salome Keller.

So lässt sich am Beispiel der im Workshop des Reallabors Schorndorf diskutierten Buseinsatzzeiten zeigen, dass Visualisierungen dabei helfen, Ergebnisse zu den bzw. Unterschiede zwischen den einzelnen Nutzertypen herauszustellen. Die visuelle Darstellung in Form von Markierungen im Wochenkalender kann als zusammenfassendes Ergebnis der Kleingruppen verstanden werden (s. Abb. 9). 


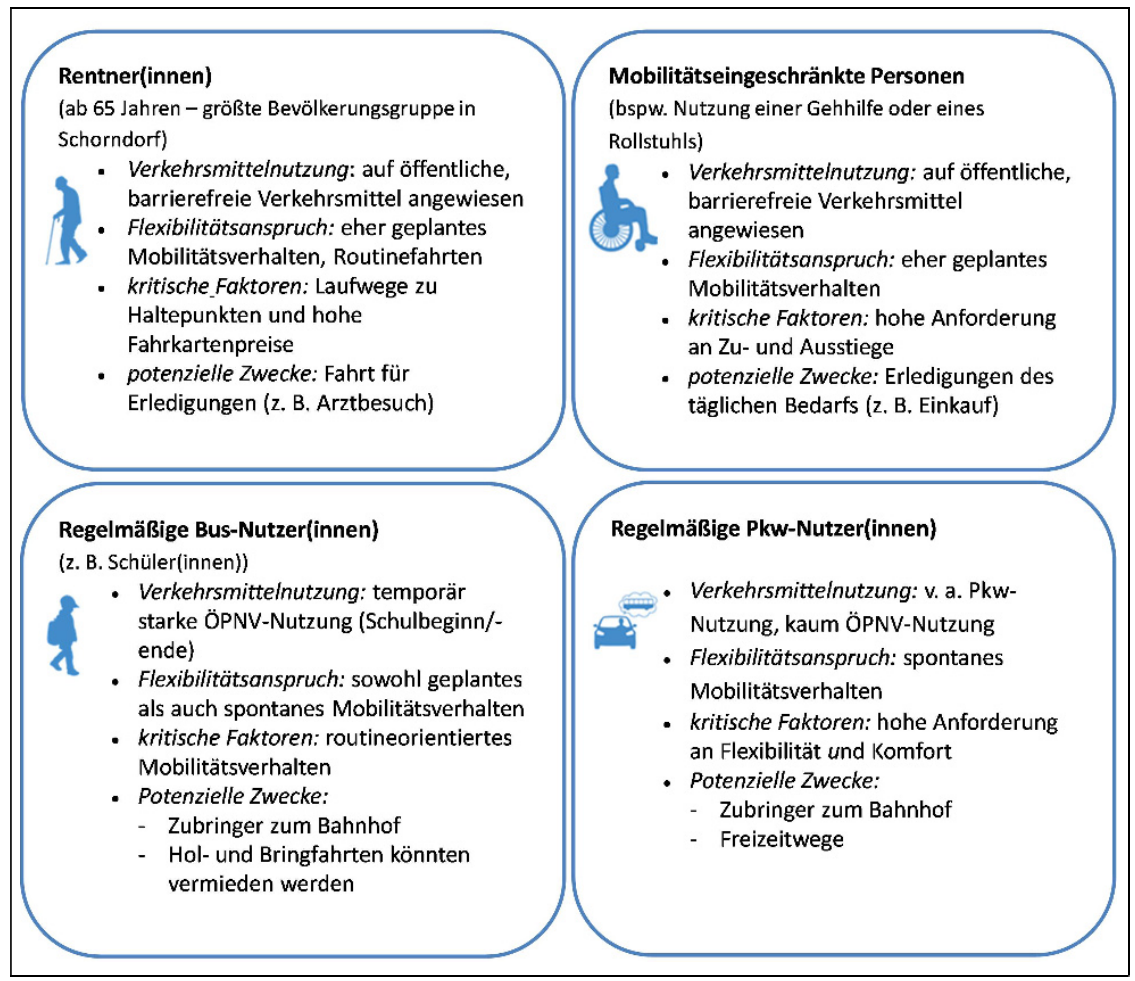

Abbildung 10: Beschreibung der identifizierten Nutzertypen anhand ausgewählter Charakteristika im Reallabor Schorndorf. (C) Laura Gebhardt, Alexandra König.

Die im Nachgang zum Workshop des Reallabors Schorndorf vorgenommenen Beschreibungen der Nutzertypen anhand ausgewählter Charakteristika (hier Verkehrsmittelnutzung, Flexibilitätsanspruch, kritische Faktoren und potenzielle Zwecke) zeigen beispielhaft, wie eine Aufbereitung der gewonnenen Ergebnisse aussehen kann (s. Abb. 10). Sie helfen dem transdisziplinären Team, ein möglichst konkretes Bild der Nutzergruppen vor Augen zu haben.

\subsubsection{Verwertung und Kommunikation der Ergebnisse}

Es empfiehlt sich, die Workshopergebnisse in Form der gemeinsam erstellten visuellen Dokumentationen sowie anhand der Beobachtungen (Protokoll, Tonund Videoaufnahmen) im Projektteam zu diskutieren und zu strukturieren. Hierbei helfen das bereits erwähnte Leitplankendokument sowie das Spezifikationsheft (s. hierzu Abschnitt 2.1). Im Leitplankendokument werden Fragen, die sich im Workshop als relevant herausgestellt haben, tabellarisch gesammelt und mit 
den Projektbeteiligten diskutiert, priorisiert und bearbeitet. Innerhalb des Projektteams beschlossene Antworten auf die offenen Fragen werden als Leitplanken festgehalten und dann in das Spezifikationsheft überführt. Das Spezifikationsheft dient der Dokumentation aller Erkenntnisse und ist letzten Endes die Grundlage für die Ausgestaltung des Systems. Zudem werden hier Argumentationsstränge und die Begründung der Parameterfestlegung festgehalten. Nach dem Workshop werden das Leitplankendokument und das Spezifikationsheft durch die Projektpartner anhand der Ergebnisse aktualisiert.

Neben der Kommunikation innerhalb des Projektteams gilt es, die Erkenntnisse des Workshops für die Teilnehmer(innen) sowie ggf. in der Öffentlichkeit transparent zu machen. Dies kann zum einen über ein Schreiben an die Teilnehmer(innen) des Workshops (inkl. einer Danksagung für die Teilnahme) geschehen, zum anderen durch das Publizieren der zentralen Erkenntnisse auf der Projekthomepage und in lokalen Medien (z. B. im örtlichen Wochenblatt).

Zwischen dem ersten und dem zweiten Workshop, der im Folgenden beschrieben wird, sollten mindestens anderthalb Monate Zeit eingeplant werden, damit die Ergebnisse des ersten Workshops in ausreichender Tiefe ausgewertet und für den anschließenden Workshop aufbereitet werden können.

\subsection{Ideen und Konzepte gemeinsam entwickeln- Co-Creation-Workshop II}

In Abhängigkeit vom Forschungsgegenstand und dessen Komplexität bietet es sich an, statt einem mehrere solcher Workshops durchzuführen, die sich jeweils einer Auswahl von Teilaspekten widmen. Um die Teilnehmer(innen) nicht zu überfordern, wird empfohlen, nicht mehr als fünf Teilaspekte je Workshop zu bearbeiten. Im Reallabor Schorndorf war ein Teilaspekt der Buchungsprozess des Bedarfsbusses, ein weiterer war das Innenraumkonzept des Bedarfsbusses. Ein wichtiges Entscheidungskriterium für die Workshopanzahl bietet also der Umfang der Teilaspekte.

\subsubsection{Workshopziele}

Ziele des Workshops sind die Entwicklung von ersten Ideen zur Ausgestaltung des zu entwickelnden Systems und die Bewertung von konkreten Konzeptideen. Zudem soll am Ende des Workshops ein Prototyp vorliegen, der das Konzept anschaulich darstellt. Dabei kann der Prototyp auch ein Ablaufschema darstellen, muss also kein haptisches Produkt sein. Wie bereits in Kapitel 1 erwähnt, kann nicht vorausgesetzt werden, dass sich die zukünftigen Nutzer(innen) ihrer eigenen Anforderungen an das zu entwickelnde System umfassend bewusst sind und diese adäquat äußern können. Mit dem Workshop II wird daher das Ziel verfolgt, die 
eher impliziten Denkweisen und Bedürfnisse der zukünftigen Nutzer(innen) zutage zu fördern und mittels Kreativtechniken in konkrete Konzeptideen und -entwürfe zu übersetzen (für eine Übersicht an Kreativtechniken, s. Degele et al. 2009). Die TraSy-Methode generiert also im Co-Creation-Workshop II mehrere Ergebnisse:

1) Entwicklung von Ideen zur konkreten Systemausgestaltung

2) Entwicklung und Bewertung von konkreten Konzeptentwürfen

3) Umsetzung der vielversprechendsten Konzeptentwürfe als Prototyp

Im Reallabor Schorndorf wurden in Phase 3 (Ideen und Konzepte gemeinsam entwickeln) Co-Creation-Workshops zur Entwicklung von Ideen und Konzeptentwürfen für den Innenraum des Bedarfsbusses sowie für den Bestell- und Buchungsprozess des Busses durchgeführt.

\subsubsection{Vorphase: Bisher identifiziertes Wissen nutzbar machen}

Die Ergebnisse der beiden vorherigen Phasen (Nutzer(innen) im alltäglichen Kontext verstehen (Abschnitt 2.1) und Nutzertypen identifizieren und beschreiben (Abschnitt 2.2), in denen die Nutzerprofile und die Szenarien erstellt wurden, werden, wie in Abb. 11 dargestellt, als Input für den Workshop verwendet. An dieser Stelle im Ablauf der TraSy-Methode enthält das Spezifikationsheft alle bisher definierten Angaben zur Charakterisierung der Nutzertypen. Zudem werden hier die Nutzungsszenarien detailliert beschrieben. Das Spezifikationsheft liefert den Rahmen für die Arbeit im Workshop, indem es die nicht mehr veränderbaren Kriterien beschreibt. Diese werden den Teilnehmer(inne)n des Workshops präsentiert, um der kreativen Arbeit einen Rahmen zu geben. Das Leitplankendokument enthält noch unbeantwortete Fragen in Bezug auf die Charakterisierung der Nutzertypen und deren spezifischen Anforderungen, die im Rahmen des Work-

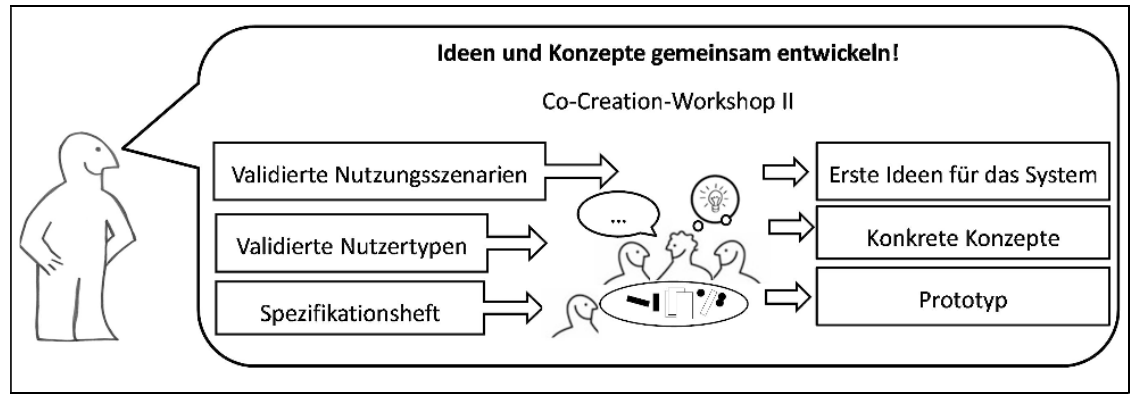

Abbildung 11: Co-Creation-Workshop II zur Entwicklung von konkreten Ideen zur Gestaltung des Systems. (C) Laura Gebhardt, Alexandra König. 
shops zu beantworten sind. Im Falle des Reallabors Schorndorf war eine solche Frage beispielsweise: "Wie können auch Nutzer(innen) des Nutzertyps ,Rentner(innen)' ohne Mobiltelefon den Bus von unterwegs buchen oder Fahrtdetails wie die Abfahrtszeit erfragen?"“

\subsubsection{Inhaltliche Vorbereitung}

Für die Durchführung des Workshops sind einige Vorarbeiten notwendig: Das Leitplankendokument enthält offene Fragen, die im Rahmen des Workshops zu adressieren sind. Aus dem Spezifikationsheft müssen jene Anforderungen an das System extrahiert werden, die für das Thema des Workshops von Relevanz sind. Diese Anforderungen sollten von den Wissenschaftler(inne)n in enger Absprache mit den Praxispartnern so aufgearbeitet werden, dass sie für die Teilnehmer(innen) des Workshops verständlich sind. Auf die Verwendung von Fachsprache sollte verzichtet werden. Wenn dies nicht möglich ist, sollten Fachbegriffe in allgemeinverständlicher Weise definiert und beschrieben werden. Die Anforderungen sind Faktoren, die im Rahmen des Vorhabens nicht beeinflussbar sind; sie ergänzen die oben erwähnten und auf die Nutzertypen und Nutzungsszenarien bezogenen Kriterien. Beide Arten von Restriktionen müssen den Workshopteilnehmer(inne)n zu Beginn des Workshops kommuniziert werden. Es empfiehlt sich, dafür eine grafische Darstellung zu nutzen, die den Teilnehmer(inne)n jederzeit während des Workshops verfügbar ist.

Zudem gilt es natürlich, das Wissen, das in Phase 1 und insbesondere in Phase 2 generiert wurde, so aufzubereiten, dass es den Workshopteilnehmer(inne)n präsentiert werden kann. Dafür müssen die im Workshop I gemeinsam mit den Teilnehmer(inne)n erarbeiteten Nutzerprofile und Nutzungsszenarien (s. Abb. 6 und 7) inhaltlich und grafisch bearbeitet werden. Die Aufbereitung der Nutzerprofile und Nutzungsszenarien übernehmen die Wissenschaftler(innen) im Team.

Des Weiteren sind für die Workshopvorbereitung dieselben Punkte in Bezug auf die Raumwahl zu beachten, die in Abschnitt 2.2.3 zur Vorbereitung des Co-Creation-Workshops I beschrieben wurden.

\subsubsection{Ansprache und Gewinnung der Teilnehmer(innen)}

Der Co-Creation-Workshop II dauert einen ganzen Tag. Dies macht die Gewinnung von Teilnehmer(inne)n nicht einfach. Daher ist ein frühzeitiger Rekrutierungsstart wichtig. Am Co-Creation-Workshop II können dieselben Personen teilnehmen wie am Co-Creation-Workshop I, die Gruppe der teilnehmenden Personen kann aber auch ganz oder in Teilen anders sein. Für die Rekrutierung der Teilnehmer(innen) gilt dasselbe wie für den Co-Creation-Workshop I (s. Abschnitt 2.2.4). Ziel sollte es sein, eine Teilnehmerzahl von mindestens 12 Perso- 
nen zu erreichen, so dass vier Kleingruppen mit mindestens je drei Teilnehmer(inne)n gebildet werden können.

Die Einladung der Teilnehmer(innen) erfolgt schriftlich mehrere Wochen vor dem Workshop. Die Einladung enthält neben Angaben zu Ort, Zeit und Dauer des Workshops auch eine kurze und verständliche Beschreibung der Ziele und des groben Workshopablaufs.

\subsubsection{Gruppeneinteilung und Moderation}

Auch im Co-Creation-Workshop II gibt es neben Diskussionsphasen in der Gesamtgruppe Phasen der Kleingruppenarbeit. Jede Kleingruppe setzt sich aus drei bis sechs Teilnehmer(inne)n zusammen. Die Kleingruppen können, im Unterschied zum Workshop I, aus zufällig ausgewählten Teilnehmer(inne)n des Workshops gebildet werden, da die persönliche Zugehörigkeit zu einem der Nutzertypen nicht erforderlich ist. Zur zufälligen Gruppeneinteilung kann beispielsweise eine Packung von Celebrations-Süßigkeiten genutzt werden. Durch das Ziehen der verschiedenen Süßigkeiten wird die Zuordnung der Teilnehmer(innen) zu den Gruppen bestimmt. Auch diesmal ist in jeder der Kleingruppen ein(e) Wissenschaftler(in) aus dem Projektteam sowie ein(e) Moderator(in) anwesend. Falls im Projektteam keine Mitglieder vorhanden sind, die über Moderationskompetenzen verfügen, so dass externe Moderator(inn)en engagiert werden, müssen diese mit dem Thema so vertraut sein, dass sie die Gruppenarbeit auch inhaltlich gut unterstützen können, und dafür ggf. durch die beteiligten Wissenschaftler(innen) entsprechend vorbereitet werden.

\subsubsection{Durchführung des Workshops}

Der Workshop dauert ungefähr acht Arbeitsstunden. Eine beispielhafte Agenda des Workshops ist in Tab. 2 zu sehen, die neben dem zeitlichen und inhaltlichen Workshopablauf auch zeigt, welche Agendapunkte im Plenum und welche in der Gruppe stattfinden. Um die Einhaltung der straffen Agenda sicherzustellen, sind mehrere Rahmenbedingungen zu beachten: Es hat sich gezeigt, dass vor allem eine gute Vorbereitung des Arbeitsmaterials von großer Bedeutung für eine flüssige Durchführung des Workshops ist. Zudem hat sich erwiesen, dass es für die Einhaltung der Agenda wichtig ist, dass die Teilnehmer(innen) gut auf die Arbeit im Workshop eingestimmt werden. Je mehr Zeit am Anfang für die Einführung eingeplant wird, desto weniger Fragen und Schwierigkeiten ergeben sich während des Prozesses. Für die Einhaltung der Agenda ist zudem die Rolle der Moderation hervorzuheben, auf die im Weiteren genauer eingegangen wird.

$\mathrm{Zu}$ Beginn des Workshops ist eine Vorstellungsrunde essenziell, um eine angenehme und vertraute Atmosphäre zwischen den Teilnehmer(inne)n zu schaffen. 
Tabelle 2: Agenda des Co-Creation-Workshops II (Fassung für das Projektteam).

\begin{tabular}{|l|l|}
\hline \multicolumn{2}{|l|}{ Agenda des Co-Creation-Workshops II } \\
\hline 08:30 & Begrüßung (Plenum) \\
09:40 & Vorstellungsrunde (Plenum) \\
09:40 & Einführung, Leitplankenvorstellung und Kleingruppeneinteilung (Plenum) \\
10:35 & Präsentation der Nutzerprofile und der Nutzungsszenarien (Kleingruppe) \\
$10: 50$ & Formulierung des systembezogenen Teamauftrags (Kleingruppe) \\
$11: 15$ & Generierung von Ideen und konkreten Konzepten (Kleingruppe) \\
$12: 00$ & Mittagspause \\
$13: 15$ & Erstellen von Prototypen (Kleingruppe) \\
$14: 20$ & Vorbereitung der Prototypenpräsentation (Kleingruppe) \\
$14: 40$ & Kaffeepause \\
$14: 55$ & Präsentation der Prototypen (Plenum) \\
$15: 55$ & Bewertung der Restriktionen (Plenum) \\
$16: 25$ & Zusammenfassung und Abschluss (Plenum) \\
\hline
\end{tabular}

Um bereits in dieser frühen Workshopphase eine kreative Stimmung zu erzeugen, eignet sich eine spielerische Selbstpräsentation mithilfe von Metaphern. Der Arbeitsauftrag könnte lauten: „Nennen Sie ein Tier oder eine Pflanze, mit dem/ der Sie sich identifizieren, und beschreiben Sie in zwei bis drei Sätzen, warum Sie dieses Tier oder diese Pflanze gewählt haben." Hierbei geht es vor allem darum, dass die häufig zu bemerkende anfängliche Zurückhaltung der Teilnehmer(innen) überwunden wird.

Um die Teilnehmer(innen) zu Beginn auf das Workshopthema einzustimmen, werden sie durch die anwesenden Vertreter(innen) des Projektteams über die Projektziele, die bisherigen Ergebnisse und die weiteren Schritte informiert. Zudem ist es wichtig, den Teilnehmer(inne)n die Workshopziele so vorzustellen, dass möglichst eine einheitliche Erwartungshaltung entsteht. Auf die Ziele und den groben Ablauf des Workshops einzugehen, ist auch dann sinnvoll, wenn diese schon in der schriftlichen Einladung aufgeführt wurden. Erfahrungsgemäß sind mindestens 15 Minuten für die Vermittlung der Zielstellung und der methodischen Herangehensweise des Workshops einzuplanen. 


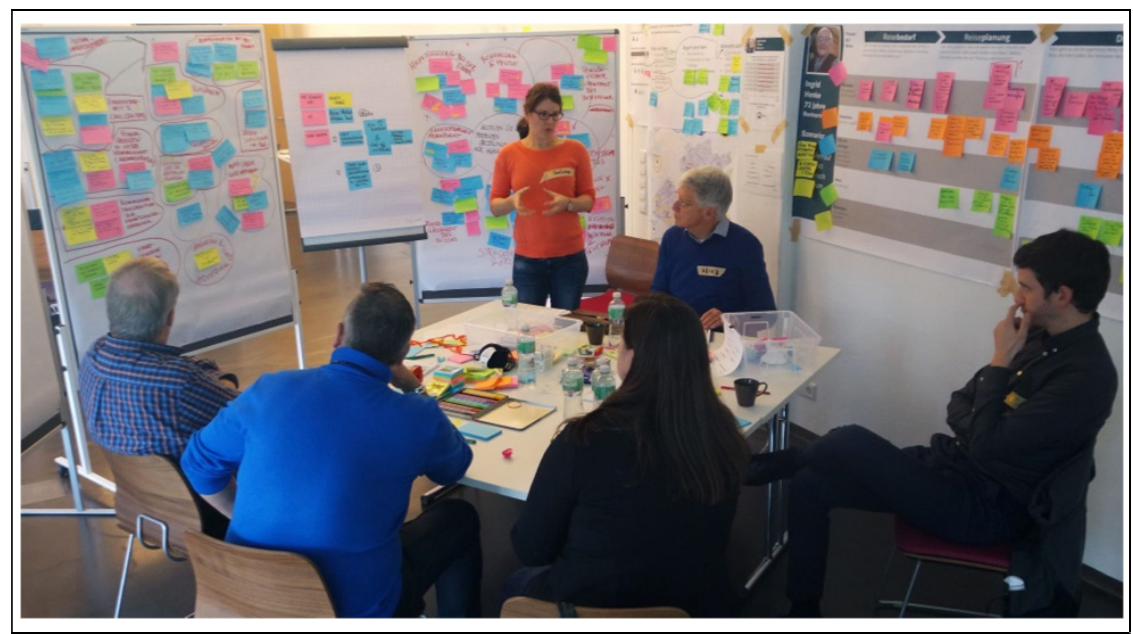

Abbildung 12: Kleingruppenarbeit innerhalb des Co-Creation-Workshops II in der Ideengenerierungsphase. Die bisherigen Ergebnisse sollten, wie hier in Form der Flipcharts und Metaplanwände, stets für alle sichtbar sein. (C) Salome Keller.

Schließlich werden im Rahmen der Einführung im Plenum die inhaltlichen Restriktionen vorgestellt, die im Workshop zu beachten sind (s. Abschnitt 2.3.3). Jede Restriktion, die eine Rahmenbedingung für das System beschreibt, wird möglichst präzise erklärt. Die Teilnehmer(innen) sollten ermutigt werden, Nachfragen zu formulieren, falls Unklarheiten bestehen, denn es ist wichtig, dass jede(r) Teilnehmer(in) die Rahmenbedingungen des Systems kennt und versteht.

Die eigentliche kreative Arbeit innerhalb des Workshops findet in den Kleingruppen statt. Hierfür sollten geeignete Räume vorhanden sein, um die einzelnen Kleingruppen voneinander zu trennen und die genügend Platz bieten, so dass jede Kleingruppe sowohl eigene Ergebnisse auf ausreichend vielen Pinnwänden festhalten kann, als auch von den Visualisierungen der bisherigen Ergebnisse ,umgeben' sein kann (s. Abb. 12). Ein(e) mit dem Thema vertraute(r) Moderator(in) (bestenfalls aus dem Projektteam stammend) kontrolliert den zeitlichen Rahmen der Teamarbeit, steht den Teammitgliedern für Nachfragen zur Seite und regt, wenn nötig, die Kreativität der Teilnehmer(innen) durch gezielte Nachfragen an. Jedoch sollte die Moderation möglichst wenig in die Selbstorganisation der Gruppe eingreifen. Konkret bedeutet dies, dass der bzw. die Moderator(in) den Workshopteilnehmer(inne)n nicht das Gefühl vermitteln darf, er/sie fühle sich aufgrund der eigenen wissenschaftlichen Qualifikationen überlegen. Zudem muss die Moderation sich zurückhaltend und neutral verhalten, also die Einschätzungen der Teilnehmer(innen) nicht bewerten. Für die Gruppenarbeit sollten einige Grund- 
regeln festgelegt werden, die das Verhalten untereinander festlegen. Sie sollten möglichst zu Beginn der Gruppenarbeit an alle Teilnehmer(innen) kommuniziert werden. Als besonders relevant werden die folgenden Regeln erachtet: Wir lassen einander aussprechen! Alle Ideen sind wertvoll! Lassen Sie auch unkonventionelle Gedanken zu! Bewerten Sie nicht die Ideen der anderen Teilnehmer(innen)! Bauen Sie auf den Ideen der anderen Teilnehmer(innen) auf und ,spinnen' Sie den Faden weiter!

Der Workshop baut auf den in Phase 2 erarbeiteten Nutzertypen und Nutzungsszenarien auf. Jede Kleingruppe widmet sich dabei einem Nutzertyp. Die Nutzerprofile und Nutzungsszenarien werden den Teilnehmer(inne)n durch die Moderation anhand der Steckbriefe und Szenarien präsentiert, die in Phase 2 erarbeitet und in Vorbereitung auf den Workshop von den Wissenschaftler(inne)n aufbereitet wurden (s. Abschnitt 2.3.3). Um sich noch besser in den Nutzertyp hineinversetzen zu können, überlegt sich jede Kleingruppe unter Anleitung der Moderation Ziele und Motive des ihr zugewiesenen Nutzertyps, zum Beispiel anhand vorbereiteter Fragen (s. Abb. 13 links), die sich einem Teilaspekt widmen und möglichst konkret zu formulieren sind. Erfahrungsgemäß regt die Arbeit mit Fragen die Diskussion innerhalb der Kleingruppe an und erzeugt konkrete Antworten.

Nach einer Kaffeepause, die zugleich die Möglichkeit zum weiteren Austausch innerhalb und zwischen den Kleingruppen bietet, kommen die Kleingruppen erneut zusammen. Nun wird der auf das System bezogene Gruppenauftrag formuliert. Dieser geht von dem der Gruppe zugewiesenen Nutzerprofil und Nutzungsszenario aus: „Entwerfen Sie ein System Z, das so gestaltet ist, dass Nutzertyp X seine Ziele $Y_{1}, Y_{2}, \ldots$ erreicht.“ Der Auftrag sollte möglichst präzise formuliert und für alle sichtbar notiert werden.

Die Teilnehmer(innen) werden also auf der Grundlage der Ziele und Motive der Nutzertypen aufgefordert, ein System zu entwickeln, das den Nutzertyp bei der Zielerreichung und der Bedürfnisbefriedigung unterstützt. In einem ersten Schritt werden die Ideen aller Gruppenmitglieder eingeholt. Diese werden alle aufgeschrieben und angeheftet. Hierbei gilt das Prinzip, dass möglichst viele unterschiedliche Ideen gesammelt werden sollen. Die Arbeit mit selbstklebenden Postits, wie auf Abb. 13 rechts zu sehen, bietet sich an, um individuelle Ideen aufzuschreiben, den Teilnehmer(inne)n die Angst vor dem weißen Blatt zu nehmen und Ideen anschließend flexibel umsortieren zu können. Wenn keine neuen Ideen mehr genannt werden, werden die gesammelten Ideen in einem zweiten Schritt durch die Teilnehmer(innen) zuerst geclustert und dann bewertet. Für die Bewertung eignen sich Klebepunkte - zum Beispiel jeweils drei pro Teilnehmer(in) -, die die Abstimmungsergebnisse visualisieren und quantifizieren. Der freien Ideengenerierung folgt dann in einem dritten Schritt die Formulierung konkreter 


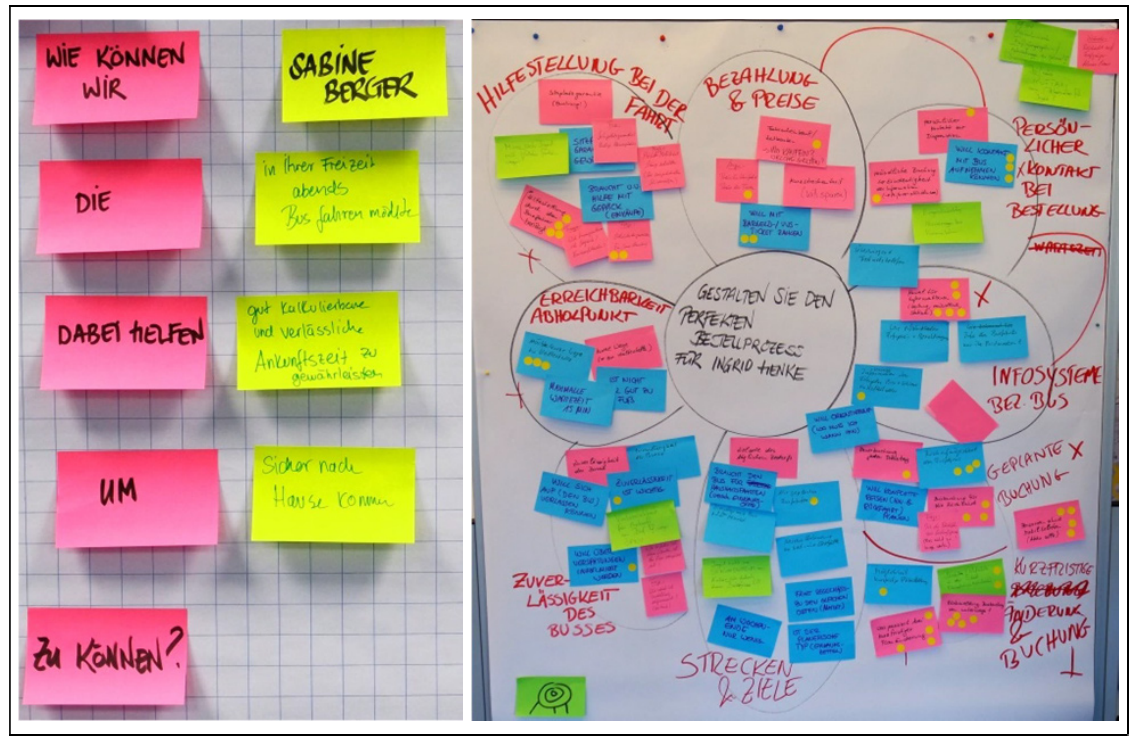

Abbildung 13: Links: Mithilfe einer vorgegebenen Frage werden die Bedürfnisse eines Nutzertyps (hier: Sabine Berger) in konkrete Anforderungen übersetzt. Rechts: Darstellung der Ergebnisse der Kleingruppenarbeit (hier die Cluster als Blütenblätter dargestellt) und Bewertung der Ideen durch die Teilnehmer(innen) mithilfe von gelben Klebepunkten. (C) Salome Keller.

Konzepte anhand der am besten bewerteten Ideen. Diese Konzepte beschreiben einen Teilaspekt des zu entwickelnden Systems möglichst konkret. Im Reallabor Schorndorf war das beispielsweise der Bezahlungsprozess auf einer Fahrtenbuchungswebseite. Die Moderation stellt sicher, dass ideale Konzepte beschrieben werden, die möglichst alle Anforderungen des Nutzertyps an das System erfüllen.

Nachdem in der Kleingruppe zuerst Ideen und dann Konzepte entwickelt wurden, ist die Übersetzung des vielversprechendsten Konzepts in einen Prototyp die nächste Aufgabe der Kleingruppe (s. Abb. 14 links). Für die Auswahl bietet sich wiederum die Priorisierung und Abstimmung mithilfe von Klebepunkten an. Für die Erstellung des Prototyps sollten den Teilnehmer(inne)n möglichst viele Materialien, wie verschiedenfarbige Stifte, LEGO ${ }^{\circledR}$-Figuren und -Bausteine, Papier, Knetmasse etc. zur Verfügung gestellt werden, um die plastische Umsetzung zu ermöglichen. Die Teilnehmer(innen) sollten auch angeregt werden, ihre Visionen grafisch in kleinen Skizzen zu visualisieren. Die plastische und grafische Darstel- 


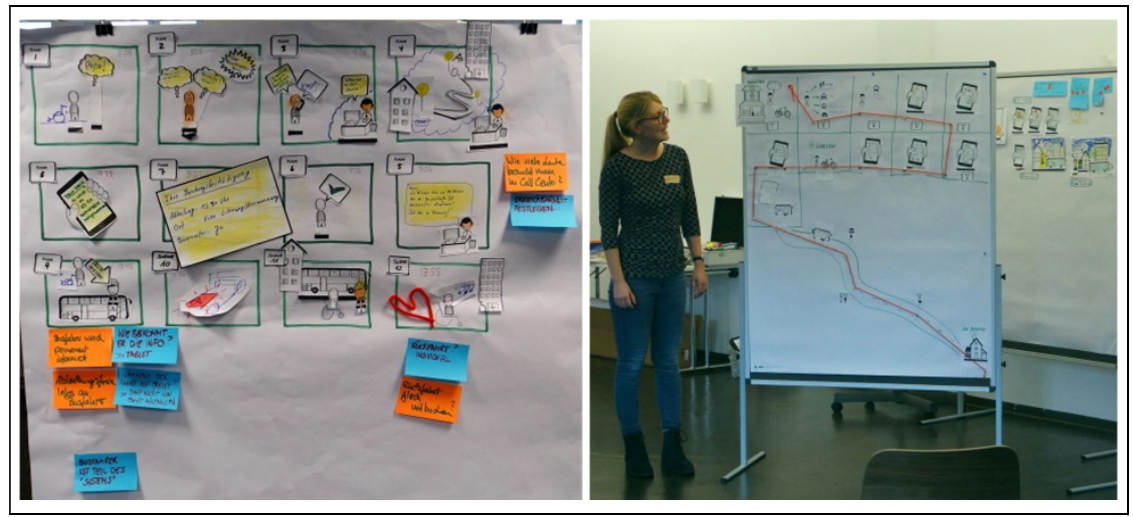

Abbildung 14: Links: Das Ergebnis der Kleingruppenarbeit, der Prototyp, muss kein anfassbares Produkt sein, sondern kann, wie hier, auch die Form eines Ablaufdiagramms haben. Wichtig ist die plastische und grafische Aufbereitung der Ideen, die eine Diskussion anregen soll. Rechts: Ein wichtiger methodischer Baustein des Workshops ist die Präsentation der Ergebnisse aus den Gruppenarbeiten im Plenum. C Salome Keller.

lung der Ergebnisse ermöglicht zum Ersten die Diskussion innerhalb der Kleingruppe und veranschaulicht zum Zweiten die Ergebnisse für die anschließende Präsentation im Plenum. Das Präsentieren der Ergebnisse der Kleingruppenarbeit im Plenum stellt ein wichtiges methodisches Element dar, weil es dazu zwingt, Argumentationsstränge zu artikulieren, und erlaubt, mit den anderen Workshopteilnehmer(inne)n über offene Fragen und potenzielle Herausforderungen zu diskutieren.

Für die Präsentation der Ergebnisse aus der Kleingruppenarbeit kommen alle Teilnehmer(innen) des Workshops wieder im Plenum zusammen. Nacheinander stellen sich die Gruppen gegenseitig ihre Ergebnisse anhand des angefertigten Prototyps vor (s. Abb. 14 rechts). Alle Teilnehmenden werden durch die Moderation ausdrücklich aufgefordert und ermuntert, Fragen zu stellen sowie Ideen und Anmerkungen zum Prototyp zu äußern. Weiterhin werden nach jeder einzelnen Präsentation die Grenzen des Prototyps besprochen. Hierbei können Fragen wie diese leiten: „Warum kann das System X nicht Y?“ (wobei Y eine Nutzeranforderung sein könnte, die sich im vorherigen Workshop und in der Arbeit mit den Nutzertypen als relevant herausgestellt hatte), „Unter welchen Umständen könnte es beim Einsatz oder der Nutzung des Systems X Schwierigkeiten geben?" usw.

Der Workshoptag schließt mit einer Zusammenfassung der Ergebnisse und einem Ausblick auf die nächsten Schritte innerhalb des Projekts durch die Moderation. 


\subsubsection{Workshopdokumentation}

Wie bereits in Bezug auf Workshop I beschrieben (s. Abschnitt 2.2.7), ist es empfehlenswert, bereits im Rahmen der Ansprache und Gewinnung der Teilnehmer(innen) zu kommunizieren, dass es Bild- und Tonaufnahmen geben wird, die lediglich für interne Zwecke verwendet werden. Innerhalb des Workshops wird dies den Teilnehmer(inne)n noch einmal schriftlich vorgelegt, mit der Bitte, sich durch ihre Unterschrift damit einverstanden zu erklären.

Die Video- oder Audioaufnahmen werden erstellt, damit die Argumentationsstränge in den Diskussionen im Nachhinein nachvollzogen werden können, ohne dass alles während des gesamten Workshops schriftlich protokolliert werden muss. Sämtliche Ergebnisse der Kleingruppenarbeit (z. B. Notizen auf den Flipcharts, alle Post-its) sind abzufotografieren. Natürlich sind auch alle erarbeiteten Visualisierungen, wie zum Beispiel die Prototypen, die im Rahmen des Workshops gemeinsam erarbeitet werden, Teil der Dokumentation der Ergebnisse.

Weiterhin ist es die Aufgabe der Moderation, während der Kleingruppenarbeit und den Diskussionen im Plenum in einem Protokoll festzuhalten, wenn es zu Problemen, Verzögerungen oder Schwierigkeiten bei der Durchführung eines der Schritte oder zu Konflikten zwischen den Teilnehmer(inne)n kommt.

\subsubsection{Verwertung und Kommunikation der Ergebnisse}

Im Anschluss an den Workshop werden die Ergebnisse innerhalb des Projektteams diskutiert, aufgearbeitet und ausgewertet. Allen Projektteammitgliedern muss Zugang zu den Workshopergebnissen verschafft werden, damit sich auch diejenigen, die nicht am Workshop teilgenommen haben, einen Überblick über den Verlauf und die Ergebnisse erarbeiten können. Die Auswertung der Workshopergebnisse wird zwischen den wissenschaftlichen Projektteammitgliedern aufgeteilt, und zwar so, dass jede(r) einen Teil der Auswertung übernimmt, bevor diese dann zu einem Gesamtergebnis zusammengefügt werden. Die Ergebnisse sind dabei möglichst umfangreich grafisch aufzuarbeiten und zu strukturieren. Wichtig ist, dass nicht nur eine sachlich-thematische Auswertung erfolgt (,X Workshopteilnehmer(innen) haben Bedenken geäußert, dass Nutzer(innen) bei der Nutzung des Prototyps Y Schwierigkeiten mit Z haben könnten"), sondern auch eine Auswertung der Argumentationsstränge. Dies ist wichtig für die spätere Nachvollziehbarkeit und Transparenz der Ergebnisse.

Die im Projektteam diskutierten, sich auf offene Fragen beziehenden Ergebnisse werden in das Leitplankendokument aufgenommen. Fragen, die vor dem Workshop noch nicht beantwortet werden konnten, können im Idealfall anhand der Workshopergebnisse nun beantwortet werden. Wenn sich auf Basis des Workshops neue Fragen ergeben haben, werden diese auch in das Leitplankendokument aufgenommen. Die Anforderungen an das System, die von den Projektbetei- 
ligten ,abgesegnet" werden, werden in das Spezifikationsheft übernommen. In diesem werden auch die Argumentationsstränge und Begründungen der Parameterfestlegung festgehalten. Mit der Aktualisierung des Leitplankendokuments und des Spezifikationshefts ist die Auswertung des Co-Creation-Workshops II abgeschlossen.

Anschließend gilt es, wie schon beim Workshop I, die Ergebnisse des Workshops den Workshopteilnehmer(inne)n sowie der interessierten Öffentlichkeit zu kommunizieren, zum einen in Form eines Schreibens an die Teilnehmer(innen) des Workshops (inkl. eines schriftlichen Dankeschöns für die Teilnahme), zum anderen durch die Publikation zentraler Ergebnisse auf der Projekthomepage und in lokalen Medien (z. B. im örtlichen Wochenblatt).

Im Anschluss an die hier präsentierten Phasen der TraSy-Methode muss im weiteren Projektverlauf die Erprobung des entwickelten Systems im Realkontext stattfinden (s. Abb. 1). Hierfür ist ein mehrmonatiger Pilotbetrieb des Systems vorzusehen, der weitere Erkenntnisse zur Nutzerfreundlichkeit des entwickelten Systems liefert. Die Ergebnisse aus qualitativen und quantitativen Nutzerbefragungen sollten dann wiederum ins Leitplankendokument und ins Spezifikationsheft einfließen. Im Reallabor Schorndorf wurde ein Pilotbetrieb des Bedarfsbusses von März bis Dezember 2018 durchgeführt. Dabei wurde das System durch die Nutzer(innen) getestet, evaluiert und kontinuierlich verbessert. Für detaillierte Angaben dazu siehe Brost et al. (2018).

\section{Reflexion und Empfehlungen zum Einsatz der TraSy-Methode}

In diesem Beitrag wurde eine Methode zur transdisziplinären Entwicklung von soziotechnischen Systemen vorgestellt, d. h. von Systemen, die das alltägliche Handeln von Menschen betreffen, also in konkrete gesellschaftliche Kontexte eingebettet sind. Das Zusammenführen von Ziel- und Systemwissen von Praxisakteuren aus der Zivilgesellschaft ermöglicht die bedarfsgerechte Entwicklung eines solchen Systems.

Die Anwendung der Methode sowie damit verbundene Kommunikationsprozesse sollten immer auf die Gegebenheiten vor Ort bezogen werden, was bedeutet, dass entsprechende Anpassungen im Vorgehen vorgenommen werden müssen.

Im Rahmen der Entwicklung und Anwendung der TraSy-Methode im Reallabor Schorndorf haben sich folgende Herausforderungen an die TraSy-Methode gezeigt, die es bereits bei der Planung von Projekten dieser Art sowie kontinuierlich im Projektverlauf zu reflektieren gilt. 


\subsection{Erwartungshaltungen der Praxispartner}

Die Erwartungshaltung aller Akteure zu Projektbeginn stellt eine wichtige Determinante für den weiteren Projektverlauf und dessen Bewertung dar. Im Sinne der geforderten „Transparenz nach innen“ bei transdisziplinären und transformativen Ansätzen (Quint et al. 2018, S. 71) wird empfohlen, der gemeinsamen Entwicklung von Zielsetzungen und Vorgehensweisen zu Beginn des Projekts ausreichend Beachtung zu schenken. Bei der Umsetzung der TraSy-Methode ist es deshalb entscheidend, frühzeitig zu kommunizieren, an welchen Stellen die Praxispartner Einfluss auf das Projekt nehmen können, um keine falsche Erwartungshaltung aufzubauen (Brandies et al. 2017). Wie von Seebacher et al. (2018) gefordert, darf aus dem „Partizipationskontinuum“ (Meyer-Soylu et al. 2016) ,[...] keine Art ,Pflicht zu andauernder Partizipation“ aller Akteure bei allen Themen und Aktivitäten abgeleitet werden" (Seebacher et al. 2018, S. 103). Bei der Durchführung der TraSy-Methode ist das ,[...] Abwägen zwischen Interessen der Praxispartner sowie forschungsbezogenen Zielen, mit Erwartungen an schnelle praxisnahe und öffentlichkeitswirksame Ergebnisse einerseits und möglicherweise als langsam wahrgenommene Produktion von Forschungserkenntnissen andererseits, hierbei häufig eine zentrale Herausforderung" (Brandies et al. 2017, S. 13).

\subsection{Die interdisziplinäre Verständigung und Einigung}

Die Entwicklung eines soziotechnischen Systems stellt besondere Herausforderungen an die Entwicklung einer gemeinsamen, disziplinübergreifenden Sprache. So hatten beispielsweise im Reallabor Schorndorf die beteiligten Psycholog(inn)en und Verkehrsingenieur(inn)e(n) ein unterschiedliches Bild vom Begriff Bedienkonzept. Die kontinuierliche gemeinsame Arbeit am Spezifikationsheft (s. Kapitel 2), in dem zentrale Termini definiert und festgehalten werden, kann dabei unterstützen, eine gemeinsame Sprache zu finden. Zudem dient das Spezifikationsheft dazu, Zielsetzungen an geeigneter Stelle zu fixieren, um der Dynamik des interdisziplinären Arbeitens zu begegnen.

Der Austausch und die Reflexion innerhalb des interdisziplinären Projektteams sind von großer Bedeutung für den Erfolg der TraSy-Methode. Eine Herausforderung dabei ist es, einerseits durch kontinuierliche Abstimmungen redundante Arbeiten, Verantwortungsdiffusion und Informationsmängel zu vermeiden und andererseits schnelle und tragfähige Entscheidungen zu treffen (Brandies et al. 2017). Diese Herausforderung wiederum kann mit der Erstellung und kontinuierlichen Pflege des Spezifikationsheftes begegnet werden, das als eine Art ,Leitwerk' die Zusammenarbeit im Projektteam strukturiert. 
Ein relevanter Aspekt für die Umsetzung der TraSy-Methode ist die Wertschätzung der Methoden und der Denkschulen der zu beteiligenden Disziplinen. Diesen liegen sehr verschiedene Methodologien zugrunde, da zwingend sozialwissenschaftliche und ,technische' Wissenschaftskulturen aufeinandertreffen. Das Spezifikationsheft kann auch helfen, dieser Herausforderung zu begegnen. Als eine Plattform zum gemeinsamen Wissensaustausch dient es der Übersetzung qualitativer Ergebnisse aus den sozialwissenschaftlichen Elementen der TraSyMethode in Anforderungen an das System. Das Spezifikationsheft stellt damit ein wichtiges Instrument zur Synthesebildung dar.

\section{Dank}

Die Autorinnen danken Svenja Kück und Christian Schmutz für die Diskussionen über eine frühere Textfassung. Insbesondere möchten sie Christina West und Belen Zevallos sowie zwei anonymen Gutachter(inne)n für die konkreten Hinweise zur Verbesserung des Textes im Rahmen des internen und externen Reviews danken. Besonderer Dank gebührt Marcel Bednarz, Christian Borowski, Dijana Hammans, Salome Keller, Christian Kuhn, Martha Miosga und Christina Zissaki für ihre Unterstützung in der Planung und Durchführung der Co-CreationWorkshops. Schließlich danken die Autorinnen den beiden Herausgebenden, Rico Defila und Antonietta Di Giulio, für ihre Rückmeldungen zum Text.

\section{Literatur}

Achtziger, A., \& Gollwitzer, P. M. (2009). Rubikonmodell der Handlungsphase [Rubicon model of action phases]. In V. Brandstätter \& J. H. Otto (Hrsg.), Handbuch der Allgemeinen Psychologie: Motivation und Emotion (S. 150-156). Göttingen: Hogrefe.

Bergold, J., \& Thomas, S. (2010). Partizipative Forschung. In G. Mey \& K. Mruck (Hrsg.), Handbuch Qualitative Forschung in der Psychologie (S. 333-344). Wiesbaden: Springer VS.

Bergold, J., \& Thomas, S. (2012). Partizipative Forschungsmethoden: Ein methodischer Ansatz in Bewegung. Forum Qualitative Sozialforschung/Forum: Qualitative Social Research, 13 (1). doi: 10.17169/fqs-13.1.1801.

Brandies, A., König, A., Viergutz, K. K., Fraedrich, E., Gebhardt, L., Ulmer, F., Sippel, T., \& Dotzauer, M. (2017). Transdisziplinäre Mobilitätsforschung unter Verwendung von Reallaboren: Integration von Stakeholderbedürfnissen und -anforderungen in die Entwicklung von Systemen bedarfsorientiert und vollautomatisiert fahrender Quartiersbusse. Vorgestellt auf der Konferenz: AAET 2017: Automatisiertes \& Vernetztes Fahren 2017, Braunschweig. https://elib.dlr.de/106113/. Zugegriffen am 01.03.2019. 
Brost, M., Gebhardt, L., Steiner, T., Neidhardt, E., Brandies, A., Karnahl, K., \& Ademeit, A.-M. (2018). Results from the real-world-laboratory based pilot operation of a demand responsive bus system. Vorgestellt auf der European Transport Conference (ETC) 2018, 10.-12.10.2018, Dublin, Irland. https://elib.dlr.de/123300/. Zugegriffen am 01.03.2019.

Büscher, C., \& Schippl, J. (2013). Die Transformation der Energieversorgung: Einheit und Differenz soziotechnischer Systeme. Technologiefolgenabschätzung - Theorie und Praxis, 22 (2), (S. 11-19).

CASS \& ProClim- (1997). Forschung zu Nachhaltigkeit und Globalem Wandel - Wissenschaftspolitische Visionen der Schweizer Forschenden. Bern: ProClim-/SANW. https:// naturwissenschaften.ch/service/publications/75640-visionen-der-forschenden. Zugegriffen am 22.02.2019.

Defila, R., \& Di Giulio, A. (2018a). Partizipative Wissenserzeugung und Wissenschaftlichkeit - ein methodologischer Beitrag. In R. Defila \& A. Di Giulio (Hrsg.), Transdisziplinär und transformativ forschen. Eine Methodensammlung (S. 39-67). Wiesbaden: Springer VS. doi: 10.1007/978-3-658-21530-9_2.

Defila, R., \& Di Giulio, A. (2018b). Reallabore als Quelle für die Methodik transdisziplinären und transformativen Forschens - eine Einführung. In R. Defila \& A. Di Giulio (Hrsg.), Transdisziplinär und transformativ forschen. Eine Methodensammlung (S. 9-35). Wiesbaden: Springer VS. doi: 10.1007/978-3-658-21530-9_1.

Degele, N., Kesselhut, K., \& Schneickert, C. (2009). Sehen und Sprechen: zum Einsatz von Bildern bei Gruppendiskussionen. Zeitschrift für Qualitative Forschung, 10 (2), (S. 363-379).

Gebhardt, L., Brost, M., \& Steiner, T. (2019). Bus on demand - ein Mobilitätskonzept mit Zukunft. Das Reallabor Schorndorf zieht nach dem Pilotbetrieb Bilanz. GAIA, 28 (1), (S. 66-68).

Gebhardt, L., Klemme, M., \& Wiegandt, C.-C. (2014). Bürgerbeteiligung und Bürgerengagement in Zeiten der Digitalmoderne - drei Thesen. disP - The Planning Review, 50 (3), (S. 11-120). doi: 10.1080/02513625.2014.979050.

Girtler, R. (2002). Methoden der Feldforschung. Wien: Böhlau.

Grosvenor, T. (2000). Qualitative Research in the Transport Sector. Vorgestellt auf der Konferenz: Transport Surveys: Raising the Standard, Grainau (24.-30.05.1997).

Hennen, L. (2012). Why do we still need participatory technology assessment?. Poiesis \& Praxis, 9 (1-2), (S. 27-41). doi: 10.1007/s10202-012-0122-5.

Hunecke, M. (2015). Mobilitätsverhalten verstehen und verändern. Psychologische Beiträge zur interdisziplinären Mobilitätsforschung. Wiesbaden: Springer VS.

Hügli, A., \& Lübcke, P. (1991). Philosophielexikon. Reinbek bei Hamburg: Rowohlt.

Jaeger-Erben, M., Nagy, E., Schäfer, M., Süßbauer, E., \& Zscheischler, J. (2018). Von der Programmatik zur Praxis: Plädoyer für eine Grounded Theory transformationsorientierter Forschung. GAIA, 27 (1), (S. 117-121). doi: 10.14512/gaia.27.1.5. 
Joss, S., \& Bellucci, S. (2002). Participatory Technology Assessment in Europe: Introducing the EUROPTA Research Project. In S. Joss \& S. Bellucci (Hrsg.), Participatory Technology Assessment: European Perspectives (S. 3-14). London: Center for the Study of Democracy.

Klötzke, M., Brost, M., Fraedrich, E., Gebhardt, L., Karnahl, K., Kopp, G., König, A., Ademeit, A., Müller, A., Sippel, T., \& Ulmer, F. (2018). Reallabor Schorndorf. Bürgernahe Entwicklung eines haltestellenlosen Quartiersbussystems. In H. Proff \& T. Fojcik (Hrsg.), Mobilität und digitale Transformation (S. 295-309). Wiesbaden: Springer Gabler. doi: 10.1007/978-3-658-20779-3_18.

König, A., Karnahl, K., Gebhardt, L., \& Klötzke, M. (2018). Reallabor Schorndorf - Bedarfsgesteuerte Mobilität gemeinsam gestalten. Vorgestellt auf der Konferenz: 12. Deutscher Nahverkehrstag, Koblenz (24.-26.04.2018) https://elib.dlr.de/120468/. Zugegriffen am 01.03.2019.

Kucharski, A., \& Merkel, S. (2018). Partizipative Technikentwicklung von Gerontotechnologie: Ansätze für mehr Akzeptanz in der Zielgruppe. Forschung Aktuell, Institut Arbeit und Technik (IAT), 6/2018.

Lösch, A. (2012). Techniksoziologie. In S. Massen, M. Kaiser, M. Reinhart \& B. Sutter (Hrsg.), Handbuch Wissenschaftssoziologie (S. 251-264). Wiesbaden: Springer VS.

Lüders, C. (2003). Teilnehmende Beobachtung. In R. Bohnsack, W. Marotzki, \& M. Meuser (Hrsg.), Hauptbegriffe Qualitativer Sozialforschung (S. 151-153). Opladen: VS Verlag für Sozialwissenschaften.

Luhmann, N. (1984). Soziale Systeme: Grundriß einer allgemeinen Theorie. Frankfurt a. M.: Suhrkamp.

Luhmann, N. (1997). Die Gesellschaft der Gesellschaft. Frankfurt a. M.: Suhrkamp.

Mayas, C., Hörold, S., \& Krömker, H. (2012). Meeting the Challenges of Individual Passenger Information with Personas. In N. Stantin (Hrsg.), Advances in Human Aspects of Road and Rail Transportation (S. 822-831). Boca Raton: CRC Press.

Meyer-Soylu, S., Parodi, O., Trenks, H., \& Seebacher, A. (2016). Das Reallabor als Partizipationskontinuum. Erfahrungen aus dem Quartier Zukunft und Reallabor 131 in Karlsruhe. Technikfolgenabschätzung - Theorie und Praxis, 25 (3), (S. 31-40).

Mobilität in Deutschland (MID) (2008). Bundesministerium für Verkehr und digitale Infrastruktur, Berlin. http://daten.clearingstelle-verkehr.de/223/. Zugegriffen am 02.03.2019.

Müller, J., Renyi, M., \& Kunze, C. (2014). Partizipative Technikentwicklung für ein selbstbestimmtes Leben im Alter - ein Exkurs. Participartory technology development for an independent life for senior citizens. Transdisziplinäre Konferenz zum Thema „Technische Unterstützungssysteme, die die Menschen wirklich wollen“, Hamburg (15.-16.12.2014). https://www.researchgate.net/publication/269703236_Partizipative_ Technikentwicklung fur_ein_selbstbestimmtes_Leben_im_Alter_- ein_Exkurs Participartory_technology_development_for_an_independent_life_for_senior_citizens. Zugegriffen am 04.03.2019. 
Plattner, H., Meinel, C., \& Weinberg, U. (2009). Design Thinking - Innovation lernen Ideenwelten öffnen. München: mi-Wirtschaftsbuch.

Pruitt, J., \& Adlin, T. (2006). The persona lifecycle: Keeping people in mind throughout product design. San Francisco: Morgan Kaufmann Publishers.

Quint, A., Alcántara, S., \& Seebacher, A. (2018). Der Partizipationsmythos „Partizipation in Reallaboren ist per se transparent und muss es auch sein“. In R. Defila \& A. Di Giulio (Hrsg.), Transdisziplinär und transformativ forschen. Eine Methodensammlung (S. 69-73). Wiesbaden: Spinger VS. doi: 10.1007/978-3-658-21530-9_3.

Røe, P. G. (2000). Qualitative research on intra-urban travel: an alternative approach. Journal of Transport Geography, 8, (S. 99-106). doi: 10.1016/S0966-6923(99) 00039-3.

Sanders, E. B. N. (2003). From user-centered to participatory design approaches. In J. Frascara (Hrsg.), Design and the social sciences (S. 18-25). Abingdon: Taylor \& Francis Books Limited.

Scheiner, J. (1998). Aktionsraumforschung auf phänomenologischer und handlungstheoretischer Grundlage. Geographische Zeitschrift, 86 (1), (S. 50-66).

Seebacher, A., Alcántara, S., \& Quint, A. (2018). Der Partizipationsmythos „Partizipation bedeutet, alle immer an allem zu beteiligen“. In R. Defila \& A. Di Giulio (Hrsg.), Transdisziplinär und transformativ forschen. Eine Methodensammlung (S. 101-104). Wiesbaden: Spinger VS. doi: 10.1007/978-3-658-21530-9_5.

Selle, K. (2013). Über Bürgerbeteiligung hinaus: Stadtentwicklung als Gemeinschaftsaufgabe? Analysen und Konzepte. Detmold: Verlag Dorothea Rohn.

Van Someren, M. W., Barnard, Y. F., \& Sandberg, J. A. C. (1994). The Think Aloud Method - A Practical Guide to Modelling Cognitive Processes. Amsterdam, Boston: Academic Press.

Verband Region Stuttgart (2011). Begleituntersuchungen zur Fortschreibung des Regionalverkehrsplans - Band 1: Mobilität und Verkehr in der Region Stuttgart 2009/2010. Regionale Haushaltsbefragung zum Verkehrsverhalten. https://www.region-stuttgart. org/information-und-download/veroeffentlichungen/schriftenreihe/. Zugegriffen am 08.03.2019. 


\section{Anhang}

Anlage a: Leitfaden für die Expertengespräche im Reallabor Schorndorf - (am Beispiel eines Interviews mit einem Vertreter des Seniorenforums):

Frage 1: Besteht bei Senior(inn)en ein Bedarf für eine bessere Mobilität?

Frage 2: Welche Vor- bzw. Nachteile werden durch einen bedarfsgerechten ÖPNV erwartet?

Frage 3: Ist die Anforderung eines Busses via App für Senior(inn)en realistisch bzw. welche anderen Lösungsansätze sind vorstellbar? Wie könnte ein einfaches Bedienkonzept aussehen?

Frage 4: Inwieweit ist zu erwarten, dass die Senior(inn)en Schorndorfs aufgeschlossen gegenüber Neuerungen/neuen Konzepten sind?

Frage 5: Welche Anforderungen bestehen an die neuen Busse? Bspw. in Form von Fahrrad- und Rollstuhlmitnahme.

Frage 6: Welche Einsatzzwecke bzw. -gebiete sehen Sie für ein solches Bussystem in Bezug auf die Bedürfnisse der Senior(inn)en Schorndorfs?

Frage 7: Wie bzw. wo sollte solch ein System bei Senior(inn)en beworben werden? 
Anlage b: Fragebogen für Feedback nach dem Co-Creation-Workshop I

Wir bitten Sie, die folgenden Fragen möglichst vollständig zu beantworten. Die Erhebung dient ausschließlich wissenschaftlichen Zwecken. Die Auswertung erfolgt anonym.

Wie häufig benutzen Sie die folgenden Verkehrsmittel? (bitte ankreuzen, Mehrfachnennungen möglich)

\begin{tabular}{|l|c|c|c|c|}
\hline & $\begin{array}{c}\text { Täglich/ } \\
\text { fast täglich }\end{array}$ & $\begin{array}{c}\text { Häufig/ } \\
\text { mind. 1x } \\
\text { pro Woche }\end{array}$ & $\begin{array}{c}\text { Selten/ } \\
\text { ca. 1x pro } \\
\text { Monat }\end{array}$ & $\begin{array}{c}\text { Nie bzw. } \\
\text { fast nie }\end{array}$ \\
\hline Pkw, als Fahrer(in) & $\square$ & $\square$ & $\square$ & $\square$ \\
\hline Pkw, als Mitfahrer(in) & $\square$ & $\square$ & $\square$ & $\square$ \\
\hline Carsharing-Pkw & $\square$ & $\square$ & $\square$ & $\square$ \\
\hline $\begin{array}{l}\text { Öffentliche Verkehrsmittel } \\
\text { (Bus, Bahn, etc.) }\end{array}$ & $\square$ & $\square$ & $\square$ & $\square$ \\
\hline $\begin{array}{l}\text { Motorrad, Roller, Mofa, } \\
\text { o. ä. }\end{array}$ & $\square$ & $\square$ & $\square$ & $\square$ \\
\hline Fahrrad & $\square$ & $\square$ & $\square$ & $\square$ \\
\hline $\begin{array}{l}\text { Zu Fuß (ohne Wege zum/ } \\
\text { vom Auto oder S-Bahn etc.) }\end{array}$ & $\square$ & $\square$ & $\square$ & $\square$ \\
\hline anderes Verkehrsmittel: & $\square$ & $\square$ & $\square$ & $\square$ \\
\hline
\end{tabular}

Welches Verkehrsmittel benutzen Sie am liebsten, welches am häufigsten? (bitte ankreuzen)

\begin{tabular}{|l|c|c|}
\hline & ...am liebsten & ...am häufigsten \\
\hline Pkw & $\square$ & $\square$ \\
\hline Öffentliche Verkehrsmittel (Bus, Bahn, etc.) & $\square$ & $\square$ \\
\hline Motorrad, Roller, Mofa, o. ä. & $\square$ & $\square$ \\
\hline Fahrrad & $\square$ & $\square$ \\
\hline Zu Fuß & $\square$ & $\square$ \\
\hline anderes Verkehrsmittel: & $\square$ & $\square$ \\
\hline
\end{tabular}




\begin{tabular}{|c|c|c|c|c|c|c|c|}
\hline \multicolumn{4}{|c|}{$\begin{array}{l}\text { Besitzen Sie oder andere Personen in } \\
\text { Ihrem Haushalt ein eigenes Auto? (bitte } \\
\text { ankreuzen) }\end{array}$} & \multicolumn{4}{|c|}{$\begin{array}{l}\text { Teilen Sie sich das Auto mit anderen } \\
\text { Personen? (bitte ankreuzen) }\end{array}$} \\
\hline$\square$ & $\mathrm{Ja}$ & $\square$ & Nein & $\square$ & $\mathrm{Ja}$ & $\square$ & Nein \\
\hline
\end{tabular}

Angaben zur Person:

\begin{tabular}{|c|c|c|c|c|c|c|c|c|c|c|c|}
\hline \multicolumn{7}{|l|}{ Wohnort (Teilort) } & & & & & \\
\hline \multicolumn{12}{|l|}{ Alter } \\
\hline \multicolumn{12}{|l|}{ Geschlecht } \\
\hline \multicolumn{12}{|l|}{ Höchster Bildungsabschluss } \\
\hline \multicolumn{12}{|c|}{ Anzahl der im Haushalt lebenden Kinder (unter 18) } \\
\hline \multirow[t]{2}{*}{ Derzeitiger Erwerbsstatus } & \multicolumn{2}{|c|}{ 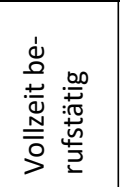 } & \multicolumn{2}{|c|}{ 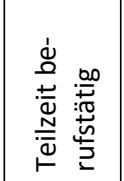 } & 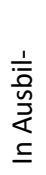 & \multicolumn{3}{|c|}{ 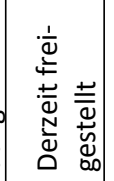 } & 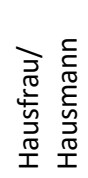 & 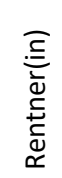 & $\begin{array}{l}\frac{n}{0} \\
\frac{0}{\mathscr{n}} \\
\frac{0}{2} \\
\frac{0}{2}\end{array}$ \\
\hline & $\square$ & & ᄃ & & $\square$ & & $\square$ & & $\square$ & $\square$ & $\square$ \\
\hline \multirow[t]{2}{*}{$\begin{array}{l}\text { Monatliches Nettoeinkom- } \\
\text { men (gesamtes Einkommen } \\
\text { nach Abzug von Steuern } \\
\text { und Sozialversicherungsbei- } \\
\text { trägen) }\end{array}$} & $\begin{array}{c}\text { Un- } \\
\text { ter } \\
500 €\end{array}$ & $\begin{array}{r}50 \\
b \\
\text { un } \\
1.0 \\
€\end{array}$ & & $\begin{array}{l}1.00 \\
€ b \\
\text { unt } \\
2.00 \\
€\end{array}$ & & $\begin{array}{l}2.00 \\
€ \text { bi } \\
\text { unte } \\
3.00 \\
€\end{array}$ & & $\begin{array}{l}3.000 \\
€ \text { bis } \\
\text { unter } \\
4.000 \\
€\end{array}$ & & & $\begin{array}{l}\text { Über } \\
6.000 €\end{array}$ \\
\hline & $\square$ & c & & $\square$ & & $\square$ & & $\square$ & & & $\square$ \\
\hline
\end{tabular}


1. Welche Erwartungen hatten Sie an den Workshop?

2. Was hat Ihnen an diesem heutigen Tag besonders gut gefallen?

3. Was hat Ihnen an diesem heutigen Tag überhaupt nicht gefallen?

4. Was möchten Sie uns noch mitteilen, was heute nicht thematisiert wurde?

5. Konnten Sie sich mit dem Nutzerprofil und dem Szenario der Busnutzung identifizieren?

6. Wären Sie grundsätzlich bereit, auch am zweiten Workshop teilzunehmen?

$\square$ Ja, ich möchte gerne teilnehmen $\quad \square$ Nein, ich habe kein Interesse

Vielen Dank für Ihre Teilnahme! 
Open Access Dieses Kapitel wird unter der Creative Commons Namensnennung 4.0 International Lizenz (http://creativecommons.org/licenses/by/4.0/deed.de) veröffentlicht, welche die Nutzung, Vervielfältigung, Bearbeitung, Verbreitung und Wiedergabe in jeglichem Medium und Format erlaubt, sofern Sie den/die ursprünglichen Autor(en) und die Quelle ordnungsgemäß nennen, einen Link zur Creative Commons Lizenz beifügen und angeben, ob Änderungen vorgenommen wurden.

Die in diesem Kapitel enthaltenen Bilder und sonstiges Drittmaterial unterliegen ebenfalls der genannten Creative Commons Lizenz, sofern sich aus der Abbildungslegende nichts anderes ergibt. Sofern das betreffende Material nicht unter der genannten Creative Commons Lizenz steht und die betreffende Handlung nicht nach gesetzlichen Vorschriften erlaubt ist, ist für die oben aufgeführten Weiterverwendungen des Materials die Einwilligung des jeweiligen Rechteinhabers einzuholen.

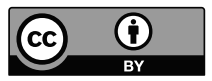




\title{
„Barcamp adapted“6 - gemeinsam zu neuem Wissen
}

\author{
Editha Marquardt \& Ulrike Gerhard
}

\section{$1 \quad$ Einleitung}

In Reallaboren wie in anderen transformativen Forschungszusammenhängen ist die Anfangsphase von großer Bedeutung. Ein Grund dafür liegt in den besonderen Akteurskonstellationen, die solche Forschungsformate aufweisen. Hier arbeiten nicht nur Beteiligte aus einer Disziplin, sondern es ist - im Gegenteil - ein wichtiges Merkmal transformativer Forschung, in transdisziplinären Zusammenhängen zu agieren. Transformative Forschung stellt sich den großen gesellschaftlichen Herausforderungen unserer Zeit: sie will etwas anstoßen, Veränderungen initiieren, Verantwortlichkeiten übernehmen - und damit über ,klassische' Forschung hinausgehen. Hier reichen disziplinäre und auch interdisziplinäre Ansätze, bei denen verschiedene Wissenschaftsdisziplinen zusammenarbeiten, nicht mehr aus. Unter Transdisziplinarität ist dabei der Einbezug von Akteuren aus anderen Disziplinen, über die eigene Universität oder Hochschule hinaus, und von außerwissenschaftlichen Akteuren zu verstehen. Damit weisen Reallabore und andere transdisziplinäre und transformative Forschungsformate eine hohe Heterogenität und Komplexität auf. Sehr unterschiedliche Partner arbeiten gemeinsam an einem Ziel. Sie kommen aus unterschiedlichen Arbeitskontexten und bringen je eigenes Experten- und Erfahrungswissen mit. So können in Reallaboren überaus unterschiedliche Welten aufeinanderstoßen und es ist eine große Herausforderung, das Verbindende herauszuarbeiten. Deshalb spielen dialogische Verfahren, die die Kommunikation in einer Gruppe befördern, eine große Rolle. Bereits in der Anfangsphase werden nun die Weichen gestellt für eine erfolgreiche Zusammenarbeit im gemeinsamen Projekt.

Um diese Phase gewinnbringend zu gestalten, bedarf es des Einsatzes spezifischer Methoden, um die verschiedenen Beteiligten in den Prozess einzubeziehen. Im Vorfeld des Reallabors „Urban Office - Nachhaltige Stadtentwicklung in der Wissensgesellschaft" an der Universität Heidelberg (Reallabor Urban Office, s. auch Steckbrief im Anhang zu diesem Buch sowie Marquardt und West 2016; 
Gerhard und Marquardt 2016) erwies es sich als besonders wirksam, bereits im Vorfeld des eigentlichen Projekts gemeinsam an wichtigen Fragestellungen zu arbeiten.

Unter Einbeziehung aller voraussichtlichen Partner, der Inputs weniger geladener Gäste und unter der Leitung eines externen Moderator(inn)enteams gelang es, eine Frage soweit zu bearbeiten, dass Ergebnisse noch am selben Abend in einer öffentlichen Veranstaltung vorgestellt werden konnten. Doch der Mehrwert lag mitnichten nur auf der Sachebene, sondern vor allem auch darin, dass es gelang, sich durch die gemeinsamen Diskussionen kennenzulernen, das Interesse an gleichen Problemstellungen zu erkennen und die Bereitschaft zu weiterer Zusammenarbeit zu fördern. Die Wahl einer nicht zum typischen Spektrum der ,traditionellen' Forschung zählenden Methode erwies sich als enorm bereichernd in Hinblick auf die beginnende Kooperation im geplanten Reallabor. Die zukünftigen Partner waren sich , auf Augenhöhe ' begegnet, erfuhren gegenseitige Wertschätzung für die eingebrachte Expertise und konnten einen entscheidenden Schritt in Richtung Offenheit für das Gegenüber gehen. Das Feedback einer breiteren Öffentlichkeit konnte direkt in die Ergebnissicherung einbezogen werden.

Die für diesen Schritt gewählte Methode soll nun im folgenden Beitrag vorgestellt werden. „Barcamp adapted“ ist die Weiterentwicklung eines sehr offenen Formats, das hervorragend dazu geeignet ist, unterschiedliche Akteure in einen Arbeitsprozess einzubeziehen. Es stellt eine Methodenkombination aus einem semioffenen Workshop und einer öffentlichen Veranstaltung dar. Der Einsatz eines „Barcamps adapted“ ist besonders in der Entstehungs- und Anfangsphase eines Forschungsprojekts zu empfehlen. Es ist hilfreich, um erste Kooperationen zu initiieren, zukünftige Partner und deren Interessen und Erfahrungen kennenzulernen, kann aber auch in späteren Projektphasen genutzt werden, um neue Ideen zu generieren und dazu zusätzliche Impulse von außen in die eigene Forschungsgruppe einzubeziehen. Eine solche Co-Produktion von Wissen durch die Zusammenarbeit unterschiedlicher, auch wissenschaftsexterner Akteure ist ein wichtiges Merkmal transformativer Forschung und kennzeichnet die Arbeit und Vorgehensweise in transdisziplinär und transformativ ausgerichteten Konstellationen, wie dies zum Beispiel in einem Reallabor der Fall ist (für eine vertieftere Diskussion der Merkmale von Reallaboren s. Arnold und Piontek 2018; Gerhard und Marquardt 2017; Beecroft et al. 2018). Im vorliegenden Beitrag wird zunächst die Methode des Barcamps kurz vorgestellt (s. Kapitel 2), um dann den idealtypischen Verlauf der Methodenkombination „Barcamp adapted“ sowie Merkmale des Gelingens zu thematisieren (im Kapitel 3 wird auf die Planung eingegangen, im Kapitel 4 auf die Durchführung). 


\section{Das „Barcamp adapted“ als Methode transdisziplinärer Forschung}

Eine fruchtbare Zusammenarbeit zwischen verschiedenen Akteuren aus Wissenschaft, Wirtschaft, Bildung, Politik, Verwaltung und Öffentlichkeit in einem gemeinsamen Forschungsprojekt stellt eine große Herausforderung dar. Jeder Partner kommt aus einem eigenen Kontext und hat eine spezifische Sichtweise auf gesellschaftliche Fragestellungen. Zugleich bringen die verschiedenen Beteiligten unterschiedliche Expertisen in das entstehende oder beginnende Projekt ein. Damit verbunden sind differierende Erwartungen an das gemeinsam verfolgte Projekt. Diese Erwartungen und daran angebundene Ziele zu erfragen und offenzulegen, ist eine entscheidende Aufgabe am Anfang des Forschungsprozesses, um die kommende Kooperation von Anfang an auf eine gute Basis zu stellen. Deshalb sollte für diesen Anfangsprozess ausreichend Zeit eingeplant werden, damit alle Beteiligten sich kennenlernen sowie ihre Ideen und Anliegen einbringen können. Auf diese Weise kann ein gemeinsamer Forschungsprozess in Gang gesetzt werden. Die Entwicklung der Forschungsidee und des daran anschließenden Forschungsprozesses sollte im Co-Design erfolgen, wozu ein besonderes methodisches Setting erforderlich ist. Notwendig ist ein offenes Format, das durch gemeinschaftliches Arbeiten an einem relevanten Thema den Prozess eröffnet und Begegnungen der unterschiedlichen Beteiligten ermöglicht. Damit kann es gelingen, einen Mehrwert auf zwei Ebenen zu erzielen: die gemeinsame Arbeit sollte auf inhaltlichen Erkenntnisgewinn zielen, zugleich sollte sie eine gemeinsame Grundlage für die zukünftige Kooperation legen bzw. eine bereits laufende $\mathrm{Zu}$ sammenarbeit stabilisieren.

\subsection{Was ist überhaupt ein Barcamp?}

Um die oben genannten Ziele zu erreichen, erweist sich die Nutzung des Barcamp-Ansatzes als vielversprechend, insbesondere wenn man ihn in Hinblick auf transdisziplinäres wissenschaftliches Arbeiten weiterentwickelt. Ein Barcamp ist eine Methode, die von der Mitwirkung aller Teilnehmer(innen) an einer Veranstaltung lebt. Hier geht es um Wissensaustausch und darum, das Wissen und die Erfahrungen aller in die Diskussion einzubeziehen. Eine Vielfalt der Mitwirkenden, die an einem gemeinsamen Thema interessiert sind - aus unterschiedlichen Disziplinen und Praxiskontexten sowie mit unterschiedlichen Positionen - ist hier von Vorteil und bereichert nicht nur die Diskussion, sondern kann zu überraschenden Einsichten und Ergebnissen führen. Das Barcamp ist mit der Methode des Open Space verwandt, Übergänge sind hier fließend. Hinsichtlich des Verlaufs gibt es große Übereinstimmungen, jedoch kann ein Barcamp in kleineren 
Zusammenhängen eingesetzt werden und bedarf damit weniger Vorbereitung als die Planung und Durchführung einer Open-Space-Konferenz. Aufgrund seiner Herkunft aus der IT-Entwicklung, in der Barcamps bis heute häufig eingesetzt werden, spielen oftmals digitale Medien - Twitter etc. - eine Rolle, über die neben der persönlichen Kommunikation ebenfalls kommuniziert wird. Im Unterschied zum Open Space sind die Zeiten der einzelnen Diskussionssessions kürzer, denn es geht stärker um einen Wissensaustausch als um die Erarbeitung von fertigen Lösungen. In der Praxis sind die beiden Methoden jedoch nicht immer eindeutig voneinander abzugrenzen, wie zum Beispiel die Diskussion im Internet zeigt (s. zu Barcamp oder Open Space: Kolenaty o. J.).

Die Idee des Barcamps ist noch relativ neu, das erste Barcamp fand 2005 in Palo Alto, Kalifornien, statt. Ursprünglich wurde diese Methode insbesondere für die Softwareentwicklung oder zur Bearbeitung unterschiedlicher Medienthemen genutzt (Dittrich-Brauner et al. 2013, S. 160). Besonderes Kennzeichen ist, dass die besprochenen Themen von den Teilnehmer(inne)n selbst vorgeschlagen werden. Es gibt in der Regel keine Rednerliste, die vorab geplant ist, sondern die Inputs und die Leitung der Gruppen ergeben sich erst im Laufe des Tages. Deshalb werden Barcamps manchmal auch als „Unkonferenzen“ (Doppler 2016, S. 83) bezeichnet, da sie sich dadurch ganz wesentlich von konventionellen Konferenzen unterscheiden. Es geht nicht um die Präsentation einschlägiger Redner(innen) und die Vermittlung von deren individuellem Wissen, sondern um die gemeinsame Erarbeitung neuer Ideen und somit schließlich um das Erlangen gemeinsamer Wissensbestände. Seit 2005 steigt die Zahl von Barcamps im globalen Kontext, aber auch in Deutschland, rasant an, weshalb von einigen Autor(inn)en bereits von einer Bewegung gesprochen wird (Hellmann 2007, S. 108). Inzwischen findet die Methode Anwendung in vielerlei thematischen Kontexten, angefangen bei der Stadtentwicklung über Themen aus Kultur und Literatur bis zu Public-HealthThemen. Der Erfolg der Methode liegt in ihrer breiten Anwendbarkeit begründet: Sie kann von einer Gruppe bereits zusammenarbeitender Personen ebenso genutzt werden wie von Menschen, die zuvor noch nie zusammengesessen haben, sich nun aber bewusst zum Dialog zusammenfinden. Ziel ist es immer, unterschiedliche Akteure in Verbindung zu bringen. Diese Akteure stehen während der Durchführung des Barcamps in keinem hierarchischen Verhältnis zueinander abgeleitet aus Renommee, Einfluss und Vorkenntnissen -, sondern erhalten die Möglichkeit, gleichberechtigt ihre Expertise in den Diskussionsprozess einzubringen.

Ein Barcamp verläuft in der Regel folgendermaßen (s. Doppler 2016, S. 83-85 oder Hellmann 2007): Es wird zu einem Event mit dem Fokus auf ein bestimmtes Thema oder eine bestimmte Fragestellung eingeladen. Die Teilnahmemöglichkeit 
ist offen, meist wird breit in der (jeweiligen) Öffentlichkeit eingeladen, es können aber auch gezielt Teilnehmer(innen) bzw. Expert(inn)en eingeladen werden. Das ist abhängig vom Kontext, in dem das Barcamp stattfindet. Zu Veranstaltungsbeginn haben alle Teilnehmer(innen) die Möglichkeit, ihre Sicht auf das Thema bzw. die Fragestellung in kurzer Form (wenige Minuten) vorzutragen. Aus diesem Grund könnte man sagen, dass es bei einem Barcamp keine Zuschauer(innen) gibt, sondern nur aktive Teilnehmer(innen). Über die Themen, die dann diskutiert werden, wird abgestimmt, es werden also nicht alle bearbeitet, und sie werden auch nicht, wie beim Open Space, grundsätzlich alle angeboten. Jede(r) hat dann die Möglichkeit, sich frei einem der ausgewählten Themen und damit einer Diskussionsgruppe zuzuordnen. Dabei geht es nicht um Lösungen, die am Ende feststehen müssen, sondern primär um den Wissensaustausch mit einem thematischen Fokus aus unterschiedlichen Perspektiven. Das Zusammentragen der Ergebnisse und die Abrundung des Barcamps sind ebenfalls anspruchsvolle Teile im Ablauf. Denn von der Ergebnispräsentation hängt ab, mit welchem Gefühl die Teilnehmer(innen) nach Hause gehen: Hat dieser Tag etwas gebracht? Wurde wieder einmal nur geredet? Welche Umsetzungschancen haben meine Ideen?

\subsection{Weiterentwicklung zum „Barcamp adapted“"}

Diese hier kurz vorgestellte Methode lässt sich durch einige Adaptionen überaus gut in transdisziplinären Arbeitskontexten anwenden. Die Weiterentwicklung des Barcamps erfolgt dabei auf zwei Ebenen. Zum Ersten betrifft sie die Veränderung der Einladung und damit der Teilnehmergruppe. $\mathrm{Zu}$ einem Barcamp wird in der Regel offen eingeladen. Da diese Methode im Hinblick auf den transdisziplinären Prozess jedoch zielgerichtet eingesetzt werden soll, sollte hier unbedingt gezielt eingeladen werden. $\mathrm{Zu}$ empfehlen ist der Einbezug aller möglichen Partner, die sich am geplanten Projekt beteiligen wollen. Weiterhin können Interessent(inn)en aus dem Projektumfeld angesprochen werden. Das können Akteur(inn)e(n) sein, die an ähnlichen Fragestellungen arbeiten oder deren Input vielversprechend für die Thematik des geplanten Projekts sein kann. Hierzu sollten auch Empfehlungen der Partner eingeholt werden. Im Unterschied zum ,klassischen' Barcamp ist es in wissenschaftlichen Zusammenhängen sinnvoll, auch Expert(inn)en von außerhalb der bereits bestehenden Gruppe einzuladen. Diese können sowohl kurze thematische Inputs geben als auch die Diskussion in den Arbeitsgruppen durch ihre Expertise bereichern.

Zum Zweiten erfolgt eine Weiterentwicklung durch die Kombination mit einer sich unmittelbar daran anschließenden öffentlichen Veranstaltung. Während durch die erste Adaption eine Schließung des Teilnehmerkreises erfolgt, gelingt 
es durch diesen Schritt, den Kreis wieder zu öffnen. Gleichzeitig können die Ergebnisse schnell in einen breiteren Kontext gestellt werden, so dass eine öffentliche Diskussion dazu eröffnet werden kann. Somit wird ein wichtiger Schritt zum Transfer des erarbeiteten Wissens gegangen, das so seinen Weg in eine größere Öffentlichkeit findet. Im Folgenden soll nun die Methodenkombination detailliert vorgestellt werden.

\section{Einsatz des „Barcamps adapted“ im transdisziplinären Forschungsprozess}

Das „Barcamp adapted“ lässt sich aufgrund seines offenen Charakters besonders effektiv zu Beginn eines gemeinsamen Forschungsprozesses einsetzen. Es eröffnet Möglichkeiten des Kennenlernens, neue Wege der Zusammenarbeit und kann zur Vorbereitung und Entwicklung eines kollaborativ angelegten Forschungsprojekts dienen (s. Praxisbeispiel Urban Office Nr. 1 in diesem Kapitel). Aber auch im weiteren Verlauf kann ein „Barcamp adapted“ gewinnbringend eingesetzt werden - etwa zur Aufbereitung von (nicht) erfüllten Erwartungen oder gegen Ende zu Fragen rund um Verstetigung und Fortführung.

Die Dauer der Veranstaltung ist gerade beim Arbeiten in transdisziplinären $\mathrm{Zu}-$ sammenhängen ein wichtiges Thema, denn es gilt, Partner aus verschiedenen gesellschaftlichen Bereichen einzubinden. Deshalb ist die Dauer genau abzuwägen, mit Blick auf die anvisierten Teilnehmer(innen), um eine hohe Mitwirkungsbereitschaft zu erzielen. Bereits ein ganzer Tag bedeutet einen hohen Zeitaufwand und kann eventuell nicht von allen Partnern, die man gerne dabeihätte, ermöglicht werden. Dennoch sollte diese Zeit möglichst nicht unterschritten werden, um relevante Ergebnisse erzielen zu können: da es sich um ein offenes und hochkommunikatives Format handelt, muss ausreichend Zeit für die verschiedenen Schritte eingeplant werden. Als Dauer für ein „Barcamp adapted“ sind demnach ein bis zwei Tage empfehlenswert. Der genaue Zeitraum hängt von der geplanten Teilnehmerzahl, den vorhandenen Ressourcen sowie der Themenanzahl, die bearbeitet werden soll, ab. Bereits die Einstiegsphase in den Workshop ist entscheidend, um einen vertrauensvollen Umgang miteinander zu ermöglichen. Dies sollte bei der Zeitplanung berücksichtigt werden. Ebenso sind ausreichend Pausen, in denen ungezwungene Gespräche entstehen können, einzurechnen. Bei einer Teilnehmerzahl von ungefähr 20 Personen lassen sich an einem Tag vier bis fünf Themen intensiv bearbeiten. 


\section{Praxisbeispiel Urban Office Nr. 1: „Barcamp adapted“ in der Vorbereitung eines Projekts}

Die Idee des Reallabors „Urban Office - Nachhaltige Stadtentwicklung in der Wissensgesellschaft" (Reallabor Urban Office) der Universität Heidelberg wurde zusammen mit zwei außeruniversitären Praxispartnern, der Stadtverwaltung Heidelberg und der Internationalen Bauausstellung Heidelberg (IBA), sowie weiteren inner- und außeruniversitären Akteuren aus den Bereichen Architektur und Soziologie entwickelt. Diese Zusammenarbeit begann also bereits vor Beginn der Arbeitsaufnahme des Reallabors. In dieser sensitiven Phase - nach zahlreichen Runden Tischen und Gesprächen - wurde ein Barcamp in Verbindung mit einer öffentlichen Veranstaltung durchgeführt, mit den Zielen, Ideen und Konzepte für Partizipationsmöglichkeiten im geplanten Reallabor zu erarbeiten, weitere mögliche Praxispartner in den Forschungsprozess des zukünftigen Reallabors zu involvieren sowie Interessierte aus der Stadtgesellschaft zu informieren und einzubeziehen. Dazu wurde die Methode des Barcamps angepasst und als eine eintägige Veranstaltung in Form eines Workshops mit öffentlicher Abendveranstaltung konzipiert. Insgesamt wurde ein ganzer Tag eingeplant, der erste Teil wurde von 9 bis 17 Uhr durchgeführt, daran schloss sich nach einer einstündigen Pause eine zweistündige öffentliche Diskussion der Ergebnisse an.

Der erste Teil des „Barcamps adapted“, der Workshop, kann hinsichtlich seiner Teilnehmerzahl recht flexibel gestaltet werden, da die Themen in kleineren Gruppen bearbeitet werden. Limitiert wird die Größenordnung lediglich durch die zur Verfügung stehenden Räumlichkeiten (mindestens ein größerer Raum für Plenumsdiskussionen sowie zahlreiche kleine für die Arbeit in den Kleingruppen). Um sinnvolle Arbeit in Kleingruppen zu ermöglichen, ist jedoch eine Mindestteilnehmerzahl von 20 Personen empfehlenswert, ebenso erscheint eine maximale Begrenzung auf 40 Teilnehmer(innen) sinnvoll. Diese Zahl ermöglicht immer wieder neue Gruppenzusammensetzungen und eine Vielzahl von Gesprächen, bleibt aber zugleich noch überschaubar.

Wie oben bereits angesprochen, ist die Auswahl der Teilnehmer(innen) im Vergleich zum ,klassischen“ Barcamp eingeschränkter und muss genau durchdacht werden (s. Praxisbeispiel Urban Office Nr. 2 in diesem Kapitel). Sie ist wesentlich, wenn die Veranstaltung die Eröffnung eines transdisziplinären Forschungsprozesses anstoßen soll. Deshalb müssen die verschiedenen Akteure, deren Mitarbeit im weiteren Projektverlauf gewünscht ist, die Möglichkeit zu einer Teilnahme erhalten. Auf diese Weise wird sichergestellt, dass die unterschiedlichen Sichtweisen, die im Projekt einbezogen werden sollen, sowie die verschiedenen Wissensbestände und Erfahrungen von Anfang an mitbedacht werden können. Angestrebt wird, eine projektbezogene Kommunikation zu eröffnen und gleichzeitig über eine initiierende Kerngruppe hinaus zu erweitern. Deshalb ist es empfehlenswert, noch weitere Personen einzuladen, die sich mit dem Thema befassen und am geplanten Projekt interessiert sind, und so andere Perspektiven einzubin- 
den, um die Grenzen des eigenen Denkens zu überwinden und neue Strategien zu entwickeln. Auf diese Weise kann das Netzwerk, auf dem ein transdisziplinäres Forschungsprojekt aufbaut, von Anfang an erweitert werden.

Elementar erscheint darüber hinaus, zusätzlich gezielt auch einige Expert(inn)en einzuladen, die zu der zu bearbeitenden Thematik einen Input aus bisher noch nicht berücksichtigten Perspektiven leisten können. Unter Expert(inn)en sind dabei nicht nur Wissenschaftler(innen) zu verstehen, sondern es können Vertreter(innen) aus ganz unterschiedlichen Bereichen, auch Bürger(innen), hinzugezogen werden. Diese ergänzende Expertise garantiert, dass substanzielles Wissen von außen in das bereits bestehende Netzwerk getragen wird, und führt zu neuen Anregungen sowie zum Überdenken bisheriger Strategien. Zugleich können diese Expert(inn)en auf manche der im Workshop neu aufgeworfenen Fragen auch mögliche Antworten aufzeigen, die die Mitglieder der bestehenden Gruppe sowie die übrigen Workshopteilnehmer(innen) nicht gefunden hätten. Durch das zusätzlich involvierte Expertenwissen kann der Workshop über ein Anreißen von Fragen sowie ein bloßes Brainstorming hinausgehen. Die Diskussion kann so entscheidend bereichert werden, um zu Ergebnissen zu kommen und Impulse für den künftigen Forschungsprozess zu geben.

\section{Praxisbeispiel Urban Office Nr. 2: Auswahl der Workshopteilnehmer(innen)}

In Vorbereitung des Heidelberger Reallabors Urban Office wurden für das geplante Barcamp zu neuen Partizipationsmöglichkeiten für eine nachhaltige Stadtentwicklung gezielt Teilnehmer(innen) eingeladen. Ausgehend von der Fragestellung des Barcamps wurden externe Expert(inn)en angefragt, die sich bereits seit längerem mit Partizipationsangeboten im urbanen Kontext beschäftigen. Sie sollten neue Impulse aus den eigenen Forschungen und praktischen Erfahrungen in anderen räumlichen Kontexten einbringen. Mit der Einladung wurden sie gebeten, kurze Inputs vorzubereiten. Dabei wurde jeweils das Inputthema, auf das sie sich vorbereiten sollten, abgesprochen. Ein Experte beschäftigte sich beispielsweise seit längerem mit Gamification als partizipatives Format in der Stadtentwicklung. Unter Gamification lässt sich die Übertragung von Spieldesign und Spielmechaniken auf andere Bereiche, hier auf Bürgerpartizipation in Stadtentwicklungskontexten, verstehen - ein Ansatz, der so im Heidelberger Kontext noch nicht ausprobiert worden war und als spannender neuer Zugang verstanden wurde (zum Ansatz s. Deterding et al. 2011). Zudem wurden nebst den Partnern des zukünftigen Reallabors weitere Interessent(inn)en eingeladen, zu denen bereits erste Kontakte bestanden.

Es gab also keine offene Einladung der breiten Öffentlichkeit für den Workshop, sondern gezielte persönliche Einladungen. Es war jedoch allen offengestellt, weitere Personen mitzubringen. Auf diese Weise ergab sich eine Teilnehmerzahl von rund 25 Personen, darunter die drei geladenen Expert(inn)en von außerhalb, verschiedene Personen aus dem geplanten Reallabor selbst sowie Student(inn)en und Akteure, die bisher nicht direkt am Reallaborprozess beteiligt waren. Dieser Personenkreis war eine überaus gute Voraussetzung für fruchtbare und zielführende Diskussionen. 
Um das „Barcamp adapted“ möglichst gewinnbringend zu gestalten, ist zur Koordination der Gesamtveranstaltung (Workshop und öffentliche Veranstaltung) der Einsatz von Moderator(inn)en, die selbst nicht in die inhaltliche Diskussion involviert sind, notwendig. Dazu ist der Einsatz eines Moderator(inn)enteams empfehlenswert, je nach Größe der Veranstaltung können auch mehr als zwei Personen für diese Aufgabe eingeplant werden. Es ist unbedingt darauf zu achten, dass die Moderator(inn)en bereits mit diesem oder zumindest mit ähnlichen Formaten (wie Open Space) Erfahrung haben. Von Vorteil ist es, wenn die Moderator(inn)en selbst ebenfalls in einem vergleichbaren thematischen Kontext arbeiten, um die Wahl der Themen während des Workshops kompetent leiten zu können (s. auch Praxisbeispiel Urban Office Nr. 3 in diesem Kapitel).

Die Moderator(inn)en koordinieren während des Workshops die Auswahl der zu diskutierenden Themen und die Bildung der Kleingruppen, sie unterstützen die Arbeit der Kleingruppen und leiten das Zusammentragen der Ergebnisse. Sie sind darüber hinaus für die Zeiteinhaltung und andere organisatorische Fragen zuständig. Es obliegt den Moderator(inn)en, die Ergebnisse aus dem Workshop zur Präsentation in der anschließenden öffentlichen Veranstaltung aufzubereiten. Damit kommt ihnen eine exponierte Rolle zu, sie sind also mitentscheidend für den Erfolg oder Misserfolg eines „Barcamp adapted“. Deshalb ist unbedingt auf eine professionelle Moderation zu achten - es kommen für diese Aufgabe nur Personen mit ausgewiesener Moderationserfahrung in Frage. Es muss gelingen, eine zugleich freundlich-offene und arbeitsintensive Atmosphäre zu erzeugen, die Kommunikation der Teilnehmer(innen) untereinander zu fördern und die Prozesse zielgerichtet ablaufen zu lassen.

\section{Praxisbeispiel Urban Office Nr. 3: Moderation}

Die Leitung des „Barcamps adapted“ (Workshop sowie Abendveranstaltung) wurde in die Hände zweier Moderatoren gelegt, die unterschiedliche Erfahrungen aus Veranstaltungen im urbanen Kontext mitbrachten und bereits in mehreren Projekten zusammengearbeitet hatten. Ein Moderator war insbesondere im Bereich der Förderung von Kultur- und Kreativwirtschaft tätig. Der andere leitete ein kleineres Stadtentwicklungsunternehmen, das die Einbindung von Bürger(inne)n in Planungsprozesse zum Ziel hatte. Dabei waren sie selbst im Bereich Bürgerpartizipation tätig und brachten auch inhaltlich Vorkenntnisse aus Heidelberg sowie aus anderen Städten mit. Die beiden Moderatoren teilten die Aufgaben untereinander auf: So moderierte einer im Workshop die Eingangsphase mit Themenwahl, der andere die Ergebnissammlung in der Plenumsphase am Nachmittag. Beide betreuten gemeinsam die Kleingruppen und standen für Gespräche in den Pausen zur Verfügung. Gemeinsam arbeiteten sie die Präsentation für die öffentliche Abendveranstaltung aus, die sie später auch moderierten. 
Für eine erfolgreiche interaktive Veranstaltung sind auch die Räumlichkeiten von Bedeutung. Räume wirken auf die Diskussion und spielen gerade in innovativen Prozessen eine zentrale Rolle. Deshalb sollte sorgsam überlegt werden, wo das „Barcamp adapted“ durchgeführt werden soll (s. Praxisbeispiel Urban Office Nr. 4 in diesem Kapitel). Dabei ist das Vorhandensein von ausreichenden Räumen für den Workshop (ein gemeinsamer Plenarraum sowie mindestens so viele kleinere Räume, wie Kleingruppen gebildet werden sollen) eine Grundvoraussetzung. Auch sollte bei einem ganztägigen Workshop ein Raum für informelle Gespräche vorgesehen sein, in dem Getränke und eventuell Kleinigkeiten zum Essen zur Verfügung gestellt werden können. Dies trägt wesentlich zu einer gelösten Atmosphäre bei und fördert den Austausch. Außerdem muss natürlich ein geeigneter Raum für die öffentliche Veranstaltung zur Verfügung stehen.

\section{Praxisbeispiel Urban Office Nr. 4: Räume}

Für das „Barcamp adapted“ im Reallabor Urban Office wurden Räume in einem Heidelberger Kreativzentrum genutzt, die sich deutlich von Universitätsräumen und üblichen Büro- und Tagungsräumen unterscheiden. Deren besondere Atmosphäre wirkte bereichernd und erzeugte eine kreative Stimmung unter den Teilnehmer(inne)n. Dabei handelte es sich um einen größeren Co-Working-Space, in dem die Plenardiskussionen am Anfang und am Ende des Workshops abgehalten werden konnten und in dem auch zwei Diskussionsgruppen Platz fanden. Hinzu kamen drei kleinere Räume für die anderen Gruppen. Außerdem stand im Flur eine Küchenecke zur Verfügung. Hier konnte Kaffee getrunken und informell miteinander gesprochen werden. Im selben Gebäude gab es zudem einen großen Saal, der für die öffentliche Abendveranstaltung genutzt werden konnte.

\section{Ablauf eines „Barcamps adapted“6}

\subsection{Themenfindung für die Gruppenarbeit}

Der erste Veranstaltungsteil, der Workshop, startet mit einer kurzen Vorstellung von Ziel, Thema sowie Veranstaltungskontext durch die Moderator(inn)en. Außerdem sollten sich alle Teilnehmer(innen) kurz vorstellen: Wer sie sind, inwieweit sie sich mit dem Thema beschäftigen, welche Kenntnisse sie mitbringen und was sie vom Workshop erwarten. Dabei muss darauf geachtet werden, dass diese Vorstellung wirklich kurz ausfällt (ein bis maximal zwei Minuten). Die eingeladenen externen Expert(inn)en werden im Vorfeld aufgefordert, Impulse vorzubereiten, deren Thema mit ihnen abgesprochen wird. Damit geben sie fachlichen Input und zeigen den Teilnehmer(inne)n zugleich, wo ihre Expertise liegt. Es ist wichtig, dass diese Impulse ebenfalls kurzgehalten werden, es sollte des- 
halb eine Maximaldauer vorgegeben werden (drei bis fünf Minuten). Alle Kurzvorstellungen und Impulse dienen zur Anregung der Themenfindung, die im nächsten Schritt erfolgt.

Nun beginnt mit der Nennung von offenen Fragen und besonderen Anliegen rund um das Workshopthema der eigentliche Themenfindungsprozess für die Gruppenarbeit. Daran beteiligen sich alle Teilnehmer(innen). So wird sichtbar, welche Inhalte in den Arbeitsgruppen im Workshop bearbeitet werden könnten. Dabei gibt es keine inhaltlichen Vorgaben, so dass sich die Themen für die Arbeitsgruppen aus den Interessen und Perspektiven aller Anwesenden ergeben. Allerdings sollten sie auf die übergeordnete Themenstellung des „Barcamp adapted“ fokussieren. Dies wird durch die Moderator(inn)en deutlich gemacht. Bei der Anmoderation werden diese Regeln kurz erläutert und es wird darum gebeten, Fragen und Anliegen auf Kärtchen zu schreiben. Wer etwas geschrieben hat, kann nach vorne treten und seine Karte anstecken. Fragen und Anliegen werden kurz erläutert, damit alle verstehen können, worauf sie konkret abzielen. Bei Unklarheiten sind Nachfragen möglich (s. Abb. 1).

Aus diesem Vorgehen bezieht das „Barcamp adapted“ seine Offenheit: es kann durchaus vorkommen, dass bei diesem Schritt unerwartete Inhalte aufkommen. Die so an der Wand platzierten Fragen und Anliegen werden von den Moderator(inn)en - in offenem Dialog mit den Teilnehmer(innen) - geclustert. Dieses Sortieren der Karten an der Pinnwand erfolgt in Diskussion und Abstimmung mit allen Teilnehmer(inne)n. Dabei werden zunächst ähnliche Fragen und Anliegen zueinander gelegt, woraus sich eine visuelle Ordnung der Kärtchen an der Pinnwand ergibt, aus der wiederum sichtbar wird, welche Fragen und Anliegen möglicherweise zusammengefasst werden können und welche eventuell identisch sind. Auch dieses Zusammenlegen erfolgt in Abstimmung mit der Gesamtgruppe der Teilnehmer(innen). Diese Cluster können nun mit einem Etikett versehen werden, das den Inhalt schlagwortartig, aber treffend wiedergibt und als ,Überschrift' wiederum auf Karten notiert und an der Pinnwand angebracht wird. Dieses Vorgehen ist notwendig, da oft viele unterschiedliche Fragen und Anliegen genannt werden, die nicht alle in Kleingruppen bearbeitet werden können. Die ,Überschriften', die nun an der Tafel sichtbar sind, sind die Themen, die im weiteren Workshopverlauf bearbeitet werden können. Danach werden alle Teilnehmer(innen) aufgefordert, mit drei Klebepunkten an der Pinnwand zu kennzeichnen, zu welchem Thema sie gern diskutieren möchten. Es ist möglich, alle Punkte für ein einziges Thema oder jeden Punkt für ein je anderes Thema zu vergeben. Nur die Themen, die viele Interessenspunkte erhalten, werden Gegenstand der weiterführenden Bearbeitung in Kleingruppen. 


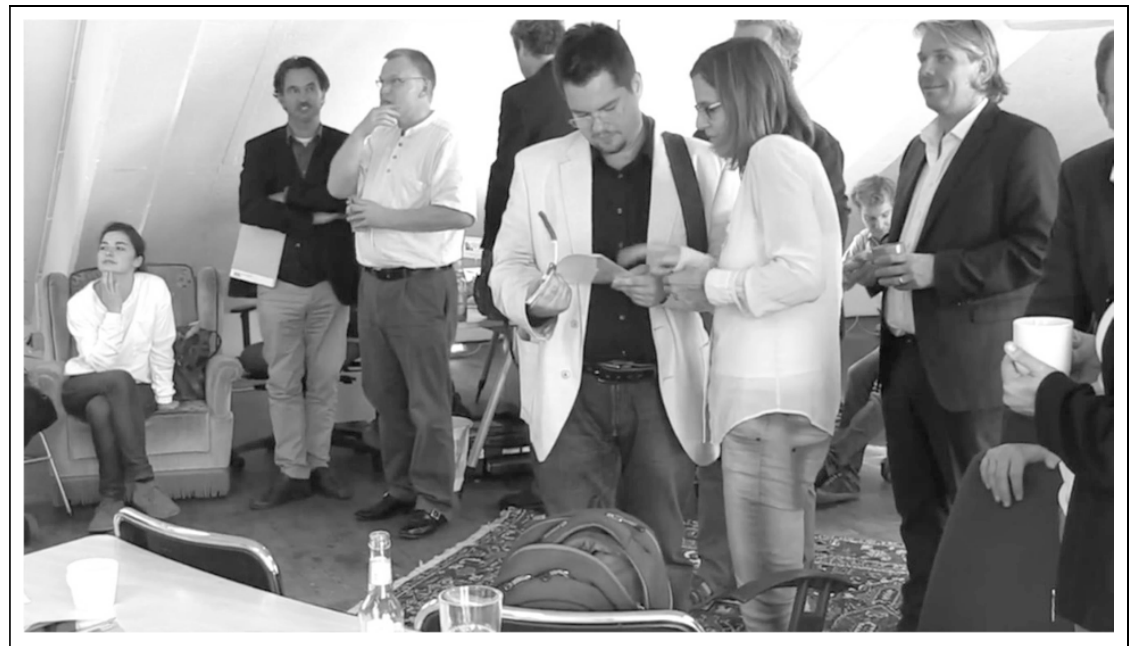

Abbildung 1: Alle Teilnehmer(innen) haben die Möglichkeit, ihre Interessen in die Themenfindung einzubringen, und sind aufgefordert, sich daran zu beteiligen, aus diesen individuellen Fragen und Anliegen die möglichen Themen für die Gruppendiskussionen zu bilden - hier beim „Barcamp adapted“ des Reallabors Urban Office in Heidelberg. (C) Reallabor Urban Office.

Die gewählten Themen werden noch einmal benannt. Dabei wird gleichzeitig erfragt, wer einzelne Aspekte, die in dieses Thema eingeflossen sind, eingebracht hat und wer von diesen Personen bereit ist, eine Gruppenleitung zu übernehmen. Anschließend ordnen sich die Teilnehmer(innen) einer Gruppe zu, die sie besuchen möchten. Die speziell eingeladenen Expert(inn)en können hierbei genau wie die anderen Teilnehmer(innen) agieren und sich sowohl bereit erklären, eine Gruppe zu leiten, als auch sich einfach einer Gruppe als Teilnehmer(in) anschließen. Auf diese Weise finden sich überaus heterogene Gruppen zu den verschiedenen Themen zusammen. Durch diese Form der Auswahl kommt es in der Regel dazu, dass nicht alle Themen - und damit auch nicht alle Fragen und Anliegen, die die Teilnehmer(innen) ,mitgebracht haben " - bearbeitet werden: das entscheiden die Teilnehmer(innen) dadurch, dass sich niemand einem bestimmten Thema zuordnet, so dass keine Gruppe entsteht. Die Moderator(inn)en teilen dann den Gruppen je einen Raum zu. Dabei sollten sie auf die Größen der Gruppen reagieren, falls diese unterschiedlich ausfallen. Diese Phase bis zum Arbeitsbeginn in den Gruppen nimmt insgesamt erfahrungsgemäß 90 bis 120 Minuten in Anspruch. 


\section{Praxisbeispiel Urban Office Nr. 5: Themenfindung für die Gruppenarbeit}

Das „Barcamp adapted“ zum Thema „Neue Formen der Partizipation zur nachhaltigen Stadtentwicklung" in Heidelberg startete nach einer Vorstellungsrunde mit kurzen Impulsreferaten der Expert(inn)en zur Bedeutung von und Erfahrung mit Partizipation in der Stadtentwicklung sowie mit einer fokussierten Diskussionsmöglichkeit. So wurde zum Beispiel der Ansatz der Gamification als ein Partizipationsformat vorgestellt (s. Praxisbeispiel Urban Office Nr. 2 in Kapitel 3). Darüber hinaus konnten auch die anderen Teilnehmer(innen) kurze Erklärungen zu ihrem beruflichen Kontext und ihrem Interesse (Fragen, Anliegen, besondere Expertise) rund um das Thema beitragen und aufzeigen, auf welchen Gebieten sie tätig waren oder welche Erfahrungen und Perspektiven für die Findung und Diskussion der Themen hier versammelt war. Deutlich wurde die Gruppendiversität: neben Wissenschaftler(inne)n der Universität Heidelberg und anderer Orte waren Vertreter(innen) aus der Stadtverwaltung, Student(inn)en, Architekt(inn)en und freiberufliche Soziolog(inn)en anwesend, die alle das Interesse am Thema der partizipativen Stadtentwicklung teilten. Aus der Themenfindung und Themenwahl für die Arbeit in Kleingruppen entstanden vier Kleingruppen, die im weiteren Verlauf des Workshops die folgenden Themen bearbeiteten: "Genius und Diskurse in der Stadtplanung", „Orte von Bürger(inne)n machen lassen“, "Gamification als Partizipationsmöglichkeit“ und „Planung eines Reallabors Urban Office“.

\subsection{Gruppenarbeit und Plenum im Workshop}

Die Moderator(inn)en informieren nun über den weiteren Ablauf der Gruppenarbeit - Räume, Zeitvorgaben, Mittagspause, Ergebnissicherung und Zusammenkommen im Plenum (für ein Beispiel eines Programms s. Praxisbeispiel Nr. 6 in diesem Abschnitt). Bevor es in die Gruppenräume geht, wird durch die Moderator(inn)en noch einmal an das Thema des gesamten „Barcamps Adapted“ erinnert. Das ist deshalb wichtig, weil es in den Kleingruppen nicht allein um die Einzelfragen gehen sollte, die sich aus den Themen ergeben, die in den Gruppen diskutiert werden, sondern immer darum, diese so zu diskutieren, dass sich daraus ein Beitrag für das übergeordnete Thema ergibt. Dann begeben sich die Gruppen in die vorbereiteten Räumlichkeiten. Dort stehen Materialien wie Stifte und Karten sowie Pinnwände, Scheren, Klebestifte etc. bereit. Wer die Leitung einer Kleingruppe übernommen hat, formuliert nochmals deren Thema und eröffnet damit das Gespräch. Die Länge der Gruppendiskussion beträgt zweimal 45 bis 60 Minuten, mit einer Pause dazwischen, wenn das „Barcamp adapted“ einen Tag dauert (bei einer längeren Dauer können entweder die Gruppenarbeitszeiten ausgedehnt oder es kann eine zweite Runde mit Themenfinden, Themenwahl und Gruppendiskussion vorgesehen werden). Danach - zum Beispiel mittags - ist eine längere Pause von mindestens einer Stunde empfehlenswert. Dies eröffnet einen Raum für informelle Gespräche auch zwischen den Gruppen. 
Die Ergebnisse der Gruppen - Hypothesen und Vorschläge zu deren Bearbeitung im geplanten Forschungsprojekt - müssen am Ende der Gruppenarbeit für das abschließende Plenum des Workshops zusammengetragen und dokumentiert werden (auf Karteikarten, bunten Zetteln oder Aufklebern, in Form von Grafiken oder Fotos etc.), um dort zur Diskussion gestellt werden zu können. Es ist die Aufgabe der Moderator(inn)en, zwischendurch auf die Ergebnissicherung aufmerksam zu machen. Die Form der Ergebnissicherung ist den einzelnen Gruppen überlassen und kann somit unterschiedlich sein. Der Kreativität sollten hier keine Grenzen gesetzt werden. Verantwortlich für das Festhalten der Diskussion und der Ergebnisse sind die Leiter(innen) der Gruppen, die Form der Ergebnisse und die Wege dahin werden jedoch gemeinsam in der Gruppe erarbeitet.

Der Workshop schließt nach Beendigung der Gruppenarbeitsphase mit einer Plenumssitzung ab, in der die Ergebnisse der Gruppenarbeit vorgestellt werden. Diese Ergebnisse werden nun gemeinsam diskutiert und ergänzt. Die Form, in der die Ergebnisse der Plenumsdiskussion festgehalten werden, hängt von der Ergebnispräsentation der jeweiligen Gruppe ab. Möglich ist beispielsweise eine Ergänzung durch weitere Karten, die ebenfalls angepinnt und fotografisch dokumentiert werden. Wurde ein Flipchart erstellt, können Kommentare direkt auf diesem vermerkt werden. Solche Ergänzungen werden entweder durch die Präsentierenden oder durch die Moderator(inn)en festgehalten.

\section{Praxisbeispiel Urban Office Nr. 6: Ablaufplan und Ergebnissicherung}

Das „Barcamp adapted“ im Heidelberger Reallabor Urban Office war für einen Tag konzipiert, d. h. die Gruppenarbeit wurde bis zum Mittag abgeschlossen. Der Nachmittag diente dann der Diskussion der Ergebnisse im größeren Plenum.

Ablaufplan:

09:30 Eintreffen

09:45 Begrüßung und Vorstellung

10:00 Themenfindung und Themenwahl

11:00 Bearbeitung der Themen in Arbeitsgruppen

13:00 Gemeinsames Mittagessen

14:00 Vorstellung der Ergebnisse der Gruppen und Diskussion im Plenum

16:00 Pause / Vorbereitung Abendveranstaltung durch die Moderation

17:30 Interne Besprechung des Ablaufs der Abendveranstaltung mit den

Teilnehmer(inne)n des ersten Teils

18:00 Beginn der Abendveranstaltung

18:15 Vorstellung der Idee eines Urban Office als Schnittstelle zwischen Universität, Stadt, IBA und Einwohnerschaft

19:00 Vorstellung der Ergebnisse aus dem Workshop und Diskussion

20:40 Abschlussdiskussion

21:00 Ende 


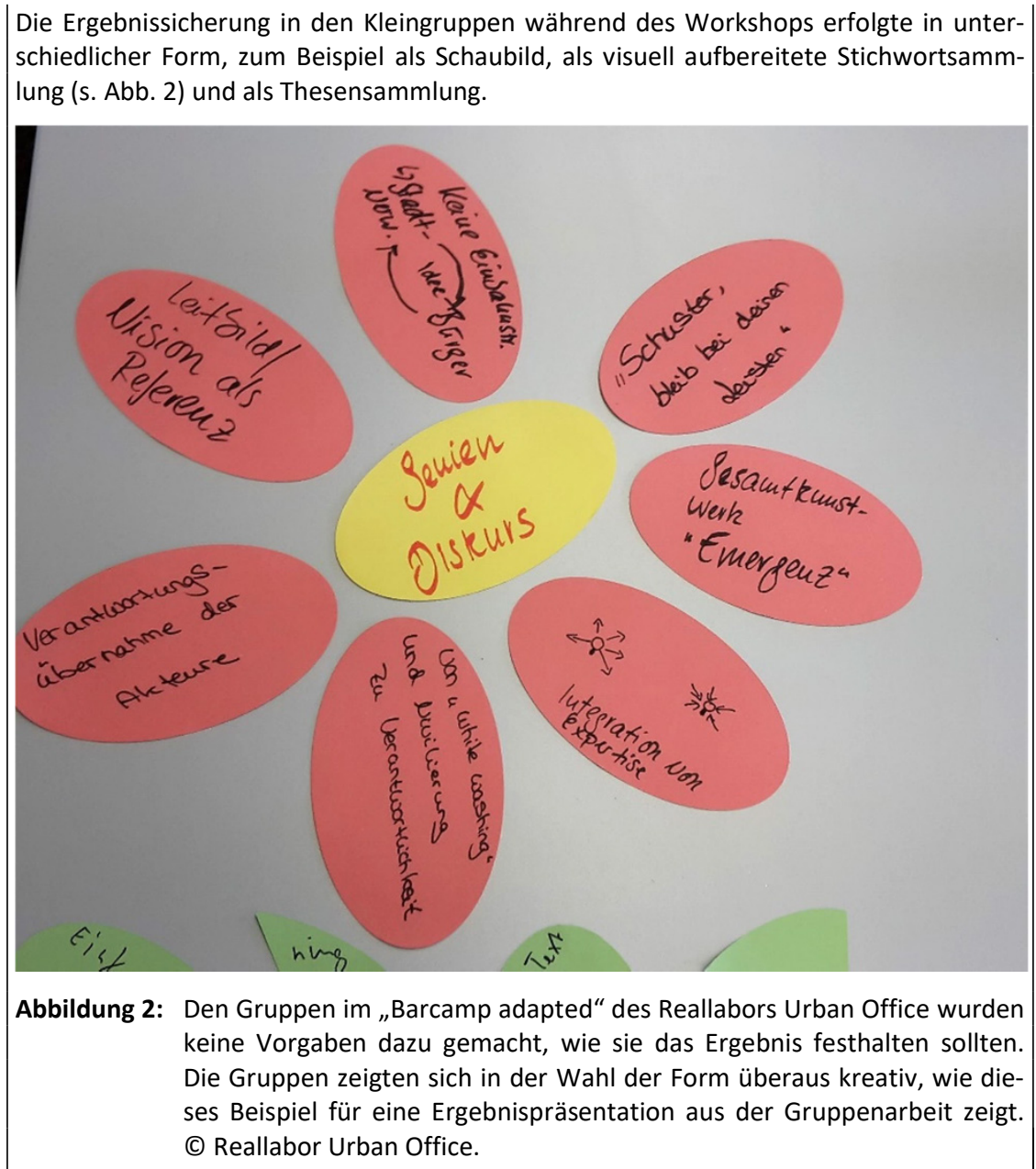

Für die anschließende öffentliche Veranstaltung müssen die Ergebnisse des Workshops weiter aufbereitet werden, um sie für eine Präsentation nutzen zu können. Dieser Schritt, der von den Moderator(inn)en in der Zeit zwischen dem Ende des Workshops und dem Beginn der öffentlichen Veranstaltung geleistet wird, dient zugleich einer Ergebnissicherung des Workshopteils von „Barcamp adapted“. Die Moderator(inn)en sammeln die Ergebnisse aus den Gruppen und bereiten diese so auf, dass sie den Teilnehmer(inne)n des Workshops zur Verfügung gestellt werden können - zum Beispiel als Kurzdokumentation in digitaler und auszudruckender Form. Die Kurzdokumentation kann zugleich für die öffentliche 
Veranstaltung genutzt werden. Weiterhin wird aus jeder Gruppe ein(e) Redner(in) ausgewählt, der/die dann während der öffentlichen Veranstaltung auf der Bühne Platz nimmt.

\section{3 Öffentliche Veranstaltung}

Die öffentliche Veranstaltung nach dem Workshop dient der Präsentation und Diskussion der Ergebnisse des Workshops in bzw. mit einer breiteren Öffentlichkeit. Zwischen Workshop und öffentlicher Veranstaltung sollte eine mindestens einstündige ,Verschnaufpause ' vorgesehen werden. Für diese Veranstaltung muss öffentlich eingeladen werden - wie zu anderen öffentlichen Angeboten auch. Dies erfolgt zum Beispiel über die Lokalzeitung, über soziale Medien, Flyer oder Verteilerlisten. Es sind unterschiedliche Formen der Veranstaltung denkbar. Geeignet sind zum Beispiel eine Ergebnispräsentation in Form eines Vortrags mit anschließender Diskussion, eine Podiumsdiskussion mit starkem Fokus auf einer Publikumsdiskussion bis hin zu interaktiveren Methoden wie Fish Bowl, eine demokratische Diskussionsform, bei der es kein Podium mehr gibt, sondern alle Teilnehmer(innen) im Kreis um die Erstredner(innen) gruppiert sind mit der Möglichkeit, sich selbst in den inneren Kreis zu begeben (zur Methode s. Flor et al. 2013).

Durch diese Kombination aus Workshop und öffentlicher Veranstaltung im „Barcamp adapted" erfolgt ein direkter und schneller Transfer der Ergebnisse, außerdem wird so ermöglicht, schnell ein Feedback von Personen zu erhalten, die nicht selbst an den Diskussionen im Workshop beteiligt waren. Auf diese Weise kann hier der geschlossene Teil des „Barcamp adapted“ für die Öffentlichkeit geöffnet werden. Ein weiterer Vorteil besteht darin, dass die Teilnehmer(innen) des Workshops selbst an dieser sich anschließenden Veranstaltung anwesend sind, was sich weniger leicht realisieren ließe, wäre diese zeitlich versetzt an einem zweiten Termin. Dies wiederum ist eine wichtige Voraussetzung, um tatsächlich einen Austausch mit Vertreter(inne)n beispielsweise aus der Stadtgesellschaft - Bürger(innen), Vertreter(innen) verschiedener Einrichtungen, der kommunalen Verwaltung etc. - in Gang zu setzen.

Ziel des öffentlichen Teils ist es, möglichst viele der Anwesenden in die Diskussion einzubeziehen. Die Diskussion kann zum Beispiel folgendermaßen gestaltet werden: Die Beteiligten des Workshops begeben sich in den Publikumsraum und gesellen sich zu den weiteren Teilnehmer(inne)n dazu. Dabei platzieren sie sich möglichst an unterschiedlichen Orten im Raum. Jeweils ein(e) Vertreter(in) jeder Gruppe nimmt auf der Bühne Platz. Zunächst wird durch die Moderator(inn)en kurz und prägnant präsentiert, was in den verschiedenen Gruppen während des Workshops erarbeitet wurde. In einem zweiten Schritt äußern sich die Sprecher(innen) der Gruppen kurz dazu, ergänzen oder werfen Fragen auf, um so den 
Dialog mit dem Publikum zu eröffnen. Dadurch, dass Beteiligte des Workshops mit im Publikum sitzen und somit Teil des Publikums sind, dabei die Aussagen des Podiums aufgreifen und aus dem Publikum heraus antworten können, entsteht schnell eine offene Atmosphäre, in der substanzielle Diskussionen möglich werden.

Auch das Feedback und die Diskussion dieser öffentlichen Veranstaltung müssen dokumentiert werden. Wichtige Fragen und Themen sollten festgehalten und verschriftlicht werden. Dazu muss im Vorhinein festgelegt werden, wer protokolliert. Von Vorteil ist es, hierzu jemanden aus dem Teilnehmerkreis des Workshops oder eine(n) der Moderator(inn)en zu wählen, da diese die Diskussion aus dem vorangegangenen Teil kennen, was das Verfolgen der Diskussion erleichtert. Von Nachteil ist jedoch, dass diese Person sich dann nur in geringem Maße selbst am Gespräch beteiligen kann. Durch die Diskussionsdokumentation wird gesichert, dass das Feedback aufgenommen werden kann und wichtige Ideen, Anregungen und Kommentare nicht verloren gehen.

\subsection{Dokumentation und Kommunikation der Gesamtergebnisse von „Barcamp adapted“"}

Die Resultate aus beiden Teilen des „Barcamps adapted“ müssen im Nachgang durch die Moderator(inn)en zusammengeführt werden. Alle gesammelten Prozesseindrücke und Ergebnisse (Dokumentation der Gruppenarbeit, Verschriftlichungen von Argumenten, visuelle Eindrücke, Tondokumente etc.) werden von den Moderator(inn)en möglichst zeitnah aufbereitet (unbedingt einen Termin setzen!) und der Kerngruppe des (geplanten) Projekts sowie den Workshopteilnehmer(inne)n zur Verfügung gestellt. Außerdem sollte sichergestellt werden, dass die wichtigsten Ergebnisse auch über diesen Kreis hinaus öffentlich verbreitet werden. Das ist dann Aufgabe der Kerngruppe des (geplanten) Projekts. Dazu können die Resultate beispielsweise in einer Ergebnisdokumentation zusammengefasst werden, die auf der Homepage der Projektgruppe aufgerufen werden kann. Die Veranstaltung in Heidelberg wurde u. a. in einem Kurzfilm dokumentiert, der über YouTube den Teilnehmer(inne)n und der interessierten Öffentlichkeit zugänglich gemacht wurde ${ }^{1}$.

All das ermöglicht zum einen eine Dissemination in die Öffentlichkeit, zum anderen wird nur durch eine umfassende und klare Dokumentation ermöglicht, dass die Ergebnisse des „Barcamps adapted“ den weiteren Verlauf des Forschungsprozesses unterstützen. Die Dokumentation stellt somit einen wichtigen Schritt für die Verbreitung und weitere Nutzung der Ergebnisse dar.

1 s. YouTube-Channel Urban Office, http://youtu.be/rifZpdEn3mY, zugegriffen am 01.04.2019. 


\section{Fazit}

Die beschriebene Kombination aus Workshop und einer anschließenden öffentlichen Veranstaltung in einem „Barcamp adapted“ hat sich als geeignete Methode erwiesen, um die Zusammenarbeit für ein geplantes transdisziplinäres Forschungsvorhaben zu initiieren bzw. zu stärken, um Impulse für dieses Vorhaben zu erhalten und um weitere Kreise für das Vorhaben zu interessieren. Das Vorgehen verhilft dazu, Wissen unterschiedlicher Akteure von Anfang an in den Forschungsprozess zu integrieren und diesen selbst partizipativ zu gestalten - über den Kreis der direkt an der Entwicklung des Forschungsprojekts Beteiligten hinaus. Die Kombination aus geladenen und spontanen Gästen des Workshops sowie die weiterführende Diskussion in einer öffentlichen Veranstaltung mit einem nochmals erweiterten Kreis eröffnen unterschiedliche Kommunikationsebenen, die unterschiedliche Perspektiven in die Vorbereitung eines solchen Vorhabens einbringen kann. Damit erscheint die Methode insbesondere für die Startphase sehr gut geeignet. Die Synthese der eingebrachten Wissensbestände unterschiedlicher Akteure führt zu einem Mehrwert, der durch disziplinäres und auch interdisziplinäres Arbeiten nicht zu erreichen ist (s. Marquardt und West 2016).

Die intensive Arbeitsphase im kleineren Kreis des Workshops ermöglicht ein kollaboratives Arbeiten an Themen, die für das geplante Forschungsvorhaben essenziell sind. Durch die gemeinsame Arbeit wird ermöglicht, sich bereits vor der eigentlichen Zusammenarbeit im Projekt über Begriffe zu verständigen und unterschiedliche Arbeits- und Denkweisen kennenzulernen - eine wichtige Voraussetzung für eine zukünftige Zusammenarbeit. Als besonders positiv kann dabei bewertet werden, dass dieser Prozess als Teil einer inhaltlichen Diskussion stattfindet und somit bereits zum geplanten Forschungsvorhaben gehört. Der Übergang in die öffentliche Veranstaltung ist dann ein Übergang aus einer ,Semiöffentlichkeit' in eine breitere Öffentlichkeit.

Der Schritt in die Öffentlichkeit ist entscheidend zur Abrundung des Tages sowie für den Wissenstransfer und die ,Validierung' des Erreichten. Die öffentliche Veranstaltung ist ein Ereignis, über das auch in der Presse berichtet und weiter diskutiert werden kann. Die Workshopergebnisse werden hier zur Diskussion gestellt und kritisch mit anderen Akteuren, insbesondere mit Bürger(inne)n und anderen Beteiligten aus der Zivilgesellschaft, diskutiert. In der breiteren Öffentlichkeit sind sowohl die Wahrnehmung als auch das Verständnis von bestimmten Themen ganz anders ausgeprägt als in dem - wenn auch deutlich geöffneten Expertenkreis des Workshops. Es können Spannungen zutage treten, die durch unterschiedliche Interessen, die die verschiedenen Akteure mitbringen, entstehen. Daher ist eine solche Auseinandersetzung mit der Öffentlichkeit an dieser Stelle 
von zentraler Bedeutung, da so ein weiterer, neuer ,Wissensraum ${ }^{2}$ entsteht, in dem ganz unterschiedliche Wissensbestände zusammengeführt werden können. In einer Wissensgesellschaft ist ein solches vernetztes Wissen eine entscheidende Ressource. Die partizipative Vorgehensweise wirkt sowohl auf die außeruniversitären als auch auf die universitären Teilnehmer(innen), weil alle eingeladen werden, eigene Denkmuster zu hinterfragen (zur Wirkung partizipativer Angebote in die Gesellschaft s. Goldschmidt et al. 2012). Schließlich ermöglicht es diese Öffnung, Spannungen rund um das Thema des geplanten Projekts frühzeitig zutage zu bringen, die durch unterschiedliche Interessen, aber auch durch Erfahrungen mit früheren und nicht geglückten Partizipationsprozessen entstanden sein können. Solche Ereignisse sind nicht selten mit Frustrationen („Hier geschieht eh nichts!") oder anderen negativen Erfahrungen belegt, und diese können sich zu einem späteren Zeitpunkt negativ auf das geplante Projekt auswirken.

Die vorgestellte Methode ist leicht einsetzbar, es bedarf jedoch einer gut organisierten Vorbereitung und eines hohen Commitments der Teilnehmer(innen), um zu relevanten Ergebnissen zu führen. Interaktion steht im Mittelpunkt dieses Ansatzes: es werden unterschiedliche Beteiligte zusammen- und in einen Austauschprozess gebracht. Die Grenze zwischen ,Agierenden' und Publikum wird weitgehend aufgehoben. Auf diese Weise kann ein lebendiger Wissensaustausch entstehen, der von den Beiträgen aller Teilnehmer(innen) profitiert und von dem umgekehrt auch alle einen Nutzen haben. Die umgehende Aufbereitung der Ergebnisse stellt sicher, dass diese nicht verloren gehen oder erst mit einer großen Zeitverzögerung zur Verfügung stehen, und umgeht so eine der Gefahren interaktiver Formate.

Risiken für eine erfolgreiche Durchführung liegen auf mehreren Ebenen. So ist die Einladung für den Workshop genau zu durchdenken, um einen möglichst hohen Nutzen für den Forschungsprozess zu erhalten. Weiterhin kommt den Moderator(inn)en eine hohe Wichtigkeit zu, denn sie haben unterschiedliche und umfangreiche Aufgaben wahrzunehmen. Deshalb sind Moderator(inn)en wichtig, die sich in der Thematik selbst auskennen und die willens sind, sich auch inhaltlich auf das Projektthema einzulassen. Die öffentliche Veranstaltung wiederum lebt von einer breiten Beteiligung. Die üblichen Probleme solcher partizipativ angelegten Veranstaltungen (wer kommt, wer redet mit, werden möglichst heterogene Akteure erreicht etc.) spielen auch hier eine Rolle (s. Meier 2018; Selle 2013), weshalb großes Augenmerk auf die Einladungen gelegt werden muss, um eine breite Beteiligung zu ermöglichen.

2 Mit Wissensräumen sind an dieser Stelle gesellschaftliche Räume gemeint, in denen sich eine offene Atmosphäre der Wissensproduktion, der Wissensdistribution und der Kommunikation bilden kann und die so einen Austausch von unterschiedlichen Wissensbeständen und Interaktionen zwischen verschiedenen Akteuren ermöglichen (s. z. B. Eigenbrodt und Stang 2014). 
Der richtige Einsatz der vorgestellten Methode kann zu einer Co-Produktion von Wissen beitragen, die einen neuen Weg zur Wissensgenerierung in einer Wissensgesellschaft darstellt. Zukünftig und insbesondere im Kontext komplexer Themenstellungen und transformativer Fragestellungen, deren Bearbeitung nur in transdisziplinären Konstellationen möglich ist, wird dies einen immer wichtigeren Stellenwert erhalten, um den Forscher(innen) kaum mehr herumkommen werden. Dazu dürfte die Nutzung solch interaktiver Methoden wie der hier beschriebenen für das wissenschaftliche Arbeiten zunehmend wichtiger werden.

\section{Dank}

Die Autorinnen danken Sophia Alcántara, Thomas Becker, Barbara Koch, Hanna Noller, Rasmus Prieß und Christina West für die Diskussionen über frühere Textfassungen. Insbesondere möchten sie Raphael Dietz und Colette Waitz sowie zwei anonymen Gutachter(inne)n für die konkreten Hinweise zur Verbesserung des Textes im Rahmen des internen und externen Reviews danken. Schließlich danken die Autorinnen den beiden Herausgebenden, Rico Defila und Antonietta Di Giulio, für ihre Rückmeldungen zum Text.

\section{Literatur}

Arnold, A., \& Piontek, F. M. (2018). Zentrale Begriffe im Kontext der Reallaborforschung. In R. Defila \& A. Di Giulio (Hrsg.), Transdisziplinär und transformativ forschen. Eine Methodensammlung (S. 143-154). Wiesbaden: Springer VS. doi: 10.1007/ 978-3-658-21530-9_8.

Beecroft, R., Trenks, H., Rhodius, R., Benighaus, C., \& Parodi, O. (2018). Reallabore als Rahmen transformativer und transdisziplinärer Forschung: Ziele und Designprinzipien. In R. Defila \& A. Di Giulio (Hrsg.), Transdisziplinär und transformativ forschen. Eine Methodensammlung (S. 75-100). Wiesbaden: Springer VS. doi: 10.1007/978-3-65821530-9_4.

Deterding, S., Khaled, R., Nacke, L. E., \& Dixon, D. (2011). Gamification: Toward a Definition. Tampere: ACM Press. http://hci.usask.ca/uploads/219-02-Deterding,-Khaled,Nacke,-Dixon.pdf. Zugegriffen am 28.10.2018.

Dittrich-Brauner, K., Dittmann, E., List, V., \& Windisch, C. (2013). Interaktive Großgruppen. Change-Prozesse in Organisationen gestalten. Berlin: Springer Link.

Doppler, S. (2016). B2B-Eventmarketing. Konstanz: UTB.

Eigenbrodt, O., \& Stang, R. (Hrsg.). (2014). Formierungen von Wissensräumen: Optionen des Zugangs zu Information und Bildung. Berlin: De Gruyter. 
Flor, P., De Meulemeester, A., Allen, T., \& Isaksson, K. (2013). Use of the fishbowl method for a discussion with a large group. Journal of the European Association for Health Information and Libraries, 9, (S. 24-25).

Gerhard, U., \& Marquardt, E. (2017). Reallabore als innovatives Forschungsformat zur Untersuchung nachhaltiger Stadtentwicklung. Eine kritische Reflexion. Berichte. Geographie und Landeskunde, 91 (1), (S. 97-111).

Gerhard, U., \& Marquardt, E. (2016). The Greener, the Happier? Urban Sustainability in the Knowledge City: Policies, Programs and Practices in the German Context. In D. Wilson (Hrsg.), The Politics of Urban and Regional Sustainability. Appraising the Concept and Process (S. 65-86). Champaign: Common Grounds Publishing.

Goldschmidt, R., Scheel, O., \& Renn, O. (2012). Zur Wirkung und Effektivität von Dialogund Beteiligungsformaten. Stuttgarter Beiträge zur Risiko- und Nachhaltigkeitsforschung, 23. doi: 10.18419/opus-5552. Zugegriffen am 01.03.2019.

Hellmann, K. J. (2007). Die Barcamp Bewegung. Bericht über eine Serie von ,Unconferences‘. Forschungsjournal Soziale Bewegungen, 20 (4), (S. 107-110).

Kolenaty, E. (o. J.). Barcamp oder Open Space. http://www.transformation.at/article 212.htm. Zugegriffen am 01.03.2019.

Marquardt, E., \& West, C. (2016). Co-Produktion von Wissen in der Stadt. Reallabor „Urban Office - Nachhaltige Stadtentwicklung in der Wissensgesellschaft" an der Universität Heidelberg. Technikfolgenabschätzung - Theorie und Praxis, 25 (3), (S. 26-31).

Meier, G. (2018). Stadt und Partizipation. Eine Analyse zur Bedeutung und Wirksamkeit von Bürgerbeteiligung in der Stadtentwicklung. Heidelberger Geographische Arbeiten, 136. Heidelberg: Selbstverl. des Geographischen Instituts der Universität Heidelberg.

Selle, K. (2013). Über Bürgerbeteiligung hinaus. Stadtentwicklung als Gemeinschaftsaufgabe? Analysen und Konzepte. Detmold: Verlag Dorothea Rohn.

Open Access Dieses Kapitel wird unter der Creative Commons Namensnennung 4.0 International Lizenz (http://creativecommons.org/licenses/by/4.0/deed.de) veröffentlicht, welche die Nutzung, Vervielfältigung, Bearbeitung, Verbreitung und Wiedergabe in jeglichem Medium und Format erlaubt, sofern Sie den/die ursprünglichen Autor(en) und die Quelle ordnungsgemäß nennen, einen Link zur Creative Commons Lizenz beifügen und angeben, ob Änderungen vorgenommen wurden.

Die in diesem Kapitel enthaltenen Bilder und sonstiges Drittmaterial unterliegen ebenfalls der genannten Creative Commons Lizenz, sofern sich aus der Abbildungslegende nichts anderes ergibt. Sofern das betreffende Material nicht unter der genannten Creative Commons Lizenz steht und die betreffende Handlung nicht nach gesetzlichen Vorschriften erlaubt ist, ist für die oben aufgeführten Weiterverwendungen des Materials die Einwilligung des jeweiligen Rechteinhabers einzuholen.

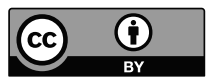




\title{
„UrbanUtopiaLAB“" - einen Möglichkeitsraum zur Produktion von Transformationswissen schaffen
}

\author{
Christina West \& Svenja Kück
}

\section{Einleitung: From Knowledge to Action}

Die Zukunftsfähigkeit von Städten, verstädterten Regionen und Gesellschaften hängt heute maßgeblich davon ab, wie es gelingt, urbane Transformationsprozesse zukunftsfähiger zu gestalten und somit auch über den künftigen Gesellschaftsvertrag nachzudenken. Die Methode und das Format UrbanUtopiaLAB setzt an dem Punkt an, dass es dazu zwar oftmals umfassende Hinweise darauf gibt, welche Ziele angestrebt werden sollen und wo die Herausforderungen liegen (z. B. United Nations 2015), das Wie jedoch, also die Frage, mit welchen Prozessen diese zu erreichen sind, kaum thematisiert wird.

Die Ursprungsidee und Methodik des UrbanUtopiaLABs basieren auf dem Konzept der Transversalen Stadt und Transtopia (West 2014a, 2014b, 2017, 2019), mit dem nicht nur der zunehmenden Komplexität von Gesellschaft Rechnung getragen wird, sondern auch Möglichkeiten angesprochen werden, neue Perspektiven in Forschung, in politisch-administrativen Zusammenhängen, Architektur und Stadtplanung, in unterschiedlichen Bildungszusammenhängen und bei der Wissensherstellung zu entwickeln. Das UrbanUtopiaLAB ist ein Vorgehen, das einen ,Mög-

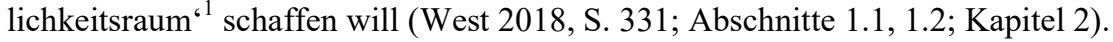

1 Möglichkeitsräume sind eng mit dem Experimentieren und mit dem von Robert Musil (2002, S. 16) beschriebenen „Möglichkeitssinn“ verbunden, der für ihn die Fähigkeit ist, ,[...] alles, was ebenso gut sein könnte, zu denken und das, was ist, nicht wichtiger zu nehmen als das, was nicht ist.“ Denn ,[...] es könnte wahrscheinlich auch anders sein.“ Diejenigen, die Möglichkeitsräume identifizieren, sind bereit, unterschiedliche Wirklichkeitswahrnehmungen zu erkennen, zu zerlegen, die Elemente dieser Wirklichkeiten zu etwas Neuartigem zu synthetisieren, um so zu erkennen, dass das Mögliche und somit noch nicht Seiende ebenso wichtig und bedeutsam - und damit vergleichbar notwendig - sein kann wie das Existierende. 
Das im vorliegenden Beitrag vorgestellte Vorgehen „UrbanUtopiaLAB | Experimenting Utopia: Past ... Present ... Future" (kurz: UrbanUtopiaLAB) ist auf die Co-Produktion von Wissen und auf die Entwicklung einer ,Kultur des Austauschs' (Abschnitt 2.2) ausgerichtet und somit anschlussfähig an die Forderung nach einer transdisziplinären, zukunftsorientierten und zukunftsfähigen Entwicklung des Urbanen. Das Verfahren des UrbanUtopiaLABs kombiniert unterschiedliche Methoden, die sowohl einzeln als auch in ihrer Gesamtheit Teil eines transdisziplinär-transformativen Forschungsprojekts (wie z. B. ein Reallabor) sein können. Es zielt auf die Entwicklung vor allem in verstädterten, urbanisierten Kontexten (Quartier, Stadt, Region), in denen häufig multiple Zuschreibungen und Raumerfahrungen auf engem Raum koexistieren und unterschiedliche Vorstellungen über die Nutzung des öffentlichen Raums bestehen. Deshalb eignet sich das Vorgehen insbesondere für Handlungsfelder und Fragestellungen, die einen direkten Bezug zu Stadt- und Siedlungsentwicklung, Stadtplanung, Städtebau, Architektur, Urban Design, Urban Governance, Stadtgestaltung oder Stadtnutzung haben. Es lässt sich jedoch auch gut im suburbanen oder ländlichen Raum sowie im regionalen Kontext durchführen.

\subsection{UrbanUtopiaLAB: Entstehungskontext und Entwicklung der Methode}

Eine kontinuierliche Ausarbeitung und Weiterentwicklung des hier vorgestellten Vorgehens für transdisziplinär-transformative Forschung fand im Rahmen des Teilprojekts „Dezentrales Wohnen“'2 des Reallabors „Asylsuchende in der RheinNeckar-Region“ (Reallabor Asylsuchende, s. auch Steckbrief im Anhang dieses

2 Im wissenschaftlichen Diskurs sowie von Integrationsfachkräften wird die sogenannte „dezentrale Unterbringung“ nahezu übereinstimmend als erstrebenswerte Option bei der Unterbringung von geflüchteten Personen gesehen (Schammann und Kühn 2016; Wendel 2014). „Dezentrale Unterbringung“ ist jedoch nicht eindeutig definiert, da ,dezentral“" sowohl in Bezug auf die räumliche Verteilung, die Lage des Standorts der Unterbringung als auch auf die Anzahl der Personen, die sich eine Wohnung oder Unterbringung teilen, verstanden werden kann. In Kommunen wird mit „dezentral“ in der Regel die Absicht bezeichnet, die gemäß des „Königsteiner Schlüssel“ zugeteilten geflüchteten Personen im Stadtgebiet zu verteilen und zudem die Anzahl der Geflüchteten in einer Wohneinheit deutlich niedriger als in einer Gemeinschaftsunterkunft (GU) zu halten, bei einer gleichzeitig höheren Quadratmeterzahl Wohnfläche pro Person. Aufgrund fehlender rechtlicher Standards ist die ,dezentrale Unterbringung“ in den Kommunen aber ganz unterschiedlich und je nach individuellen Ressourcen der Kommune ausgestaltet. Für die Stadtverwaltung Heidelberg entspricht das sog. „Heidelberger Modell“ einer „dezentralen Unterbringung“ von Flüchtlingen und Asylsuchenden. Diese soll den dort lebenden Menschen eine Anbindung an das Stadtteilleben ermöglichen, was als Grundlage für eine gelingende Integration gesehen wird. 
Buches) statt. Zusammen mit dem Reallabor-Praxispartner „Each1Teach1 e. V.“ entwickelten die Wissenschaftler(innen) des Teilprojekts anhand der Leitfrage „Wie wollen wir in Zukunft miteinander leben?" einen auf Kooperation, Kollaboration und Co-Kreation angelegten Möglichkeitsraum (West 2018, S. 331), eben das UrbanUtopiaLAB, mit dem bisher schwerpunktmäßig in Heidelberg gearbeitet wurde. Die Laufzeit des UrbanUtopiaLABs wurde auf 23 Monate angelegt (Mai 2017 bis März 2019). Die Beteiligten der beiden Partnerinstitutionen waren gleichermaßen verantwortlich für Konzeption und Realisierung. Synergien ergaben sich durch jeweils spezifische Erfahrungen und Kenntnisse sowie gemeinsame Interessen und vorhandene Netzwerke in der Stadt.

Die Wissenschaftlerinnen des Teilprojekts „Dezentrales Wohnen“ analysierten unter anderem die Bedeutung kommunaler Strukturen bei der Umsetzung dezentraler Wohnformen sowie die Verankerung der Themen Flucht, Asyl und soziale Teilhabe in der lokalen politischen Agenda. Schwerpunkte lagen auf der Analyse der Partizipation von Nicht-Geflüchteten bei der Umsetzung dezentraler Wohnformen, den Aushandlungsprozessen zwischen Kommunen und Bürger(inne)n in den sich entwickelnden politischen Partizipations- und Governanceprozessen, den selbst- und co-organisierten Formaten, Kommunikationsstrukturen und Prozessen gesellschaftlicher Teilhabe von Geflüchteten in Heidelberg sowie der Ermittlung von Wohlfühlorten, Nicht-Wohlfühlorten und Aktionsräumen von Geflüchteten und Nicht-Geflüchteten in der Stadt. Mit den Erkenntnissen und Methoden wurde der Ansatz „Transversale Gesellschaft - Transversale Stadt“ (West 2014a, 2014b, 2017, 2019) weiterentwickelt, empirisch überprüft und auf Anwendungstauglichkeit im Reallabor getestet.

Im Fokus des Teilprojekts „Dezentrales Wohnen“ wie des UrbanUtopiaLABs in Heidelberg stand dabei die grundsätzliche Frage: Wie wird das Urbane produziert? Durch das transdisziplinäre Vorgehen beim Co-Design und bei der Co-Produktion von Wissen über die Prozesse während der Produktion des Urbanen wurden die Weiterentwicklung und Ausdifferenzierung des zugrunde gelegten Nachhaltigkeitsverständnisses u. a. in den Dimensionen Soziales, Kultur, Organisation und Politische Prozesse möglich, wodurch System-, Ziel- und Transformationswissen erzeugt wurde (West 2019). Das im Reallabor Asylsuchende entwickelte UrbanUtopiaLAB leistete als Experimentierfeld Pionierarbeit im Rahmen einer transdisziplinär-transformativ erarbeiteten Perspektive in der Forschung zu Flucht und Migration. Es basiert auf den Annahmen, dass etablierte politisch-administrative und statistische Differenzierungskategorien die Komplexität unserer Gesellschaft nicht mehr abbilden und dass die Rolle der Forscher(innen) unter forschungsethischen Gesichtspunkten neu diskutiert werden muss. 


\subsection{UrbanUtopiaLAB: Charakteristik und Anwendungskontexte}

Das UrbanUtopiaLAB kann auch in anderen thematischen Kontexten als denjenigen, um die es im Reallabor Asylsuchende ging, zum Einsatz kommen: Nämlich immer dann, wenn das Urbane mit Hilfe einer - raumbezogenen - Utopie von der Zukunft her gedacht werden soll. Die konzeptionelle Basis dafür ist eine ,Kultur des Austauschs' (s. Abschnitt 2.2). Diese gelingt durch den Einbezug relevanter und heterogener Praxisakteure sowohl im Planungsprozess als auch in der konkreten Durchführung sowie durch den Einsatz von geschulten Personen bei der Prozessleitung und in der Moderation. Angestrebt wird die gemeinsame Wissensproduktion auf Augenhöhe, um schließlich konkrete Veränderungspotenziale aufzuzeigen und politische Prozesse anzustoßen. Die dabei entstehenden konkreten Impulse finden zum einen (in der Tradition des „Policy Briefs“) Eingang in allgemein formulierte Handlungsempfehlungen zu den adressierten Fragestellungen, zum anderen wird in diesem Prozess auch die individuelle Neuorientierung der Denkroutinen der Akteure angestrebt.

Im Möglichkeitsraum, der durch UrbanUtopiaLAB geschaffen wird, besteht im Rahmen von Interventionen und Diskussionen die Gelegenheit, auszuprobieren, neu oder anders Gedachtes weiterzuentwickeln und Anwendungsmöglichkeiten im eigenen Praxisfeld auszuloten. Damit eröffnet das UrbanUtopiaLAB auch eine innovative Perspektive auf Politikberatung: als eine Form inter- und transdisziplinärer Kollaboration und Co-Kreation liefert das UrbanUtopiaLAB eine Antwort auf aktuelle steuerungspolitische Herausforderungen und den Formwandel des Regierens in Richtung transdisziplinärer Governance.

Das UrbanUtopiaLAB wurde erfolgreich in dem potenziell konfliktreichen Themenbereich Flucht und Migration eingesetzt. Ein Einsatz im Kontext konfliktträchtiger Themen ist also möglich, verlangt allerdings eine grundlegende Bereitschaft zu achtsamer, konstruktiver und kreativer Zusammenarbeit zwischen den Teilnehmer(inne)n. Einzelne Personen, die sich nicht an die ,Spielregeln' (s. Abschnitt 4.2.1) halten (wollen), können durch die Moderator(inn)en mit Hinweis auf die verbindlich aufgestellten Spielregeln sowie über entsprechende Mittel der Moderation (z. B. Hinweis auf und Einfordern des Einhaltens von Redezeiten, die aktive Strukturierung des Gesprächs und Fokussierung auf die Themensetzung des UrbanUtopiaLABs) meist eingebunden werden. Sofern das nicht gelingt, muss die Bereitschaft da sein, Personen notfalls auszuschließen. Dagegen ist eine Anwendung des Vorgehens nicht anzuraten, wenn die Mehrzahl der Teilnehmer(innen) in einem Konflikt oder in einem ablehnenden Verhältnis zueinanderstehen, die ein konstruktives Miteinander unmöglich oder aussichtslos erscheinen lassen. Wie viel Konfliktpotenzial im UrbanUtopiaLAB konstruktiv verarbeitet werden kann, hängt sowohl von den einzelnen Teilnehmer(inne)n als auch von Persönlichkeit, Fähigkeit und Stil der Moderator(inn)en ab. 


\subsection{Gliederung des Beitrags}

Den Schwerpunkt dieses Beitrages bilden die ersten beiden Phasen des Urban UtopiaLABs (s. Abschnitte 3.1, 3.2). Diese sind durch eine weitgehend themenunabhängige, aber methodisch nachvollziehbare Vorgehensweise charakterisiert und wurden bereits mehrmals in verschiedenen Kommunen in unterschiedlichen thematischen Kontexten eingesetzt (in Heidelberg und Sinsheim für die soziale Teilhabe von Geflüchteten, Wohnen und Stadtentwicklung; in Mannheim zur Bürgerbeteiligung bei der Entwicklung des Nutzungskonzepts für die unter Denkmalschutz stehende „Multihalle“, die größte freitragende Holzgitterschalenkonstruktion der Welt). In Kapitel 2 werden zunächst das grundlegende Design, die Ziele sowie allgemeine Hinweise für die Umsetzung des UrbanUtopiaLABs aufgezeigt. Kapitel 3 fokussiert auf die Beschreibung der insgesamt vier Phasen des UrbanUtopiaLABs:

- Phase 1: StadtERFORSCHEN mit Emo/Action-Mapping

- Phase 2: StadtERKUNDEN

- Phase 3: StadtEXPERIMENTIEREN

- Phase 4: StadtMITENTSCHEIDEN

Da Phase 3 und Phase 4 inhaltlich, methodisch und in ihrem Verlauf maßgeblich von den Themensetzungen, Akteuren und Ergebnissen in Phase 1 und Phase 2 bestimmt werden, sind sie hier lediglich in allgemeinerer Weise skizziert. In Kapitel 4 wird die Durchführung der ersten zwei Phasen eines UrbanUtopiaLABs als idealtypische Tagesveranstaltung konkretisiert. In Kapitel 5 schließlich findet sich eine Reflexion zu den Grenzen, Bedingungen und Besonderheiten eines UrbanUtopiaLABs.

\section{UrbanUtopiaLAB: Grundlagen, Ziele und Prozessdesign}

Die Bezeichnung UrbanUtopiaLAB steht für einen kollaborativ/co-kreativen und ergebnisoffenen Prozess. Dieser geht von individuellen Utopien und konkreten Wünschen zum zukünftigen Leben in der Stadt aus, von einer vielschichtigen Urbanität, der emotionalen Wahrnehmung und Kodierung von Orten und Wegen in der Stadt sowie von Erfahrungen der Ausgrenzung, aber auch der Teilhabe. Auf dieser Grundlage sollen in mehreren Phasen schließlich praktisch umsetzbare Vorhaben oder politische Forderungen und Handlungsempfehlungen für Politik, Verwaltung, Planung und Wissenschaft entstehen, die u. a. in Form von Policy Briefs festgehalten und kommuniziert werden können. 
Das ,Utopische“ und das Transformative beziehen sich auf den Raum ebenso wie auf das Denken und die Handlungsroutinen. Das ,Urbane' steht dafür, dass die Stadt (und analoge Räume) nicht nur funktional, also als gebaute, physisch-materielle Struktur verstanden werden kann. Mit dem ,Urbanen' wird die andere Seite (in) der Stadt in den Vordergrund der Betrachtung gerückt: die Begegnungen und das Zufällige, also das nicht immer intendierte oder nicht ausschließlich zielgerichtete Aufeinandertreffen von Menschen. Dort, wo durch Begegnungen Aushandlungsprozesse entstehen, wird gemeinsam öffentlicher Raum erzeugt, an dem jede(r) teilhaben und den jede(r) gestalten kann.

Mit dem Begriff „Utopia“ wird dazu aufgefordert, sich zunächst auf die eigenen Wunschvorstellungen, Visionen, den ,besseren Ort', der paradiesähnlich und unrealisiert in den Köpfen der Menschen existiert (Foucault 1966/2005), einzulassen. Dabei geht es aber nicht um die großen gesamtgesellschaftlichen Utopien, sondern um ,intentionale Utopien“ (Landauer 1907; Bloch 1990). Hier weicht der geschlossen-statische und anti-individualistische Charakter der klassischen Utopie einem prozessualen Verständnis, das die Veränderlichkeit der formulierten Utopie selbst miteinbezieht, wodurch die jeweils eigene Vision bedeutender wird. In diesem Prozess wird zum schon vorhandenen Systemwissen (s. Abschnitt 3.3) in hohem Maß Zielwissen für die Entwicklung von Visionen generiert, die wiederum, wenn sie umgesetzt werden in transdisziplinär-transformative Interventionen und Experimente, zur Entstehung von Transformationswissen führen können (s. Abschnitte 3.2.2, 3.2.3 3.3, 3.4; Abb. 2; CASS und ProClim- 1997; West 2019). Im UrbanUtopiaLAB werden alle Beteiligten bewusst dazu aufgefordert, Stadt und Stadtentwicklung nicht gleich vom Machbaren und Funktionalistischen her $\mathrm{zu}$ denken und zu diskutieren, sondern erst einmal Visionen zu entwickeln und von dort aus Möglichkeiten zu suchen, wie diese in der Stadtentwicklung wirksam werden können. Fragen, die in diesem Prozess aufgeworfen werden, lauten beispielsweise: Was wäre für mich der ideale Ort? Wie wäre meine ideale Stadt beschaffen? Welche Qualitäten müssten Orte in der Stadt haben, damit das Gemeinsame funktioniert? Welche Organisationsmodi und Finanzierungskonzepte könnte ich mir vorstellen?

Die Methodik des UrbanUtopiaLABs basiert, wie gesagt, auf dem Konzept der Transversalen Stadt und Transtopia (West 2014a, 2014b, 2019): Transtopia ist der Raum der Improvisation, des Experimentierens, der Co-Produktion. Es ist der Raum, in dem durch transversale Praktiken und Transgression, also durch den Akt des Überschreitens und Aufbrechens von bisherigen Ordnungen, Rollenverständnissen und Zuschreibungen, Entwicklung und damit Systeminnovation möglich wird. Die urbane Sphäre bleibt in Bewegung, Demokratie und neue politische Prozesse werden exploriert und die physisch-materielle Struktur der Stadt wird co-designed. Dabei werden auch Möglichkeiten aufgezeigt, neue Perspektiven für Planung und politisches Handeln zu entwickeln. Utopien der Moderne 
(Foucault 1966/2005, S. 9), die als ortlose Ideen, als unausführbare Leitbilder, Visionen einer zukünftigen Gesellschaftsordnung entwerfen, werden durch ein Denken in Möglichkeiten ersetzt. Damit wird ein Möglichkeitsraum geschaffen, in dem ,intentionale Utopien“ für die gemeinsame Stadt der Zukunft neu erdacht und zur Umsetzbarkeit weiterentwickelt werden.

Im Prozess des UrbanUtopiaLABs ist das Kombinieren von wissenschaftlich-akademischem Wissen und nicht-wissenschaftlichem Praxiswissen zentral. Das UrbanUtopiaLAB ist also durch eine inter- und transdisziplinäre Vorgehensweise geprägt. Die Aufgabe der Wissenschaftler(innen) ist dabei nicht nur die Bereitstellung und Einspeisung des jeweiligen relevanten Systemwissens, das meist als disziplinär geprägtes faktenbasiertes Wissen vorliegt. Vielmehr gehört auch dazu, dass Diskussionen, Optionen und Strategien kritisch reflektiert und in übergeordnete Zusammenhänge eingeordnet werden. Entstehende Dynamiken werden diskutiert und mögliche nicht-intendierte negative wie positive Folgen werden aufgezeigt. Damit wird eine Diskussionsgrundlage gleichermaßen für Entscheidungen im weiteren Verlauf des UrbanUtopiaLABs wie für die Aushandlungsprozesse beim Co-Design von für die Akteure relevanten Forschungsfragen generiert, aus der sich eine agile Transferkultur und ,Kultur des Austauschs' (s. Abschnitt 2.2) für den übergeordneten transformativ-transdisziplinären Forschungsprozess entwickeln kann. Voraussetzung für die Anschlussfähigkeit zwischen wissenschaftlichem und nicht-wissenschaftlichem Wissen ist die Aufbereitung der wissenschaftlichen Erkenntnisse in einer Sprache, dass diese für die jeweils anderen wissenschaftlichen Disziplinen wie auch für Praxisakteure nachvollziehbar sind, verstanden werden und gegebenenfalls an die eigenen Wissensbestände angedockt werden können. Insgesamt kommt den Wissenschaftler(inne)n im UrbanUtopiaLAB damit auch nicht nur eine moderierende Rolle zu. Die Forscher(innen) verlassen die postulierte neutrale Beobachterposition, sie überschreiten Grenzen zwischen Wissenschaft, Politik, Verwaltung und Zivilgesellschaft, indem sie sich aktiv in den Prozess einbringen - und werden so Teil der Utopie.

\subsection{Prozessdesign: Leitung und Moderation}

Für ein UrbanUtopiaLAB ist mindestens eine Person nötig, die die Prozessleitung übernimmt. Sie ist verantwortlich für das Design des gesamten Prozesses, für die Vorgehensweisen mit den beteiligten Akteuren sowie für die Hauptmoderation und gegebenenfalls die Adaption des Prozessdesigns. Daneben muss für die Einzelschritte (z. B. Emo/Action-Mapping, s. Abschnitt 3.1) ein Team von Moderator(inn)en und Organisator(inn)en gebildet werden, das von der Prozessleitung aus den verfügbaren Akteuren (dazu gehören auch Praxispartner, engagierte Teilnehmer(innen) von früheren Veranstaltungen etc.) zusammengestellt wird. Diese 
werden, bevor sie zum Einsatz kommen, sowohl bezüglich der Methode selbst als auch zur Bedeutung und zum Hintergrund eines UrbanUtopiaLABs geschult.

Die Umsetzung eines UrbanUtopiaLABs erfordert bestimmte Kenntnisse auf Seiten der Prozessleitung, wie sie typischerweise in einer akademischen gesellschaftswissenschaftlichen Ausbildung vermittelt werden. Kenntnisse des Konzepts „Transversale Stadt - Transtopia - Transversale Gesellschaft“ (Kapitel 1, 2) sind von Vorteil, ebenso ein fundiertes Verständnis transdisziplinär-transformativer Forschung. Solche Kenntnisse sind hilfreich sowohl beim Prozessdesign eines UrbanUtopiaLABs wie auch bei der Beobachtung und Beurteilung der stattfindenden Prozesse. Sie erlauben es außerdem, auf unvorhergesehene Probleme und inhaltliche Entwicklungen adäquat und im Sinne der Gesamtzielsetzung zu reagieren. Erforderlich ist auch ein tiefergehendes Verständnis der wissenschaftlichen Produktionsweise gesellschaftsbezogenen Wissens. Daher wird die Position der Prozessleitung gewöhnlich von entsprechend ausgebildeten Wissenschaftler(inne)n übernommen. Werden bei einzelnen Veranstaltungen oder Schritten (wissenschafts-)externe Moderator(inn)en zur Unterstützung hinzugezogen, müssen auch diese im Vorfeld je nach Einsatzbereich entsprechend eingewiesen oder geschult werden.

\subsection{Kultur des Austauschs}

Das gesamte Prozessdesign des UrbanUtopiaLABs zielt auf den Aufbau einer Kultur des Austauschs ab. Entsprechend sind die einzelnen Abschnitte und Vorgehensweisen durch eine wertschätzende, achtsame, sich an Regeln orientierende Kommunikation (s. Abschnitt 4.2.1) und ein respektvolles Miteinander auf Augenhöhe geprägt. Das UrbanUtopiaLAB bringt Menschen zusammen, die im Alltag oftmals nicht miteinander kommunizieren. Im UrbanUtopiaLAB tauschen sie sich aus, zeigen sich gegenseitig ihre Stadt und ihre damit verbundenen Emotionalitäten, sie offenbaren Sehnsüchte und Geschichten, suchen Anknüpfungspunkte und entwickeln spielerisch und experimentell Neues (s. Abschnitte 3.1, 3.2, 4.2). Durch den Austausch, die Diskussionen und Aushandlungen öffnen sie einen Raum des sich gegenseitigen Erkennens und Anerkennens. Der Austausch im Rahmen des UrbanUtopiaLABs erfordert Offenheit, und dies wiederum muss in kurzer Zeit ,hergestellt' werden, was eine gewisse Herausforderung für die Moderation darstellt.

Die Methode ist so konstruiert, dass sie sich sowohl für überaus heterogene Gruppen - bezüglich Alter, Herkunft, Weltanschauung, Bildungstiefe, Kenntnisse in der Verkehrssprache, Aktionsradius, Mobilität, Zugehörigkeit zu Funktionssystemen (Verwaltung, Politik, Bildung, Wirtschaft, Wissenschaft, Kirche etc.) eignet wie auch für eher homogene Gruppen, sowohl für ,spontane' Gruppen als auch für solche, die auf Einladung oder Bewerbung zusammenkommen. 
Den Organisator(inn)en und Moderator(inn)en muss es in allen Phasen und ungeachtet der Gruppenzusammensetzung gelingen, eine Atmosphäre entstehen zu lassen, in der unterschiedliche oder sogar gegensätzliche Positionen, Perspektiven, Ideen, frei geäußert und konstruktiv diskutiert werden können. Eine solch konstruktive Atmosphäre wird insbesondere durch das Verfahren des Emo/ Action-Mappings (s. Abschnitt 3.1) befördert, das deshalb das ,Herzstück ${ }^{6}$ in der ersten Phase eines UrbanUtopiaLABs ist. Darüber hinaus kann es aber auch ganz gezielt in allen weiteren Phasen des UrbanUtopiaLABs immer wieder eingesetzt werden.

\section{Phasen des UrbanUtopiaLABs - Überblick, Ziele und Hinweise zur Durchführung}

Der Gesamtprozess eines UrbanUtopiaLABs lässt sich in vier Phasen einteilen (s. Abb. 1). Diese Phasenabfolge muss nicht eingehalten werden, Iterationen einzelner Phasen oder Segmente sind durchaus möglich. Der exakte inhaltliche Ablauf des UrbanUtopiaLABs muss also nicht bis ins Detail vorausgeplant werden, da Erkenntnisse aus der einen Phase die Inhalte der nächsten Phase bestimmen. Die exakten Rahmenbedingungen, die sich einfindende Akteurskonstellation und die sich daraus ergebenden thematischen Fokussierungen sind nicht von vornherein bekannt, sondern ergeben sich erst im Prozess. Eine minutiöse Planung des Gesamtprozesses würde dem Prinzip der (experimentellen) Ergebnisoffenheit sogar widersprechen.

Daher können die Phasen auch in verschiedener Reihenfolge kombiniert werden. So kann auf StadtERFORSCHEN (Phase 1) StadtERKUNDEN (Phase 2) folgen und darauf dann StadtEXPERIMENTIEREN (Phase 3). StadtERKUNDEN (Phase 2) und StadtEXPERIMENTIEREN (Phase 3) können aber auch miteinander verbunden werden (s. Kapitel 4, Box 1). Alle Phasen können mehrmals wiederholt werden, zudem ist die Erarbeitung des Policy Briefs in StadtMITENTSCHEIDEN (Phase 4) als iterativer Prozess gedacht, der von den Erkenntnissen aus allen Phasen gespeist wird und nicht zwingend (nur) die Schlussphase darstellen muss.

Im Rahmen der transdisziplinären Entwicklung von Ideen für die Zukunft wird aus dem Wissen über Strukturen, Prozesse und Variabilitäten in Räumen (Systemwissen) Zielwissen generiert, aus dem wiederum durch transdisziplinär-transformative Interventionen ein Wissen entstehen kann, das sich als Transformationswissen in konkreten Handlungsempfehlungen niederschlägt und damit auch allgemein zugänglich gemacht werden kann (s. CASS und ProClim- 1997; West 2018). 


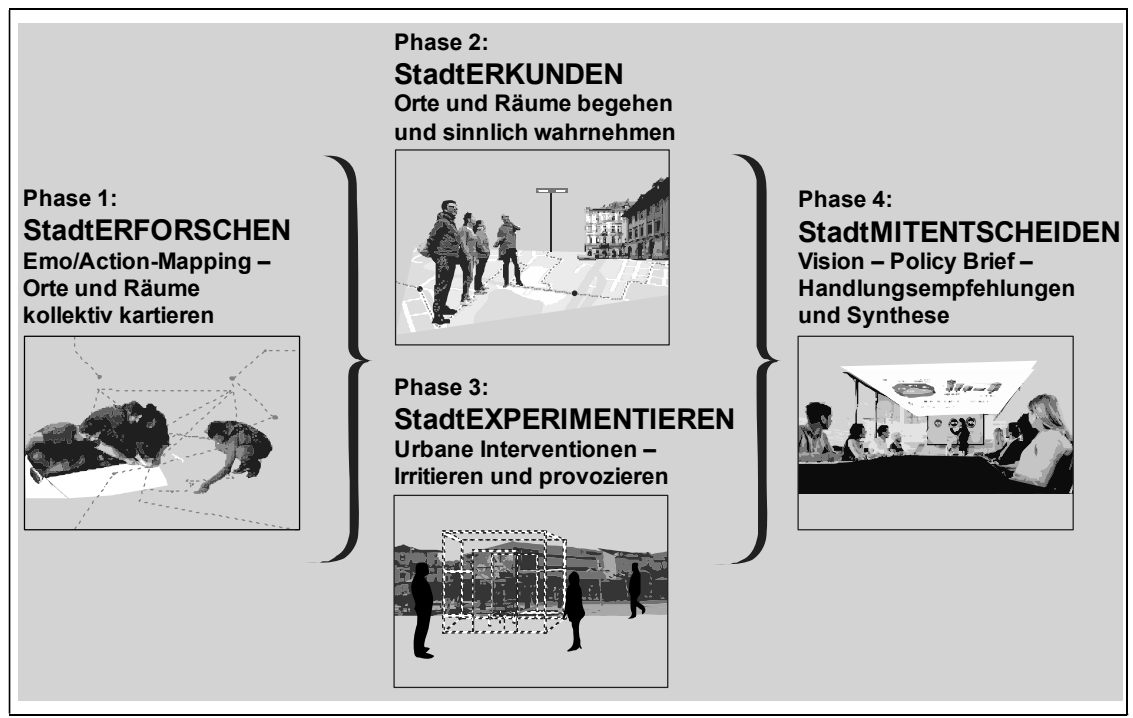

Abbildung 1: Phasen eines „UrbanUtopiaLABs | Experimenting Utopia: Past ... Present ... Future“. Während die Phasen 1 und 2 themenunabhängig, aber methodisch nachvollziehbar sind, werden Phasen 3 und 4 inhaltlich, methodisch und im Ablauf von den Themensetzungen, Akteuren und Ergebnissen in den Phasen 1 und 2 bestimmt. Die Abfolge der vier Phasen kann durch Iterationen einzelner Phasen und Segmente variiert werden. (C) Krishan Rajapakshe, Christina West, Heidelberg 2018.

\subsection{Phase 1: StadtERFORSCHEN: Emo/Action-Mapping - Orte und Räume kollektiv kartieren}

Phase 1 ist das Emo/Action-Mapping, also das kollektive Kartieren, das Verorten von individuellen Emotionen und Aktivitätsräumen, das gemeinsame Stadt ERFORSCHEN. Dieses kann in einem Reallabor oder einem anderen transdisziplinär-transformativen Projekt zu verschiedenen Zeitpunkten eingesetzt werden. Es kann als eintägiges Event (s. Box 1) geplant oder auch als ein mehrteiliger Prozess eingesetzt werden. Es dient dazu, Kreativität zu fördern für eine Vision zum Zusammenleben, zu begreifen, dass sich Raum und das Urbane als alltäglich co-produziert verstehen lassen. Es kann mit einer, geschlossenen' Gruppe realisiert werden, es kann aber auch umgesetzt werden, um neue Teilnehmer(innen) aus der Stadtgesellschaft in den laufenden Prozess einzubinden. Für letzteres können Mapping-Events auch im öffentlichen Raum durchgeführt werden. 
Von den Teilnehmer(inne)n werden außer einer Ergebnisoffenheit keine besonderen Voraussetzungen oder Kenntnisse erwartet. Beim Emo/Action-Mapping geht es weniger um eine ,klassische“ Kartierung von Funktionen einer Stadt, wie sie vor allem in den universitären Disziplinen Geographie und Kartographie gelehrt werden, sondern insbesondere um die persönliche Codierung einer Stadt, eines Quartiers oder einer Region: Dazu gehören sowohl die persönlichen Wohlfühlorte als auch die Räume oder Routen, die gemieden werden, wie auch individuelle Aktionsräume und Alltagspraktiken zur Aneignung des Raums.

Beim Emo/Action-Mapping richtet sich der Fokus der Arbeit auf die gemeinschaftliche ,Karte'. Dabei potenzieren sich unterschiedliche individuelle Formen des Erfassens und Codierens, indem verschiedene Symbole und Icons zur Verfügung gestellt (s. Abschnitte 4.1.1, 4.1.3) und neue Icons generiert werden, die wiederum zur Kreation von Collagen und Zeichnungen sowie zur Formulierung von Erzählungen, Forderungen, Wünschen anregen, und somit die Entfaltung unterschiedlicher Produktionsmodi fördern. Dies unterstützt auch die Wahrnehmung von und die Offenheit für die Diversität der ökologischen, ökonomischen, sozialen, kulturellen und politischen Aspekte und macht diese damit auch kommunizier-, aushandel- und gestaltbar.

Durch die Verknüpfung verschiedener Zugänge zum eigenen und gemeinsamen Leben in der Stadt oszillieren die Teilnehmer(innen) zwischen einem realen Raumbezug zur Stadt, zum Quartier oder zur Region im eigenen Erinnerungsraum sowie auf einer Karte zum einen und dem emotionalen Raum, angeregt und vermittelt über die Emo/Action-Icons, zum anderen. Dadurch werden die Teilnehmer(innen) zu einem völlig neuen, spielerischen Zugang zu ,ihrer' Stadt angeregt. Während des Mappings werden eine Vielzahl von mit einem Ort verbundenen Geschichten, Gerüchen, Geräuschen und Emotionalitäten erinnert. Zwischen der Wahl des dazu passenden Icons und dessen Platzieren auf der Karte wird in einem kurzen Augenblick entschieden, was dazu erzählt wird. Diese Herausforderung führt zu Momenten der Distanzierung und Entfremdung vom eigentlich vertrauten Raum, was wiederum einen bzw. mehrfache Perspektivenwechsel fördert. Die Phase des Emo/Action-Mappings führt zur Konstruktion eines kollektiven Horizonts, ohne dass dabei die individuellen Wahrnehmungen der beteiligten Menschen verloren gehen.

Das Emo/Action-Mapping ist darauf ausgerichtet, Menschen miteinander ins Gespräch zu bringen, die in der alltäglichen Begegnung in der Regel nicht miteinander kommunizieren, nur übereinander reden oder sich sogar niemals begegnen würden. Da in Gruppen zusammen an einer maßstabsgetreuen Karte gearbeitet wird, ermöglicht das Emo/Action-Mapping sowohl, in visionäre Diskussionen mit den anderen Teilnehmer(inne)n zu kommen, als auch, diese Diskussionen ,räumlich zu verorten' und damit gemeinsam die Entwicklung der realen Stadt von der Zukunft her zu denken. 
Die Emo/Action-Icons dienen dazu, Aktionsräume zu ,markieren' und sich gestützt darauf gegenseitig individuell angeeigneten Raum zu zeigen. Das erfolgt nicht immer konfliktfrei, sondern kann auch zu Reibungen führen, die zuzulassen sind, weil konfliktbehaftete Aneignungsprozesse Gegenwart wie Zukunftsideen und Visionen mitbestimmen. Es geht also um den Austausch und das Zusammenbringen der diversen Wahrnehmungen, Einordnungen und ,Emotionalisierungen des Raums. Daraus sowie aus den individuellen Erzählungen zu einzelnen Orten ergeben sich kollektive Geschichten über diese Orte. Mit dem Emo/Action-Mapping können sich Kommunikations- und Interaktionsformen der Einzelnen zu mehr Transversalität und Transgressivität (s. Kapitel 1,2) verändern, indem beispielsweise Grundüberzeugungen zugunsten komplexerer Sichtweisen aufgegeben werden oder indem die Fähigkeit, fremde Perspektiven einzunehmen und ohne Wertung mit der eigenen zu vergleichen, gefördert wird oder indem die Bereitschaft geweckt wird, anderen in die Assoziationen ihrer Utopie zu folgen. Die zentrale Frage „Wie wollen wir in Zukunft miteinander leben?“ wird zum Ausgangspunkt für die individuellen Erzählungen und zum Diskussionsrahmen für die kollektive Aushandlung.

\subsection{Phase 2: StadtERKUNDEN: Orte und Räume begehen und sinnlich wahrnehmen}

Bei StadtERKUNDEN zeigen sich die Teilnehmer(innen) gegenseitig ihre Orte im realen Raum der Stadt, des Quartiers, der Region. Handlungsleitend in dieser Phase 2 des UrbanUtopiaLABs ist, diesen Raum gemeinsam zu erkunden und aus den im Emo/Action-Mapping neu gewonnenen Blickwinkeln $\mathrm{zu}$ beobachten. Idealerweise erfolgt StadtERKUNDEN als Fortsetzung zu StadtERFORSCHEN, dann werden die verschiedenen Perspektiven, die auf der zweidimensionalen Karte des Emo/Action-Mappings erfasst sind, in ihrer realweltlichen Komplexität besonders deutlich, und die entsprechende Aufnahmebereitschaft ist erhöht und geschärft. Dies trägt dazu bei, auch scheinbar Vertrautes neu zu sehen oder mit allen Sinnen wahrzunehmen, und dazu, sich über die Aha-Momente und neu gewonnenen Erkenntnisse auszutauschen und auseinanderzusetzen. Stadt ERKUNDEN kann aber auch eigenständig oder zusammen mit StadtEXPERIMENTIEREN durchgeführt werden.

In StadtERKUNDEN werden die Teilnehmer(innen) erneut aufgefordert, sich , spielerisch' und mit offenem Ausgang auf die Stadt einzulassen, diese wortwörtlich mit allen Sinnen zu ERKUNDEN, also explizit sinnessensibel ,ins Feld‘ zu starten. Die Teilnehmer(innen) sollen also den Raum ganz bewusst sehen, hören, riechen, schmecken, tasten, fühlen. Die sich dabei einstellenden „Urban Emotions" und sinnlichen Wahrnehmungen im physischen Raum werden von den Teilnehmer(innen) artikuliert, aufgezeichnet und mit den anderen Teilnehmer(innen) 
ausgetauscht. Sie können den bekannten alltäglichen Zugang zur Stadt, zum Quartier, zur Region verändern, ,blinde Flecken' offenlegen und die Wahrnehmung und Formulierung von Veränderungspotenzialen unterstützen.

\subsection{Phase 3: StadtEXPERIMENTIEREN: Urbane Interventionen- Irritieren und provozieren}

In StadtEXPERIMENTIEREN, in Phase 3 eines UrbanUtopiaLABs, werden, auf der Grundlage der kollektiv entstandenen Emo/Action-Karten Orte in der Stadt, im Quartier oder in der Region ausgewählt und mit (kleinen) urbanen Interventionen im öffentlichen Raum bespielt, die ganz bewusst überraschen, irritieren oder auch provozieren sollen, also absichtlich ,Brüche" hervorrufen. Diese Interventionen sollen die Planung, Nutzung, Aneignung und Kommerzialisierung des öffentlichen Raums, Inklusionen und Exklusionen kommentieren, kritisieren und dadurch (neu) sichtbar machen. Im Unterschied zu den Phasen 1 und 2 (s. Abschnitte 3.1, 3.2) geht es dabei aber nicht (nur) um das Erforschen und Beobachten, sondern vor allem um eine gezielte Konfrontation der Bevölkerung mit den bis dahin im UrbanUtopiaLAB erarbeiteten kollektiven Wahrnehmungen, Einsichten, Visionen und Veränderungsideen. Alternativ oder zusätzlich werden Ausstellungen, Diskussionsrunden o. Ä. sowie Emo/Action-Mapping-Events im öffentlichen Raum durchgeführt, in deren Rahmen zum Beispiel neue Emo/ Action-Karten produziert werden.

Damit werden die kollektiven Emo/Action-Karten Teil der urbanen Interventionen oder von Ausstellungen u. Ä. und erzeugen Anschlussfähigkeit zwischen diesen und sich zufällig begegnenden Menschen und deren (Welt-)Perspektiven. Daraus können dann neben Diskussionen auch (realisierbare) Ideen für die Transformation der Stadt, des Quartiers, der Region entstehen. Umgekehrt können die in StadtEXPERIMENTIEREN erzielten Ergebnisse auch die Basis für Veranstaltungen in StadtERFORSCHEN bilden (s. Abschnitt 3.1). Ein gelungenes Beispiel für eine Intervention im Sinne von StadtEXPERIMENTIEREN stellt das studentische Projekt ,„Wissen to Go ${ }^{6}$ - Bringt Eure Zukunft ins Rollen! Einkaufswagen als Wissens-Hotspots im Neuenheimer Feld" dar, in dem transdisziplinär entwickelte und produzierte mobile Wissensorte aus umgestalteten Einkaufswagen im Stadtraum platziert und beforscht wurden (für eine Beschreibung dieses „Reallabors im Kleinen“"s. West 2018).

Das Ziel in dieser Phase ist es, Reflexion und Diskussion freizusetzen, um die inter- und transdisziplinäre Entwicklung konkreter Handlungsempfehlungen an Politik, Verwaltung, Planung und Wissenschaft zu formulieren (s. Abschnitt 3.4). Deshalb ist es spätestens zu Beginn dieser Phase notwendig, sich auf ein gemeinsames Verständnis zu einigen, in welchem Verhältnis einerseits einzelne Interventionen und Experimente, aber andererseits die verschiedenen Wissensforma- 
tionen zueinander stehen, um schließlich Klarheit darüber zu erhalten, welche Funktion Interventionen und Experimente für die Generierung von Systemwissen, Zielwissen und Handlungsoptionen sowie Transformationswissen haben. Erst so wird es möglich, eine gemeinsame transdisziplinäre Kommunikationsebene für die Entwicklung des Gesamtdesigns herzustellen und das transdisziplinäre Problemverständnis zu formulieren.

Diese Diskussionen werden für alle Beteiligten leichter nachvollziehbar, wenn die Prozessabläufe und Interdependenzen der einzelnen ,Bausteine ' während der Diskussion visualisiert werden, zum Beispiel gemeinsam am Flipchart, an einem Whiteboard, auf einer Tafel oder Braunpapier. Dabei ist es wichtig, dass alle Wissenschaftler(innen) und Praxisakteure - nicht nur reden und diskutieren, sondern auch zeichnen dürfen und sollen. Ziel dieses Vorgehens ist, dass alle Beteiligten ein gemeinsames Verständnis über den idealtypischen Verlauf eines Transformationsprozesses entwickeln, der mit der Durchführung eines UrbanUtopiaLABs angestrebt wird. Abb. 2 zeigt den während der Entwicklung der Methode „UrbanUtopiaLAB“ im Reallabor Asylsuchende mit den Praxisakteuren ausdiskutierten und gemeinsam entwickelten schematischen Ablauf, der je nach Problemstellung eines UrbanUtopiaLABs mit den konkreten Themen, Forschungsfragestellungen und Interventionen gefüllt werden kann und sollte.

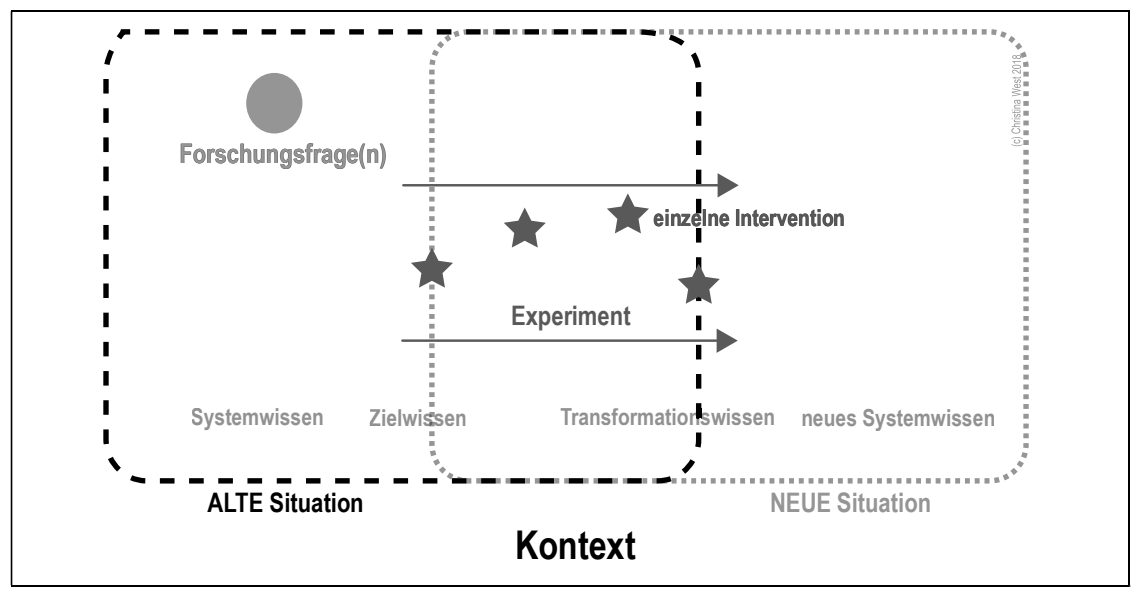

Abbildung 2: Visualisierung des gemeinsamen Verständnisses über den idealtypischen Verlauf eines Transformationsprozesses, die im Reallabor Asylsuchende entstand. Deutlich wird, welche zentrale Position Interventionen bei der Produktion von Transformationswissen haben und dass ein gewisses Maß an Zielwissen zwischen den Akteuren abgestimmt sein muss, bevor ein Experiment - oftmals zusammengesetzt durch einzelne Interventionen beginnt. (O) Jaro Eiermann, Christina West, Heidelberg 2018. 
Der in Abb. 2 dargestellte idealtypische Verlauf kann selbstverständlich mehrmals wiederholt werden, da sich mit jedem Ablauf und somit auch dem Durchlaufen der Phase StadtEXPERIMENTIEREN das transdisziplinäre Problemverständnis verändert und neues Systemwissen hinzukommt.

\subsection{Phase 4: StadtMITENTSCHEIDEN: Policy Brief- Handlungsempfehlungen und Synthese}

In StadtMITENTSCHEIDEN, in Phase 4 eines UrbanUtopiaLABs, werden die Materialien und Ergebnisse der vorangegangenen Phasen zusammengetragen. Auf dieser Basis formulieren das für das UrbanUtopiaLAB zuständige Projektteam sowie die in die verschiedenen Phasen eingebundenen Teilnehmer(innen) in der Tradition des Policy Briefs konkrete Handlungsempfehlungen an Politik, Verwaltung, Planung und Wissenschaft. Diese Handlungsempfehlungen werden anschließend mit politisch Verantwortlichen, weiteren Akteuren in der Stadt sowie Verbänden diskutiert und nach Möglichkeit bezüglich ihrer Umsetzbarkeit weiterentwickelt (s. Kapitel 5).

\section{Idealtypische Tagesveranstaltung im Rahmen eines UrbanUtopiaLABs}

Im Folgenden wird eine idealtypische sechsstündige Tagesveranstaltung (s. Box 1) vorgestellt, die sich in dieser oder ähnlicher Form bewährt hat, und in der die Phasen 1 und 2 des UrbanUtopiaLABs umgesetzt werden. Diese kann in ihrem Ablauf je nach Fragestellung und Akteurskonstellation oder auch bei wiederholter Durchführung - mit oder ohne Veränderung der Zusammensetzung der Gruppe der Teilnehmer(innen) - variiert werden, indem die zeitliche Dauer der einzelnen Abschnitte angepasst wird. Denkbar ist, dass bei wiederholter Durchführung und nahezu gleichbleibendem Kreis an Teilnehmer(innen) die einzelnen Emo/ActionKarten (Phase 1; s. Abschnitt 3.1, Abb. 1) nicht neu erstellt, sondern nur noch ergänzt werden, wodurch mehr Gewicht auf StadtERKUNDEN (Phase 2; s. Abschnitt 3.2, Abb. 1) gelegt wird bzw. die Veranstaltung um StadtEXPERIMENTIEREN (Phase 3; s. Abschnitt 3.3, Abb. 1) ergänzt werden kann. Gegebenenfalls kann auch die Dauer auf 4 bis 5 Stunden reduziert werden. 


\section{StadtERFORSCHEN und StadtERKUNDEN}

Übergeordnete Frage: Wie wollen wir in Zukunft in der Stadt X [im Quartier X, in der Region X] miteinander leben?

Dauer: $6 \mathrm{~h}$, vorzugsweise samstags

09:45-10:00 Ankommen

10:00-10:10 Begrüßung

Begrüßung der Anwesenden; Vorstellung des Projektteams;

Organisatorisches und Zeitplan

10:10-10:30 Einführung UrbanUtopiaLAB

Einführung in Leitfragen und Ablauf des UrbanUtopiaLABs; ggf. Vorstellungsrunde; Verteilung der Teilnehmer(innen) auf die Diskussionsinseln

StadtERFORSCHEN (Phase 1)

10:30-11:00 Emo/Action-Mapping: Orte und Räume kollektiv kartieren in Kleingruppen an den Diskussionsinseln; fließender Übergang in

11:00-11:45 Emo/Action-Mapping: Offene Diskussion in Kleingruppen an den Diskussionsinseln; fließender Übergang in

11:45-12:00 Emo/Action-Mapping: Clustern und Priorisierung von Themen in Kleingruppen an den Diskussionsinseln

12:00-12:30 Kurzvorstellung Emo/Action-Karten der einzelnen Diskussionsinseln im Plenum

12:30-13:00 Pause - Zeit für Essen und Gespräche

StadtERKUNDEN (Phase 2)

13:00-14:45 StadtERKUNDEN (in Kleingruppen)

14:45-15:00 Emo/Action-Karten der Diskussionsinseln ergänzen (in Kleingruppen)

15:00-15:55 Zusammenfassung und nächste Schritte (im Plenum)

Präsentation StadtERKUNDEN - Einblicke aus den Kleingruppen

Diskussion/Einordnung - mit Blick auf die übergeordnete Frage

What's next? - wie, wann und mit wem geht es weiter?

15:55-16:00 Abschied

Dank und Verabschiedung

$16: 00$

Veranstaltungsende

anschl.: Möglichkeit zur Fortsetzung der Diskussionen und Gespräche

Während des gesamten UrbanUtopiaLABs:

Plakatwände, auf denen die Teilnehmer(innen) ihre Ideen hinterlassen können

Box 1: Idealtypischer Ablauf: „UrbanUtopiaLAB | Experimenting Utopia: Past ... Present ... Future“ (Phasen 1 und 2) 


\subsection{VORBEREITUNG | UrbanUtopiaLAB - StadtERFORSCHEN (Phase 1) und StadtERKUNDEN (Phase 2)}

\subsubsection{Inhaltliche Vorbereitung}

Die übergeordnete Frage des UrbanUtopiaLABs, bezogen auf eine Stadt, ein Quartier, eine Region, lautet: „Wie wollen wir in Zukunft miteinander leben?“ Diese Frage kann im UrbanUtopiaLAB selbstverständlich auf ganz unterschiedliche inhaltliche Themen fokussiert werden. Ist der thematische Rahmen für das Emo/Action-Mapping durch das übergeordnete Forschungsprojekt noch nicht ausreichend detailliert festgelegt oder gibt es Zweifel, dass ausreichendes Interesse für das sich daraus ergebende Thema existiert, empfiehlt sich eine Voruntersuchung. Dabei führt ein hierfür zusammengestelltes Vorbereitungsteam mit ausgewählten Expert(inn)en ebenso wie mit Bewohner(inne)n Interviews, die das Ziel verfolgen, wichtige Themen zu identifizieren, diese in das Thema einzuordnen, dem das übergeordnete Projekt gewidmet ist, sowie einige diesbezügliche Einschätzungen zur Zukunftsfähigkeit von Stadt, Quartier oder Region zu erhalten. $\mathrm{Zu}$ all diesen Themen können gegebenenfalls spezifische UrbanUtopiaLABIcons (s. Abschnitte 4.1.3, 4.2.2) entwickelt werden, die später beim Emo/ActionMapping (s. Abschnitt 4.2.2) eingesetzt werden.

Denkbar ist es dann auch, dass die einzelnen Diskussionsinseln beim Emo/Action-Mapping je mit einem dieser ermittelten Themen bzw. den daraus abgeleiteten zukunftsbezogenen Fragen belegt werden und sich die Teilnehmenden dann interessensgeleitet für eine ,Themeninsel ${ }^{`}$ entscheiden (s. Abschnitt 4.2.1). Wichtig ist, dass die Formulierung der Teilfragen oder -aspekte auch in diesem Fall immer entlang der übergeordneten Leitfrage „Wie wollen wir in Zukunft miteinander leben?" erfolgt.

\subsubsection{Auswahl der Räumlichkeiten und deren Ausstattung, Anzahl der Teilnehmer(innen)}

Prinzipiell ist Phase 1 StadtERFORSCHEN des UrbanUtopiaLABs (s. Abschnitt 3.1, Abb. 1) so gestaltet, dass sie in unterschiedlichen Raumsituationen durchgeführt werden kann: sowohl in einem großen Raum bzw. in einer Halle oder in einer Kombination aus einem größeren Raum für das Plenum und kleineren Arbeitsräumen für die Gruppendiskussionen - bei Bedarf oder je nach Lust und Laune auch unter freiem Himmel. Dadurch lässt sich das Vorgehen flexibel an Gruppengrößen zwischen 5 und 100 Teilnehmer(innen) anpassen, wobei immer Kleingruppen mit jeweils 5 bis 10 Teilnehmer(inne)n gebildet werden. Wird die Methode mit mehr als 5 Kleingruppen durchgeführt, dann müssen die in Box 1 festgelegten Zeiten für die Plenumsabschnitte ungefähr verdoppelt werden. 
Wird Phase 1 des UrbanUtopiaLABs in einem großen Raum oder in einer Halle durchgeführt, dann werden die 2 bis 10 Diskussionsinseln für die Gruppendiskussionen in einem gebührenden Abstand zueinander aufgebaut. Diese Diskussionsinseln bestehen aus einer quadratischen Tischformation mit 5 bis 12 Stühlen und einer Moderationswand zum Befestigen von Moderationskarten sowie aus Standardmoderationsmaterial (Stifte, Moderationskarten, Pins, Klebepunkte, Kreppband, Schere, Braunpapier, Wollknäul etc.). Hinzu kommt dann noch die Formation für das Plenum. Diese kann sich ,klassisch` aus mehreren Stuhlreihen und einem Projektor mit Leinwand und Mikrofon zusammensetzen. In Abhängigkeit von der Anzahl der Teilnehmer(innen) kann auch eine U-förmige Bestuhlung für das Plenum gewählt werden, da eine solche meist die Diskussion zwischen den Teilnehmer(innen) fördert. Alternativ oder unter freiem Himmel kann mit Moderationskarten oder Braunpapier auch an einer (Haus-)Wand o. Ä. anstelle von Moderationswänden gearbeitet und statt den Stuhlreihen kann eine Amphitheater-ähnliche Situation geschaffen werden, in der auf dem Boden, auf Stühlen oder alternativen Sitzgelegenheiten sitzend sowie im Stehen diskutiert wird.

Die Durchführung einer ganztägigen UrbanUtopiaLAB-Veranstaltung kann für manche Teilnehmer(innen) ermüdend sein. Das Projektteam sollte daher auch kurze Pausen zwischendurch einplanen und für genügend Verpflegung sorgen, die während des gesamten Tages zugänglich ist.

\subsubsection{Vorbereitung der Materialien}

Für die Durchführung des Emo/Action-Mappings müssen verschiedene Materialien vorbereitet werden. Zusätzlich zum Standardmoderationsmaterial wird jede Diskussionsinsel (s. Abschnitt 4.2.2) mit einem ausreichenden Vorrat von Emo/Action-Icons (s. Abb. 3) ausgestattet sowie einer großmaßstäblichen Karte, mindestens im Format DIN A0, die den Ausschnitt der Stadt, des Quartiers oder der Region wiedergibt, auf den im UrbanUtopiaLAB fokussiert wird. Je nach disziplinärer Zusammensetzung des Projektteams können Erstellung, Druck oder Beschaffung dieser Karten zeitintensiv und kostenaufwendig sein. Ist niemand mit kartografischen Kompetenzen im Team, muss die Erstellung der Karten extern in Auftrag gegeben werden.

Ein Set UrbanUtopiaLAB-Icons (s. Abb. 3) besteht aus rund 35 bis 40 verschiedenen vom Projektteam für das Emo/Action-Mapping entwickelten farbigen Icons. Die 3 x $3 \mathrm{~cm}$ großen Icons werden auf stabiles weißes Papier oder leichten Karton gedruckt, und für jede Diskussionsinsel sind 8 bis 9 vollständige Sets nötig. Die kleinen Bilder umfassen sowohl abstrakte Darstellungen wie zum Beispiel ein rotes Herz, einen gelben Stern, einen roten Punkt oder mehrere in die Luft gestreckte Hände wie auch gegenständliche Darstellungen, beispielsweise ein Fahrrad, ein Auto, eine Uhr. Es können sowohl bekannte wie auch unbekann- 


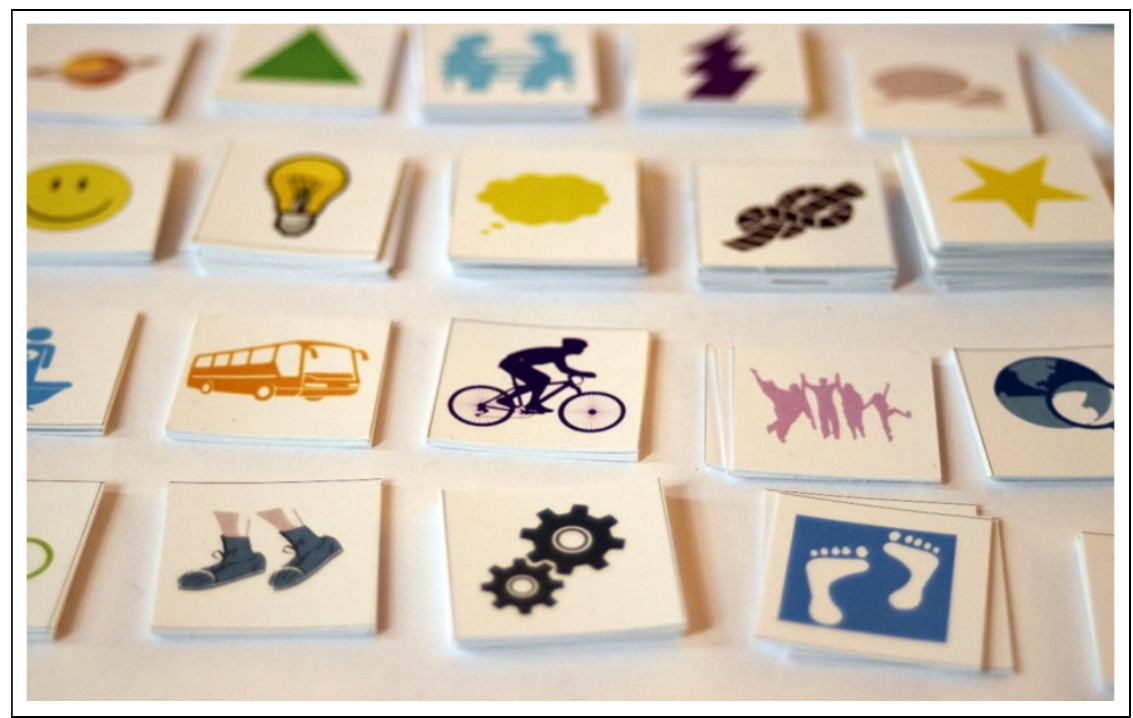

Abbildung 3: UrbanUtopiaLAB-Icons: Die UrbanUtopiaLAB-Icons - hier eine exemplarische Auswahl - sind ein wichtiger Bestandteil des Emo/Action-Mappings, das ,Herzstück‘ der ersten Phase eines UrbanUtopiaLABs, in der sich eine Kultur des Austauschs beginnt zu entwickeln. Mit den Emo/ Action-Icons markieren die Teilnehmer(innen) ihre Aktionsräume und zeigen sich gegenseitig ihre individuell angeeigneten Räume. (C) Krishan Rajapakshe, Heidelberg 2018.

te Piktogramme verwendet werden. Die Bedeutung eines Icons ist nicht notwendigerweise eindeutig festgelegt, sondern kann von den Teilnehmer(inne)n selbst definiert werden. Zusätzlich werden Blanko-Icons bereitgestellt, die von den Teilnehmer(innen) bemalt bzw. beschriftet werden können (zur Ergebnissicherung, s. Abschnitt 4.2.4).

Gibt es eine Voruntersuchung (s. Abschnitt 4.1.1), können die Ergebnisse der geführten Interviews mit Blick auf Hinweise darauf analysiert werden, ob spezifische Bedarfe nach speziellen Symbolen und damit Icons bestehen. Plant ein Projektteam mehrere Emo/Action-Mappings, kann auch, als Teil der Nachbereitung des Emo/Action-Mappings in StadtERFORSCHEN und/oder des StadtERKUNDENs das archivierte Material von den Wissenschaftler(inne)n und Praxispartnern des Projekts gesichtet und systematisch dahingehend ausgewertet werden, welche Ortszuschreibungen, Funktionen, Tätigkeiten, Emotionen, Wertschätzungen, Wertorientierungen, Visionen etc. genannt oder diskutiert wurden. Auf dieser Grundlage können dann die bestehenden Icons weiterentwickelt und ergänzt werden. Dafür empfiehlt sich eine Zusammenarbeit mit Grafiker(inne)n. Eben- 
falls kann durch das Projektteam geprüft werden, ob Icons aus dem Set der UrbanUtopiaLAB-Icons herausgenommen werden können und sollen, da sie von den Teilnehmer(innen) nicht benutzt wurden.

Für die Begehungen in StadtERKUNDEN werden die Moderator(inn)en bzw. alle Kleingruppen mit Plänen der Stadt, des Quartiers, der Region auf Papier sowie mit technischen Geräten für Foto- und Filmaufnahmen (dafür ist fast jedes Mobiltelefon geeignet) ausgestattet. Bei der Präsentation der Gruppenergebnisse von StadtERKUNDEN im Plenum (s. Box 1, Abschnitt 4.2.3) geben die einzelnen Gruppen Einblicke in ihre Rundgänge. Dafür müssen eine Infrastruktur, die eine Datenübertragung von mobilen Film- und Tonaufnahmegeräten auf ein Notebook ermöglicht (in der Regel ein Datentransportkabel mit den entsprechenden Anschlüssen), ein Projektionsgerät (Beamer o. Ä.), eine Projektionsfläche (Leinwand, weiße Wand o. Ä.) und Lautsprecher vorhanden sein.

\subsubsection{Rekrutierung und Einladung potenzieller Teilnehmer(innen)}

Die Ansprache möglicher Teilnehmer(innen) ist ein wichtiger Aspekt der Vorbereitungen zum Emo/Action-Mapping, da eine zu geringe Teilnehmerzahl oder eine einseitige Auswahl der Teilnehmer(innen) dem Ziel der ergebnisoffenen, auf transdisziplinäre Co-Produktion von Wissen ausgerichteten Methode entgegenläuft.

Neben den üblichen Wegen wie Social Media, Pressemitteilungen, Presseartikel, Flyer etc. erweisen sich insbesondere die persönliche Ansprache sowie die direkte Einladung von möglichen Teilnehmer(inne)n zum Beispiel bei Interviews oder vorausgehenden Veranstaltungen des Projekts (wie z. B. Interventionen oder öffentlichen Emo/Action-Mappings unter freiem Himmel) als erfolgreich, wenn auch hierbei eine bewusste oder unbewusste Vorselektion der Teilnehmer(innen) nicht ausgeschlossen werden kann. Sinnvoll ist es zudem, die Kontakte oder Verteiler nicht nur der Praxispartner im Projekt, sondern auch weiterer, dem Projekt thematisch verwandter Organisationen in Anspruch zu nehmen. Im Rahmen des Reallabors Asylsuchende waren es vor allem die Kontakte des Praxispartners Each1Teach1 e. V., über die viele Teilnehmer(innen), Geflüchtete und nicht Geflüchtete, auf die Veranstaltung aufmerksam wurden, Informationen in ihren jeweiligen Netzwerken verbreiteten und teilnahmen. 


\subsection{DURCHFÜHRUNG | UrbanUtopiaLAB -StadtERFORSCHEN (Phase 1) und StadtERKUNDEN (Phase 2)}

\subsubsection{Begrüßung und Einführung}

Insgesamt 30 Minuten sind für die Begrüßung und Einführung in das UrbanUtopiaLAB vorgesehen (s. Box 1). Dieser Begrüßungsteil wird in der Regel von einem Mitglied des Projektteams übernommen, dieses kann aus dem Kreis der Wissenschaftler(innen) oder aus dem Kreis der Praxispartner stammen. In den ersten 10 Minuten werden die Anwesenden begrüßt, das Projektteam sowie der Zeitplan der Veranstaltung und organisatorische Details vorgestellt (Hinweis auf die Teilnehmerlisten, wo die Getränke stehen, wie die Mittagspause organisiert ist u. Ä.). Insbesondere der Hinweis auf Ton- und Filmaufnahmen während der gesamten Veranstaltung sowie das Einholen des Einverständnisses zur internen Nutzung dieser Daten zum Zweck der wissenschaftlichen Auswertung (am besten durch Unterzeichnung einer entsprechenden Erklärung) müssen am Anfang der Veranstaltung stehen, sofern dies nicht bereits im Zuge von Einladung und Anmeldung erfolgte.

Direkt anschließend werden die verbindlichen ,Spielregeln' für die Veranstaltung kommuniziert. Es empfiehlt sich, diese nicht nur vorzulesen, sondern gleichzeitig für alle schriftlich zu visualisieren. Je nach zur Verfügung stehenden Möglichkeiten können diese digital auf eine Fläche projiziert oder auf einem großen Plakat o. Ä. gezeigt werden. Der Vorteil eines Posters ist, dass die Spielregeln während des ganzen Workshops präsent sind und jederzeit darauf verwiesen werden kann. In der Regel ist es ausreichend, grundlegende Gesprächsregeln festzulegen, bei Bedarf können auch andere Regeln hinzugenommen werden. Bewährt haben sich acht Regeln, die so aufgebaut sind, dass sie nicht nur einschränken oder verbieten, sondern Begründungen liefern und Alternativen aufzeigen (s. Box 2).

Bei Bedarf können von den Teilnehmer(innen) am UrbanUtopiaLAB noch andere Regeln dazu genommen werden, wobei mehr als 10 Regeln unübersichtlich sind und überfrachten, und dadurch hemmend oder beschränkend wirken (können). Wichtig ist es, deutlich zu formulieren, dass diejenigen, die sich nicht an die verbindlichen Spielregeln halten, vom UrbanUtopiaLAB ausgeschlossen werden können. 


\begin{tabular}{|c|c|}
\hline Nr. & Spielregel \\
\hline 1 & Jede Stimme zählt. \\
\hline 2 & $\begin{array}{l}\text { Ausreden lassen, den anderen zuhören und nicht ins Wort fallen - nur eine(r) } \\
\text { spricht. }\end{array}$ \\
\hline 3 & Jede(r) spricht für sich selbst. \\
\hline 4 & So präzise wie möglich - Verallgemeinerungen vermeiden. \\
\hline 5 & Monologe vermeiden - andere wollen auch etwas sagen. \\
\hline 6 & $\begin{array}{l}\text { Kritisches Nachfragen erlaubt - aber am besten gleich mit Alternativen oder } \\
\text { Lösungsvorschlägen. }\end{array}$ \\
\hline 7 & $\begin{array}{l}\text { Eigene Frustration oder Enttäuschung nicht in persönliche Angriffe oder Kil- } \\
\text { lerphrasen verwandeln - stattdessen gern als Fragen mit den anderen teilen } \\
\text { und gemeinsam Veränderungsmöglichkeiten finden. }\end{array}$ \\
\hline 8 & $\begin{array}{l}\text { Gemeinsam Verantwortung für das Ganze übernehmen - damit lassen sich } \\
\text { mehr Möglichkeiten denken und entwickeln. }\end{array}$ \\
\hline
\end{tabular}

Box 2: $\quad$ Acht ,Spielregeln“ für die Phasen 1 und 2 im UrbanUtopiaLAB.

Für die Einführung in das UrbanUtopiaLAB (s. Box 1), also für die Erläuterung des Vorgehens, der Leitfrage(n), des Projektkontextes und der verfolgten Absichten sind rund 20 Minuten vorzusehen. Es hat sich bewährt, bei einer Anzahl von bis zu 10 Teilnehmer(innen) eine kurze Vorstellungsrunde durchzuführen, die dann im Zeitplan berücksichtigt werden muss. Die Teilnehmer(innen) nennen dabei ihren Namen und ihren Herkunftsort. Wichtig ist, dass ein Bezug zu der jeweiligen Stadt, zum Quartier oder zur Region hergestellt wird, in dem das Urban UtopiaLAB durchgeführt wird. Schließlich sagen die Teilnehmer(innen), weshalb sie zum UrbanUtopiaLAB gekommen sind.

Am Ende dieses Veranstaltungsteils werden die Teilnehmer(innen) auf die Diskussionsinseln aufgeteilt. Beabsichtigt ist es, in möglichst heterogenen Gruppen zu diskutieren, in denen die Teilnehmer(innen) sich untereinander kennenlernen. Deshalb erfolgt die Gruppenbildung am besten nach dem Zufallsprinzip. Verfahren wie das Abzählen der Reihe nach sind schnell, einfach und transparent. Die Anwesenden zählen von 1-X, die Teilnehmer(innen) mit der gleichen Zahl bilden eine Gruppe, wobei X die Anzahl der Diskussionsinseln ist (s. Abschnitt 4.1.2). Falls im Zuge der Vorbereitungen (s. Abschnitt 4.1.1) entschieden wurde, ,Themeninseln' zu bilden, erfolgt die Gruppeneinteilung nicht nach dem Zufallsprinzip, sondern interessengeleitet. In diesem Fall sollte es aber mindestens eine Diskussionsinsel geben, die eine themenoffene Diskussion anbietet, also einen Austausch, der nicht vordefinierten Themen folgt. 
Diese erste Ansprache zu Beginn darf in ihrer Bedeutung nicht unterschätzt werden, da hier die wissenschaftlichen Grundlagen, die Ziele, das Vorgehen und die Funktion des Emo/Action-Mappings im Rahmen des UrbanUtopiaLABs sowie die Aufgaben der Teilnehmer(innen) erläutert werden. Diese Ansprache zielt darauf $a b$, dass die Teilnehmer(innen) eventuelle Vorbehalte ablegen und sich von Anfang an für die Herangehensweise öffnen, indem ihnen die Bedeutung und Zielrichtung des Ansatzes verständlich wird. Dadurch soll auch von Anfang an eine Atmosphäre des gegenseitigen Respekts und der Offenheit geschaffen werden, in der es nicht um die Bestimmung von richtig oder falsch geht, sondern um Austausch, Kommunikation und ein gegenseitiges Zeigen der jeweiligen Alltagswelt - weniger aus einer funktionalen Perspektive, sondern vielmehr aus einer emotionalen Verwobenheit mit Orten, Plätzen oder Routen in der Stadt, im Quartier, in der Region.

Zentral in der inhaltlichen Einführung ist auch die Erläuterung des Namens UrbanUtopiaLAB (s. Kapitel 2), weil die Erfahrung gezeigt hat, dass die Bezeichnung UrbanUtopiaLAB bei einzelnen Teilnehmer(innen) Irritationen hervorrufen kann. Das wiederum ist durchaus intendiert, da damit ein erstes Nachdenken angeregt werden kann, was auch schon die erste Auseinandersetzung mit den Inhalten und Zielen darstellt. Dabei kann die Erläuterung entlang der miteinander kombinierten Einzelbegriffe ,Urban “ - ,Utopia “-,LAB“ (s. Kapitel 2) erfolgen, womit gleichzeitig nochmals Ziel und Idee der Methode kommuniziert werden können. Voraussetzung ist natürlich, dass die einführende Person mit den zugrunde gelegten Konzepten ausreichend vertraut ist. Wie die Erfahrungen zeigen, wirken solche theoretischen Erläuterungen zu Beginn der Veranstaltung nicht abschreckend auf die Teilnehmer(innen). Vielmehr können sie sogar dazu beitragen, dass alle Anwesenden die gleiche Verständnisgrundlage teilen und dadurch eine respektvolle Begegnung gefördert wird wie auch die Bereitschaft, sich auf neues Denken einzulassen und die ,Köpfe zu öffnen'.

\subsubsection{METHODE (Phase 1) | StadtERFORSCHEN: Emo/Action-Mapping: Orte und Räume kollektiv kartieren}

Durchgeführt wird das Emo/Action-Mapping an den Diskussionsinseln mit den vorgefertigten UrbanUtopiaLAB-Icons (s. Abschnitt 4.1.3, Abb. 3, 4), wobei fehlende Begriffe oder Symbole von den Teilnehmer(inne)n während des Emo/ Action-Mappings auf den bereitgestellten Blanko-Icons grafisch oder schriftlich dargestellt werden können.

Bei jeder Diskussionsinsel ist eine(n) Moderator(in) (s. Abschnitt 2.1). Die Moderator(inn)en fordern die Teilnehmer(innen) nacheinander auf, ihre persönlich bedeutsamen Orte, Plätze, Räume etc. mit einem Icon des UrbanUtopiaLAB-Sets zu markieren und kurz zu erläutern, warum dieser Ort mit diesem Icon belegt 


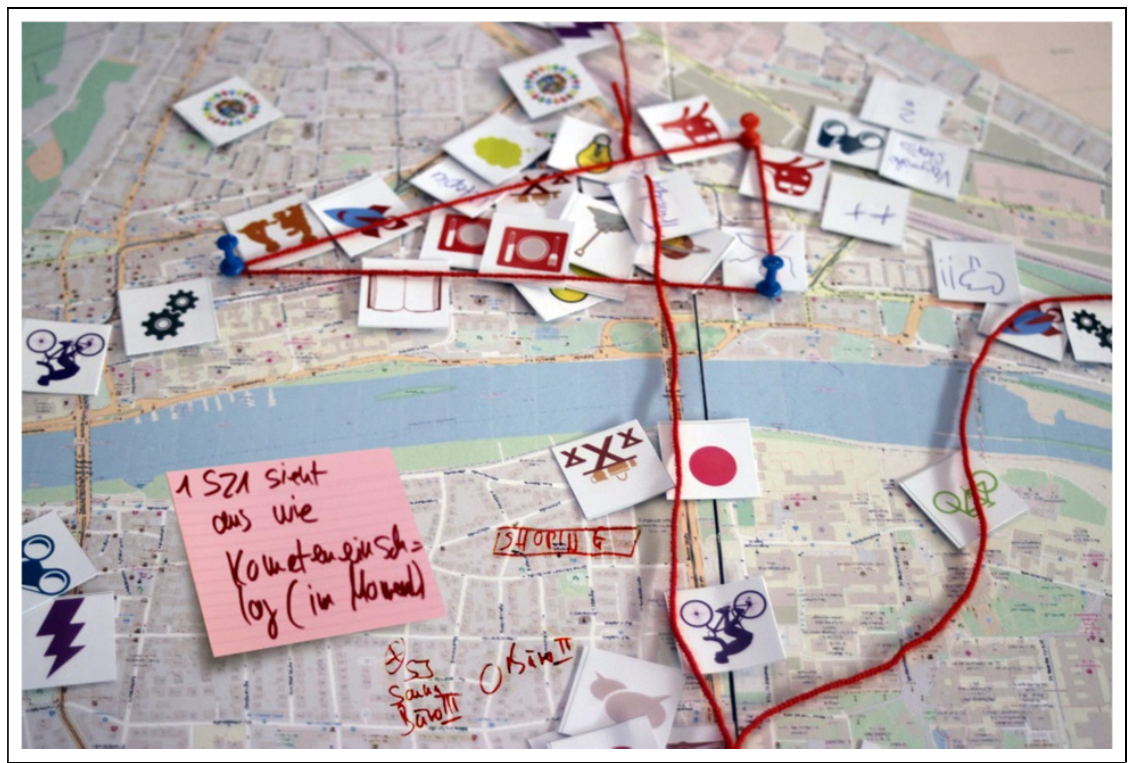

Abbildung 4: Emo/Action-Map mit UrbanUtopiaLAB-Icons: Die Karte zeigt einen Ausschnitt der Stadt Heidelberg im Prozess des Emo/Action-Mappings während eines UrbanUtopiaLABs. Zu sehen sind ein roter Wollfaden, mit dem die genutzten Wege in der Stadt gekennzeichnet sind, verschiedene Urban UtopiaLAB-Icons mit Ausführungen zu ihren Bedeutungen auf Moderationskarten sowie handschriftliche Ergänzungen und Erläuterungen direkt auf der Grundkarte, wodurch die Aktionsräume der Teilnehmer(innen) mit ihren bedeutsamen Orten sichtbar, kommunizierbar und aushandelbar werden. (C) Krishan Rajapakshe, Heidelberg 2018.

wird. Ergänzt werden die markierten Orte durch Strecken oder Wege, die mit Stiften direkt auf der Karte eingezeichnet oder mit Wollfäden markiert werden, wodurch alle Teilnehmer(innen) Einblicke in die Aktionsräume der anderen erhalten (s. Abb. 4).

Alle Emo/Action-Icons (vorgefertigte oder selbst erstellte) können dabei entsprechend ihrer individuellen positiven, negativen oder neutralen Bewertung des markierten Ortes von den Teilnehmer(inne)n mit einem $(+),(-)$ oder $(+/-)$ markiert werden. Auch die Strecken oder Wege können entlang dieses Einordnungsschemas bewertet werden. 
Die Markierungen werden entlang des folgenden Schemas eingeordnet und kommentiert:

- (+) Wohlfühlorte: Orte, die aus einem Grund positiv belegt sind, mit kurzer Erläuterung dazu, warum diese Orte positiv belegt sind (bspw. Funktionen, Tätigkeiten, Socialising, Freunde, Familie).

- (-) Orte, die (eher) negativ belegt sind oder die gemieden werden, ebenfalls mit kurzer Erläuterung der Gründe.

- (+/-) Orte, die aus einem Grund (immer wieder oder nicht mehr) aufgesucht werden, aber vielleicht doch nicht eindeutig emotionalisiert sind. Nach Möglichkeit sollten die markierten Orte nicht als völlig neutral charakterisiert werden. Gegebenenfalls muss ein(e) Teilnehmer(in) von der Moderation aufgefordert werden, den Ort zu kontextualisieren oder eine kurze Geschichte dazu zu erzählen.

- $\mathrm{Zu}$ beachten ist auch, dass ein und derselbe Ort im Tagesgang oder im Wochen- bzw. Jahresgang mit unterschiedlichen Emotionalitäten belegt sein kann. Dies soll dann zusätzlich auf dem gewählten Icon oder durch die Kombination mehrerer Icons kenntlich gemacht werden.

Je nach Anzahl der Teilnehmer(innen) pro Diskussionsinsel (s. Abschnitt 4.1.2) haben die Einzelnen während des Emo/Action-Mappings nur einen begrenzten Zeitrahmen zur Verfügung, um ihre Orte auf der Karte mit einem oder mehreren Emo/Action-Icons zu markieren und eine dazugehörige Erläuterung oder Geschichte zu kommunizieren. Der Zeitrahmen pro Teilnehmer(in) wird von den Moderator(inn)en zu Beginn des Mappings festgelegt und mitgeteilt. Generell obliegt es den Moderator(inn)en, die zur Verfügung stehende Zeit pro Teilnehmer(in) zu kontrollieren - diese ergibt sich abhängig von der Anzahl der Teilnehmer(innen) bei der Diskussionsinsel.

Den Moderator(inn)en kommt zudem die Aufgabe zu, während des Emo/ActionMappings die Teilnehmer(innen) dazu aufzufordern, ihre Ausführungen zu ihren Orten sowie weiterführende Ideen zur übergeordneten Leitfrage „Wie wollen wir in Zukunft miteinander leben?" stichwortartig auf Moderationskarten zu schreiben und an die Moderationswände zu pinnen. Damit auch im Nachhinein der Zusammenhang zwischen den Icons auf der Karte und den Moderationskarten an der Pinnwand hergestellt werden kann, werden die Orte von denjenigen, die sie markieren, mit fortlaufend aufsteigenden Zahlen nummeriert. Die zu den Orten gehörenden Moderationskarten werden ebenfalls mit der entsprechenden Nummer markiert. Die Ideen und Aussagen an den Moderationswänden werden fortlaufend thematisch geclustert. Da am Ende des Emo/Action-Mappings die Ideen und Aussagen nochmals geclustert werden, ist in diesem Moment eine ungefähre Zuordnung ausreichend. 
$\mathrm{Zu}$ Dokumentations- und Auswertungszwecken und mit dem Einverständnis aller Teilnehmer(innen) können die Gespräche an den einzelnen Diskussionsinseln auch in Form von Ton- oder Filmaufnahmen aufgezeichnet werden (s. Abschnitt 4.2, 4.3). Ergänzend können die Moderator(inn)en weitere als wichtig oder interessant erachtete Aussagen der einzelnen Teilnehmer(innen) auf Moderationskarten festhalten und diese an die Moderationswände pinnen. Diese Karten werden dann entweder den thematischen Clustern zugeordnet, die schon an der Pinnwand vorhanden sind, oder es werden dafür neue eröffnet. Zusätzlich steht allen Teilnehmer(inne)n immer die Möglichkeit offen, Gedanken, Ideen oder Informationen auf der Tischdecke aus Braunpapier zu notieren und, falls eindeutig zuordenbar zu schon mit Icons markierten Orten, diese ebenfalls mit den entsprechenden Nummern zu versehen.

Während des Mappings sind Zwischenfragen und Kommentare anderer Teilnehmer(innen) zugelassen. Falls sich daraus eine umfassende Diskussion ergibt, ist es Aufgabe der Moderator(inn)en, nach angemessenem Austausch und entlang eines kontrollierten Zeitmanagements das Wort wieder zurück an die Person zu geben, die gerade an der Reihe ist. Meist wächst der Diskussionsbedarf in dem Maße, in dem die entstehenden kollektiven (Stadt-)Karten und Aussagen auf den Moderationskarten an Detail gewinnen bzw. je detaillierter diese von den jeweiligen Sprecher(inne)n ausgeführt und festgehalten werden. Zeigt sich bei der Durchführung des Mappings, dass von Teilnehmer(innen) immer wieder Symbole im Set der UrbanUtopiaLAB-Icons vermisst werden, fragen die Moderator(inn)en nach Möglichkeit direkt während oder im Anschluss an das Mapping bei den Teilnehmer(innen) nach, wie diese Icons aussehen könnten. In jedem Fall notieren sie, was vermisst wurde, und geben diese Information im Nachgang der Veranstaltung an das Projektteam, damit gegebenenfalls weitere Icons für nächste Emo/ Action-Mappings entwickelt werden können.

Nachdem alle Beteiligten Gelegenheit hatten, ihre persönlichen Orte mit Icons zu kartieren und zu erläutern sowie ihre eigenen Utopien zu skizzieren, lässt der/die Moderator(in) den fließenden Übergang zu in die offene Diskussion an der Diskussionsinsel. Diese Diskussion sollte sich um die übergeordnete Leitfrage „Wie wollen wir in Zukunft miteinander leben?" drehen sowie um die daran konsequenterweise direkt anknüpfende Frage „Wie können wir das erreichen?“ Auf der Grundlage der im Prozess des Emo/Action-Mappings entstandenen kollektiven Karte sowie der Aussagen auf den Moderationskarten ist es den Moderator(inn)en an den Diskussionsinseln möglich, bei Bedarf diese Diskussion immer wieder anzustoßen bzw. voranzutreiben sowie schon formulierte Gedanken nochmal in die Diskussion einzubringen. Dabei werden die beim Mapping geschilderten individuellen Wahrnehmungen und Utopien mit anderen Perspektiven in der Gruppe konfrontiert und kritisch reflektiert, um so Schritt für Schritt entweder gemeinsame Positionen und Utopien zu entwickeln oder Unvereinbarkeiten zu schärfen. 
Letzteres geschieht, um einerseits herauszufinden, wie unvereinbar sie tatsächlich sind, oder ob es nicht doch Anknüpfungspunkte zwischen solchen Positionen gibt, und um andererseits das Unvereinbare in Positionen möglichst genau zu benennen. Dadurch werden Veränderungspotenziale herausgearbeitet, die wiederum Ausgangspunkte für urbane Interventionen (s. Abschnitt 3.3) und den Policy Brief (s. Abschnitt 3.4) werden. Da das Emo/Action-Mapping eine entscheidende kreative Phase des Neudenkens und der gemeinsamen Formulierung von gesellschaftlich relevanten Fragestellungen darstellt, soll genügend Zeit- und Freiraum für die offene Diskussion gewährt werden.

15 bis 20 Minuten vor Ende der Emo/Action-Mapping-Phase (s. Box 1) werden die sich aus den formulierten Aussagen, Utopien und Mappings ergebenden übergeordneten Themenfelder identifiziert und geclustert, und es werden Argumentationslinien rekonstruiert. Auch wenn dieser Schritt mit allen in der Gruppe gemeinsam ausgehandelt werden soll, müssen sich die Moderator(inn)en während des vorausgehenden Emo/Action-Mappings darauf einstellen, dass sie wichtige Impulse der Erzählungen und Diskussionen nochmals wiedergeben und Vorschläge für die Bündelung von Ideen und die Entwicklung von Argumentationslinien geben müssen.

Im Anschluss an diesen Schritt priorisieren die Teilnehmenden der Diskussionsinsel die einzelnen Nennungen mittels einer Mehrpunkteentscheidung (Verteilung von Klebepunkten). Die Priorisierung erfolgt entlang der konkreten Frage „Welches sind die für mich interessantesten Themen, die zu Stellschrauben werden für die Beantwortung der übergeordneten Leitfrage: Wie wollen wir in Zukunft miteinander leben?" Dabei erhält jede(r) Teilnehmer(in) mindestens fünf Klebepunkte, höchstens jedoch so viele Punkte, die der Hälfte der Anzahl Nennungen entspricht (z. B. bei 20 Nennungen: 10 Klebepunkte pro Person). Die Klebepunkte dürfen von den Einzelnen auch kumuliert verteilt werden, müssen sich jedoch auf mindestens drei Nennungen verteilen. Eine Weitergabe der Punkte an andere Teilnehmende ist nicht gestattet. Diese Priorisierung führt nicht dazu, dass Nennungen oder Themen wegfallen, sondern sie dient einer Orientierung für das Projektteam bei der Überlegung, für welche Themen sich eventuell Arbeitsgruppen finden lassen, mit denen die Entwicklung einer urbanen Intervention (s. Abschnitt 3.3) möglich ist, und welche Themen im Policy Brief (s. Abschnitt 3.4) besonders stark berücksichtigt werden sollten.

Die abschließende Vorstellung im Plenum soll nach Möglichkeit durch eine(n) von der Gruppe bestimmte(n) Teilnehmer(in) erfolgen, kann aber auch durch die Moderator(inn)en vorgenommen werden. Sie umfassen die Erzählungen an der Diskussionsinsel, die besondere Einsichten in die ,Stadt der anderen' gegeben haben, und die Aha-Momente, hervorgerufen durch die verschiedenen Veränderungsideen und Utopien. 


\subsubsection{METHODE (Phase 2)| StadtERKUNDEN: Orte und Räume begehen und sinnlich wahrnehmen}

Phase 2 findet in denselben Kleingruppen statt wie Phase 1, d. h., beim zweistündigen Stadtgang erkunden die Teilnehmer(innen) die Stadt in der Regel in den Gruppen, in denen sie das Emo/Action-Mapping durchgeführt haben, da hier schon Ideen, Beobachtungen und Gefühle offenbart und ausgetauscht wurden, was für die Phase des StadtERKUNDENs eine gute Basis ist. Auch beim StadtERKUNDEN zeigen sich die Teilnehmer(innen) gegenseitig ihre Orte - jetzt allerdings im realen Raum der Stadt, des Quartiers, der Region. Handlungsleitend ist, den Raum gemeinsam zu erkunden und ihn aus den im Emo/Action-Mapping neu gewonnenen Blickwinkeln zu beobachten. Dabei erfolgt eine Fortsetzung der Diskussion vom Vormittag, gleichzeitig aber auch ein Perspektivenwechsel von der zweidimensionalen Kartenansicht des Emo/Action-Mappings zur realweltlichen Komplexität und zur Wahrnehmungserweiterung durch die Aktivierung aller Sinne. Dies trägt dazu bei, auch scheinbar Vertrautes neu zu sehen bzw. mit allen Sinnen wahrzunehmen und schafft eine nochmal neue Grundlage, um sich über die Aha-Momente und neu gewonnenen Erkenntnisse aus dem Mapping auszutauschen.

Die Route und die aufgesuchten Orte werden in der Regel von den jeweiligen Gruppen selbst gewählt. Damit müssten die Moderator(inn)en also keine fertigen Routen für den Stadtgang vorbereiten. Falls aber keine Vorschläge aus der Gruppe kommen und allgemeine Ratlosigkeit herrscht, sollten sie Vorschläge bereit halten oder eine Entscheidung für eine Route herbeiführen. Liegen die ausgewählten Orte oder Areale nicht fußläufig vom Veranstaltungsort entfernt, können die Gruppen, sofern es der zeitliche Rahmen erlaubt, mit dem Rad, öffentlichen Verkehrsmitteln o. Ä. dorthin gelangen.

An den besuchten Orten werden die Teilnehmer(innen) aufgefordert, sich spielerisch und experimentell auf den Ort einzulassen und diesen wortwörtlich mit allen Sinnen zu erkunden, d. h., sinnessensibel ,ins Feld‘ zu gehen. Dabei sollen sie den Ort ganz bewusst sehen, hören, riechen, schmecken, tasten, fühlen und dazu einen Sinn nach dem anderen an die erste Stelle der Reiz- und Informationsaufnahme setzen. Steht der Geruchssinn an erster Stelle, dann konzentriert sich die Person darauf und die Augen werden geschlossen, die Ohren zugehalten und so weiter. Unterstützend kann hierbei ein Arbeitsblatt ausgeteilt werden, das die fünf Sinne abbildet sowie dazu passende Fragen stellt (z. B. „Was schmeckst du an diesem Ort?"). Die Urban Emotions und sinnlichen Wahrnehmungen im Raum werden von den Teilnehmer(innen) als Eindrücke und Gefühle artikuliert und als digitale Sprachnachricht oder als Filmaufnahme aufgezeichnet. Mit den Urban Emotions und sinnlichen Wahrnehmungen verändert sich der bekannte, alltägliche Zugang zum Raum nochmals, wodurch ,blinde Flecken' offengelegt und Veränderungspotenziale erkannt werden können. 
Wichtig ist es, die Strecke und markante Orte, an denen in der Gruppe diskutiert wurde, fotografisch oder filmisch zu dokumentieren, um diese dann im Plenum vorstellen und diskutieren zu können. Auch wenn heute üblicherweise fast alle ein technisch dazu geeignetes Mobilgerät in der Tasche tragen, sollte die Moderation vorsorglich entsprechend ausgerüstet sein (s. Abschnitt 3.2). Je nach Thema und besuchtem Ort kann auch eine Konfrontation von Passant(inn)en oder Anwohner(inne)n Teil des Rundgangs sein. Diese kann in Form von Interviews oder kleinen Interventionen stattfinden, für die es natürlich keine Blaupause gibt: Hier ist die Kreativität der Moderator(inn)en sowie der Teilnehmer(innen) selbst gefragt und die Dokumentation überaus wichtig.

Zum Schluss, nach der Rückkehr der Gruppen vom Rundgang, sollte den Teilnehmer(inne)n zunächst die Gelegenheit gegeben werden, die Eindrücke des Rundgangs durch Ergänzungen auf den Emo/Action-Karten an den Diskussionsinseln festzuhalten. Dann werden im Plenum die Eindrücke und Einsichten der einzelnen Gruppen ausgetauscht und diskutiert. Dies kann anhand von Filmaufnahmen, digitalen Sprachnachrichten, Fotos und natürlich mündlichen Berichten erfolgen.

Die Aufgabe der Moderator(inn)en ist es hierbei, darauf zu achten, dass während der Diskussion auch eine Einordnung der Beobachtungen in den übergeordneten thematischen Zusammenhang des UrbanUtopiaLABs und des Projekts erfolgt. Die Frage, wie es weitergeht, sollte ebenfalls adressiert werden. Dabei sollte allerdings vermieden werden, die Teilnehmer(innen) in eine vorgefertigte Agenda zu drängen, denn von ihrer Initiative und Eigenmotivation hängt es schließlich ab, ob und wie das UrbanUtopiaLAB weitergehen kann. Oft entstehen jedoch konkrete Pläne zur Weiterarbeit nicht im Abschlussplenum selbst, sondern in kleineren Runden erst nach dem offiziellen Ende des UrbanUtopiaLABs und der Verabschiedung der Teilnehmer(innen). Besonders weniger extrovertierte bzw. durchsetzungsfähige Teilnehmer(innen) sind oftmals in einem intimeren Rahmen eher bereit, ihre im Laufe des Tages gereiften Ideen mitzuteilen, zu diskutieren und Ideen für zukünftige Aktivitäten zu entwickeln. Deshalb ist es außerordentlich wichtig, für eine solche ,After-LAB'-Fortsetzung einen geeigneten Raum zur Verfügung zu stellen, am besten vor Ort. Das Bereitstellen von Speisen und Getränken kann diesen Prozess ungemein fördern.

\subsection{DOKUMENTATION | UrbanUtopiaLAB - StadtERFORSCHEN (Phase 1) und StadtERKUNDEN (Phase 2)}

Eine umfassende Dokumentation ermöglicht eine Weiterarbeit mit dem, was an Argumenten, Erzählungen und Einsichten, Interventions- und Veränderungsideen, Utopien, Beobachtungen und Wahrnehmungen während des Emo/ActionMappings (s. Phase 1 StadtERFORSCHEN, Abschnitte 3.1, 4.2.2) und während 
des Raumerkundens (s. Phase 2 StadtERKUNDEN, Abschnitte 3.2, 4.2.3) mündlich und schriftlich formuliert wurde. Im weiteren Verlauf des UrbanUtopiaLABs sind diese Ergebnisse dann wichtige Bausteine für die Konzipierung und Realisierung der urbanen Interventionen (s. Phase 3 StadtEXPERIMENTIEREN, Abschnitt 3.3) und für die Entwicklung und Formulierung des Policy Briefs (s. Phase 4 StadtMITENTSCHEIDEN, Abschnitt 3.4).

Um die Äußerungen und Ergebnisse inhaltlich festzuhalten und später nachvollziehen zu können, werden deshalb alle visualisierten Ergebnisse, also die kollektiv erstellten Emo/Action-Karten, die von den Teilnehmer(inne)n und Moderator(inn)en beschriebenen Moderationskarten sowie das Braunpapier auf den Tischen getrennt nach Diskussionsinseln fotografisch dokumentiert sowie physisch gesammelt und archiviert, auch die Ton- und Filmaufnahmen werden gesichert. Fotodokumentation wie Ton- und Filmaufnahmen ermöglichen die Weiterbearbeitung, Auswertung und Dokumentation durch die Wissenschaftler(innen) des Projektteams wie auch die Aufbereitung der so erzeugten Ergebnisse für die urbanen Interventionen und den Policy Brief. Da es sich hierbei um große Mengen und vor allem sensible Daten handelt, deren Schutz gewährleistet sein muss, ist es ratsam, passende und entsprechend gesicherte Archive und Speichermedien einzurichten.

Damit die Teilnehmer(innen) auch für die nächsten Phasen des UrbanUtopiaLABs eingeladen und Aussagen über die soziodemografische Zusammensetzung gemacht werden können, werden die Teilnehmer(innen) gebeten, sich in vorbereitete Teilnehmerlisten mit Name und E-Mail-Adresse (falls nicht vorhanden alternativ mit Postadresse, Social Media-Identität oder Handynummer) einzutragen und gegebenenfalls zusätzlich ein Kästchen anzukreuzen, womit die Zustimmung gegeben wird, dass Ergebnisse, weitere Informationen und Einladungen aus dem Projekt zugeschickt werden dürfen.

\section{$5 \quad$ Reflexion und Fazit}

Mit der Methode des UrbanUtopiaLABs wird es möglich, die Geschichte vom „selbst Sprechen und Gehört werden“ neu zu entwickeln und dadurch eine neue soziale Realität zu kreieren, in der eben jede Stimme gehört werden kann, mit dem Ziel, eine auf den Raum des gelebten Miteinanders bezogene politische und soziale Transformation herbeizuführen. Über Instrumente wie die der urbanen Intervention (s. Abschnitt 3.3) und des Policy Briefs (s. Abschnitt 3.4) werden Ideen und umsetzungsbezogene Ergebnisse generiert, immer mit dem Ziel, sie direkt mit Verantwortlichen zu diskutieren und weiterzuentwickeln. Ziel des UrbanUtopiaLABs ist es, diffuse Wahrnehmungen und Einstellungen, die Vergangenheit, eine daraus resultierende Pfadabhängigkeit und Gegenwart zu reflek- 
tieren und zukunftsorientierte Diskurse in der Gesellschaft hervorzurufen. So soll eine Form der Öffentlichkeit geschaffen werden, die oszilliert zwischen der ,Bühne', einem Sichtbarwerden durch den erzeugten öffentlichen Raum, und der ,Werkstatt', in der mit einer überschaubaren Gruppe intensiv, konzentriert und zielgerichtet reflektiert wird. In Gang gesetzt wird ein Prozess, indem weniger über andere gesprochen wird als vielmehr miteinander.

In den Feedbackrunden im Plenum oder in informellen Einzelgesprächen, die Teil der Abschlussrunde eines UrbanUtopiaLABs sind, wurde deutlich, dass die Teilnehmer(innen) sich von UrbanUtopiaLAB angesprochen fühlten, dass sie die Grundidee schätzten und dass sie bei sich selbst und bei anderen eine Veränderung in der Perspektive, im Denken und im Engagement feststellten. Hervorgehoben wurden die Art und Weise der Diskussion und die inhaltliche Kommunikationsqualität, die als wertschätzend, achtsam und respektvoll empfunden und meist mit dem Emo/Action-Mapping in Verbindung gebracht wurden. Moderator(inn)en berichteten von deutlich gelasseneren und weniger konfrontativen Diskussionen der Teilnehmer(innen) im Vergleich zu anderen partizipativen Formaten. Hervorzuheben ist außerdem die umfassende Eigenständigkeit bei der Entwicklung von Ideen sowie, dass zu den Ideen direkt Umsetzungsmöglichkeiten entwickelt würden.

Bislang liegen noch keine expliziten Erfahrungen zu Situationen vor, in denen aufgrund $\mathrm{zu}$ hoher Konfliktpotenziale das Vorgehen nicht erfolgreich durchgeführt werden konnte. Allerdings sollten generell Konstellationen vermieden werden, in denen zwischen den Teilnehmer(inne)n stark verhärtete Fronten verlaufen, die einen konstruktiven Austausch und damit einen gemeinsamen kreativen Prozess im Vorhinein unmöglich machen.

Das Vorgehen ist darauf ausgelegt, dass die Möglichkeit geschaffen wird, dass jede(r) mit jeder $/ \mathrm{m}$ direkt sprechen kann und nicht von Anfang an bestimmte Kommunikationsformen festgelegt werden. Trotzdem spielt die Bedeutung des sprachlichen Ausdrucks keine untergeordnete Rolle. Das UrbanUtopiaLAB wurde in einem Team aus geflüchteten und nicht-geflüchteten Personen entwickelt und war in der Phase seiner Entwicklung schwerpunktmäßig, wenn auch nicht ausschließlich, Fragen des Zusammenlebens in der Stadt in Zeiten von Migration und Flucht gewidmet. Da sich nicht alle Teilnehmer(innen) in den Verkehrssprachen Deutsch oder Englisch auf dem gleichen sprachlichen Niveau befanden, wurde im Plenum zweisprachig gesprochen oder aber eine Flüsterübersetzung in Deutsch oder Englisch - je nach Zusammensetzung der Gruppe - angeboten. In den Kleingruppen, beispielsweise während des Emo/Action-Mappings, wurde Gesagtes meist direkt durch die Teilnehmer(innen) selbst oder aber andere Gruppenmitglieder ins Deutsche oder Englische übersetzt, wodurch auch lange und komplexe, teils konfliktbehaftete Gesprächseinheiten bewältigt werden konnten. Der aufmerksame Umgang mit Sprache kann Sichtbarkeit und Handlungsmacht 
für diejenigen schaffen, deren Stimmen noch mehr Gehör finden sollten. Auch bei der ,Übersetzung' realweltlicher Erfahrungen in das Format einer maßstabsgetreuen Karte halfen sich die Teilnehmer(innen) gegenseitig.

Die Absicht des UrbanUtopiaLABs ist es nicht, eine große Einheitsutopie zu generieren, sondern vielmehr die Vielfalt und Diversität von Positionen und Ideen herauszuarbeiten und gegebenenfalls miteinander in Beziehung zu setzen. Dies soll die Grundlage schaffen für eine ,Kultur des Austauschs` zwischen allen Involvierten, und die so gefundenen und formulierten ,Denkstellen' sollen öffentlich diskutierbar gemacht werden. Das UrbanUtopiaLAB stellt eine Möglichkeit inter- und transdisziplinärer Politikberatung dar, die der Komplexität einer transversalen Stadt und Gesellschaft Rechnung trägt.

\section{Dank}

Die Autorinnen danken Bernd Böttger und Alexandra König für die Diskussionen über frühere Textfassungen. Insbesondere möchten sie Martina Hilligardt und Christian Schmutz sowie zwei anonymen Gutachter(inne)n für die konkreten Hinweise zur Verbesserung des Textes im Rahmen des internen und externen Reviews danken. Besonderer Dank gebührt Jaro Eiermann, Doris Mayer und Krishan Rajapakshe, die mit ihrer Praxisexpertise die Entwicklung des UrbanUtopiaLAB kritisch reflektierend, ideenreich und tatkräftig mitgestaltet haben. Schließlich danken die Autorinnen den beiden Herausgebenden, Rico Defila und Antonietta Di Giulio, für ihre Rückmeldungen zum Text.

\section{Literatur}

Bloch, E. (1990). Das Prinzip Hoffnung. 3 Bde., 3. Aufl. Frankfurt a. M.: Suhrkamp.

CASS \& ProClim- (1997). Forschung zu Nachhaltigkeit und Globalem Wandel - Wissenschaftspolitische Visionen der Schweizer Forschenden. Bern: ProClim-/SANW. https:// naturwissenschaften.ch/service/publications/75640-visionen-der-forschenden. Zugegriffen am 21.01.2018.

Foucault, M. (1966/2005). Die Heterotopien. In M. Foucault, Die Heterotopien - Der utopische Körper. Radiovortrag von 1966 (S. 9-22). Frankfurt a. M.: Suhrkamp.

Landauer, G. (1907). Die Revolution. In M. Buber (Hrsg.), Die Gesellschaft. Sammlung sozialpsychologischer Monographien. Bd. 13. Frankfurt a. M.: Rütten \& Loening.

Musil, R. (2002). Der Mann ohne Eigenschaften. Bd. 1. Erstes und Zweites Buch. 16. Aufl. (Hrsg. von Adolf Frisé). Reinbek bei Hamburg: Rowohlt. 
Schammann, H., \& Kühn, B. (2016). Kommunale Flüchtlingspolitik in Deutschland. Gute Gesellschaft - soziale Demokratie \#2017 plus. Bonn. https://ibrary.fes.de/pdf-files/ wiso/12763.pdf. Zugegriffen am 25.01.2019.

United Nations (2015). Transforming our World: The 2030 Agenda for Sustainable Development. https://sustainabledevelopment.un.org/content/documents/21252030\%20Agenda $\% 20$ for\%20Sustainable\%20Development\%20web.pdf. Zugegriffen am 25.01.2019.

Wendel, K. (2014). Unterbringung von Flüchtlingen in Deutschland: Regelungen und Praxis der Bundesländer im Vergleich. https://www.proasyl.de/wp-content/uploads/2014/ 09/Laendervergleich_Unterbringung_2014-09-23_02.pdf. Zugegriffen am 25.01.2019.

West, C. (2014a). Zwischen kulturellem Pluralismus und Transkulturalität - Postmoderne Momente im Migrationsdiskurs. In P. Gans (Hrsg.), Räumliche Auswirkungen der internationalen Migration (S. 92-126). Hannover: Forschungsberichte der ARL 3. http:// nbn-resolving.de/urn:nbn:de:0156-07540. Zugegriffen am 07.03.2019.

West, C. (2014b). Deutschland in der Integrationsfalle? - Integrationspolitische Perspektivenwechsel, Herausforderungen und Chancen für Wissenschaft, Politik und Planung. In P. Gans (Hrsg.), Räumliche Auswirkungen der internationalen Migration (S. 292-310). Hannover: Forschungsberichte der ARL 3. http://nbn-resolving.de/urn:nbn:de:0156-07 540. Zugegriffen am 07.03.2019.

West, C. (2017). Dimensionen des individuellen und gesellschaftlichen Wandels: Die grundlegenden Orientierungen, geschlossen-offen' und ,konkret-abstrakt' als Determinanten der Stadtentwicklung in Barcelona (1986-2005). Mannheim: MADOC. https://ub-madoc.bib.uni-mannheim.de/42814. Zugegriffen am 21.01.2018.

West, C. (2018). „Wissen to Go“ - transdisziplinär-transformative Lehre als „Reallabor im Kleinen“. In R. Defila \& A. Di Giulio (Hrsg.), Transdisziplinär und transformativ Forschen. Eine Methodensammlung (S. 329-373). Wiesbaden: Springer VS. doi: 10.1007/ 978-3-658-21530-9 15.

West, C. (2019). Transversal City and Transtopia - Reflecting and Analysing Migration, the City, and the Urban after the Postmigrant City. Special edition Social Geography: Die ,postmigrantische Stadt“ - urbanes Zusammenleben aus der Perspektive der Migration. Geographia Helvetica. (im Druck).

Open Access Dieses Kapitel wird unter der Creative Commons Namensnennung 4.0 International Lizenz (http://creativecommons.org/licenses/by/4.0/deed.de) veröffentlicht, welche die Nutzung, Vervielfältigung, Bearbeitung, Verbreitung und Wiedergabe in jeglichem Medium und Format erlaubt, sofern Sie den/die ursprünglichen Autor(en) und die Quelle ordnungsgemäß nennen, einen Link zur Creative Commons Lizenz beifügen und angeben, ob Änderungen vorgenommen wurden.

Die in diesem Kapitel enthaltenen Bilder und sonstiges Drittmaterial unterliegen ebenfalls der genannten Creative Commons Lizenz, sofern sich aus der Abbildungslegende nichts anderes ergibt. Sofern das betreffende Material nicht unter der genannten Creative Commons Lizenz steht und die betreffende Handlung nicht nach gesetzlichen Vorschriften erlaubt ist, ist für die oben aufgeführten Weiterverwendungen des Materials die Einwilligung des jeweiligen Rechteinhabers einzuholen.

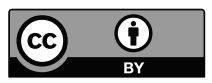




\title{
Das „Transformative Projektseminar“ - didaktische Ansätze und methodische Umsetzung
}

\author{
Richard Beecroft
}

\section{Anwendungskontext}

\subsection{Der Erfahrungshintergrund}

Die hier vorgestellte Methodik des „Transformativen Projektseminars“ (TraPS) basiert auf Erfahrungen der „Karlsruher Schule der Nachhaltigkeit“ (KSN), von der sechs TraPS in einem Zeitraum von drei Jahren durchgeführt wurden. Das Konzept des TraPS wurde in der Zeit iterativ weiterentwickelt, die hier vorgestellte Form stellt einen Idealtyp auf Basis dieser Erfahrungen dar. Die sechs Kurse im Einzelnen und wesentliche Anpassungsschritte in der Struktur sind im Anhang zu diesem Beitrag dargestellt.

Eine Besonderheit war die Einbettung dieser sechs TraPS in ein Reallabor (im Folgenden als „Reallabor Karlsruhe“ bezeichnet, s. auch Steckbrief im Anhang zu diesem Buch): Es setzt sich zusammen aus mehreren Projekten, beginnend im Jahr 2012 mit dem „Quartier Zukunft - Labor Stadt“. Mit dem Projekt „Reallabor 131: KIT findet Stadt“" (2015-2017, 2018-2019) wurden die Aktivitäten das erste Mal explizit als Reallabor gerahmt. Gemeinsamer Zielhorizont der Aktivitäten war die Nachhaltigkeitstransformation eines Karlsruher Stadtteils, der Oststadt (s. Waitz et al. 2018; Trenks et al. 2018; Parodi et al. 2016; Beecroft und Parodi 2016; Meyer-Soylu et al. 2016). Wichtig in der Architektur des Reallabors ist eine Trennung zwischen a) dem Labor als Infrastruktur, b) übergreifenden Aufgaben des Laborbetriebs und c) den transdisziplinären Projekten, die darin stattfinden (Beecroft et al. 2018). Neben Realexperimenten und kleineren partizipativen Veranstaltungsformaten waren dies im Reallabor Karlsruhe u. a. die TraPS.

Ein Kernelement der Infrastruktur ist der seit 2015 bestehende „Zukunftsraum für Nachhaltigkeit und Wissenschaft", ein multifunktionaler Projektraum mitten im Stadtquartier, der als Büro, Treffpunkt, Lernumgebung und Eventraum genutzt 
wird, sowohl vom Forschungsteam des Reallabors selbst als auch von unterschiedlichen Praxispartnern des Reallabors, insbesondere zivilgesellschaftlichen Gruppen. Die Wechselwirkung der TraPS mit dem Reallabor Karlsruhe wurde anhand der sechs bislang durchgeführten TraPS untersucht (Beecroft 2018), siehe auch Abschnitt 5.3.

Die Einbettung der TraPS im Reallabor stellte eine Einbettung in die Forschung dar, die die Potenziale für Nachhaltige Entwicklung auf Quartiersebene, insbesondere auch in kultureller und sozialer Hinsicht, untersuchte. Das Reallabor hatte auch ein deutliches Profil in der Kooperation mit zivilgesellschaftlichen Initiativen im Quartier und der Stadt Karlsruhe, so dass die Praxiseinbettung der TraPS in der Regel durch die Kooperation mit den jeweiligen Praxispartnern gegeben war. Die Lehreinbettung der TraPS lag beim Zentrum für Angewandte Kulturwissenschaft und Studium Generale (ZAK) am Karlsruher Institut für Technologie. Das ZAK bot u. a. ein freiwilliges „Begleitstudium Nachhaltige Entwicklung" für alle Student(inn)en an. Dieses wurde gemeinsam mit der KSN konzipiert und umfasste neben einem Einführungs- und einem Wahlpflichtmodul auch ein transdisziplinäres Projektmodul, für das insbesondere ein TraPS belegt werden kann.

Das TraPS ist keine grundsätzlich neue Lehrform, sondern integriert mehrere Ansätze aus dem State of the Art fachübergreifender, aktiver Lehre mit einer Reihe ausgewählter methodisch-didaktischer Elemente $\mathrm{zu}$ einer transdisziplinären Arbeitsweise mit transformativem Anspruch. In den folgenden Abschnitten (1.2-1.4) werden zunächst geeignete Anwendungsfälle für ein TraPS, die Akteurskonstellation und Voraussetzungen benannt. In Kapitel 2 wird die Phasenstruktur des TraPS beschrieben. In Kapitel 3 werden die didaktischen Ansätze, die der TraPS-Konzeption zu Grunde liegen, vorgestellt und in Kapitel 4 um didaktische Einzelmethoden und Themen ergänzt, die für die Umsetzung eines TraPS besonders zu beachten sind. Kapitel 5 reflektiert die Erfahrungen mit TraPS anhand einiger zentraler Themen.

\subsection{Wann eignet sich das Transformative Projektseminar?}

Das TraPS ist ein Lehrformat, um parallel Bildungs-, Praxis- und Forschungsziele zu verfolgen. Es verbindet mehrere didaktische Ansätze zu einem transdisziplinären Prozess, der zeitlich in sechs Phasen strukturiert ist.

Bildungs-, Praxis- und Forschungsziele beschreiben zunächst nur die Zieldimensionen, um die es geht. Welche spezifischen Ziele in den einzelnen Dimensionen verfolgt werden, muss für jedes TraPS neu festgelegt werden: Ein TraPS kann im Bereich der Praxis dazu eingesetzt werden, Praxispartner eines Reallabors oder eines vergleichbaren Forschungsprojekts mit einer Reihe von kleineren Maßnah- 
men zu unterstützen. Dies bietet sich zum Beispiel an bei vielschichtigem oder diffusem Bedarf, um umfassendere Transformationsprozesse zu unterstützen, sowie indirekt, um langfristige Kooperationen anzubahnen, auch zwischen Praxispartnern. Es dient explizit nicht dazu, als Einzelmaßnahme Transformationsprozesse zu initiieren, sondern dazu, existierende zu verstärken.

Im Bereich der Bildung dient es dazu, verantwortliche Eigenaktivitäten und Reflexionsvermögen der Student(inn)en zu fördern, zum Beispiel gerahmt als Bildung für Nachhaltige Entwicklung oder Bildung durch Engagement. Darüber hinaus ist möglich, dass Praxispartner und Forschungspartner im Projekt auch eigene Bildungsziele verfolgen. Zumindest implizite Bildungswirkungen sind für alle Partner zu erwarten.

Im Hinblick auf die Forschung bietet das TraPS die Möglichkeit, erstens ein neues Feld zu erschließen, zweitens eine mehrdimensionale Problemanalyse und Lösungssuche durchzuführen (z. B. unter verschiedenen Nachhaltigkeitsgesichtspunkten) sowie drittens kleinskalige, eher risikoarme Interventionen durchzuführen und zu dokumentieren. Unter Forschungsgesichtspunkten ist das TraPS gut an unterschiedliche wissenschaftliche Kontexte adaptierbar, um beispielsweise unterschiedlichen disziplinären Anforderungen zu entsprechen, bestimmte Modelle oder Theorien zu verwenden oder einen bestimmten Gegenstand in den Mittelpunkt zu stellen. In jedem Fall bietet es einen Anlass zur kontinuierlichen interdisziplinären Kooperation. Die Transformationswirkung des TraPS basiert nicht auf einer großen Intervention, sondern auf mehreren kleineren Beiträgen, die parallel von Studierendenteams erarbeitet werden, aus denen sich in der praktischen Anknüpfung einzelne als zentral erweisen können, während andere keine weitere Dynamik entfalten.

Im Rahmen größer angelegter transdisziplinärer Projekte ist das TraPS insbesondere geeignet für eine Phase, in der noch Kooperationen stabilisiert werden sollen, aber schon erste Interventionen beginnen. Aufgrund seiner dreifachen Zielsetzung ist das TraPS recht flexibel einsetzbar.

Ein Transfer in andere Bildungskontexte (eher berufliche und Weiterbildung als Schulbildung) ist denkbar. Eine regelmäßige Anwendung mit denselben Partnern ist denkbar, falls im Projekt Praxispartner mit regelmäßig neuen Bedarfen beteiligt werden können. Das TraPS kann im Prinzip über mehrere Semester gestreckt, allerdings nicht wesentlich gekürzt werden.

Die wichtigste Einschränkung eines TraPS liegt darin begründet, dass die Freiheit der Student(inn)en im Sinne des forschenden Lernens (s. Abschnitt 3.2) in Konkurrenz dazu geraten kann, die im TraPS zu erarbeitenden Ergebnisse im Voraus zu planen. Das TraPS steht und fällt mit einer Gruppe engagierter Student(inn)en. Wenn nur geringe Aussichten bestehen, Student(inn)en zu gewinnen, beispiels- 
weise wegen fehlender Anerkennung, unattraktivem Thema oder zu vielen vergleichbaren Kursen, sollte auf das Format eher verzichtet werden. Es ist nicht geeignet für ein Lehrteam ohne jede Lehrerfahrung in projektförmiger Lehre.

\subsection{Voraussetzungen}

Voraussetzungen für die Durchführung eines TraPS sind:

1) Praxispartner mit Anliegen, die sich innerhalb eines Semesters bearbeiten lassen. Solche Anliegen der Praxispartner dürfen heterogen und unspezifisch sein. Im Rahmen der Karlsruher TraPS waren insbesondere kleine zivilgesellschaftliche Gruppen mit Nachhaltigkeitsbezug beteiligt, die bestimmte Aktivitäten nicht aus eigener Kraft bewältigen konnten.

2) Student(inn)en, die im Rahmen ihres Studiums oder freiwillig am TraPS teilnehmen können. Es ist ideal, wenn sie aus unterschiedlichen Fachrichtungen, aber mit einem gewissen gemeinsamen Grundwissen teilnehmen (im Fall der Karlsruher TraPS nahmen viele Student(inn)en des freiwilligen Begleitstudiums Nachhaltige Entwicklung Teil, die das TraPS in der Regel erst nach einigen einführenden Kursen besuchten). Für andere Studierendengruppen muss die Struktur gegebenenfalls angepasst werden.

3) Ein wissenschaftlicher Projektrahmen, der Impulse aus dem Seminar inhaltlich aufgreifen kann. Im Karlsruher Fall war dies das Reallabor. Andere, längerfristige Rahmen (Fachbereiche, Arbeitsstellen, Forschungsprojekte mit hinreichend langer Laufzeit, Wissenschaftsläden, städtische Wissenschaftsbüros etc.) bieten ein ähnliches Potenzial. Wichtig ist, dass Ressourcen auch für die Zeit nach dem Seminar vorgesehen sind, im Einzelfall kann dies auch ein Praxispartner übernehmen.

Wenn eine der drei Bedingungen nur knapp erfüllt ist, kann ein TraPS (leicht angepasst) durchgeführt werden, wenn hingegen mehrere Bedingungen nicht solide gegeben sind, ist die Sinnhaftigkeit dieser Arbeitsweise überaus fraglich. ${ }^{1}$

1 Die in Kapitel 3 dargestellten didaktischen Ansätze bieten ggf. eine Auswahl an anderen Zugängen, die jeweils spezialisierter eine der Zielsetzungen in den Mittelpunkt rücken. 


\subsection{Beteiligte Akteure und deren Rollen}

Das TraPS beteiligt im Kern drei Gruppen in jeweils mehreren Rollen:

1) Wissenschaftler(innen), die im wissenschaftlichen Projektrahmen tätig sind, wenn möglich als Lehrteam aus zwei Personen. Von diesen geht zumeist die Initiative für das TraPS aus, sie beteiligen die anderen Partner.

2) Student(inn)en als Seminarteilnehmer(innen), ideal 12 bis 24 Personen, sowie evtl. als Hilfskräfte. Die Student(inn)en arbeiten in den Phasen III bis V in Teams zusammen, in den Phasen II und VI sind sie eher als Einzelpersonen beteiligt.

3) Praxispartner, die am Projekt beteiligt sind, wie Mitglieder von zivilgesellschaftlichen Gruppen (Vereine, Kirchengemeinden, Initiativen), Mitarbeiter(innen) aus kommunalen Einrichtungen (Agendabüros, Schulen, Ämter, Kultureinrichtungen) oder Unternehmen (sowie z. B. Branchenverbände, Kammern). Es sollten idealerweise 2 bis 3 Personen beteiligt sein.

Ob die Motivation, ein TraPS durchzuführen, in der Lehrplanung durch Wissenschaftler(innen), in einem Forschungsprojekt mit transformativem Anspruch oder in einem praktischen Anliegen gründet, ist unerheblich, solange letztendlich die Einbettung in Bildungs-, Forschungs- und Praxiskontexte möglich ist und die oben genannten Voraussetzungen gegeben sind. Die Erfahrung mit zivilgesellschaftlichen Gruppen war im Reallabor Karlsruhe besonders gut, was zumindest teilweise am Elan der dort Engagierten lag. Gegebenenfalls kann auch mehr als eine Gruppe von Praxispartnern beteiligt werden, sofern die Themenstellung dies rechtfertigt und die Rollen klar sind. Bei einem Karlsruher TraPS, in dem ein „Nachhaltigkeitsspaziergang“ entwickelt wurde, waren beispielsweise Vertreter(innen) der Stadtverwaltung - primär als Expert(inn)en - und eines eingetragenen Vereins, der alternative Stadttouren anbietet - als Expert(inn)en und Adressat(inn)en -, beteiligt. Die Phasenstruktur erlaubt es zudem, relativ leicht weitere Wissenschaftler(innen) in geringem Umfang zu involvieren, beispielsweise durch Gastvorträge oder als Interviewpartner(innen). Damit ergibt sich die Akteurskonstellation in Abb. 1, dargestellt für die Phasen III bis V, in denen die Student(inn)en in Teams zusammenarbeiten.

Man darf aus dieser Konstellation nicht die Schlussfolgerung ziehen, die drei Gruppen hätten jeweils nur eine der drei Zieldimensionen im Auge. Es ist keineswegs so, dass Student(inn)en nur zum Lernen, Praxispartner nur für die Förderung ihrer Praxis und Wissenschaftler(innen) nur für die Forschung stehen - dann wäre ein gemeinsamer Prozess auch schwer umzusetzen. Vielmehr nehmen Personen aus allen drei Gruppen bezogen auf diese Zieldimensionen unterschiedliche Rollen ein. 


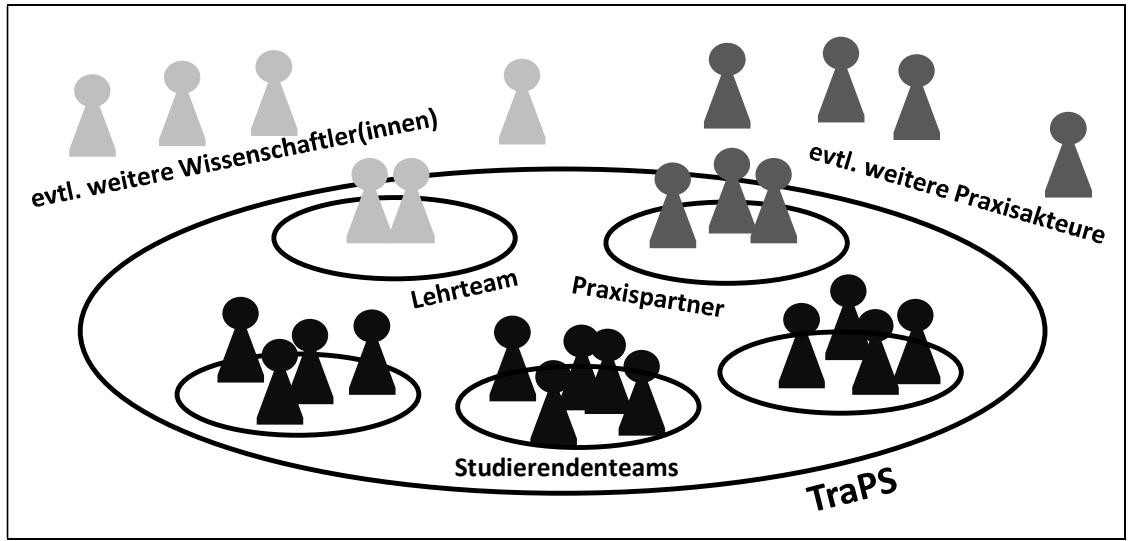

Abbildung 1: Akteurskonstellation im Transformativen Projektseminar (Phasen III-V). (C) Richard Beecroft.

- Student(inn)en können zum Beispiel ihre Rolle als Lernende verbinden mit denen als Change Agents - also als Vorreiter(innen) oder aktive Treiber(innen) von Transformationsprozessen -, als Forschende, die recherchieren, experimentieren, auswerten und abstrahieren, als kritische Beobachter(innen), als Vermittler(innen), als Betroffene oder als politisch interessierte Bürger(innen).

- Den Lehrenden kommen schon klassischerweise verschiedene Rollen zu: Als Expert(inn)en für Methoden und Gegenstände, als Leitung mit Verantwortung wie für den Lernort und die Zeitplanung, als Beobachter(innen) der Lernprozesse, als Bewerter(innen) der Leistungen der Student(inn)en. In transdisziplinären Settings kommen weitere Rollen hinzu, insbesondere als Moderator(inn)en von Gruppenprozessen, als Gesprächspartner(innen) zur Reflexion, als Mediator(inn)en bei Konflikten, als wissenschaftliche Partner gegenüber den Praxispartnern, als Vermittler(innen) von Kontakten zu weiteren Akteuren, als Lernende und als Evaluator(inn)en.

- Die Praxispartner sind auf jeden Fall als Expert(inn)en für ihr jeweiliges Themenfeld und als Adressat(inn)en für das Ergebnis der Student(inn)en beteiligt, aber andere Rollen umfassen die als Advokat(inn)en für ihr Anliegen, als Gesprächspartner(innen) für die Student(inn)en, als Mitbewerter(innen) der studentischen Leistungen, als Werber(innen) für ihre Initiativen, als Vermittler(innen) von weiteren Kontakten und als Lernende.

Durch die Gleichzeitigkeit von Forschungs-, Bildungs- und Praxiszielen, durch die gezielt herbeigeführten Erfahrungen mit Neuem und die intensive Zusammenarbeit werden immer wieder Rollenverschiebungen nötig. Einige Rollen hängen 
von der jeweiligen Phase des TraPS ab. Die Lehrenden treten etwa tendenziell am Anfang vor allem als Leiter(innen) und Expert(inn)en, während der Projektarbeit vor allem als Moderator(inn)en, Gesprächspartner(innen) und Beobachter(innen), zum Schluss als Bewerter(innen) und als Ansprechpartner(innen) für die Praxispartner in Erscheinung. Sowohl eingangs, bei der Formulierung der Bedarfe, als auch abschließend bei der Übergabe von Resultaten kommt es oft zu Rollenwechseln. Andere Rollen, wie die Rolle der Lehrenden als Verantwortliche für den Rahmen, bleiben durchgängig relevant. Eine Form der Rollenverschiebung, die sich in den sechs TraPS in Karlsruhe zeigte, waren Rollenwechsel zwischen den drei Gruppen, zum Beispiel:

- Praxispartner wurden zu Co-Lehrenden.

- Student(inn)en wurden zu Mitgliedern der Initiativen, die als Praxispartner beteiligt waren.

- Student(inn)en waren Mitglieder anderer zivilgesellschaftlicher Gruppen, die als zusätzliche Praxispartner einbezogen wurden.

Neben Rollenverschiebungen kommt es im TraPS immer wieder auch zu Rollenüberschneidungen. Wie in vielen anderen lebensweltlichen Situationen decken sich die Erwartungen der anderen beteiligten Personen an einen nicht immer mit der je eigenen Rollenvorstellung, und auch jede(r) einzelne Beteiligte(r) kann in ihrem/seinem Selbstverständnis zwischen mehreren Rollenvorstellungen schwanken. Problematisch werden solche Rollenüberschneidungen aber erst dann, wenn die Rollen nicht vereinbare Aktivitäten erforderlich machen. Weiterhin können Rollen außerhalb des TraPS-Kontextes in die Rollenvielfalt hineinspielen. Eine Praxispartnerin mit sozialwissenschaftlichem Beruf freute sich beispielsweise zu Beginn eines Karlsruher TraPS mit ungefähr diesen Worten: „Cool, jetzt bin ich Praxispartnerin - sonst habe ich immer welche!“ Ein typischer Rollenkonflikt kann auch zwischen beruflichem und ehrenamtlichem Engagement der Beteiligten aufbrechen.

Sowohl Rollenverschiebungen als auch Rollenüberschneidungen sind nicht per se problematisch, sollten aber gegebenenfalls explizit angesprochen werden, um unangemessene Erwartungen aneinander zu vermeiden - oft reicht hierzu ein kurzer Hinweis („Als Wissenschaftler(in) finde ich diesen Punkt sehr spannend, als Lehrende(r) muss ich Sie aber an Ihren Zeitplan erinnern ..."). In der Reflexion des Lehrteams zwischen den Terminen sollten Rollenkonflikte oder unerwünschte Rollenverschiebungen jeweils thematisiert werden. 


\section{Idealtypischer Ablauf eines Transformativen Projektseminars}

Ein TraPS folgt einem Ablaufschema von sechs Phasen, die jeweils unterschiedlichen Zielen dienen und in denen unterschiedliche Arbeitsweisen zum Einsatz kommen (s. Abb. 2).

Phasen II bis V entsprechen einem normalen Semestertakt mit 14 wöchentlichen Terminen à 90 Minuten und eigenständiger Arbeit dazwischen. Phasen I und VI rahmen die Seminarphasen ein; sie dienen insbesondere dem transdisziplinären Charakter des TraPS. Ihre Länge kann nicht allgemein beschrieben werden, da sowohl die Anbahnung einer Kooperation als auch die In-Wert-Setzung der Ergebnisse und Produkte wesentlich von bestehenden Kooperationsbeziehungen abhängen. Bei der Anbahnung sollte immer die Möglichkeit mitgedacht werden, dass keine Kooperation zustande kommt - zumindest nicht in Form eines TraPS.

Abb. 2 verdeutlicht auch die Bedeutung und den Aufwand der Arbeitsschritte zwischen den Seminarsitzungen für die Student(inn)en. Die Zeile, die mit „PP“ markiert ist, zeigt, an welchen Terminen die Praxispartner unbedingt $(++)$, eher $(+)$ oder eher nicht $(-)$ anwesend sein sollten, In der Zeile „St.“ ist ausgewiesen, wann die Student(inn)en in parallelen Teams arbeiten.

Im Folgenden werden für jede der Phasen die idealtypisch aufeinander folgenden Arbeitsschritte benannt. Zwischen den Terminen ist es ratsam, dass sich das Lehrteam zumindest kurz darüber austauscht, welche Entwicklungen in der Seminargruppe als Ganzes, in den einzelnen Teams, gegebenenfalls bei einzelnen Studierenden sowie in der Zusammenarbeit mit den Praxispartnern aufgefallen sind sowohl auf der Ebene der Gruppendynamik als auch auf der sachlichen Ebene. In diesen Reflexionsgesprächen muss abgewogen werden, ob eine Intervention oder ein inhaltlicher oder methodischer Input nötig ist. 


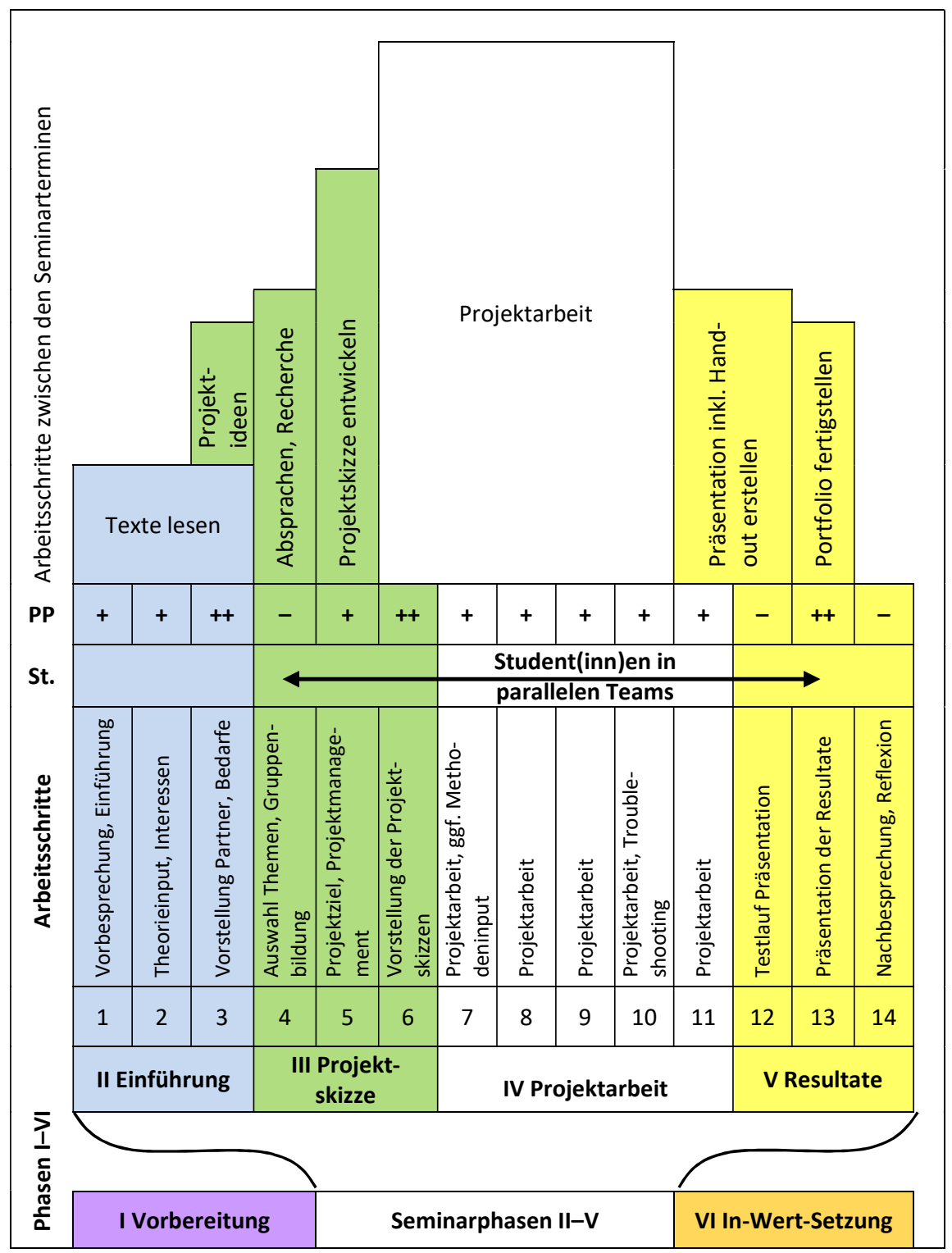

Abbildung 2: Phasen und Arbeitsschritte im Transformativen Projektseminar (idealtypisch, 14 Termine mit Student(inn)en umfassend). Die Zeile „PP“ zeigt, in welchen Phasen und Schritten die Praxispartner involviert sind, die Zeile „St.“ bezieht sich auf die Student(inn)en. (O Richard Beecroft. 


\subsection{Phase I: Vorbereitung}

In dieser Phase gilt es, ausgehend von einer vorläufigen Idee für das Seminar, den Einstieg in die gemeinsame Arbeit für alle beteiligten Gruppen zu gestalten: Auf Seiten der wissenschaftlichen Partner muss entschieden werden, wer aus dem wissenschaftlichen Projektrahmen als Lehrteam die Veranstaltung übernimmt. Wenn das TraPS u. a. der Verstetigung einer Kooperation mit bestimmten Praxispartnern dient, kann diese Entscheidung auch von der $\mathrm{Zu}$ - oder Absage der infrage kommenden Praxispartner abhängen. Es hat sich bewährt, dass im Lehrteam zumindest eine Person Erfahrung in projektförmiger Lehre hat und zumindest eine Person tiefergehende Kenntnisse im Gegenstandsfeld mitbringt. Das Lehrteam sollte Zeit dafür vorsehen, sich breit inhaltlich einzuarbeiten (da man nie genau weiß, wie die Projekte der Student(inn)en ausgerichtet sein werden) und die Rollen und Verantwortlichkeiten zu klären: Wer ist für die Student(inn)en für welche Fragen Ansprechperson, wer hält den Kontakt zu den Praxispartnern, wer bereitet welche Inputs vor, wer arbeitet sich in welche Themen tiefer ein? Gegebenenfalls können auch weitere Partner aus der Wissenschaft als Expert(inn)en eingebunden werden, da diese Rolle in der Regel eingespielt und unproblematisch anschlussfähig ist, solange sie nicht zu aufwendig wird.

Des Weiteren gilt es, Praxispartner einzubinden. Zunächst werden der thematische Rahmen und die Ziele, die im TraPS bearbeitet werden sollen, mit dem zu beteiligenden Praxispartner vereinbart. Im Anschluss muss vonseiten des Praxispartners (bzw. der Praxispartner, falls mehrere mitwirken werden) festgelegt werden, welche 1 bis 3 Personen sich im TraPS einbringen. Es sollte in Phase I verbindlich vereinbart werden, an welchen Terminen die Anwesenheit erforderlich ist und welche Inputs, insbesondere zu Beginn des Seminars, erfolgen sollen; eventuell kann hierfür ein Lehrauftrag oder ein Vortragshonorar vereinbart werden. Der Aufwand für die Praxispartner muss sich auch an deren Kapazitäten anpassen. Nur an drei Terminen eines TraPS ist die Anwesenheit unerlässlich: Bei der Vorstellung der Praxispartner (in Phase II), bei der Vorstellung der Projektskizzen (in Phase III) und bei der Präsentation und Übergabe der Resultate (in Phase V). Weiterhin gilt es zu entscheiden, wann und über welche Kommunikationsmittel die Praxispartner für die Student(inn)en erreichbar sein werden. Es sollte vereinbart werden, wann weitere Mitglieder der Akteursgruppen zu einzelnen Terminen im TraPS hinzukommen - dies ist insbesondere bei der Präsentation der Ergebnisse sinnvoll. Im Rahmen dieser vorbereitenden Absprachen sollten auch die Erwartungen seitens der Praxispartner diskutiert und zu hohe oder falsche Erwartungen korrigiert werden (z. B., dass eine reine Beratungs- oder Dienstleistungstätigkeit in Aussicht steht). 
Mit Blick auf die Student(inn)en muss in dieser Phase das Informations- und gegebenenfalls Werbematerial zum TraPS erstellt werden. Dies sollte mit großer Sorgfalt geschehen, da die Erwartungen, die sich aus den Veranstaltungsankündigungen ergeben, für die Teilnahmeentscheidung und die Zufriedenheit mit der Veranstaltung wichtig sind. Sowohl das Thema als auch die spezifische Arbeitsweise sollten deutlich herausgestellt werden. Daneben müssen natürlich alle normalen Vorbereitungen für eine Lehrveranstaltung erfolgen (z. B. Teilnahmelisten erstellen, Texte auf eine Lernplattform hochladen). Hierbei gilt es immer, darauf $\mathrm{zu}$ achten, dass die Praxispartner nicht außen vor bleiben bei der Nutzung universitätsinterner Infrastrukturen (z. B. Zugriffsrechte auf die Lernplattform). Ein rechtzeitiges und verbindliches Anmeldesystem erleichtert die Planung deutlich.

Sofern die Möglichkeit hierzu besteht, sollte in dieser Phase auch die Lernumgebung vorbereitet werden (s. Abschnitt 4.3), indem überlegt und entschieden wird, in welchen physischen Räumen, gegebenenfalls auch über welche virtuellen Plattformen, die Kooperation gestaltet werden soll und welche Materialien von Anfang an verfügbar sein sollten. Typische Fragen dieser Art in den Karlsruher TraPS betrafen etwa:

- Sollen die Student(inn)en die frei verfügbare Projektmanagementsoftware der Praxispartner nutzen, um den Anschluss an die Praxis zu erleichtern?

- Wo erhält man einen Stadtplan in DIN A0?

- Trifft man sich zu ausgewählten Terminen in den Räumen der Praxispartner?

- Mit welcher Cloud-Lösung dürfen Mitarbeiter(innen) der Kommune arbeiten?

- Wo lagern wir die erarbeiteten Materialien in einem vielfältig genutzten Raum?

Der Vorlauf und der Zeitaufwand für Phase I ist äußerst kontextabhängig. Eine vorausgegangene oder kontinuierlich bestehende Kooperation mit einem Praxispartner kann Phase I eines TraPS deutlich erleichtern. Wenn das TraPS verpflichtend in der Lehre verankert ist und dementsprechend nicht ausfallen darf, muss die Planung dafür weit im Voraus erfolgen - eventuell kann nicht jeder Praxispartner so weit im Voraus verbindliche Zusagen treffen.

\subsection{Phase II: Einführung}

Phase II beginnt mit der ersten regulären Seminarsitzung (90 Minuten, Uhrzeit in Abstimmung mit Praxispartnern) und umfasst drei Wochen (Termine 1 bis 3). Nachstehend werden die inhaltlichen Schwerpunkte der drei Termine geschildert. Dabei gilt jedoch, dass diese relativ einfach getauscht werden können, um beispielsweise eine Veranstaltung der Praxispartner besuchen zu können oder um 
,trockenen' theoretischen Input auf mehrere Termine zu verteilen. Die Praxispartner müssen unbedingt zu ihrer Vorstellung erscheinen, zu den anderen Terminen ist die Anwesenheit erwünscht, aber nicht erforderlich.

\section{Termin 1}

Die Student(inn)en erhalten eine Einführung in den übergreifenden thematischen Horizont und den wissenschaftlichen Rahmen (in den Karlsruher TraPS z. B.: Nachhaltigkeit und die Bedeutung von Städten, Bezug zum jeweiligen Themenfeld, z. B. nachhaltiger Konsum, sowie eine anschauliche Vorstellung des Reallabors). Hierzu werden auch Texte zur Verfügung gestellt, die von den Student(inn)en zwischen den Terminen gelesen werden sollen. Die Student(inn)en werden eingeführt in die transdisziplinäre Methodik des TraPS. Die zu erbringenden Studienleistungen - Projektergebnis, Lernportfolio und gegebenenfalls Hausarbeit (s. Abschnitt 4.1) - müssen erläutert werden. Hierzu typische formale Fragen zu Fristen, Bedingungen für Studienleistungen etc. müssen mit den Student(inn)en geklärt werden. Insbesondere sollte der hohe Arbeitsaufwand in Phase IV von Anfang an deutlich gemacht werden. Sofern der Kurs nicht verpflich-

tend ist, sollte seitens der Student(inn)en bis zum Ende von Phase II eine definitive Entscheidung über die Teilnahme erfolgen.

\section{Termin 2}

Am zweiten Termin werden theoretische Grundlagen knapp eingeführt, die für die Bearbeitung der Themenstellung unerlässlich sind. Bei Bedarf können auch grundlegende Wissensbestände dargestellt werden, die die Relevanz des Projekts deutlich machen (z. B. Informationen über Lebensmittelverschwendung zur Kooperation mit einer Tafel, Diskussion der Kritik an geplanter Obsoleszenz als Grundlage zur Kooperation mit einem Reparaturcafé).

Ein Element aus dem forschenden Lernen (s. Abschnitt 3.2), das sich in den TraPS besonders bewährt hat im Anschluss an theoretischen Input, ist das Explizieren und Reflektieren von eigenen Bildungszielen und Interessen durch die Student(inn)en. Dies wird durch eine kurze Phase individueller Reflexion, eventuell gefolgt von kurzen Zweiergesprächen zum Aktivieren und dann einem Austausch im Plenum erreicht. Hierbei kann die oben eingeführte Unterscheidung in Bildungs-, Praxis- und Forschungsziele als Reflexionsfolie verwendet werden, indem die Student(inn)en dazu aufgefordert werden, sich mit den eigenen Interessen in diesen Zieldimensionen zu positionieren (z. B. visualisiert mit Klebepunkten und Schlagworten in einem ,Zieldreieck' auf einem Flipchart). Die Student(inn)en halten ihre Ziele und Erwartungen individuell schriftlich fest und legen sie im Lernportfolio ab (s. Abschnitt 4.1), um sie in der Abschlussreflexion (Phase V) nutzen zu können. Wenn Praxispartner bei diesem Schritt anwesend 
sind, sollten sie sich weitgehend zurückhalten. Für das Lehrteam ist eine sokratische Gesprächsführung (s. Abschnitt 4.2) zielführend, eventuell verbunden mit einer Rollenverteilung unter den Lehrenden (z. B. Protokollieren und Moderieren oder Fokus auf die unterschiedlichen Zieldimensionen).

\section{Termin 3}

Die Praxispartner stellen ihre Arbeit vor, im Idealfall sowohl durch einen Vortrag als auch im Rahmen einer Exkursion, und beschreiben ihre Bedarfe. Gegebenenfalls können hierzu unterschiedliche Quellen genutzt werden, nicht nur klassische wissenschaftliche Texte (z. B. im TraPS zu „Gemeinwohlökonomie in Karlsruhe?" ein Video mit dem Gründer der GWÖ und eine Broschüre mit einer radikalen Kritik des Ansatzes durch eine wirtschaftsliberale Stiftung). Es kann passieren, dass sich relevante Probleme als nicht bearbeitbar für die teilnehmenden Student(inn)en herausstellen: In der Zusammenarbeit mit einer der Karlsruher Tafeln wurde etwa das Problem angesprochen, dass viele ältere Kunden nur wenige Einkäufe mit der Straßenbahn transportieren können, da deren Stufen zu hoch sind für Einkaufsroller. Da die Tafel bereits sehr gute und vertrauensvolle Kontakte zur Stadt hat und aus vielen Gründen in ihren gegenwärtigen Räumen bleiben muss, war durch die Student(inn)en kein Ansatzpunkt für einen barrierefreien Zugang zur Tafel zu identifizieren.

\subsection{Phase III: Projektskizze}

Ziel von Phase III ist es, dass die Student(inn)en Projektideen entwickeln und Studierendenteams bilden, um jeweils eine Projektidee zu einer Projektskizze auszuarbeiten, die realisierbar, relevant und interessant ist (und in diesem Sinne Potenzial bietet für Praxis-, Forschungs- und Bildungsziele). Phase III umfasst drei Seminartermine (Termine 4 bis 6). Direkt im Anschluss an den letzten Seminartermin von Phase II erhalten die Student(inn)en den Auftrag, individuell mehrere Projektideen zu formulieren, und zwar unabhängig davon, ob sie diese selbst bearbeiten wollen. Auch unvollständige Projektideen sind hier willkommen, die beispielsweise nur das Thema, aber kein Vorgehen beschreiben.

\section{Termin 4}

Der vierte Termin ist für den Projektverlauf besonders wichtig. Soweit irgend möglich, sollten alle Student(inn)en anwesend und notfalls bereit sein, etwas zu überziehen. Je nach Anzahl und Qualität der individuell gesammelten Projektideen ist noch ein gemeinsames Brainstorming nötig, zumindest sollte kurz Gelegenheit gegeben werden, neu aufkommende Ideen einzubringen. Alle Projektideen werden auf Moderationskarten festgehalten und thematisch gruppiert. 


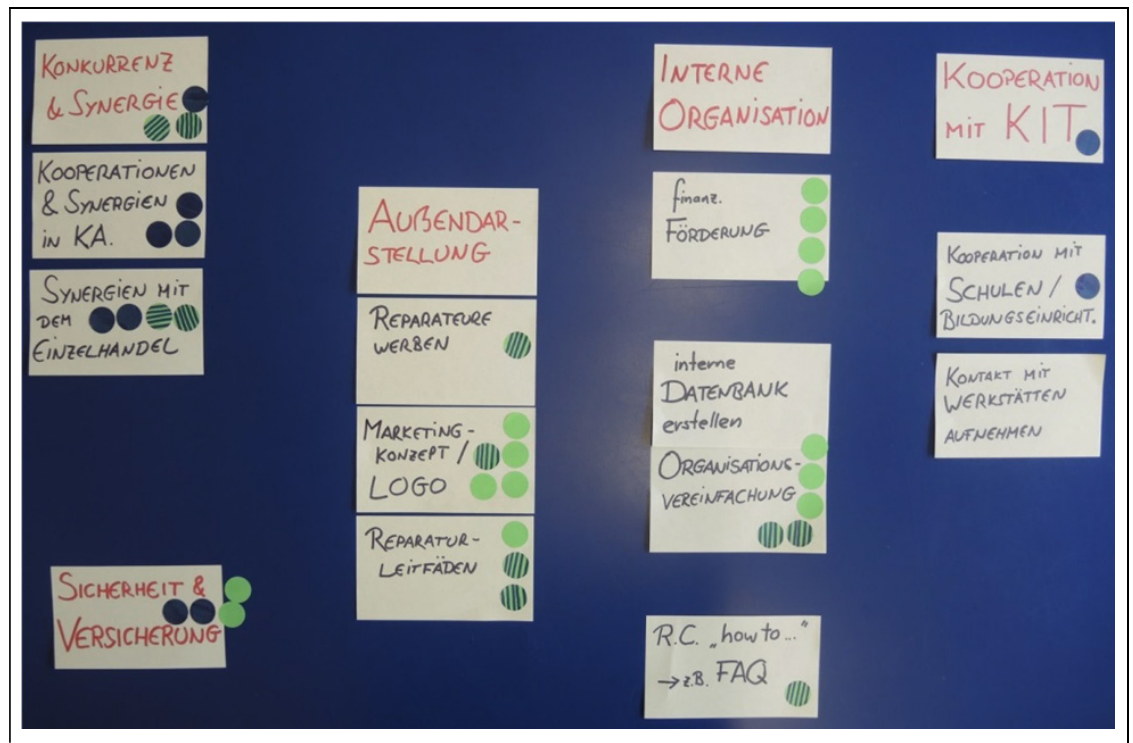

Abbildung 3: Geclusterte Projektideen, bewertet nach Bildungspotenzial (gestreift), Forschungspotenzial (dunkelblau) und Praxispotenzial (hellgrün). (C) Richard Beecroft.

Gegebenenfalls kann anhand dieser übersichtlicheren Sammlung noch einmal nach Themen gefragt werden, die noch fehlen, insbesondere auch im Hinblick auf die eingeführte Theorie und den Bedarf der Praxispartner.

Die so erarbeitete Ideensammlung wird nun durch die Student(inn)en nach drei Aspekten bewertet: Nach der (individuellen) Relevanz für den je eigenen Bildungsprozess, nach dem angenommenen praktischen Potenzial (in der Regel für den Praxispartner) und nach der wissenschaftlichen Relevanz der Themen (gegebenenfalls erfolgt diese Bewertung durch das Lehrteam, falls die Student(inn)en wenig einschlägige wissenschaftliche Vorkenntnisse mitbringen). Abb. 3 zeigt ein Beispiel für diesen Auswahlprozess. Auffälligkeiten in Bezug auf die Verteilung der Punkte werden gemeinsam diskutiert. Erst ausgehend von dieser Themenkarte finden sich dann Studierendenteams zusammen, die eine (oder gegebenenfalls auch mehrere zusammenpassende) dieser Ideen bearbeiten möchten. Wenn möglich sollten die Studierendenteams sich auf mehrere Cluster verteilen, zwingend ist aber nur, dass die Projekte hinreichend unterschiedlich sind - das kann auch gegeben sein, wenn mehrere Teams dasselbe Thema bearbeiten (z. B. erstellte ein Studierendenteam für das ReparaturCafé Karlsruhe, das vor einer Vereinsgründung stand, eine Best-Practice-Studie für Reparaturcafés in Deutschland, ein weiteres Studierendenteam ein Planspiel zum Test, ob die für das ReparaturCafé 
geplante Vereinssatzung die relevanten Fragen und Probleme behandelt; beide Projekte bezogen sich auf die Projektidee „Entwicklung der Satzung unterstützen"). In der Weiterentwicklung der Projektidee zur Projektskizze sollten die Student(inn)en Forschungs-, Bildungs- und Praxisziele berücksichtigen, auch wenn hierzu zunächst keine Punkte vergeben wurden.

Die Studierendenteams sollten eher klein sein (4 bis 5 Personen), um die Logistik für Treffen in der Projektphase nicht zu erschweren. Die Priorisierung der Themen- vor der Gruppenfindung erlaubt es, Interessen und Kompetenzen bestmöglich zu verteilen. Zudem besteht bei einem freiwilligen Kursangebot immer eine gewisse Gefahr, dass Student(inn)en doch auf die weitere Teilnahme verzichten oder erst spät zum Seminar dazustoßen, so dass eine zu frühe Gruppenzuordnung riskant ist. Durch die relativ späte Teambildung erhalten die Student(inn)en genug Zeit, sich kennenzulernen und die Seminargruppe als Ganzes zu erleben, nicht als konkurrierende Teams.

Es hat sich bewährt, den sich findenden Gruppen etwas Zeit zu geben, um miteinander abzustimmen, wer das Seminar für sich wie hoch priorisiert und wie viel Zeit und Energie bei den Einzelnen für die Projektarbeit zur Verfügung stehen hierzu sollten das Lehrteam und die Praxispartner möglichst den Raum verlassen, damit die Student(inn)en offen auch über eine eher geringe Beteiligung sprechen können. Insbesondere bei einer Gruppe, in der einige Student(inn)en keine Bescheinigung brauchen, andere die Veranstaltung aber als Prüfungsleistung in ihr Studium einbringen wollen, können sich Spannungen ergeben, die sich zu Beginn der Gruppenzusammensetzung am besten adressieren lassen.

$\mathrm{Ab}$ diesem Termin arbeiten die Student(inn)en als Teams zusammen. Damit ist die Akteurskonstellation im Seminar fertig aufgebaut (s. Abb. 1). Die Studierendenteams sollten sofort festlegen, durch welche Medien und in welcher Form (Treffen, Datenaustausch, Videokonferenzen etc.) sie zusammenarbeiten wollen; für Aspekte der Datensicherheit lohnt sich eventuell die Nutzung geschützter Cloud-Dienste, übliche Lernplattformen sind dagegen oft zu unflexibel zur Unterstützung von Projektarbeit.

\section{Termin 5}

Ausgehend von den von ihnen gewählte(n) Idee(n) arbeitet jedes Studierendenteam eine realistische Projektskizze aus. Diese umfasst die verfolgten Ziele, das angestrebte konkrete Ergebnis, die wichtigsten Arbeitspakete, die zu erwartenden Hürden und die Verteilung von Verantwortlichkeiten. Hierbei lohnt es sich, einerseits mit jeder Gruppe einzeln ihre Skizze durchzugehen (das Lehrteam sollte sich hierzu aufteilen), andererseits im Plenum übergreifende Fragen und Berührungspunkte zwischen den Projektskizzen (Synergien nutzen, Doppelarbeit vermeiden) anzusprechen - prinzipiell sind auch direkt aufeinander bezogene Projekte von 
Studierendenteams möglich, solange sie nicht zeitlich voneinander abhängen, also parallel bearbeitet werden können. Die Projektskizzen sollten alle einer festgelegten Form genügen (z. B. Handout von 2 Seiten, das die oben genannten Punkte aufgreift und separat Fragen ausweist, die mit den Praxispartnern zu klären sind). Bei manchen Arbeitsweisen (z. B. Fragebogenerstellung, Projektplanung) ist gegebenenfalls eine intensivere Unterstützung der Student(inn)en nötig. Bei diesem Termin sollten die Praxispartner eher nicht dabei sein.

\section{Termin 6}

Die Projektskizzen aller Studierendenteams werden den Praxispartnern gemeinsam vorgestellt, jeweils anhand eines Handouts, vorzugsweise mit einer kurzen Präsentation (jeweils 3 bis 4 Folien), die derselben Struktur wie das Handout folgt. $\mathrm{Zu}$ diesem Termin ist es von Vorteil, wenn die Praxispartner weitere Mitglieder ihrer Akteursgruppe einladen und eventuell auch weitere Wissenschaftler(innen) anwesend sind. Die Projektskizzen werden einzeln vorgestellt (ca. 10 Min./Projekt) und mit allen Anwesenden diskutiert (ca. 10 Min./Projekt), wobei die Praxispartner ausreichend zu Wort kommen sollten. Die Diskussionsergebnisse sollte jedes Studierendenteam für sich festhalten. Die (gegebenenfalls modifizierten) Projekte sind ab diesem Moment festgeschrieben, weitere Veränderungen müssen mit dem Lehrteam abgesprochen werden (solche sind, als Folge unerwarteter Entwicklungen in den Projekten, eher die Regel als die Ausnahme).

\subsection{Phase IV: Projektarbeit}

Die Projektarbeit umfasst fünf Wochen (Termine 7 bis 11). Das ist die Phase, in der die Studierendenteams ihre Projekte realisieren. In dieser überaus intensiven Phase dominiert ein selbstorganisierter Arbeitsstil, die gemeinsamen Seminartermine dienen primär der Unterstützung der Studierendenteams durch das Lehrteam sowie der Bearbeitung übergreifender Fragen oder möglicher Synergien, dem Austausch zwischen den Studierendenteams und kurzen methodischen Inputs seitens des Lehrteams, die mehrere Studierendenteams betreffen.

\section{Termine 7-11}

An diesen Seminarterminen wird in der Regel in einer kurzen Runde zu Beginn jedes Termins der Stand der Projekte vorgestellt und gegebenenfalls bestehender Klärungsbedarf benannt. Die einzelnen Studierendenteams erhalten insbesondere am 7. Termin methodische Beratung, ansonsten nach Bedarf Rückmeldung zu Zwischenergebnissen, inhaltliche Inputs oder Gelegenheit zum Austausch mit den Praxispartnern. Danach teilen sich die Student(inn)en wieder in ihre Teams auf, an mehrere Tische oder in getrennte Räume. 
Im gedrängten Semester stellt das Zeitmanagement oft die größte Herausforderung in dieser Phase dar. Dies ist insbesondere heikel, wenn Informationen von Dritten nötig sind und diese spät oder nicht zur Verfügung gestellt werden. Wenn die Praxispartner in dieser Phase nicht anwesend sind, sollten sie zumindest gut erreichbar sein (das Lehrteam natürlich ebenso). Die Student(inn)en müssen in dieser Phase in engem Austausch miteinander stehen; wenn ein Studierendenteam aber angekündigt einen Seminartermin nicht wahrnimmt, um etwa die Zeit für einen gemeinsamen Termin mit Dritten zu nutzen, ist dies in der Regel unproblematisch. Fehlt dagegen ein(e) Teilnehmer(in), ohne mit dem eigenen Team gesprochen und gegebenenfalls benötigte Zwischenergebnisse übergeben zu haben, ist das ein Alarmzeichen. Gelegentlich sind Interventionen im Gruppenprozess einzelner Teams nötig, beispielsweise eine Aussprache über Unmut über den Projektverlauf oder die Teamdynamik. Dabei hat die Erfahrung in den TraPS in Karlsruhe aber kein wiederkehrendes Muster gezeigt, wann oder aus welchen Anlässen ein Tief im Gruppenprozess eines Teams entstand. Das Team Teaching (s. Abschnitt 3.3) kann während der Projektarbeit dazu genutzt werden, den einzelnen Studierendengruppen ausreichend Raum zum Austausch mit den Lehrenden zu bieten, indem diese den Studierendenteams einzeln zur Verfügung stehen (und sich dann zwischen den Seminarterminen abstimmen). In dieser Phase kann es auch immer wieder nötig sein, die Studierendenteams durch sokratische Gesprächsführung (s. Abschnitt 4.2) zu unterstützen, ohne ihnen dabei das Heft aus der Hand zu nehmen. Sofern Student(inn)en im Rahmen des Seminars Hausarbeiten anfertigen, besteht in der Projektphase Gelegenheit, mögliche Themen hierfür abzusprechen, um Synergien zwischen Projektarbeit und Hausarbeiten rechtzeitig einzuplanen.

\subsection{Phase V: Resultate}

Die Phase V dauert drei Wochen (Termine 12 bis 14). Direkt im Anschluss an Phase IV, zwischen dem 11. und 12. Termin, erstellen die Studierendenteams jeweils eine Präsentation zu ihrem Projektergebnis (ca. 10 Min., bei wenigen Studierendenteams im TraPS auch etwas länger). Die Präsentation wird begleitet von einem kurzen Handout, das die wichtigsten Aspekte des Resultats enthält. Dieses soll so gestaltet sein, dass die Praxispartner es nutzen und weitergeben können. Wenn das Resultat selbst die Form eines kurzen Textes hat, ist kein zusätzliches Handout notwendig. Manche Resultate haben die Form von Prozessen oder Aktivitäten (etwa: eine Schulungsveranstaltung in Phase VI) und erfordern klare Absprachen zwischen den Praxispartnern und der Studierendengruppe. Die Präsentation wird erst nach der Projektarbeit (Phase IV) erstellt, damit nicht die bei Student(inn)en gut eingespielte Arbeitsweise des Vorbereitens von Vorträgen Energie von der eigentlichen Projektarbeit abzieht. Das Lehrteam muss deutlich machen, dass die Präsentation nicht selbst das Ergebnis ist, sondern nur dazu dient, das Resultat der Projektarbeit vorzustellen. 


\section{Termin 12}

Beim Testlauf der Präsentation sollte seitens des Lehrteams insbesondere darauf geachtet werden, dass die für die Praxispartner relevanten Informationen klar dargestellt sind und dass die Beschreibung, wie die Studierendenteams zu ihrem jeweiligen Resultat gekommen sind, nicht zu viel Raum einnimmt. An diesem Termin sollten die Student(inn)en auch angeregt werden, sich gegenseitig Feedback zu geben. Die Situation vor der echten Präsentation ist besonders günstig, konstruktives Feedback zu geben, da es direkt umgesetzt werden kann. Je nach Nähe der Projekte zueinander muss abgesprochen werden, welches Studierendenteam überlappende Inhalte präsentiert. Pragmatische Fragen zur Präsentation („Wer druckt die Handouts?“, „Welche Anschlüsse hat der Beamer?") sollten an diesem Termin final geklärt werden. Die Praxispartner sind an diesem Termin möglichst nicht anwesend.

\section{Termin 13}

Die Resultate werden den Praxispartnern präsentiert, weitere Personen können aber gut einbezogen werden. Im Idealfall sind die Praxispartner zu diesem Termin mit weiteren Personen vertreten, um eine praktische Nutzung der Resultate zu fördern. Der Ort und die Zeit für die Abschlusspräsentation müssen sorgfältig auf den Bedarf der Praxispartner abgestimmt werden: Soll es ein Abendtermin sein oder soll der Termin während der Arbeitszeit stattfinden? Steht ohnehin ein Treffen an, das sich mit dem Seminar verbinden lässt, oder sollte man konkurrierenden Terminen ausweichen?

Sofern ein materielles Produkt erarbeitet wurde, wird dieses übergeben. An jede Präsentation schließt sich eine zweigeteilte Diskussion an: Zunächst steht die Anwendung der Ergebnisse durch die Praxispartner im Vordergrund. Dabei reicht die Spanne der möglicherweise zu diskutierenden Themen von kleinen praktischen Fragen (z. B. „Wer lädt den Film auf welche Plattform hoch?“) über Fragen zum Anwendungsprozedere (z. B. „Wenn wir Ihr Planspiel nutzen, kann jemand von Ihnen moderieren?") bis hin zu grundlegenden Fragen zum Projektansatz. Wenn Projekte nicht vollends fertig geworden sind, sollte an diesem Punkt der Weg bis zum Abschluss geklärt werden. In einem zweiten Teil der Diskussion geben die Praxispartner den Studierendenteams Rückmeldung zur praktischen Relevanz ihrer Projektergebnisse.

Für die Student(inn)en stellt die Ergebnispräsentation den Kulminationspunkt ihres Engagements im Seminar dar. Alles, was danach noch stattfindet, sollte gut begründet werden, eine entspannte Atmosphäre bieten und wieder ganz unter der Regie des Lehrteams stattfinden. Unbedingt sollte eine Nachbesprechung mit den Student(inn)en stattfinden, um eine Reihe von Punkten zu bearbeiten (s. Termin 14). Dies sollte schon zu Beginn des TraPS angekündigt werden, um deutlich zu 
machen, dass es nicht nur um einen symbolischen Abschluss geht (in den Worten eines Studenten in einem Karlsruher TraPS: „Labern wir nur oder passiert da noch was?"). Wenn zwischen Präsentation der Resultate und Reflexion zwei bis drei Wochen (ohne Seminartermine) liegen, ist das eher vorteilhaft.

\section{Termin 14}

An diesem letzten regulären Termin sollten folgende Punkte jeweils knapp bearbeitet werden:

- Reflexion der Zusammenarbeit mit den Praxispartnern: Wie wurde die Kooperation erlebt, wie standen die Erfahrungen in Relation zu den Erwartungen, gab es unausgesprochene Konflikte? Grundlage hierzu sind die eingangs seitens der Student(inn)en individuell formulierten Ziele und Erwartungen (Phase II).

- Reflexion des Bildungsprozesses: Anhand des eigenen Lernportfolios (s. Abschnitt 4.1) können Student(inn)en den eigenen Lernprozess reflektieren. Ein methodischer Weg, um in diesem Arbeitsschritt auf das Individuum zu fokussieren, ist, die Student(inn)en vor einem Austausch im Plenum drei Reflexionsfragen in wenigen Sätzen schriftlich (fürs Lernportfolio) beantworten zu lassen. Diese Fragen sollten sich möglichst konkret auf den Prozess beziehen und Aspekte aufgreifen, die dem Lehrteam wesentlich erscheinen (eher „Inwieweit war die kritische Rückmeldung der Praxispartner auf Ihre Projektskizze für Sie hilfreich?“ als „Welche Rolle spielte Kritik in Ihrem Prozess?“). Die damit finalisierten Lernportfolios werden an diesem Termin abgegeben.

- Rückmeldung zum Seminarverlauf und Einordnung in den Forschungskontext: Die Student(inn)en erwarten in der Regel, dass sie zumindest in groben Zügen erfahren, wie ihre Projekte im Forschungskontext aufgegriffen werden, wie ihre Aktivitäten in einem weiteren Forschungshorizont einzuordnen sind und was das Lehrteam im Prozess gelernt hat. Diese Rückmeldung kann, muss aber nicht eine Bewertung der einzelnen Teams umfassen. Wenn es in oder zwischen den Studierendenteams Spannungen gab, kann hierzu auch eine Aussprache darüber im Plenum und/oder eine Reihe von getrennten Gesprächen mit den Studierendenteams erfolgen. In einigen Fällen fordern die Student(inn)en auch eine Gesamtbeurteilung der Lehrenden ein („Wie waren wir denn?"). Diese Frage lässt sich recht gut entlang der Phasen beantworten.

- Abschließend ist auch eine klassische Lehrevaluation der Lehrveranstaltung durch die Student(inn)en sinnvoll - diese ist in den meisten Universitäten und Hochschulen hoch formalisiert, so dass hier nicht im Detail darauf eingegangen wird. Sie kann durch eine kurze Abschlussrunde ergänzt werden, die persönliche Rückmeldung an die Lehrenden gibt und/oder strukturierten Leitfragen folgt. 
- Sofern Student(inn)en im Rahmen des Seminars Hausarbeiten anfertigen, sollten am Reflexionstermin hierzu Absprachen getroffen werden: Was soll bis wann bearbeitet werden? Dient die Hausarbeit primär Forschungszielen oder sollen auch Bildungs- und Praxisziele verfolgt werden? Soll die Hausarbeit nur an das Lehrteam gehen oder ist sie auch für die Praxispartner von Interesse?

\subsection{Phase VI: In-Wert-Setzung}

Diese Phase gerät leicht aus dem Blick, es ist aber äußerst riskant, hierfür keine zeitlichen Ressourcen einzuplanen. Sie dient dazu, die im TraPS verwobenen Stränge von Praxis, Forschung und Bildung wieder so voneinander zu lösen, dass die Bilanz für alle Zieldimensionen positiv ausfällt, zumindest aber in keiner Schaden entsteht.

Es gilt zunächst, die Fertigstellung oder Optimierung derjenigen Ergebnisse und Produkte zu begleiten, die noch nicht vollständig praxistauglich sind (ein typischer Fall: Ein Informationsflyer wird erstellt, aber die Klärung der Bildrechte ist nicht abgeschlossen). Teilweise müssen dabei auch die Eigentumsverhältnisse und die zukünftige Trägerschaft ausgehandelt werden. Wer hat zum Beispiel welche Rechte an einem im Seminar produzierten Film: die Student(inn)en, die ihn erstellten, die Praxispartner, für die er erstellt wurde, oder das Lehrteam, an das die externen Anfragen zur Nutzung des Films gestellt werden? In manchen Fällen ist es nötig, dass hierbei noch Student(inn)en - zumindest per E-Mail - involviert bleiben.

Es liegt in aller Regel in der Hand der Praxispartner, die erarbeiteten Ergebnisse zu nutzen. Dies kann sehr einfach (z. B. Aufhängen eines Infoposters) oder sehr aufwendig sein (z. B. Kooperation mit durch ein Studierendenteam rekrutierten Schulpartnern). Zum Abschluss sollte eine Nachbesprechung des Lehrteams mit den Praxispartnern durchgeführt werden, um die praktische Relevanz der Ergebnisse zu beurteilen, gegebenenfalls weitere Schritte in der Zusammenarbeit zu planen und sich - soweit gewünscht - wechselseitig Feedback über den Prozess zu geben.

Die normalen Lehraufgaben müssen abgeschlossen werden, wie das Betreuen der Hausarbeiten, das Benoten von Studienleistungen oder die Durchführung mündlicher Prüfungen.

Die erarbeiteten Ergebnisse gilt es auch in den Forschungsprozess einzuspeisen. $\mathrm{Zu}$ den Arbeiten, die hierzu nötig sein können, gehören die Datenintegration (wie 
z. B. das Eintragen von Punkten in einer Online-Karte), die Entwicklung von Nutzungsstrategien für ausgearbeitete partizipative Methoden oder das Aufgreifen bislang unberücksichtigter Theoriekonzepte.

Einzelne Student(inn)en suchen schließlich nach dem Seminar auch nach Gelegenheiten, weiter mit dem Lehrteam bzw. dessen Institution (bei den Karlsruher TraPS: dem Reallabor Karlsruhe) in Kontakt zu bleiben, beispielsweise über studentische oder zivilgesellschaftliche Initiativen - so kann das Seminar auch zur Entwicklung des Netzwerks von Akteuren beitragen.

Der Verlauf des TraPS muss immer wieder angepasst werden: Feiertage, Termine der Praxispartner, Exkursionswochen oder unabgeschlossene Arbeitsschritte machen immer wieder Anpassungen gegenüber dem idealtypischen Verlauf nötig, ebenso Sprünge zurück in frühere Phasen, wenn einzelne Punkte nicht abgeschlossen wurden. Es hat sich aber bewährt, diesen idealtypischen Ablaufplan im Seminar trotzdem sichtbar zu halten, nicht zuletzt, um den hohen Arbeitsaufwand während der Projektphase deutlich zu machen und gegebenenfalls bei den Student(inn)en einfordern zu können.

\section{Didaktische Ansätze als Grundlage im TraPS}

Das TraPS integriert vier Ansätze aus der allgemeinen Didaktik und Hochschuldidaktik (die in sich noch keine Lehrkonzeptionen darstellen), um transdisziplinäres Arbeiten mit transformativem Anspruch zu ermöglichen:

- Service Learning beschreibt Lehre, die den Student(inn)en einen Lernprozess durch Erbringen gesellschaftlich relevanter Leistungen eröffnet (s. Abschnitt $3.1)$.

- Im Forschenden Lernen verfolgen die Student(inn)en eigene Interessen und Ideen in einer wissenschaftlichen Arbeitsweise (s. Abschnitt 3.2).

- Im Team Teaching gestalten Lehrende mit unterschiedlichen Perspektiven, unterschiedlichem Wissen und unterschiedlichen Rollen gemeinsam einen Bildungsprozess (s. Abschnitt 3.3).

- Durch Soziales Lernen können die unterschiedlichen Beteiligten im Austausch von- und miteinander lernen (s. Abschnitt 3.4). 
Diese Ansätze werden in diesem Kapitel kurz vorgestellt, wobei zuerst ihre spezifische Funktion im TraPS anhand von Qualitätskriterien erläutert wird und anschließend das Zusammenspiel der didaktischen Ansätze dargestellt wird (s. Abschnitt 3.5). Im folgenden Kapitel werden drei didaktische Einzelthemen angesprochen, die sich im TraPS als wichtig herausgestellt haben (s. Kapitel 4):

- Die Vermeidung von negativen Rückwirkungen der Prüfungsform, u. a. mit Lernportfolios.

- Das Sokratische Gespräch als eine Art der Gesprächsführung, in der das Lehrteam durch Fragen die Student(inn)en unterstützt, ohne ihren Ergebnissen vorzugreifen.

- Die Lernumgebung so zu gestalten, dass sie die Prozesse im TraPS unterstützt.

\subsection{Service Learning}

Der Ansatz Service Learning wurde in den 2000er Jahren aus den USA nach Deutschland gebracht, unter anderem unter dem Begriff „Lernen durch Engagement", wobei er sich im Schul- und Hochschulkontext relativ unabhängig voneinander weiterentwickelte. Clayton Hurd definiert Service Learning wie folgt:

„As pedagogy, service learning emphasizes meaningful student learning through applied, active, project-based learning that draws on multiple knowledge sources (academic, student knowledge and experience, and community knowledge) and provides students with ample opportunities for ethical and critical reflection and practice." (Hurd 2008, S. 44).

Service Learning erreicht eine positive Wirkung im Lebensumfeld der Student(inn)en, indem diese einüben, Verantwortung zu übernehmen. Dabei ist das Wechselspiel von Erfahrung und Reflexion zentral. Im deutschsprachigen Diskurs finden die Qualitätskriterien von Seifert et al. (2012) weitgehend Zustimmung. Tab. 1 zeigt die Kriterien und deren jeweilige Umsetzung im TraPS. 
Tabelle 1: Elemente von Service Learning (nach Seifert et al. 2012) im TraPS.

\begin{tabular}{|c|c|c|}
\hline $\begin{array}{l}\text { Qualitätskriterien für } \\
\text { Service Learning }\end{array}$ & Umsetzung im TraPS & Phase \\
\hline $\begin{array}{l}\text { Das Engagement der } \\
\text { Lernenden reagiert auf } \\
\text { einen realen Bedarf in } \\
\text { der Gemeinschaft. }\end{array}$ & $\begin{array}{l}\text { - Praxispartner mit bearbeitbaren Bedarfen } \\
\text { - Bedarfe aus der Praxis werden vorgestellt } \\
\text { und diskutiert }\end{array}$ & I \\
\hline $\begin{array}{l}\text { Service Learning ist mit } \\
\text { den Inhalten der Lehr- } \\
\text { pläne verknüpft und } \\
\text { greift diese explizit auf. }\end{array}$ & $\begin{array}{l}\text { - Möglichst Einbettung in einen Studiengang, } \\
\text { ein fachübergreifendes Zertifikatsprogramm } \\
\text { o. Ä. }\end{array}$ & 1 \\
\hline $\begin{array}{l}\text { Es findet eine regelmäßi- } \\
\text { ge und bewusste Reflexi- } \\
\text { on der Erfahrungen statt. }\end{array}$ & $\begin{array}{l}\text { - Reflexion der individuellen Ziele } \\
\text { - Reflexion als Teil des Prozesses } \\
\text { - Abschlussreflexion ohne Praxispartner }\end{array}$ & $\begin{array}{l}\text { II } \\
\text { III, IV } \\
\text { V }\end{array}$ \\
\hline $\begin{array}{l}\text { Die Lernenden sind aktiv } \\
\text { in die Konzeption, Um- } \\
\text { setzung und Nachberei- } \\
\text { tung des Service Learn- } \\
\text { ing-Vorhabens eingebun- } \\
\text { den. }\end{array}$ & $\begin{array}{l}\text { - Abstimmung der Projektideen mit den Pra- } \\
\text { xispartnern } \\
\text { - } \text { Eigenständige Umsetzung durch die Stu- } \\
\text { dent(inn)en } \\
- \text { Diskussion der Umsetzbarkeit/Umsetzung } \\
\text { der Ergebnisse mit Praxispartnern } \\
- \text { Nachbereitung: Portfolios und Hausarbeiten }\end{array}$ & $\begin{array}{l}\text { III } \\
\text { VI, V } \\
\text { II, III, } \\
\text { VI }\end{array}$ \\
\hline $\begin{array}{l}\text { Das Engagement der Ler- } \\
\text { nenden findet gemein- } \\
\text { sam mit Partnern außer- } \\
\text { halb des (Hoch-)Schul- } \\
\text { kontextes statt. }\end{array}$ & $\begin{array}{l}\text { - Einbindung von Praxispartnern } \\
\text { - Gegebenenfalls Third Space zwischen Univer- } \\
\text { sität und Gesellschaft nutzen, z. B. Reallabor }\end{array}$ & $\begin{array}{l}\mathrm{I} \\
\mathrm{I}-\mathrm{VI}\end{array}$ \\
\hline $\begin{array}{l}\text { Die Lernenden erhalten } \\
\text { im Prozess und am Ende } \\
\text { des Service Learning- } \\
\text { Projekts konstruktives } \\
\text { Feedback und Anerken- } \\
\text { nung. }\end{array}$ & $\begin{array}{l}\text { - Feedback zu den Projektskizzen } \\
\text { - Begleitendes Feedback f. Studierendenteams } \\
\text { - Wechselseitiges Feedback: Testlauf Präsenta- } \\
\text { tion } \\
\text { - Feedback zu den Resultaten durch das Lehr- } \\
\text { team und die Praxispartner } \\
\text { - Feedback in der Abschlussreflexion }\end{array}$ & $\begin{array}{l}\text { III } \\
\text { IV } \\
\text { V } \\
\text { V }\end{array}$ \\
\hline
\end{tabular}




\subsection{Forschendes Lernen}

Unter dem Begriff Forschendes Lernen (learning through inquiry) werden zwei Ansätze - teils übereinstimmend, teils divergierend - diskutiert: zum einen die selbstbestimmte und aktive Einführung von Lernenden in das wissenschaftliche Arbeiten, zum anderen die didaktische Nutzung der strukturellen Parallelen des erfahrungsbasierten Lernens mit der Forschung (Mieg und Lehmann 2017). Beides ist für das TraPS interessant. Eine ausdifferenzierte Typologie forschungsbezogener Lehre bieten Rueß et al. (2016), einen Vorschlag zur Abgrenzung des forschenden Lernens vom forschungsbasierten oder forschungsorientierten Lernen macht Huber (2014). Folgende Definition beschreibt den Kern forschenden Lernens:

„Forschendes Lernen zeichnet sich vor anderen Lernformen dadurch aus, dass die Lernenden den Prozess eines Forschungsvorhabens, das auf die Gewinnung von auch für Dritte interessanten Erkenntnissen gerichtet ist, in seinen wesentlichen Phasen - von der Entwicklung der Fragen und Hypothesen über die Wahl und Ausführung der Methoden bis zur Prüfung und Darstellung der Ergebnisse in selbstständiger Arbeit oder in aktiver Mitarbeit in einem übergreifenden Projekt - (mit)gestalten, erfahren und reflektieren.“ (Huber 2009, S. 11).

Wegen der Verankerung des Diskurses zum Forschenden Lernen auch in der Schulpädagogik kommt der professionellen Reflexion der Lehrendenrolle darin besondere Bedeutung zu. Reitinger (2013) formuliert Qualitätskriterien für Forschendes Lernen, die in Tab. 2 dargestellt und den didaktischen Umsetzungen im TraPS gegenübergestellt sind (s. auch Reitinger et al. 2016). Abweichend vom klassischen Forschenden Lernen tritt im TraPS an die Stelle der Hypothesenbildung ein lösungsorientierter Ansatz, wie es Vilsmaier und Meyer (2017) für Forschendes Lernen in der Nachhaltigkeitsforschung vorgeschlagen haben. 
Tabelle 2: Elemente Forschenden Lernens (nach Reitinger 2013 und Reitinger et al. 2016) im TraPS.

\begin{tabular}{|c|c|c|}
\hline $\begin{array}{l}\text { Qualitätskriterien für } \\
\text { Forschendes Lernen }\end{array}$ & Umsetzung im TraPS & Phase \\
\hline $\begin{array}{l}\text { Neugierde/ } \\
\text { Forschungs- } \\
\text { interesse }\end{array}$ & $\begin{array}{l}\text { - Explikation der individuellen Interessen und } \\
\text { Ideen für Projekte } \\
\text { - Freie Themenwahl in Hausarbeiten }\end{array}$ & VI \\
\hline $\begin{array}{l}\text { Lernwege finden und } \\
\text { mitbestimmen }\end{array}$ & $\begin{array}{l}\text { - Einbezug kritischer Perspektiven, um Dogmatik } \\
\text { zu vermeiden } \\
\text { - Wahl des Projektthemas } \\
\text { - Themenfindungsprozess integriert Ideensamm- } \\
\text { lung, Bewertung, Auswahl und Gruppenbildung } \\
\text { - Erstellung der Projektskizze } \\
\text { - Selbstständige Projektrealisierung }\end{array}$ & $\begin{array}{l}\text { II } \\
\text { III } \\
\text { III } \\
\text { III } \\
\text { III, IV }\end{array}$ \\
\hline $\begin{array}{l}\text { Vermuten auf der } \\
\text { Basis von Erfahrung }\end{array}$ & $\begin{array}{l}\text { - Im Kern kein (!) hypothesenprüfendes Vorge- } \\
\text { hen, stattdessen Suche nach realistischen Prob- } \\
\text { lemlösungen } \\
\text { - Einbezug von Erfahrungen der Praxispartner }\end{array}$ & $\begin{array}{l}\text { III-V } \\
\mathrm{I}-\mathrm{VI}\end{array}$ \\
\hline $\begin{array}{l}\text { Authentizität, Auto- } \\
\text { nomie und Explora- } \\
\text { tion }\end{array}$ & $\begin{array}{l}\text { - Freiheit in der Projektgestaltung } \\
\text { - Spanne von explorativen bis zu konstruktiven } \\
\text { Projekten }\end{array}$ & $\begin{array}{l}\text { III, IV } \\
\text { III-V }\end{array}$ \\
\hline $\begin{array}{l}\text { Reflektierte Kommu- } \\
\text { nikation durch das } \\
\text { kritische Gespräch }\end{array}$ & $\begin{array}{l}\text { - Nutzung Sokratischer Gesprächsführung (s. } \\
\text { Abschnitt 4.2) } \\
\text { - Wechselseitige Rückmeldung zwischen Studie- } \\
\text { rendenteams } \\
\text { - Formative Evaluationsrunden, Abschlussreflexi- } \\
\text { on }\end{array}$ & $\begin{array}{l}\text { II-V } \\
\text { IV, V } \\
\text { V }\end{array}$ \\
\hline $\begin{array}{l}\text { Anwenden und Mit- } \\
\text { teilen (conclusion- } \\
\text { based transfer) }\end{array}$ & $\begin{array}{l}\text { - Präsentation und Ergebnisübergabe an die } \\
\text { Praxispartner } \\
\text { - Beteiligung an der In-Wert-Setzung }\end{array}$ & $\mathrm{V}$ \\
\hline
\end{tabular}




\subsection{Inter- und transdisziplinäres Team Teaching}

Team Teaching ist ein Ansatz, der kooperative Lehre durch mehrere Lehrende beschreibt. Auf Basis einer Literaturanalyse führen Welch et al. (1999) vielfältige Definitionen von Team Teaching und verwandten Ansätzen folgendermaßen zusammen:

„We define team teaching as the simultaneous presence of two educators in a classroom setting who share responsibility in the development, implementation, and evaluation of direct service in the form of an instructional or behavioral intervention to a group of students with diverse needs." (Welch et al. 1999, S. 38)

Im Team Teaching war die Beziehung zwischen den Lehrenden ursprünglich oft hierarchisch angelegt (Michael 1963), heute aber wird sie in der Regel kooperativ gedeutet. Besondere Bedeutung kommt dem Team Teaching in der interdisziplinären Lehre zu (DeZure 2010), sowie im Einbezug von externen Partnern (Higgins und Litzenberg 2015).

Im Fall des TraPS gilt es zwei Ebenen der Zusammenarbeit im Team Teaching zu berücksichtigen, erstens die zwischen den Wissenschaftler(inne)n im Lehrteam und zweitens deren Kooperation mit Praxispartnern. Auf diese Weise werden sowohl eine interdisziplinäre Perspektivenvielfalt im Lehrteam als auch eine transdisziplinäre Erweiterung ermöglicht. Im interdisziplinären Team Teaching sollten nicht nur die disziplinären Kompetenzen der Lehrenden berücksichtigt werden, sondern auch deren weitere Kompetenzen (z. B. Moderationsfähigkeiten, Softwarekenntnisse, Erfahrungen mit ähnlichen Projekten). Im transdisziplinären Team Teaching gilt es darüber hinaus zu berücksichtigen, wie viele theoretische Beiträge die Praxispartner beisteuern können und wollen - die Erfahrungen in den Karlsruhe TraPS waren hier sehr unterschiedlich. Darüber hinaus erlaubt das Team Teaching auch die Weitergabe von Erfahrungen im Lehrteam sowie wechselseitige Beobachtung und Rückmeldung. Die folgenden Charakteristika eignen sich dazu, gutes Team Teaching zu beschreiben, und lassen sich der Umsetzung in einem TraPS gegenüberstellen (s. Tab. 3). 
Tabelle 3: Charakteristika von Team Teaching (nach Murata 2002, S. 73f., eigene Übersetzung) im TraPS.

\begin{tabular}{|c|c|c|}
\hline $\begin{array}{l}\text { Charakteristika von } \\
\text { Team Teaching }\end{array}$ & Umsetzung im TraPS & Phase \\
\hline $\begin{array}{l}\text { Gemeinsame Lehr- } \\
\text { philosophie (com- } \\
\text { mon philosophy) }\end{array}$ & $\begin{array}{l}\text { - Normativer Orientierungsrahmen, z. B. Nachhal- } \\
\text { tigkeit } \\
\text { - Frühe Abstimmung der Ziele im Lehrteam } \\
\text { - }\end{array}$ & $\begin{array}{l}1 \\
1\end{array}$ \\
\hline $\begin{array}{l}\text { Betonung (über- } \\
\text { greifender) Kon- } \\
\text { zepte (emphasis } \\
\text { on concepts) }\end{array}$ & $\begin{array}{l}\text { - Übergeordnetes Thema, z. B. nachhaltige Stadt- } \\
\text { entwicklung als integrativ wirkendes Konzept } \\
\text { - Einführung von Schlüsselbegriffen } \\
\text { - Suche nach gemeinsamen Schlüsselkonzepten mit } \\
\text { den Praxispartnern (was nicht immer gelingt) }\end{array}$ & $\begin{array}{l}\mathrm{I}-\mathrm{VI} \\
\mathrm{II}\end{array}$ \\
\hline $\begin{array}{l}\text { Unterschiede } \\
\text { als Stärken } \\
\text { (differences as } \\
\text { strengths) }\end{array}$ & $\begin{array}{l}\text { - Aufstellung eines Lehrteams mit komplementären } \\
\text { Kompetenzen } \\
\text { - Darstellung der jeweiligen Kompetenzen gegen- } \\
\text { über den Student(inn)en } \\
\text { - Aufteilung, um unterschiedliche Lern- und Arbeits- } \\
\text { stile der Student(inn)en besser betreuen zu können }\end{array}$ & II, IV \\
\hline $\begin{array}{l}\text { Gemeinsame Pla- } \\
\text { nung (team plan- } \\
\text { ning) }\end{array}$ & $\begin{array}{l}\text { - Vor- und Nachbereitung jedes Seminartermins } \\
\text { - Gemeinsame Planung federt Fehlzeiten von Mit- } \\
\text { gliedern des Lehrteams gut ab } \\
\text { - Möglichkeit wechselseitiger Rückmeldung }\end{array}$ & $\begin{array}{l}I I-V \\
I I-V\end{array}$ \\
\hline $\begin{array}{l}\text { Offenheit für Ver- } \\
\text { änderungen (open- } \\
\text { ness to change) }\end{array}$ & $\begin{array}{l}\text { - Vor- und Nachbereitung erlaubt kontinuierliche } \\
\text { Kurskorrekturen im Seminarverlauf. }\end{array}$ & $\mathrm{II}-\mathrm{V}$ \\
\hline
\end{tabular}

\subsection{Soziales Lernen}

Die Diskurse zum Sozialen Lernen (social learning, societal learning) sind vielfältig und heterogen (s. Beiträge in Wals 2007), zu weiten Teilen auch ohne pädagogisch-didaktische Perspektive. Je nach Lesart bedeutet Soziales Lernen, dass unterschiedliche Personen miteinander, voneinander, in gemeinsamer Aktivität lernen oder dass Institutionen, Organisationen, Netzwerke oder die Gesellschaft als Ganzes ,lernen'. Für das TraPS sind zwar all diese Lesarten potenziell einschlägig, aber vier davon lassen sich didaktischen und methodischen Elementen eines TraPS gegenüberstellen (s. Tab. 4). 
Tabelle 4: Elemente Sozialen Lernens im TraPS.

\begin{tabular}{|c|c|c|}
\hline Soziales Lernen & Umsetzung im TraPS & Phase \\
\hline inder & $\begin{array}{l}\text { - Unterschiedlicher Hintergrund im Lehrteam, unter- } \\
\text { schiedliche Praxispartner } \\
\text { - Wenn möglich: Kooperation Student(inn)en ver- } \\
\text { schiedener Studiengänge } \\
\text { - } \\
\text { - Kustausch der Studierendenteams untereinander } \\
\text { Kompetenzen unter den Teilnehmer(inne)n explizie- } \\
\text { ren } \\
\text { - Einbezug nicht-akademischer Kompetenzen aller Be- } \\
\text { teiligten }\end{array}$ & $\begin{array}{l}\text { II } \\
I-I I I \\
I I I-V \\
I I, I I I\end{array}$ \\
\hline $\begin{array}{l}\text { Ler } \\
\text { mit }\end{array}$ & 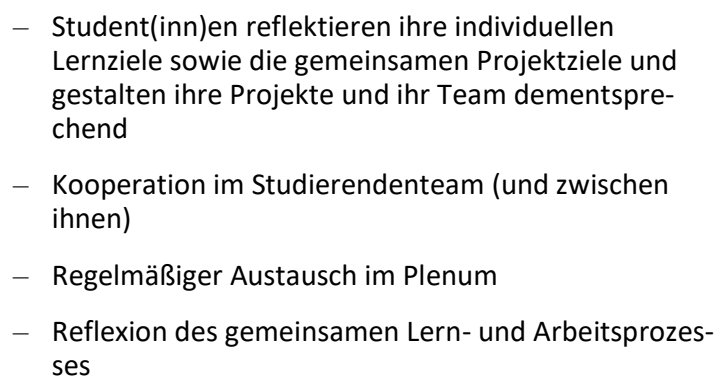 & $\begin{array}{l}I I I-\mathrm{V} \\
\mathrm{II-V} \\
\mathrm{III-V}\end{array}$ \\
\hline $\begin{array}{l}\text { Lernen in } \\
\text { gesellschaft- } \\
\text { lichen Zusam- } \\
\text { menhängen }\end{array}$ & $\begin{array}{l}\text { - Übergreifender Theorierahmen, z. B. nachhaltige } \\
\text { Entwicklung } \\
\text { - Reale gesellschaftliche Problemlagen als Referenz- } \\
\text { punkt } \\
\text { - Spezifische Bedarfe der Praxispartner } \\
\text { - Erleben der eigenen Wirksamkeit in praktischen } \\
\text { Kontexten }\end{array}$ & $\begin{array}{c}\text { I, V, VI } \\
\text { I, V, VI } \\
\text { I, II } \\
\text { III-VI }\end{array}$ \\
\hline $\begin{array}{l}\text { Alle Beteiligten } \\
\text { lernen im Trans- } \\
\text { formations- } \\
\text { prozess }\end{array}$ & $\begin{array}{l}\text { - Offenheit der Lehrenden, selbst zu lernen; Zuge- } \\
\text { ständnis eigener Wissenslücken und fehlender Kom- } \\
\text { petenzen } \\
\text { - Gelegenheit für die Praxispartner, aus der Seminarer- } \\
\text { fahrung zu lernen }\end{array}$ & $\mathrm{I}-\mathrm{VI}$ \\
\hline
\end{tabular}


Der erste Punkt, das Lernen voneinander, stellt in TraPS immer wieder eine besondere Herausforderung für die Planung dar, da nicht absehbar ist, mit welchen Fähigkeiten und welchem Wissen - und komplementär mit welchen Lernbedarfen - Student(inn)en in den Prozess eintreten werden.

\subsection{Integration der Ansätze}

Die oben vorgestellten Ansätze werden in einem TraPS verbunden. Die Vielfalt der didaktischen Elemente dient dazu, unterschiedliche Lernformen und Typen von Studierendenprojekten zu ermöglichen, aber auch als Sicherheitsnetz, denn sie bietet so viel Flexibilität, dass zumindest einige der Lehr- und Lernansätze sowie der angebotenen Herangehensweisen gelingen sollten und dass unterschiedliche Elemente den jeweiligen Interessen und Vorlieben der Student(inn)en entgegenkommen.

Abb. 4 verdeutlicht, wie die didaktischen Ansätze Verbindungen zwischen den drei Zieldimensionen des TraPS ermöglichen. Inter- und transdisziplinäres Team Teaching stellt eine Brücke zwischen Forschungs- und Praxiszielen dar, Forschendes Lernen greift eine an Forschungszielen orientierte methodische Arbeits-

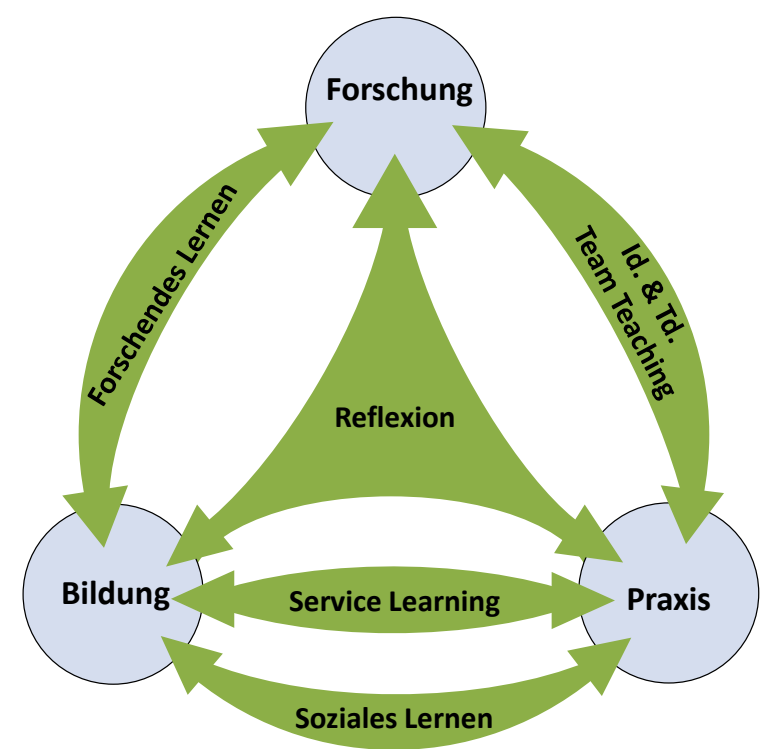

Abbildung 4: Die didaktischen Ansätze im TraPS verbinden die drei Zieldimensionen. (C) Richard Beecroft. 
weise auf und verbindet sie mit Bildungszielen, Service Learning wiederum richtet Bildungsprozesse an Praxiszielen aus. Soziales Lernen - in einer seiner Lesarten - nutzt die gemeinsame Praxis dafür, dass die Beteiligten von- und miteinander lernen können. In allen genannten didaktischen Ansätzen ist Reflexion der Erfahrungen essenziell. Sie stellt zugleich (durch Abstraktion) einen Mechanismus der Forschung und (z. B. durch kritisches Hinterfragen der eigenen Rolle) einen Mechanismus der Bildung dar.

Tab. 5 gibt eine Übersicht über die Ansätze und die jeweils im Detail übernommenen Kriterien und Anforderungen. Diese wurden hierfür sprachlich angeglichen und zu fünf Clustern zusammengefasst, die zentrale didaktische Felder im TraPS beschreiben. Für Lehrende, die nicht mit allen dieser Ansätze vertraut sind, dient die Tabelle dazu auszuloten, welche didaktische Vorbereitung noch nötig ist. Rechts zeigt die Tabelle, in welcher Phase das jeweilige Kriterium von besonderer Relevanz ist. Anhand der Tabelle wird deutlich, dass die Ansätze nicht einzelnen Phasen zugeordnet sind, sondern jeweils mehrere Phasen im Seminarablauf betreffen. Wenn Schwierigkeiten in einer der Phasen auftreten, bietet die Tabelle eine schnelle Zuordnung, auf welche Elemente der jeweiligen didaktischen Ansätze zurückgegriffen werden kann.

Um das Zusammenspiel der Ansätze zu realisieren, werden auch einige didaktische Methoden und Elemente eingesetzt, die vielleicht nicht allgemein bekannt sind. Sie sollen im Folgenden dargestellt werden. 
Tabelle 5: Übersicht über die im TraPS verbundenen didaktischen Ansätze (SeL: Service Learning, FL: Forschendes Lernen, TT: Team Teaching, SoL: Soziales Lernen).

\begin{tabular}{|c|c|c|c|c|c|c|c|c|c|c|c|}
\hline \multirow{2}{*}{\multicolumn{2}{|c|}{ Elemente }} & \multicolumn{4}{|c|}{$\begin{array}{c}\text { Didaktischer } \\
\text { Ansatz }\end{array}$} & \multicolumn{6}{|c|}{$\begin{array}{l}\text { TraPS } \\
\text { Phase }\end{array}$} \\
\hline & & Sel & FL & TT & Sol & 1 & II & III & IV & $\mathbf{v}$ & VI \\
\hline \multirow{3}{*}{$\begin{array}{l}\text { Dreifacher } \\
\text { Ausgangs- } \\
\text { punkt }\end{array}$} & Realer Bedarf & $x$ & & & $x$ & & & & & & \\
\hline & Wiss. Fragestellung & & $x$ & & & & & & & & \\
\hline & Neugierde & & $x$ & & $x$ & & & & & & \\
\hline \multirow{3}{*}{$\begin{array}{l}\text { Dreifache } \\
\text { Einbettung }\end{array}$} & Curriculare Einbettung & $x$ & $x$ & & & & & & & & \\
\hline & Einbettung in Forschung & & $x$ & & & & & & & & \\
\hline & Praktische Einbettung & $x$ & & & $x$ & & & & & & \\
\hline \multirow{3}{*}{$\begin{array}{l}\text { Aktive Rolle } \\
\text { der Lernen- } \\
\text { den }\end{array}$} & (Co-)Design & $x$ & $x$ & & & & & & & & \\
\hline & Durchführung & $x$ & $x$ & & $x$ & & & & & & \\
\hline & Anwenden/Weitergeben & $x$ & $x$ & & & & & & & & \\
\hline \multirow{9}{*}{$\begin{array}{l}\text { Vielfältige } \\
\text { Mechanismen } \\
\text { des Lernens }\end{array}$} & Instruktion/Input & & & $x$ & & & & & & & \\
\hline & Übergreifende Konzepte & & & $x$ & & & & & & & \\
\hline & Kritisches Gespräch & & $x$ & & & & & & & & \\
\hline & Reflexion & $x$ & $x$ & & $x$ & & & & & & \\
\hline & Hypothesen/Lösungsideen & & $x$ & & & & & & & & \\
\hline & Erfahrung & $x$ & $x$ & & & & & & & & \\
\hline & Wissenschaftl. Methoden & & $x$ & & & & & & & & \\
\hline & Feedback & $x$ & $x$ & & & & & & & & \\
\hline & Austausch/Differenz & $x$ & & $x$ & $x$ & & & & & & \\
\hline \multirow{5}{*}{$\begin{array}{l}\text { Gemeinsame } \\
\text { und reflexive } \\
\text { Aufgaben der } \\
\text { Lehrenden }\end{array}$} & Gemeins. Lehrphilosophie & & & $x$ & & & & & & & \\
\hline & Gemeinsame Planung & & & $x$ & & & & & & & \\
\hline & Offenheit f. Veränderung & & & $x$ & & & & & & & \\
\hline & Reflexion Lehrendenrolle & & $x$ & & & & & & & & \\
\hline & Evaluation & $x$ & & & & & & & & & \\
\hline
\end{tabular}




\section{Einzelne didaktische Methoden und Elemente im TraPS}

\subsection{Bewerten ohne negative Rückwirkungen}

Bewerten stellt ein zentrales - und nicht immer geliebtes - Thema jeder Lehre dar. Immer wieder kommt die Sorge auf, dass die Form der Bewertung einen negativen Effekt auf die Erreichung der Bildungsziele hat (auch im deutschen Diskurs oft als Washback oder Backwash bezeichnet). Im Fall eines TraPS muss darüber hinaus vermieden werden, dass negative Rückwirkungen gegenüber den Praxis- und Forschungszielen auftreten. Es gilt, folgende Kriterien zu erfüllen:

1) Bildungs-, Forschungs- und Praxisziele werden gleichermaßen in der Bewertung abgebildet.

2) Die Bewertungsgrundlage verursacht möglichst wenig zusätzlichen Aufwand für die Lernenden.

3) Die Bewertung tariert die Gruppenleistung (in den Studierendenteams) und die individuelle Leistung (die Unterschiede im Eingangsniveau berücksichtigen kann) aus.

In einem TraPS werden, um diesen Anforderungen gerecht zu werden, zwei oder drei gleich gewichtete Leistungen erbracht (s. Tab. 6):

- In einem Lernportfolio ordnen Student(inn)en jeweils individuell alle Dokumente ein, die sie im Seminarverlauf bearbeitet bzw. erstellt haben: Fragen und Kommentare zu einführenden Texten, Projektideen, die Projektskizze, Rechercheergebnisse, Kurzinterviews, Protokolle von Treffen und individuelle Beiträge zum Ergebnis. Diese Texte (und Skizzen etc.) entstehen ohnehin im Arbeitsprozess, ihre systematische Sammlung stellt nur einen geringen Mehraufwand dar. Nur zwei Dokumente werden spezifisch für das Lernportfolio erstellt: Die Reflexion der eigenen Lernziele zu Beginn (Phase II) und die Abschlussreflexion (Phase V). Insgesamt dient das Lernportfolio primär dazu, dass die Student(inn)en ihren individuellen Bildungsprozess kontinuierlich dokumentieren und reflektieren. Lernportfolios, obwohl vielen Lehrenden unbekannt, stellen inzwischen eine recht anerkannte Prüfungsform dar, so dass bei Bedarf hierzu hochschuldidaktische Beratung an fast jeder Hochschule verfügbar sein sollte. Wesentliche Kriterien zur Bewertung sind die vollständige Abbildung des Projekts, die Dokumentation relevanter Projektbeiträge und die Tiefe der Abschlussreflexion.

- Das Resultat, inklusive der Präsentation, als Gruppenleistung. Wenn das Projektergebnis nicht selbst textförmig ist, sondern etwa ein Film, wird ein 
Tabelle 6: Passung der Studienleistungen im TraPS zu den Zieldimensionen.

\begin{tabular}{|l|l|l|}
\hline Bewertete Studienleistung & Sozialform & passt insbesondere zu \\
\hline Portfolio & Individuell & Bildungsziele \\
\hline Projektergebnis inkl. Präsentation & Team & Praxisziele \\
\hline Hausarbeit (ergänzend) & Individuell oder zu zweit & Forschungsziele \\
\hline
\end{tabular}

kurzes, ausformuliertes Handout mit abgegeben, das auch eine bessere Grundlage zur Bewertung darstellt. Bei der Benotung wird die praktische Wirksamkeit - vorzugsweise beurteilt von den Praxispartnern - mit einbezogen. Für diese Leistung sollte man von vorneherein anbieten, $50 \%$ der Note durch individuelle mündliche Prüfungen zu ersetzen, beispielsweise falls ein Gruppenprozess scheitert. In den sechs TraPS in Karlsruhe gab es keinen einzigen Fall, in dem Student(inn)en davon tatsächlich Gebrauch machten. Allein diese Option zu haben, hat aber mehrfach Studierendenteams geholfen, entspannter mit Konflikten umzugehen.

- Für Student(inn)en, die die maximale Zahl an Leistungspunkten (LP) erwerben wollen (in Karlsruhe: 6 statt 4 LP), besteht die Möglichkeit, eine (individuelle) Hausarbeit zu erstellen. Diese darf auf der eigenen Projektarbeit aufbauen, und sie muss nicht zwingend für die Praxispartner relevant sein, sondern soll primär wissenschaftlich gehaltvoll und interessant für die Student(inn)en sein.

Dieser Bewertungsansatz erlaubt es, einer äußerst heterogenen Gruppe von Student(inn)en aus allen Studiengängen, im Bachelor- oder Masterstudium, die unterschiedliche (benotete oder unbenotete) Scheine benötigen, gerecht zu werden. Je nach der Einbettung des TraPS in Studiengänge und fachübergreifende Programme muss dieses Bewertungssystem so angepasst werden, dass die drei eingangs im Abschnitt genannten Punkte (1.-3.) erreicht werden. Selbstverständlich muss es den Student(inn)en von Beginn an transparent gemacht werden.

\subsection{Das Sokratische Gespräch}

Das Sokratische Gespräch ist eine Unterrichtsmethode, die sich aus der philosophischen Tradition des dialogischen Argumentierens ableitet. Während in der Philosophie zumeist nur zwei Personen beteiligt sind, eignet es sich als didaktische Methode auch für Gruppen (Raupach-Strey 2002). Im Sokratischen Gespräch kommt einer Person - im Fall der TraPS einem Mitglied des Lehrteams die Rolle zu, durch systematisches Fragen anderen, insbesondere Student(inn)en, bei der Entwicklung ihrer eigenen Gedanken zu helfen. Die Fragen werden dabei so gestellt, dass die fragende Person keine eigenen Ideen einbringt und die Be- 
fragten inhaltlich nicht leitet. Die Fragen dienen vielmehr den Befragten dazu, ihre eigenen Überlegungen zu vertiefen, weiterzutreiben, Widersprüche zu entdecken oder, im Fall einer Gruppe, sich zu verständigen. Bei der Arbeit mit einer Gruppe können sich dabei Fragen, wie sie auch in der Moderation üblich sind („Sehen Sie das auch so wie X?" „Haben Sie verstanden, was Y meint?“), abwechseln mit solchen, die zu einem tieferen Verständigungsprozess hinleiten („Was meinen Sie mit ,nützlich“?" „Wenn Sie beide von ,Vertrauen“ sprecht, meinen Sie dasselbe - wer vertraut wem?"). Das Sokratische Gespräch unterstützt auf diese Weise analytisches und kritisch-reflexives Denken sowie Kreativität (s. Stenning et al. 2016). Es eignet sich insbesondere dazu, einen spezifischen Fall im Licht von theoretischen Konzepten zu diskutieren (z. B. in der genetischexemplarisch-sokratischen Methode, s. Wagenschein 1999).

Das Sokratische Gespräch muss im Rahmen eines TraPS umsichtig eingesetzt werden, da unterschiedliche Rollenerwartungen an das Lehrteam gestellt werden: Wenn dieses etwa in seiner Expertenrolle angesprochen wird oder weil es verantwortlich für den Rahmen ist, kann sokratisches Nachfragen statt klarer Ansagen und Auskünfte schnell zu Irritationen führen. Nichtsdestotrotz ist das Sokratische Gespräch ein wichtiges Mittel, um die Studierendenteams zu unterstützen, aber zugleich das jeweilige Projekt in deren Verantwortung zu lassen.

\subsection{Die Gestaltung der Lernumgebung}

Sofern die Möglichkeit dazu besteht, sollte die Lernumgebung (Learning Environment, s. Jonassen und Land 2012) für ein TraPS passend zu den spezifischen Arbeitsformen vorbereitet werden. Dies hat eine offensichtliche praktische und eine subtile Seite.

Praktisch gilt es, die Räume für die unterschiedlichen Arbeitsformen im Seminar auszustatten: Je nach Phase und Arbeitsschritt werden ein Plenum, Gruppentische, Platz für flüsternde Zweiergespräche oder individuelle Stillarbeit benötigt. Teilweise sind Visualisierungen (Beamer, Flipchart) nötig, teilweise würden sie ablenken. Laptops, Tablets und Smartphones sind zur Recherche unerlässlich, stören aber in anderen Arbeitsschritten. Alle Materialien, die zuvor im TraPS erarbeitet wurden, sollten jederzeit wieder greifbar sein (typischerweise z. B. Flipchartblätter, Karten). Es lohnt sich, Stauraum dafür vorzusehen und auf systematische Beschriftungen zu achten. Je nach Projekt der Student(inn)en besteht auch noch weiterer Bedarf nach Infrastruktur (z. B. Zugriff auf einen Drucker), auf den die Lernumgebung möglichst flexibel ausgerichtet sein sollte. Wenn ein Programm zur virtuellen Unterstützung der Kooperation im Seminar eingesetzt wird, sollte auch dieses auf seine Tauglichkeit als Lernumgebung hin überprüft und gegebenenfalls gestaltet werden. In den Karlsruher TraPS fungierte der Zukunftsraum im Reallabor Karlsruhe als Lernumgebung (s. Anhang zu diesem Beitrag). 
Subtiler ist die Bedeutung des Raums für die Arbeitsatmosphäre, für den ,Stil ‘ der Veranstaltung. TraPS können, ob geplant oder nicht, ,unter die Haut gehen', unbewusste Selbstverständlichkeiten, Gewohnheiten und Ansichten zum Vorschein bringen, herausfordern oder auch schlummernden Veränderungswillen anstoßen. Im Gruppenprozess gilt es, vermeintlich dumme Nachfragen, Kritik, das Erkennen von Irrwegen in der Projektarbeit und die Bearbeitung von Spannungen zu ermöglichen. Je näher den Student(inn)en (sowie dem Lehrteam und den Praxispartnern) die Themen liegen, desto mehr kann die Arbeit deren Selbstverständnis berühren, desto sensibler sind auch die Gruppenprozesse. Es ist ein wichtiger Teil der Lehraufgaben im TraPS, hierfür einen geschützten Rahmen zu bieten. Der physische Raum, in dem dies alles stattfindet, sollte (gleichermaßen wie die Haltung des Lehrteams) diesem Bedarf Rechnung tragen. Er sollte offen, inspirierend und einladend sein, zugleich aber auch genug Ruhe und Ernsthaftigkeit bieten, um solche Prozesse ungestört gestalten zu können. Das Lehrteam (und evtl. die Praxispartner) sollten sich im physischen Raum möglichst ,zu Hause' fühlen und mit geübter Selbstverständlichkeit alle Möglichkeiten des Raumes nutzen können.

\section{Reflexion}

Die Erfahrung mit den TraPS (s. Anhang) wird hier zunächst anhand von zwei zentralen Aspekten reflektiert, die von allgemeiner Relevanz sind: Zum Ersten anhand des Umgangs mit Zielkonflikten (s. Abschnitt 5.1), zum Zweiten anhand des Umgang mit Erwartungen und Trägerschaft (s. Abschnitt 5.2). Beide bergen das Risiko, Spannungen im Gruppenprozess hervorzurufen, lassen sich aber nicht allein auf der Ebene der Gruppendynamik bearbeiten, da sie eng mit den Inhalten, dem transformativen Anspruch und der transdisziplinären Arbeitsweise verbunden sind. Abschließend wird die Erfahrung mit der spezifischen Situation, TraPS in einem Reallabor zu verankern, dargestellt (s. Abschnitt 5.3).

\subsection{Vorausschauender und entspannter Umgang mit Zielkonflikten}

Ein TraPS dient dazu, Ziele in drei Dimensionen zu verfolgen, Forschungs-, Praxis- und Bildungsziele (s. Beecroft et al. 2018) - dieser Zielhorizont kann zu Zielkonflikten führen. Das bedeutet, dass nicht nur verschiedene Beteiligte unterschiedliche Interessen verfolgen (Interessenkonflikte, die es gegebenenfalls auch auszuhandeln und auszuhalten gilt), sondern dass die Beteiligten gemeinsam Ziele in unterschiedlichen Dimensionen anstreben, diese Ziele aber in Konkur- 
renz zueinander geraten können. ${ }^{2}$ Solche Zielkonflikte sollten vorausschauend identifiziert und unaufgeregt thematisiert werden.

Es lohnt sich, die individuellen Ziele aller Beteiligten zu explizieren, um bereits von Anfang an mögliche Konflikte aufzuspüren. Dies geschieht in Phase I mit den Praxispartnern, in den Phasen II und III mit den Student(inn)en, die gemeinsam in den Studierendenteams einen Zielhorizont für ihr jeweiliges Projekt festlegen. Eventuell gelingt es bereits zu diesem frühen Zeitpunkt, eine Handhabe für die Zielkonflikte zu finden, zumindest die Prioritäten der Ziele zu benennen und damit in den Projekten auch zu überprüfen, was essenziell ist.

In einem TraPS ist ein recht entspannter Umgang mit den Zielen ratsam: Nicht jedes Studierendenprojekt wird Ziele in allen drei Dimensionen im selben Maß erreichen können. Wenn von einem Studierendenteam nur in zwei der drei Zieldimensionen Ziele erreicht werden, ist das völlig ausreichend. Gegebenenfalls ist immer noch möglich, durch ein ergänzendes, komplementäres Element (z. B. eine Hausarbeit, einen Workshop, ein separates Dokument) in Phase VI ein Ziel zu verfolgen, das sonst in den Hintergrund gerückt wäre. Schließlich stellt die Fähigkeit, Zielkonflikte zu erkennen und zu bearbeiten, auch eine wichtige Kompetenz dar, die die Student(inn)en in einem TraPS erwerben können - so dass ihre Bearbeitung selbst schon einem Bildungsziel dient.

Positiv gewendet lässt sich sagen, dass ein TraPS als Ansatz gerade auch dann interessant ist, wenn schon die Ausgangsbedarfe des Praxispartners explizit oder implizit Zielkonflikte aufweisen: In solchen Fällen können die parallel arbeitenden Studierendenteams Einzelbeiträge liefern, die zusammengenommen allen Zieldimensionen dienlich sind.

\subsection{Erwartungen managen und Trägerschaft mitdenken}

Der dreifache Zielhorizont kann es mit sich bringen, dass die verschiedenen Beteiligten nicht im Einklang stehende Erwartungen aneinander und an das TraPS insgesamt haben, die gleichzeitig nicht selten diffus und implizit bleiben. In den Karlsruher TraPS hat es sich bewährt, den Zielhorizont und die damit verbundenen Erwartungen sehr explizit zum Thema zu machen, und zwar zunächst in Phase I in einer Diskussion mit den Praxispartnern, in Phase II in einer Diskussion mit den Student(inn)en und indem in Phase III den Studierendenteams dann Gelegenheit gegeben wird, eigene Ansprüche zu formulieren.

2 Natürlich kommt es mitunter auch innerhalb der Forschungs-, Bildungs- und Praxisziele zu Zielkonflikten; in diesen Fällen sind die Ziele aber vergleichbar und eine Abwägung dadurch deutlich leichter möglich. 
Die Erwartungen der Praxispartner sind besonders zu Beginn oft diffus und nicht übermäßig hoch. Die Student(inn)en wiederum bringen zunächst Erwartungen mit ins TraPS, die sich auf den Titel und den Veranstaltungskommentar beziehen. Ungünstige Beschreibungen können von Anfang an falsche Erwartungen wecken. Vorsicht ist auch geboten in Bezug auf Versprechungen, dass das TraPS ,Spaß macht ${ }^{\star}$. Insbesondere in der Projektarbeit kommt es immer wieder zu Situationen, die weniger Spaß machen. Andere Motivationen, etwa die Möglichkeit, eigenen Interessen $\mathrm{zu}$ folgen oder praxisrelevante Arbeitstechniken zu erlernen, sind in dieser Hinsicht robuster.

Bei der Erstellung der Projektskizzen (Phase III) neigen die Student(inn)en zu hohen, teils auch unrealistischen Erwartungen an ihr eigenes Projekt, wobei die Bildungs- und Forschungsziele tendenziell weniger klar gefasst sind als die Praxisziele. Dabei können übersteigerte Erwartungen an die eigene Leistungsfähigkeit, an die Qualität des geplanten Resultats und an dessen Nutzung durch die Praxispartner Hand in Hand gehen. Möglicherweise ist dies bei TraPS im Rahmen eines Fachstudiums anders, da die Student(inn)en dort vielleicht klarere Vorstellungen mitbringen in Bezug auf die eigenen Bildungsziele, den wissenschaftlichen Anspruch des Fachs und den Aufwand der einzusetzenden Methoden. Gelegentlich kann ein Projekt eines Studierendenteams in praktischer Hinsicht gänzlich scheitern; in diesem Fall ist es besonders wichtig, die Erwartungen in den anderen beiden Zieldimensionen im Blick zu behalten. Gegenüber den Praxispartnern sollte die Möglichkeit, dass Projekte in Bezug auf die Praxisziele scheitern können, bereits in Phase I deutlich gemacht werden.

Als besonders relevant haben sich Erwartungen herausgestellt, die die Trägerschaft betreffen, also die mittelfristige Verantwortung für und Verfügung über die Resultate: Jedes Projekt eines Studierendenteams ist verbunden mit einer Vorstellung darüber, wer in Folge was damit tun soll. Manche Ergebnisse können ohne Aufwand genutzt werden (z. B. ein Infoposter), andere erfordern aber intensive Beschäftigung und eine klare Umsetzungsabsicht (z. B. ein Planspiel). Wenn die Trägerschaft nicht schon während der Erstellung der Projektskizzen mitbedacht und in der Projektarbeit verbindlich gemacht wird, kann es nach der Übergabe der Ergebnisse zu Enttäuschungen seitens der Student(inn)en kommen. Genauso wie die Praxispartner ihnen den Freiraum zugestehen müssen, die eigenen Projekte zu skizzieren, müssen die Studierendenteams ihr Projektergebnis am Ende loslassen. Die explizite Diskussion und Reflexion der Ziele und Erwartungen aller Beteiligten kann solchen Enttäuschungen entgegenwirken und die Sensibilität dafür erhöhen, welche Akteure welche Art Freiheit und Autonomie brauchen. Gerade in der Kooperation mit kleinen zivilgesellschaftlichen Initiativen muss mit der Erwartung, dass diese die Trägerschaft übernehmen, vorsichtig umgegangen werden: Die Student(inn)en können im Extremfall gemeinsam über 4.000 Arbeitsstunden (bei 6 ECTS und 24 Student(inn)en) in die Projekte investieren - eine kleine Initiative kann nur einen Bruchteil dessen für die langfristige Trägerschaft aufwenden. 


\subsection{Besonderheiten der Einbettung eines TraPS in einem Reallabor}

In Karlsruhe waren die TraPS in ein Reallabor eingebettet (s. Abschnitt 1.1). Auch wenn diese Einbettung nicht notwendig ist für ein TraPS, bietet ein Reallabor doch eine wertvolle Unterstützungsstruktur, von der vielleicht einzelne Elemente in andere Projektrahmen übertragen werden können. Da ein Reallabor selbst Forschungs-, Praxis- und Bildungsziele verfolgt, ist die Passung zu einem TraPS leicht herzustellen. Die in Beecroft et al. (2018) skizzierten Designprinzipien können beispielsweise herangezogen werden, um die Passung zwischen dem Reallabor - oder einem vergleichbaren Vorhaben im Grenzbereich von Wissenschaft und Praxis - und dem TraPS zu optimieren. Dabei gilt es zu bedenken, dass ein TraPS darauf ausgelegt ist, von einer Rahmung zu profitieren, die transformative Prozesse unterstützt, und umgekehrt selbst zu einer solchen Rahmung beizutragen.

Erstens stellt ein Reallabor einen Forschungskontext dar, in dem transdisziplinäre Projekte stattfinden. Es ist möglich, einzelne Studierendenprojekte im TraPS an übergreifende Themen und Fragen im Reallabor anzuschließen und im TraPS entsprechende Theoriebezüge, Evaluationsmechanismen, eine Dokumentationsinfrastruktur usw. zu nutzen. Das interdisziplinäre Reallaborteam wiederum stellt eine wichtige Ressource für das TraPS dar, wenn weitere Expert(inn)en zu Teilfragen oder spezifischen Methoden gesucht werden. Das TraPS seinerseits kann zur Forschung im Reallabor beitragen, indem entweder eine systematische Integration von Fallstudien im Reallabor angelegt ist oder auch Forschungsfragen in das TraPS bereits in der Konzeption mit eingespeist werden.

Zweitens bietet das Reallabor mit den von ihm gepflegten Kooperationen mit Praxisakteuren einen Praxiskontext für das TraPS. Das Reallabor zielt darauf ab, Transformationsprozesse zu initiieren und zu begleiten. In diesem Kontext können auch die Praxisziele eines TraPS verfolgt werden. Das Reallabor unterhält ein Netzwerk von Akteuren, aus dem sich Praxispartner für ein TraPS gewinnen lassen. Das TraPS kann umgekehrt dazu eingesetzt werden, bestehende Kooperationen für ein Reallabor zu vertiefen.

Drittens stellt das Reallabor eine Lernumgebung dar. Diese umfasst die physische und virtuelle Infrastruktur des Reallabors, die Räume, Materialien, Arbeitsplätze etc., aber ebenso den Untersuchungsraum des Reallabor, über den im Reallabor bereits ein erheblicher Wissensschatz zur Verfügung steht. Da das Reallabor darauf ausgerichtet ist, iterative Lernprozesse zu ermöglichen, können umgekehrt die Erfahrungen aus einem TraPS in die Weiterentwicklung des Reallabors selbst und in die Lernprozesse aller Beteiligten einfließen. In Form eines TraPS kann universitäre Lehre in gesellschaftliche Lernprozesse eingebettet werden - zum beiderseitigen Vorteil. 


\section{Dank}

Der Autor dankt Charlotte Eller für die Diskussionen über eine frühere Textfassung. Insbesondere möchte er Marius Albiez, Andri König, Astrid Ley und Thomas Potthast sowie zwei anonymen Gutachter(inne)n für die konkreten Hinweise zur Verbesserung des Textes im Rahmen des internen und externen Reviews danken. Schließlich dankt der Autor den beiden Herausgebenden, Rico Defila und Antonietta Di Giulio, für ihre Rückmeldungen zum Text.

\section{Literatur}

Beecroft, R., \& Parodi, O. (2016). Reallabore als Orte der Nachhaltigkeitsforschung und Transformation. Einführung in den Schwerpunkt. Technikfolgenabschätzung - Theorie und Praxis, 25 (3), (S. 4-8).

Beecroft, R., Trenks, H., Rhodius, R., Benighaus, C., \& Parodi, O. (2018). Reallabore als Rahmen transformativer und transdisziplinärer Forschung: Ziele und Designprinzipien. In R. Defila \& A. Di Giulio (Hrsg.), Transdisziplinär und transformativ forschen. Eine Methodensammlung (S. 75-100). Wiesbaden: Springer VS. doi: 10.1007/978-3-65821530-9_4.

Beecroft, R. (2018). Embedding Higher Education into a Real-World Lab: A ProcessOriented Analysis of Six Transdisciplinary Project Courses. Sustainability, 10 (10), (Nr. 3798). doi: 10.3390/su10103798.

DeZure, D. (2010). Interdisciplinary pedagogies in higher education. In R. Frodeman, J. Klein \& R. Pacheco (Hrsg.), The Oxford handbook of interdisciplinarity (S. 372-386). Oxford: Oxford University Press.

Higgins, L., \& Litzenberg, K. (2015). Transferring experience through team teaching: The chance of a lifetime. College teaching, 63 (3), (S. 105-111).

Huber, L. (2009). Warum Forschendes Lernen nötig und möglich ist. In L. Huber, J. Hellmer \& F. Schneider (Hrsg.), Forschendes Lernen im Studium. Aktuelle Konzepte und Erfahrungen (S. 9-35). Bielefeld: Universitäts-Verlag.

Huber, L. (2014). Forschungsbasiertes, Forschungsorientiertes, Forschendes Lernen: Alles dasselbe? Ein Plädoyer für eine Verständigung über Begriffe und Unterscheidungen im Feld forschungsnahen Lehrens und Lernens. Das Hochschulwesen, 62 (1/2), (S. 32-39).

Hurd, C. A. (2008). Is service-learning effective? A look at current research. In S. Shalini (Hrsg.), Service-learning: Perspectives and applications (S. 44-60). Punjagutta: ICFAI University Press. Manuskript vom Juni 2006 verfügbar unter http://fresnostate.edu/ craig/depts-programs $/ \mathrm{mktg} /$ documents/Is $\quad$ S.L. Effective-.pdf. Zugegriffen am 09.03.2019. 
Jonassen, D., \& Land, S. (Hrsg.). (2012). Theoretical foundations of learning environments. Routledge: New York.

Meyer-Soylu, S., Parodi, O., Trenks, H., \& Seebacher, A. (2016). Das Reallabor als Partizipationskontinuum. Erfahrungen aus dem Quartier Zukunft und Reallabor 131 in Karlsruhe. Technikfolgenabschätzung - Theorie und Praxis, 25 (3), (S. 31-40).

Michael, L. (1963). Team teaching. The bulletin of the National Association of Secondary School Principals, 47 (283), (S. 36-63).

Mieg, H., \& Lehmann, J. (Hrsg.). (2017). Forschendes Lernen: Wie die Lehre in Universität und Fachhochschule erneuert werden kann. Frankfurt, New York: Campus.

Murata, R. (2002). What does team teaching mean? A case study of interdisciplinary teaming. The Journal of educational research, 96 (2), (S. 67-77).

Parodi, O., Albiez, M., Beecroft, R., Meyer-Soylu, S., Quint, A., Seebacher, A., Trenks, H., \& Waitz, C. (2016). Das Konzept „Reallabor“ schärfen: Ein Zwischenruf des Reallabor 131: KIT findet Stadt. GAIA, 25 (4), (S. 284-285). doi:10.14512/gaia.25.4.11.

Raupach-Strey, G. (2002). Sokratische Didaktik. Die didaktische Bedeutung der Sokratischen Methode in der Tradition von Leonard Nelson und Gustav Heckmann. Münster: Lit.

Reitinger, J. (2013). Forschendes Lernen. Theorie, Evaluation und Praxis. Theorie und Praxis der Schulpädagogik, Band 12. Immenhausen bei Kassel: Prolog.

Reitinger, J., Haberfellner, Ch., \& Keplinger, G. (2016). An Overview of the Theory of Inquiry Learning Arrangements (TILA). In J. Reitinger, C. Haberfellner, E. Brewster \& M. Kramer (Hrsg.). Theory of inquiry learning arrangements: research, reflection, and implementation (S. 1-11). Kassel: Universitätsverlag Kassel.

Rueß, J., Gess, C., \& Deicke, W. (2016). Forschendes Lernen und forschungsbezogene Lehre - empirisch gestützte Systematisierung des Forschungsbezugs hochschulischer Lehre. Zeitschrift für Hochschulentwicklung, 11 (2), (S. 23-44).

Seifert, A., Zentner, S., \& Nagy, F. (2012). Praxisbuch Service-Learning: „Lernen durch Engagement" an Schulen. Mit Materialien für Grundschule und Sekundarstufe I + II. Weinheim: Beltz.

Stenning, K., Schmoelz, A., Wren, H., Stouraitis, E., Scaltsas, T., Alexopoulos, C., \& Aichhorn, A. (2016). Socratic dialogue as a teaching and research method for cocreativity? Digital Culture \& Education, 8 (2), (S. 154-168).

Trenks, H., Waitz, C., Meyer-Soylu, S., \& Parodi, O. (2018). Mit einer Realexperimentreihe Impulse für soziale Innovationen setzen - Realexperimente initiieren, begleiten und beforschen. In R. Defila \& A. Di Giulio (Hrsg.), Transdisziplinär und transformativ forschen. Eine Methodensammlung (S. 233-268). Wiesbaden: Springer VS. doi: 10.1007/978-3-658-21530-9_12. 
Vilsmaier, U., \& Meyer, E. (2017). Forschendes Lernen in der Nachhaltigkeitswissenschaft. In H. Mieg \& J. Lehmann (Hrsg.). Forschendes Lernen: Wie die Lehre in Universität und Fachhochschule erneuert werden kann (S. 357-366). Frankfurt/New York: Campus.

Wagenschein, M. (1999). Verstehen lehren: genetisch, sokratisch, exemplarisch. Weinheim: Beltz.

Waitz, C., Quint, A., Trenks, H., Lezuo, D., Jäkel, A., Wäsche, H., \& Parodi, O. (2018). Das Reallabor als Motor für nachhaltige Quartiersentwicklung - Erfahrungen aus dem Karlsruher Experimentierraum. Berichte. Geographie und Landeskunde, 91 (1), (S. 67-80).

Wals, A. (Hrsg.). (2007). Social learning towards a sustainable world: Principles, perspectives, and praxis. Wageningen: Wageningen Academic Pub.

Welch, M., Brownell, K., \& Sheridan, S. (1999). What's the score and game plan on teaming in schools? A review of the literature on team teaching and school-based problemsolving teams. Remedial and Special Education, 20 (1), (S. 36-49). 


\section{Anhang}

\section{Die Erfahrungen mit sechs TraPS in Karlsruhe}

Die untenstehende Tabelle gibt eine Übersicht über die sechs TraPS, die in Karlsruhe innerhalb von drei Jahren durchgeführt wurden. Die Teilnehmerzahl spiegelt die Anzahl der Student(inn)en, die bis zum Ende des TraPS beteiligt blieben. Da die Teilnahme freiwillig war, gab es immer wieder Teilnehmerschwund, insbesondere in den ersten Wochen, was auch auf die Kollision mit Pflichtveranstaltungen zurückzuführen war, deren Termine zuerst noch nicht feststanden. Die folgende Darstellung ist eine Übersetzung und Zusammenfassung einer qualitativen Analyse der sechs TraPS (Beecroft 2018, S. 11-14).

Das erste TraPS - „Besser Altern in Karlsruhe“ - wurde gemeinsam mit einem frühen Praxispartner des Reallabors Karlsruhe, einer Gemeindeinitiative zur Verbesserung der Lebensbedingungen in einer benachbarten Gemeinde, und mit sehr wenigen Student(inn)en durchgeführt. Nach den ersten Wochen veränderte sich die Kooperation im Kurs wesentlich, als die Praxispartner ein Thema einbrachten, zu dem sie Unterstützung suchten (die Planung und Durchführung einer Infoveranstaltung zu Notknöpfen). Mit diesem Bedarf waren die Praxisziele gesetzt und es entwickelte sich im Wesentlichen eine Service-Learning-Dynamik. Die Student(inn)en fanden einen weiteren Praxisakteur (einen Anbieter von Notknöpfen), die

Tabelle: Die sechs Karlsruher TraPS.

\begin{tabular}{|c|c|c|c|c|}
\hline Semester & Titel & Veranstalter & Praxispartner & TN \\
\hline $\begin{array}{l}\text { WS } \\
2014 / 15\end{array}$ & Besser Altern in Karlsruhe & $\begin{array}{l}\text { Beecroft, } \\
\text { Albiez }\end{array}$ & $\begin{array}{l}\text { Gemeindeinitia- } \\
\text { tive Gut Altern }\end{array}$ & 3 \\
\hline $\begin{array}{l}\text { SS } \\
2015\end{array}$ & Zu Tisch! & $\begin{array}{l}\text { Albiez, } \\
\text { Beecroft }\end{array}$ & $\begin{array}{l}\text { Karlsruher Tafel } \\
\text { e. V. }\end{array}$ & 7 \\
\hline $\begin{array}{l}\text { WS } \\
2015 / 16\end{array}$ & $\begin{array}{l}\text { Reparieren, Wieder- } \\
\text { verwerten, Selbstmachen! }\end{array}$ & $\begin{array}{l}\text { Tamm, } \\
\text { Beecroft }\end{array}$ & $\begin{array}{l}\text { ReparaturCafé } \\
\text { Initiative Karls- } \\
\text { ruhe }\end{array}$ & 14 \\
\hline $\begin{array}{l}\text { SS } \\
2016\end{array}$ & $\begin{array}{l}\text { Gemeinwohlökonomie für } \\
\text { Karlsruhe? }\end{array}$ & $\begin{array}{l}\text { Beecroft, } \\
\text { Tamm }\end{array}$ & $\begin{array}{l}\text { GWÖ-Initiative } \\
\text { Karlsruhe }\end{array}$ & 12 \\
\hline $\begin{array}{l}\text { WS } \\
\text { 2016/17 }\end{array}$ & $\begin{array}{l}\text { Bildung für Nachhaltige } \\
\text { Entwicklung planen }\end{array}$ & $\begin{array}{l}\text { Beecroft, } \\
\text { Friedrichs }\end{array}$ & ZAK, KIT & 3 \\
\hline $\begin{array}{l}\text { SS } \\
2017\end{array}$ & $\begin{array}{l}\text { Nachhaltigkeitsspaziergang } \\
\text { Karlsruhe }\end{array}$ & $\begin{array}{l}\text { Tamm, } \\
\text { Beecroft }\end{array}$ & $\begin{array}{l}\text { stattreisen e. V. } \\
\text { Stadt Karlsruhe }\end{array}$ & 16 \\
\hline
\end{tabular}


Praxispartner warben intensiv in ihrem Umfeld für die Infoveranstaltung, mit mäßigem Erfolg, und die Student(inn)en betteten das vorgegebene Thema in einen weiteren Kontext ein. Es war essenziell, mit den Student(inn)en ein Nachtreffen ohne die Praxispartner durchzuführen, um die Rollen und den Seminarverlauf zu reflektieren. Dieser Kurs war der ,Prototyp ' für die hier vorgestellte Struktur, die erst in Folge formalisiert und weiterentwickelt wurde.

Das zweite TraPS - „Zu Tisch!“ - in Kooperation mit einer der Karlsruher Tafeln fokussierte auf die sozialen Fragen nachhaltiger Ernährung. Nach einem Einstieg, in dem herausgearbeitet werden musste, dass die Veranstaltung kein Rekrutierungsevent für den Praxispartner ist, starteten die Student(inn)en eine kritische Diskussion über die Angemessenheit von Tafeln. Diese konnte in die beiden Studierendenprojekte teilweise einbezogen werden, die sich auf Informationen zur Nahrungsmittellagerung für die Kundschaft und das Abfallmanagement der Tafel bezogen. Letzteres stellte sich als analytisch anspruchsvolles Thema heraus, führte aber zu dem unspektakulären Ergebnis, dass das bestehende System nahezu ideal sei. Die Praxispartner blieben zurückhaltend, die Student(inn)en im laufenden Betrieb in die Tafel einzuladen, um keine Irritation bei der Kundschaft hervorzurufen. Die (hochschulweit standardisierte) Evaluation der Veranstaltung machte mit einem kritischen Ergebnis die Orientierungslosigkeit deutlich, die zu Beginn der Projektphase eingetreten war. Eine Reflexion mit den Student(inn)en am Ende des TraPS war wesentlich, um unausgesprochene Erwartungen (z. B. bezüglich der Rolle der Lehrenden), Fragen der Gerechtigkeit bei sehr unterschiedlichen Projekten und Zielkonflikte zwischen praktischer Wirksamkeit und wissenschaftlicher Relevanz aufzuarbeiten. Die Erfahrung mit diesem Kurs führte zu einer systematischen Explikation der Forschungs-, Bildungs- und Praxisziele zu Beginn der Veranstaltung und zur Einführung eines Probelaufs der Präsentation.

Das dritte TraPS - „Reparieren, Wiederverwerten, Selbstmachen!“ - mit einem Fokus auf Konsum und Nachhaltigkeit unterstützte die ReparaturCafé-Initiative in Karlsruhe, die selbst aus einem Event des Reallabors hervorgegangen war und dann eng mit dem Reallabor kooperierte. Zum Zeitpunkt des Kurses war die Initiative dabei, einen eigenständigen eingetragenen Verein zu gründen. Die ursprüngliche Hoffnung des Praxispartners, insbesondere zu Versicherungs- und Haftungsfragen Unterstützung zu erhalten, musste enttäuscht werden, da die Student(inn)en keinerlei einschlägige Kenntnisse hatten. Nichtsdestotrotz konnten die vier Studierendenteams mehrere wertvolle Beiträge liefern: einen Imagefilm, um auch online Sichtbarkeit für die Initiative und ihre konsumkritischen Themen herzustellen, ein Planspiel zum Test der geplanten Vereinssatzung, ein Flugblatt mit Hinweisen zu Fachhändlern im Quartier, die Ersatzteile verkaufen, sowie mehrere analytische Beiträge zu Kooperationspotenzialen und zur Vergleichbarkeit mit anderen Initiativen. Die vielfältigen Ergebnisse waren für den Praxis- 
partner nur knapp zu bewältigen, dementsprechend wurden Fragen der Trägerschaft seit diesem Kurs in das Konzept eines TraPS aufgenommen. Es war der erste Kurs, der den frisch eingeweihten „Zukunftsraum“ als Lernumgebung nutzen konnte. In diesem Kurs fand die Ausdifferenzierung der Studienleistungen die in diesem Beitrag vorgestellte Form, und die Vorstellung der Projektskizzen erfolgte erstmals systematisch.

Das vierte TraPS - „Gemeinwohlökonomie (GWÖ) für Karlsruhe?“ - mit der lokalen GWÖ-Initiative thematisierte nachhaltiges Wirtschaften. Mit einem CoLehrenden aus der GWÖ-Initiative und einer Reihe von weiteren Mitgliedern der Initiative, die bei mehreren Seminarterminen präsent waren, bildete sich eine enge Zusammenarbeit heraus. Die dadurch verursachte Vermutung der Student(inn)en, von ihnen würde erwartet, die Position der GWÖ zu nachhaltigem Wirtschaften ohne Kritik zu übernehmen, musste direkt angesprochen und ausgeräumt werden. Die kritischen Fragen konnten so in zwei der vier Studierendenprojekte einbezogen werden, was zu mehr Tiefe in der Auseinandersetzung mit den Projektthemen führte. Als lokaler Zweig eines aktiven internationalen Netzwerks konnten die Praxispartner weitere Spezialist(inn)en aus dem GWÖ-Netzwerk einbeziehen, und auch die Ergebnisse konnten so weiter verbreitet werden. Eine Studierendengruppe erstellte eine erste (minimale) GWÖ-Bilanz eines Vereins und konnte so Unterschiede zum Einsatz in Unternehmen ausweisen. Ein Informationsfilm zur GWÖ wurde - Jahre später - eine der meistgenutzten deutschsprachigen Online-Ressourcen zur Information über den Ansatz. In diesem Kurs wurde die Verwendung eines Online-Projekttools getestet, mit dem die Praxispartner arbeiten - dieses wurde aber nicht als fester Baustein in das Seminarkonzept aufgenommen, da die Studierendenprojekte zu unterschiedlich gelagert waren für ein einheitliches System. An diesem Kurs wurden die Überlegungen zur langfristigen Trägerschaft verfeinert und die Rollenklärungen mit den Praxispartnern systematisiert.

Das fünfte TraPS - „Bildung für Nachhaltige Entwicklung planen“ - mit einer kleinen Gruppe von Student(inn)en stellte einen Planungsprozess für eine große Einführungsveranstaltung zu Nachhaltiger Entwicklung dar, in Kooperation mit dem Zentrum für Angewandte Kulturwissenschaft und Studium Generale (ZAK). $\mathrm{Da}$ diese Veranstaltung (die inzwischen unter dem Namen „Frühlingstage der Nachhaltigkeit" auf rund 300 Teilnehmer(innen) ausgelegt ist) von Anfang an als Kooperation mit dem ZAK geplant war, unterschieden die Student(inn)en nicht zwischen den Rollen als Lehrende und als Praxispartner. Dies belastete den Kurs nicht, es mussten aber immer wieder Fragen nach der Offenheit des Prozesses und nach dem Verhältnis zwischen den Bildungs- und Praxiszielen angesprochen und geklärt werden. Dieser Kurs profitierte sehr von den Kompetenzen der Mitglieder des Reallaborteams, die nicht im Lehrteam waren, und vom weiteren wissenschaftlichen Netzwerk des Reallabors. 
Das sechste TraPS - „Nachhaltigkeitsspaziergang Karlsruhe“ - war eingebettet in ein internationales Vergleichsprojekt zu Stadt-Universitäts-Kooperationen. Als Praxispartner waren Vertreter(innen) von fünf Ämtern der Stadt Karlsruhe sowie eines nichtkommerziellen Anbieters von Stadtführungen (Verein) beteiligt. Ein ehemaliger Praktikant des Reallabors führte als Masterarbeit an einer anderen Universität eine Begleitforschung durch. Das Seminar hatte statt einer Skizzenund einer Projektphase zwei Projektphasen: In der ersten gruppierten sich die Student(inn)en zu übergreifenden Fragen (z. B. Zielgruppe, mögliche Route, Nachhaltigkeitstheorien). Nach einer Entscheidung über diese allgemeinen Fragen gemeinsam mit den Praxispartnern arbeiteten die Student(inn)en in neu gemischten Teams Informationen zu einzelnen Stationen aus und bezogen diese auf die Nachhaltigkeitsziele der Vereinten Nationen. Im Projektverlauf wurde die Rolle des zivilgesellschaftlichen Vereins immer aktiver, so dass dieser die Trägerschaft für eines der Produkte, eine geführte Nachhaltigkeitstour, übernahm. Zugleich engagierten sich dann zwei der Student(inn)en dort als Reiseführer. Die Phase der In-Wert-Setzung war, da mehrere parallele Produkte (Audioguide, Online-Karte, Broschüre, geführte Tour) entwickelt wurden, besonders aufwendig und dauerte, auch durch technische Verzögerungen bedingt, lange an. Ein Teil der Ergebnisse wurde zum Teil der Infrastruktur des Reallabors. Das Seminar hatte eine komplexe Architektur, die nicht 1:1 in das in diesem Beitrag vorgestellte Vorgehen für ein TraPS übernommen wurde, da sie kaum ohne ein Reallabor als unterstützenden Rahmen realisierbar ist. In diesem Kurs war erstmals die vielfältige Einbettung ins Reallabor (lokale Netzwerke ausbauen, Infrastruktur entwickeln, Sichtbarkeit erhöhen, wissenschaftliche Vernetzung fördern u. a.) von Anfang an Teil der Detailplanung des TraPS. Durch die Einbettung in ein internationales Vergleichsprojekt wurde die theoretische Aufarbeitung des transdisziplinären Charakters des Seminarablaufs und des transformativen Anspruchs deutlich vorangebracht.

Open Access Dieses Kapitel wird unter der Creative Commons Namensnennung 4.0 International Lizenz (http://creativecommons.org/licenses/by/4.0/deed.de) veröffentlicht, welche die Nutzung, Vervielfältigung, Bearbeitung, Verbreitung und Wiedergabe in jeglichem Medium und Format erlaubt, sofern Sie den/die ursprünglichen Autor(en) und die Quelle ordnungsgemäß nennen, einen Link zur Creative Commons Lizenz beifügen und angeben, ob Änderungen vorgenommen wurden.

Die in diesem Kapitel enthaltenen Bilder und sonstiges Drittmaterial unterliegen ebenfalls der genannten Creative Commons Lizenz, sofern sich aus der Abbildungslegende nichts anderes ergibt. Sofern das betreffende Material nicht unter der genannten Creative Commons Lizenz steht und die betreffende Handlung nicht nach gesetzlichen Vorschriften erlaubt ist, ist für die oben aufgeführten Weiterverwendungen des Materials die Einwilligung des jeweiligen Rechteinhabers einzuholen.

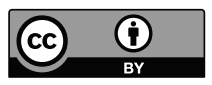


Anhang 


\section{Steckbriefe der BaWü-Labs Asylsuchende, R131, Schorndorf, SRB, Urban Office}

Auf den folgenden Seiten stellen sich diejenigen der im Rahmen der beiden Förderlinien „Reallabore“ und „Reallabore Stadt"“1 des Ministeriums für Wissenschaft, Forschung und Kunst Baden-Württemberg (MWK) geförderten Reallabore („BaWü-Labs“) vor, die eine Methodenbeschreibung zu Teil 2 dieses Buches beigesteuert haben:

BaWü-Lab Asylsuchende

BaWü-Lab R131

BaWü-Lab Schorndorf

BaWü-Lab SRB

BaWü-Lab Urban Office
„Asylsuchende in der Rhein-Neckar-Region“"

„Reallabor 131: KIT findet Stadt“ (gemeinsam mit „Quartier Zukunft - Labor Stadt“ das „Reallabor Karlsruhe" bildend)

„Reallabor Schorndorf: Zukunftsweisender Öffentlicher Verkehr - Bürgerorientierte Optimierung der Leistungsfähigkeit, Effizienz und Attraktivität im Nahverkehr“"

„STADT-RAUM-BILDUNG - Reallabor für die nachhaltige Planung von Bildungslandschaften und die Integration von Aus- und Umbauten von Schulgebäuden“"

„Urban Office - Nachhaltige Stadtentwicklung in der Wissensgesellschaft"

Die Steckbriefe enthalten eine Kurzbeschreibung der BaWü-Labs. Sie äußern sich zur Leitung/Koordination, nennen die Forschungs- und Praxispartner, geben Auskunft über die Ziele der BaWü-Labs, listen die Teilprojekte und/oder die wichtigsten Realexperimente und partizipativen Formate auf und weisen auf ausgewählte Publikationen und in der Praxis unmittelbar nutzbare Produkte hin.

1 Für Informationen zu den Förderlinien „Reallabore“ und „Reallabore Stadt“ sowie eine Kurzbeschreibung aller geförderten BaWü-Labs siehe http://www.reallabore-bw.de (zugegriffen am 20.05.2019). 
Für die Darstellung ihrer Ziele orientieren sich die BaWü-Labs an den drei Zieldimensionen gemäß Beecroft et al. $2018^{2}$ und unterscheiden Forschungsziele (also die Erzeugung neuen Wissens), Praxisziele (also das Anstoßen und Begleiten von Transformationsprozessen) und Bildungsziele (also das Anregen und Unterstützen von Lernprozessen).

Wer die Beiträge in diesem Buch liest, merkt, dass die Vielfalt, in der das Format Reallabor in den BaWü-Labs umgesetzt wurde, groß ist. Diese Vielfalt kann in Steckbriefen, die naturgemäß schematisch sein müssen, um einen schnellen Überblick zu erlauben, nur sehr bedingt abgebildet werden. Komplexe Forschungsverbünde wie die BaWü-Labs über einen Leisten zu schlagen, wird ihrer Individualität, der spezifischen Art, wie sie sich organisieren und ihre Beziehungen zu den für sie relevanten Akteuren gestalten, nur bedingt gerecht.

So zeigt sich zwar die große Vielfalt der Akteurskonstellationen und der Forschungs- und Partizipationsformate in den entsprechenden Listen. Diese spiegeln aber nur einen Ausschnitt aus diesen BaWü-Labs, d. h., es finden sich hier z. B. bei Weitem nicht alle für die BaWü-Labs wichtigen Praxisakteure. Mit Blick auf den Umfang der Steckbriefe musste insbesondere auf die Nennung unorganisierter zivilgesellschaftlicher Akteure, wie z. B. eine Quartiersbevölkerung oder die Bürgerinnen und Bürger, die an Workshops mitwirkten, verzichtet werden. Die Beschränkung auf organisierte Akteure ist allein der gebotenen Kürze der Steckbriefe geschuldet; damit soll keineswegs ausgedrückt werden, die hier nicht genannten Praxisakteure hätten weniger zu den BaWü-Labs beigetragen. Ähnliches gilt für die in den Steckbriefen genannten Publikationen und Produkte. Die Vorgabe an die BaWü-Labs war hier, nur eine kleine Auswahl an Publikationen und Produkten aufzuführen - Publikationen, die am ehesten einen Überblick über die Ergebnisse erlauben, bzw. Produkte, die unmittelbar in der Praxis genutzt werden können. ${ }^{3}$

Gleichwohl haben die Herausgeberin und der Herausgeber darum gebeten, solche Steckbriefe zur Verfügung zu stellen. Sie bitten denn auch alle um Nachsicht, die eines dieser BaWü-Labs gut kennen und sich oder ihre Anliegen in den nachstehenden Ausführungen vielleicht nicht in der ihnen angemessen scheinenden Weise wiederfinden. Für diejenigen wiederum, die diese BaWü-Labs weniger gut kennen, sollen die Steckbriefe Anregung dafür sein, sich intensiver mit ihnen zu befassen und den Hinweisen für weiterführende Informationen zu diesen BaWüLabs nachzugehen.

2 Beecroft, R., Trenks, H., Rhodius, R., Benighaus, C., \& Parodi, O. (2018). Reallabore als Rahmen transformativer und transdisziplinärer Forschung: Ziele und Designprinzipien. In R. Defila \& A. Di Giulio (Hrsg.), Transdisziplinär und transformativ forschen. Eine Methodensammlung (S. 75-100). Wiesbaden: Springer VS.

3 Die Beiträge der BaWü-Labs im vorliegenden Buch und im Buch von 2018 (Defila und Di Giulio 2018, s. Fn. 2) sind in diesen Listen ebenfalls nicht enthalten. 


\section{BaWü-Lab Asylsuchende „Asylsuchende in der Rhein-Neckar-Region“"}

Das Reallabor analysierte Erfolgsfaktoren für eine nachhaltige gesellschaftliche Teilhabe von geflüchteten Menschen in der Region, in Ausbildung und Beschäftigung. Untersucht wurden die schulischen und beruflichen Werdegänge von geflüchteten Menschen, insbesondere bzgl. der Entwicklung von sprachlichen, mathematischen und beruflichen Kompetenzen, die die Teilhabe von Asylsuchenden am sozialen und gesellschaftlichen Leben sowie am Arbeitsmarkt unterstützen. Reflektiert und diskutiert wurden neben dem Beitrag der beruflichen Bildungsangebote auch zivilgesellschaftliche Initiativen und Unterstützungsangebote von Kommunen und öffentlichen Stellen sowie sozialräumliche Aufnahmebedingungen: das Wohnumfeld im Quartier, das bürgerschaftliche Engagement vor Ort und die allgemeine Integrationsbereitschaft einer Stadtgesellschaft.

Förderperiode MWK: 2016-2019

www.reallabor-asyl.de

\section{Leitung/Koordination}

\section{Direktorium}

Prof. Dr. Birgit Werner, Pädagogische Hochschule Heidelberg

PD Dr. Friedhelm Pfeiffer, Leibniz-Zentrum für Europäische Wirtschaftsforschung GmbH Mannheim

Dr. Christina West, Geographisches Institut der Universität Heidelberg und Hochschule Darmstadt

Dr. Georg Mildenberger, Centrum für Soziale Investitionen und Innovationen, Universität Heidelberg

Geschäftsführung

Dr. Monika Gonser (2016-2017), Pädagogische Hochschule Heidelberg

Mareike Bahn (2017-2019), Pädagogische Hochschule Heidelberg

\section{Forschungspartner}

Pädagogische Hochschule Heidelberg: Prof. Dr. Birgit Werner; Prof. Dr. Anne Berkemeier (Westfälische Wilhelms-Universität Münster); Prof. Dr. Petra Deger; Prof. Dr. Havva Engin; Dr. Oksana Kovtun-Hensel; Rebecca Höhr; Corinna Uebel

Leibniz-Zentrum für Europäische Wirtschaftsforschung GmbH Mannheim: PD Dr. Friedhelm Pfeiffer; Dr. Katrin Sommerfeld; Martin Lange 
Geographisches Institut der Universität Heidelberg: Dr. Christina West (s:ne | Systeminnovation für Nachhaltige Entwicklung: Zukunftsorientierte Stadtentwicklung, Hochschule Darmstadt); Svenja Kück

Centrum für Soziale Investitionen und Innovationen (CSI), Universität Heidelberg: Prof. Dr. Adalbert Evers; Dr. Georg Mildenberger; Verena Schmid

\section{Praxispartner}

Agentur für Arbeit Heidelberg

Amt für Chancengleichheit Heidelberg,

Stadt Heidelberg

Amt für Schule und Bildung, Stadt

Heidelberg

Anpfiff ins Leben e. V.

Arbeitskreis Asyl Heidelberg

BBQ Berufliche Bildung gGmbH

Heidelberg/Sinsheim

Berufliche Bildung $\mathrm{GmbH}$

Berufsschullehrerverband

Each1Teach1 e. V. Heidelberg

Flüchtlingspartner Ziegelhausen

Hotelfachschule Heidelberg (Ursula Hummel)
IHK Mannheim

IHK Rhein-Neckar

Integrationsbeauftragte, Stadt Sinsheim

Flüchtlingsbeauftragter, Stadt Sinsheim

Julius-Springer-Schule (Kaufmännische Schule Heidelberg, VABO

Fachbereichsleitung Christel Herzog, StD'in) in Kooperation mit der Stabstelle Integration, Landratsamt RheinNeckar-Kreis

Stadt Wiesloch

Stift Sunnisheim Sinheim

vhs Heidelberg

\section{Ziele}

a) Forschungsziele

- Erkenntnisse erlangen dazu, welche Faktoren auf kommunaler Ebene die wirtschaftliche wie soziale Integration und Teilhabe von Asylsuchenden fördern oder hemmen. Mit Blick auf dieses Ziel wurden u. a. folgende Fragestellungen bearbeitet:

- Welche Faktoren/Determinanten beeinflussen die Alltagsaktivitäten von Asylsuchenden?

- Welche Aktionsräume und raumbezogene Aneignungsstrategien entwickeln Asylsuchende? 
b) Praxisziele

- Selbstbestimmung und Teilhabe von Neuankommenden/Menschen mit Fluchterfahrung/Asylsuchenden stärken.

- Sozial- und Arbeitsmarktanschluss von geflüchteten Menschen unterstützen.

- Neue Formen der Kooperation im Feld des zivilgesellschaftlichen Engagements erhalten und stärken.

- Anstöße geben und Methoden entwickeln für eine zukunftsorientierte, nachhaltige, resiliente und inklusive Quartiers- und Stadtentwicklung.

- Einen Beitrag leisten dazu, die Form des (gesellschaftlichen) Experimentierens als Bestandteil von (Planungs-)Kulturen zu etablieren.

c) Bildungsziele

- Methoden des Co-Designs und der Co-Produktion einer zukunftsorientierten, nachhaltigen und inklusiven Stadtentwicklung trainieren und reflektieren im Hinblick auf die Förderung entsprechender methodischer und kommunikativer Kompetenzen bei Wissenschaftler(inne)n, Studierenden, Praxisakteuren und Asylsuchenden.

- Lehr- und Lernformate entwickeln, anwenden und bewerten, die den komplexen Herausforderungen gerecht werden, mit denen Asylsuchende sowie Lehrende und Bildungseinrichtungen konfrontiert sind.

- Geflüchtete Menschen zum eigenständigen Lernen und Spracherwerb befähigen.

\section{Teilprojekte und wichtigste partizipative Formate}

a) Teilprojekte

- Diagnose und Förderung sprachlicher und mathematischer Kompetenzen von berufsschulpflichtigen jugendlichen Asylsuchenden

- Wege in den Arbeitsmarkt

- Stadtgeographische, stadtgesellschaftlich-demographische und migrationssoziologische Dimensionen dezentraler Unterbringung

- Bürgerschaftliches Engagement und trisektorale Kooperation 
b) Die wichtigsten partizipativen Formate

- Entwicklung des partizipativen Formats „UrbanUtopiaLAB“

- Interaktive Ausstellung „Learning from Journeys“ (Lebensgeschichten, Fluchtwege und Visionen von geflüchteten Personen in Heidelberg)

- Transdisziplinäre interaktive Workshop-Reihe „Learning from Journeys“ (mit „Interaktiver Ausstellungsparty“, „Mini-Documenta“, „Offene Stadt - Utopie und Realität")

\section{Zentrale Publikationen und Produkte}

a) Zentrale Publikationen auf der Ebene des Gesamtprojekts

Deger, P., Gonser, M., Kolb, M., Kück, S., Lange, M., Mildenberger, G., Pfeiffer, F., Sommerfeld, K., \& West, C. (2017). Integrationspotenziale: Ausgewählte Ergebnisse der Reallabor-Befragungen unter Geflüchteten. ZEW, Mannheim.

Gonser, M., Jesske, B., \& Pfeiffer, F. (2017). Werkstattbericht Reallabor-Befragungen von Geflüchteten in der Rhein-Neckar-Region. infas Bonn, Mannheim.

Bahn, M., Berkemeier, A., Deger, P., Engin, H., Evers, A., Höhr, R., Kovtun-Hensel, O., Kück, S., Lange, M., Mildenberger, G., Pfeiffer, F., Schmid, V., Sommerfeld, K., Uebel, C., Werner, B., \& West, C. (2019). Reallabor Asylsuchende in der Rhein-NeckarRegion: Analysen und Handlungsempfehlungen. https://www.reallabor-asyl.de/file admin/user_upload/Redaktion/reallabor-asyl/Ver\%C3\%B6ffentlichungen/Handlungs empfehlungen_Reallabor_Asylsuchende.pdf. Zugegriffen am 28.05.2019.

Bahn, M., Berkemeier, A., Deger, P., Engin, H., Evers, A., Höhr, R., Kovtun-Hensel, O., Kück, S., Lange, M., Mildenberger, G., Pfeiffer, F., Schmid, V., Sommerfeld, K., Uebel, C., Werner, B., \& West, C. (2019). Reallabor Asylsuchende in der Rhein-NeckarRegion: Vielfalt an Problemen - Vielfalt an Lösungen. http://ftp.zew.de/pub/zew-docs/ policybrief/pb04-19.pdf. Zugegriffen am 28.05.2019.

West, C. (2019). Transversal City and Transtopia - Reflecting and Analysing Migration, the City, and the Urban after the Postmigrant City. Special edition Social Geography: Die ,postmigrantische Stadt" - urbanes Zusammenleben aus der Perspektive der Migration. Geographia Helvetica. (im Druck).

b) Produkte, die in der Praxis unmittelbar genutzt werden können

- Format und Methode „UrbanUtopiaLAB“

- Interaktive Stadtkarten (analog)

- Kompetenzbeobachtungsbogen: theoriegeleitetes und evaluiertes Diagnoseund Lehrmittel (www.reallabor-asyl.de/registrierung-kompetenzbeobachtungsbogen)

- Selbstlernmaterialien für geflüchtete Menschen, zur Nutzung in der Erstaufnahmeeinrichtung 


\section{BaWü-Lab R131 \\ „Reallabor 131: KIT findet Stadt“" (gemeinsam mit „Quartier Zukunft - Labor Stadt" das „Reallabor Karlsruhe“6 bildend)}

Das BaWü-Lab „Reallabor 131: KIT findet Stadt“ (R131) (2015-2019) ist eingebettet in das Reallabor „Quartier Zukunft - Labor Stadt“" (QZ) (seit 2012). Gemeinsam bilden die beiden - ergänzt um weitere transdisziplinäre Projekte ( $\mathrm{ab}$ 2018) - das „Reallabor Karlsruhe“ (RK). Dessen Aufgabe ist es, in einem transdisziplinären Prozess mit Bürgerschaft, Universität und Stadtverwaltung eine nachhaltige Quartiersentwicklung in der Karlsruher Oststadt zu initiieren und zu begleiten. Gemeinsam verfolgen sie das langfristige Ziel, eine Kultur der Nachhaltigkeit zu etablieren. Das R131 baute in seiner Arbeit auf den Aktivitäten und Erkenntnissen des QZ auf und vertiefte die Themenbereiche Energie, Konsum, Mobilität sowie Soziales und Raum. Im Bereich der Bildung für Nachhaltige Entwicklung und in der Gestaltung gesellschaftlicher Lernprozesse arbeitet das RK eng mit der „Karlsruher Schule der Nachhaltigkeit“ (KSN) am KIT zusammen. RK und KSN stellen den Kern des in Gründung befindlichen „Karlsruher Transformationszentrums für nachhaltige Zukünfte und Kulturwandel“" (KAT) dar, mit dem der Ausbau und die Verstetigung des RK erfolgen sollen.

Förderperiode MWK R131: 2015-2019

www.quartierzukunft.de

\section{Leitung/Koordination}

Dr. Oliver Parodi und Dr. Andreas Seebacher, beide Karlsruher Institut für Technologie (KIT), Institut für Technikfolgenabschätzung und Systemanalyse (ITAS)

\section{Forschungspartner}

European Institute for Energy Research (EIFER)

- Dr. Andreas Koch; Dr. Syed Monjur Murshed; Dr. Beata Sliz-Skliniarz; Dr. Jochen Wendel

Karlsruher Institut für Technologie (KIT)

- Fachgebiet Bauphysik \& Technischer Ausbau (fbta): Prof. Dr. Andreas Wagner; Dr. Amar Abdul-Zahra

- Fachgebiet Immobilienwirtschaft, Lehrstuhl Ökonomie und Ökologie des Wohnungsbaus (ÖÖW): Prof. Dr.-Ing. habil. Thomas Lützkendorf; Maria Balouktsi 
- Institut Entwerfen von Stadt und Landschaft (IESL): Dr. Dagmar Lezuo

- Institut für Entwerfen, Kunst und Theorie (EKUT): Dr.-Ing. Angelika Jäkel; Lisa Schneider; Hannes Siefert

- Institut für Industriebetriebslehre und Industrielle Produktion (IIP): Dr. Russell McKenna; Kai Mainzer; Elias Naber; Prof. Dr. rer. pol. Frank Schultmann

- Institut für Sport und Sportwissenschaft (IfSS): Dr. Hagen Wäsche

- Institut für Verkehrswesen (IfV): Dr.-Ing. Bastian Chlond; Dr. Tamer Soylu; Prof. Dr. Peter Vortisch

- Zentrum für Angewandte Kulturwissenschaft und Studium Generale (ZAK): Miriam Friedrichs; Ines Bott; Sarah Bloesy

\section{Praxispartner}

Stadt Karlsruhe (mit vielen Fachämtern)

Beete \& Bienen

Bikes without Borders e. V.

Bürgerstiftung Karlsruhe

Bürgerverein Oststadt

FabLab Karlsruhe e. V.

Gemeinwohlökonomie Karlsruhe

Haus und Grund Karlsruhe

Karlsruher Energie und Klimaschutzagentur
Karlsruher Tafel e. V.

Kinder- und Jugendhaus Oststadt

Kreativ Salon

Living Knowledge Network

Luthergemeinde Oststadt

Oststadt-Nachbarschaft Karlsruhe

ReparaturCafé Karlsruhe e. V.

Second Future

Stadtwerke Karlsruhe

\section{Ziele}

a) Forschungsziele

- Generieren, Bereitstellen und Testen von Wissen für eine Transformation in Richtung Nachhaltigkeit im urbanen Kontext

- Weiterentwicklung des Formats „Reallabor“ als Forschungsinfrastruktur sowie als Bildungs- und Transformationsformat 
b) Praxisziele

- Nachhaltigkeitstransformationen (in Karlsruhe) anstoßen und diese sichtbar, spürbar und erlebbar werden lassen

- Ermächtigung von Praxisakteuren für eine nachhaltige Stadtentwicklung

- Erzeugen einer „dichten Nachhaltigkeit“ und Etablierung einer „Kultur der Nachhaltigkeit"“

c) Bildungsziele

- Wissenschaftliche Akteure, Praxisakteure und Studierende an transdisziplinäres Arbeiten und realweltliche Transformationsprozesse heranführen

- Kompetenzen für eine Nachhaltige Entwicklung erarbeiten, entsprechendes Wissen vermitteln und eine auf Nachhaltigkeit bezogene Selbstreflexion erhöhen

- Institutionelle und informelle Bildungsangebote für Nachhaltige Entwicklung entwickeln und verankern

\section{Teilprojekte und Realexperimente}

a) Teilprojekte

- Energiekonzept Oststadt

- Nachhaltige Mobilität

- Nachhaltiger Konsum: Veranstaltungsreihe „KonsumCafe““

- Soziales und Raum

- Vollerhebung: Gebäudebestand und Akteure

- Methodengestützte Nachhaltigkeitsanalyse und -bewertung der Karlsruher Oststadt

b) Realexperimente

- Dein NachhaltigkeitsExperiment 1: „Beete \& Bienen“

- Dein NachhaltigkeitsExperiment 2: „Kreativ-Salon“

- Dein NachhaltigkeitsExperiment 3: „Oststadt-Treff“

- Dein NachhaltigkeitsExperiment 4: „Second Future“ 


\section{Zentrale Publikationen und Produkte}

a) Zentrale Publikationen

Beecroft, R. (2018). Embedding Higher Education into a Real-World Lab: A ProcessOriented Analysis of Six Transdisciplinary Project Courses. Sustainability, 10 (10), (Nr. 3798). doi: 10.3390/su10103798.

Beecroft, R., \& Parodi, O. (Hrsg.). (2016). Reallabore als Orte der Nachhaltigkeitsforschung und Transformation [Schwerpunkt]. Technikfolgenabschätzung - Theorie und Praxis, 25 (3).

Meyer-Soylu, S., Parodi, O., Trenks, H., \& Seebacher, A. (2016). Das Reallabor als Partizipationskontinuum. Erfahrungen aus dem Quartier Zukunft und Reallabor 131 in Karlsruhe. Technikfolgenabschätzung - Theorie und Praxis, 25 (3), (S. 31-40).

Parodi, O., Albiez, M., Beecroft, R., Meyer-Soylu, S., Quint, A., Seebacher, A., Trenks, H., \& Waitz, C. (2016). Das Konzept „Reallabor“ schärfen. Ein Zwischenruf des Reallabor 131: KIT findet Stadt. GAIA, 25 (4), (S. 284-285).

Parodi, O., Beecroft, R., Albiez, M., Quint, A., Seebacher, A., Tamm, K., \& Waitz, C. (2017). The ABC of real-world lab methodology - From "action research" to "participation" and beyond. TRIALOG, 126/127 (3-4), (S. 74-82).

Parodi, O., Ley, A., Fokdal, J., \& Seebacher, A. (2018). Empfehlungen für die Förderung und Weiterentwicklung des transformativen Formats „Reallabor“ - Erkenntnisse aus der Arbeit der BaWü-Labs. GAIA, 27 (1), (S. 178-179).

Parodi, O., Waitz, C., Bachinger, M., Kuhn, R., Meyer-Soylu, S., Alcántara, S., \& Rhodius, R. (2018). Insights into and recommendations from three real-world laboratories: An experience-based comparison. GAIA, 27 (S1), (S. 52-59).

Singer-Brodowski, M., Beecroft, R., \& Parodi, O. (2018). Learning in real-world laboratories: A systematic impulse for discussion. GAIA, 27 (S1), (S. 23-27).

Withycombe Keeler, L., Beaudoin, F., Wiek, A., John, B., Lerner, A. M., Beecroft, R., Tamm, K., Seebacher, A., Lang, D., Kay, B., \& Forrest, N. (2018). Building actorcentric transformative capacity through city-university partnerships, Ambio (2018). doi: $10.1007 / \mathrm{s} 13280-018-1117-9$.

b) Produkte, die in der Praxis unmittelbar genutzt werden können

- Zukunftsraum für Nachhaltigkeit und Wissenschaft (Stadtteilbüro)

- Digitale interaktive Nachhaltigkeitskarte (www.quartierzukunft.de/vor-ort/nachhaltigkeitskarte/)

- Nachhaltigkeitsspaziergang Karlsruhe (nachhaltigkeitsspaziergangka.wordpress.com/ueber)

- Interaktive Stadtkarte (analog)

- ReparaturCafé Karlsruhe (verstetigt als e.V.; www.reparaturcafe-karlsruhe.de) 
- Leihladen „leih.lokal“ (ein Projekt der Bürgerstiftung Karlsruhe; www.buergerstiftung-karlsruhe.de/leihlokal)

- Urban-Gardening-Beet auf zentralem Platz

- Schwerlastenrad (als Infomobil und zum Verleih)

- Fotobox der Nachhaltigkeit, inkl. Wanderausstellung (www.mensch-und-technik.kit.edu/ksn_fotobox)

- Stadtentwicklungsspiel Karlsruhe, angepasst vom Spiel „FutureShocks and City Resilience“ der Arizona State University

- Spiel „Lebe Global“ zu Personaler Nachhaltigkeit

- Methodenvernissage (Veranstaltungsformat zu transformativen Methoden)

- Kurzfilme zu Reallaborkonzept, Gemeinschaft, Entschleunigung, ReparaturCafé und Gemeinwohlökonomie

- Webseite Quartier Zukunft - Labor Stadt (www.quartierzukunft.de)

- Leporello „Wie Nachhaltigkeit möglich ist“ (Deutsch, Englisch, Türkisch, Portugiesisch; www.quartierzukunft.de/wp-content/uploads/2016/05/12_2016_ web_Leporello_IKONE_de.pdf)

- Projekt-Broschüre „Das Morgen zum Mitmachen“ 


\section{BaWü-Lab Schorndorf „Reallabor Schorndorf: Zukunftsweisender Öffentlicher Verkehr - Bürgerorientierte Optimierung der Leistungs- fähigkeit, Effizienz und Attraktivität im Nahverkehr“6}

Im Reallabor Schorndorf entwickelten Wissenschaftler(innen) gemeinsam mit Praxispartnern und Bürger(inne)n der Stadt Schorndorf ein flexibles, bedarfsgerechtes und digital gestütztes Bussystem. Busfahren nach Bedarf statt nach Fahrplan, so erprobten die Schorndorfer(innen) von März bis Dezember 2018 unter Realbedingungen den neuen Bedarfsbus. Er ersetzte von Freitag- bis Sonntagabend zwei reguläre Buslinien und konnte per Smartphone-App, Telefon, PC oder über lokale Partner wie Geschäfte oder Cafés bestellt werden.

Förderperiode MWK: 2016-2019

www.reallabor-schorndorf.de

\section{Leitung/Koordination}

Mascha Brost (Dipl.-Ing. MDes.), Deutsches Zentrum für Luft- und Raumfahrt (DLR), Institut für Fahrzeugkonzepte, und stv. Laura Gebhardt (M.Sc. Geographie), Deutsches Zentrum für Luft- und Raumfahrt (DLR), Institut für Verkehrsforschung

\section{Forschungspartner}

Deutsches Zentrum für Luft- und Raumfahrt e. V. (DLR)

Universität Stuttgart, Zentrum für interdisziplinäre Risiko- und Innovationsforschung (ZIRIUS)

Hochschule Esslingen

\section{Praxispartner}

Verkehrs- und Tarifverbund Stuttgart (VVS)

Stadtverwaltung Schorndorf

Kommunikationsbüro Ulmer $\mathrm{GmbH}$

Knauss Reisen Dieter Frank GmbH \& Co. KG 


\section{Ziele}

a) Forschungsziele

- Generieren und Testen von Wissen für eine Transformation urbaner Mobilität. Mit Blick auf dieses Ziel wurden u. a. folgende Fragestellungen bearbeitet:

- Welche Faktoren/Determinanten beeinflussen die Alltagsaktivität unterschiedlicher Zielgruppen?

- Wie kann ein nachhaltiges, bedarfsgerechtes Buskonzept aussehen?

- Wie können die Attraktivität des öffentlichen Verkehrs und seine Verknüpfung mit anderen Mobilitätsangeboten gesteigert werden?

- Welche Anforderungen an ein zukünftiges Bedienkonzept haben unterschiedliche Gruppen von Nutzer(inne)n?

- Wie kann ein innovatives Fahrzeug für den Einsatz in diesem Bedienkonzept aussehen?

b) Praxisziele

- Die Zivilgesellschaft in die Diskussion und Gestaltung zukünftiger Mobilitätskonzepte einbeziehen.

- Die Entwicklung eines an die Bedürfnisse der Nutzer(innen) angepassten Mobilitätskonzepts.

- Schaffung eines attraktiven Angebots im öffentlichen Personennahverkehr (ÖPNV), das eine Verlagung vom Individualverkehr (MIV) zum öffentlichen Verkehr (ÖV) fördert.

c) Bildungsziele

- Anstoßen von Bewusstseins- und Verhaltensänderungen durch die Erprobung eines neuen Mobilitätsangebots unter Realbedingungen und des damit verbundenen Diskurses.

- Eine Debatte zur Zukunft städtischer Mobilität anstoßen durch zahlreiche dialogische Verfahren mit zivilgesellschaftlichen Akteuren (Workshops, Marktstände etc.).

- Generierung von methodischem Wissen zu Co-Creation im Bereich Verkehr. 


\section{Realexperiment}

- Neunmonatiger Testbetrieb des entwickelten neuen Bussystems unter Realbedingungen.

\section{Zentrale Publikationen und Produkte}

\section{a) Zentrale Publikationen}

Reallabor Schorndorf (2019). Reallabor Schorndorf - Entwicklung eines bedarfsgerechten Bussystems mit Testbetrieb in Schorndorf (Baden-Württemberg). Projektbericht. Stuttgart. (im Druck).

Gebhardt, L., Brost, M., \& Steiner, T. (2019). Bus on demand - ein Mobilitätskonzept mit Zukunft. Das Reallabor Schorndorf zieht nach dem Pilotbetrieb Bilanz. GAIA, 28 (1), (S. 66-68).

Gebhardt, L., \& Lenz, B. (2019). „On demand“ statt Fahrplan. Baustein eines zukünftigen Mobilitätsmanagements? Information zur Raumentwicklung, 46 (1), (S. 98-111).

Brost, M., Klötzke, M., Kopp, G., Deisser, O., Fraedrich, E., Karnahl, K., Sippel, T., Müller, A., \& Beyer, S. (2018). Development, Implementation (Pilot) and Evaluation of a Demand-Responsive Transport System. World Electric Vehicle Journal, 9 (1), (Nr. 4).

Klötzke, M., Brost, M., Fraedrich, E., Gebhardt, L., Karnahl, K., Kopp, G., König, A., Ademeit, A., Müller, A., Sippel, T., \& Ulmer, F. (2018). Reallabor Schorndorf. Bürgernahe Entwicklung eines haltestellenlosen Quartiersbussystems. In H. Proff \& T. Fojcik (Hrsg.), Mobilität und digitale Transformation (S. 295-309). Wiesbaden: Springer Fachmedien.

Brandies, A., König, A., Viergutz, K., Fraedrich, E., Gebhardt, L., Ulmer, F., Sippel, T., \& Dotzauer, M. (2017). Transdisziplinäre Mobilitätsforschung unter Verwendung von Reallaboren: Integration von Stakeholderbedürfnissen und-anforderungen in die Entwicklung von Systemen bedarfsorientiert und vollautomatisiert fahrender Quartiersbusse. AAET 2017: Automatisiertes \& vernetztes Fahren 2017 (S. 165-185). Braunschweig: ITS automotive nord e. V.

b) Produkte, die in der Praxis unmittelbar genutzt werden können

- Webseite (www.reallabor-schorndorf.de)

- Anleitungsbroschüre zum Bussystem für die Nutzer(innen)

- Entwickelte App zur Bestellung des Busses

- Dispositionsapp (optimierte Routenerstellung)

- Navigationsapp für die Busfahrer(innen) 


\section{BaWü-Lab SRB}

\section{„STADT-RAUM-BILDUNG - Reallabor für die nachhaltige Planung von Bildungslandschaften und die Integration von Aus- und Umbauten von Schulgebäuden“"}

Im Reallabor wurden Transformationsprozesse an Schulen und in deren Kontext nicht nur analysiert, sondern auch initiiert. Im Sinne der sozialen Nachhaltigkeit standen der Austausch zwischen und die Co-Produktion mit den Praxisakteuren bei der Planung von Schulen im Vordergrund. Es wurden gemeinsam Lösungsansätze gesucht, wie neue pädagogische Lehr- und Lernmethoden in bestehenden Schulgebäuden mit nur wenigen baulichen Eingriffen umgesetzt werden können, wie der Ganztagesbetrieb der Schulen zu einer stärkeren Vernetzung von Schule und Quartier führen kann und wie diese Aspekte frühzeitig im Planungsprozess von Aus-, Um- und Erweiterungsbauten mitgedacht werden und dauerhaft Wirkung entfalten können. Bezugsraum des Reallabors: Kommunen in Baden-Württemberg.

Förderperiode MWK: 2015-2018

www.stadt-raum-bildung.de

\section{Leitung/Koordination}

Projektleitung: Prof. Dr. Marc Kirschbaum und Prof. Andreas Bartels, beide SRH Hochschule Heidelberg, School of Engineering and Architecture

Projektkoordination: Mandana Alimardani, SRH Hochschule Heidelberg, School of Engineering and Architecture

Projektkoordination: Charlotte Eller, Universität Stuttgart, Städtebau-Institut

\section{Forschungspartner}

SRH Hochschule Heidelberg, School of Engineering and Architecture: Prof. Dr. Marc Kirschbaum; Prof. Andreas Bartels; Mandana Alimardani; Belen Zevallos

\section{Universität Stuttgart}

- Städtebau-Institut (SI): Prof. Dr. Helmut Bott; Prof. Dr. Astrid Ley; Charlotte Eller; Prof. Dr. Thorsten Erl; Martina Hilligardt; Christian Schmutz

- Institut für Raumkonzeptionen und Grundlagen des Entwerfens (IRGE): Prof. Markus Allmann; Charlotte Eller

- Internationales Zentrum für Kultur- und Technikforschung (IZKT): Dr. Elke Uhl; Martina Hilligardt 
Pädagogische Hochschule Heidelberg, Institut für Erziehungswissenschaft: Prof. Dr. Albrecht Wacker; Karin Haupt-Mukrowsky

Institut für Schulentwicklung: Dr. Otto Seydel

Compare Consulting: Christina Benighaus; Ludger Benighaus

\section{Praxispartner}

Stadt Heidelberg

Hochbauamt (ehemals Gebäude-

management)

Amt für Schule und Bildung

Stadtplanungsamt

Geschwister-Scholl-

Gemeinschaftsschule

Marie-Marcks-Schule/Wilckensschule

Waldparkschule

weitere Schulen in Heidelberg

Stadt Müllheim

Baudezernat u. a.

Michael-Friedrich-Wild-Grundschule

Adolph Blankenhorn Gemeinschaftsschule

Heilpädagogisches Förderzentrum (HPF)

weitere Schulen in Müllheim
Stadt Stuttgart

Referat Jugend und Bildung

Schulverwaltungsamt

Heusteigschule

Schickhardt Schule

weitere Schulen in Stuttgart

Weitere Schulen, deren Akteure vor Ort sowie das zuständige Amt

Christliche Schule im Hegau in Hilzingen

Alemannenschule in Wutöschingen

Gemeinschaftsschule Gebhard in Konstanz

Gemeinschaftsschule Steißlingen

Gemeinschaftsschule Neuenstein

Ernst-Reuter-Schule in Karlsruhe

Gemeinschaftsschule in Ludwigsburg

\section{Ziele}

a) Forschungsziele

Erkenntnisse gewinnen über die Verknüpfung von Raum und Pädagogik vor allem hinsichtlich der Umsetzung neuer pädagogischer Lehr- und Lernformen, der Einführung und Gestaltung des Ganztagesunterrichts, der Sicherstellung von Inklusion und der Digitalisierung. Mit Blick auf dieses Ziel wurden u. a. folgende Fragestellungen zum Thema Schulumbau bearbeitet: 
- Welche Zusammenhänge gibt es zwischen dem gebauten Lernraum und der darin stattfindenden Pädagogik aus architektonischer, pädagogischer und raumsoziologischer Sicht, wo entstehen Unvereinbarkeiten und Spannungsfelder, und wie können diesbezüglich Verbesserungen erreicht werden?

- Welche Umbaupotenziale bestehen in Schulen unterschiedlichster Entstehungszeiten (Schulbauepochen), um heutige pädagogische Ziele des individuellen und aktivierenden Lernens besser zu erreichen?

- Welche Handlungsspielräume existieren bei der Transformation des städtischen Bausteins Schule, um Potenziale für soziale Nachhaltigkeit im Quartier entfalten zu können?

- Wie müssen zukünftige Planungsprozesse im Schulbau gestaltet bzw. verbessert werden, damit trotz hohen Handlungsdrucks qualitativ gute und nachhaltige Umbaukonzepte realisiert werden können?

b) Praxisziele

- Aufbau und Konstitution einer lokalen Schulbau-AG im jeweiligen kommunalen Teilprojekt zum Zweck der Transparenz und des Schaffens von Vertrauen unter den beteiligten Akteuren.

- Aufbau und Pflege einer Plattform (im Rahmen der Veranstaltungsreihe „Old school - Neues Lernen“) für ein regionales Akteursnetzwerk zum Zweck des Lernens von ,Peer to Peer" und des Nutzens von Multiplikatoreneffekten.

c) Bildungsziele

- Studierende lernen Modelle des forschenden Lernens kennen und entwickeln kommunikative Kompetenzen.

- Inwertsetzung studentischer Arbeit für Forschung und Praxis.

- Akteure aus der Wissenschaft, Studierende und Praxispartner lernen den Umgang auf Augenhöhe.

Teilprojekte und wichtigstes partizipatives Format

a) Kommunale Teilprojekte „Old School - Neues Lernen“

- Heidelberg: Geschwister-Scholl-Schule

- Stuttgart: Heusteigschule Stuttgart

- Müllheim: Bildungscampus Müllheim 
b) Wichtigstes partizipatives Format

- Veranstaltungsreihe „Old School - Neues Lernen“

\section{Zentrale Publikationen und Produkte}

a) Zentrale Publikationen

Eller, C., Erl, Th., Kirschbaum, M., \& Wacker, A. (2020). Reallabor STADT-RAUM-BILDUNG. Ergebnisse und Handlungsempfehlungen. Heidelberg: Heidelberger Hochschulverlag. (in Vorbereitung).

Hilligardt, M., Erl, Th., \& Schmutz, C. (2018). Next Generation Schule. In: Schulbau Magazin 04 | 2018, Neue Lernsettings (S. 10-14).

Kirschbaum, M., \& Wacker, A. (2018). Den Schul(um)bau sozial-ökologisch gestalten. GAIA, 27 (4), (S. 396-397).

b) Produkte, die in der Praxis unmittelbar genutzt werden können

- Dokumentationen der Entwurfsstudien der jeweiligen kommunalen Teilprojekte (diese können den Schulbauträgern vor Ort dienen bei der Definition der Aufgabenstellung für einen städte- oder hochbaulichen Wettbewerb im Schulbau und bei der Ausarbeitung eines Schulsanierungsplans):

Erl, Th., Schmutz, C., \& Bartels, A. (2019). Kommunale Lernlandschaft. Bestandaufnahme von Sekundarschulen in Heidelberg. Heidelberg: Heidelberger Hochschulverlag. (in Vorbereitung).

Schmutz, C., Eller, C., \& Erl, Th. (2019). Old School - Neues Lernen. Entwurfsstudien zum Umbau der Geschwister-Scholl-Schule in Heidelberg. Heidelberg: Heidelberger Hochschulverlag. (in Vorbereitung).

Eller, C., \& Erl, Th. (2019). Old School - Neues Lernen. Entwurfsstudien zum Umbau der Heusteigschule in Stuttgart. Heidelberg: Heidelberger Hochschulverlag. (in Vorbereitung).

Hilligardt, M., Schmutz, C., \& Eller, C. (2019). Old School - Neues Lernen. Entwurfsstudien zum Umbau des Schulzentrums II in Müllheim. Heidelberg: Heidelberger Hochschulverlag. (in Vorbereitung).

- Das kleine Schulbaulexikon:

Seydel, O. (2018). Das kleine Schulbaulexikon. 50 Begriffe, über die es eine Verständigung zwischen Planer(inne)n und Pädagog(inn)en braucht. Unter Mitarbeit von A. Bartels, A. Wacker, B. Zevallos, M. Kirschbaum, T. Erl, M. Hilligardt, C. Schmutz, C. Benighaus, M. Alimardani. https://stadt-raum-bildung.de/aktuell/daskleine-schulbaulexikon. Zugegriffen am 20.05.2019. 


\section{BaWü-Lab Urban Office „Urban Office - Nachhaltige Stadtentwicklung in der Wissensgesellschaft"}

Das Reallabor reagierte auf die zunehmende Bedeutung von Städten bei der Transformation zur Wissensgesellschaft, indem es inter- und transdisziplinär nachhaltige Prozesse der Stadtentwicklung ergebnisoffen in Gang setzte und erforschte. Dafür konzeptualisierte und implementierte das Urban Office Organisationsnetzwerke, Kommunikationsstrukturen und Aktivitäten zur inter- und transdisziplinären Wissensproduktion sowie zum Wissenstransfer.

Förderperiode MWK: 2015-2018 www.uni-heidelberg.de/urbanoffice

\section{Leitung/Koordination}

Projektleitung: Prof. Dr. Ulrike Gerhard und Dr. Editha Marquardt, beide Universität Heidelberg, Geographisches Institut

Projektkoordination: Dr. Christina West, Universität Heidelberg, Geographisches Institut; Urban Office

\section{Forschungspartner}

\section{Universität Heidelberg}

- Centrum für soziale Investitionen \& Innovationen: Dr. Georg Mildenberger

- Diakoniewissenschaftliches Institut: Prof. Dr. Johannes Eurich; Dr. Stefanie Wiloth

- Institut für Gerontologie: Prof. Dr. Dr. Andreas Kruse

- Geographisches Institut: Prof. Dr. Ulrike Gerhard; Dr. Editha Marquardt; Dr. Christina West; Kerstin Fröhlich

- Geoinformatik/GIScience: Prof. Dr. Alexander Zipf; Michael Auer; Sebastian Döring; Lukas Loos

- Max-Weber-Institut für Soziologie: Christina Herrmann

Deutsche Universität für Verwaltungswissenschaften Speyer: Lehrstuhl für Hochschul- und Wissenschaftsmanagement: Prof. Dr. Michael Hölscher

IFEU - Institut für Energie- und Umweltforschung Heidelberg GmbH: Dr. Martin Pehnt; Miriam Dingeldey 
Universität Stuttgart: SI - Städtebau-Institut, Lehrstuhl Internationaler Städtebau: Prof. Dr. Helmut Bott; Prof. Dr. Thorsten Erl; Theresa Eitel

\section{Praxispartner}

Amt für Stadtentwicklung und Statistik Heidelberg: Joachim Hahn $\dagger$

Der Bahnstadttreff LA 33: Stefanie Ferdinand

Frey Architekten, Freiburg: Wolfgang Frey

IBA - Internationale Bauausstellung Heidelberg „Wissen I schafft I Stadt“: Prof. Michael Braum; Carl Zillich

Stadtplanungsamt Heidelberg: Annette Friedrich; Christoph Czolbe; Prof. Dr.-Ing. Henning Krug; Margit Sachtlebe

Stadtwerke Heidelberg GmbH: Michael Teigeler

Stadtteilverein Bahnstadt

Stadtteilverein Südstadt

Urban Innovation Center HD \& Urban Innovation - Stadt neu denken! e. V.

\section{Ziele}

a) Forschungsziele

- Implementierung von inter- und transdisziplinärer Forschung und von Forschungsformaten, die zu einer nachhaltigen Stadtentwicklung in der Wissensgesellschaft beitragen; kritische Reflexion von gesellschaftlich relevanten Forschungsfragen und des Rollenverständnisses wissenschaftlicher und außerwissenschaftlicher Akteure.

- Forschung zu Aspekten urbaner Transformationen in der Wissensgesellschaft und Beiträge zur Transformation nachhaltiger Wissensproduktion.

b) Praxisziele

- Implementierung, Etablierung, Verstetigung des „Urban Office“ als Organisations- und Schnittstelle zwischen Universität Heidelberg, Stadtverwaltung Heidelberg, IBA Heidelberg, Bewohner(inne)n und weiteren Akteuren. Ziele des „Urban Office“ sind: inter- wie transdisziplinäres Co-Design, Co-Creation und Co-Produktion von Forschungsfragen und -ergebnissen mit Praxispartnern; Verknüpfung von Forschungsergebnissen, Syntheseleistungen, Qualitätssicherung; Transfer und Kommunikation von Forschungsergebnissen; Sicherung (ergebnis-)offener, transparenter Forschungsprozesse; Entwickeln 
und Evaluation von transdisziplinären Forschungs- und Kommunikationsformaten, Methoden, Interventionen, Realexperimenten.

- Verknüpfen von Aspekten einer nachhaltigen Stadtentwicklung mit konkreten IBA-Stadtentwicklungsprojekten.

c) Bildungsziele

- Schaffung und Etablierung von spezifischen Lehr- und Forschungsformaten, mit denen Reallaborforschung und experimentelle Arbeitsweisen gelehrt und reflektiert werden.

- Theoretische und praktische Auseinandersetzung mit den verschiedenen Modi der Wissensproduktion im Zusammenhang mit den Paradigmen nachhaltiger Stadtentwicklung.

- Vermittlung und Weiterentwicklung von disziplinären sowie inter- und transdisziplinären Kompetenzen bei Studierenden und Akteuren der Stadtgesellschaft.

\section{Teilprojekte und Realexperimente}

a) Teilprojekte

- Transformation von Stadtteilen durch neue Wissensorte

- Innovative Partizipationsstrukturen und nachhaltige Wohnkonzepte angesichts des demografischen Wandels

- Die Bedeutung von Netzwerken relevanter Wissensakteure

- Wissen schaffen für die Stadt - neue Methoden der Bürgerbeteiligung durch interaktive Stadtplanung im Web 2.0 am Beispiel der Energiewende

b) Wichtigste Realexperimente

- Wissen to Go \& FairteilerMobile

- fUrOre - Sustainable Spots erFAHREN

- Urban Innovation Center HD \& Urban Innovation - Stadt neu denken! e. V.

- MovingLABs (z. B. ReaLABarcelona, UrbanUtopiaLAB (Kooperation mit BaWü-Lab Asylsuchende), utc - urban thinkers camp „Multihalle“ in Kooperation mit Urban Thinkers Campus Mannheim), „Neue Formen der Teilhabe in der Stadtentwicklung“ - transdisziplinäre Masterarbeit mit MovingLABs in Kooperation mit N-E-U (Netzwerk für experimentellen Urbanismus) 


\section{Zentrale Publikationen und Produkte}

a) Zentrale Publikationen

Fröhlich, K., \& Gerhard, U. (2017). Wissensbasierte Stadtentwicklung - ein Erfolgskonzept auch für Nachhaltigkeit? Einblicke in die Entwicklung der Heidelberger Südstadt aus Reallaborperspektive. Berichte. Geographie und Landeskunde, 91 (1), (S. 13$33)$.

Gerhard, U., \& Marquardt, E. (2017). Reallabore als innovatives Forschungsformat zur Untersuchung nachhaltiger Stadtentwicklung. Eine kritische Reflexion. Berichte. Geographie und Landeskunde, 91 (1), (S. 97-111).

Gerhard, U., \& Marquardt, E. (2015). The Greener, the Happier? Urban Sustainability in the Knowledge City. Policies, Programs and Practices in the German Context. In D. Wilson (Hrsg.), The Politics of Urban and Regional Sustainability. Appraising the Concept and Process (S. 65-86). Champaign: Common Grounds Publishing.

Gerhard, U., Marquardt, E., \& West, C. (2017). Reallabore in der Stadtforschung. Eine Einführung. Berichte. Geographie und Landeskunde, 91 (1), (S. 5-12).

Hermann, C., \& Hölscher, M. (2017). Innere Suburbanisierung als Antwort auf die Wissensgesellschaft? Die Heidelberger Bahnstadt im theoretischen Spannungsfeld von Urbanität und Suburbanität. Berichte. Geographie und Landeskunde, 91 (1), (S. 49-65).

Marquardt, E. (2019). Hochschule und Stadt als Partner in Reallaboren. Neue Wege für ein konstruktives Miteinander. Beiträge zur Hochschulforschung, 41 (1), (S. 108-123).

Marquardt, E., \& West, C. (2016). Co-Produktion von Wissen in der Stadt. Reallabor „Urban Office - Nachhaltige Stadtentwicklung in der Wissensgesellschaft" an der Universität Heidelberg. Technikfolgenabschätzung - Theorie und Praxis, 25 (3), (S. 26-31).

West, C., Marquardt, E., \& Gerhard, U. (2017). Co-Design und Co-Produktion von nachhaltigem Wissen in der Stadt. Das Reallabor Urban Office in Heidelberg. GAIA, 26 (1), (S. 58-59). doi: https://doi.org/10.14512/gaia.26.1.13.

Wiloth, S., \& Eurich, J. (2017). Auf dem Weg zu Sorgestrukturen für ältere Menschen in der Bahnstadt Heidelberg - eine explorative Studie zur Erfassung zentraler Rahmenbedingungen. Berichte. Geographie und Landeskunde, 91 (1), (S. 35-47).

b) Produkte, die in der Praxis unmittelbar genutzt werden können

- Broschüre „Die lernende Stadt. Nachhaltige Stadtentwicklung in der Wissensgesellschaft - 15 Heidelberger Thesen“ (erschienen als RNZ-Beilage am 21.04.2018)

- Urban Office an der Universität Heidelberg (über die Förderperiode hinaus), mit festen Öffnungszeiten für Publikumsverkehr und interessierte Bürger(innen) (www.uni-heidelberg.de/urbanoffice)

- Webseite Urban Office (www.uni-heidelberg.de/urbanoffice) 
- Facebook-Auftritt Urban Office Heidelberg (www.facebook.com/UrbanOfficeHD)

- YouTube-Channel Urban Office (Urban Office HD) (www.youtube.com/channel/UCBs4guVf6dOISRpAMvLakzw)

- FairteilerMobile Heidelberg

- Interaktive Klimaschutzkarte Deutschland (www.klimaschutzkarte.de)

- Prozessanalyse IBA-Projekt „Der Andere Park“ - Begleitforschung

- Netzwerkanalyse IBA-Akteure

- Prozessgraphik „Haus des Lernens B ${ }^{3}$ - Genese eines Quartierzentrums“ (Universität Stuttgart, Städtebau-Institut)

- Urban Innovation Center HD (www.uic-hd.com) \& Urban Innovation - Stadt neu denken! e. V. (www.urbaninnovation.de)

- Podcast mit Shownotes: West, C., \& Schweizerhof, M. (2017). Perspektiven auf soziales Engagement, heute: Malte und Christina zum „Fairteiler mobil“. Das Urban Office Heidelberg im Herzen der Nachhaltigkeitsdebatte. In: Schönborn, J. karmajob vom 24.10.2017. Heidelberg.

(https://karmajob.de/?s=urban+office, zugegriffen am 29.05.2019) 


\section{Autorinnen und Autoren}

\section{Marius Albiez}

Dipl., Geoökologie. Wissenschaftlicher Mitarbeiter am Institut für Technikfolgenabschätzung und Systemanalyse (ITAS) am Karlsruher Institut für Technologie (KIT), zuvor bis Ende 2018 Internationales Zentrum für Ethik in den Wissenschaften (IZEW) der Universität Tübingen. Forschungsschwerpunkte: Nachhaltigkeitsforschung in Reallaboren; nachhaltige Energiewende und Partizipation; Bildung für Nachhaltige Entwicklung (BNE). In der Förderlinie „Reallabore Stadt" (MWK Baden-Württemberg): Bildungsprozesse; Analyse und Kriterien einer nachhaltigen Energiewende; transdisziplinäre Forschung im BaWü-Lab Energielabor Tübingen ${ }^{1}$.

\section{Mandana Alimardani}

M.Sc., Architektur. Mitarbeiterin im Schulverwaltungsamt Stuttgart, Neu- und Erweiterungsbauten, zuvor bis Mitte 2018 Wissenschaftliche Mitarbeiterin an der SRH Hochschule Heidelberg School of Engineering and Architecture. Forschungsschwerpunkte: Innovativer Schulbau; Schule als Lern- und Lebensraum - eine Planungsaufgabe. In der Förderlinie „Reallabore Stadt“ (MWK Baden-Württemberg): Projektkoordination; beteiligt an den Arbeitspaketen „Lernräume“ und „Planungsverfahren“; Durchführung Interdisziplinärer Feldforschung und Mitarbeit an Kolloquien und Symposien im BaWü-Lab SRB ${ }^{2}$.

1 „Energielabor Tübingen - Potenziale, Partizipation, Perspektiven“ („BaWü-Lab Energielabor Tübingen").

2 „STADT-RAUM-BILDUNG - Reallabor für die nachhaltige Planung von Bildungslandschaften und die Integration von Aus- und Umbauten von Schulgebäuden“ (,BaWü-Lab SRB“). 


\section{Richard Beecroft}

Dr. phil. (des.), Nachhaltigkeitswissenschaft; Dipl.-Ing., Materialwissenschaft. Wissenschaftlicher Mitarbeiter am Institut für Technikfolgenabschätzung und Systemanalyse (ITAS) am Karlsruher Institut für Technologie (KIT), Co-Leiter Karlsruher Schule der Nachhaltigkeit. Forschungsschwerpunkte: Transdisziplinäre Methodologie; Didaktik der Technikfolgenabschätzung; Integration von Transformativer Forschung und Lehre; Bildung für Nachhaltige Entwicklung. In der Förderlinie „Reallabore“ (MWK Baden-Württemberg): Gesellschaftliche Lernprozesse und Bildung im BaWü-Lab R131 ${ }^{3}$.

\section{Christina Benighaus}

Dipl. Geogr., Leiterin Bereich „Kommunikation: Information, Dialog, Partizipation" der DIALOGIK gGmbH für Kommunikation und Kooperationsforschung sowie COMPARE Consulting, Senior Researcher in Projekten zur Risiko- und Krisenkommunikation und zur Partizipation. Forschungsschwerpunkte: Konfliktschlichtungs- und Partizipationsverfahren; Mediation; Risikomanagement; Risiko-, Krisen- und Umweltkommunikation. In der Förderlinie „Reallabore“ (MWK Baden-Württemberg): Beratung, Konzeptentwicklung und Moderation von Kolloquien, Symposien und Workshops im BaWü-Lab RNM.

\section{Ludger Benighaus}

Dipl. Geogr., Wissenschaftlicher Mitarbeiter der DIALOGIK gGmbH für Kommunikation und Kooperationsforschung sowie COMPARE Consulting. Forschungsschwerpunkte: Moderation und Mediation von Beteiligungsverfahren von Bürger(inne)n und Stakeholdern; Konzeption und Planung von Konfliktschlichtungs- und Partizipationsverfahren; Wahrnehmung und Kommunikation von Risiken in unterschiedlichen Themenfeldern (Geothermie, Energie, Ernährung, Bergbau, Umwelt, Radioaktivität); Aufbau von Dialog- und Kommunikationsstrukturen; Modelle der Kommunikation; E-Learning, Blended Learning, virtuelle Lehre; Gerontologie und demografische Entwicklung. In der Förderlinie „Reallabore Stadt" (MWK Baden-Württemberg): Beratung, Konzeptentwicklung und Moderation von Kolloquien, Symposien und Workshops im BaWü-Lab RNM.

3 „Reallabor 131: KIT findet Stadt“(„BaWü-Lab R131“). 
Rico Defila

Fürspr., Rechtswissenschaft. Stv. Leiter interdisziplinäre und interuniversitäre Forschungsgruppe Inter-/Transdisziplinarität (FG Id/Td), Senior Researcher am Programm Mensch-Gesellschaft-Umwelt (MGU) der Universität Basel, Departement Umweltwissenschaften. Forschungsschwerpunkte: Gestaltung inter-/transdisziplinärer Prozesse in Forschung und Lehre; Organisationsentwicklung interdisziplinärer Institutionen; nachhaltiger Konsum. In der Förderlinie „Reallabore“ (MWK Baden-Württemberg): Co-Leiter Begleitforschungs-Projekt „Reallabore vernetzen, verstehen, verstetigen“"(BF-Team Basel).

\section{Antonietta Di Giulio}

Dr. phil., Philosophie. Leiterin interdisziplinäre und interuniversitäre Forschungsgruppe Inter-/Transdisziplinarität (FG Id/Td), Senior Researcher am Programm Mensch-Gesellschaft-Umwelt (MGU) der Universität Basel, Departement Umweltwissenschaften. Forschungsschwerpunkte: Gestaltung inter-/transdisziplinärer Prozesse in Forschung und Lehre; gutes Leben und nachhaltiger Konsum; Bildung und Nachhaltige Entwicklung. In der Förderlinie „Reallabore“ (MWK Baden-Württemberg): Co-Leiterin Begleitforschungs-Projekt „Reallabore vernetzen, verstehen, verstetigen“ (BF-Team Basel).

\section{Raphael Dietz}

Dipl.-Ing., Architektur und Stadtplanung. Wissenschaftlicher Mitarbeiter am Städtebau-Institut, Lehrstuhl Internationaler Städtebau der Universität Stuttgart, Mitarbeiter bei asp Architekten Stuttgart GmbH. Forschungsschwerpunkte: Nachhaltige Transformation des öffentlichen Raums; Konzeption und Durchführung transdisziplinärer Forschungs- und Lehrformate; urbane Potenziale der Energiewende; Stadt am Fluss. In der Förderlinie „Reallabore“ (MWK Baden-Württemberg): Koordination der Realexperimente sowie der städtebaulichen Visualisierung für den Visionsworkshop des BaWü-Labs RNM ${ }^{4}$.

4 „Future CityLab Stuttgart - Reallabor für nachhaltige Mobilitätskultur“ („BaWü-Lab RNM“). 


\section{Jochen Eckart}

Prof., PhD, Civil and Environmental Engineering. Professur für Verkehrsökologie im Studiengang Verkehrssystemmanagement an der Hochschule Karlsruhe Technik und Wirtschaft, Studiendekan Studiengang Verkehrssystemmanagement Master, stv. Leiter Institut für Verkehr und Infrastruktur. Forschungsschwerpunkte: Nachhaltige Mobilität; Fußverkehrsförderung; Radverkehrsförderung; wassersensible Straßenraumgestaltung; Lärmminderung; Luftreinhaltung. In der Förderlinie „Reallabore Stadt“ (MWK Baden-Württemberg): Mitglied Projektleitung BaWü-Lab GO Karlsruhe ${ }^{5}$.

\section{Charlotte Eller}

Dipl.-Ing., Architektur und Stadtplanung. Wissenschaftliche Mitarbeiterin am Städtebau-Institut, Lehrstuhl Internationaler Städtebau, sowie am Institut für Raumkonzeptionen und Grundlagen des Entwerfens der Universität Stuttgart. Forschungsschwerpunkte: Schulforschung; Nachhaltige Stadtentwicklung; urbane Transformation; Konzeption und Durchführung transdisziplinärer Forschungsund Lehrformate. In der Förderlinie „Reallabore Stadt“ (MWK Baden-Württemberg): Koordination (Schwerpunkt auf Kooperationen mit Kommunen); Lehre im Rahmen der kommunalen Kooperationsprojekte; Konzeption und Mitarbeit im „Labor 2: Bildungslandschaft“ im BaWü-Lab SRB.

\section{Thorsten Erl}

Prof. Dr.-Ing., Architektur, Städtebau und Stadtplanung. Professur für Städtebau an der Universität Siegen, Departement Architektur der Fakultät Bildung, Architektur, Künste. Forschungsschwerpunkte: Nachhaltige Stadtentwicklung; urbane Transformation; stadtstrategische und integrale Planung; Co-Design und Co-Produktion. In den Förderlinien „Reallabore“ und „Reallabore Stadt“ (MWK BadenWürttemberg): Im BaWü-Lab Urban Office ${ }^{6}$ beteiligt am Teilprojekt „Haus des Lernens B ${ }^{3}$, Genese eines Quartierszentrums“ (Teil von „Die Bedeutung von Netzwerken relevanter Wissensakteure"); im BaWü-Lab SRB in den Arbeitspaketen zu Bildungslandschaften und zu kommunalen Teilprojekten.

5 „GO Karlsruhe“ - Partizipative Forschung für den Fußgängerverkehr („BaWü-Lab GO Karlsruhe").

6 „Urban Office - Nachhaltige Stadtentwicklung in der Wissensgesellschaft“ („BaWüLab Urban Office"). 


\section{Laura Gebhardt}

M.Sc., Geographie. Wissenschaftliche Mitarbeiterin und Projektleiterin am Institut für Verkehrsforschung des Deutschen Zentrums für Luft- und Raumfahrt e. V. (DLR). Forschungsschwerpunkte: Sozialwissenschaftliche Mobilitäts- und Verkehrsforschung, insbesondere zur partizipativen Entwicklung zukünftiger Mobilitätskonzepte. In der Förderlinie „Reallabore Stadt“ (MWK Baden-Württemberg): Entwicklung und Durchführung der Stakeholder-Partizipation im BaWü-Lab Schorndorf?

\section{Ulrike Gerhard}

Prof. Dr., Geographie. Professorin am Geographischen Institut sowie Heidelberg Center for American Studies der Universität Heidelberg. Forschungsschwerpunkte: Nachhaltige Stadtentwicklung; aktuelle Prozesse der Stadtentwicklung in den USA; vergleichende Stadtforschung; Partizipation und Stadt. In der Förderlinie „Reallabore“ (MWK Baden-Württemberg): Leitung des BaWü-Labs Urban Office sowie des Teilprojekts „Transformation von Stadtteilen durch neue Wissensorte“.

\section{Monika Gonser}

Dr. rer. pol., Sozialwissenschaften. Wissenschaftliche Mitarbeiterin im Transferzentrum der Pädagogischen Hochschule Heidelberg. Forschungsschwerpunkte: Fluchtforschung; Integrationsforschung; Knowledge Mobilization; Sozialwissenschaftliche Transformationsforschung; Arbeitsbeziehungen. In der Förderlinie „Reallabore Stadt“ (MWK Baden-Württemberg): Projektkoordination und Geschäftsführung im BaWü-Lab Asylsuchende ${ }^{8}$.

\section{Elke Häußler}

M.A., Geographie und Ethnologie. Wissenschaftliche Mitarbeiterin am Institut für Verkehr und Infrastruktur (IVI) der Hochschule Karlsruhe - Technik und Wirtschaft. Forschungsschwerpunkte: nachhaltige Quartiersentwicklung; Akteursanalyse in partizipativen Prozessen; nachhaltige Mobilität, v. a. Fußverkehr. In der Förderlinie „Reallabore Stadt“ (MWK Baden-Württemberg): Mitarbeiterin im BaWü-Lab GO Karlsruhe.

7 „Reallabor Schorndorf: Zukunftsweisender Öffentlicher Verkehr - Bürgerorientierte Optimierung der Leistungsfähigkeit, Effizienz und Attraktivität im Nahverkehr“ (,BaWü-Lab Schorndorf").

8 „Asylsuchende in der Rhein-Neckar-Region“ („BaWü-Lab Asylsuchende“). 


\section{Martina Hilligardt}

M.Sc., Architektur und Stadtplanung. Wissenschaftliche Mitarbeiterin am Städtebau-Institut, Lehrstuhl Internationaler Städtebau sowie Internationales Zentrum für Kultur- und Technikforschung der Universität Stuttgart; Mitarbeiterin sander.hofrichter Architekten GmbH, Ludwigshafen (Rhein). Forschungsschwerpunkte: interdisziplinäre Schul- und Lernraumentwicklung. In der Förderlinie „Reallabore Stadt“ (MWK Baden-Württemberg): Beteiligt am Arbeitspaket „Planungsverfahren“ und kommunale Teilprojekte; Konzeption und Durchführung transdisziplinärer Forschungs- und Lehrformate; Entwicklung und Durchführung von Kolloquien und Symposien im BaWü-Lab SRB.

\section{Katharina Köglberger}

Dipl.-Ing. Bakk. Phil., Architektur und Städtebau sowie Romanistik. Aktuell freischaffend tätig, zuvor bei German University of Cairo, Staatliche Akademie der Bildenden Künste Stuttgart und Technische Universität Graz. Forschungsschwerpunkte: Fragestellungen an den Schnittstellen zwischen Architektur, Soziologie (Gesellschaftlicher Wandel) und Kunst; Wohnbau; Gestaltung und Entwurf. In der Förderlinie „Reallabore“ (MWK Baden-Württemberg): Konzeption, Antrag, Wissenschaftliche Mitarbeiterin während der Durchführung im BaWü-Lab Space Sharing 9 .

\section{Alexandra König}

M.Sc., Psychologie. Doktorandin am Institut für Verkehrssystemtechnik des Deutschen Zentrums für Luft- und Raumfahrt e. V. (DLR). Forschungsschwerpunkte: Erhebung von Nutzeranforderungen an bedarfsorientierte Bedienkonzepte des ÖPNV und nutzerzentrierte Systemgestaltung. In der Förderlinie „Reallabore Stadt" (MWK Baden-Württemberg): Anforderungserhebung an das Bedienkonzept des Bedarfsbusses im BaWü-Lab Schorndorf.

\section{Svenja Kück}

M.A., Geographie. Wissenschaftliche Mitarbeiterin und Doktorandin am Geographischen Institut der Universität Heidelberg. Forschungsschwerpunkte: Geographische Migrationsforschung; Heimat; Biographieforschung; transdisziplinäre Forschung. In der Förderlinie „Reallabore Stadt“ (MWK Baden-Württemberg): Mitarbeiterin im BaWü-Lab Asylsuchende ${ }^{10}$, TP III „Dezentrales Wohnen“ und „UrbanUtopiaLAB“.

9 „Space Sharing - Nutzungsintensivierung des Gebäudebestands durch Mehrfachnutzung und dynamische Programmierung“ („BaWü-Lab Space Sharing“).

10 „Asylsuchende in der Rhein-Neckar-Region“ („BaWü-Lab Asylsuchende“). 


\section{Editha Marquardt}

Dr., Geografie. Wissenschaftliche Mitarbeiterin an der Universität für Verwaltungswissenschaften Speyer, Leitung des Projekts „Town \& Gown. Optimierung der Kooperation von Stadtverwaltungen mit regionalen Wissenschaftseinrichtungen“ im Verbundprojekt „Wissens- und Ideentransfer in der Verwaltung (Witi)“, „Förderlinie Innovative Hochschule“ (BMBF). Forschungsschwerpunkte: Transdisziplinäre Stadtforschung; nachhaltige Stadtentwicklung; Stadt in der Wissensgesellschaft. In der Förderlinie „Reallabore“ (MWK Baden-Württemberg): Leitung des BaWü-Labs Urban Office sowie im Teilprojekt „Transformation von Stadtteilen durch neue Wissensorte“.

\section{Felix M. Piontek}

M.Sc., Wirtschaftschemie. Wissenschaftlicher Mitarbeiter am Institut für Nachhaltige Unternehmensführung der Universität Ulm. Forschungsschwerpunkte: Life Cycle Assessment; Product-Service Systems. In der Förderlinie „Reallabore“ (MWK Baden-Württemberg): Mitarbeiter im BaWü-Lab Dietenheim zieht an ${ }^{11}$.

\section{Thomas Potthast}

Univ.-Prof. Dr., Biologie und Philosophie. Professor für Ethik, Theorie und Geschichte der Biowissenschaften und Sprecher des Internationalen Zentrums für Ethik in den Wissenschaften (IZEW) der Universität Tübingen. Forschungsschwerpunkte: Inter- und transdisziplinäre Ethik; Biodiversität und Nachhaltige Entwicklung; Naturphilosophie; Wissenschaftstheorie und Geschichte der Lebenswissenschaften und des Naturschutzes. In der Förderlinie „Reallabore Stadt“ (MWK Baden-Württemberg): Leitung des Teilprojekts des IZEW im BaWü-Lab Energielabor.

\section{Jan Riel}

Prof. Dr.-Ing., Verkehrsplaner. Professur für Verkehrsplanung und Verkehrstechnik, Sprecher des Instituts für Verkehr und Infrastruktur, Hochschule Karlsruhe Technik und Wirtschaft. Forschungsschwerpunkte: Stadtverträgliche Mobilität; autonomes Fahren; Verkehrssicherheit; Straßenraumgestaltung; Schnittstelle Stadt - Verkehr - Energie. In der Förderlinie „Reallabore Stadt“ (MWK BadenWürttemberg): Mitglied Projektleitung BaWü-Lab GO Karlsruhe.

11 „Nachhaltige Transformation der Textilwirtschaft am Standort Dietenheim“ („BaWüLab Dietenheim zieht an"). 


\section{Christina West}

Dr. phil., Geographie und Deutsche Philologie. Senior Researcher Hochschule Darmstadt, University of Applied Sciences. Co-Leiterin „Zukunftsorientierte Stadtentwicklung“ im forschungsbasierten Innovations- und Transferprojekt ,s:ne | Systeminnovation für nachhaltige Entwicklung", Co-Gründerin und Vorsitzende „Urban Innovation - Stadt neu denken! e. V., Heidelberg“. Forschungsschwerpunkte: Stadt-/Regional-/Urbanitätsforschung; Migration/Flucht/Integration; Kritische Geographie; Transformation/Nachhaltige Entwicklung/Systeminnovation; transdisziplinäre Forschung und Lehre; Partizipation und Governance; Utopie/ Heterotopie/Transtopie; Wertorientierungen; Gesellschaft und Digitalisierung; demographischer Wandel und Wohnungsmärkte. In den Förderlinien „Reallabore“ und „Reallabore Stadt“ (MWK Baden-Württemberg): Wissenschaftliche Koordinatorin und Geschäftsführerin BaWü-Lab Urban Office, Direktoriumsmitglied und Projektleiterin im BaWü-Lab Asylsuchende, TP III „Dezentrales Wohnen“ und „UrbanUtopiaLAB“.

\section{Belen Zevallos}

M.Sc., Architektur. Wissenschaftliche Mitarbeiterin an der SRH Hochschule Heidelberg School of Engineering and Architecture, Ko-Gründerin des interdiziplinären Kollektives Space Transcribers. Forschungsschwerpunkte: Inter- und transdisziplinäre Schulentwicklung; Schule als Lern- und Lebensraum; innovative Lernräume und Lernsettings. In der Förderlinie „Reallabore Stadt“ (MWK Baden-Württemberg): Beteiligt am Arbeitspaket „Lernräume“; Durchführung Interdisziplinärer Feldforschung; Mitarbeit an Kolloquien und Symposien im BaWüLab SRB. 


\section{Externe Reviewerinnen und Reviewer}

Mit einem großen Dank dafür, dass sie mit ihrer Expertise zur Qualität dieses Buches beigetragen haben:

Dr. Stephanie Bock, Deutsches Institut für Urbanistik, Forschungsbereich Stadtentwicklung, Recht und Soziales

Prof. Dr. Antje Bruns, Universität Trier, Governance and Sustainability Lab

Dr. Marion Dreyer, DIALOGIK gemeinnützige Gesellschaft für Kommunikations- und Kooperationsforschung $\mathrm{mbH}$

Prof. Dr.-Ing. Agnes Förster, RWTH Aachen, Lehrstuhl für Planungstheorie und Stadtentwicklung

Prof. Dr. Jasmin Godemann, Universität Gießen, Professur für Kommunikation und Beratung in den Agrar-, Ernährungs- und Umweltwissenschaften

Dr. Niklas Gudowsky, Institut für Technikfolgen-Abschätzung (ITA) der Österreichischen Akademie der Wissenschaften

Prof. Dr.-Ing. Helga Kanning, Leibniz Universität Hannover, Institut für Umweltplanung

Dr.-Ing. Daniela Karow-Kluge, Stadt Aachen und RWTH Aachen, Lehrstuhl für Planungstheorie und Stadtentwicklung

Prof. em. Dr. Ruth Kaufmann-Hayoz, Universität Bern, Allgemeine Ökologie

Dr. Bettina König, Humboldt-Universität zu Berlin, Integrative Research Institute on Transformations of Human-Environment Systems (IRI THESys)

PD Dr. habil. Kerstin Krellenberg, Leibniz-Institut für ökologische Raumentwicklung (IÖR)

Prof. i.R. Dr. Wolfgang Krohn, Universität Bielefeld, Institute for Interdisciplinary Studies of Science $\left(\mathrm{I}^{2} \mathrm{SoS}\right)$ 
Prof. Dr. Benjamin Nölting, Hochschule für Nachhaltige Entwicklung Eberswalde, Fachgebiet Governance regionaler Nachhaltigkeitstransformation

Prof. Dr. Dr. Martina Schäfer, Technische Universität Berlin, Zentrum Technik und Gesellschaft (ZTG)

Dr. Mandy Singer Brodowski, Freie Universität Berlin, Institut Futur

Apl. Prof. Dr. Ulli Vilsmaier, Leuphana Universität Lüneburg, Methodenzentrum und Institut für Ethik und Transdisziplinäre Nachhaltigkeitsforschung (IETSR)

Prof. em. Dr.-Ing. Hille von Seggern, Leibniz Universität Hannover, Institut für Freiraumentwicklung, und Studio Urbane Landschaften Hamburg/Hannover

Prof. em. Dr. Dr. h.c. Johannes Wildt, Technische Universität Dortmund, Hochschuldidaktisches Zentrum (heute Zentrum für HochschulBildung)

Petra Wlasak, PhD, Karl-Franzens-Universität Graz, Regional Centre of Expertise Graz-Styria 